\title{
WHOI-81-102 1981
}

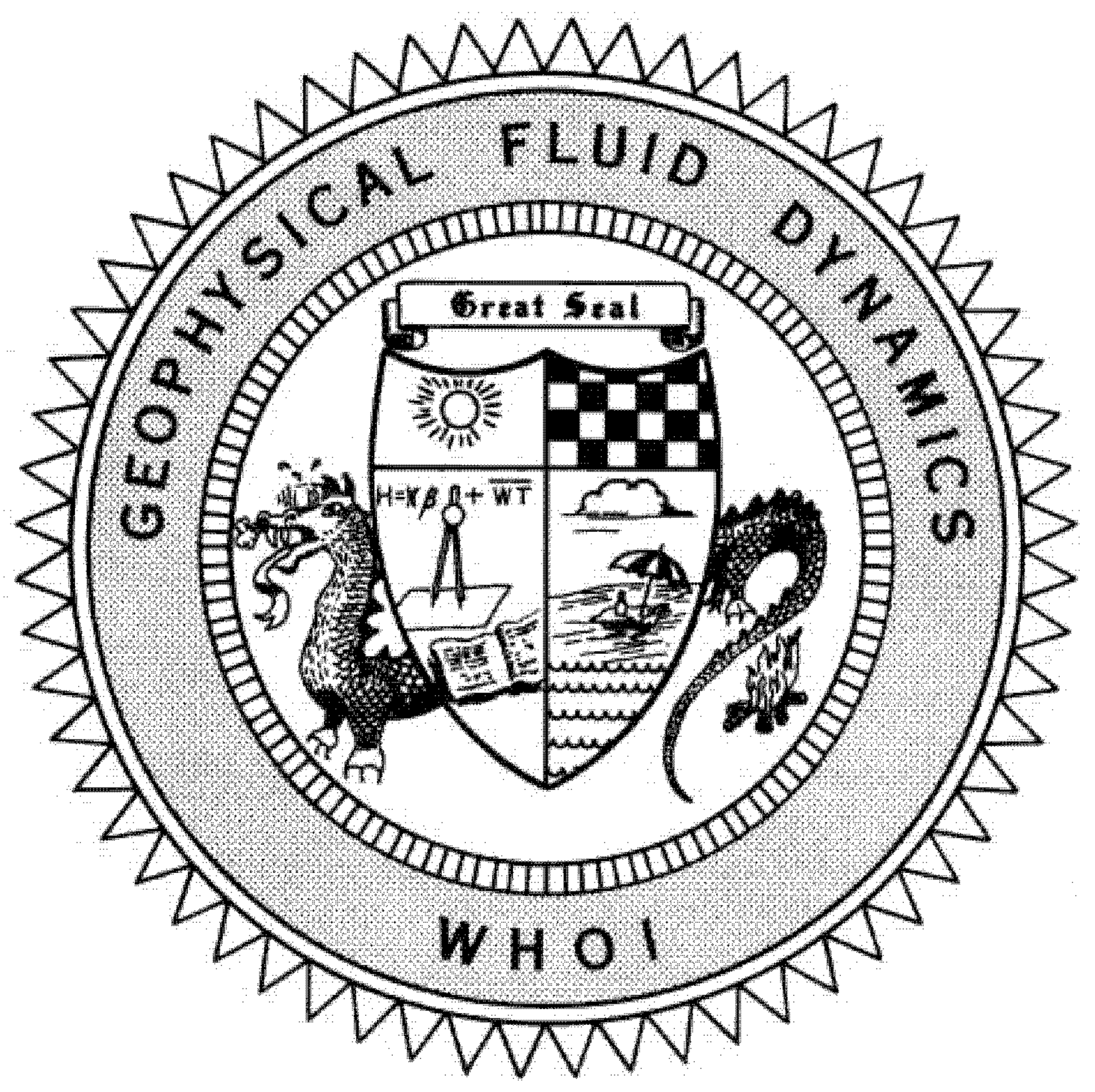

COURSE LECTURES

SEMINARS

ABSTRACTS OF SEMINARS

LECTURES OF THE FELLOWS 
WHOI-81-102

\author{
1981 SUMMER STUDY PROGRAM \\ IN \\ GEOPHYSICAL FLUID DYNAMICS \\ THE WOOSS HOLE OCEANOGRAPHIC INSTITUTION \\ PHYSICS OF CONVECTION
}

by

Willem V. R. Malkus, Director
Florence K. Mellor, Editor

WOOSS HOLE OCEANOGRAPHIC INSTITUTION

Woods Hole, Massachusetts 02543

November 1981

\title{
TECHNICAL REPORT
}

Prepared for the Office of Naval Research mder Contract N00014-81-G-0089.

Reproduction in whole or in part is permitted for any purpose of the United States Government. This report should be cited as: Woods Hole Oceanog. Inst. Tech. Rept. WHOI81-102.

Approved for public release; distribution zonlimited.

Approved for Distribution

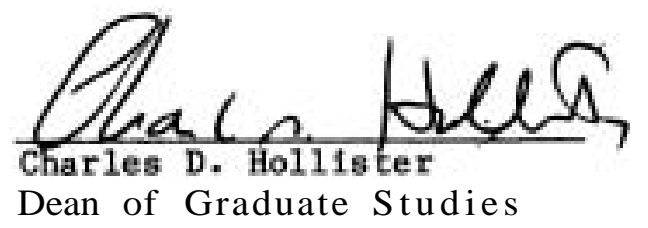


1981 SUMMER STUDY PROGRAM

in

GEOPHYSICAL FLUID DYNAMICS

at

THE WOODS HOLE OCEANOGRAPHIC INSTITUTION

PHYSICS OF CONVECTION 
GEPHYSICAL FUID DYNAMICS RROCRAM

Summer 1981 - STAFF

\section{STAFF AND VISITORS}

Booker, John

Busse, Friedrich A. Cushman-Roisin, Benoit

Foster, Theodore D.

Fowler, Andrew

Gollub, Jerry

Gough, Douglas

Guckenheimer, John

Howard, Louis $\mathrm{N}$.

Knobloch, Edgar

K rishnamurti, Ruby

Landahl, Marten

Libchaber, Albert J.

Loper, David

Lumley, John I.

Malkus, Willem V. R.

Marcus, Philip

Meiss, James

Moatroll, Elliott

Morris, Stephen

Pedlosky, Joseph

Siggia, Eric E.

Spiegel, Edward A.

Stern, Melvin E.

Thompson, Rory

Topham, David R.

Veronis, George

Weiss, Nigel

Welander, Pierre

Whitehead, John A.
Geophysics, Univ. of Washington

Geophysics, U. C. - Los Angeles

Oceanography, Univ. of Washington

Oceanography, U. C. - San Diego

Applied Mathematics, M. I. T.

Haverford College, Pennsylvania

Astronomy, Univ. Cambridge

Nat. Science, U. C. - Santa Cruz

Applied Mathematics, M. I. T.

Physics, U. C. - Berkeley

Oceanography, Florida State Univ.

M. I. T.

Physics, Ecole Normale Superieure

Mathematics, Florida State Univ.

Engineering, Cornel1 Univ.

Applied Mathematics, M. I. T.

Applied Mathematics, M. I. T.

Physics, Univ. Texas - Austin

Inst. of Phys. Science and Tech.

Univ. of Maryland

Engineering, U.C. - Berkeley

G. F. D. - W. H. O. I.

Physics, Corne11 Univ.

Astronomy, Columbia Univ.

Oceanography, Univ. Rhode Is land

CSIRO, Australia

Institute of Ocean Sciences, Canada

Oceanography, Yale University

Mathematics, Univ. Cambridge

Oceanography, Univ. Washington

G. F. D. - W. H. O. I.

\section{FELOWS}

Bretherton, Christopher S.

Cattaneo, Fausto

Coullet, Pierre H.

Fishbein, Evan F.

Honda, Satoru

Long, Bruce

Roberts, Anthony J.
Applied Mathematics, M. I. T. Applied Mathematics, Univ. Cambridge Physics, Ecole Normale Superieure Geophysics, U. C. - Los Angeles Geophysics, Univ. Tokyo Oceanography, Univ. Washington Applied Mathematics, Univ. Cambridge 


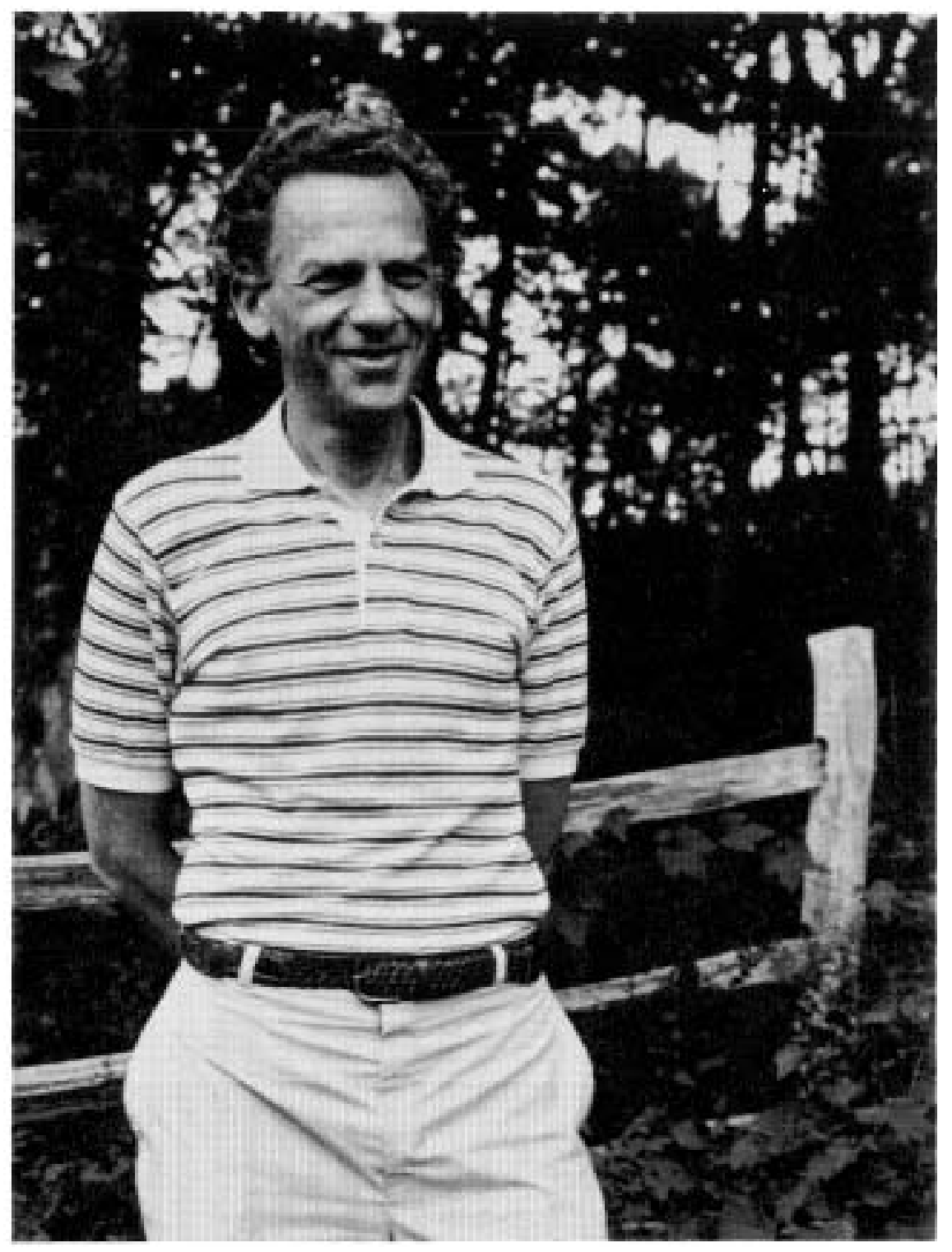

OUR PRINCIPAL LECTURER - EDWARD A. SPIEGEL 


\section{Editor's Preface}

Reexploring convection and its various transitions to chaotic behavior were the central themes of GD 1981. Our principal lecturer, Dr. Edward A. Spiegel, provided both a rich historical picture and stimulating hours at the current frontiers of this topic. Before the summer was out his research lecture on "A Tale of Two Methods" elegantly merged Pierre Coullet's canonical formalism for studying dynamical systems in a central manifold and the more traditional two-timing amplitude expansions near critical points. Other lecture sequences on convection and its relation to simpler dynamical systems ranged from the fine presentations of John Guckenheimer on bifurcation theory to Fritz Busse's survey of his immense contributions to our understanding of nonlinear convection. The list of other lectures found on the following pages attests to our summer-long exposure to convection in the ocean, the atmosphere, the earth's core and mantle, and in the sun. August brought lectures on new observations of convection in the laboratories of physicists. Albert Libchaber's precise experiments on the many routes convection can take to turbulence, with parallel laboratory and numerical experiments described by J. Gollub and E. Siggia, added much to our language of inquiry.

The Fellowship lectures reported at the end of the program represent the first visible creative results of our summer research efforts. These reports are to be treated as unpublished manuscripts. Readers desiring to quote from the material should seek the permission of the author. As in past years, these reports are often reworked for publication or are incorporated into doctoral theses.

We are indebted to Horace Hoffman of the Office of Naval Research and to James Greenberg of the National Science Foundation for finding financial support for the program. This report would not have been assembled without the skillfull guidance of Florence Mellor and her assistant Gail P. Jackson. Special thanks are due Dr. William R. Young and Lynne D. Talley for mathematical editing. We are also grateful to A. L. Peirson for his thoughtful administration of our program. 


\section{TABLE OF CONTENTS}

PHYSICS OF CONVECTION

EDWARD A. SPIEGEL

Page No.

The History and Physics of Buoyancy in $g 1 u i d s, \ldots \ldots \ldots \ldots \ldots \ldots \ldots \ldots$

Small Nondissipative Motions and the Anelastic Approximation, .,.,....5

Convective Equilibrium and the Warm-up Problem....................9

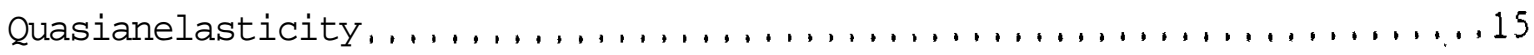

Dissipative Boussinesq Dynamics $\ldots \ldots \ldots \ldots \ldots \ldots \ldots \ldots \ldots \ldots \ldots \ldots$

Convection on Very Large Horizontal Scales.....................28

Mildly Boussinesq Convection with Fixed $s 14 x \ldots \ldots \ldots \ldots \ldots \ldots \ldots \ldots \ldots$

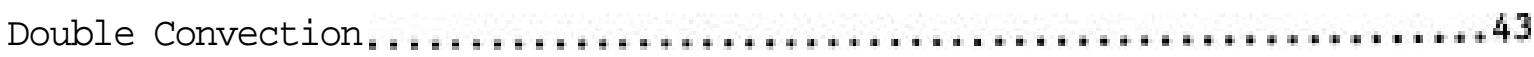

Reconstitution Method for a Simple Example and the Long

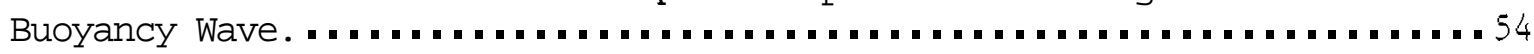

Long Buoyancy Waves and Solar Variability; A Geometrical

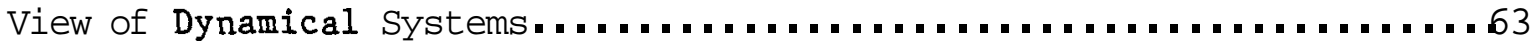


SEMINARS and ABSTRACTS OF SEMINARS

Page No.

Pattern Generation and Transition to Turbulence in Thermal

Convection

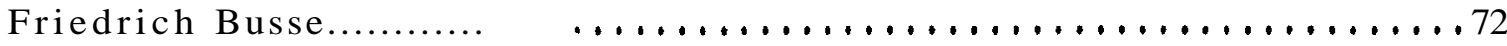

An Introduction to Chaotic Motion and Strange Attractors

John Guckenheimer.. ..............................

Period Doubling and the Transition to Chaos for Simplest Dynamical

System

Pierre Coullet......................................

Bifurcation and Chaos

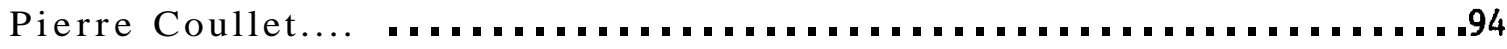

Slowly Varying Waves and Convection

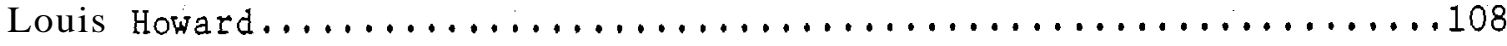

Nonlinear Oscillations in Doubly Diffusive Convection

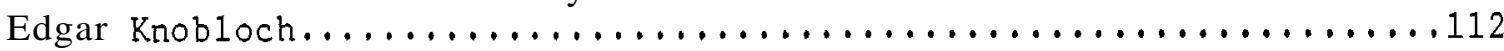

Who is Turbulence?

Melvin E. Stern.....................................

A Numerical Method for Simulating Environmental Fluid Mechanics

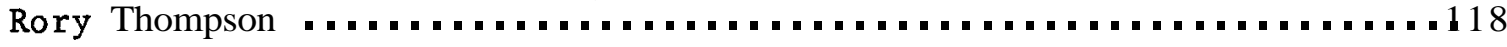

Convection and Magnetic Fields in Stars

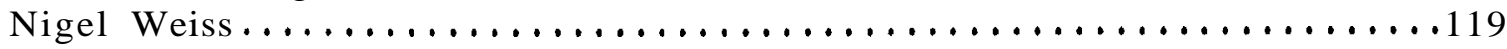

A Measurement of Convection at High Rayleigh Number

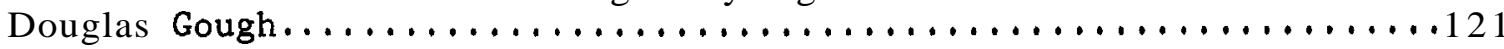

Some Observations of Turbulent Structures in Large Scale

Forced Convection

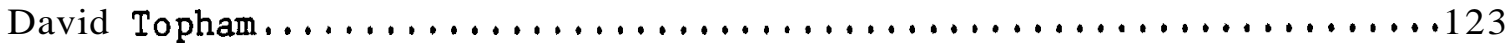

High Rayleigh Number Convection Modelled as a Two Fluid System, with

Application to Convection in Atmospheric Boundary Layer

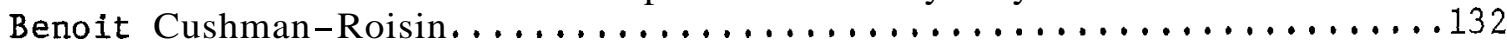

Measurements of Large Scale Flow in a Turbulent Convecting Fluid

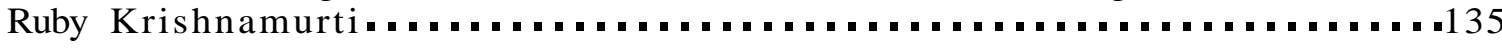

Bifurcations in Double Diffusive Convection

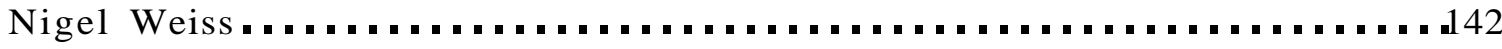

Modal Truncations

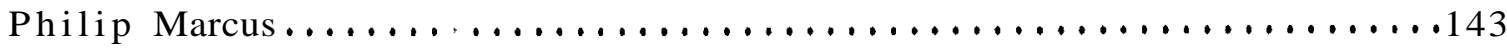

Troublemakers in a Fourier Series Approach to Transitions

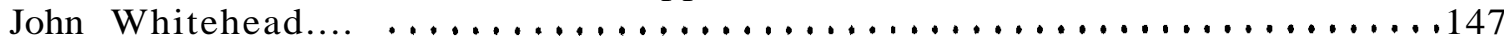


Page No.

A Description of the Lorenz Attractor at High Prandtl Number

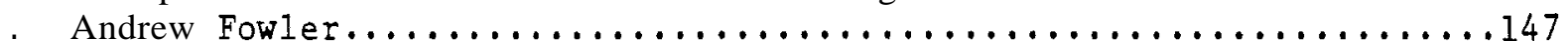

On Thermal Convection in a Fluid with Strongly Temperature-Dependent Viscosity

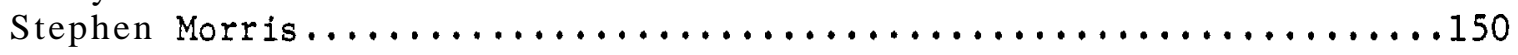

A Discussion of Compositional Convection

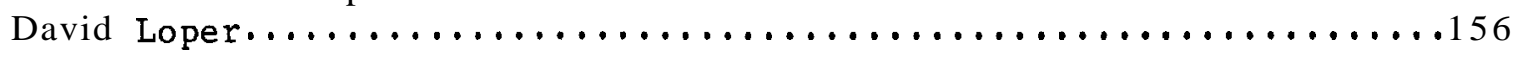

Chaotic Behavior of Mildly Unstable Baroclinic Waves

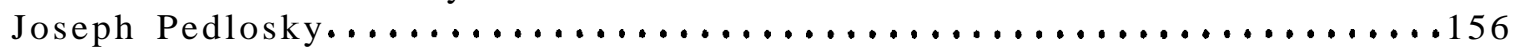

Stability Bounds on the Amplitude and Structure of Turbulent

Transport Processes

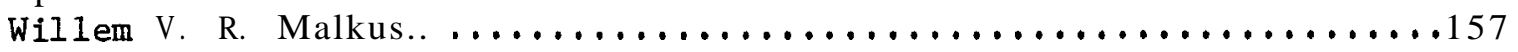

Inverse Methods for Oceanography

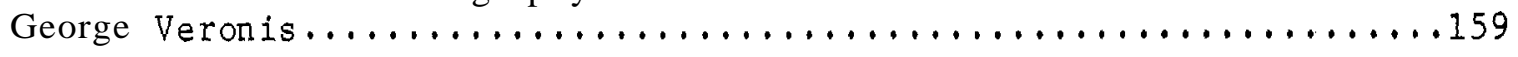

Slow Box-Model Oscillators

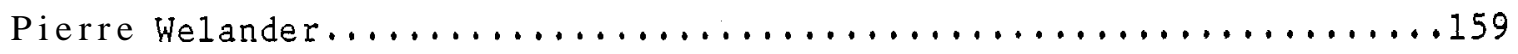

Large-Amplitude Convection in Porous Media

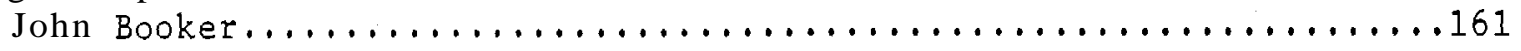

Pattern Selection in Rayleigh-Benard Convection Near Threshold

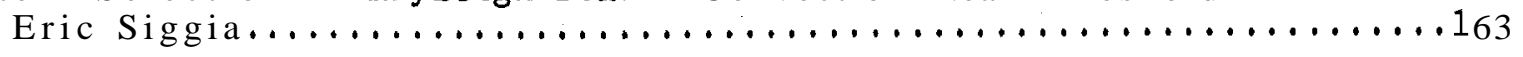

Weak Turbulence in Small Aspect Ratio Cells: The Case of Liquid

Helium

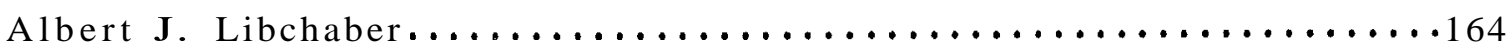

Convection at Very High Rayleigh Numbers

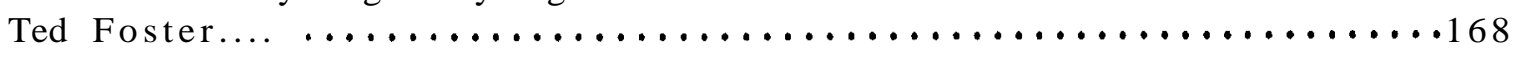

Turbulent Convection

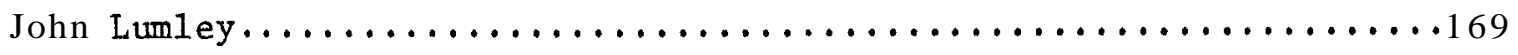

Examples of Reverse Cascades in Turbulent Phenomena

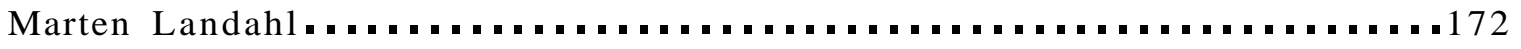

Studies of the Transition to Turbulent Convection Using Scattered

Laser Light

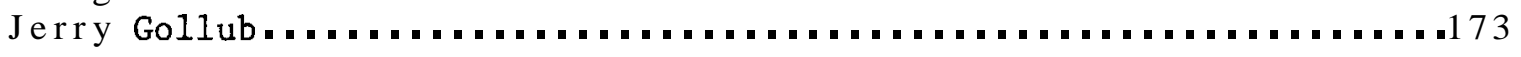

Statistical Properties of Mappings by the Characteristic Function

Formalism

James Meiss........................................ 
Fixed Flux Penetrative Convection

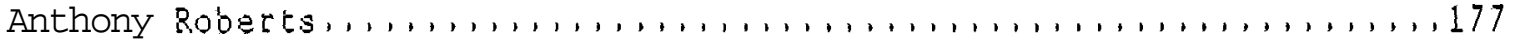

An Instability of Gravity Waves

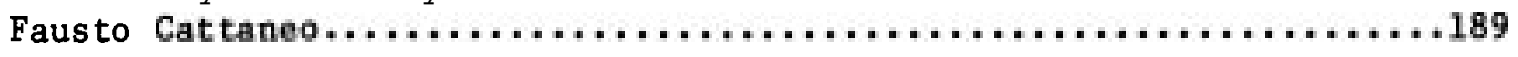

Double Diffusion in a Long Box

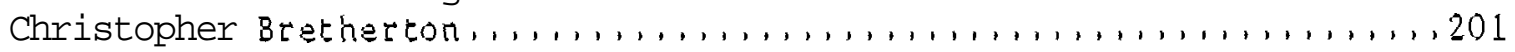

On Horizontal Advection in the Rayleigh-Benard Convection

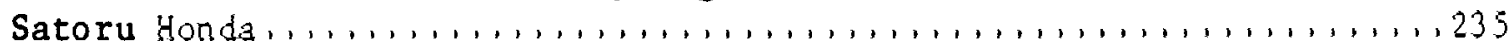

Spatially Varying Plan-Forms: Perpendicular Systems of Rolls

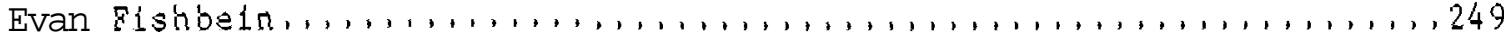

The Spin-Up Problem with a Radial Channel on the Bottom

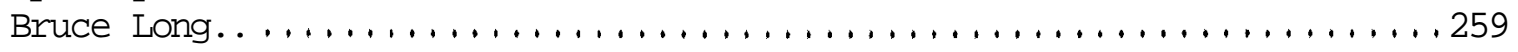

A Tale of Two Methods

Pierre Coullet and Edward Spiegel .......................276 


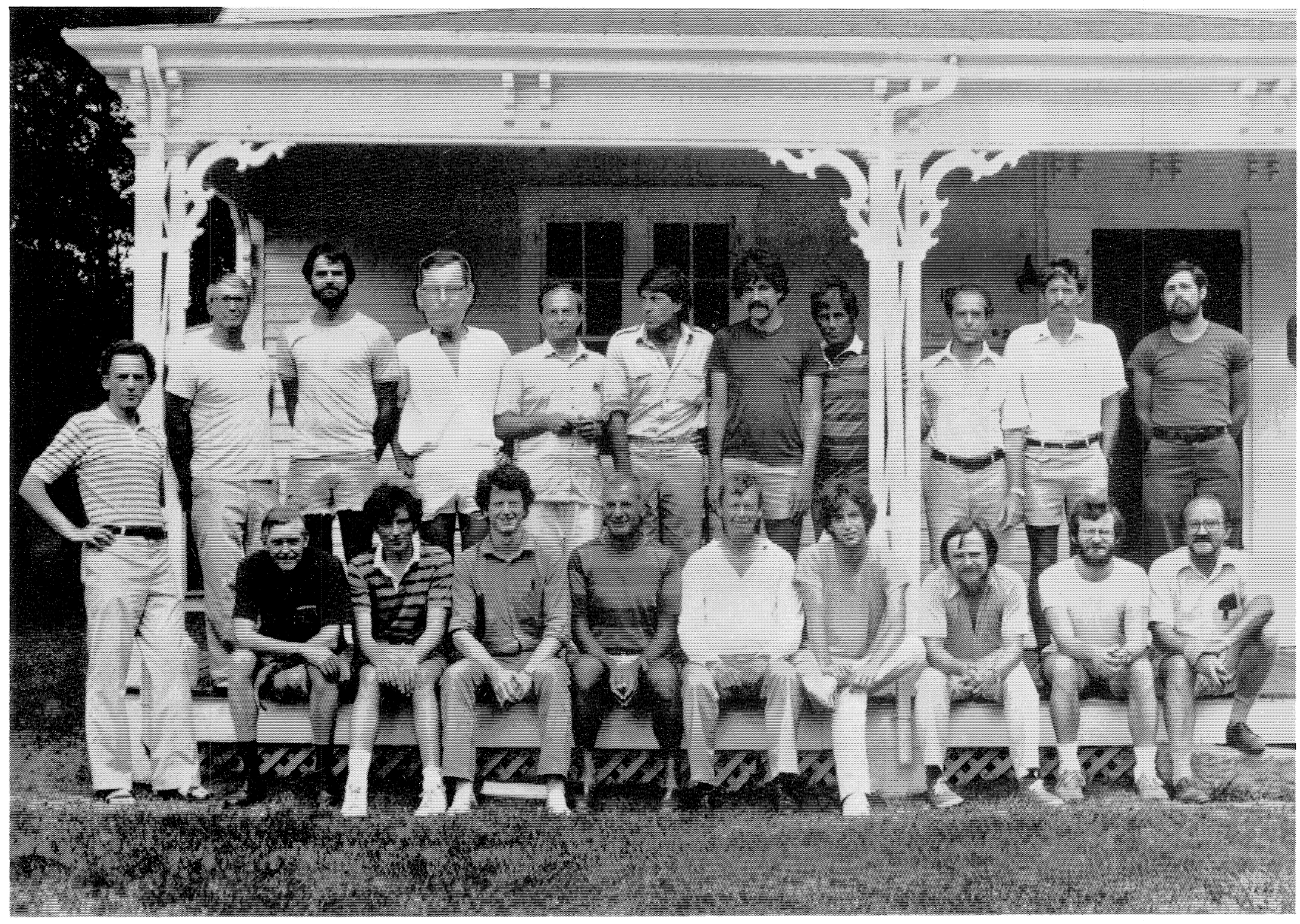

Row 1:

Spiege1, Howard, Bretherton, Roberts, Veronis, Booker, Cattaneo, Knobloch, Fowler, Stern.

Back Row:

Malkus, Gregory-Allen, Busse, Libchaber, Coullet, Long, Greenberg, Guckenheimer, Siggia, Fishbein.

Absentees:

Cushman-Roisin, Foster, Gollub, Honda, Krishnamurti, Landah1, Loper, Lumley, Marcus, Meiss, Morris, Pedlosky, Rhines, Thompson, Topham, Weiss, Welander, Whitehead. 


\section{THE HISTORY AND PHYSICS OF BUOYANCY I N FLUIDS}

Edward A. Spiegel

The study of convection is a venerable field. Before discussing any detailed work, let us embark on an historical sketch.

\subsection{Historical Sketch}

c.250 BC Archimedes discovers the principle sf specific gravity and thus quantifies the idea of buoyancy,

1657

Rinaldi demonstrates convection in experiments designed to disprove Aristotles's ideas on the flow of heat (Middleton, 1908).

Ben Franklin gives a geophysical application - the motion of air i n a thunderstorm (Middleton, 1968).

1798 Rumford (in trying to discover why soup cools faster than apple pie)does experiments with convection in which a trace (yellow resin) is introduced for the first time (Brown, 1979).

1834

Pruitt coined the word "convection" (Brown, 1979).

1840

1861

Rayleigh, Espy and others looked at the instability of an unstably stratified fluid and derived the "Schwartzchild" interior.

Lord Kelvin introduced convective equilibrium (Lamb).

1900

Jevons discussed double diffusive convection.

Benard observed hexagonal convection cells upon heating a thin layer of fluid above the critical Rayleigh number. Unfortunately, he probably did not realize surface tension had a very important influence on his results.

1903 Boussinesq's approximate equations for convection in a thin, almost adiabatic layer were published (1903, in his Theorie Analytique de la Chaleus").

1916 Lord Rayleigh discussed marginal stability of Boussinesq convection and introduced the bey stability parameter.

1926 Jeffreys (See Saltzman, 1962) looked at the case of insulating top and bottom and found that the most unstable modes were horizontally infinite in extent.

Up to 1940 Better B.C.'s and better calculations of the critical Rayleigh number and wave length at convective instability were done culminating in a paper of Pellev and Southwell (1940) (See Saltzman, 1962). Their results are summarized in Figure 1. 
These were of two kinds. Rotation could couple modes with vertical vorticity to horizontal motion and produce overstability (later done by nonlinear terms with no rotation by Busse, Busse and Clever), Computing instabilities like magnetic and thermal effects could produce overstability in a generic way. This opened the quantitative study of computing instabilitie s continued by Townsend (JFM, 1959), Stern (1961), and onward.

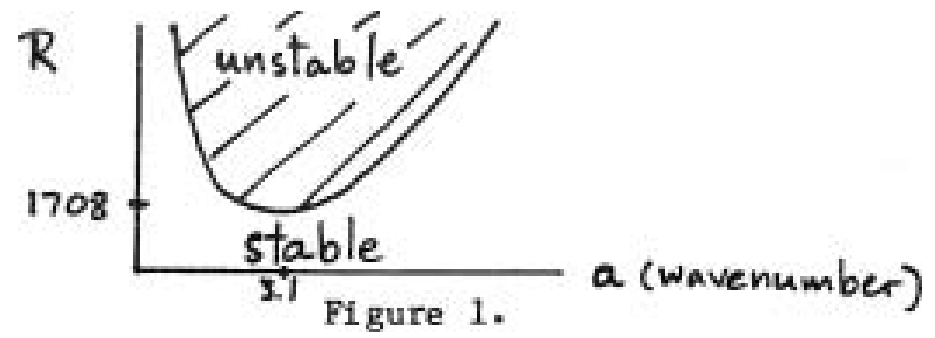

Malkus and Veronis found for R slightly greater than $R_{C}$, that steady small amplitude convection motions took place and found their form by perturbation theory.

1965

Busse took this analysis to higher order to show that the only stable steady convective solutions were two-dimensional rolls. Above a second critical Rayleigh number $R_{b}$ these become unstable. Unlike the initial instability this bifurcation depends on the Prandtl number $=\frac{\nu}{k_{*}}$. For $\sigma>\sigma_{\epsilon}$, the steady solution bifurcates into two steady solutions. For $<\sigma_{c}$, it bifurcates into oscillatory modes. The picture is as in Figure 2, in which we are looking at the amplitude $\|\underline{w}\|=\mathbf{A}$ of the motion vs R.

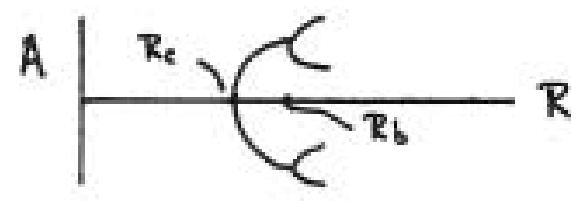

Figure 2.

\subsection{A Motivation in Astrophysics}

For the most part, the study of convection has been an outcast of GFD, since most geophysical processes involve high Rayleigh number, turbulent convection, which has generally been modelled as a small-length scale mixing process whose effects on larger scales has been treated as an eddy viscosity. However, we will try to keep in mind a real problem which clearly involves convection and several interacting length scales, namely the transport of heat from the solar interior.

Photographs of the apparent surface of the sun in visible light reveal "granulation" in the form of cellular patterns of about $1000 \mathrm{~km}$ diameter. These cells clump into globs of about $5000 \mathrm{~km}$, and the surface velocity shows "super-granularity" at a $30,000 \mathrm{~km} \mathrm{scale.} \mathrm{Superimposed} \mathrm{on} \mathrm{these} \mathrm{convective}$ patterns are sunspots, magnetic flux tubes usually in pairs of positive and negative polarity. In the sunspots, the strong magnetic field inhibits convection and so they are cooler at the surface, and therefore a prominent feature of pictures of the sun. 
Sunspots obey an 11-year cycle of abundance; between cycles the maximum number of sunspots varies dramatically* At some times, such as the Maunder Minimum of $1550-1600$, rediscussed by Jack Eddy, almost no sunspots were seen. The goal of this course will be to try to provide a model which possesses similar behavior.

We now go back and try to capture some of the features of buoyancy forces i n fluids by looking a t a simple model. It will be the scope of future lectures to show how the set of equations describing such a model is relevant to the general problem of convection.

We adopt a Lagrangian viewpoint and focus our attention on the motion of an idealized fluid particle through a surrounding fluid. The fluid particle has mass $m$, volume $V$, density $\rho$ and is uniquely identified by its position $z$. The forces on such particles are due to gravity, buoyancy and drag and can be written in the equation of motion as:

$$
m \ddot{z}=-g\left(\rho-\rho_{0}\right) \vee-\mu \dot{z}
$$

We have chosen $g$ to act i $n$ the negative $z$ direction. We can write $m=\rho V$ and if the fluid is almost incompressible we can approximate $m$ by writing $m \simeq \rho_{\mathrm{e}} V$. Dividing through we get:

$$
\ddot{z}=-g\left(\frac{\rho-\rho_{0}}{\rho_{0}}\right)-\nu \dot{z}
$$

Where, in the spirit of the Boussinesq approximation, we choose $\boldsymbol{\nu}$ to be a constant. This is the first of our equations. If the fluid is completely incompressible $\rho, \rho_{o}=$ const and (1) has solution:

$$
z(t)=k e^{-\nu t}-\frac{g}{\nu}\left(\frac{\rho-\rho_{0}}{\rho_{0}}\right) t
$$

After the transient decays (2) describes the motion of a particle moving at constant velocity. The effective buoyancy force is equal to the viscous drag and the direction of motion depends on the sign of $\left(p-\rho_{\circ}\right)$.

More generally we expect the density of the fluid particle to depend on the thermodynamic state and to obey some equation of state like $p=\rho(p, T)$. If we assume that the temperature and pressure of the particle deviate only slightly from the ambient temperature and pressure we can approximate the equation of state by:

$$
\rho=\rho_{0}\left[1+\kappa_{T}\left(P-P_{0}\right)-\alpha\left(T-T_{0}\right)\right]
$$

Where we have defined the isothermal compressibility and the thermal expansivity $\alpha=-\frac{1}{\rho}\left(\frac{\partial \rho}{\partial T}\right)=$

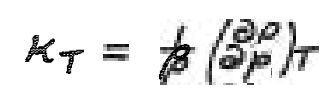

Again, for a Boussinesq fluid the variations induced by pressure differences can be shown to be negligible and the equation of state reduces to

$$
\rho=\rho_{0}\left[1-\alpha\left(T-T_{0}\right)\right]
$$

We now consider the heat exchanged between the fluid particle and its surroundings; assuming this obeys Newton's Law of cooling we write

$$
\dot{T}=-\sigma\left(T-T_{0}\right)+\partial_{t} T_{0}
$$

for some constant $q$ The background temperature $T_{0}$ depends on $z$, and 
possibly t. If we introduce a temperature excess $\boldsymbol{\theta}=\left(\mathrm{T}-\mathrm{T}_{\mathrm{O}}\right)$ and $\boldsymbol{\beta}=-\partial T_{0} / \partial z$ the last equation becomes

$$
\dot{\theta}=\beta \dot{z}-g \theta
$$

This is the second of our equations. Equations (1) and (3) cannot, as yet, be solved as we made no assumptions regarding the evolution of $\mathrm{s} A$ possibility is to assume $T_{0}$ to be linearin $z$ and $\boldsymbol{\beta}=\beta_{\theta}=$ const and then the equations are linear. For such a set of equations a solution of the form $z \propto e^{\natural}$ exist provided $\eta$ satisfied:

$$
\eta\left[(\eta+8)(\eta+\nu)-g \alpha \beta_{0}\right]=0
$$

We notice that the translational invariance of the system shows up with 7 as one of the factors.

If $\nu=0$ we have no viscous dissipation, and for $q^{2} \gg g \alpha \beta_{0}$ (i.e., the rate of exchange of heat is much larger than the rate at which buoyancy does work) then the solution has

$$
\eta \simeq \frac{g \alpha \beta_{0}}{q}
$$

which shows that heat conduction slows down but does not prevent the runaway of the fluid particle.

If $\nu \neq 0$ there exists a marginal mode provided

$$
\pi=\frac{g \times \beta}{g^{\nu}}=1
$$

and solutions become unstable for $\pi>1$. If $\beta$ is not constant we can get our third and last equation by, for example, writing the evolution of $\beta$ as
an expansion in powers of the heat transport. To first order:

$$
\dot{\beta}=c\left[\dot{z} \theta-\kappa\left(\beta-\beta_{0}\right)\right]
$$

for some constant $C$ and $K$. The term $z$ in (5) is the advection of heat and the term $K \beta$ is the conduction.

Equations (1), (3) and (5) for variables z $\quad \theta$ are known as the Lorenz equations. Their analysis is an interesting topic in itself, which will be dealt with by other lecturers.

\section{REFERENCES}

Brown, S. C., 1979. Benjamin Thomson, Count Rumford. MIT Press.

Middleton, W. E. K., 1968. Physics, 10, 299.

Saltzman, B., 1962. Selected Papers on the Theory of Thermal Convection. Dover Pub1. Co.

Chandrasekhar, 1961. Hydrodynamie and Hydromagnetic Institute, Oxford. 
Edward A. Spiegel

\section{$\underline{\text { Equations }}$}

We first recall a few technical points in the study of fluid (and other) dynamics. The fluid is often pictured as a continuum of particles obeying Newton's Laws. A fluid particle or element has an orbit in space that is paraneterized by time. The distinction among orbits is made by other (Lagrangian) parameters. Thus $x(t)$, a particular orbit, contains in its description parameters (not written explicitly here) which distinguish it from the others. By $\mathbb{z}$ we mean we $\partial x / \partial t$ evaluated on an orbit, and to emphasize this we write

$$
\dot{x}=\frac{D x}{D t}
$$

For any function defined on an orbit

$$
\left.\partial_{t} f\right|_{x \text { fixed }}+\dot{x} \quad \partial_{x} f I_{t} \text { fixed }=\frac{D f}{D t} \text {. }
$$

Finally, if we can express the parameters which characterize the orbit in terms of $x$ and $t$, we can write

$$
x(t, \text { parameters })=(x, t)
$$

So

$$
\begin{aligned}
& \frac{D x}{D t}=\underset{\sim}{u}(x, t) . \\
& \frac{D f}{D t}=f_{t}+(\underset{w}{u} \cdot z) f,
\end{aligned}
$$

we shall also write $\underset{\boldsymbol{A}}{\boldsymbol{A}}=\partial_{\mathrm{t}} \mathrm{f}=\mathrm{f}_{\mathrm{t}}$ 。

We now introduce the three conservation equations that are the basis of this course:

$$
\begin{aligned}
& p_{t}+\underset{\sim}{\nabla}\left(\rho_{\sim}\right)=0
\end{aligned}
$$

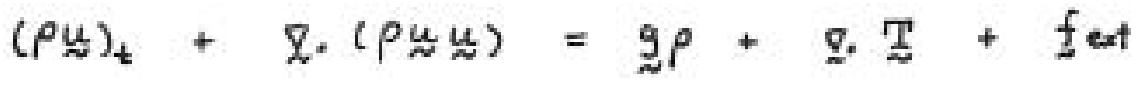

$$
\begin{aligned}
& (\rho S)_{t}+\nabla \cdot(\rho u s)=\dot{Q} / T \rho
\end{aligned}
$$

where $\rho$ is density, ${ }^{2}$ is velocity, $\mathbf{T}$ is the fluid stress tensor, $f_{e}$ is any externally applied force field in addition to gravity (whose acceleration is $g$ ), $S$ is the specific entropy and Q / TP is a vague notation for thermal dissipative processes. I use Malkus-Veronis notation, which is based on the idea that a reader can distinguish among $T, T, T$ and the like.

The simple model used in what follows is

$$
T_{i j}=-p \delta_{i j}+\mu\left(\frac{\partial u_{i}}{\partial x_{j}}+\frac{\partial u_{j}}{\partial x_{i}}\right)-\frac{2}{3} \mu(\underset{v}{\sim} \underset{\sim}{u})
$$

and we shall stay always i n Cartesian coordinates. The thermodynamic equation will be written using $\mathrm{dH}=\mathrm{Tds}+$ specific enthalpy. Also we write

$\frac{d p}{p}$ where $\mathrm{T}$ is temperature and $\mathrm{H}$ is

$$
d H=C_{p} d T
$$


and get (with some further conventional assumptions)

$$
\rho C_{P} \frac{D T}{D t}-\frac{D P}{D t}=\dot{Q}=\nabla \cdot \underset{\sim}{F}+\Phi
$$

where $F$ be heat flux and $\Phi$ be viscous dissipation into heat. We note that

$$
\Phi=\frac{\partial u_{i}}{\partial x_{j}}\left(T_{i j}+p \delta_{i j}\right)
$$

We take $E=-K \mathbb{Z} T$. The equations of state that arise in convection problems are varied but, in this course, we shall not deal with Messrs. Saha and Gruneisen. We consider the simplest gas as an example:

$$
\mathrm{p}=\mathrm{R} P \mathrm{~T}
$$

with R constant. The entropy for a perfect gas is

$$
\mathrm{S}=\mathrm{C}_{\mathrm{v}} \ln \left[\mathrm{P} / \mathrm{\rho}^{1 / T}\right]
$$

where $\gamma$ is the ratio of specific heats. For small perturbations, $(\mathrm{p}, \quad, \mathrm{P})$ about the static state $\left(\rho_{0}, U_{\sim}, P_{0}\right)$,

$$
\begin{aligned}
& \rho_{t}+\nabla \cdot\left(\rho_{0}, u\right)=0 \\
& \left(\rho_{0} \underline{u}\right)_{t}=-\nabla p+\rho g \\
& P_{t}+v \cdot \nabla P_{0}=c^{2}\left(\rho_{t}+\psi v \cdot \nabla \rho\right)
\end{aligned}
$$

where $c^{2}=\gamma P_{*} / \rho_{o}$

These equations are combined to obtain a wave equation

$$
u_{t t}=\underset{\sim}{\nabla}\left(c^{2} \nabla \cdot u+\underset{\sim}{u} \underset{\sim}{g}\right)+c^{2} \underset{\sim}{\partial}\left\{\ln \frac{p_{0}}{p_{0} / T}\right\} \nabla \cdot \underset{\sim}{u}
$$

For an isothermal atmosphere

$$
p_{0} \propto p_{0} \propto e^{-z / H}
$$

where $\mathrm{H}=\mathrm{RT}_{\mathrm{o}} / \mathrm{g}$ is the scale height.

Note that

and

$$
c^{2}=\gamma R T_{0}
$$

$$
\underset{\sim}{\nabla} \frac{P_{0}}{\rho_{0}^{1 / \sigma}}=\left(\frac{1-\gamma}{r H}\right) \hat{z}
$$

are both independent of $(x, y, z)$. We seek wave solutions,

$$
\underset{\sim}{u}=\underset{\sim}{u}(z) e^{i(\ell x+m \jmath+n z-\omega t)}
$$


and find the approximate dispersion relations

$$
\begin{aligned}
& \omega_{0}^{2}=c^{2}\left(k^{2}+n^{2}+\frac{1}{4 H^{2}}\right) \\
& \sigma_{g}^{2}=\left(\frac{\gamma-1}{\gamma H}\right)\left(\frac{g k^{2}}{k^{2}+m^{2}+\frac{1}{4 H^{2}}}\right) \\
& k^{2}=l^{2}+m^{2}
\end{aligned}
$$

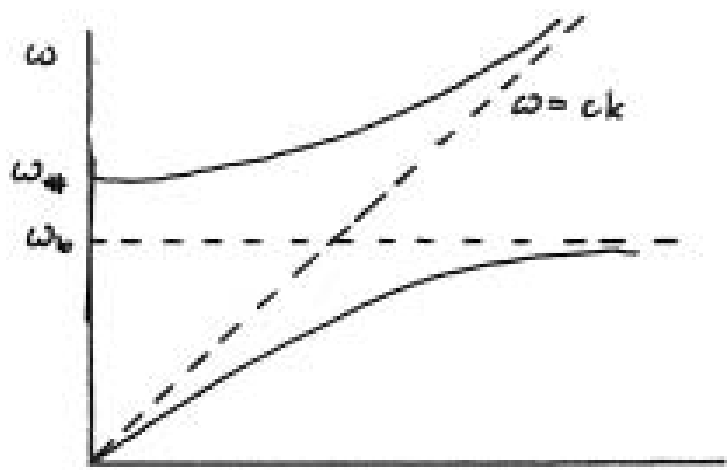

where

$$
\begin{aligned}
& \omega_{*}=\frac{\gamma g}{4 H}=\frac{c^{2}}{4 H^{2}} \\
& \omega_{b}=\frac{(\gamma-1) g}{\gamma H}
\end{aligned}
$$

Convective instabilities are associated with gravity modes where $\omega_{g}^{2}<\mathbf{I}$ Choosing a long time scale, relative to $\omega_{*}$ is one way to separate out gravity waves. This is roughly equivalent to letting

$$
\gamma \rightarrow \infty, \omega_{\#} \Rightarrow \infty, \omega_{b} \rightarrow \frac{g}{H}
$$

For the polytropic atmosphere

$$
\frac{d T}{d z}=-\frac{T_{0}}{z_{0}}
$$

the dispersion curves look something like

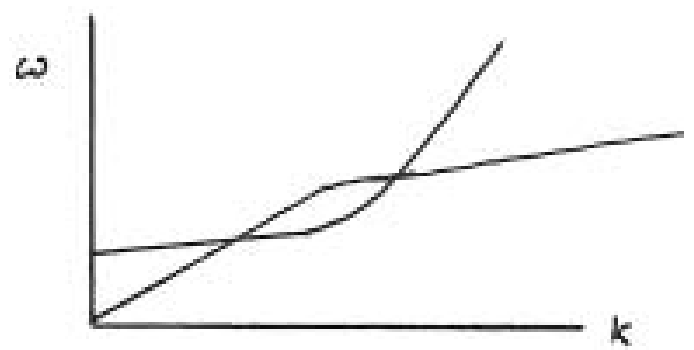

The time scales for the two modes in general do not separate, hence it is not easy to filter out one kind of motion and study the other. If the temperature profile is

$$
T(z)=T_{*}\left(1-\frac{z}{z_{*}}\right)
$$


Scale heights can be defined

$$
H(z)=H_{*}\left(1-\frac{z}{z_{*}}\right)
$$

The static state is

$$
\begin{aligned}
& p(z)=P_{*}\left(1-\frac{z}{z_{*}}\right)^{m-1} \\
& \rho(z)=P_{*}\left(1-\frac{z}{z_{r}}\right)^{m}
\end{aligned}
$$

Where the polytropic index

$$
\begin{gathered}
m=1+\frac{z_{*}}{H_{v}} \\
\text { In the limit } z / z_{*} \Rightarrow 0(m \rightarrow \infty) \\
p(z) \propto \rho(z) \propto e^{-z / H_{*}}
\end{gathered}
$$

Hence is the $1 \mathrm{imit}$ of large $z *$, the atmosphere can be treated locally as isothermal. The atmosphere characterized by

$$
p \propto e^{\Gamma}
$$

with entropy profile given by

$$
\frac{d S}{d z}=(\Gamma-r) \frac{d \ln p}{d z}
$$

where $\Gamma=$ is the polytropic constant. The atmosphere is said to be in convective equilibrium where $\Gamma=\gamma, i \circ e_{0}, \underline{m}=\frac{1}{\gamma-1}$.

Wave solutions $y(z) e^{i(\omega t-2 x-m y)}$ admit gravity modes (see Lamb, 1931; Spiegel and Unno, 1962) with the dispersion relation

$$
\frac{2 \mu \omega^{2}}{(m+2) g} M(\mu+1 ; m+2 ; 2 k)+\left[\frac{(m+1) \omega^{2}}{g k}-\frac{\left(\omega^{2}-2 g\right)}{g k}\right] M(\mu ; m+2 ; 2 k)=0
$$

where

$$
2 \mu=\frac{m+1}{\nu}\left(\frac{g k}{\omega^{2}}-\frac{\omega^{2}}{g k}\right)-\frac{2 g m}{\omega^{2}}+m+2
$$

and $M(a ; b ; c)$ is the confluent hypergeometric function (see Magnus and oberhettinger, 1954). The relation is obtained by assuming rigid boundaries at $z=$ 01 . This law has the property

$$
\operatorname{sign}\left(\omega^{2}\right)=\operatorname{sign}\left(\boldsymbol{T}^{\mathbf{T}}-1\right)
$$

from which it follows $\omega^{2}=0$ for Hence if the atmosphere is in approximate convective equilibrium, the nongravity modes are suppressed by setting

$$
P_{t}=P_{t}=0
$$

This is the idea behind the anelastic approximation of Charney (see Ogura $S$. Phillips, 1962):

$$
\text { V. }(\rho, \underline{y})=0
$$


In order to understand how the gravity modes become unstable consider the energetics of a displaced fluid element

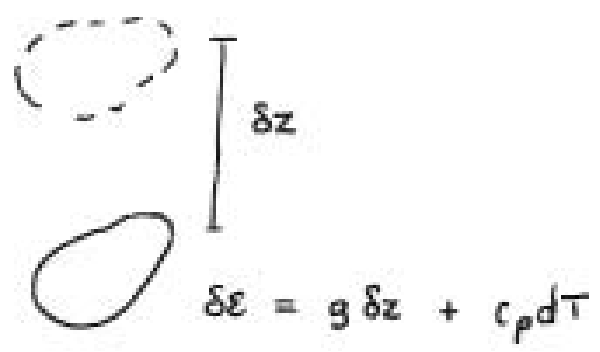

The Schwartzchild criterion for instability is $\delta \varepsilon<0$ or

$$
\frac{d T}{d z}+\frac{g}{c_{p}}<0
$$

from which using $P=R \rho T$ we obtain

$$
\frac{d S}{d z}<0
$$

a s a necessary condition for instability. Hence, if the atmosphere is marginally unstable, $d S / d z=0, i . e, \Gamma=\gamma$, and the anelastic approximation can be invoked to filter out the acoustic modes while retaining the convective instability.

\section{REFERENCES}

Cough, D. 0., 1969. The anelastic approximation for thermal convection. IAS, $26,448-456$.

Lamb, H., 1945. Hydrodynamics. 6th ed., Dover Publishers, N. Y.

Magnus, W, and F. Oberhettinger, 1954. Formulas and theorems for the functions of mathematical physics. Chelsea Publ. Co., N. Y.

Ogura, Y. and Phillips, N. A., 1962. Scale analysis of deep and shallow convection in the atnosphere. IAS, 19, 173-179.

Spiegel, E. A. and W. Unno., 1962. On convective growth-rates i n a polytropic atmosphere. Astr. Soc. of Japan, 14, 1, 28-32.

NOTES SUBMITTED BY

EVAN FISHBEIN and

PIERRE COULLET

\section{CONVECTIVE EQUILIBRIUM AND THE WARM-UP PROBLEM}

Edward A. Spiegel

Convective Equilibrium

Consider a hydrostatic atmosphere of layer thickness $z_{0}$ with a linear vertical temperature profile of gradient $\beta=T_{*} / z_{Q^{*}}$ The vertical 
coordinate $z$ is defined positive downwards, with $z=0$ at the top of the layer (Fig*1).

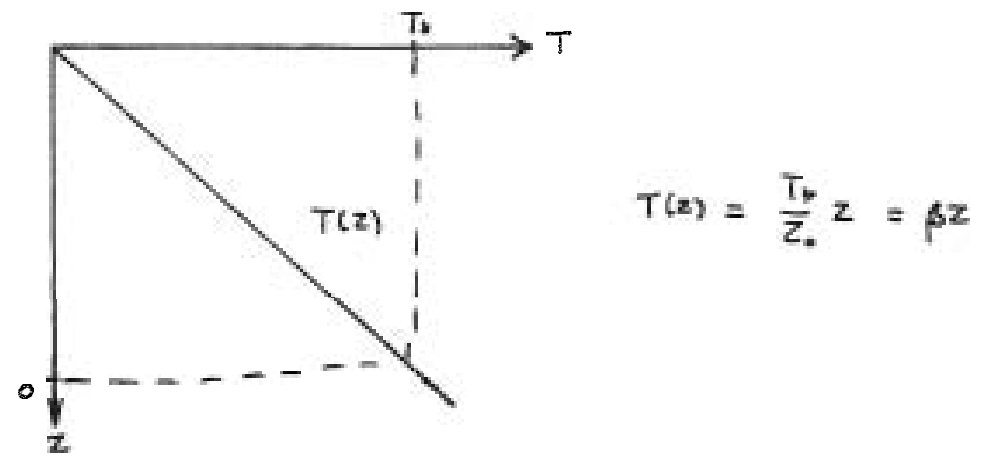

FIGURE 1.

In the static state, the pressure, density, and temperature satisfy

$$
\begin{array}{ll}
\frac{\partial p}{\partial z}=g \rho & \text { hydrostatic equation } \\
P=R \rho T & \text { equation of state for ideal gas } \\
T=\beta z & \text { linear temperature profile. }
\end{array}
$$

where $\mathbb{R}$ and g are constant.

We can solve for the pressure and density of this polytropic atmosphere as a function of $z$

$$
\begin{aligned}
& p=\pi\left(\frac{z}{z_{0}}\right)^{m+1} \\
& \rho=\frac{\pi}{R T_{0}}\left(\frac{z}{z_{0}}\right)^{m}
\end{aligned}
$$

where $\pi$ is the pressure at $z=z_{0}$ and $m$, the polytropic index, is given by

$$
m=\frac{g}{R \beta}-1=\frac{z_{0}}{H \rightarrow}-1
$$

$\mathrm{H}_{*}$ being the pressure scale height $\left(\mathrm{H}_{*}=\frac{R T_{*}}{g}\right)$. In terms of the polytropic exponent, defined as

$$
\Gamma=\frac{m+1}{m}
$$

$\mathrm{p}$ and

$$
\begin{aligned}
& \rho \text { are related by an equation of the form } \\
& \mathrm{p}=\text { const. } \rho \text {. }
\end{aligned}
$$

In a state of convective equilibrium, the temperature gradient is equal to the so-called adiabatic lapse rate

$$
\beta=\beta \text { adiabatic }=g / c_{p}
$$


where $c_{p}$ is the specific heat at constant pressure. In this case

$$
m=\frac{6}{y-1}
$$

and

$$
r=\gamma
$$

where $\gamma$ is the ratio of specific heats at constant pressure and volume $\gamma=$ $c_{p} / c_{y}$ ): Using the Schwartzchild discriminant, we see that for convective equilibrium

$$
\frac{1}{T}\left(\frac{d T}{d z}-\frac{g}{c_{p}}\right)=\frac{1}{c_{p}} \frac{d s}{d z}\left(\equiv \frac{d \ln \theta}{d z}\right)=0
$$

i.e., the entropy $S$ and the potential temperature $\theta$ are constant with height.

The sign of $d S / d z$ gives us information about the stability of a layer. We expect convective instability if $d s / d z>0$. Foranidealgaswith constant specific heats

Thus

$$
\begin{aligned}
S & =c_{v} \ln (p / \rho \gamma) & & + \text { const } \\
& =c_{v}\left(\frac{\Gamma-\gamma}{\Gamma}\right) \ln p & & + \text { const } .
\end{aligned}
$$

$$
\frac{d s}{d z}=c_{v}\left(\frac{r-\gamma}{\Gamma}\right) \frac{d \ln p}{d z}
$$

and for instability $\Gamma$ must be greater than $\boldsymbol{r}$, since $d \mathbf{l n p} / \mathrm{dz}>0$, Just as the conductive heat flux can be written in terms of a thermal conductivity as

$$
\mathcal{F} \text { conductive }=-\mathrm{K} \nabla \mathrm{T}
$$

we may conjecture in this case that

$$
\mathcal{F}_{\text {convective }}=-\kappa_{+} \nabla \theta \propto-\kappa_{+} \nabla S
$$

where $k_{+}$is a sort of eddy conductivity.

As an example, consider the sun, where the profile of $s$ with distance from the center looks something Pike:

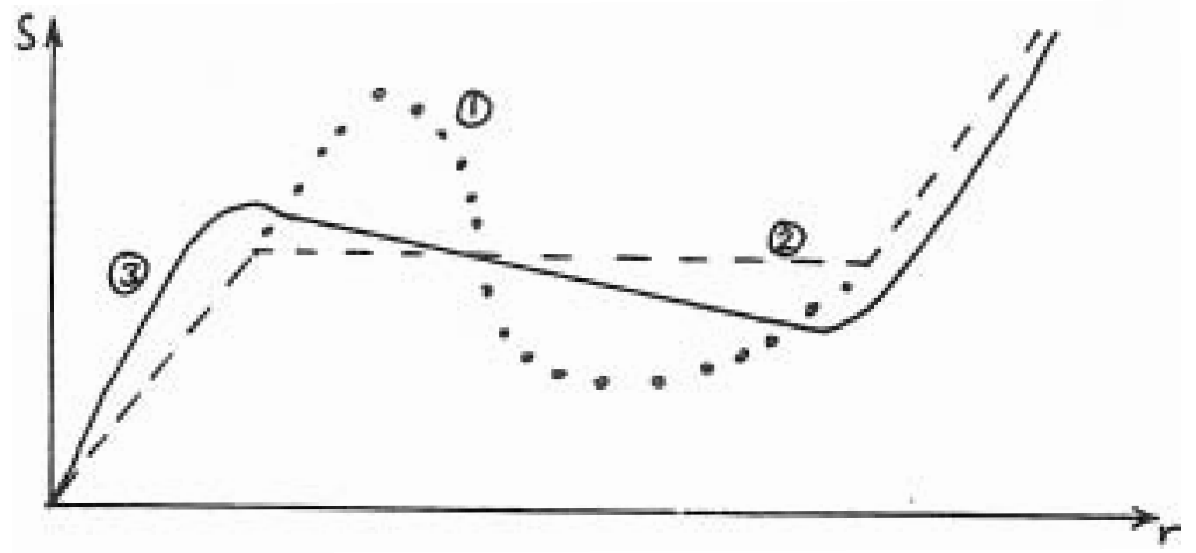


Here we assume that $\Gamma$, which is evaluated locally, has a variation with height. Curve 1 is the profile in the absence of convection, computed assuming that radiative diffusion is the only mechanism for heat transport from the interior to the surface. There is a convectively unstable region where $d s / d r<0$. If convection tends to bring the profile to convective equilibrium we would expect a profile like curve 2 , where ds/dr $=0$ in the former convective zone. The actual. profile must be slightly super-adiabatic i n order for convection to be maintained (curve 3 ).

Response Time for Perturbation from Convective Equilibrium - the Warm-up Problem.

Suppose that we change the temperature profile of an atmosphere initially i n convective equilibrium by heating it from "the side", that is, we introduce a small perturbation to the temperature profile at time $t=0$ by uniformly heating the atmosphere from a vertical wall such that

$$
\mathrm{T}(\mathrm{z}) \rightarrow(1+\varepsilon) \mathrm{T}(\mathrm{z})=(1+\varepsilon) \beta \mathrm{Z}
$$

where $\xi$ is assumed small. What is the characteristic "warm-up"time $\boldsymbol{\sigma}_{\Perp}$ for the atmosphere to reach a new state of equilibrium with this new temperature profile? This warm-up problem is analogous to the spf n-up problem i n rotating fluids and to the heat-up problem in stably stratified incompressible fluids (Veronis, 1970). We introduce:

a) The dynamical time $\tau$ dyn for this problem as the time needed for a sound wave (gravity waves are excluded i n convective equilibrium) to propagate vertically across the atmosphere. The scale height is given by

$H_{*}=z_{0} /(m+1)$, and for $m$ of order one, H* $\sim z_{0} \cdot$ Thus

$$
\tau_{\text {dyn }}=z_{o} / c \sim H_{*} / c
$$

where $c$ is the speed of sound $\left(c^{2}=\gamma \mathrm{gH} *\right)$. We anticipate that an atmosphere subject to thermal disturbances alters its hydrostatic structure on a time scale $\tau_{d y n}$.

b) The thermal time of the atmosphere $\tau_{w} \approx z_{\%}^{2} / \kappa$, where $\kappa$ is roughly the mean of thermal diffusivity over $z$, weighted in favor of smaller values.

In the warm-up problem, the region that is at first affected by thermal perturbation is that adjacent to the side wall on which the temperature perturbation is made. Nothing very significant occurs until a time $\tau_{\text {dyn }}$ passes. In that interval the side wall disturbance has had time to diffuse horizontally and establish a thermal boundary layer whose thickness, $\delta_{2}$ th is given by

$$
\frac{H_{*}}{C} \sim \frac{\delta_{H}}{K}
$$

where $\kappa$ is the thermal diffusivity. We obtain for the boundary layer thickness

$$
\delta_{\text {th }} \sim\left(\frac{K H_{r}}{c}\right)^{1 / 2}
$$


This is the analog of the Ekman layer thickness in the spin-up problem.

The physical response of the system to the perturbation may be summarized a s follows::

Initially sound waves are excited from the wall. These sound waves are presumably of little dynamical importance, much like the inertial waves which are the initial response in the spin-up problem. In the thermal boundary layer near the wall the vertical extent of the atmosphere locally changes by a n amount

$$
\delta H_{*} \sim \delta z_{0}=\varepsilon z_{0} .
$$

This change in thickness is caused by a vertical velocity in the boundary layer which is

$$
W \sim \frac{\delta z_{0}}{H_{F} / C} \sim \frac{\varepsilon z_{0} C}{H_{F}}
$$

From continuity this vertical motion must also engender a horizontal suction velocity u in the direction of the wail

$$
\frac{u}{\delta_{m}} \sim \frac{\omega}{H_{*}}+\frac{\omega}{z_{0}}=\frac{\omega}{H_{*}}\left(1+\frac{H_{*}}{z_{0}}\right)
$$

It is this induced horizontal circulation which ultimately determines the warm-up time. Consider a ring of material of radius a with its axis perpendicular to the wall (Fig, 3). The ring feels the effect of the suction long before it feels the effect of the direct diffusion of heat from the wa11. As the ring is drawn towards the wall it expands isentropically

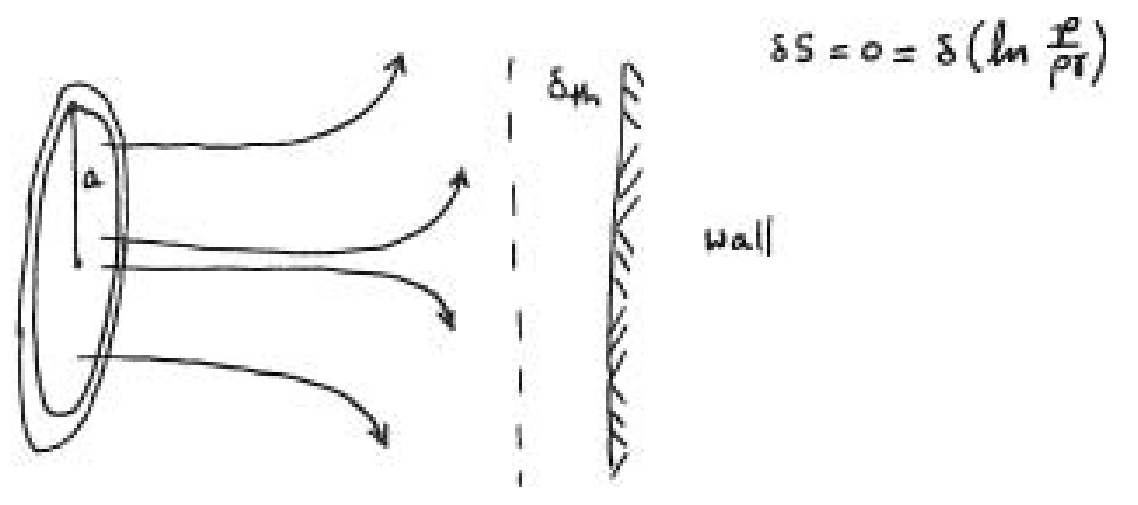

FIGURE 3 .

Using the ideal gas law and the relationship $\delta T / T=\varepsilon$, we have for the relative change in the ring's density

$$
\frac{\delta \rho}{\rho} \sim \frac{\varepsilon}{\gamma-1}
$$

The relative change in the radius of the ring is given by

$$
\frac{\delta a}{a} \sim \frac{\delta \rho}{\rho}
$$


Assuming a is of the same order as $z_{0}$

$$
\delta a \sim \frac{\varepsilon z_{0}}{\tau-1}
$$

The characteristic warm-up time for this problem is given by the time it takes for the ring to come to the new equilibrium dictated by the altered sidewall condition. Since the distance traversed by the ring is of the same order as da

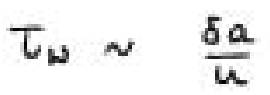

Substituting in the previously derived expressions for sa and $u$

$$
\tau_{\omega} \approx\left(\frac{1}{\gamma-1}\right)\left(\frac{z_{0}}{z_{0}+\mu_{\mu}}\right)\left(\frac{H_{\gamma}^{3}}{c \alpha}\right)^{1 / 2}
$$

For $z_{0} \sim H_{*}, \tau$, is approximately given by the geometric mean between the dynamical time $\tau_{\mathrm{dyn}}=\mathrm{H} * / \mathrm{c}$ and the thermal time for the whole atmosphere $\tau_{\text {therm }}=H_{*}^{2} / \mathcal{K} \quad$ Generally

$$
\tau_{\text {dyn }}<\tau_{\omega}<\tau_{\text {therm }}
$$

Just as in the spin-up problem, a characteristic response time calculated assuming only simple diffusion without consideration of the induced circulation is much too long -- the induced circulation brings the fluid to equilibrium much more quickly than diffusion alone could.

As an example of where this distinction between response times is important, consider a two-layer model of the sun consisting of the base of the convective zone and the top of the inner radiative zone. The two layers adjust to small perturbations on different time scales. In the upper turbulent layer $\tau_{\text {there }}$ is about one month and $\tau_{\text {dyn }}$ is about half an hour, yielding a warm-up time $t_{w}$ of a few hours. In the lower diffusive layer the thermal time is very long -- about 106 years, while $\tau$ dyn is again about one-half hour. Thus $\tau_{W}$ is on the order of a few years in the subconvective layer. There is now a strong suspicion that the luminosity of the sun is not constant -- it appears to vary in consort with the solar cycle of 11 years. Wh should we observe a phenomenon with a period of a few years? Both the dynamical and thermal times of the diffusive layer are of completely different orders of magnitude, but the warm-up time fits the bill, and we should expect the sun to be very responsive on this time scale. A model has been proposed where "ropes" of hot material are pulled up from below by magnetic effects, engendering thermal changes in solar magnetocline which manifest themselves i n a variable solar luminosity with a period of about $\tau$ w.

Finally, we note that several other characteristic times may be defined. Just as the thermal time $z_{0}^{2} / k$ represents the time for heat to diffuse across a layer of thickness $z_{0}$, the viscous time $z b / v$ represents the 
time needed for momentum diffusion in a fluid with kinematic viscosity $r$. The convective time is the time it takes for a parcel of fluid to "fall" across the layer

$$
\tau_{\text {convective }} \sim\left(z_{0} / g \varepsilon\right)^{1 / 2}
$$

where $g \varepsilon$ isthereducedgravity due tothebuoyancyforce. Inthenext lecture we will study the anelastic mode when we move slightly off convective equilibrium. This "quasi-anelastic" approximation is analogous to the quasigeostrophic approximation. Following Ogura and Phillips (1962) (see references in ":Small Nondissipative Motions and the Anelastic Approximation"), this relevant time scale for the perturbation equations is

$$
\tau \text { convective As long as } \tau \text { convective } \gg \tau_{\mu} \text { we are near }
$$

convective equilibrium, and sound waves are effectively filtered from the system.

\section{REFERENCES}

Veronis, Go, 1970. The analog between rotating and stratified fluids. Ann. Rev. F1uid Mech. 2, 37-66.

\section{NOTES SUBMITTED BY \\ SATORU HONDA AND \\ BRUCE LONG}

\section{QUASIANELASTICITY}

Edward A. Spiege 1

In many geophysical situations, we are trying to model a system in which rapid vertical mixing is taking place. We would like to scale the equations of fluid motion so as to use the nearness of the system to convective equilibrium. The result of such a scaling is the anelastic approximation.

We will, for simplicity, restrict ourselves to an ideal gas whose thermodynamic properties -- $K$, the thermal conductivity; $\mu$, the viscosity; $C p$, the specific heat at fixed pressure; $R$, the gas constant normalized with the molecular weight; $\boldsymbol{\gamma}$, the ratio of specific heats, and local gravity $g$-are constant. Our first task is to identify static equilibrium states of the gas, which must obey:

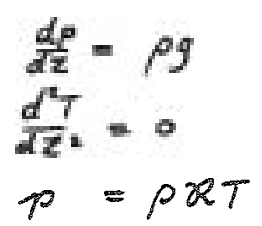

With the geometry of Figure 1, we can see that the solutions to the above must have a linear temperature gradient and thus must be polytropic:

$$
\begin{aligned}
& p=\pi p_{1}(z) \\
& \rho=\rho \rho_{1}(z) \\
& T=\Theta T_{1}(z)
\end{aligned}
$$


where $\pi$, and (QS are the pressure, density, and temperature at the bottom of the gas, and

$$
\begin{aligned}
& \pi=Q P \theta \\
& \theta=g z_{0} / m \vec{R} \\
& \rho_{0}(z)=\left(z / z_{0}\right)^{m+1}, \rho_{1}(z)=\left(z / z_{0}\right)^{m}, T_{1}(z)=z / z_{0}
\end{aligned}
$$
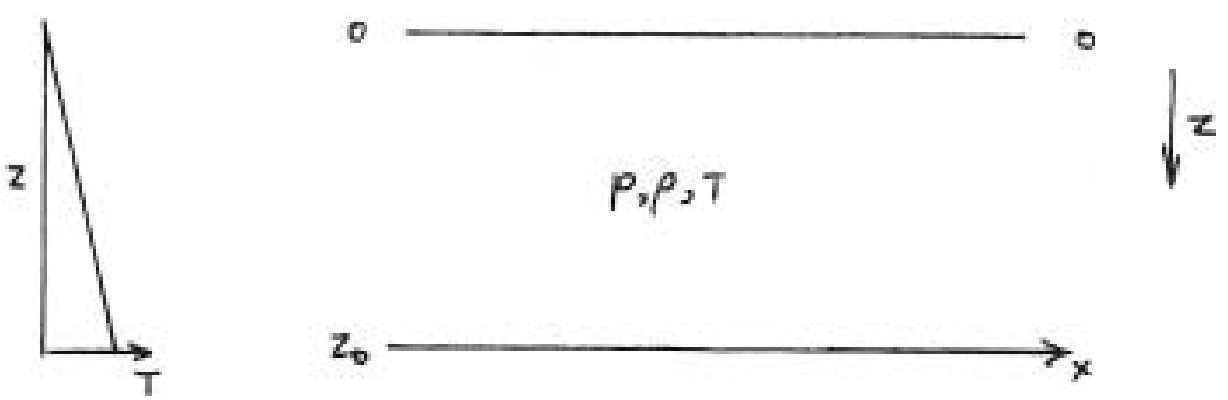

FIGURE 1. THE Geometry of the Gas

To isolate the gravitationally driven motions we assume small vertical entropy gradients, that is, that the atmosphere is nearly in convective equilibrium. This condition,

$$
\frac{d}{d z} \ln \frac{\rho}{\rho r} \approx 0
$$

implies

$$
m=\frac{1}{\gamma-1}
$$

and thus that vertical temperature gradient is near its adiabatic value

$$
\beta_{c}=\left.\frac{d T}{d z}\right|_{m=\frac{1}{\gamma=1}}=\frac{g}{Q}\left(\frac{\gamma-1}{\gamma}\right)
$$

Imagire a real gas in motion. We would like to consider the motion as a perturbation away from an adiabatic $(M=1 / \gamma-1)$ static state described by (1). Which state do we pick? Out of the two parameter family, we can pick, for instance, the static state which preserves the average mass and average basal pressure of a fluid column:

$$
\begin{aligned}
& \pi=\overline{\rho\left(x, z_{x}, t\right)} \\
& P \int_{t}^{z_{0}} \rho_{1}(z) d z=\overline{\int_{0}^{z_{t}} \rho(x, z, t) d z}
\end{aligned}
$$

where $p$ and $\rho$ are the pressure and density in the real gas, $z_{\infty}$ its depth, 
and - is a horizontal and temporal average. If

$$
\tau=\left|\frac{p\left(x, z_{r}, t\right)-\pi p_{i}(z)}{\pi p_{1}(z)}\right| \leqslant 1
$$

the above atmosphere is near convective equilibrium.

The time scale of convective motions then becomes $\tau_{\text {conv }}-\left(\frac{z_{\theta}}{g \varepsilon}\right)^{1 / 2}$ which is much longer than the other time scales in the problem:

$$
\tau_{\text {conv }} \sim \bar{\varepsilon}^{-1 / 2} \gg \tau_{\text {warm }} \sim \varepsilon^{-1 / 4} \gg \tau_{\text {sound }} \sim \varepsilon^{0}
$$

Thus, on the convective time scale other adjustments are effectively instantaneous, sound waves just being an ignorable background sea of noise. by (1)

Consider the small, order $\varepsilon$, perturbations to the basic state as given

$$
\begin{aligned}
& \rho=\pi\left(\rho_{1}+c \tilde{\rho}\right) \\
& \rho=\rho\left(\rho_{1}+\varepsilon \tilde{\rho}\right) \\
& T=B\left(T_{1}+\varepsilon \tilde{T}\right)
\end{aligned}
$$

and nondimensionalize the kinematic variables with respect to the reference length $z_{0}$ and the reference time $\tau_{\text {conv }}=\left(z_{0} / g_{\varepsilon}\right)^{\sqrt{2 z}}$.

Upon the neglect of relatively small terms, the momentum equation becomes

$$
\rho_{i} \frac{D \mu}{\partial t}=-\left(\frac{\gamma-1}{\gamma}\right) \nabla \tilde{p}-\tilde{\rho}^{\hat{z}}+\left(\frac{\nu_{p}^{2}}{z_{0}^{3} g^{\varepsilon}}\right)^{r_{2}} \nabla \cdot \underset{\psi}{\gamma}
$$

here the nondimensional viscous stress tensor is given by

$$
{\underset{\approx}{*} i j}_{i j}=\frac{\partial u_{i}}{\partial x_{j}}+\frac{\partial u_{j}}{\partial x_{i}}-\frac{2}{3} \frac{\partial u_{k}}{\partial x_{k}} \delta i_{j}
$$

and the effective kinematic viscosity $\quad \nu_{*}=\bar{\rho} \quad$ Similarly, the entropy equation is simplified to

$$
\rho_{1} \frac{D \tilde{T}}{D t}-\left(\frac{\tau-1}{\gamma}\right) \alpha T_{1} \frac{D \tilde{\rho}}{D t}=\left(\frac{\kappa}{\nu_{r}}\right)\left(\frac{\nu_{v}{ }^{2}}{z_{0}^{3} g^{\varepsilon}}\right)^{1 / 2} \frac{1}{\rho c_{p}} \nabla^{2} \tilde{T}+\left(\frac{\nu_{r}^{2}}{z_{0}^{3} g c}\right)^{1 / 2} \Phi
$$

where $\Phi$ is the nondimensionalized mechanical dissipation. The substitution of (2) and the rescaling of the mass conservation equation and the equation of state is straightforward. In equations (3) and (4) some nondimensional constants appear. The factor $\frac{r-f}{r}$ is just a number of $O(1)$. The constant $\mathrm{Gr}=z_{0}^{3} g c / \nu_{*}^{2}$, called the Grashof number, also appears and must be assumed to be $O(1)$ so that viscous dissipation can be included. For thermal diffusion to be retained we must also have the Prandt 1 number $\sigma=y_{*} / K$ of $O(1)$. Only when these two numbers are $O(1)$ are the convective, thermal and viscous timescales of the same order of magnitude. 
Having established which terms are neglected in the quasi-anelastic approximation we can now return to the dimensional form of the equations. In their general form, allowing for the variation of some of the thermodynamic properties of the gas, they read

$$
\begin{aligned}
& \nabla \cdot\left(\rho_{c} \underset{\sim}{u}\right)=0 \\
& P=p_{c}\left[1-\alpha_{c}\left(T-T_{c}\right)+K\left(T_{c}\right)\left(P-P_{c}\right)\right] \\
& \rho_{c} \frac{D \underline{\partial}}{D t}=-\nabla p-\rho g \hat{z}+\nabla \cdot \frac{P}{\not t} \\
& \rho_{c} c_{p c} \frac{D T}{D t}-\alpha_{c} T_{c} \frac{D P}{D t}=\underset{\sim}{\nabla} \cdot\left[\kappa_{c} \underset{\sim}{\nabla}\left(T-T_{c}\right)+\left(\kappa-\kappa_{c}\right) \nabla T_{c}\right] \\
& +\Phi+\left(\alpha-\alpha_{c}\right) T_{c} \frac{\partial p_{c}}{\partial z}-\alpha_{c}\left(T-T_{c}\right) \frac{\partial p_{c}}{\partial z}+\left\{-C_{p c}\left(\rho-p_{c}\right) w \frac{d T_{c}}{d z}\right. \\
& \text { - } \left.p_{c}\left(c_{p}-c_{p c}\right) w \frac{\partial \tau_{c}}{\partial z}\right\}
\end{aligned}
$$

The original equations have essentially been modified by ignoring the effect of the small density deviations from convective equilibrium in the inertia and in mass conservation. The equations have been linearized in $T, p$ and $\rho$, but the problem is still nonlinear due to advection of momentum, temperature, and pressure.

\section{The Boussinesq Approximation}

In many applications the layer of convecting fluid covers only a small fraction of a scale height. This is used in the shallow layer approximation which will be dealt with later. If additionally the horizontal scales are assumed to be of the same scale as the vertical motions, then the Boussinesq approximation follows.

Long horizontal scales can be generated in the Boussinesq approximation*. In such cases the approximation needs to be reexamined. It is important to remember that the Boussinesq approximation may not be a consistent scaling of some convection problems, e.g. , convection at large Prandtl number, and even as a model may not have the correct bifurcation structure.

The Boussinesq approximation follows from

$$
\delta=\frac{d}{H_{0}} \ll 1
$$

where $d$ is the convecting layer depth and $H_{0}$ is the minimum scale height in the layer (note that $\underset{Z}{\mathbb{Z}}$ is now vertically upwards). All the previously defined timescales are replaced by changing the atmospheric height $z_{0}$ by the layer depthd, the amplitude of the motion being determined by a balance between the new convective and dissipative timescales. Using this assumption we find that

$$
\left.\rho_{c}=\rho_{c}(0)+\alpha \delta\right)
$$

and thus $\rho_{c}$ may be taken to be a constant $\rho_{d}$ The pressure perturbations in the layer are also small and can be ignored in the equation of state.

*For example, by constant flux boundary conditions, large scale modulations of small scale structures or in cells much longer in one direction than another. 
In the heat equation we find that the dissipation is small compared with the diffusive term (at least for $O(1)$ Prandt 1 number) and that to leading order

$$
\frac{D P}{D t}=\frac{D P_{c}}{D t}=-\rho_{0} g w
$$

Thus the Boussinesq version of equation (5) is just

$$
\begin{aligned}
\rho_{0} \frac{D U}{D t} & =-\nabla \rho+\rho g \hat{z}+\mu \nabla^{2} u \\
\rho_{0} c_{p} \frac{D T}{D t} & =-\rho_{0} g \omega+\nabla \cdot(K \nabla T) \\
\rho & =\rho_{0}\left[1-\alpha\left(T-T_{\forall}\right)\right] \\
\underset{\sim}{\nabla} u & =0
\end{aligned}
$$

where $T_{*}$ is some reference temperature at which $\rho=\rho_{0}$.

The boundary conditions to be used at $z=0, d$ are somewhat arbitrary. No simple and physical choice is obvious. Usually used are rigid boundaries:

$$
u=0 \text { and } T=\text { given; }
$$

or stress free boundaries

$$
\underset{\sim}{u} \cdot \hat{n}=u_{Z} x \hat{n}=0 ; T=\text { given. }
$$

Note that at a rigid boundary the above could be changed to $u+C \frac{\partial u}{\partial \tilde{z}} \times \hat{n}=0$ where $c$ measures the mean free path of the fluid's constituent particles. If an eddy viscosity is used, then perhaps $c$ should be related to the eddy size.

Nondissipative Boussinesq Linear Dynamics.

To simplfy the problem even further we consider the inviscid non-diffusive Boussinesq equation ( $\mu=k=0$ ) for small velocities. This allows the nonlinearity in the velocity to be neglected. The analysis is relevant to the stability of the purely conducting trivial solution.

Let $T_{0}(z)$ be some initial given temperature field and introduce the dependent variable $\theta=T-T_{0}$ to replace $T$. The set of equations (6) reduce to

where

$$
\begin{aligned}
\rho_{0} \frac{\partial u}{\partial t} & =-\nabla p+g \alpha \theta \hat{z} \\
\frac{\partial \theta}{\partial t} & =\beta w \\
\nabla_{0} u & =0
\end{aligned}
$$

$$
\beta=-\left(\frac{d T_{0}}{d z}+\frac{g}{c_{p}}\right)
$$

Taking the $z$-component of the $\underset{\sim}{\nabla} \underset{\sim}{\nabla} \mathrm{x}$ of the first equation in (7) gives the following simple equation

$$
\nabla^{2} \frac{\partial W}{\partial t}=\nabla_{1}^{2}(g \alpha \theta)
$$


Try for a solution of the form

$$
\left(\begin{array}{l}
w \\
0
\end{array}\right)=e^{\eta t} f(x, y)\left(\begin{array}{c}
W(z) \\
\Theta(z)
\end{array}\right)
$$

and the above equations give the following equation for $\varepsilon$

$$
\nabla_{1}^{2} f=-k^{2} f
$$

where $k$ is a measure of the horizontal wave number. This equation guides the choice of the horizontal geometry of convection, the question being: how to tessellate the plane? Usually a planform of regular polygons is preferred.

The expression for the growth rate of the modes is

$$
y^{2}=\frac{\operatorname{gos} \beta k^{2}}{k^{2}+n^{2} a^{2}}
$$

where $n$ is the vertical wave number. The solid line in the following figure is a graph of $\mathrm{n}^{2}$ as a function of $\mathrm{k} 2$ :

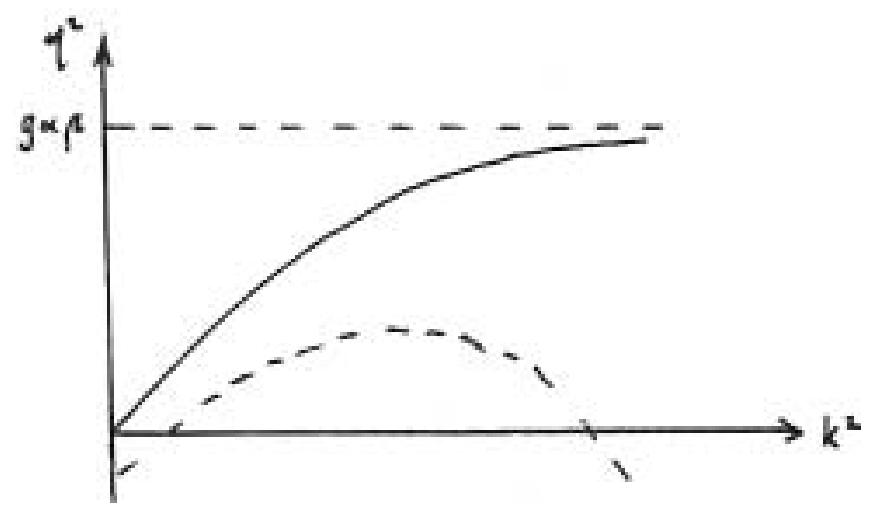

Notice that in the limit of convective equilibrium $(\beta \Rightarrow 0)$ there is no growth. The upper limit of the growth rate is given by $g \propto \beta$ instead of $O$ ( $)$ ), and is due to geometric constraints rather than the fluid's compressibility. The inclusion of the dissipative terms, which extract energy preferentially from short wave lengths, causes the growth rate to be maximized at some finite wave number $k$, and gives rise to the dotted line of the figure.

\section{REFERENCES}

Anelastic Approximation:

Gough, D. 0.., 1964. JAS, 26. 448 .

Kovshov, V. I., 1978, S o . Astron. J., 22, 288.

Malkus, W.V.R., 1967. Unpublished manuscript in WHOI Notes.

Ogura and Phillips, 1962. IASA, 19, 137.

Spiegel E. A. and G. Veronis, 1960. ApI. 131, 442. 


\section{DISSIPATIVE BOUSSINESQ DYNAMICS}

Edward A. Spiege 1

We want to investigate how the inclusion of dissipative terms alters the behavior of the fluid at the onset of convective instability,

We know that for a given horizontal wave number a, there is a value of the Rayleigh number $R_{O}$, above which motion on the corresponding horizontal scale becomes possible. Our first task is to determine the relationship between $R$ and a.

The full Boussinesq equations are:

$$
\begin{aligned}
& \partial_{+} \underline{\mu}+\underline{u} \cdot \nabla \underline{u}=-p_{\theta}^{-1} \nabla p+g \alpha \theta \hat{\varepsilon}+v \nabla^{2} y \\
& \underset{\sim}{u} \underset{\sim}{u}=0 \\
& \partial_{t} \theta+\mu \cdot \nabla \theta=\beta \omega+k \nabla^{2} \theta
\end{aligned}
$$

where $\underline{\mathrm{u}}=(\mathrm{u}, \mathrm{v}, \mathrm{w})$, the temperature $\mathrm{T}=\mathrm{T}_{\text {static }}+\boldsymbol{\theta}$ and $\beta=\left(\frac{d T_{\mathbf{s}}}{d \mathrm{z}}+\frac{\mathrm{g}}{\mathrm{C}_{\mathrm{p}}}\right)$

At the onset of convective instability we expect the velocity and temperature fluctuations to be small. We may therefore determine the initial time dependence from the linearized equations with the understanding that if we find instability the linearization quickly loses validity. If we omit $\underline{\underline{1}}$. $\underline{\mathrm{u}}$ from (1) and apply $\underset{\sim}{\nabla} \nabla \times$ we find

$$
\left(\partial_{t}-\nu \nabla^{2}\right)_{\underline{L}}=g \alpha \nabla^{2}(\theta \underline{\underline{z}})-g \alpha \nabla\left(\frac{\partial \theta}{\partial z}\right)
$$

where $\underline{\underline{z}}$ is a unit vector. Equation (4) has the components

$$
\begin{aligned}
& \left(\partial_{t}-v \nabla^{2}\right) \nabla^{2} u=-g \alpha \partial_{k}^{2} \theta \\
& \left(\partial_{t}-\nu \nabla^{2}\right) \nabla^{2} v=-g \alpha \nabla_{y}^{2} \theta \theta \\
& \left(\partial_{t}-\nu \nabla^{2}\right) \nabla^{2} \omega=g \alpha \nabla_{1}^{2} \theta
\end{aligned}
$$

while the linearized version of (3) is

$$
\left(\partial_{t}-k \nabla^{2}\right) \theta=\beta \omega
$$

Before we proceed further we discuss the kinematic boundary conditions:

A number of possibilities are available but it is usual to restrict the choice to:

$\left.\begin{array}{ll}\text { rigid boundary } & w=\underline{\underline{u}} \cdot \underline{\underline{\underline{Z}}}=0 \\ \text { free boundary } & w_{2} \partial_{2} u_{3} \quad \partial_{2} v=0,4\end{array}\right\}$ top and bottom 
We postpone the choice of the thermal boundary conditions. If we take

$\partial y(5 a)-\partial_{x}(5 b)$ and define the vertical vorticity $\zeta=\partial_{x} v-\partial_{y} u$

we get:

$$
\left(\partial_{t}-\nu \nabla^{2}\right) \nabla^{2} \zeta=0
$$

We can seek solutions of the form:

$$
\zeta \propto e^{s t} f_{s}(x, y) Z(z)
$$

Then we get $\quad \nabla_{1}^{2} f_{5}=-k^{2} f_{5}$ and

$$
\left[s-v\left(D^{2}-k^{2}\right)\right]\left(D^{2}-k^{2}\right) Z(z)=0
$$

where

$$
\nabla^{2}=\nabla_{1}^{2}+D^{2}, \nabla_{t}^{2}=\frac{\partial^{2}}{\partial x^{2}}+\frac{\partial^{2}}{\partial y^{2}}, D=\frac{\partial}{\partial z}
$$

If $z=0$ and $z=d$ are the top and bottom, the free B. C.'s give $\zeta(a)$, $Z(d)=0$ and we get

$$
S=-\nu\left(\frac{n^{2} \pi^{2}}{d^{2}}+k^{2}\right)
$$

which implies that in the linear regime the vertical vorticity decays. Clearly we expect it to couple to other modes at finite amplitude, but for the moment we leave it at that.

In a similar manner we may separate the horizontal structure from (5a) and 5(d) to obtain

$$
\begin{aligned}
{\left[\partial_{t}-\nu\left(D^{2}-k^{2}\right)\right]\left[D^{2}-k^{2}\right] \hat{\omega} } & =-g \alpha k^{2} \hat{(H)} \\
{\left[\partial_{t}-k\left(D^{2}-k^{2}\right)\right] \hat{\omega} } & =\beta \hat{\omega} \\
\nabla_{t}^{2} f & =-k^{2} f
\end{aligned}
$$

where $\hat{W}$ and $\hat{\theta}$ are functions of $z$ and $\mathbf{t}$ only.

We choose d as the unit of length, $\mathrm{d}^{2} / \mathrm{k}$ as the unit of time and $\Delta \mathrm{T}=$ $\beta d$ as the unit of temperature, and $1 \mathrm{et}$

$$
R=\frac{g \propto \Delta T d^{3}}{\nu \kappa} \quad \sigma=\frac{\nu}{k}
$$

(the Rayleigh and Prandtl numbers respectively),

The nondimensional equations are

$$
\begin{aligned}
& \left(\sigma^{-1} \partial_{t}-\Delta\right) \Delta \hat{\omega}=-R a^{2} \oplus \\
& \left(\partial_{t}-\Delta\right) \hat{\oplus}=\hat{\omega}
\end{aligned}
$$




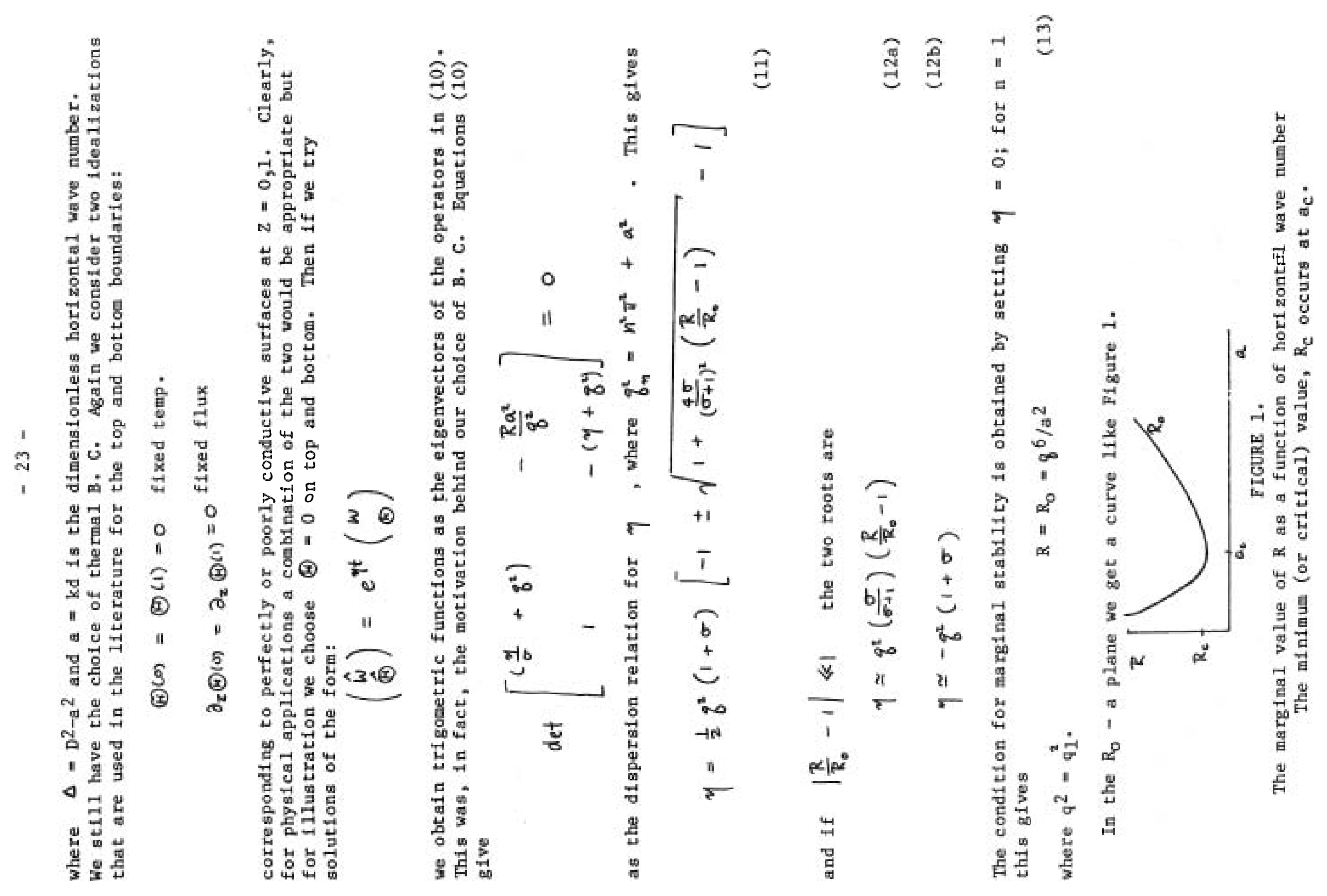


We now want to find how the curve in Figure 1 is modified by a change in the thermal B. C. This time we fix the flux and consider the equations governing the steady state:

$$
\begin{aligned}
& \Delta^{2} \omega=R a^{2} \Theta \\
& \Delta \Theta=-w
\end{aligned}
$$

The boundary conditions are DQ = o top and bottom. In effect, this problem was partly solved by Jeffrey, though its meaning was not appreciated until the $1960^{\circ}$ s by Sani and Hurle, Jakeman and Pike (1967). The essential point is that $R$ has its minimum at $a=0$. Since a is small, we rescale W; let $Q=W / a^{2}$. Integrating (14b) from $Z=0$ to $Z=1$ we find that

$$
a^{2} \int_{0}^{1} \Theta d z=\int_{0}^{1} w d z
$$

To zeroth order we find

$$
\begin{aligned}
& D^{2} \Theta_{0}=0 \\
& D^{4} \Omega_{0}=R_{0} \Theta_{0}
\end{aligned}
$$

This gives $\Theta 0$ const $\Omega_{0}=R_{0} \Theta_{0} P(z)$ where $P(Z)$ is a 4 th order polynomial such that $\mathrm{P}^{\prime \prime}(\mathrm{z})=\mathrm{l}$. The next order gives

$$
D^{2} \Theta_{2}=\mathscr{A}_{0}\left(1-R_{0} P(z)\right)
$$

which if integrated from 0 to 1 , becomes, on use of (15)

$$
R_{0}=\left[\int_{0}^{1} P(z) d z\right] \Rightarrow R_{0}=5 !
$$

It can be shown that the linear stability curve becomes Figure 2.

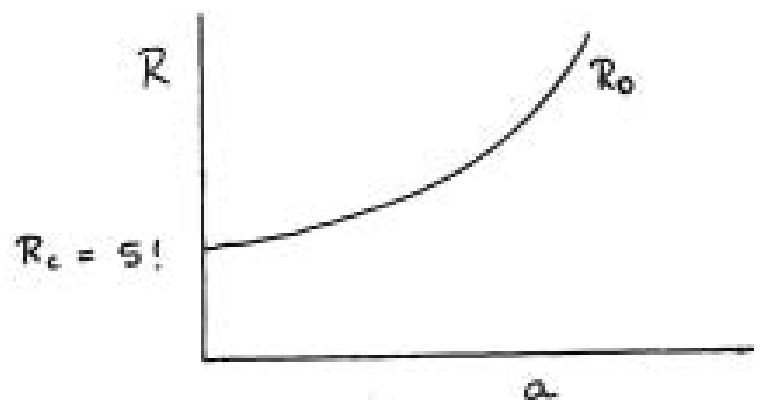

FIGURE 2.

Linear stability calculations are a useful tool to determine the value of $R_{0}$ and the initial structure of the motion. They are clearly unsatisfactory when evolved in time as they predict that the amplitude of the motion will grow exponentially without limit. To get a more reasonable picture we must include the nonlinear properties of the equations. These describe the reaction of the stable modes to the exponential growth of an unstable one. We expect these modes to provide, eventually, a way to prevent the unlimited growth of the unstable modes. 
If we describe the amplitude of the motion by $A$ we expect that for $R$ just supercritical the evolution of $\mathbf{A}$ is governed by the Landau equation

$$
\partial_{t} A=\underset{\substack{\exp \\ \text { growth }}}{\xi \mathrm{A}^{3}}
$$

(Terms of order $A^{2}$ are omitted as the equations are invariant under $A-S-A$ ).

We now seek to derive the coefficients of the Landau equation for the case of $2 D$ convection. The velocity is solenoidal and can be written in terms of a stream function:

$$
u=\left(-\psi_{r}, 0, \psi_{\boldsymbol{z}}\right)
$$

The equations for $\psi$ and $\theta$ become

$$
\begin{aligned}
& \left(\partial_{t}-\sigma \nabla^{2}\right) \nabla^{2} \psi=-\sigma R \theta x+J\left(\psi, \nabla^{2} \psi\right) \\
& \left(\partial_{t}-\nabla^{2}\right) \theta=-\psi_{x}+J(\psi, \theta)
\end{aligned}
$$

Where $J(f, g)=f_{x} g_{z}-f_{z E x}$. We $f i x$ the temperature concentrate on top and bottom and assume that on the sidewalls of the cells ( $x=0,2 \pi / a)$ that there is no heat flux $\left(\theta_{x}=0\right)$ and tangential viscous stress $\left(\psi_{n}=0\right)$. On top and bottom, $\theta \quad \nabla^{2} \psi$ all vanish. Let $R$ be slightly above marginal:

$$
\mathrm{R}=\mathrm{R}_{0}\left(1+\varepsilon^{2}\right)
$$

The natural time variable would be $\eta^{t}$ but suppose that $\sigma \sim 1$ and use $\mathcal{E}^{2} t$. We rescale the variables:

$$
\begin{array}{cc}
t \rightarrow t / \varepsilon^{2}, \psi \rightarrow \varepsilon \psi & \theta \rightarrow \varepsilon \theta / \lambda \\
R=\lambda^{2}>\lambda=\lambda_{0}+\varepsilon^{2} \lambda_{2}
\end{array}
$$

and get

$$
\mathbb{E}=\mathbb{I}
$$

where

$$
\begin{aligned}
& \mathbb{E}=\left(\begin{array}{cc}
\nabla^{4} & -\lambda_{0} \partial_{x} \\
-\lambda_{0} \partial_{k} & \nabla^{2}
\end{array}\right) \quad \mathbb{E}^{+}=\left(\begin{array}{cc}
\nabla^{4} & \lambda_{0} \partial_{k} \\
\lambda_{0} \partial_{\mu} & \nabla^{2}
\end{array}\right) \\
& \mathbb{I}=\mathcal{E}\left[\begin{array}{c}
\sigma^{-1} J\left(\psi, \nabla^{2} \psi\right) \\
J(\psi, \theta)
\end{array}\right]+\mathcal{E}^{2}\left[\begin{array}{cc}
\sigma^{-1} \partial_{t} \nabla^{2} & \lambda_{s} \partial_{x} \\
\lambda_{2} \partial_{x} & \partial_{t}
\end{array}\right] V
\end{aligned}
$$


and

$$
V=\left(\begin{array}{l}
\Psi \\
\theta
\end{array}\right)
$$

We than expand TI i n powers of $\varepsilon: V=V_{0}+\varepsilon V_{1}+\varepsilon^{2} v_{2}$. and find

$$
\mathbb{I}=\Pi_{0}+\varepsilon \Pi_{1}+\varepsilon^{2} \Pi_{2}
$$

At each order $n$ in powers of $\varepsilon$ in order to solve for $V_{n}$ we must impose

$$
\left(\tilde{V}, \mathbb{I}_{n}\right)=0
$$

where $\mathbb{E}^{+} \ddot{V}=0$. This is known as the solvability condition in classical perturbation theory, the removal of resonant terms in the suppression or the suppression of secularities.

By inspection we see that $I_{0}=0 ;$ also it is easy to deduce

$$
\begin{aligned}
& \mathbb{I}_{1}=\left[\begin{array}{c}
\sigma^{-1} J\left(\psi_{0}, \nabla^{2} \psi_{0}\right) \\
J\left(\psi_{0}, \theta_{0}\right)
\end{array}\right]=\left[\begin{array}{c}
0 \\
J\left(\psi_{0}, \theta_{0}\right)
\end{array}\right] \\
& I_{2}=\left[\begin{array}{cc}
\sigma^{-1}\left[J\left(\psi_{0}, \nabla^{2} \psi_{1}\right)+J\left(\psi_{1}, \nabla^{2} \psi_{0}\right)\right. \\
J\left(\psi_{0}, \theta_{0}\right)+J\left(\psi_{1}, \theta_{0}\right)
\end{array}\right]+\left[\begin{array}{cc}
\sigma^{-1} \nabla^{2} \partial_{t} & \lambda_{2} \partial_{x} \\
\lambda_{2} \partial_{x} & \partial_{t}
\end{array}\right] V_{Q}
\end{aligned}
$$

To zeroth order we recover the linear theory result $\mathbb{E} V_{0}=0$

$$
V_{0}=\left(\begin{array}{l}
A \sin \sin \sin \pi z \\
B \cos a x \sin \pi z
\end{array}\right) \quad \quad \quad \quad V_{0}=\left(\begin{array}{cc}
q^{4} & \lambda_{0} \\
-\lambda_{0} a & -q^{2}
\end{array}\right) V_{0}=0
$$

$\Rightarrow \lambda_{0}=\delta^{3} / a$ choosing the $+w e$ root and $B=-9 A$. $A=A(t)$ and is arbitrary thus far. Clearly we also have $\tilde{V} \propto\left(\begin{array}{l}\operatorname{sinax} \sin \pi z \\ \cos a x \sin \pi z\end{array}\right)$ We plug $v_{0}$ into our expression for $\mathbb{I}_{1}$, and get

$$
I_{1}=-\frac{A^{2}}{2} q a \pi\left[\begin{array}{c}
0 \\
\sin 2 \pi z
\end{array}\right]
$$

Condition (18) is satisfied for all A and we find

$$
V_{1}=\left(\begin{array}{c}
0 \\
c \sin 2 \pi z
\end{array}\right)+V_{0} \quad c=\frac{A^{2} a q}{8 \pi}
$$


Wew go to the next order and calculate

$$
I_{\boldsymbol{I}}=\left[\begin{array}{l}
-\sin a x \sin \pi\left(\sigma^{-i} \dot{A} q^{2}-\lambda_{2} A q a\right) \\
-\cos a x \sin \pi z\left(\delta \dot{A}-\lambda_{1} a A+\pi a A C\right)+\pi a A C \cos a x \sin 3 \pi x
\end{array}\right]
$$

Condition (18) now gives an equation for A, namely

$$
\dot{A}-\frac{2 a \sigma}{q(1+\sigma)} A+\frac{a^{2} \sigma}{8(\sigma+1)} A^{3}=0
$$

This describes the bifurcation of steady solutions from the static one at $R=R_{c}$. Schematically the result is displaced in Figure 3.

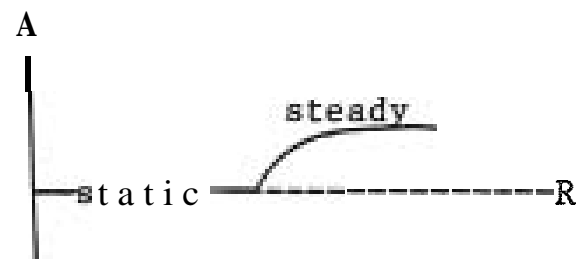

stable

FIGURE 3.

One of the characteristics of this result is that the value at which steady solutions bifurcate is independent of $\sigma$. By following the evolution of the steady solution Busse has shown that at $\sigma=\infty$ two more growing branches bifurcate from the stable steady ones and for $\sigma$ finite two overstable branches appear. Near $\sigma_{\varepsilon}$ we may expect codimension two behavior (see below).
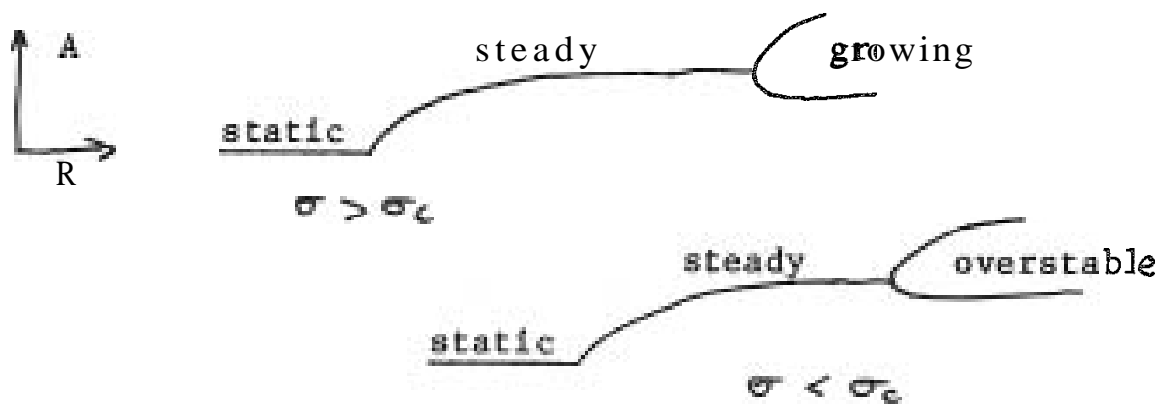

REFERENCES

Expansion in a: Childress, Levanclousky, Spiegel, 1975. JFM.

Fixed-flux convection (linear theory): Hurle, D.J.G., E. Jakeman and E. R. Pike, 1967. Numerically. PRS, A296.

Landau Equation KageIman and Keller, 1972. SIAM. 
Linear modes (including vertical vorticity modes) for fixed temperature and

slippery boundaries. Ledaux, P., M. Schwarztchild, E. A. Spiegel. Ap.J.

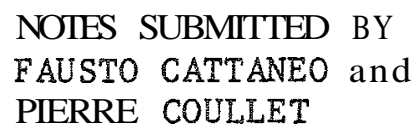

\section{CONECTION ON VERY LARGE HORZONTAL SCALES}

Edward A. Spiegel

1) What are the realistic boundary conditions?

The determination of boundary conditions is not a trivial matter, especially thermodynamic ones. Usually a constant temperature is imposed at the boundaries, thus assuming perfectly conducting walls and inhibiting large scale horizontal motions. Consider the more realistic situation (see Fig. 1) of a liquid layer bordered by two thick conducting plates

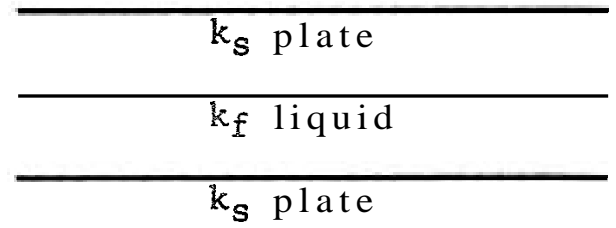

FIGURE 1。

(constant conductivity $k_{f}, k_{s}$ respectively). In the plates heat is transported only by conduction, hence, in the static state $\nabla^{2} \theta_{\boldsymbol{\varepsilon}}=0$, where $@ \$$ is the temperature profile. (We consider only the case of the upper plate; the arguments can be applied to the lower plate as well.) The horizontal dependence can be decomposed in periodic solutions of horizontal wave number a. The vertical axis $Z$ is positive upward, the origin is the lower surface. The solution is

$$
\theta_{s}=A(x, y) \cosh (a z)+B(x, y) \sinh (a z)
$$

where $A$ and $B$ are combinations of sinusoid all with wave length a. The upper boundary (not in contact with the fluid) is a constant temperature, hence

$$
A(x, y)=-B(x, y) \tanh (a h)
$$

At the interface of the fluid and plate $\theta_{s}=A(x, y)$. It is natural to assume that flux and temperature are continuous. Thus, we have

$$
\begin{gathered}
\theta_{s}(0)=\theta_{f}(0) \\
k_{b} \frac{d \theta_{s}}{d z}(0)=k_{F} \frac{d \theta_{F}}{d z}(0)
\end{gathered}
$$


where $\theta_{F}$ represents the temperature in the fluid. Then,

$$
\begin{gathered}
A=\theta_{F}(0) \\
k_{s} a B=k_{F} \frac{\partial \theta_{F}(0)}{\partial z}
\end{gathered}
$$

Eliminating A by using the former equation and the boundary condition, we get

$$
\theta_{F}(0)=-\frac{t_{F}}{\lambda_{S}} \frac{\tanh (a h)}{a} \frac{d \theta F(0)}{d z}
$$

or

$$
\theta_{F}(0)+\mathcal{B}_{k} \frac{d \theta_{F}(0)}{d z}=0 \quad \text { where } \quad \mathcal{B}_{k}=\frac{k_{F}}{l_{s}} \frac{\ldots}{a}
$$

A similar calculation shows that, at the upper surface of the lower plate, we get

$$
\theta_{F}(0)-B_{k} \frac{d \theta}{d z}=0
$$

The change of sign results from the presumed antisymmetry of the geometry.

As a result, we are able to generalize the boundary condition a the interface to

$$
\begin{aligned}
\mathrm{B} \theta^{+}+\mathrm{C} \theta_{\mathrm{x}}^{+}=0 & \text { at the upper boundary } \\
\text { and } \quad \mathrm{B} \theta^{-}-\mathrm{C} \theta_{z}^{-}=0 & \text { at the lower boundary }
\end{aligned}
$$

where $B$ and $C$ are appropriately determined functions of $x, y, a$ and the geometry of the plates. It is interesting to consider two limiting cases of the above equations. That is, when $\mathrm{C} \Rightarrow 0$, we obtain the constant temperature condition, and then, when $B \rightarrow 0$, we obtain constant heat flux case which was already discussed by Hurle et. al. (1967) in the case of the Boussinesq problem (see "Dissipative Boussinesq Dynamics", this volume). The change of boundary condition greatly affects the stability curve (Hurle et. a1., 1967). Such a change is schematically illustrated in Figure 2.

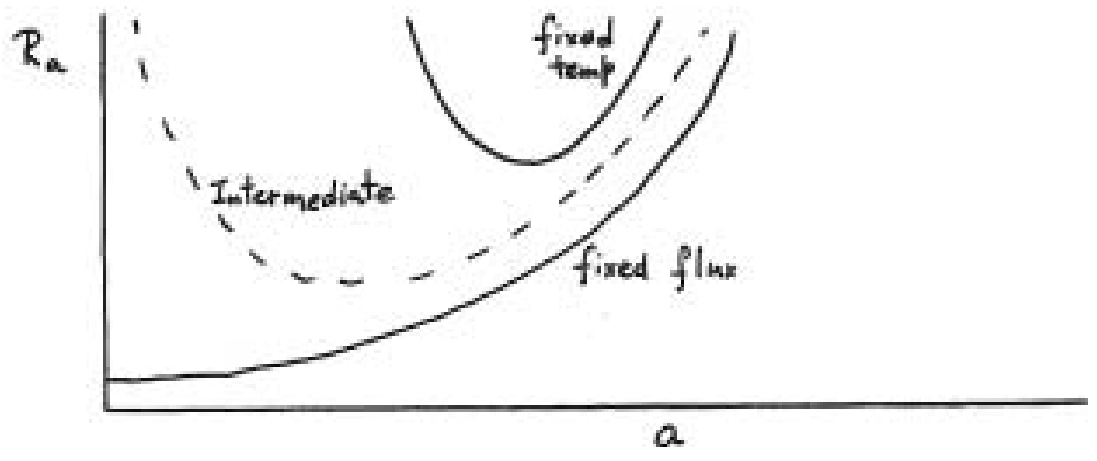

FIGURE 2.

As the boundary becomes a poorer heat conductor the critical Rayleigh number becomes smaller and the wave number of the most unstable mode approaches zero (in the case of fixed heat flux condition it becomes zero). These phenomena can be understood as the thermal penetration of the convection layer into its 
boundaries. If the boundary is perfectly conducting, the thermal penetration does not occur. However, on the contrary, if the boundary becomes a poorer conductor, it will penetrate deep into the boundary and in case of fixed heat flux, it will do so indefinitely (see Fig. 3).
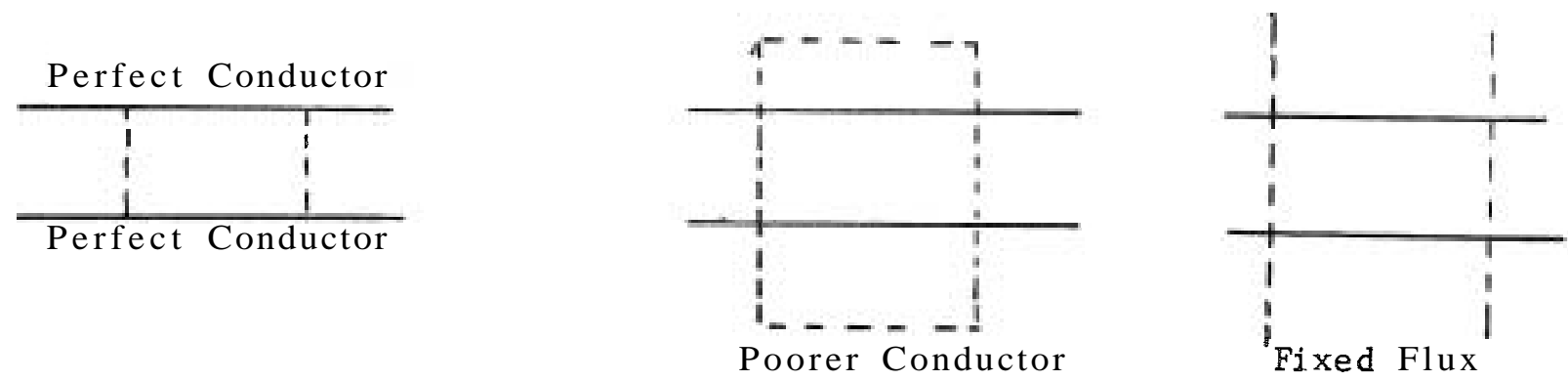

FIGURE 3 .

This is the reason why the large horizontal scale motion may be preferred in linear theory with fixed-flux boundary conditions.

2) Some Examples of NonBoussinesq Convection

As an example of nonBoussinesq convection, we consider a fluid containing microorganisms, (such as tetrahymena pyroformis) which are negatively geotactic, i.e., like to swim upward. The density is expressed as,

$$
\rho=\rho_{0}[1+\alpha[c]]
$$

where $\mathrm{C}$ is the concentration, satisfying the equation of motion,

$$
\begin{aligned}
& c_{t}+\nabla \cdot[c U \hat{z} \quad \kappa \nabla c+c u] \\
& \text { upward dispersion advection } \\
& \text { swimming }
\end{aligned}
$$

where $-U$ is the geotactic velocity, $K(c, Z)$ is dispersion coefficient that models random swimming, and $y$ is the fluid velocity. If $k / \mu=f(e)<c^{k}$ you get a polytropic solution, $c \times z^{k} \quad$ The convection arises as high concentration fluid descends and organisms swim up to maintain the concentration gradient.

Another example of nonBoussinesq convection very similar to bio-convection are: "chromium plated" stars which have an excess of ( $\mathrm{Cr}, \mathrm{Mg},---)$ in their spectra. These elements sense the radiation force, and are levitated to the surface, giving rise to a positive concentration gradient, in an analogous fashion to the negative-geotactic microorganisms. A similar instability occurs as shown by Lin (1980, Columbia dissertation).

\section{3) NonBoussinesq Convection on a Very Large Horizontal Scale}

When we consider convection having large horizontal scale, we can find that nonBoussinesq terms may become significant. In other words, to guarantee the validity of the Boussinesq approximation, the condition that horizontal scale be much smaller than a critical value is also necessary when the heat flux is fixed on the boundaries (Depassier and Spiegel, 1981). 
Consider convection having large horizontal scale. We suppose that the thermal boundary conditions at the upper and lower surfaces are those of fixed flux. We find, from the first order perturbation analysis, that the most unstable mode has the wave length of infinite length (Hurle et.al., 1967).

Assume the equation of state is slightly nonBoussinesq,

$$
\rho=\rho_{0}\left[1-\alpha\left(T-T_{0}\right)+\alpha \delta \delta\left(T-T_{0}\right)\right]
$$

q is an arbitrary function with $q(0)=0$, and $\xi$ is a measure of the deviation from the Boussinesq condition, assumed small. The basic equations are

$$
\begin{aligned}
& P_{0}\left(\underline{\sim}_{t}+\underline{u} \cdot \nabla \underline{z}\right)=-\nabla p-g \rho \hat{z}+\mu \nabla^{2} \underline{u} \\
& \nabla \cdot u=0 \\
& \rho_{0} c_{p}\left(T_{t}+y \cdot \nabla T\right)=\underset{\sim}{\nabla} \cdot(k \nabla T)
\end{aligned}
$$

We restrict ourselves to two-dimensional flow, and introduce a stream function $\psi$ - After nomalization d (depth of convective layer) as a unit length, $\psi_{\rho_{0}} c_{\rho} d^{2} / \ell_{0}$ as a unit of time, $\rho_{*}$ as a unit of density, $\boldsymbol{F d} / \mathcal{K}_{*}$ as a unit of temperature (we use conventional symbols of fluid mechanics). We obtain

$$
\begin{aligned}
& \nabla^{4} \psi=R\left[1-\delta \xi^{\prime}(\theta-z)\right] \theta x+\sigma^{-1}\left[\nabla^{2} \psi_{t}+\frac{\partial\left(\nabla^{*} \psi, \psi\right)}{\partial(x, z)}\right] \\
& \nabla^{2} \theta=\theta_{t}-\psi_{x}+\frac{\partial(\theta, \psi)}{\partial(x, z)}
\end{aligned}
$$

where the primed means $\partial / \partial z, R=g \times d^{4} \rho_{0}^{2} c_{p} F / k^{2} \mu, \sigma=\frac{c_{p} \mu}{\ell}$ and is a perturbed temperature defined by $T=T_{0}-z+\theta$. We take the coordinate as indicated in Figure 4.

$$
\begin{aligned}
& Z=1 / 2 d \quad \text { fixed flux }=F \\
& Z=0-1 / 2 d \quad \text { fixed flux }=F \\
& \text { FIGURE } 4 .
\end{aligned}
$$


The boundary conditions are

$$
\begin{aligned}
& \psi=0 \\
& \psi_{z}=0 \\
& \theta_{z}=0
\end{aligned} \quad \text { at } z= \pm \frac{1}{2} d
$$

We rescale the above three equations as follows:

$$
\xi=\varepsilon \times, S=\varepsilon^{4} t, \psi=\varepsilon \Psi, \quad, \quad=\varepsilon^{2}
$$

and obtain

$$
\begin{aligned}
& \theta_{z z}=\varepsilon^{2}\left[\theta_{5 \xi}-\Psi_{\xi}-\Psi_{z} \theta_{r}+\Psi_{\xi} \theta_{z}+\varepsilon^{4} \theta_{s}\right] \\
& \Psi_{z z z z}=R\left[1-\varepsilon^{2} q^{\prime}(\theta-z)\right] \theta_{\xi}-2 \varepsilon^{2} \Psi_{5 z z}+\frac{\varepsilon^{2}}{\sigma} \frac{\partial\left(\xi_{j}, \psi_{z z}\right)}{\partial(\xi, z)}
\end{aligned}
$$

Integrating Equation ( 9 ) over $z$ from $-1 / 2$ to $+1 / 2$ we find

$$
\varepsilon^{4}\left\langle\theta_{s}\right\rangle=\langle\theta\rangle_{\xi \xi}+\langle\Psi\rangle_{\xi}-\left\langle\Psi \theta_{x}\right\rangle_{\xi}
$$

where $\langle\cdots\rangle=\int_{-\frac{1}{\frac{1}{2}}}^{\frac{1}{2}} \cdots d z$

In the process of integration, we used the temperature boundary conditions at $z= \pm 1 / 2$ of (7). Equation (11) is an evolution equation for $\langle\theta\rangle=$ We expand

$$
\begin{aligned}
& \Psi=\Psi_{0}+\varepsilon^{2} \Psi_{1}+\varepsilon^{4} \Psi_{2}+\cdots \\
& \theta=\theta_{0}+\varepsilon^{2} \theta_{1}+\varepsilon^{4} \theta_{2}+\cdots \\
& R=R_{0}+\varepsilon^{2} R_{1}+\varepsilon^{4} R_{2}+\cdots
\end{aligned}
$$

and substitute these into Equations (9) and (18). We obtain the lowest order linear equations

$$
\begin{aligned}
& \theta_{0 z z}=0 \\
& \Psi_{0 z z z z}=R_{0} \theta_{0 \xi}
\end{aligned}
$$

Considering the boundary conditions, we have

$$
\theta_{0}=f(\xi, s)
$$


Then we can write as

$$
\Psi_{0}=R_{0} f_{\xi} P(z)
$$

where

$$
P^{(i v)}=1
$$

At this point we use the kinematic boundary condition of (17), that is,

$$
P=0, P^{\prime}=0 \text { on } z= \pm \frac{1}{2}
$$

We have

$$
P=\frac{1}{4 !}\left(z^{4}-\frac{1}{2} z^{2}+\frac{1}{16}\right)
$$

Now, the problem is to derive the equation of $f$ by using (11). Putting Equation (15) and (18) into (11) we get $\mathrm{fxx}_{\mathrm{x}}=0$ or $\mathrm{R}_{\mathrm{o}}=6$ : in the leading term. We need the computation of the next order. Selecting the case of $R_{0}$ $=6$ ? which is coincident with the critical value of $\mathrm{R}$ derived from the Boussinesq approximation, we find

$$
f_{5}+r f_{55}+k f_{5555}-\mu\left(f_{5}^{3}\right)_{5}-F_{55}=0
$$

where $\quad=R_{1} / R_{0}=R_{1} / 6 !, F(f)=R_{0}\left(Q_{5}-\frac{1}{2} Q_{4}+\frac{1}{12} Q_{3}\right), \mu=\frac{10}{7}$ and

$K=\frac{11}{462} \quad Q$ is defined as

$$
Q_{n}=\int_{-\frac{1}{2}}^{\frac{1}{2}} d \xi_{1} \int_{-\frac{1}{2}}^{\Sigma_{1}} \cdots \int_{-\frac{1}{2}}^{\xi_{n-1}} q\left(f-\xi_{n}\right) d \xi_{n}
$$

If we assume that $\left(T-T_{0}\right) \propto\left(T-T_{0}\right)^{2}$, that is, the expression which we can expand by the Taylor series, we find that $F=f Z+$ const. We have the evolution equation as follows:

$$
f_{s}+r f_{55}+k f_{\text {I5 } 5}-\mu\left(f_{\varepsilon}^{3}\right)_{\xi}-\lambda\left(f^{2}\right)_{5 \xi}=0
$$

$\left(\right.$ note $\left.F=\lambda \mathrm{f}^{2}\right)$.

In this equation, $\lambda$ represents the nonBoussinesq contribution. In solving equation (21) we must require suitable boundary conditions. They may be $\theta_{n}=0$ and $\Psi_{n}=0$ at the end points describing no heat flux from the neighbor and shear stress free. These conditions are converted to

$$
\begin{aligned}
& f_{Y}=0 \\
& f_{I S S}=0 \quad \text { on } \xi= \pm \frac{\pi}{\alpha}
\end{aligned}
$$

Another important condition derived from (22) is

$$
\bar{f}_{s}=0
$$


where

$$
\bar{f}=\frac{\alpha}{2 \pi} \int_{-\frac{\pi}{\alpha}}^{\frac{\pi}{\alpha}} f d \xi
$$

To the order of our present approximation conservation of mass gives

$$
\bar{f}=0
$$

To solve (21) with conditions (223 and (24) we expand $f$ by a new small parameter $\hat{\delta}$, that is,

$$
\begin{aligned}
f(\xi, s) & =\hat{f}_{0}+\hat{\delta} f_{1}\left(\xi, \hat{\delta}^{2} s\right)+\hat{\delta}^{2} f_{2}\left(\xi, \hat{\delta}^{2} s\right) \\
& =r_{0}+\hat{\sigma} r_{1}+\hat{\delta}^{2} r_{2}+\cdots
\end{aligned}
$$

In leading term we have

$$
f_{0}=A\left(\hat{\delta}^{2} s\right) \cos (\alpha \xi)
$$

with $r_{0}=k \alpha^{2}$

$$
\text { (1.e., } \left.R=R_{m}=6 !\left(1+t^{2} k \alpha^{2}\right)=R_{C}\left(1+k \alpha^{2}\right)\right)
$$

In higher order we find a Landau equation,

$$
\dot{A}=\alpha^{2} r_{2} A-\frac{3}{4}\left(\mu \alpha^{4}-\frac{2}{9} \frac{\lambda^{2}}{\kappa}\right) A^{3}
$$

where $r_{2}$ measures $R-R_{m}$ " Equation (28) states that bifurcation from $R=$ $R_{m}$ to the subcritical area exist, if the wave number $\alpha$ is less than a certain transition wave number $\alpha_{0}$.

$$
\alpha_{0}^{4}=\frac{2 \lambda^{2}}{9 \mu k} \quad \text { or } \quad \alpha_{0}=1.43 \sqrt{|\lambda|}
$$

The rough sketch of this situation is shown in Figure 5.

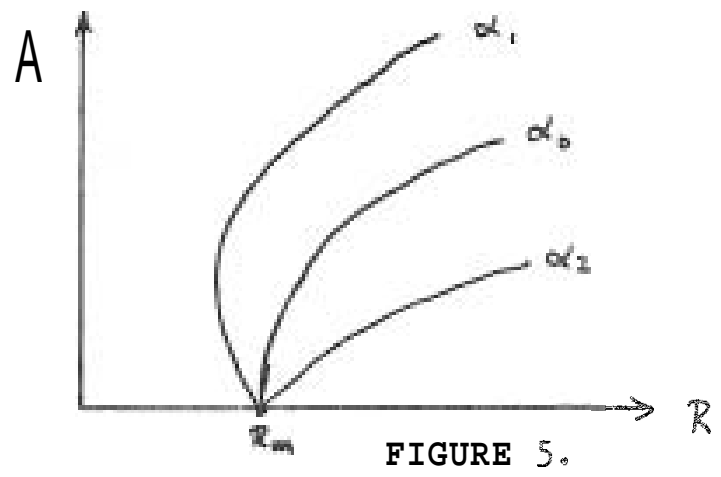

It is clear that, if we do not take the nonBoussinesq term into account $(\lambda=0)$, we cannot find such a solution. This may be interpreted as follows: 
The small parameter \& may be considered as $\mathcal{L}$ where His a vertical characteristic length associated to a nonBoussinesq effect, To guarantee the Boussinesq approximation we require the condition

$$
\frac{d}{\alpha_{0}} \sim \frac{d}{\sqrt{B}} \sim \sqrt{H d}
$$

must be sina 11.

\section{REFERENCES}

Mildy NonBoussinesq Case:

Depassier, M. C. and E. A. Spiege1, 1981. Manuscript. Convection with heat flux prescribed on the boundaries of the system. I. The effect of temperature dependence of material properties.

Linear Theory:

Hurle, D. T. J., E. Jakeman and E. R. Pike, 1967. On the solution of Benard problem with boundaries of finite conductivity. Proc. Roy. Soc. Lon., A296, 469.

\section{Using Boussinesq Theory:}

Chapman and M. J. Procter, 1980. JEM.

Using NonBoussinesq Theory:

Childress et.a1., 1978. (Submitted to IFM).

NOTES SUBMITTED BY

SATORU HOMDA and

EVAN FISHBEIN

\section{MILDLY NONBOUSSINESO CONVECTION WITH FIXED FLUX}

Edward A. Splege1

Using the scaling

$$
\xi=\varepsilon x, \quad S=\varepsilon^{4} t \quad \psi=\varepsilon \Psi
$$

we have arrived at the evolution equation of two-dimensional fixed-flux convection for the leading order temperature perturbation $\theta=f(\xi, s)+$ $O\left(\Sigma^{2}\right)$. It is

$$
f_{5}+k f_{\xi \xi \xi \xi}+r f_{\xi \xi}=\nu\left(f_{\xi}^{3}\right)_{\xi}+\lambda\left(f^{2}\right)_{\xi \xi}
$$

where the Rayleigh number of the flow is determined by $r$ in the relation

$$
R=R_{0}\left(1+\varepsilon^{2} r\right)
$$

and $\mathrm{K}$ and $\gamma$ are constants depending upon the boundary conditions $(\mathrm{K}=17 / 462$, $=10 / 7$ for rigid boundaries, and is a parameter ineasuring the non- 
Boussinesq effectso Equation (1) is solved on an interval in $\xi$ of length $2 \pi / k$ subject to zero velocity ( no neat heat flux) and no viscous stress boundary conditions at $\xi=0,2 \pi / k$.

Now suppose that we are interested in that part of the parameter regime where the behavior $s f$ is weakly nonlinear. Hence $f$ can be represented as an asymptotic sequence in some small parameter h that measures the nonlinearity in f. Thus substitute

$$
\begin{aligned}
& \tau=h^{2} s \\
& f=h F_{0}(\xi, \tau)+h^{2} F_{1}(\xi, \tau)+\cdots \\
& r=r_{0}+h r_{1}+h^{2} r_{2}+\cdots
\end{aligned}
$$

into equation ( $($ ), group like powers of h and solve the resultant recursive set of equations.

The first order equation is

$$
T_{0} F_{055}+k F_{0555}=0
$$

with solution

$$
F_{0}=A(\sigma) \cos (k), \quad r_{0}=k k^{2}
$$

where the condition $r_{0}=k^{2}$ implies that near linear behavior of $f$ is only found for values of $r$ near this particular $r_{0}$. The equation at second order is

$$
r_{0} F_{15 \xi}+\kappa F_{15 \xi \xi \xi}=r \cdot A k^{2} \cos (k \xi)-2 \lambda A^{2} k^{2} \cos (2 k \xi)
$$

To eliminate the secular forcing term in $\cos (k)$ we must choose $r_{I}=0$. Thus the second order solution is

$$
F_{1}=B(\tau) \cos (k \xi)-\frac{\lambda A^{2}}{6 k k^{2}} \cos (2 k \xi)
$$

Substituting the solutions of the first and second order problems into the third order equation we find that it has the form

$$
\begin{aligned}
r_{0} F_{35 \xi}+k F_{35 \xi \xi \xi}= & {\left[-\dot{A}+r_{2} k^{2} A+\left(\frac{\lambda^{2}}{6 k}-\frac{3 \nu}{4} k^{4}\right) A^{3}\right] \cos k \xi } \\
& +[\cdots] \cos (2 k \xi)+\{\cdots\} \cos (3 k \xi)
\end{aligned}
$$

where $\AA=A_{t}$. To eliminate the secular term in the solution for $F_{3}$ we must again have that if the coefficient of $\cos (k$,$) ) in the righthand side is$ zero, hence defining

$$
k_{0}^{4}=\left(\frac{2}{4 k v}\right) \quad \lambda^{2}
$$


we end up with the following Landau equation for $A(t)$

$$
A=r_{2} k^{2} A+\left(\frac{15}{14}\right)\left(k_{0}^{4}-k^{4}\right) A^{3} .
$$

From this equation we see that the amplitude of the steady solution is

$$
A_{S t}{ }^{2}=\frac{r_{2} k^{2}}{k^{4}-k_{0}{ }^{4}}
$$

It is then also easy to see that for $k<k_{0}$ we must have $r_{2}<0$ and the steady solution is unstable; while for $k>k_{0}$ we must have $r_{2}>0$ and the steady solution is stable, see Figure 1 .

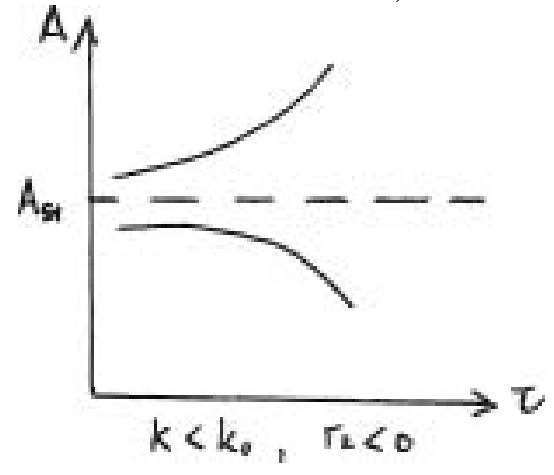

FIGURE 1.

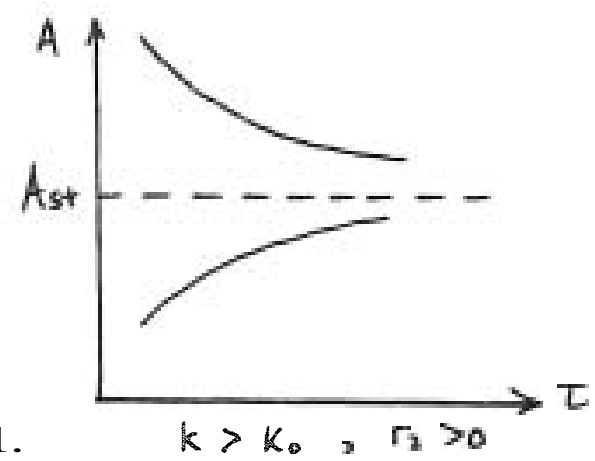

We now turn our attention to the steady nonlinear solutions of the evolution, equation (1). Firstly, note that since $\overline{\mathrm{f}}_{\mathrm{s}}=0, \overline{\mathrm{f}}$ is constant in time and mass conservation requires that this constant be zero to the accuracy of the Boussinesq approximation. Define new variables $\varnothing$ and $y$ and new parameters $\rho$ and $\alpha$ by

$$
\begin{array}{cc}
\phi(y)=\left(\frac{2 \nu}{k}\right)^{1 / 2} \operatorname{sgn}(\lambda) f(\xi), y=k \cdot \xi \\
\rho=\frac{r}{k k_{0}^{2}}, \alpha=k / k_{0}
\end{array}
$$

where $k_{0}$ is defined by equation (2) as before. Substituting into equation (1) and integrating once we find the following nonlinear ordinary differential equation for $\varnothing$

$$
\ddot{\phi}-\frac{1}{2} \dot{\phi}^{2}-3 \phi \dot{\phi}+\rho \dot{\phi}=0
$$

where denotes $d / d y$. Note that there is now no $\lambda$ dependence appearing explicitly in the problem, it does, however, occur implicitly through the definition of $k_{0}$.

Replace by the new parameter $P$ where

$$
p=\alpha^{2}+p
$$

then for P near zero equation (3) has solutions that are only weakly 
nonlinear. In terms of this new parameter the Rayleigh number is given by

$$
R=R_{0}\left[1+K_{a}^{2}+P\right]
$$

where $a=\varepsilon k$ and $a_{0}=c k_{0}$ are wave numbers i $n$ the $x$ coordinate.

Thus we are looking at solutions for Rayleigh numbers near the marginal $R_{m}=$ $R_{0}\left(1+K_{a}{ }^{2}\right)$ for the given wave number, the difference from $R_{m}$ is measured by $P$.

Equation (3) can be integrated twice by introducing the function

$$
G(\varnothing)=\dot{\phi}
$$

which transforms equation (3) to the equation

$$
\frac{d^{2}}{d \phi^{2}} G^{2}=G^{2}+6 \varnothing-2 \rho
$$

which can be solved to give

$$
\dot{\phi}^{2}=2 A \cosh \phi+2 B \sinh \phi-6 \phi+2 \rho
$$

where $A$ and $B$ are integration constants. This equation can be written in the form of a particle in a potential well. Let

$$
\begin{gathered}
\phi_{0}=-\min _{y} \phi(y) \quad \phi_{1}=\max _{y} \phi(y) \\
\Phi=\phi_{0}+\phi_{1} \\
x(y)=\phi(y)+\phi_{0}
\end{gathered}
$$

then $\Phi$ measures the amplitude of the motion and $X$ represents the '"Particle's height". Equation (4) can then be written as

$$
\frac{1}{2} \dot{x}^{2}+v(x)=0
$$

where

$$
\begin{aligned}
& V=3 X+E(\cosh x-1)+C \sinh X \\
& c=[E(1-\sinh \Phi)-3 \Phi] / \sinh \Phi \\
& E=\rho+3 \phi_{0} \\
& \bar{X}=\phi_{0}
\end{aligned}
$$

Observe that the shape of the potential well varies as the "energy" $E$ is changed. The outcome is that there are no solutions unless

$$
\rho=P+\alpha^{2}>-3 \cdot \frac{(\sinh \Phi-\Phi)}{(\cosh \Phi-1)}
$$


This behavior is summed up in Figure 2 where we see that for $\alpha>1$ there is a supercritical blfurcation to a nonlf near steady solution: for $\alpha<1$ there is a subcritical bifurcation and the subcriticality is limited by the $\alpha=0$ asymptote at $P=-3$.

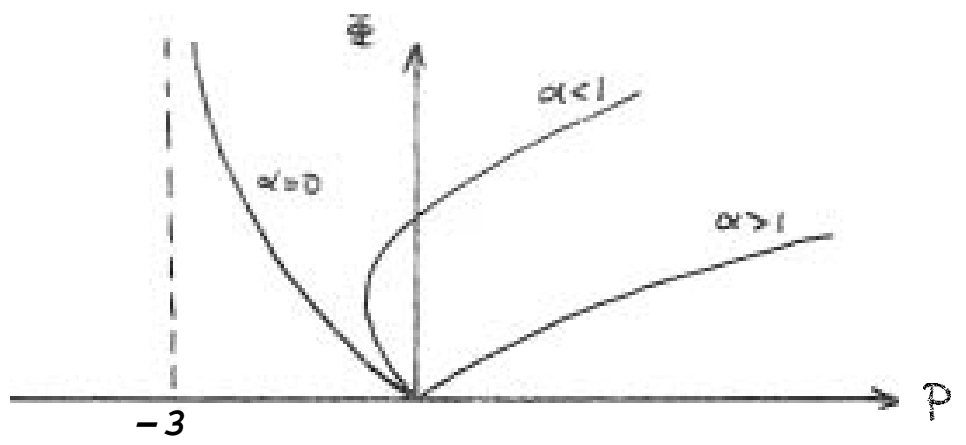

FTGURE 2.

Amplitude sf steady nonlinear solutions for various wave number parameters $\alpha=k / \mathrm{k}_{0}$.

\section{Transition to Finite Critical Wave Number}

Consider the situation

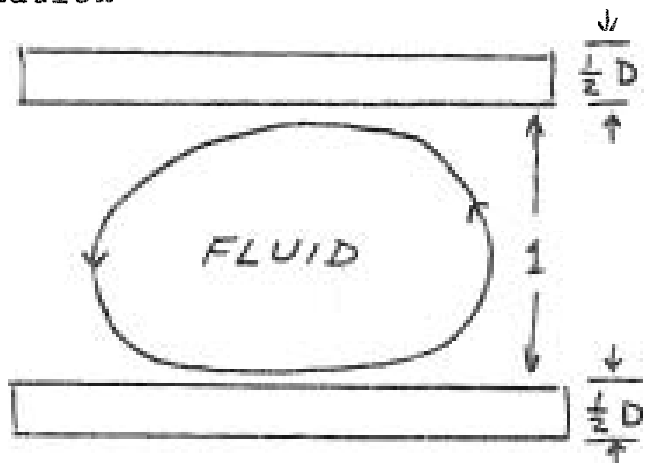

where the convecting fluid is sandwiched between two conducting plates of thickness $\frac{1}{2} D$. The fixed flux temperature boundary condition is now applied on the outside sf the plates. In the fluid we use the previously derived equations of motion while in the plates there is the purely diffusive problem

$$
\frac{\partial \theta^{t}}{\partial t}=k_{s} \nabla^{2} \theta^{ \pm}
$$

We consider the case where the temperature boundary conditions of the fluid and the plates are just

$$
\begin{aligned}
& \theta \frac{ \pm}{z}=0 \text { on } z= \pm \frac{1}{2}(1+D) \\
& \theta, k \frac{\partial \theta}{\partial z} \text { continuous on } z= \pm \frac{1}{2}
\end{aligned}
$$

As before we can obtain (Poyet, 1979) an evolution equation for the temperature perturbation which is

$$
(1+D) f_{s}+r f_{\xi \xi}+k f_{\xi \xi \xi \xi}=\nu\left(f_{\xi}^{3}\right)_{\xi}-\lambda\left(f^{2}\right)_{\xi \xi}
$$


But now $K$ depends on $D$ and $\tau=K_{\mathbf{s}} / K$ (the ratio of the plates to the fluids diffusivity). The relationship is given by

$$
k=\left[68+213 D \sigma-154(D \tau)^{2} / \sigma-262(D \tau)^{3}\right] / 1848
$$

and the regions of different behavior in the $(D, t)$ plane are shown in Figure 3.

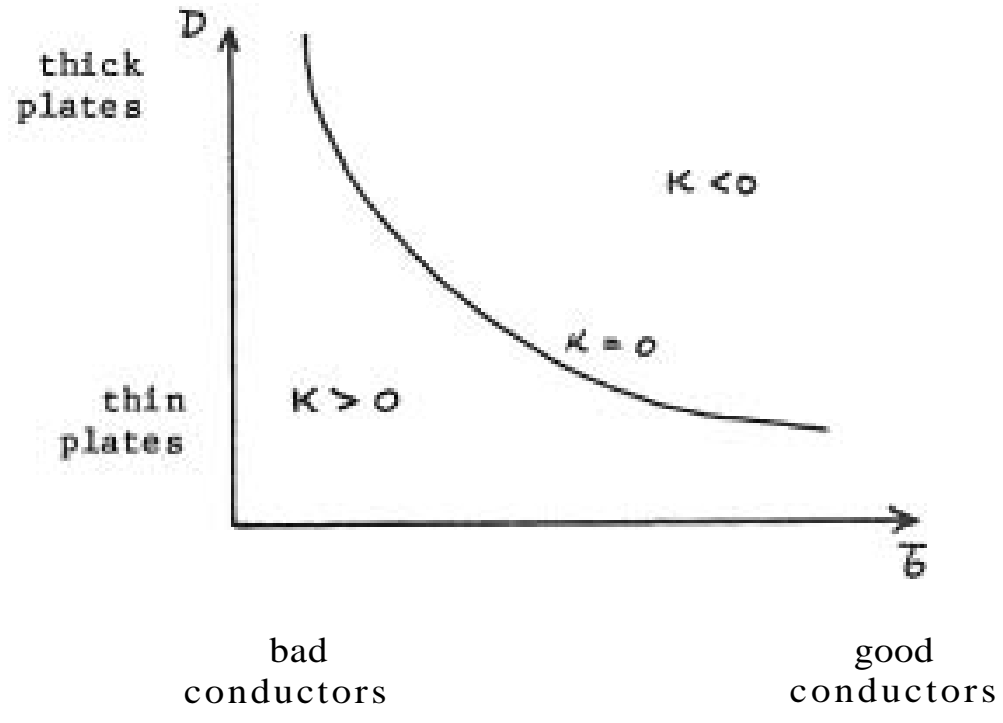

FIGURE 3.

We conclude that in the regime of plate configurations where $K \square$ Rayleigh number versus wave number marginal stability curve looks like Figure $4 a$, the situation is similar to the previous discussion and so there is a subcritical bifurcation for wave numbers smaller than some critical value. For $\mathrm{k}>0$ the Rayleigh number, wave number curve books like Figure $4 \mathrm{~b}$ and bifurcations are supercritical.

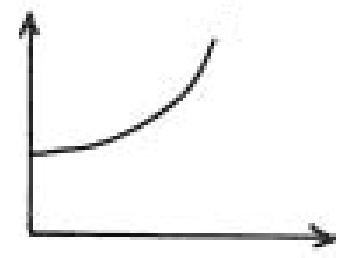

(a) $\kappa>0$

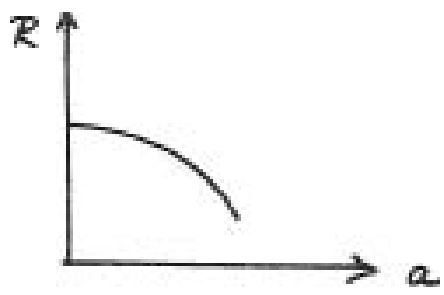

(b) $\quad N<0$

FIGURE 4*

The transition between one sort of behavior and the other, when $\mathrm{K}$ is small and of order $\varepsilon^{2}$, is also interesting. We have to go right back to the beginning, set

$$
R=R_{0}+\varepsilon^{4} R_{4}
$$

and scale the time with $\varepsilon^{6}$ and $\theta$ with $\varepsilon$ Then the analogous evolution equation to equation (1), involving a term in $\mathrm{f}_{\mathbf{5} 5 \xi \xi \xi}$, is sixth order

instead of fourth order. It turns out that WA is positive and so Figure 4b can be redrawn to give Figure 5 for values of $K$ just less than 0. 


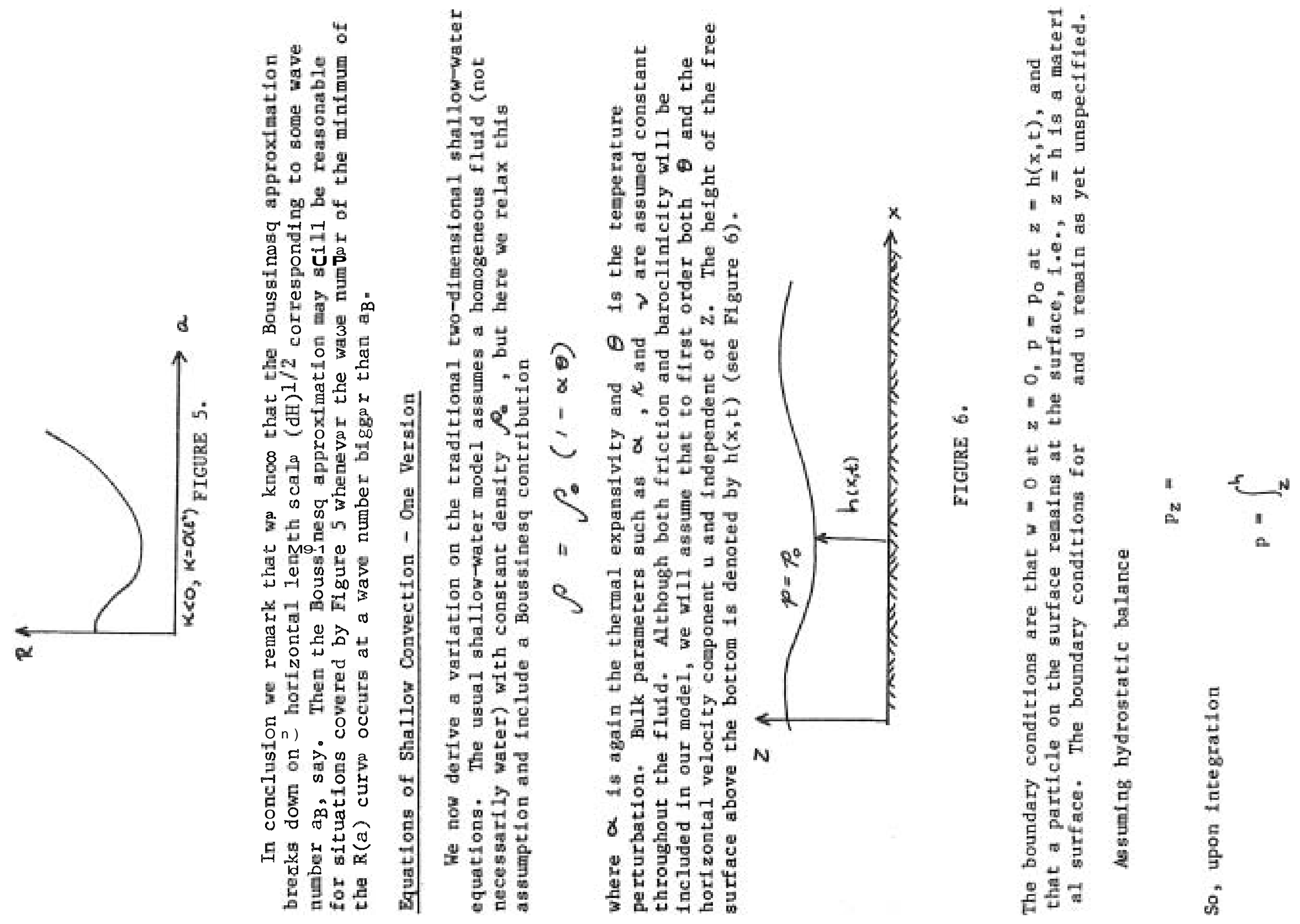


where we have used the fact that $p=p_{0}$ at $z=h$. Substituting for

$$
\mathrm{p}=\mathrm{p}_{0}+g \rho_{0}\left[\mathrm{~h}-z-\alpha \int_{z}^{h} \theta \mathrm{dz}\right] \text {. }
$$

Now using the fact that $z=h$ is a material surface, we have for $z=h$

$$
\frac{D}{D t}(h-z)=h_{t}+u h_{x}-w=0
$$

or

$$
w(z=h)=h_{t}+u h,
$$

Integrating the incompressibility condition $\nabla \cdot u_{u}^{u}=0$ vertically from $z=0$ to $z=h$

which implies

$$
\begin{aligned}
& \int_{0}^{h}\left(u_{x}+w_{z}\right) d x=0 \\
& h u_{x}+w(z=h)=0
\end{aligned}
$$

where we have employed our presumption of $O(1)$ absence of vertical shear in u. Using the result for $w(z=h)$,

$$
h_{t}+(h u)_{x}=0
$$

which is the expression for conservation of mass in the context of shallow-water theory.

In the Boussinesq approximation the $x$-component momentum equation, with the previously derived expression for the pressure inserted, is

$$
\rho_{0}\left(u_{t}+u u_{x}+\omega u_{z}\right)=-9 \rho_{0}\left[h-z-\alpha \int_{z}^{h} \theta d z\right]_{x}+\mu \nabla^{2} u
$$

This can be rewritten in flux form

$$
\begin{aligned}
u_{t}+(u u)_{x} & +(\omega u)_{z}=-g\left[h_{x}-\alpha \int_{z}^{h} \theta_{x} d z\right] \\
& +v u_{x x} \\
& + \text { higher order terms. }
\end{aligned}
$$

The higher order terms might include a boundary term in if we stray from a constant temperature $B$. $C$. on the free surface or $a v u_{z z}$ term if we have a no-slip bottom boundary layer.

Again integrating vertically and ignoring any $z$-dependence on $u$ and $\theta$

$$
\begin{aligned}
h u_{t} & +h\left(u u_{x}\right)+u\left(h_{t}+u h_{x}\right) \\
& =-g\left[h h_{x}-\frac{1}{2} \alpha \theta * h^{2}\right]+\nu h u_{* x}
\end{aligned}
$$




$$
(h u)_{t}+\left(h u^{2}\right)_{x}=-\left(\frac{1}{2} g h^{2}\right)_{x}+\nu h u_{x x}+\frac{1}{2} g \propto h^{2} \theta_{x}
$$

The thermodynamic equation can be written

$$
\theta_{t}+\nabla \cdot(y \theta)=\kappa \nabla^{2} \theta+\beta w
$$

Here

$$
\beta=-\left(\frac{d T_{0}}{d z}+\left.\frac{d T}{d z}\right|_{\text {adiabatic }}\right)
$$

where the temperature $T=T_{0}+\theta$, and $T_{0}(Z)$ is the static temperature profile. Performing another vertical integration

$$
\begin{aligned}
h \theta_{t} & +h(u)_{x}+\theta\left(h_{t}+u h_{x}\right) \\
& =h \kappa \theta_{x x}+\left(\Delta T-\frac{g h}{c_{p}}\right) w(z=h)
\end{aligned}
$$

Regrouping, we have our third governing equation for $h$, $u$ and $\theta$

$$
\begin{aligned}
& (h \theta)_{t}+(h u \theta)_{x} \\
& =k h \theta \times x+\left(\Delta T-\frac{g h}{c_{p}}\right)\left(h_{t}+u h_{x}\right)
\end{aligned}
$$

The existence of a free surface now allows the propagation of gravity waves - in particular a thermally-induced bore or shock wave might be possible if the fluid is heated from below. Another approach is to use amplitude expansions as in the derivation of Bousainesq or Kortwig-DeVries equations. That has been begun by Depassier in order to study the possible existence of convective solitary waves. The corresponding double-diffusive problem with a free surface does seem to show coherent wave-like solutions at marginal stability.

\title{
REFERENCE
}

Poyet, J.-P., 1980. Dissertation, Astronomy Department, Columbia University.

\author{
NOIES SUBMITTED BY \\ BRUCE LONG and \\ ANTHONY ROBERTS
}

\section{DOUBLE CONVECTION}

Edward A. Spiegel

\footnotetext{
"If I have seen less far than other men, it is because I have stood behind giants". - Et. al.
} 
In this lecture we will explore the codimension two bifurcation of thermohaline convection, which occurs when the heat and salt gradients are so adjusted as to make a direct and an oscillating instability both close to marginally stable. The idea is to study the mechanism by which competing instabilities bring i n complicated dynamical behavior in a situation which permits the use of current analytical techniques.

\section{A Mean Field Model}

First, let us imagine a qualitative generic physical model of double convection such a semi-convection (Moore and Splege1, 1966). Imagine a blob of fluid, volume $V(t)$, which has average density $\rho(t)$, temperature $T(t)$ and salinity $\Sigma(t)$, and which is moving vertically in a medium with density $\rho_{0}(z)$, temperature $\mathrm{T}_{0}(\mathrm{z})$, and salinity $\Sigma_{0}(z)$ (Figure 1 ). We make the following assumptions to strip the physics to its hare bones:

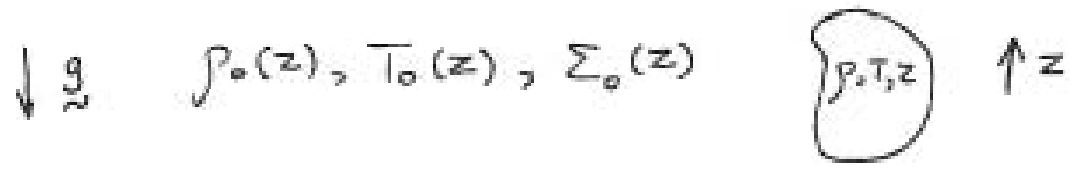

FIGURE 1.

a) Variations of $\rho_{0}, T_{0}, \sum_{0}$ are small enough so that they can be neglected except when their vertical derivatives appear explicitly. So, $P_{0}, T_{0}$ and $\Sigma_{0}$ are "constant with a nonzero derivative".

b) $P_{*}, \mathrm{~T}_{\mathrm{Q}}, \quad$ are not influenced by the parcel. We concentrate on the kinematics of the parcel, but at the expense of two more equations for changes in $d \tau_{*} / d z$ and $d \Sigma / d z$ caused by excess heat or salt transport by the parcel (see "'The History and Physics of Bouyancy in Fluids"', in this volume), we could get a fully coupled fifth order system analogous to the modal truncation of Veronis (see Welss in these proceedings), coupling the mean field back to the representative blob.

c) The drag on the parcel is negligible.

d) The rate at which salt diffuses from the blob is much slower than the heat diffusion rate, and so can be neglected (at least for a few thermal diffusion times). This is a good approximation, since $\kappa_{s} / \kappa_{r}$ $10^{-2}$ i n brine.

The momentum is:

$$
\rho V \ddot{z}=-g\left(\rho-\left|z_{1}\right|\right.
$$

Now, by assumption (a),

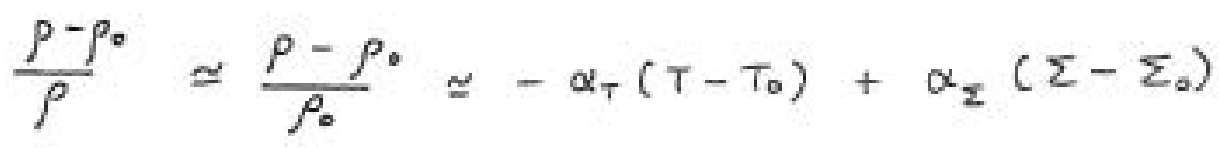

The parcel loses heat by Newtonian cooling:

$$
T=-q\left(T-T_{0}(z)\right)
$$


and keeps the initial salinity:

$$
\Sigma=\Sigma(t=0)=\text { constant }
$$

It is convenient to work with a temperature perturbation $\theta(t, z(t))=$ $T(t)-T_{0}(z)$ and a salinity perturbation $S(z(t))=\Sigma-\Sigma_{0}(z)$.

From equation (2)

$$
\dot{\theta}=-q \theta-\dot{z} \frac{d T_{0}}{d z}
$$

Rewriting the momentum equation (1) in terms of $\theta$ and $S$, we get

$$
\ddot{z}=g\left\{-\alpha_{T} \theta+\alpha_{z} s\right\}
$$

Elimination of $\theta$ gives a third order equation in time,

$$
\ddot{z}+g z \ddot{z}+g\left\{\alpha_{T} \frac{d \tau_{0}}{d z}-\alpha_{\Sigma} \frac{d \Sigma_{0}}{d z}\right\} \dot{z}+g \alpha_{\Sigma} q s(z)=0
$$

Imagine a parcel which, due to strong convection or nonBoussinesq effects has vertically varying gradients of $\mathrm{T}_{0}$ and $\Sigma_{0}$, as in Figure 2. Then (5) can, because the terms i $z$ and $\dot{z}$ are nonlinear in $z$, can produce complicated dynamical behavior since, depending on the parcel position, both, either, or neither direct or oscillating instability can be important, and the blob is kicked between different dynamical regimes.

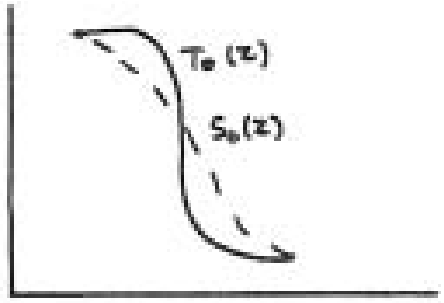

Fig. 2. Typical Mean Temperature and salinity fields.

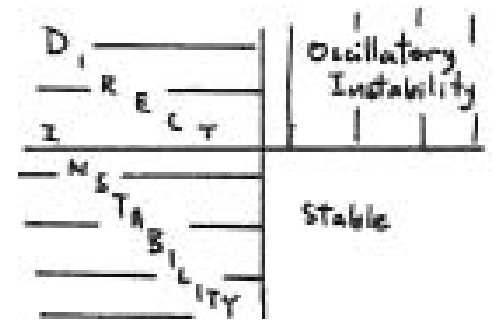

Fig. 3. Stability diagram for ideal the rmohaline convection

However, to look at the nature of the instabilities, we restrict ourselves

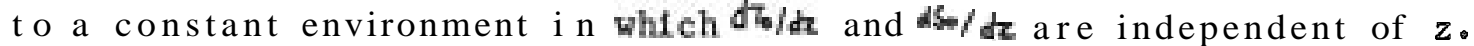
Measure $z$ from the level at which the salinity of our blob is equal to the ambient salinity $\Sigma(z)$. Then

$$
S(z)=-z \frac{d \Sigma}{d z}
$$

and (3) is a homogeneous linear equation for $z$ with eigenmodes $z(t) \propto e^{\lambda t}$, for which

$$
\lambda^{3}+q \lambda^{2}+g\left\{\alpha_{T} \frac{d T_{0}}{d z}-\alpha_{\Sigma} \frac{d \Sigma_{0}}{d z}\right\}-g \alpha_{z} z \frac{d \Sigma_{0}}{d z}=0
$$

A direct instability $(\lambda>0$, real $)$ is found if

$$
\beta^{\alpha} \alpha_{\Sigma} \frac{d \Sigma_{0}}{d z}>0 \Rightarrow \frac{d \Sigma_{0}}{d z}>0
$$


An oscillating instability ( $\operatorname{Re} \lambda>0, \lambda$ complex) is found for

$$
g \alpha_{z} q \frac{d \Sigma_{0}}{d z}<0, z\left\{\alpha_{T} \frac{d T_{0}}{d z}-\alpha_{\Sigma} \frac{d \Sigma_{0}}{d z}\right\}<-g \alpha_{\Sigma} \frac{d \Sigma_{0}}{d z} \Rightarrow \frac{d \Sigma_{0}}{d z}, \frac{d T_{0}}{d z}<0
$$

In this case, in any situation in which the potential energy can be lowered there is an instability which does it.

Physically, if $\frac{d \tau_{0}}{d z}>0$ a parcel displaced slowly downward radiates

off its excess heat and falls due to its high salinity, causing "salt

fingers". If $d \Sigma_{0} / d z<0$ and $d \bar{\tau}_{0} / d z<0$ a blob displaced down feels a strong

upward buoyancy force due to the stable density gradient, augmented by the buoyancy produced by the heat diffusing into the parcel, shooting it up faster than it came down to produce an overstable oscillation.

Clearly, when there are very small gradients of $T_{0}$ and $\Sigma_{g}$, both instabilities are nearly marginal, and small inhomogeneities in the mean field (perhaps produced by the convection itself) can bounce the parcel between regimes of oscillating and direct instability. $z(t)$ may a t different tines reflect both of these behaviors. The influence of nonzero viscosity and salt diffusion changes the particular gradients for which the instabilities compete, but the qualitative behavior near the point of competition is much the same.

\section{Reconstitution}

We will now aim to describe a co-dimensional two bifurcation in the realistic thermohaline case, by suitable recombination of the equations found by an amplitude expansion. The "reconstituted" equation, which gives a complete description of the dynamics near the bifurcation, is a Van der Pol-Duffing equation for the roll amplitude. It can be derived by a variety of means, which do not expand all variables in powers of $\varepsilon$ and thus reduce manipulation. One such technique is described in Knobloch and Proctor (preprint).

We examine Boussinesq thermohaline convection in a box with stress-free boundaries (Figure 4). The temperature and salinity are fixed on the top and bottom, while their fluxes through the horizontal boundaries are zero. Define a streamfunction $\gamma$ with $u=\psi_{x} \quad=-\Psi_{x}$. Nondimensionalize distances with $d$, times with $d^{2} / k_{T}$ and temperatures and salinities by their difference

across the layer. Work with perturbations $T$ and S fron the conduction state. Then

$$
\begin{aligned}
\nabla^{4} \psi-\nabla_{T} T_{x}+R_{6} S_{r} & =\sigma=\left\{\nabla^{2} \psi_{t}+J\left(\nabla^{2} \psi, \psi\right)\right\} \\
\nabla^{2} T-\psi_{x} & =T_{t}+J(T, \psi) \\
\tau \nabla^{2} S+\psi_{x} & =S_{t}+J(S, \psi)
\end{aligned}
$$

The nondimensional parameters are 


$$
\begin{aligned}
& \sigma=\nu / \kappa_{T} \quad \text { (the Prandtl number) } \\
& \tau=\kappa_{S} / \kappa_{T} \quad \text { (the Schmidt number) } \\
& \left.\mathcal{R}_{T}=\frac{g \alpha_{T} \Delta T d^{3}}{\nu \kappa_{T}} ; R_{S}=-\frac{g \alpha_{s} \Delta S d^{3}}{\nu \kappa_{T}} \quad \text { (the Rayle1gh numbers, with } \alpha_{T}, \alpha_{s}>0\right)
\end{aligned}
$$

Our interest centers on values of $R_{T}$ and $R_{S}$ within a small distance, call it $O\left(\varepsilon^{2}\right)$, the codimension bifurcation, that is, of the joint occurrence of the two instabilities. There are two times in the problem an $O(c)$ frequency periodic orbit due to the osclllating instability and a slow $O\left(\Sigma^{2}\right)$ frequency on which dissipation and forcing act. Thus, define

$$
8^{t_{*}}=t^{2} t
$$

so $F_{t}=\varepsilon F_{g}+\varepsilon^{2} F_{t}$

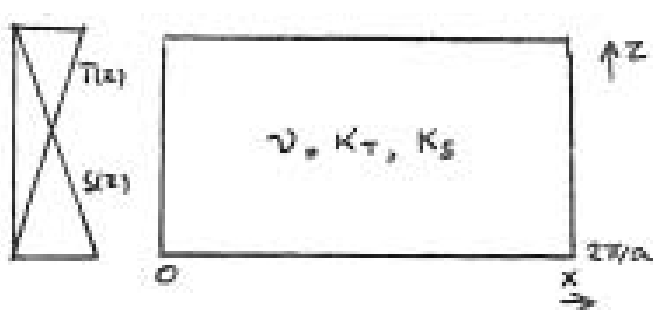

Fig. 4 The physical situation

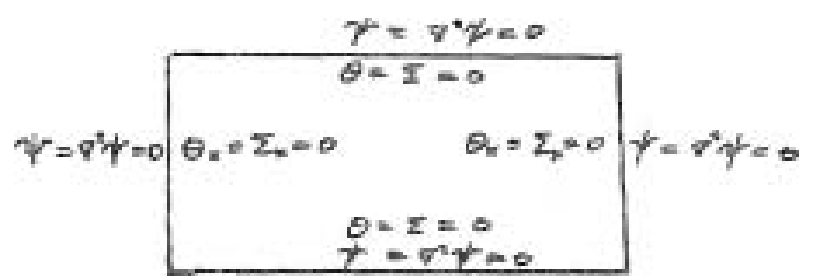

Fig. 5 Boundary condition on the scaled equation

Rescale the variables to symetrize the Inear operator and take into account the weak supercriticality

$$
\begin{aligned}
& R_{T}=M^{2}, \quad R_{S}=-\Gamma^{2} \\
& T=\frac{R}{M} \theta, \quad S=\frac{\theta}{\Gamma} \Sigma, \quad \psi=E \Psi
\end{aligned}
$$

and define a state vector

$$
\Phi=\left(\begin{array}{l}
\frac{v}{0} \\
\frac{0}{2}
\end{array}\right)
$$

The scaled equations are

$$
\begin{aligned}
\nabla^{4} \Psi-M \theta_{x}-\Gamma \Sigma_{x} & =\frac{\varepsilon}{\sigma}\left\{\nabla^{2} \Psi_{s}+\varepsilon \nabla^{2} \Psi_{t_{*}}+J\left(\nabla^{2} \Psi, Y\right)\right\} \\
\nabla^{2} \theta-M \Psi_{x} & =\varepsilon\left\{\theta_{s}+J(\Psi, \theta)+\varepsilon^{2} \theta_{t}\right\} \\
\nabla^{4} \Sigma+\Gamma \Psi_{x} & =\varepsilon\left\{\Sigma_{s}+J(\Sigma, \Psi)+\varepsilon^{2} \Sigma_{t}\right\}
\end{aligned}
$$


We expand

$$
\begin{aligned}
& \Phi=\Phi_{0}+\varepsilon \Phi_{1}+\cdots \\
& \Gamma=\Gamma_{0}+\varepsilon \Gamma_{1}+\cdots \\
& M=M_{0}+\varepsilon M_{1}+\cdots
\end{aligned}
$$

to get a sequence of linear problems, which determine $\sim$ " a $\Phi_{n}$ 's The boundary conditions are (Figure 5)

$$
\begin{array}{ll}
\psi=\nabla^{2} \psi=\theta=\Sigma=0 & \text { at } z=0,1 \\
\psi=\nabla^{2} \psi=\theta_{x}-\Sigma_{x}=0 & \text { at } z x=0, \frac{2 \pi}{a}
\end{array}
$$

$0(1)$

The analysis is pivoted on the linear operator:

$$
L=\left[\begin{array}{ccc}
\nabla^{4} & -M_{0} \partial_{\gamma} & -r_{0} \partial_{\mu} \\
-M_{0} \partial_{x} & \nabla^{z} & 0 \\
r_{0} \partial_{x} & 0 & \tau \nabla^{2}
\end{array}\right]
$$

At $O(1)$ or system (6) reduces to

$$
L \Phi_{0}=0
$$

With the given boundary conditions, we can have solutions wieh $\Phi_{0} \propto \sin n \pi z_{0}$ However, when $n \gg 1$, the system 11 be violently unstable to a sin $n a z$ mode when the $n>1$ mode is marginally stable, so we restrict ourselves to the ansatz.

$$
\Phi_{0}=\left[\begin{array}{lll}
A_{0}\left(s, t_{t}\right) & \sin a x & \sin \pi z \\
B_{0}\left(s, t_{*}\right) & \cos a x & \sin \pi z \\
C_{0}(s, t) & \cos a x & \sin \pi z
\end{array}\right]
$$

whence (8) yields a homogeneous linear system for $A_{0}, B_{0}, C_{0}$ which has a solution only if

$$
M_{0}^{2}-\frac{r_{0}^{2}}{\frac{\sigma}{6}}=\frac{q^{6}}{a^{2}}, \quad \delta^{2}=a^{2}+\pi^{2}
$$

Then the eigenvector has

$$
\Phi_{0}=A_{0}\left(s, t_{*}\right)\left[\begin{array}{cc}
1 & \sin (\operatorname{axp}) \sin (\pi z) \\
-\frac{M_{0} a}{g^{2}} & \cos (\operatorname{axpsin}(\pi z) \\
\frac{r_{0} a}{\sigma_{q^{2}}} & \operatorname{cosaxsin}(\pi z)
\end{array}\right]
$$




\section{$\underline{\text { Ad joint }}$}

For higher order in $\varepsilon$, we require a condition on the righthand side of such that a solution $\Phi_{m}$ exists. Therefore, we inspect the adjoint operator

$$
L^{+}=\left[\begin{array}{ccc}
\nabla^{4} & M_{0} \partial_{x} & -\Gamma_{0} \partial_{x} \\
M_{0} \partial_{x} & \nabla^{2} & 0 \\
\Gamma_{0} \partial x & 0 & \sigma_{\nabla^{2}}
\end{array}\right]
$$

and require $R_{n}$ be orthogonal to all solutions $\varphi$ of $L^{+} \varphi=0$ The form of

. hints that any solution $\varphi$ with vertical wave number unequal to $u$ or horizontal wave number unequal to a will automatically be orthogonal to $\Phi_{m}$ Thus only one solution is important:

$$
\underset{\sim}{\mathrm{R}_{n}}+\varphi=0\left(\begin{array}{l}
\operatorname{sinax} \sin \pi z \\
\operatorname{cosax} \sin \pi z \\
\operatorname{cosax} \sin \pi z
\end{array}\right)
$$

with the inner product defined in the natural way. Explicitly

$0=\left\langle\equiv_{n} \operatorname{sinaxsin} \pi z\right\rangle+\frac{M_{e} a}{q^{2}}\left\langle H_{n} \operatorname{cosaxsin} \pi z\right\rangle+\frac{r_{0} a}{\tau q^{2}}\left\langle z_{n} \operatorname{cosaxsin} \pi z\right\rangle$

where $<>$ is the spatial integral:

$$
\langle f\rangle=\int_{0}^{1} d z \int_{0}^{\pi / 4} d x f(x, z)
$$

$\underline{0(\varepsilon)}$

At this order the Jacoblan terms and the time dependence of the slow periodic orbit come in to produce an inhomogeneity on the righthand side, and the system (6) can be written

$$
L \Phi_{1}=\left(\begin{array}{l}
\bar{z}_{1} \\
H_{1} \\
Z_{1}
\end{array}\right)
$$

where

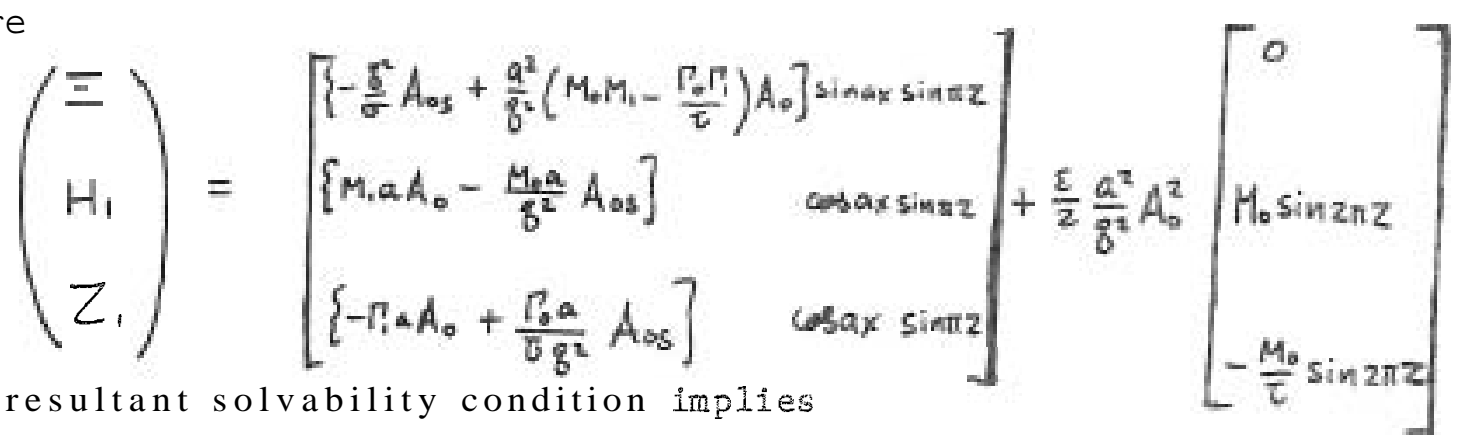

$$
M_{0}^{2}-\tau^{-1} \Gamma_{0}^{2}=-g^{6} / \sigma a^{2} \quad \text { a } \quad M_{0} M_{1}-\sigma^{-4} \Gamma_{0} \Gamma_{s}=0
$$


which, combined with the zeroth order condition (9) implies

$$
\Gamma_{0}^{2}=\sigma^{2}\left(\frac{q^{6}}{a^{2}}\right)\left(\frac{\sigma+1}{1-\tau}\right)+M_{0}^{2}=\left(\frac{\sigma^{6}}{\sigma a^{2}}\right)\left(\frac{\sigma+\tau}{1-\tau}\right)
$$

The second condition merely tells us that the only allowed $O(\varepsilon)$ changes in the Rayleigh numbers are perpendicular to the marginal stability boundary for oscillations. This is because an $0(\varepsilon)$ movement away from the doubly degenerate parameters along that boundary caused an oscillation with a frequency o( $\left.\Sigma^{1 / 2}\right)$, which is not allowed by our scaling, In fact, since we want growth and decay on a time scale $O\left(\varepsilon^{2}\right)$, and only an oscillation on time of $O(\varepsilon)$, we must choose the supercritically to be $0\left(\varepsilon^{2}\right)$, not $O(\varepsilon)$, so

$$
M_{1}=1=0
$$

We can now solve for $\Phi_{.}$. The most general solution is

$$
\Phi_{1}=\left[\begin{array}{cc}
A_{1} & \sin a x \sin \pi z \\
-\frac{a M_{0}}{\delta^{2}} A_{1}+\frac{M_{0} a}{q^{4}} A_{05} \cos a x \sin \pi z & \frac{a \Gamma_{0}}{\tau q^{2}} A_{1}-\frac{\Gamma_{0} a}{\tau^{2} q^{4}} A_{05} \cos a x \sin \pi z
\end{array}\right]+\left[\begin{array}{c}
0 \\
-\frac{a^{2}}{\delta \pi q^{2}} M_{0} A_{0}^{2} \sin 2 \pi z \\
\frac{a^{2}}{8 \pi \varepsilon^{2} g^{2}} \Gamma_{0} A_{0}^{2} \sin z \pi z
\end{array}\right]
$$

where $A_{1 \times}$ is the coefficient of the $O(E)$ contribution to the homogeneous linear problem

$\underline{O\left(\varepsilon^{2}\right)}$

One can compute from (A) the o( $\left.\varepsilon^{2}\right)$ inhomogeneity. Thus

$$
L \Phi_{2}=\left[\begin{array}{l}
\vec{U}_{2} \\
H_{2} \\
z_{2}
\end{array}\right]
$$

where we can calculate with tedious algebra

$$
\begin{aligned}
& \Xi_{2}=\left(-\frac{g^{2}}{\sigma} A_{1 s}-\frac{g^{2}}{\sigma} A_{0 t_{+}}+\frac{a^{2}}{g^{2}} Q_{21} A_{0}\right) \sin a x \sin \pi z \\
& H_{2}=\left(\frac{M_{0} a}{q^{4}} A_{0 s s}+M_{2} a A_{0}-\frac{M_{0} a}{q^{2}} A_{0} t_{*}\right) \cos a x \sin \pi z \\
& +\frac{\pi M_{0} a^{2}}{2 q^{4}}\left(-\left(1+\frac{q^{2}}{2 \pi^{2}}\right) A_{0 g} A_{0}+q^{2} A_{0} A_{1}\right) \sin 2 \pi z \\
& +\frac{M_{0} a^{3}}{4 q^{2}} A^{3} \cos a x \sin \pi z \cos 2 \pi z \\
& z_{2}=\left(-\frac{\Gamma_{0} a}{\tau^{2} q^{4}} A_{0 s s}-\Gamma_{2} a A_{0}+\frac{\Gamma_{0} a}{\tau q^{2}} A_{0 t_{*}}\right) \cos a x \sin \pi z+\frac{\pi \Gamma_{0} a^{2}}{2 \tau^{2} q^{4}}\left(\left(1+\frac{q^{2}}{2 \pi^{2}}\right) A_{0 s} A_{0}\right. \\
& \left.-\frac{g^{2}}{\tau} A_{0} A_{1}\right) \sin 2 \pi z-\frac{\Gamma_{0} a}{4 \tau^{2} \xi^{2}} A_{0}^{3} \cos a x \sin \pi z \cos 2 \pi z
\end{aligned}
$$


We have defined the symbol:

$$
Q_{m n}=M_{0} M_{n}-\frac{\Gamma_{0} P_{m}}{\tau^{n}}
$$

The solvability condition for the existence of $\Phi_{4}$ is

$$
Q_{03} A_{0 s s}+2 Q_{21} q^{4} A_{0}-\frac{1}{8} a^{2} q^{2} Q_{03} A_{0}^{3}=0
$$

Note this is a conservative equation. To leading order, energy exchanging effects on the slow time $t_{\text {do }}$ not enter the equation, because to moves around the orbits so much faster than it crosses them that the latter effect is subjugated to the next order. This system is not structurally stable, since the addition of infinitesimal dissipation destroys most of the periodic orbits of (14), so we must go to next order to recover the dissipation and get a true picture of the behavior near the bifurcation.

So, we compute $\Phi_{2}$ and use $1 \mathrm{t}$ back in (6) for the $0\left(8^{3}\right)$ behavior. To save space, its precise form will be omitted.

$\underline{O\left(\varepsilon^{3}\right)}$

The equation to be solved is

$$
L \Phi_{3}=\left(\begin{array}{l}
E_{13} \\
H_{3} \\
Z_{3}
\end{array}\right)
$$

We will only use the solvability condition, so we expand $\mathrm{m}_{3}, \mathrm{Z}_{3}, \ln$ Fourier components. The only resonant tertis is $\operatorname{sir} z / \operatorname{sinax}^{2}$, so "we isolate

$$
\left(\begin{array}{c}
\operatorname{sinax} \\
\operatorname{cosa} x \\
\operatorname{cosa} x
\end{array}\right)
$$

the coefficient of this term.

$$
\left(\begin{array}{l}
G_{3} \\
H_{3} \\
z_{3}
\end{array}\right)=\left(\begin{array}{l}
G_{3}^{(n)} \operatorname{sinaxsin} \pi z \\
H_{3}^{(4)} \operatorname{cosaxsin} \pi z \\
Z_{3}^{(1)} \operatorname{cosaxsin} \pi z
\end{array}\right)
$$

Knowing $\Phi_{2}$, we may calculate the inhomogeneous term. We find

$$
\begin{aligned}
& \Xi_{3}^{(1)}=\frac{a^{2}}{\delta^{2}} Q_{21} A_{1}-\frac{a^{2}}{q^{4}} Q_{22} A_{0 s}-\frac{g^{2}}{\sigma} A_{2 s}-\frac{g^{2}}{\sigma} A_{1 t *} \\
& H_{3}^{(m)}=M_{2} a A_{1}-\frac{M_{2} a}{q^{2}} A_{2 s}-\frac{1}{g^{2}} H_{2 s}^{(1)}+\frac{M_{2} a}{g^{2}} A_{1 t_{4}}+\frac{M_{0} a}{b^{2}} A_{0 s t_{2}} \\
& +\pi a\left[H_{1}^{(02)} A_{0}+H_{1}^{(0)} A_{1}\right]
\end{aligned}
$$




$$
\begin{aligned}
& z_{3}^{(i)}=-\Gamma_{2} a A_{1}+\left(\frac{r_{0} a}{\tau_{q^{2}}}\right) A_{2 s}-\frac{1}{\tau q^{2}} Z_{2 s}^{(i)}+\frac{\Gamma_{0} a}{\tau^{2} q^{2}} A+\frac{\Gamma_{0} a}{\tau^{2} \xi^{2}} A_{0 s t}, \\
& +\pi a\left[Z_{2}^{(02)} A_{0}+z_{1}^{(02)} A_{1}\right]
\end{aligned}
$$

$H_{m}^{(k l)}, Z_{m}^{(k l)}$ are the coskaxsinl $\pi y$ components of thand $Z_{m}$, whlch can be deduced from our earlier expressions for $\mathrm{H}_{m}$ and $Z_{\mathrm{m}} \mathrm{m}=1,2$. After gruesome agony we deduce the solvabillty condition

$$
\begin{aligned}
Q_{0 s} A_{1 s s} & +2 q^{4} Q_{21} A_{1}-\frac{3 a^{2} q^{2}}{8} Q_{03} A_{0}^{2} A_{1}=\frac{1}{q^{2}} Q_{04} A_{0 s s s} \\
+ & 2 q^{2} Q_{22} A_{0 s}-\frac{Q_{04}}{8} a^{2}\left(4+\frac{q^{2}}{2 \pi^{2}}\right) A_{0}^{2} A_{0 s}-2 Q_{03} A_{\text {ost }}
\end{aligned}
$$

Now, here we can take two tacks. First, we can find the condition ow the slow time dependence of $A_{0}$ that results from insisting that $A_{1}$ remain $O(1)$. To do this, we multiply (15) by $A_{0 g}$ and average over a large number of periods of $A_{0}$. Let $P(t *)$ be the period of the fast oscillation of $A_{0}$. Then

$$
\begin{aligned}
& \frac{1}{n} \int_{0}^{n P}\left[Q_{01} A_{1 s s}+2 q^{4} Q_{21} A_{1}-\frac{3 a^{2} q^{2}}{g} Q_{03} A_{0}^{2} A_{1}\right] A_{0 s} d s \\
& =\frac{1}{n} \int_{0}^{n p}\left[\frac{1}{q^{2}} Q_{04} \text { Aosss } A_{0 s} d s+2 \delta^{2} Q_{21} A_{0 s}^{2}-Q_{04} \frac{a^{2}}{g}\left(4+\frac{a^{2}}{2 \pi^{2}}\right) A_{0}^{2} A_{0 s}^{2}\right. \\
& \left.\quad-2 Q_{01} A_{0 s t_{+}}\right] d s
\end{aligned}
$$

Integrate the left-hand side by parts to get some boundary terms, which if $A_{1}$ remains bounded are $O(V / n)$ and an integral of the $0\left(\varepsilon^{2}\right)$ solvability condition. Thus, the left-hand side is $O(\mathrm{ln})$. The right-hand aide is simplified by substituting the of $\varepsilon^{2}$ ) solvability condition to get

$$
A_{\text {osss }}=-\left(\frac{2 Q_{21}}{Q_{01}}\right) q^{4} A_{0 S}+\frac{1}{8} a^{2} q^{4}\left(A_{0}^{3}\right)_{S}
$$

The equation (16) now reads

$$
\begin{gathered}
O\left(\frac{1}{n}\right)=\frac{1}{n} \cdot n \cdot\left\{\int_{0}^{p}\left[2 q^{2}\left(Q_{22}-\frac{Q_{21}}{Q_{05}} Q_{04}\right) A_{06}^{2}-Q_{04} \frac{a^{2}}{8}\left(1+\frac{g^{2}}{2 \pi^{2}}\right) A_{0}^{2} A_{05}^{2}\right] d s\right. \\
-\quad \int_{0}^{p} Q_{05} A_{0 s}^{2} d s
\end{gathered}
$$

or, taking the limit $n>p$,

$$
Q_{03} \frac{\partial}{\partial t y} \int_{0}^{P} A_{0 s}^{2} d s=2 \varepsilon^{2}\left\{Q_{22}-\frac{Q_{21}}{Q_{03}} Q_{04}\right\} \int_{0}^{P} A_{0 s}^{2} d s-Q_{04} \frac{a^{2}}{8}\left(1+\frac{a^{2}}{2 \pi^{2}}\right) \int_{0}^{P} A_{0}^{2} A_{0 s}^{2} d s
$$

This equation gives a recipe far the sloweffect of dissipation and forefng an 
the orbit (and can be used to find the orbit which ss asymptotically approached for large $t_{x}$ ).

An alternate way of using (15) is to use it in conjunction with the solvability condition for $A_{0},(14)$, to get an improved amplitude equation for the total amplitude $A=A_{0}+\varepsilon A_{1}$. While it is not essential for $\operatorname{small} \varepsilon$ the use of (18) to eliminate $A$ ossg from (14) puts the equation in a Van der Pol-Duffing form and reduces the order of the equation to the minimum order necessary to describe the small E physics. The time derivative is also resumed by defining the total time dexivative, in term $\mathrm{sf}$ a time $\bar{\sigma}=\boldsymbol{c} \mathbf{t}$

$$
\frac{\partial}{\partial t}=\frac{\partial}{\partial s}+e \frac{\partial}{\partial t_{*}}
$$

We add (14) to $\varepsilon$ (15), using (17), to derive the "'reconstituted' equation:

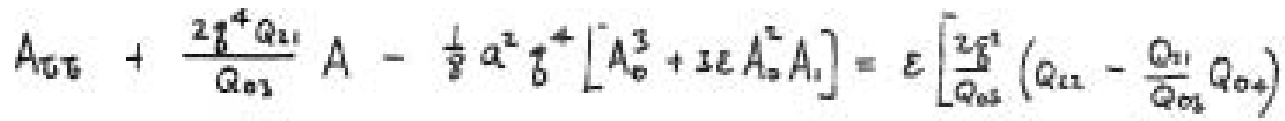

$$
\begin{aligned}
& \left.-\frac{a^{2}}{8} \frac{Q_{04}}{Q 0 \%}\left(1+\frac{8^{2}}{2 \pi^{2}}\right) A_{0}^{2}\right] A_{0 \tau}
\end{aligned}
$$

But

$$
A_{0}^{3}+3 \varepsilon A_{0}^{2} A_{1}=\left(A_{0}+\varepsilon A_{1}\right)^{3}+O\left(\varepsilon^{2}\right)
$$

and

so

$$
A=A,+O(\varepsilon)
$$

$$
\begin{aligned}
A_{\tau \tau} & +\varepsilon\left[\frac{2 a^{4}}{Q_{03}}\left[Q_{04} \frac{Q_{21}}{Q_{03}}-Q_{22}\right]+\frac{a^{2}}{8} \frac{Q_{04}}{Q_{03}}\left(1+\frac{\delta^{2}}{2 \pi^{2}}\right) A^{2}\right] A_{\tau} \\
& +2 Q_{21} q^{4} A-\frac{1}{8} a^{2} q^{2} Q_{03} A^{3}=O\left(\varepsilon^{2}\right)
\end{aligned}
$$

All the dependence of $A$ on the slow tine can be incorporated in $\frac{\partial}{\partial t} \cdot(19)$ is a "post-asymptotic equation" insofar as it combines two orders of the equation, and in fact contains both time scales of the problem. It is proposed that for $E$ not small, that (19) is the most robust description of the dynamics, even though it now neglects corrections of $O\left(\varepsilon^{2}\right) \sim O(1)$. The reason is that it represents the normal form for the co-dimension two bifurcation of thermohaline convection, and is structurally stable to the addition of higher order terms, in the sense that small perturbations make no qualitative changes in the phase plane dynamics.

\section{REFERENCES}

Arneodo, At, P. Coullet, E. A. Splegel and C. Tresser, 1981. Preprint. (See Coullet lectures in this volume).

Knobloch, E. and M* R* E. Proctor, 1981. Submitted to JWM。 
Moore, D.W. and E. A. Splege1, 1966. ApI.

Turner, J. S., 1973. Buoyancy Effects in Fluids Ch. 8, Cambridge University.

NOTES SUBMITTED BY

CHRISTOPHER BRETHERTON

and FAUSTO CATTANEO

\section{RECONSTITUTION METHOD FOR A SIMPLE EXANPLE AND THE LONG BUOYANCY WAVE}

Edward A. Splege 1

Reconstitution Method for a Simple Example.

Let us consider the third order differential equation

$\ddot{z}+\ddot{z}+\left(\alpha-a z^{2}\right) \dot{z}+\beta z-b z^{3}=0$

In near theory: $\quad z$ ne tt $^{t}$

$$
\eta^{3}+q^{2}+\alpha \eta+\beta=0
$$

According to the values of the parameters, two types of instability occur for the solution $z=0$

- Direct instability or stationary bifurcation (pitchfork due to symetry $z \rightarrow-z$ )

$$
\beta=0 \rightarrow \eta^{3}+\eta^{2}+\alpha \eta=0 \Rightarrow \eta=0
$$

- Overstability or Hopf bifurcation

$$
\alpha=\beta=\omega^{2}>0 \quad \eta^{3}+\eta^{2}+\omega^{2}(\eta+1)=0 \Rightarrow \eta= \pm i \omega(4 \eta=-1)
$$

Now the interesting point is that we can choose the values of the parameters such that the two types of instabllity occur almost simultaneously.

- Codimension two bifurcation

$$
\alpha=\beta=0 \quad \eta^{3}+\eta^{2}=0 \Rightarrow y^{2}=0 \quad(* \eta=-1)
$$
near the degenerate situation $\alpha=\beta=0$ the characteristic polynomial is
written as

$$
(\eta+1+O(\alpha))\left(\eta^{2}+(\alpha-\beta) \eta+\beta\right)
$$

such that we can expect that the dynamics near the bifurcation is then governed by a second order differential equation whose linear part is written obviously as

$$
\ddot{z}+(\beta-\alpha) \ddot{z}+\beta z=0
$$


The problem we want to solve is to find the pertinent or asymptotically relevant nonlinear cerms of this second order differential equation.

Fortunately, normal form theory tells us what the generic terms are for the interaction between two instabilities described by the characteristic polynomial

$$
\eta^{2}+\varepsilon_{1} \eta+\varepsilon_{2}=0
$$

The answer is

$$
\ddot{z}+\left(\varepsilon_{1}+\tilde{a} z^{2}\right) z+\left(\varepsilon_{2}+\tilde{b} z^{2}\right) z=0
$$

Now we try to find the correct amplitude expansion method to derive (2) from (1)

Scaling

$$
\begin{array}{ll}
\alpha=\varepsilon^{2} \mu & \beta=\varepsilon^{2} \nu \\
z=\varepsilon x & \tau=\varepsilon \omega t \\
x=x_{0}+\varepsilon x_{1}+\cdots & \\
\omega=\omega_{0}+\varepsilon \omega_{1}+\cdots &
\end{array}
$$

so at the first order we get

$$
\omega_{0}^{2} \bar{x}_{0}+\nu x_{0}-b x_{0}^{3}=0
$$

This is the Duffing equation

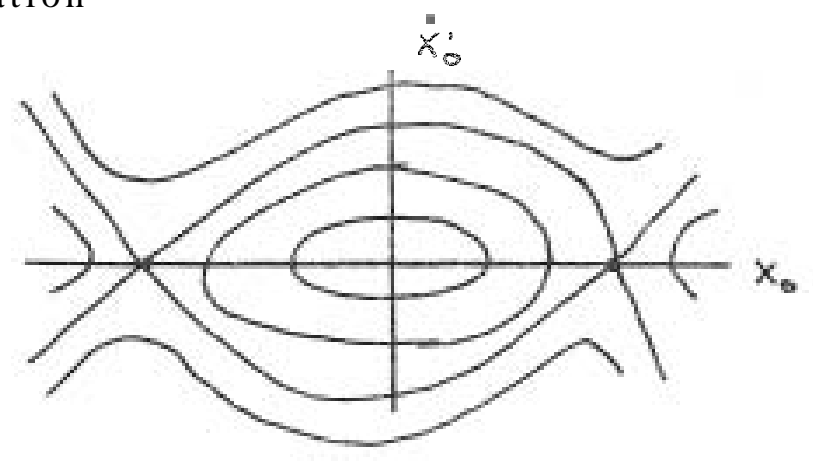

At this order the absence of dissipative terra does not allow us to determine the solutions. In orher words this equation (hamiltonian system) is not structurally stable in the set of dissipative dynamical systems. In some sense this equation is qualitatively false but for short enough times, it describes qualitatively the behavior of solutions of (1) for $\alpha, \beta$ small.

At this stage one alternative is to pick solutions of this equation and try to use the next order to determine their stability to higher order effects. In fact, we are not interested in this alternative which allows us only to determine the asymptotic solution. We wane to find the correct or generic amplitude equations. At the second order we get

$$
\omega_{0}^{2} \ddot{x}_{1}+\nu x_{1}-3 b x_{0}^{2} x_{1}=-\omega_{0}^{3} \ddot{x}_{0}-\omega_{0}\left(\mu-a x_{0}^{2}\right) \dot{x}_{0}
$$


Reconstitution Procedure.

Step 1. Remove the resonances using a solvability condition for any initial condition; $x_{0}$ is a given periodic orbit of the Duffing problem of period $\underline{\text { P. }}$

Now it is easy to verify that if we multiply (4) by $x_{0}$ and integrate over the period $P$ the left-hand side vanishes identically. This gives the solvability condition. We get at this order

$$
\omega_{0}^{2}=\frac{\int_{0}^{p}\left(\mu-a x_{0}^{2}\right) \dot{x}_{0}^{2} d \tau}{\int_{0}^{p} \ddot{x}_{0}^{2} d \tau}
$$

Step 2. In general the right-hand side of the second order equation (4) contains higher derivative in $x_{0}$. We use the first order equation to compute these derivatives

$$
-\omega_{0}^{3} \ddot{x}_{0}=\nu \omega_{0} \dot{x}_{0}-3 b x_{0}^{2} \dot{x}_{0}
$$

Then (4) becomes

$$
\omega_{0}^{2} \ddot{x}_{1}+\nu \dot{x}_{1}-3 b x_{0}^{2} x_{1}=\left[(\nu-\mu)+(a-3 b) x_{0}^{2}\right] \omega_{0} \dot{x}_{0}
$$

Step 3. Let

$$
\begin{aligned}
& \text { where } \quad x=x_{0}+\varepsilon x_{1} \\
& \text { and form } \quad x=x(e t) \\
& \text { we get } \quad(3)+c(5) \\
& \ddot{x}+\varepsilon\left[(\mu-v)-(a-3 b) x^{2}\right] \dot{x}+v x-b x^{3}=0
\end{aligned}
$$

This is the first order reconstitution of the asymptotic sequence of equation for small amplitudes. This gives the same equation as the equation obtained using the normal form procedure. The reconstitution method in the case of thermohaline convection gives the same kind of amplitude equation

$$
\ddot{A}+\varepsilon\left[k+\xi A^{2}\right] \dot{A}+\lambda A-\zeta A^{3}=0
$$

$\lambda$ and $k$ are given by

$$
\left(R_{T}\right)_{2} \text { and }\left(R_{S}\right)_{2}=\frac{R_{T, S}-R_{T, S}^{c}}{\varepsilon^{2}}
$$

$\xi$ and $\xi$ depend on the Prandt 1 numbers. the flow associated to (7) is given by

$$
\begin{aligned}
\nabla \cdot \underline{u} & =\frac{\partial \dot{A}}{\partial A}+\frac{\partial \dot{B}}{\partial B} \\
& =-\varepsilon\left(K+\xi A^{2}\right)
\end{aligned} \quad \text { where } \quad B=\dot{A}
$$


Solutions for small \& using the averaging

nondissipative case (Duffing)

integral:

$$
\ddot{A}_{0}+\lambda A_{0}-\xi A_{0}^{3}=0
$$

$$
E\left(A_{0}, \dot{A}_{0}\right)=\frac{1}{2} \dot{A}_{0}^{2}+\frac{1}{2} \lambda A_{0}^{2}-\frac{1}{4} \xi A_{0}^{4}
$$

Let $\dot{\varepsilon}=E(A, \dot{A})$

$$
\dot{\varepsilon}=-\varepsilon\left(K+\xi A^{2}\right) \dot{A}^{2}
$$

Let

$$
\bar{\varepsilon}=\frac{1}{p} \int_{0}^{p} \varepsilon(\tau) d \tau
$$

and $A_{0}=A_{0}(t, \bar{\varepsilon})$ we get $\dot{\bar{\varepsilon}}=-\varepsilon f(\bar{\varepsilon})$. This equation gives the selection of a given orbit due to the dissipation term

$$
-\varepsilon\left(K+\xi A^{2}\right) \AA
$$

Long Buoyancy Waves.

Motivation: Study long buoyancy waves in the two-dimensional the rmohaline convection with fixed flux boundary conditions.

Basic Idea: As we have seen in the previous lecture, the themohaline convection near the doubly degenerate situation with fixed temperature and salinity boundary conditions is described in terms of a second order ordinary differential equation for the most unstable mode (finite wave length): the Van der Pol-Duffing equation obtained using the reconstitution amplitude expansion method. In the fifth lecture we heard that fixed-flux boundary conditions favor large scale motions. The idea here is to derive a second order partial derivative equation using ordinary amplitude expansions, describing a wave packet with small wave number, in the case of the thermohaline convection with fixed-flux boundary conditions. This equation w1ll describe long buoyancy waves. As we shall see, the waves produced by such an equation are in some sense degenerate (lack of dissipation as in the Duffing equation). The next lecture will be a derivation of a "structurally stable" wave equation including dissipation using the reconstitution method.

Equation of the Two-Dimensional Thermaline Convection.

$$
\begin{array}{ll}
\left(\partial_{1}-\sigma \nabla^{2}\right) \nabla^{2} \psi+\sigma R \partial_{*} \theta+\sigma_{\tau} S \partial_{x} \Sigma=J\left(\psi, \nabla^{2} \psi\right) & \text { stream funtion equation } \\
\left(\partial_{t}-\nabla^{2}\right) \theta-\partial_{x} \psi=J(\psi, \theta) & \text { temperature equation } \\
\left(\partial_{t}-\tau \nabla^{2}\right) \Sigma-\partial_{x} \psi=J(\psi, \Sigma) & \text { salinity equation }
\end{array}
$$

where $R, S$ are the Rayleigh numbers

where $\sigma, \tau$ are the Prandt 1 numbers 
Boundary Conditions.

$$
z= \pm \frac{1}{2} \begin{cases}\partial_{2} \theta=\partial_{z} \Sigma=0 & \text { flux fixed } \\ \psi=\partial_{z}^{2} \psi=0 & \text { stress free but not deformable boundaries }\end{cases}
$$

Scaling and Scaled Equations.

$$
\begin{aligned}
& R=R_{0}+\varepsilon^{2} R_{2} \\
& S=S_{0}+\varepsilon^{2} S_{2}
\end{aligned}
$$

$R_{0}, S_{Q}$ are the values of the Rayleigh number which render the fluid neutrally stable and $R_{2}, S_{2}$ are arbitrary

$$
\psi=\varepsilon \tilde{\psi}>\tilde{x}=\varepsilon x>\tilde{t}=\varepsilon^{3} t
$$

We wow rewrite the equation dropping the tildes

$$
\begin{aligned}
\psi_{z z z z}= & R \theta_{x}-\tau S \Sigma_{x}+\varepsilon^{2}\left[-2 \psi_{x x z z}+\sigma^{-1}\left(\psi_{z} \psi_{z z x}-\psi_{x} \psi_{z z z}\right)\right] \\
& +\frac{\varepsilon^{3}}{\sigma} \psi_{t z z}+\varepsilon^{4}\left[-\psi_{x k x x}+\sigma^{-1}\left(\psi_{z} \psi_{x x x}-\psi_{x} \psi_{x x z}\right)\right] \\
& +\varepsilon^{5} \sigma^{-1} \psi_{t x y} \\
\theta_{z z}= & \varepsilon^{2}\left(\psi_{x}-\theta_{x x}+\psi_{z} \theta_{x}-\psi_{x} \theta_{z}\right)+\varepsilon^{3} \theta_{t} \\
\tau \Sigma_{z z}= & \varepsilon^{2}\left(\psi_{x}-\tau \Sigma_{x x}+\psi_{z} \Sigma_{x}-\psi_{x} \Sigma_{z}\right)+\varepsilon^{3} \Sigma_{t}
\end{aligned}
$$

Mow we expand $\Psi, \theta, \Sigma$ in power of $\boldsymbol{E}$

$$
\begin{aligned}
& \psi=\psi_{0}+\varepsilon \psi_{1}+c^{2} \psi_{2}+\cdots \\
& \theta=\theta_{0}+\varepsilon \theta_{1}+\varepsilon^{2} \theta_{2}+\cdots \\
& \Sigma=\Sigma_{0}+\varepsilon \Sigma_{1}+\varepsilon^{2} \Sigma_{2}+\cdots
\end{aligned}
$$

The boundary conditions are

$$
\theta_{n z}=\Sigma_{n z}=\psi_{n}=\psi_{n z z}=0 \text { at } z= \pm \frac{1}{2}
$$

Perturbation Expansion.

- Nth Order.

$$
=\left(R_{0} f-\tau S_{0} g\right)_{x} P(z) \text {, where } P(z) \text { is a given polynomial in } z
$$




$$
\begin{aligned}
& \theta_{0}=f(x, t) \\
& \Sigma_{0}=g(x, t) \\
& \quad f \text { and } g \text { are arbitrary funtions to be detemined. }
\end{aligned}
$$

- 1 st Order.

$$
\begin{aligned}
& \psi_{1}=\left(z_{0} f_{1}-\tau s_{0} g_{1}\right)_{x} P(z) \\
& \theta_{1}=f_{1}(x, t) \\
& \Sigma_{1}=g_{1}(x, t)
\end{aligned}
$$

$\mathrm{F}_{1}=\mathrm{g}_{1}$ are arbitrary functions

\section{- 2nd Order.}

The solvability condition arises at this order and gives a relation between $\mathrm{R}_{\mathrm{o}}, \mathrm{S}_{\mathrm{O}}$

$$
\left(R_{0}-S_{0}\right)=5 !
$$

This determines a critical value for the total Rayleigh number (direct instability). We have at this order a relation between $f$ and $g$

$$
f=\tau g
$$

We have for $\psi_{2}, \theta_{2}, \Sigma_{2}$

$$
\begin{aligned}
& \psi_{z}=\left[R_{0} f_{2}-\tau S_{0} g_{2}+\left(R_{2}-S_{2}\right)\right]_{x} P(z)+P_{2}(z) f_{x x}+Q_{z}(z)_{x} f_{x x} \\
& \theta_{2}=f_{2}(x, t)+H_{2}(z) f_{a x}+G_{2}(z)\left(f_{x}\right)^{2} \\
& \Sigma_{2}=g_{2}(x, t)+\tau^{-1} H_{2}(z) f_{k x}+G_{x}(z)\left(f_{x}\right)^{2}
\end{aligned}
$$

$P_{2}, Q_{2}, H_{2}=G_{2}$ are given polynomial i $z$ and where $f_{2}$ and $g_{2}$ are yet two more functions to be found.

\section{- 3 rd Order.}

The compatability condition gives

$$
R_{0}-\sigma^{-1} S_{0}=0
$$

Then we have

$$
R_{0}=\left(\frac{1}{1-\tau}\right) 5 ! \quad, S_{0}=\left(\frac{\tau}{1-\tau}\right) s !
$$

This gives the condition of the double degeneracy and we have

$$
f_{1 k x}-\tau g_{i x x}=-\left(\frac{1-\tau}{\tau}\right) f_{t}
$$

We can also compute $\psi_{3}, \theta_{3}, \Sigma_{3}$ but these expressions will be useless to compute the nonlinear wave equation for f obtained in getting the next order. 
- 4 th Order.

We get as a solvability condition the nonlinear wave equation

$$
f_{t t}-\mu \tau f_{x \times x \pi}-k \tau f_{x \times x \times x x}-\nu\left(f_{x}\right)_{x \times x}^{3}=0
$$

where

$$
\mu=\left(R_{2}-S_{2}\right) / 5 !
$$

and $k, y$ are given numerical constants. This equation is a nonlinear wave equation whose properties we are at present trying to understand.

\section{Long Buoyancy Waves.}

When the amplitude of $f$ is infinitesimal the evolution equation may be linearized and $\mathbf{i t}$ has a solution of the form

$$
f=e^{* t} \cos k x
$$

This gives us

$$
\eta^{2}=\sigma k^{6}\left(\mu-k k^{2}\right)
$$

so we have instability whenever

$$
\mu \geqslant \mu_{0}=k k^{2}
$$

if the situation is only slightly unstable, we can once again make an amplitude expansion.

Scaling and Unscaled Equations.

$$
\begin{array}{ll}
\mu=\mu_{0}+\left(\frac{\pi}{\tau}\right) \delta^{2} & \text { is an arbitrary parameter } \\
f=\delta F, \delta=\delta t &
\end{array}
$$

he get

$$
k \tau\left(F_{x x x x x x}+k^{2} F_{x x x x}\right)=\delta^{2}\left\{F_{s j}-\lambda F_{x x x x}-\nu\left(F_{x}\right)_{x x x}^{3}\right\}
$$

We expand again

$$
F=F_{0}+\delta F_{1}+\delta F_{2}+\cdots
$$

- Oth Order.

$$
F_{0 x \times x \times x x}+k^{2} F_{0 x x k x}=0
$$


with the solution

$$
\bar{F}_{0}=X(\beta) \cos (k x)+Y(\phi) \sin k x
$$

\section{- Ist Order.}

The solvability condition (orthogonally to sin and cos of the righthard side) gives two coupled equations for $X$ and $Y$.

We define

$$
\begin{aligned}
& X=A \cos \Phi \\
& Y=B \sin \Phi
\end{aligned}
$$

and get for these variables

$$
\ddot{A}-A \dot{\Phi}^{2}-\lambda k^{4} A-\frac{3}{4} v k^{5} A^{3}=0
$$

and

$$
A \dot{D}^{2}+2 \dot{A} \dot{\Phi}=0
$$

hence

$$
\dot{\Phi}=b / A^{2}
$$

where b is arkitrary. We get the equation for the amplitude

$$
\ddot{A}-\left(b / A^{2}\right)-\lambda k^{4} A-\frac{3}{4} \nu k^{5} A^{3}=0
$$

This has the integral

$$
E=\frac{1}{2} \dot{A}^{2}+V(A)
$$

where

$$
V=\frac{1}{2}\left(\frac{b^{2}}{A^{2}}-\lambda k^{4} A^{2}-\frac{3}{8} \nu k^{5} A^{4}\right)
$$

and $E$ is a constant. Solutions may be expressed in eliptic functions, but it is instructive simply to look at plots of the amplitude and phase in the following figure, here for $b=.001, \lambda=-12$ and $k=1$.

Bound solutions of this systen exiet only for negatives E and those may be expressed in terms of elliptic functions. The waves found are in some sense degenerate. Their amplitudes are arbitrary and determined by inltial condition. This is due to the fact that the amplitude equations are nondissipacive. The next step will be (next lecture) ro wge the reconstitution wethod ro get a more generic wave equation. 

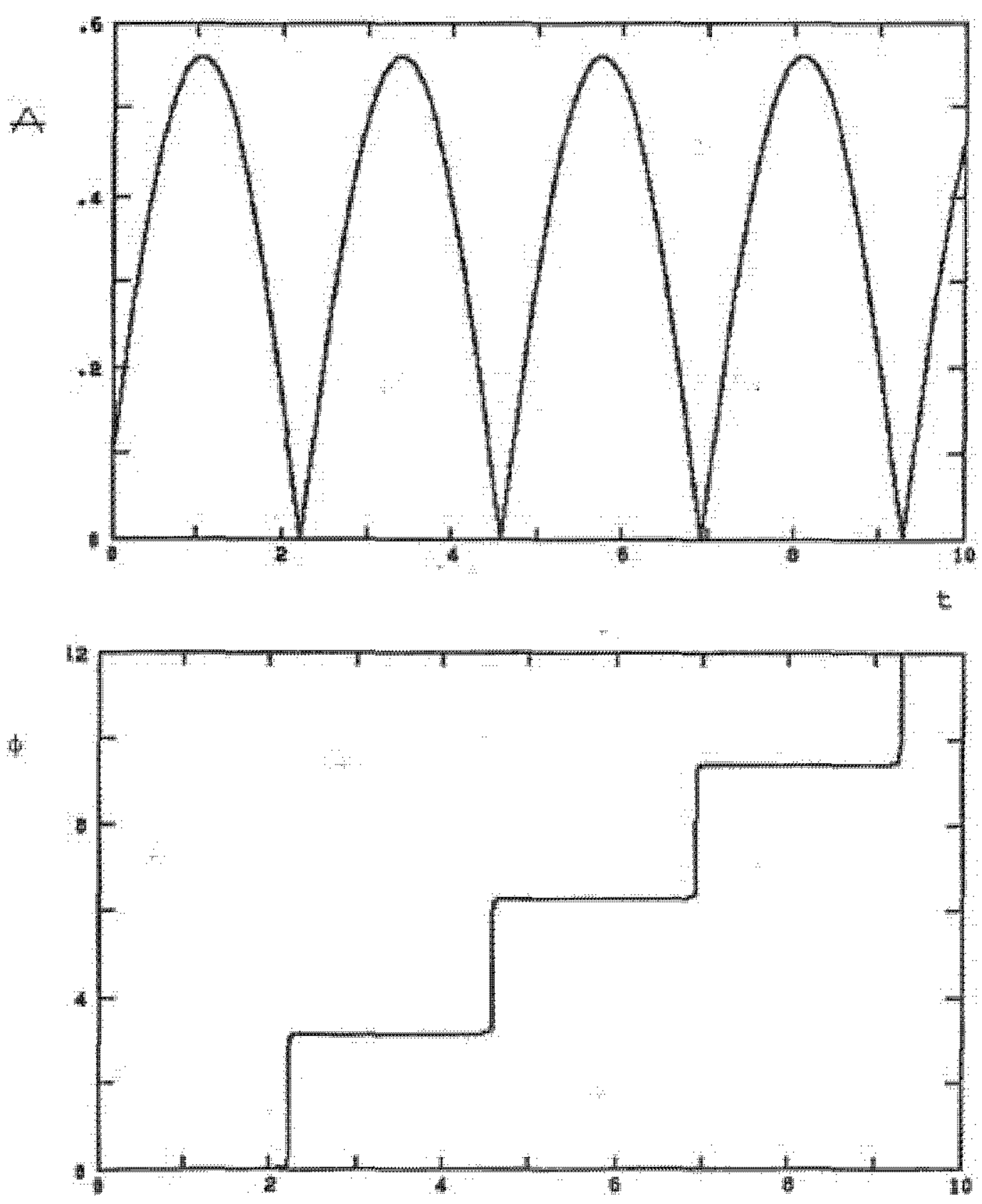

FIGURE 1. Amplitude and Phase as a Function of Time. 
Childress, S. and Spiege1, E. A., 1981. "A Prospectus for a Theory of Variable Variability", Workshop on Solar Constant Variations, NASA-CP, S-Sofia, ed.

NOTES SUBMITTED BY

PIERRE COULLET and

EVAN FISHBEIN

\section{LONG BUOYANCY WAVES AND SOLAR VARIABILITY; A GEOMETRICAL VIEW OF DYNAMICAL SYSTEMS}

\section{Edward A. Splege 1}

\section{A Mode 1 for Stellar Variability.}

One of the aims of this course has been to indicate a convective explanation of the magnetic activity cycles of the sun and certain other stars. If we look at a plot of mean annual sunspot number, we observe several important features:

1) There is a definite time scale (one crudely would say periodicity) of about 11 years -- the well-knom solar cycle.

2) There is also erratic, nonperiodic behavior.

$3)$ There is intermittency where the solar cycle is apparently turned off, as exemplified by the Maunder minimum of the late 16 th century when sunspot abundance was extresely low.

These features have usually been explained as an effect of the solar dynamo and in some sense ultimately are. 3ut we mag lmagine a model in which the underlying physical mechanisn in all of the phenomena of the activity cycle occurs as an offshoot of the main process. We focus on the Instability of a thin layer beneath the convective zone. The model permits efther monotonic growth or growing oscillations (overstabilty) of perturbations to a static state, and for Low frequencies the nearness of these instabilities in parameter space allows quite complicated dynamics. In the simplified set of third-order equations for magnetoconvection whth fixed-flux boundary conditions we will consider, the asymptotic solutions for small but finite perturbations are of the form of nonlinear waves propagating in the meridional direction. The strict Boussinesq equations require an excra rem in the heat equation, but we consider a simplified version (Spejgel and Weiss, 1981). The period of these waves is determined by the rate at which the toroidal magnetic field i forced down by penetrative convection from the overlying convective zone, and can be matched co the 11-year solat cycle. At this level of simplification we do not yet see any erratic behavior or intemittency, but the extension of the model to triple convection shows the desired chaos.

Since the fixed-flux boundary condition favors large horizontal scales, we will use the 2-D long buoyancy wave model which we developed in the preceding lecture as out starting point. 
2. Long Buoyancy Waves.

Picking up where we left off, we recall the evolution equation for the zeroth--order nondimensional temperature perturbation $f$ :

$$
f_{t t}-\mu \tau f_{x x \pi x}-\kappa_{\sigma} \sigma f_{x x x \times x x}-\nu\left[\left(f_{x}\right)^{3}\right]_{m x}=0
$$

where $\sigma$ is the ratio of magnetic to thermal diffusivity, $\mu$ is the degree of instability

$$
\mu=\frac{\left(R_{2}-S_{2}\right)}{5 !}
$$

and $K$ and $\nu$ are numerical constants. For small amplitude this nonlinear wave equation can be linearized and we obtain a solution of the form

$$
f \propto e^{\eta t} \cos k x
$$

with the dispersion relation

$$
\eta^{2}=\tau k^{4}\left(\mu-\kappa k^{2}\right)
$$

We find that whenever

$$
\mu \geq \mu_{0}=K k^{2}
$$

we have instability. If we consider only long waves of small amplitude, i.e., $\mu$ only slightly greater than $\mu_{0}$, we can again make an amplitude expansion. Letting

$$
\mu=\mu_{0}+\left(\frac{\lambda}{\tau}\right) \delta^{2}
$$

where $\delta^{2} \ll /$ and $\lambda$ is an arbitrary parameter, and scaling the amplitude $f$ and the time $t$ by $\delta$, we expand in powers of $\delta$ to obtain, after removing resonances by a set of operations analogous to those used to obtain the original $f$ equation, the zeroth-order solution

$$
f=\delta R(\delta t) \cos (k x+\theta(\delta t))
$$

Here the amplitude function $\mathcal{R}(\boldsymbol{t} t)$ and the phase $\theta(\Delta t)$ satisfy the equations

$$
\begin{gathered}
\ddot{R}-\left(b^{2} / R^{3}\right)-\lambda k^{4} R-\frac{3}{4} \nu k^{s} R^{3}=0 \\
\dot{\theta}=b / R^{2}
\end{gathered}
$$

where $b$ is another arbitrary constant and the dot denotes differentiation with respect to the slow time $\delta t$. 
The amplitude equation has the first integral

$$
\frac{1}{2} \dot{R}^{2}+V(R)=\gtrless
$$

where

$$
V=\frac{1}{2}\left[\frac{b}{R^{2}}-\lambda k^{4} R^{2}-\frac{3}{8} v k^{5} R^{4}\right]
$$

and $E$ is a constant. $E$ can be thought of as the total energy and $\forall a s$ the potential - we see a loose analogy to central force motion. Bound solutions to this system exist only for negative $E^{E}$, In terms of elliptic functions.

The form of the solutions as a function sf time is shown in Fig. 2.

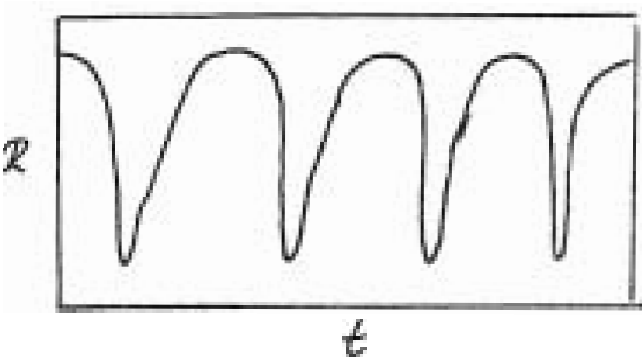

We see a sort of "solar cycle" in the periodic behavior shown by the amplitude function, while the phase diagram shows a latitude drift of magnetic activity, a magnetic curtain traveling north-south. There is no indication of any erratic behavior.

To obtain a larger class of phenomena we need to reconstitute the problem to get a more generic co-dimension two equation analogous to the Landau equation i n ordinary Rayleigh-Benard convection (Childress and Spiege1, unpublished).

We return to the basic equations as scaled in Lecture fi:

$$
\begin{aligned}
\theta_{z z}= & \varepsilon^{2}\left(\psi_{x}-\theta_{x x}+\psi_{z} \theta_{x}-\psi_{x} \theta_{z}\right)+\varepsilon^{3} \theta_{T} \\
\phi_{z z}= & \varepsilon^{2}\left(\frac{1}{\tau} \psi_{x}-\phi_{x x x}+\frac{1}{\tau} \psi_{z} \phi_{x}-\frac{1}{\tau} \psi_{x} \phi_{z}\right)+\frac{\varepsilon^{3}}{\sigma} \phi_{\tau} \\
\psi_{z z z z}= & R \theta_{x}+\tau \delta \phi_{x}+\varepsilon^{2}\left(-2 \psi_{x x z z}+\sigma^{-1} \psi_{z} \psi_{z z x}-\sigma^{-1} \psi_{x} \psi_{z z}\right) \\
& +\varepsilon^{3} \sigma^{-1} \psi_{T z z}+\varepsilon^{4}\left(-\psi_{x m x}+\sigma^{-1} \psi_{z} \psi_{x x x}-\sigma^{-1} \psi_{x} \psi_{x x z}\right)+\varepsilon^{5} \sigma^{-1} \psi_{x x T}
\end{aligned}
$$

Let

$$
\begin{aligned}
& \theta=f(x, T)+\varepsilon^{z} F(x, z, T) \\
& \phi=g(x, T)+\varepsilon^{2} G(x, z, T) \\
& \psi=(R f+\tau S g) \times P(z)+\varepsilon^{2} \chi \\
& P^{\prime v}(z)=0, \quad P\left( \pm \frac{1}{z}\right)=0, \quad P^{\prime}( \pm t)=0
\end{aligned}
$$


$-66-$

We find, on introducing

$$
\rho=R f+\sigma S g
$$

$$
\begin{aligned}
& \text { that } F_{z z}=\rho_{x x} P-f_{x x}-f_{x} \rho_{x} P^{\prime}+\varepsilon f_{T}+\varepsilon^{2}[\cdots]+\varepsilon^{3} F_{T}+\varepsilon^{4}[\cdots] \\
& \sigma G_{z z}=\rho_{x x} P-\sigma g_{x x}+g_{x} \rho_{x} P^{\prime}+\varepsilon g_{T}+\varepsilon^{2}[\cdots]+\varepsilon^{3} G_{T}+\varepsilon^{4}[\cdots] \\
& F=\left(\varepsilon f_{T}-f_{x x}\right) q^{(z)}+\rho_{x x} p_{(z)}+f_{x} \rho_{x} p^{\prime}(z)+o\left(\varepsilon^{2}\right) \\
& G=\left(\varepsilon g_{T}-\sigma g_{x x}\right) g^{(z)}+\rho_{x x} p(z)+g_{x} \rho_{x} P^{\prime}(\varepsilon)+o\left(\varepsilon^{2}\right) \\
& \text { where } \\
& q^{\prime \prime}(z)=1, \rho^{\prime \prime}=P
\end{aligned}
$$

with

$$
\begin{aligned}
& \left(\varepsilon f_{T}-f_{x x}\right) g^{\prime}\left( \pm \frac{1}{2}\right)+p_{k x} p^{\prime}\left( \pm \frac{1}{2}\right)=O\left(\varepsilon^{2}\right) \\
& \left(\varepsilon g_{T}-\tau g_{x x}\right) g^{\prime}\left( \pm \frac{1}{2}\right)+\rho_{x x} p^{\prime}\left( \pm \frac{t}{2}\right)=O\left(\varepsilon^{2}\right)
\end{aligned}
$$

Thus, $F$ and $G$ can be worked out. Then we get

$$
\begin{aligned}
X_{z 2 z z}=(R F & +\sigma S G)_{x}-2 \rho_{x x x} P^{\prime \prime}+\sigma^{-1} \rho_{x} \rho_{x x}\left(P^{\prime} P^{\prime \prime}-P P^{\prime \prime}\right) \\
& +\varepsilon \sigma^{-1} \rho_{\pi x} P^{\prime \prime}+\sigma\left(c^{2}\right)
\end{aligned}
$$

This leads to $\chi$. Putting it all together, we find

$$
\begin{aligned}
& u_{r}-u \tau v_{x x}-\kappa \tau v_{x x x x}-\left(v \tau^{2} / R_{c}^{2}\right)\left(v_{x}^{3}\right)_{x} \\
& +\varepsilon\left[(1+\sigma) \mu u_{x x}+\kappa(1+\tau) u_{x x x y}+\beta \tau \sigma^{-1} v_{7 x x}\right. \\
& \left.+2 \nu \tau R_{x}^{-2}(1+\tau)\left(v_{x}^{2} u_{x}\right)_{x}\right]=0 \\
& v_{T}-u_{x x}-\varepsilon\left[\gamma \sigma_{x x}+\tau(1+\tau) R^{-1} \nu\left(v_{x}^{3}\right)_{x}\right]=0
\end{aligned}
$$

where

and

$$
\begin{array}{ll}
u=R f+S g \quad & , v=R f+s \tau^{-1} g \\
\mu=\left(\frac{R}{R_{b}}-1\right) / \varepsilon^{2} & , \gamma=\left[R+\tau^{-1} S-\left(R_{0}+\tau^{-1} S_{0}\right)\right] / \varepsilon^{2}
\end{array}
$$

and the other quantities $\left(K_{2} \nu, \beta\right)$ are numbers that depend only on the choice of boundary conditions. 
These are the reconstituted wave equations and the waves they give have we11-determined amplitude and velocity that vary slowly,

\section{Some Speculation on Turbulence.}

What I have been trying to do in these lectures is open up topics for further research. The problems I have presented can be divided into three parts. The first category is the fundamentals. There are still veins to be tapped oven at this level, as the warm-up problem attests. At the next level we have the problems occupying the tillers of the field - how to extend the standard calculations we have become familiar with. And next we have the impossible dreams.. .

In the last category we might place the current interest in strange attractors as a model for chaos. In the usual dynamical problems we are handed a flow field $U(X)$ and asked to compute the Lagrangian variable $X$ (which we can think of as a streakline) from a set of equations of the form

$$
\dot{x}=u(x)
$$

If divU is negative, a swarm of points contracts to zero volume and we have an attractor. An example of this sort of behavior is the damped harmonic oscillator, where the dependent variables shrink to a fixed point. Other examples of an attractor are a limit cycle, or in 4-D, a torus.

A worst possible definition of a strange attractor is an attractor that is not a point, limit cycle, or torus. This does not help us much. A distinguishing characteristic of a strange attractor is the existence of erratic, nonperiodic behavior. Consider a 3-B orbit in phase space. If we insert a surface of section we get a pattern of points where the orbit intersects the surface (Fig. 3). We call this a Poincare map.

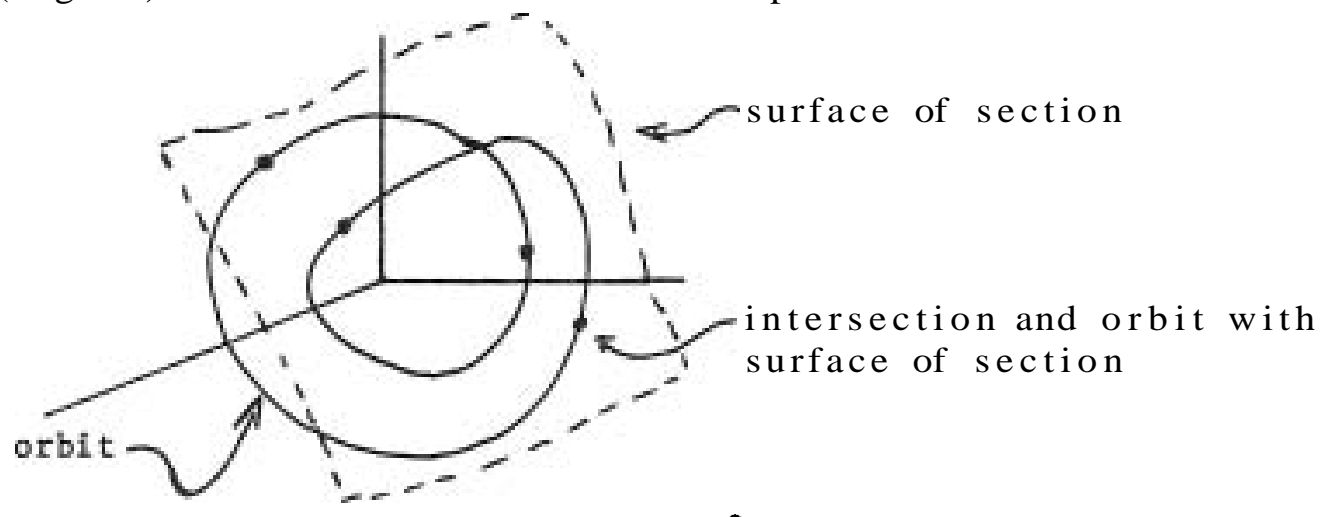

\section{FIGURE 3}

For a strange attractor we get an infinite set of points on our Poincare map, with a distribution showing a self-similar structure on all scales - a Cantor set (Fig. 4).

In the real world a system governed by a strange attractor would thus be highly sensitive to noise. Just as in the hydrogen atom, where ideally there are an infinite number of discrete energy levels, but in real life we observe a finite number of levels due to the inevitable environmental noise, the number of leaves on the Poincare map of a strange attractor is dependent on background noise. A strange attractor could be viewed as a sort of noise amplifier. Nevertheless, we do not need noise to get chaos. Erratic behavior 


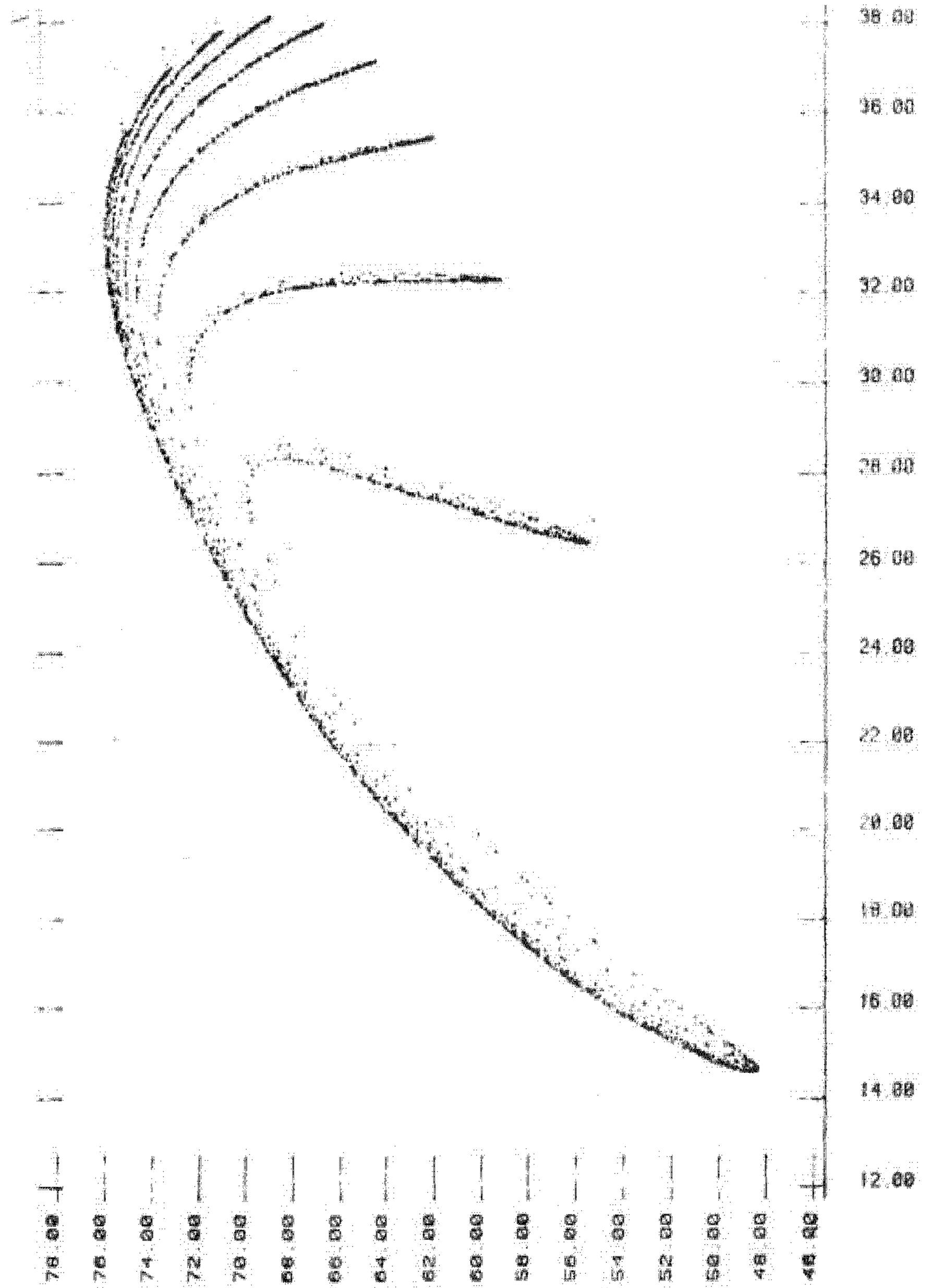

Fig. 4 Plot of Poincare Mapf $x+x+\left(\alpha-q k^{2}\right)+\beta x=0$. 
is part of the very nature of the strange attractor. This inherent chaos and that introduced by the noisy real world may both be important in realizable dynamical systems.

Some people have actually called the chaos exhibited by the strange attractor turbulence. There are others who claim that the sort of truncations that have been made on the governing equations to obtain systems with strange attractors have nothing to do with real fluid mechanics. Then what is turbulence?

The word turbulence has its root in either of the two Latin words turbo-vortex, or turba-mob. Mob is an old abbreviation for moblie. A mob of mobile vortices, if you will. Vorticity lies at the heart of turbulence. I now offer some conjectures on the nature of turbulence and the possibly related class of convection (thermohalence). These run as follows:

1) Solitary waves or objects occur in many real flows, though they are a secondary phenomenon and hard to get our hands on. We found possible solitary wave solutions in double convection; solitons can be shown to run down vortex tubes, and, vorticity being at the heart of turbulence we might expect solitary waves to be important in turbulence, too. In convection thermals are sslitary objects, so are oceanic gyres.

2) Solitary objects in an unstable situation which have "metaphorical minds". On a fast time we see a nonlinear wave on coherent structure, but on a slow time the amplitude and phase are governed by dynamical systems (ODE's) of the forn

$$
\dot{A}=f(A) ; \text { A a vector }
$$

The attractor of this systen is what $I$ mean by the metaphorical mind. The standard KdV and Schroedinger solitons are "mindless", while waves whose attractors are fixed points are "simple-minded". The waves we just studied already show interesting behavior that we might call Zitterbewegung. Certainly, waves whose minds are srrange would move chaotically. These solitary waves are the elementary objects of this vague turbulence model. An example arises when we study the reconstituted equation for triple convection with fixed flux.

3) In a fast collision our solitary objects collide more or less like solitons (particles), but on a slow time the collisions are "telepathic" in that the attractors (or metaphorical minds) interact. As a possible example of what I mean by a solitary wave with a mind of its own, the Great Red Spot of Jupiter presents itself. These are my reasonable conjectures: we enter the truly conjectural part of this lecture. If we think of solitary waves as particles, we can write theix orbits in the usual way

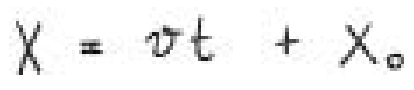

where $v$, on a long time scale, is governed by a system of equations with strange attractor and may behave chaotically. The "old one" does not need to play dice to know what such particles will do, but we have to. If we have a large number of solitary waves we essentially have a problem in statistical mechanics. Fast collisions are assumed elastic while slow collisions are strongly telepathic. Unfortunately we do not know the collision rules, but some clues ought to be calculable from the buoyancy wave theory. 
The picture this suggests is an analog of kinetic theory in which the particles can interact simply on a fast time and in which the representative points on each attractor can disturb each other. In fact, one may simply assume that all of the particles have identical minds so that we can visualize a 11 of their representative points on the same attractor. When two or more of them interact, they conceivable distort the attractor itself. So that is the kind of problem I want to study: a large number of representative points moving on an attractor, but with the attractor itself influenced or even shaped by the interactions. How caw we write a theory that allows such possibilities? Consider the system

$$
\dot{x}=u(x)
$$

The effect of the attractor is measured by $\nabla . u$; I see this as similar to a gravitational attractor. Let

$$
g_{i j}=\frac{1}{2}\left\{\frac{\partial u_{i}}{\partial x_{j}}+\frac{\partial u_{j}}{\partial x_{i}}\right\}
$$

and think of $g_{i j}$ as being like a gravitational potential. Similarly, think of $\nabla \times u$, the vortieity, as being like a magnetic field; and since $u, s$ the vector potential for vorticity, it is like the electromagnetic potential. So motion around this attractor has a loose analogy to motions in an EM + gravitational field. This should not be taken literally, but the analogy points to ways in which we can model the attractor. One description that has been used to study motion in gravitational and electric fields is to introduce Finsler geometry (Stephenson and Kilmister, 1953). That is what I do for the dissipative dynamical system.

Suppose you consider a space with coordinates $x^{i}$ and a line element

$$
d s=L\left(x^{i}, d x^{j}\right)
$$

such that

$$
L\left(x, k d x^{j}\right)=k L\left(x^{i}, d x^{i}\right)
$$

Let $x^{\prime}=\frac{d y d}{d S} \quad$. Then the geodesics given by

$$
\delta \int L\left(x^{i}, \dot{x}^{j}\right) d s=0
$$

are the orbits of a Hamiltonian system with $L$ as its Lagrangian.

Let

$$
d s=u_{i} d x^{i}+\sqrt{g_{i j} d x^{i} d x j}
$$

where units are chosen suitable to make this nondimensional. The first part of ds, uds, is the usual action. The second part corrects in some way for the effect of the attractor. The second part corrects in some way for the effect 
of the attractor. The geodesics are given by

$$
\ddot{x}^{i}+\left\{\begin{array}{c}
i \\
j k
\end{array}\right\} \dot{x}^{j} \dot{x}^{k}+g^{i j} \omega_{j k} \dot{x}^{k}=0
$$

where

$$
\left[\begin{array}{c}
i \\
j k
\end{array}\right]=\frac{1}{2} g^{i l}\left[g_{j l, k}+g_{k \ell, j}-g_{j k, \ell}\right]
$$

and

$$
\omega_{j k}=\frac{1}{2}\left(\frac{\partial u_{j}}{\partial x_{k}}-\frac{\partial u_{k}}{\partial x_{j}}\right)
$$

In other words, this geometry contains all the kinematic information of the original system and it has some close correspondences. These will have to be told in a future summer. I just want to close by noting that the model, too, can be closed by expressing gij in terms of what the particles on the attractor are doing. If there is a strange attractor for turbulence, I imagine that it will be like that.

I cannot predict this yet, but it appears that the Hamiltonian system develops caustics near where the original system has an attractor. So the particles in the associated system spend a lot of time in the right place. This opens up the possibility of a statistical mechanic for such systems when they are embedded in a heat bath. I am sorry that my time is up and I cannot tell you more about this, but you can perhaps see how it goes.

These are problems for one's dotage, perhaps. I now end the course, and propose to begin my dotage. 
SEMINARS AND ABSTRACTS OF SEMINARS

\title{
PATTERN GENERATION AND TRANSITION TO TURBULENCE I N THERMAL CONVECTION
}

\author{
Friedrich H. Busse
}

Among the fluid systems that exhibit a gradual onset of turbulence convection in a layer heated from below with a large horizontal extent is of special interest because of the approximate homogeneity and isotropy with respect to two spatial dimensions. Unlike the onset of Taylor vortices in unstable circular Couette flow or the onset of convection in small convection boxes, the linear problem is highly degenerate. Because of this degeneracy randomness is introduced when convection sets in without controlled initial conditions. The convective instability acts as a selective amplifier of microscopic noise present in the layer at the point when the critical value of Rayleigh number is exceeded. Thus the theoretically predicted solution of two dimensional rolls is realized only locally. The direction of rolls varies randomly from one patch to another and a steady state is achieved only when the influence of sidewalls finally becomes important throughout the experimental layer. In some experiments (Ahlers and Behringer, 1978) it is found that for sufficiently large aspect ratio (and low Prandtl number P) a truly steady state is never achieved. There does not seem to be available an adequate theory to deal with patchiness and long time evolution of convective patterns.

The theory of Schlutes, Lortz and Busse (1965) is capable of describing rather arbitrary patterns by considering solutions of the form

$$
u_{z}=f(z, \alpha) \sum_{n=-N}^{u} c_{n} \exp \left(i \underset{n_{n}}{z}\right)
$$

with the properties

$$
\left|k_{n}\right|=\alpha, \quad k_{m}=-k_{n}, \quad k_{m} \cdot \lambda_{\nu}=0, \quad c_{m}^{*}=c_{m}
$$

where $\underset{\sim}{\lambda}$ is the vertical unit vector. But in applying the theory the assumption is made that $\left|k_{n}-k_{m}\right| \gg L^{-1}$ for $n \neq m$ where $L$ is horizontal length of the layer. The theory and its extensions (Busse, 1967) are capable of predicting the stability regions of roll-and hexagon convection. Experiments by Krishnamurti (1968) and Ahlers (1980) have confirmed the predictions quantitatively.

In addition to the randomness introduced by the patchiness of convection there is an intrinsic origin of chaotic behavior caused by higher bifurcations. Bifurcations from the roll solution can be investigated by numerical analysis and a survey of the stability boundaries in the $R-P-\alpha$ space can be found in a recent review (Busse, 1978). Experimentally the stability boundaries have been studied by using controlled initial conditions (Chen and Whitehead, 1968; Busse and Whitehead, 1971) i n order to eliminate patchiness. The experimental observations indicate that the theoretically identified mechanisms of instability continue to be operative outside the regime for which the theoretical analysis is valid. Even after the rolls have been transformed into a three dimensional pattern, say of bimodal convection, the oscillatory instability occurs approximately as predicted by the theory. Instead of travelling waves standing waves are now realized. Similarly, the transition of oscillatory bimodal convection to spoke pattern convection is essentially the same as that described by the knot-instability of rolls. 
In the case of a nonrotating plane layer as well as in the case of a spherical convection layer a Ljapunow functional exists, the minima of which correspond to stable steady solutions. The existence of the functional insures the existence of at least one stable steady solution, although more than one may exist at the same time. This latter situation occurs when stable hexagon and roll patterns coexist in certain ranges of the Rayleigh number for convection layers with deviations from the Boussinesq approximation.

But, in a layer rotating about a vertical axis a Lfapunow functional does not exist. Indeed, a $s$ Kuppers \& Lortz (1969) have shown, all steady solutions become unstable when the Taylor number exceeds a critical value. The interesting time dependence that results can be understood by the concept of the statistical limit cycle (Busse, 1981). Because of the spatial variation of the phase of the Kuppers-Lortz instability a patch structure of rolis results. Rolls vary i n their orientation from patch to patch, and owing to the K-L instability patches propagate into each other. This patch interaction problem can be treated in the two dimensional ease. But even here numerical solution seems to be required. Qualitatively the statistical limit cycle concept is i n agreement with the experimental observations (Busse and Heikes, 1980; Heikes and Busse, 1980).

\section{REFERENCES}

Ahlers, G., 1980. J. Fluid Mech., 98, 137-148.

Ahlers, G. and R. P. Behringer, 1978. Phys. Rev. Lett. 40, 712-716.

Busse, F.H., 1967. J. Fluid Mech. 30, 625-649.

Busse, F. H., 1978. Rep. Prog. Phys. 41, 1929-1967.

Busse, F.H., 1981. "Transition and Turbulence", R. E. Meyer, ed., Academic Press, 43-61.

Busse, F. H. and K. E. Heikes, 1980. Science, 208, 173-175.

Busse, F. H. and J. A. Whitehead, 1971. J. Fluid Mech. 47, 305-320.

Chen, M. M. and J. A. Whitehead, 1968. J. Fluid Mech. 31, 1-15.

Heikes, K. E. and F. H. Busse, 1980, Ann. New York Academy of Sciences. $357,28-36$.

Krishnamurti, R., 1968. J. Fluid Mech., 33, 445-455 and 457-453.

Kuppers, G. and D. Lortz, 1969. J. Fluid Mecho, 35, 609--620.

Schluter, A., D. Lortz, and F. Busse, 1965. J. Fluid Mech., 23, 19-144.

\section{AN INTRODUCTION TO CHAOTIC MOTION AND STRANGE ATTRACTORS}

\section{John Guckenheimer}

This lecture is an overview of the mathematical ideas known as dynamical systems theory which appear to play a significant role in the transitions ro 
chaotic motion for fluid flow and similar physical systems. The adjective "significant" here should be interpreted in a very local sense in both space and time: the perspective represents only my own views a the current time. I make no pretense that these reflect a consensus, and some of the speculative comments may well be in error. When there is a choice between general, complex statements and simpler more restricted ones, I have opted for the latter. Our intention is to emphasize the relationship of the mathematics and the physics. More systematic accounts of the mathematics involved may be found elsewhere.

There is a varied set of experiments with fluids which one would like to interpret in terms of dynamical systems with low dimensional state spaces. This is not an appropriate place to give an account of these, but the recent volume edited by Swinney and Gollub (1981) provides a comprehensive survey for some of these. The experiments are ones in which the fluid motion undergoes transitions in its qualitative structure as a parameter is varied. These transitions carry the fluid from a steady state of simple motion to a state which is chaotic in time. In the typical experiment of Rayleigh-Benard convection, Gollub and Benson (1980) indicate the range of transition phenomena which are observed as a function of Rayleigh number. In all of the experiments, the test for chaotic motion has been the presence of a continuous portion of the power spectrum of a measured quantity.

The basic hypothesis which one would like to test is that the fluid transitions can be faithfully modelled by bifurcations of systems of ordinary differential equations with few variables. This hypothesis was put forward by Ruelle and Takens (1971) as a contrast to older theories which pictured turbulence as the superposition of many independent oscillatory modes. While some of the predictions of the Ruelle-Takens theory have been substantiated, it i still unclear whether low dimensional dynamical systems do provide a good representation of chaotic fluid flow. This uncertainty is due in part to the need for a better understanding of the low dimensional dynamical systems themselves. While we have achieved a good understanding of some classes of systems, we are still unable to characterize the kind of data we should expect to see if the Ruelle-Takens'hypothesis is true. This lecture is devoted to a discussion of this state of affairs.

\section{The Standard Theory Axiom A Systems.}

The most studied class of dynamical systems which possess chaotic trajectories are those which satisfy Smale's Axiom A (Smale, 1967). This class represents the archetype of chaotic behavior, and many properties have been established for these systems. We shall recall some of these because they provide a background that can be used in attempts to understand other physical systems. We shall describe everything in terms of discrete time systems in the interest of simplicity, noting that there is indeed a significant loss in ignoring the role played by singular points of continuous time systems.

Let $x=\left(x_{1} \ldots, x_{n}\right) \in \mathbb{R}^{n}$ and 1 et $f: R^{n} \rightarrow \mathbb{R}^{n}$ generate a discrete dynamical system: $\underline{x}(t+1)=\underline{f}(\underline{x}(t))$. In this section $f$ will be assumed to have an inverse $\mathrm{f}^{-1}$. A set $\Lambda \mathrm{s} \mathbb{R}^{n}$ is invariant for $\mathrm{f}$ if $\mathrm{f}(\Lambda)=\Lambda$ A hyperbolic structure for the invariant set $\Lambda$ consists of a continuous and invariant direct sum decomposition of tangent vectors based in $\wedge$ into those which are expanded and contracted by the variational mapping of $f$. More formally, there 
is a continuous decomposition $T_{\Lambda} M=E_{\Lambda}^{U} \oplus E_{\Lambda}^{S}$ and constants $\mathrm{c}>0,0<\mathrm{A} \varsigma 1$ with the properties:

$$
\begin{aligned}
& D f\left(E_{x}^{*}\right)=E_{f(x)}^{u}, \quad D f\left(E_{x}^{*}\right)=E_{f(x)}^{s} \\
& \text { If } v \in E_{x}^{5},\left|D f^{n} v\right|<c \lambda^{n}|v| \\
& \text { If } v \in E_{x}^{u},\left|D f^{n} v\right|>\left(c \lambda^{4}\right)^{-1}|v|
\end{aligned}
$$

A ciosed invariant set $\Lambda$ for is an Axiom $A$ attractor If (1) $\mathcal{A}$ has a hyperbolic structure, (2) $\Lambda$ has a nelghborhood $U$ such that $f n(x) \rightarrow \Lambda$ for a11 $x \in U$. (3) $\wedge$ has a dense orbit, and (4) periodic orbits of $A$ are dense. The first condition is the crucial one which distinguishes the uniform hyperbolicity of Axiom A attractors. In their pager, Ruelle and Takens defined a strange attractor to be one which satisfied Axiom A. Since then, the term strange attractor has been used to describe a much wider class sf invariant sets.

Many properties of Axion A systems have been proved. We mention here a few which are of relevance to our considerations. Two attractors $A,{ }_{3}$ for maps $f_{1}$ and $f_{2}$ are topologically equivalent if there is a homeomorphism $h: A,-A$, such that $h f_{1}=f_{2} h$. Colloquially, there is a change of coordinates which identifies $\Lambda$, with $\Lambda$, in a way that preserves the dynamics. An attractor $\Lambda$ for a map $f$ is structurally stable If there is a neighborhood $U$ of $A$ such that all $\left(C^{1}\right)$ perturbations $f$ of $f$ have an attractor $A$ in $U$ which is topologically equivalent to $\Lambda$ and $\pi=\bigcap_{n \geqslant 0} f^{\prime \prime}(U)$.

Theorem (Smale, 1967) Axion A attractors are structurally stable. In particular, this theorem implies that the appearance of an Axiom A attractor will be 1 ittle affected by round off errors in a numerical computation.

The second result about Axiom A atcractors which we mention involves the structure of the trajectories they contain. We shall label trajectories by the order in which they visit various sets $R_{j}$ into which the attractor has been partitioned. This process is called symbolic dynamics. Axiom A attractors possess special partitions. called Markov Dartitions. which have

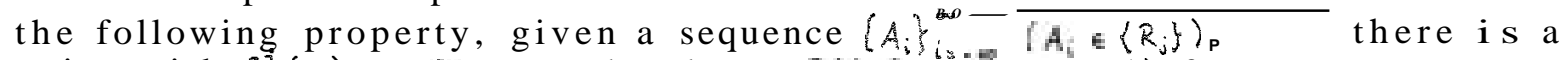
point with $f(x) \in A_{1}$ if and only if $f\left(A_{-1}\right) n A_{1+1} \neq$ ) for all 1 . In other words, the sequence of possible visits to the $\mathbb{B}_{5}$ by a paint of $A$ is determined entirely by noting which intersections of the form $f\left(R_{4}\right) \cap$ are nonempty. If one thinks of each $\mathbb{R}_{f}$ as a "course-grained state" of the system, then the map on the attractor behaves like a Markov chain for whicl there is a transition matrix describing which states can follow a given state. Theorem (Bowen, 1975) Axlom A attractors have finite Markov partitions.

The final result we mention involves the statistical properties of the attractor. This is a theorem which implies that most polnts in the attractor have the same statistical properties.

Theorem (Bowen and Ruelle, 1975) 1et $A$ be an Axiom A attractor and $U$ a neighborhood of $\Lambda$ for which $\cap f^{\prime}(U)=\Lambda$ and $f(U)<U$. Then there is a 
measure $\mu$ supported on $\Lambda$ with the following property: for a $11 \mathrm{~L}^{1}$ functions $\mathrm{g}$ and almost all $\mathrm{x} \in U$,

$$
\lim _{n \rightarrow \infty} \frac{1}{n} \sum_{i=0}^{n=1} g\left(f^{i}(x)\right)=\int_{\Lambda} g d \mu .
$$

While Axiom A attractors possess these striking attributes, it has not been easy to find them in physically defined systems. Indeed, the only examples which are known have a relatively complex geometry which seems difficult to realize in practice. Intuitively, finding a consistent grid of stable and unstable directions requires nontrivial topology because the unstable directions must be stretched and then fit back upon themselves. It appears to be much more common that a prospective field of unstable directions bends back upon itself with the uniformity of the stretching violated in the bend. Coping with this difficulty requires a consideration of systems which cannot have Axiom A attractors u t still appear chaotic.

\section{One Dimensional Mappings:}

There is an analog of the problem discussed at the end of the last section which has been analyzed thoroughly and provides insight into the prevalence of nonhyperbolic attractors. This is the question of iterating a real valued function $f \neq \mathbb{R} \rightarrow \mathbb{R}$. The examples to keep in mind are quadratic $f: f(x)=$ ax $(1-x)$. If $0<a \leqslant 4$, then $f$ maps the interval $(0,1)$ into itself in a noninvertible way. Extensive numerical calulations with these mappings and similar ones indicate the existence of invariant sets which behave much like Axiom A attractors. Though there are important differences with the Axiom A theory, the structure of these mappings and their attractors is quite well understood at this point.

Let us briefly recall the relevant results of this theory. We shall consider families of mapping $\mathrm{f}_{\mathrm{a}}$ which satisfy the following conditions:

(1) $\mathrm{f}_{\mathrm{a}}: \mathrm{I} \rightarrow \mathrm{I} ; \mathrm{I}=[0,1] ;$ a $\in\left[\mathrm{a}_{0}, \mathrm{a}_{1}\right]$

(2) $f(0)=f(1)=0$

(3) $f_{a}$ has single eritical point $c$, a maximum which is nondegenerate and independent of a.

(4) $f_{a}$ is three times differentiable and $\mathrm{Sf}_{a}=f^{\prime \prime \prime}-\frac{3}{2}\left(\frac{f^{n}}{f^{7}}\right)^{2}<0$ except a t $c$.

(5) $\mathrm{f}_{\mathrm{a}_{\mathrm{g}}}$ is onto while $\mathrm{f}_{\mathrm{a}_{e}}$ satisfies $\mathrm{f}_{a_{g}}(\mathrm{x})<\mathrm{x}$ for all $\mathrm{x} \in \mathrm{I}$.

The family $f_{a}(x)=a x(1-x), a c(0,4)$, satisfies these conditions as do $c^{3}$ perturbations of the quadratic family after coordinate changes. Families that satisfy (I) - (5) will be called full.

There is a great deal of rigidity in the structure of one dimensional mappings which is reflected in properties that are universal among full families. These include the order in which bifurcations producing new periodic orbits appear and even metric properties involving the appearance of chaotic behavior for the first time (Feigenbaum, 1977). Of importance here is the following result. 
Theorem (Guckenheimer, 1979): $\mathrm{f}_{\mathrm{a}}$ in a full family, then almost a 11 points of I have the same 1 imit set $\Lambda$, (i.e., A is the set of limit points of the sequences $\left\{f^{n}(x)\right\}$ for almostall $\left.x \in I\right)$. There are three possibilities for $\Lambda$.

(1) A is a stable periodic orbit

(2) $\Lambda$ is a Cantor set

(3) $\Lambda$ is a finite union of intervals

Only the third case for $\Lambda$ represents truly strange-attraceor behavior. The second case represents a limiting behavior which occurs when there are increasingly fine collections of intervals which are permuted among themselves. In the second case, points with nearby initial conditions do not typically have trajectories which diverge from one another. In the third case, nearby trajectories do diverge from one another and one has sensitivity to initial conditions. Indeed, there is an $\epsilon>$ o such that all pairs of trajectories are separated by $c$. The final result which we state here is the starting point for a possible resolution of the question we posed about the prevalence of strange attractors.

Theorem (Jacobson, 1981) let $\mathrm{f}_{\mathrm{a}}$ be a full family. Denote by $\mathrm{S}$ the set of a's for which the limit set $\Lambda$ of most trajectories is a union of intervals. Then $S$ has positive Lebesque measure.

The proof of this result is quite technical, but it is worthwhile giving a hint as to why the theorem is plausible. The argument is based on an iterative construction of induced mappings. If $\tilde{I}: I \rightarrow I$ and $J \subset I$ is a closed subinterval, then the induced map $f_{J}: J-J$ is defined by $f_{J}(x)=f^{n}(x)$ if $\mathrm{f}^{\mathrm{n}}(\mathrm{x}) \in J$ but $\mathrm{f}^{i}(\mathrm{x}) \& J$ for $0<\mathrm{i}<\mathrm{n}$. Roughly speaking, the construction of an induced mapping near the critical point of a map $f$ with negative Schwartzian derivative has a tendency to increase the magnitude of derivatives at the expense of introducing jump discontinuities into the map. The basic idea of the proof is to use the induced mappings as a means for detecting when there is a stable periodic orbit.

When a map $f_{a}$ fron a full family has a stable periodic orbit of period $n$, then there is an interval containing the critical point $c$ all of whose points tend to the periodic orbit. Indeed, there is another point p which lies in an unstable periodic orbit of period $n$, so that all of the points of the interval $(p, c)$ approach the stable periodic orbit. If $\bar{p} \neq \mathrm{p}$ is the point with $f(\bar{p})=$ $f(p)$, then the induced mapping on the interval $(p, \bar{p})$ is continuous and is given by $\mathrm{f}^{\mathrm{n}}$ restricted to $(\mathrm{p}, \overline{\mathrm{p}})$. Thus we want to avold situations in which there is a continuous induced mapping.

As the parameter a varies, the function $g_{\mathrm{n}}(\mathrm{a})=\mathrm{f}_{\mathrm{a}}{ }^{\prime}$ (c) tends to oscillate wildly for large n. The situations to be avoided are ones for which $1 g_{n}(a)$ - clis very small. To investigate this possibility, one type of estimate which is required places a lower bound on $\frac{d g_{n}(0)}{d a} \mid$ a $t$ parameter values which remain of interest, with $n$ chosen by the construction of an induced mapping. Ideally, we want $\mathrm{g}_{\mathrm{n}}(\mathrm{a})=\mathrm{f}_{\mathrm{a}}$ (c) to move linearly across an interval on which an induced mapping for the interval $J$ is defined, so that $\left(g_{n}\left(a_{2}\right)-g_{n}\left(a_{1}\right)\right) /\left(a_{2}-a_{1}\right)$ would be constant over the range of a's for which $f_{a}^{n}(c)=f_{J}(c)$. This ideal cannot be realized, but the departure from the ideal can be estimated and controlled.

Next, one makes a simple estimate for the quadratic function $g(x)=d x^{2}+e$ One wants to determine for which values of $e, q(0)$ falls on a point where $q^{\prime}(0)$ 
is small. Now $q^{\prime}(x)=2 d x$, so $\left|q^{\prime}(x)\right| \leqslant 1$ in the interval $\left[-2 \alpha^{-1}, 2 d^{-1}\right]$. On the other hand, $q(x)-q(\theta)=1$ when $|x|=d^{-1 / 2}$. When $d$ is large, there will be a range of $x$ for which $|\not|(x) \mid$ is large but $g(x)-g^{(0)}$ is small since $d^{-1} / d^{-4 / 3} \rightarrow 0$ as $d \rightarrow \infty$. Another much harder set of estimates guarantees that this behavior for a quadratic function carries over to the induced mappings $f_{J}$ near the critical point $c$.

The resonance conditions which one wants to avoid are situations in which $f_{J}(c)$ falls into an interval for which $\left|f_{j}\right|$ is smaller than some $\lambda>1$. If $\left|f_{Y}^{\prime}\left(f_{J}(c)\right)\right|>\lambda$ at each step of our iteration, then $f_{J_{n}}^{\prime \prime}(c)$ grows exponentially with n. The estimates carried out above for quadratic functions then guarantee that the proportion of the remaining parameter set which leads to resonances at step ndecreases at an exponential rate. This means that the measure of the parameter space which never leads to resonance will be a product

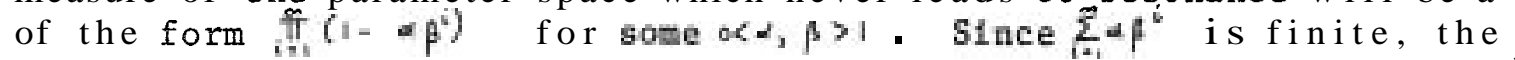
infinite product is positive. This leads to the conclusion of Jacobson's Theorem.

One would like to extend this argument of Jacobson to prove a similar result about two dimensional invertible mappings. Quadratic mappings like $F_{a, b}(x, y)=\left(y,-b x+a_{y}(1-y)\right)$ were studied numerically by Henon (1976) and appeared to have strange attractors. The map $F_{a}, b$ has a constant Jacobian $\mathrm{b}$, and yields a $\mathbf{1}$ dimensional quadratic mapping $\mathbf{l}$ the $1 \mathrm{imit} b \rightarrow \mathrm{b}$. It is apparent that some of the structure of the singular limit b $=0$ carries over to $b>0$ and that some of it does not. The question which we raise is whether the presence of strange attractors for large sets of parameter values is one of the properties that does persist for $b>0$. We believe that it does and that the basic character of the "resonance" is similar in the two cases. The issue is one that remains to be fully resolved. Indeed, there is still no example of a smooth invertible mapping of Henon type which has been proved to have a strange attractor.

\section{Experimental Data.}

The issues raised in the last two sections reveal a lack of mathematical understanding about the models with few degrees of freedom which one would like to use for the chaotic motions of fluid mechanics.

Uncertainty about the mathematical structures creates difficulty in comparing experimental results with the models. Evidence is convincing that the transition of a fluid to a state of chaotic motion follows closely the lines predicted by bifurcations of low dimensional systems. It is less clear, however, that the chaotic states which develop continue to be described adequately by systems with few degrees of freedom.

In this section we shall discuss a few ideas about ways that one can deal with chatic data. Similar techniques have been discussed by Takens (1981) and the Santa Cruz Dynamical Systems Group (Packard, et. al., 1980), but we shall put our emphasis in a slightly different place.

In a 11 cases, one makes the observation that a single time series almost always provides enough information to faithfully reconstruct an attractor of a system with a finite number of degrees of freedom. Let the given time series be $v(t)=v(x(t))$, measuring one quantity of the system along a trajectory $x(t)$. For most choices of $v$, the map from the phase space $\mathbf{P}$ of the system $\tilde{v}: \mathbb{P} \rightarrow \mathbb{R}^{n} \quad$ definedby $\tilde{v}(x)=(v(x(n)), v(x(n), \ldots, v(x(n-1)) \quad$ will embed $P$ into $\mathbb{R}^{n}$ provided $n$ is larger than twice the dimension of $\mathbb{P}$. This is an easy 
consequence of the Whitney Embedding Theorem. If the dynamics of a fluid system are reducible to one with a finite number of degrees of freedom, then this process should produce a representation of an experimental time series which looks like an attractor in $\mathbb{R}^{m}$. Moreover, the appearances and structure of this attractor should be unchanged by increasing $n$ beyond a certain $n_{0}$.

This view of a time series leads directly to a dimension which is called the capacity (or Hausdorff dimension) of the corresponding attractor. One measures the size of the attractor by counting the number of elements visited by the time series $\vec{\nabla}$ in a partition of $\mathbb{R}^{n}$ by a grid of uniform mesh size. The capacity measures the rate of exponential growth in this number with decreasing mesh size. If the growth rate stabilizes at a finite value with increasing $m$, then there is a nonlinear correlation between successive elements in the time series which allows one to predict the short-term behavior $v(x(m+n)), v(x(m+n+1)),-v(x(m+w+k))$ from $v(x(m)), v(x(m+1)),-, v(x(m+n+n)$.

If $\mathrm{k}$ grows too large, relative to the mesh size, then the sensitive dependence to initial conditions within the attractor destroys this correlation. The presence of noise within the system or an infinite dimensionality for the attractor would be reflected in grid sizes for which the growth rate in the number of partition elements visited is unbounded as $n$ increases.

There is another perspective upon distinguishing strange attractors from noise that has not been emphasized previously. In Axiom A attractors there is a uniform spreading of trajectories from one another in forward and backwards time. A typical pair of trajectories which start close to one another will diverge from each other exponentially at characteristic rates given by Liapounov exponents. The Liapounov exponents of a trajectory are defined to be the characteristic rates of exponential growth for the variational equations along the trajectory. Ruelle (1979) proves that these almost always exist. Thus, we suggest that within a strange attractor the difference between two time series which approximate each other for a number of successive terms should be approximated by a sum of two exponential functions over a substantially longer period of time. The extent to which the suggestion is true may depend upon the constancy of the exponential spreading of trajectories within the trajectory along the characteristic directions, but is manifestly correct if one of the trajectories being compared is a hyperbolic periodic orbit.

Within a system which contains noise, one expects the near approach of two trajectories to occur in a way which is much more haphazard. In the idealization of a system with superimposed Gaussian noise, the distances between successive observations will be a function which is erratic on the scale of the variance of the noise and does not resemble a sum of two exponentials. There are many gradations between the extremes of a limit cycle with superimposed noise and an Axiom A attractor, so we cannot draw a sharp dichotomy. Rather we suggest that matching trajectories which approach one another and examining the statistics of their differences should allow one to measure the effective predictability and noise level within the data, even if the underlying deterministic dynamics are chaotic. The development of statistics along these lines should help determine more conclusively whether strange attractors of low dimensional systems do provide good models for chaotic fluid motions.

\section{REFERENCES}

Bowen, Ro, 1975. Equilibrium states and the ergodic theory of Anosov diffeomorphisms, Lecture Notes in Math $\$$ \$70. 
Bowen, R. and D. Ruelle, 1975. Ergodic theory of Axiom A flows. Invent. Math. 29, $181-202$.

Feigenuaun, M*, 1977. J. Stat. Phys., 19, 25.

Go1lub, J. and S. Benson, 1980. Many routes to turbulent convection, JFM, 100, $449-470$.

Guckenweimer, J., 1977. On bifurcations of maps of the interval. Invent. Math. $\quad 39,165-178$.

Guchenheimer, J., 1979. A brief introduction to dynamical systems. Lectures i n Appl. Math. 1/17, 187-253.

Gucnenheimer, J., 1979. Sensitive dependence to initial conditions for one dimensional maps. Comm. Math. Phys.. 70, 133 .

Guckenheiner, J., 1981. Multiple bifurcations of $\sim$ dimensiawo. Proceedings Warwick Symposium. (in press).

Guckenheimer, J., J. Moser, and S. Newhouse, 1980. Dynamical Systems, Bressanone Lectures 1978, Birkhauser.

Henon, N., 1976. On a two dimensional mapping with a strange attractor. Comm. Math. Phys., 50, 69.

Jacobson, Y., 1981. Comm. Llath. Phys. $\underline{81}, 39-88$.

Packard, N., J. Crutchfield, D. Farmer, and R. Shaw, 1980. Phys. Rev. Lett., 7UA, 1 .

kuelic, D., Iy79. Ergodic theory of differentiable dynamical systems. Publ. I. H. E. S. , 50, 27-28.

Kivelle, D. and F. Takens, 1971. On the nature of turbulence. Comm. Math. Phys., 20, 167-192.

Snale, S., 1967. Cifferentiable dynamical systems. Bull. Am. Math. Soc., 73, $748-817$.

swintley, He anc J. Gollui, Eds, 1981. lydrodynamic instabilities and the transition to turbulence. Springer-verlag.

lakens, F., 1981. Proceedings Warwick Symposium. (in press).

PERIOD DOUBLING AND THE TRANSITION TO CHAOS FOR SIMPLEST DYNAMICAL SYSTEM

Pierre Coullet

\section{Introduction.}

Cascaút of period doubling bifurcations occurs in many systems as the precursor of chaotic behaviors. 
Some examples:

1) Experiment of Maurer and Libchaber (convection at small aspect ratio 1 helium). Roughly they have observed the following route to turbulence (temporal chaos).

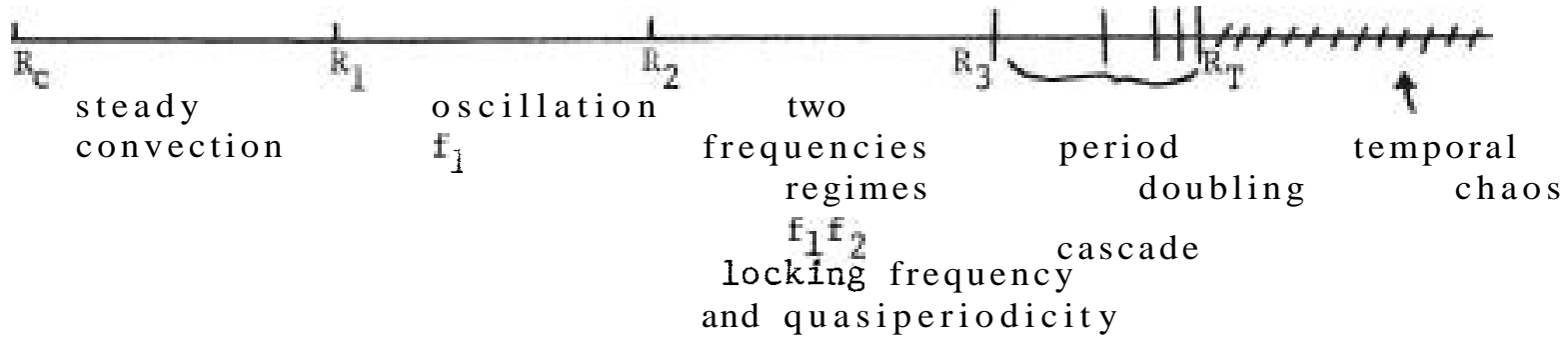

2) Various systems of ordinary differential equations in dimensions greater or equal to three (two for periodically driven systems).

Some examples:

- the normal form of the interaction between three instabilities with the characteristic equation $\lambda^{3}+\mu \lambda^{2}+\nu \lambda+\eta=0$

$$
\ddot{x}+(\beta+\alpha x) \ddot{x}+(\nu+\beta x+\gamma \dot{x}) \dot{x}+(\eta+\delta x) x=0
$$

- Lotka-Volterta equations for three species

$$
\partial_{t} N_{i}=N_{i}\left(\mu_{i}+\sum_{j} \nu_{i j} N_{j}\right) \quad i=1,3
$$

- nonlinear oscillators driven-periodically

$$
\begin{aligned}
& \ddot{x}+\nu \bar{x}+\frac{\partial \nu(x, \lambda)}{\partial x}=0 \\
& \lambda=\lambda_{0}(1+f \cos g t) \text { for various } V
\end{aligned}
$$

For example the rigid pendulum with periodic modulation of the frequency

$$
\ddot{\theta}+\nu \dot{\theta}+\omega_{0}(1+f \cos \Omega t) \sin \theta=0
$$

- nonlinear coupling between two oscillators. For example two Van Der Pol oscillators

$$
\ddot{x}_{i}+\left(\mu_{i}+\sum_{i j} \nu_{i j} x_{j}^{2}\right) \dot{x}_{i}+\omega_{i}^{2} x_{i}=0 \quad i=1,2
$$

J) Various invertible mapping of the plane

$$
\begin{aligned}
& x_{i+1}=f_{\mu}\left(x_{i}, y_{i}\right) \\
& y_{i+1}=g_{\mu}\left(x_{i}, y_{i}\right)
\end{aligned}
$$

the most popular example - the Henon mapping

$$
\begin{aligned}
& x^{\prime}=1-\mu x^{2}+\epsilon y \\
& y^{\prime}=x
\end{aligned}
$$

4. Woninvertible mappings of the interval

$$
x_{i+1}=f_{\mu}\left(x_{i}\right)
$$

example: the logistic difference equation

$$
x_{i+1}=\mu \times(1-x)
$$


II. Period Doubling Bifurcation: A Generic Bifurcation For Periodic Orbits.

Let us consider the system of O.D.E.

$$
\partial_{t} x=F_{\mu}(x) \quad x=x_{1} \ldots x_{n}
$$

Let $\varphi_{\mu}(t)$ a periodic orbit of (1) with period $T_{\mu}$

$$
\partial_{t} P_{\mu}(t)=F\left(\varphi_{\mu}(t)\right) \quad \varphi_{\mu}\left(t+\tau_{\mu}\right)=\varphi_{\mu}(t)
$$

to study the stability of $\varphi_{\mu}(t)$ we can consider the variational equation

$$
\partial_{t} y=F_{\mu}\left(y+\varphi_{\mu}\right)-F_{\gamma}\left(\varphi_{\mu}\right) \quad \text { where } y=x-\varphi_{\mu}(t)
$$

this is a nonlinear system with periodic coefficients. Its linearization near 4

$=0$ gives the information of the local stability of $\varphi_{\mu}(t)$

$$
\partial_{t} y=\left.\frac{\partial F_{\mu}}{\partial x}\right|_{\varphi_{\mu}} y
$$

Now we can apply the floquet theory. Starting with an initial condition $0=y_{1}(0)_{3} \ldots y_{n}(0)$ if we integrate (3) until $t=T_{\mu}$ we get $y\left(T_{\mu}\right)=y_{1}\left(T_{\mu}\right)_{2} \ldots y_{n}\left(T_{\mu}\right)$. we want to know the matrix $C$ which map $y(0)$ to $y\left(T_{\mu}\right)$

$$
y\left(T_{\mu}\right)=c_{y}\left(o^{\prime}\right.
$$

C can be thought of as the period advanced mapping. To do that we start with the initial condition

$$
\delta_{k}=(0,0,1,0 \ldots 0)
$$

Let us integrate (3) on a period $T_{\mu}$ - We get

$$
\Delta_{k}=\left(\Delta_{k 1}, \Delta_{k 2}, \ldots, \Delta_{k n}\right) \text {. }
$$

The matrix $C$ is then given by

$$
C=\left(\Delta_{1}^{T}, \Delta_{1}^{T} \ldots, \Delta_{k}^{T}\right)=\left(\begin{array}{ll}
\Delta_{k 1} & \Delta_{k 1} \\
\Delta_{12} & \Delta_{k 2} \\
\Delta_{1 n} & \Delta_{k m}
\end{array}\right) .
$$

The stability of $\varphi$ (linear stability) is given by the eigenvalues of C (they are independent of the basis), called floquet multipliers. If $C$ is a contraction, that is, if all the eigenvalues of $\mathrm{C}$ are inside the unit circle the orbit is locally stable. If some floquet multipliers cross the unit circle the orbit loses its stability (bifurcation). In fact, one of the floquet exponents of (3) is always equal to 1 . This is due to the orbital invariance (no dilatation or contraction for an initial perturbation along the orbit) is a solution of (3) and an eigenvector of $\mathrm{C}$ with eigenvalue $\mathbb{1}$.

Typically, there exist three ways for a periodic orbit to lose its stability.

A single multiplier crosses through 1 .

A pair of complex conjugate multipliers cross the unit circle.

A single multiplier crosses through -1 . 
The last case corresponds to the period doubling. This can be intuitively understood considering $\mathrm{C} 2=\mathrm{C}\left(2 \mathrm{~T}_{\mu}\right)$ which has a nontrivial multiplier equal to one. Just before the bifurcation an initial perturbation converges to generating a Mosbius band

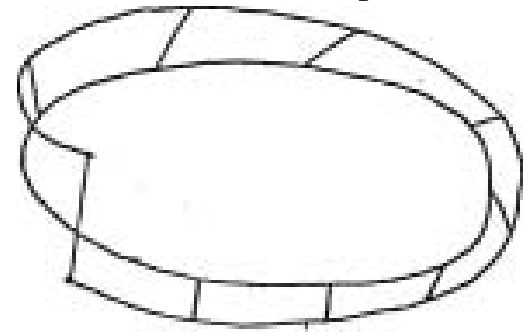

An alternative way to study the stability of a periodic orbit is to construct the Poincare map

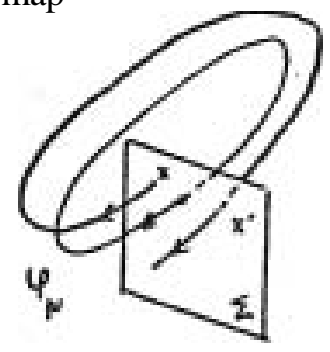

let $x=\left(x_{1}, x_{2} \ldots x_{m-1}\right)$ the coordinates on the hyperplane $\Sigma$. Let us choose the origin of the coordinate $(x=0)$ as the intersection of $\varphi_{\mu}$ and $\Sigma$.

We define a map from $\Sigma$ into $\Sigma$ in the following way:

Start with $x \approx 0$ and integrate the equations ( 1 ) until we cut $\Sigma$ with the same orientation of the velocity. Let us call $x^{\prime}$ the point of this intersection. The mapping $x^{\prime}=\Phi_{\mu}(x)$ defined in this way admits $x=0$ as fixed point. Doing this we have reduced the problem of the stability of the periodic orbit to those of the stability of $\mathrm{x}=0$ of the mapping " Let us rewrite $\bar{s}$ in the following way:

$$
\begin{aligned}
x^{\prime}=A x+F(x) \text { where } \quad A=\left.\frac{\partial \Phi}{\partial x_{i}}\right|_{x=0} \\
\quad F=\frac{x_{i} x_{j}}{2 !} \frac{\partial^{2} \frac{1}{\partial x_{i}} \partial x_{j}}{}+0\left(|x|^{3}\right)
\end{aligned}
$$

the eigenvalues of A are nothing else than the nontrivial floquet multiplier previously defined (constructing the Poincare map $\Phi$ we have avoided the problem of the existence of the trivial floquet multiplier associated to the orbital invariance). Now assume that one of the floquet multipliers is crossing the unit circle through-1 for $\mu=a$. By a linear change of coordinate, when $\mu=0, \Phi$ can be written in the following way

$$
\begin{aligned}
& Y^{\prime}=-Y+G(Y, Z) \\
& Z^{\prime}=B Z+H(Y, Z)
\end{aligned}
$$

where $Y$ represents the coordinate along the direction of the eigenvector associated to the eigenvalue 1 and $B$ is a linear contraction on the complementary space (5) can be reduced to a one dimensional mapping by computing the central manifold $Z=\psi(Y)$ (see Fig. 1).

Let us consider the quadratic terms in (5b)

$$
Z^{\prime}=B Z+H_{2}(Y, Z)+R(Y, Z) \text { where } H(Y, Z)=H_{2}(Y, Z)+R(Y, Z)
$$


Let us split $H_{2}$ in the following way $H_{2}(Y, Z)=I_{2}(Y, Z)+Q_{2}(Y)$ such as $I_{2}(Y, Z)=0$.

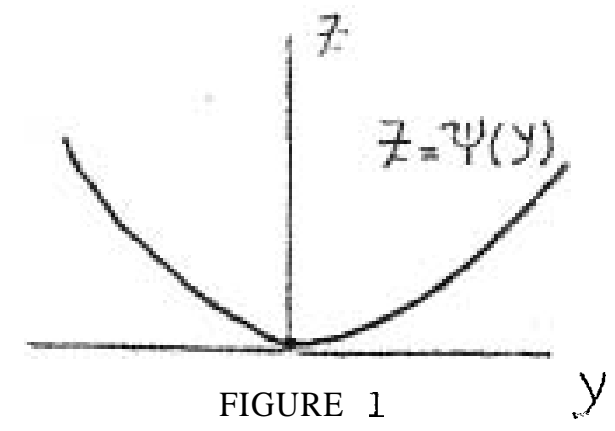

Now we introduce the change of variable

$$
\begin{array}{cc}
Z=\tilde{Z}+\psi_{2}(Y) \quad \psi_{2} \text { quadratic in } Y \\
\tilde{Z}^{\prime}+\psi_{2}\left(Y^{\prime}\right)=B \tilde{Z}+B \psi_{2}(Y)+I_{2}\left(Y_{1} \tilde{Z}+\psi_{2}(Y)\right)+Q_{2}(Y)+R\left(Y, \tilde{Z}+\psi_{2}(Y)\right)
\end{array}
$$

At the quadratic order we get

$$
\psi_{2}(-Y)=B \psi_{2}(Y)+Q_{2}(Y)+I_{2}(Y, \tilde{Z})
$$

We can croose $\psi_{2}$ such as

Since $\psi_{2}(-\gamma)=\psi_{2}(\gamma)$

$$
\psi_{2}(-Y)-B \psi_{2}(Y)=Q_{2}(Y)
$$

$$
\psi_{2}(\gamma)=(1-B)^{-1} Q_{2}(\gamma)
$$

the matrix 1- $B$ is always invertible since $\mathrm{E}$ has no eigenvalue equal to one. In the new variables (5) write

$$
\begin{aligned}
& Y^{\prime}=-Y+F\left(Y, \tilde{Z}+\psi_{2}(Y)\right) \\
& \tilde{Z}^{\prime}=B \tilde{Z}+I_{2}(Y, \tilde{Z})+\tilde{R}(Y, \tilde{Z})
\end{aligned}
$$

So that if we consider (6b) at the quadratic order, $\ddot{Z}=0$ is an invariant manifolv (stabic at least near the origin) and the dynamic on this manifold can be studied by (6a) setting $\widetilde{Z}=0$. Let us decompose $F$ in the following way:

$$
F(Y, Z)=F_{20} Y^{2}+Y Z_{i} F_{11}^{i}+Z_{i} Z_{\delta} F_{02}^{i j}+Y^{3} F_{30}+Y^{2} Z_{i} F_{21}^{i}+Y Z_{i} Z_{j} F_{12}^{i j}+Z_{i} Z_{j} Z_{k} F_{03}^{i j t}
$$

heplacing $z$ by $\Psi_{2}(\gamma)$ in this expansion $(Z=0)$ we get

$$
F\left(Y, \psi_{2}(Y)\right)=F_{20} Y^{2}+Y^{3} \psi_{2}^{i} F_{30}^{i}+Y^{3} F_{30} \quad+\text { higher order terms }
$$

so that in a consistent way the problem is reduced to study the mapping

$$
Y^{\prime}=-Y+\alpha Y^{2}+\beta Y^{3}+\text { higher order terms }
$$

where $\alpha=F_{20}, \quad \beta=F_{30}+\sum_{i} \psi_{2}^{i} F_{4}^{i}$ 


$$
\psi_{1}^{i} \text { is defined by } \psi_{2}(\gamma)=\left(\begin{array}{c}
\psi_{1}^{(i)} \gamma^{2} \\
\vdots \\
\psi_{2}^{(i)} \gamma^{2} \\
\vdots
\end{array}\right)
$$

the vector $\Psi_{z} \quad 1$ s given by $\Psi_{2}=(0-8)^{-4} Q_{2}$

$$
Q_{2}(Y)=\left(\begin{array}{c}
Q_{2}^{(1)} y^{2} \\
\vdots \\
Q_{2}^{(i)} Y^{2}
\end{array}\right)
$$

This procedure can be continued to compute the terms of order 4 and 5 i n (7) by eliminating the cubic tern constant inz(6b), but in fact we need only the cubic and quadratic terms in (7) to describe the behavior of the Poincare map near $\mu=0$,

In (7) the quadratic tern $\alpha \gamma^{2}$ can be removed by the change of variable

$$
Y^{\prime}=x+A x^{2}+O\left(x^{2}\right)
$$

(7) becomes

$$
x^{\prime}=-x+\hat{\beta} x^{8}+o\left(x^{4}\right)
$$

The cubic term in (7) cannot be removed: it is a resonant term. Now to study the behavior of (8) near the bifurcation situation, we introduce a smal1 parameter $v$ linearly related to $y$.

$$
x^{\prime}=-(1+\nu) x+\tilde{p} x^{2}+o\left(x^{4}\right)
$$

For $y<0$ the fixed point $x=0$ is stable. For $\nu=0$ it loses its stability and we have a super or subcritical bifurcation (according to the sign of giving rise to a periodic orbit of period 2.

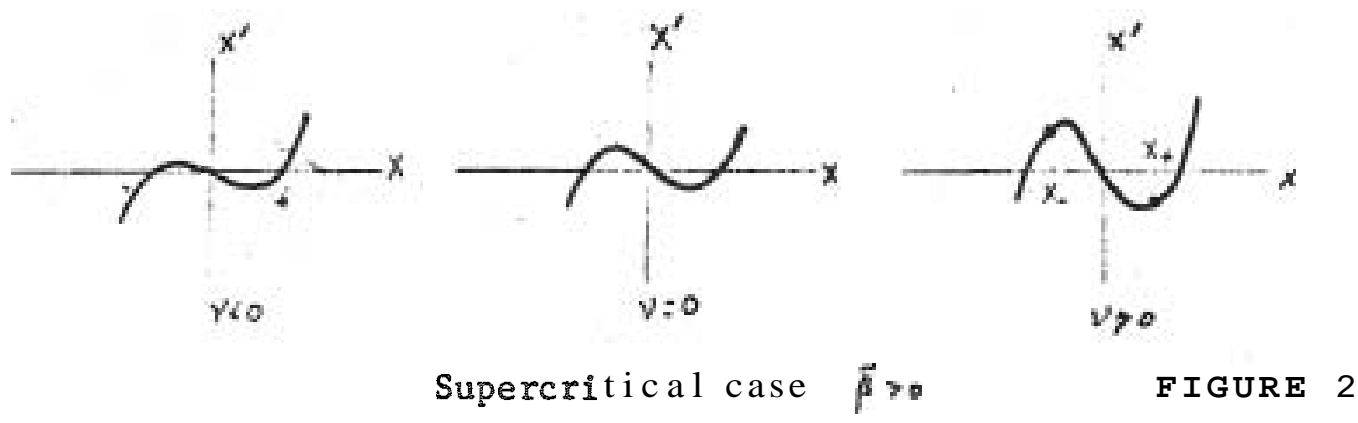

As we have already mentioned this kind of bifurcation is often observed to occur in cascade. The most simple example of such a phenomenon arises in the problem of iteration of one dimensional noninvertible map, e.g., $x^{\prime}=1-\mu x a$ 
The physical Interest of such maps is that they model quite well the behavior of more realistic (invertible) maps of the plane which at their turn are good models for Poincare maps of differential equationss

As an example, let us consider the Henon map

$$
H_{a, e} \in\left\{\begin{array}{l}
X^{\prime}=1-a X^{2}+\in Y \\
Y^{\prime}=X^{\prime}
\end{array}\right.
$$

M. Henon has proposed this map to model some numerical Poincare maps of the Lorenz equations gotten by Ibanez and Pomeau for values of $\mathbb{R}$ (Rayleigh number i $n$ the Lorenz system) where they observe the period doubling cascade. In fact this kind sf map is in some sense an analytic realization of the Smale horseshoe map and a good qualitative model for Poincare maps of any differential equations which exhibit period doubling bifurcatf on.

$H_{a, 0}$ is an area contracting mapping; $\epsilon$ is the rate of contraction ateachiteration; when $\leftrightarrow \rightarrow 0$ the Henon map becomes the noninvertible dimensional map

$$
X^{\prime}=1-a x^{2}
$$

so that for $E$ small enough the qualitative dynamics $\mathrm{sf}$ the Henon map is we11 approximated by those of this quadratic map.

\section{Qualitative Dynamies of the One Dimensiona1 Noninvertible Map.}

in the following we shall restrict to a general* class of single bumped map.

$$
X^{\prime}=f_{\mu}(x)
$$

Actually we shall consider as an example the simplest example, namely the discrete logistic equation

$$
x^{\prime}=\mu x(1-x)
$$

The type of question we shall ask is what kind of dynamical behavior occurs when $\mu$ increases?

1) Some definitions

An orbit is the collection of points

$$
x_{0}, x_{1}=f\left(x_{0}\right), \quad x_{3}=f\left(f\left(x_{0}\right)\right)=f^{(2)}\left(x_{0}\right) \ldots
$$

$x_{0}$ is called the initial point or initial condition, the practical way to construct the sequence $x_{1}, x_{2} \ldots$ is given on the following picture.

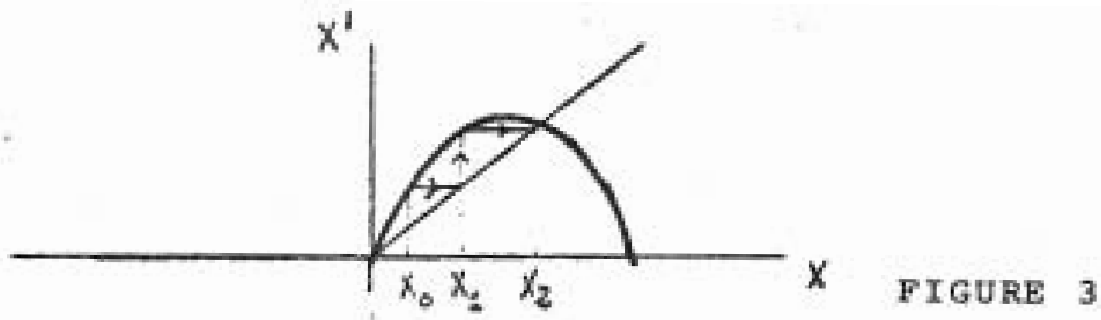

*The class of map we consider is technically defined by the negativity of the

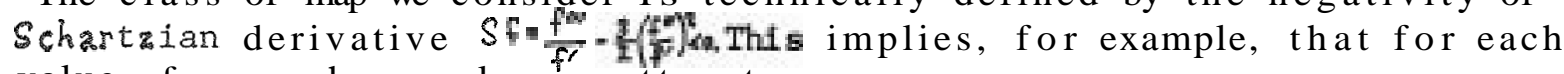
value of $\mu$ we have only one attractor. 
A periodic orbit of period $x$ is an orbit consisting in $n$ points such as

$$
x_{0}, \ldots x_{m-1}, \quad x_{n}=x_{0}
$$

A fixed point is a point such as

$$
x, f\left(x_{0}\right)
$$

Each point of a 'periodic orbit is called a perjodic point. Any periodic point of a periodic orbit is a fixed point of

$$
x_{i}=f^{(*)}\left(x_{i}\right)
$$

- Linear stability:

Let us consider the case of a fixed point $x_{0}=f\left(x_{0}\right) \quad$ If we start with an initial condition $x_{0}+(\delta x)_{0} \quad$ we get after one iteration $x_{0}+(\delta x)_{1}$

$$
(s x), \sim f^{\prime}(x,)(s x) \text {. }
$$

so that $x_{0}$ is stable if $\left|f^{\prime}\left(x_{0}\right)\right|<1$ and unstable in the opposite case. The same results apply for the stability of periodic orbits. A periodic orbit is stable (resp. unstable) if its periodic points are stable (resp. unstable) by respect to $f(x)$

$$
\begin{aligned}
& \sigma=x_{0} \ldots x_{n-1} \\
& \theta \text { is stable if }\left|f^{(n)}\left(x_{i}\right)\right| s, \quad x_{i} \in \theta
\end{aligned}
$$

It is easy to see that $f^{(n)}\left(x_{i}\right)$ is independent of $i$ (derivative chain rule).

- Bifurcations of one dimensional map of the interval:

Period doubling occurs for a periodic orbit of perfod $n$ when $f_{\mu}^{(n)}\left(x_{i}\right)=-1$. This leads to periodic orbits of period $2 n$. The technical assumption, namely the negativity of the Schartzian derivative, insures that all period doubling bifurcations are supercritical.

- Saddle node occurs for a periodic orbit of period $n$ when $f_{\mu}^{(\infty)}\left(x_{i}\right)=1$

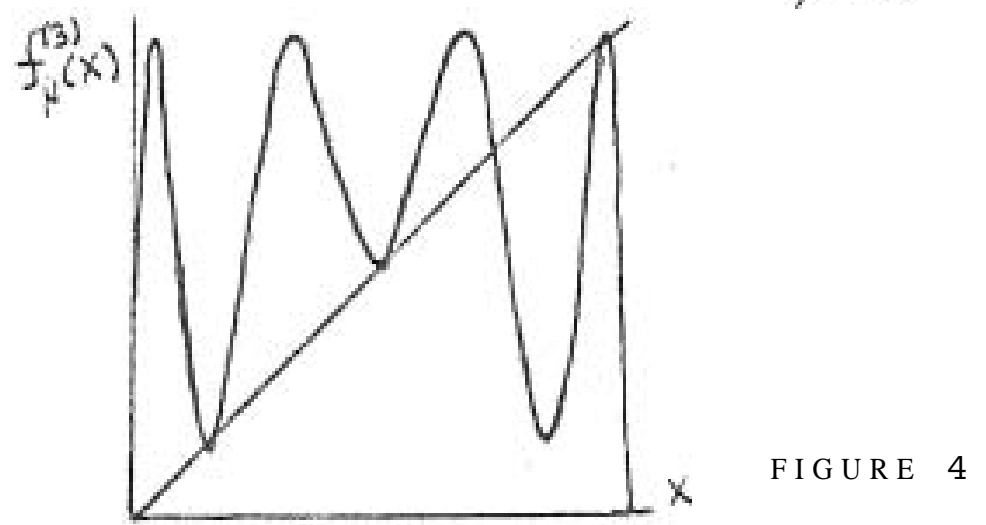

Saddle Node Bifurcation For Period Three Orbit

In this case after the bifurcation value we have the appearance of a pair of periodic orbits of period $h$, one stable the other unstable. 
2) Phenomenology

F'or the logistic equation, the interesting range of parameters is Let us consider the two extreme situations:

- For $\mu<4$ almost all initial conditions eventually escape from $(0,1)$. Actually for these parameter values the mapping realizes an infinitely contracted horseshoe map which obviously has no attractor but an invariant Cantor set with infinitely many unstable periodic orbits of any period and aperiodic ones. The construction of this Cantor set is given on the following picture.

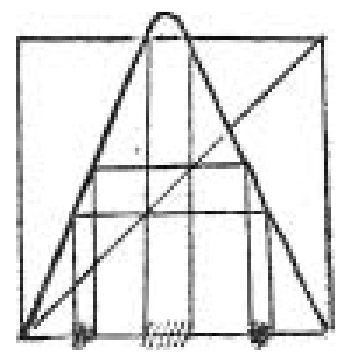

FIGURE 5

The coding of the point of the Cantor set can be done in such a way that

$$
\mathrm{f}\left(\mathrm{a}_{0} \mathrm{a}_{1} ; \cdots\right)=\mathrm{a}_{1} \mathrm{a}_{2} \cdots \quad(\mathrm{sh} \text { ift })
$$

Lach sequences of $a_{i}$ with $a_{1}=0,1$ is an element of this set, so that it contains infinitely many periodic orbits (ex.: period 3 10 1 $0110 \ldots$ ) and aperiodic ones. This provides a very simple example of what is called symbolic dynamics.

- For $\mu \simeq \circ, X=0$ is the only periodic orbit of $F$. So that the question is what happens for values of $\mu$ between 0 and 4? What kind of bifurcations of attractors do we observe?

a) The bifurcations are those described previously: saddle node and period doubling. The only exception occurs for $\mu \neq 1$. We have a transcritical vifurcation. 0 and $X^{*}$ exchange their stability $\left(X^{*}\right.$ is the other root of $X^{*}$ $=\mu X^{*}\left(1-X^{*}\right)$ negative for $\left.\mu<1\right)$

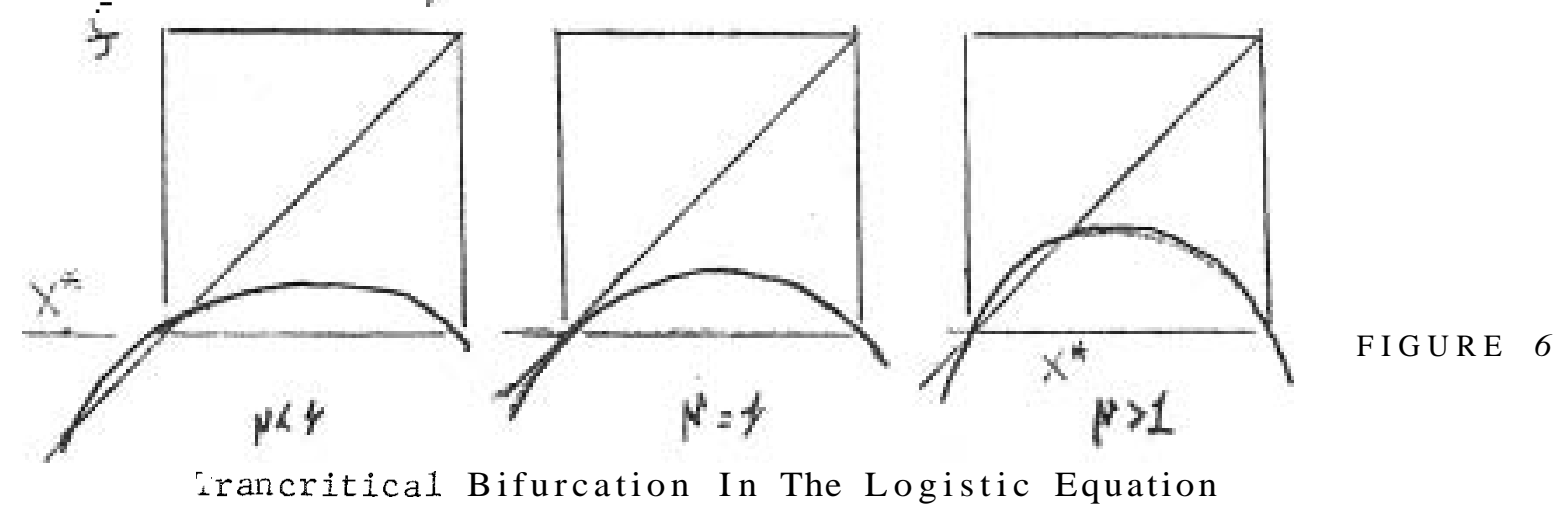

b) The attractors are either periodic orbirs which are stable for finite range of $\mu$ or strange attractors which occur for precise values of the parameter. One of the properties of these maps is that, when we have a stable periodic orbit, the orbit issues from the critical point (abscissa of the maximum $1 / 2$ for the logistic map) converges to it. Otherwise speaking, the 
critical point is always in its basin of attraction, so that when the orbit of the critical point contains an unstable periodic point we have no stable periodic orbit. For this kind of value of strange attractors occur. As a trivial example, let us consider the situation when the second iterate of the critical point is 0 .

$$
f^{(2)}\left(\frac{1}{2}\right)=0=\frac{\mu^{2}}{4}\left(1-\frac{\mu}{4}\right)
$$

This occurs for $\mu=4$ when $X=0$ is an unstable fixed point. So that for this value there is no stable periodic orbit. Actually, we have a strange attractor characterized by an invariant measure of probability continuously associated to the lebesque measure. The dynamics exhibit the property of sensitivity by respect to initial conditions.

Actually there exist infinitely many values of the parameter where this occurs. Let us give a second example which will be useful In the following. For some value of $\mu$ we have $f_{\nu}^{(2)}\left(f_{\mu^{2}}^{(z)}(1 / 2)\right)=X^{*}$ if $X^{*} \neq 1 / 2$ this nappens when this fixed point is unstable and we have again a strange attractor.

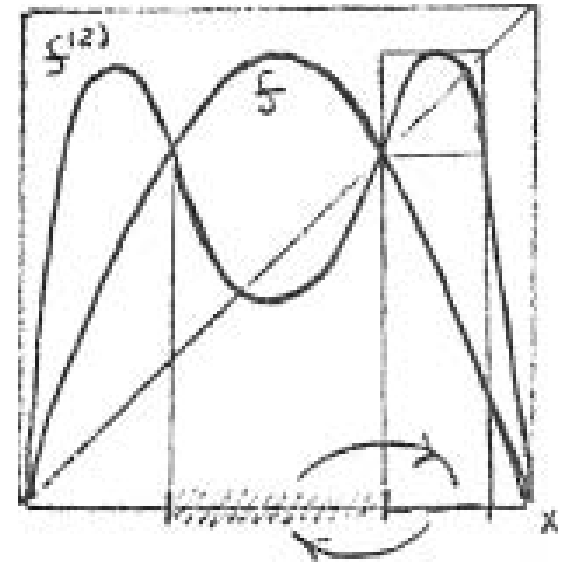

FIGURE 7

\section{A "Period" Two Strange Attractor}

c) Regular regime and chaotic regime

The parameter range $(0<\mu \leqq 4$ ) can be divided in two domains lor $\mu<\mu_{c}$. We have a finite number of unstable periodic orbits which are the tossils of the first period doubling cascace, $\mu_{c}$ is defined as the accumulation point of these cascades and the $\mu_{m}$ are the successive period doubling bifurcation values. For $\mu>$ we have an infinite number of unstable periodic orbits. The attractors observed are either periodic orbits, or strange attractors.

u) lough structure of the chaotic regine

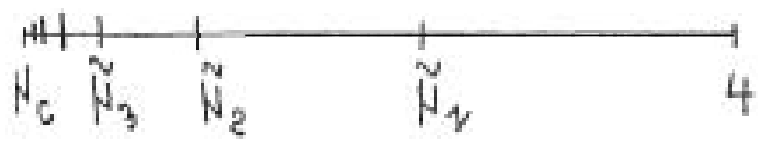

As we shall see we can define in the chaotic range of parameter a sequence $\mu_{n}$ si that for $\mu_{n}<\mu<\tilde{\mu}_{n}$ attractors have a rough periodicity $2^{n}$.

For $\mu_{n}<\mu_{c} \mu_{c}$ in the regular regime we have only periodic orbits whose period is a multiple of $2^{\mu}$. For $\mu_{e}>\mu>\vec{\mu}_{\text {w }}$ wen we have stable periodic orbits they have a period which is necessarily a multiple of 2 " For values 
where strange attractors exist, the dynamics consist in a chaotic motion wi h a gross period which is a multiple of $2^{n}$

As an example, let us consider $f^{(x)}$

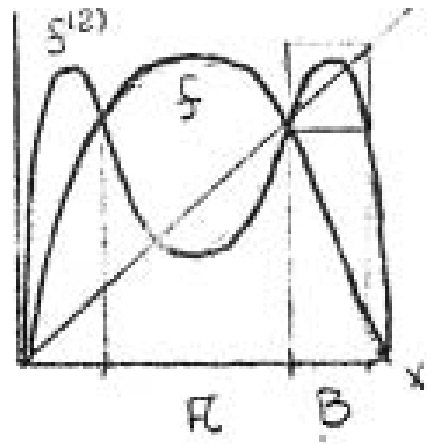

FIG URE 8

For $\mu_{1}>\mu>\tilde{\mu}_{1}, f^{(2)}$ has two invariant nonconnected sets $A, B$ which exchange themselves under

$$
f(A) \subset B \quad f(3) \subset A
$$

Now the point is that if we consider $\left.\boldsymbol{g}^{(4)}\right|_{f} \quad$ (that is the restriction of $f^{(4)}$ on A for $\mu_{1}<\mu<\tilde{\mu_{0}}$ ) we can define $\mu_{2}$ and $\tilde{\mu}_{L}$ as the range of parameter where $\left.f^{(q)}\right|_{A} \mu$ has two invariant sets $\alpha, \beta$ which exchange themselves under p(z)

$$
f^{(2)}(\alpha) \subset \beta \quad f^{(2)}(\beta) \subset \alpha \text {. }
$$

This inductive construction can be continued to generate the sequence
This gives a rough information of what happens in the chaotic regime. The sequence of $\tilde{\mu}_{a}$ is called inverse cascade

e) Fine structure of the chaotic regime.

At this point it is more convenient to consider the quadratic map

$$
x^{\prime}=1-\mu x^{2}
$$

As a model of this map we introduce the piece-wise linear map

$$
x^{\prime}=1-\mu|x|
$$

The family of map $X^{\prime}=I-\mu|X|^{1+\epsilon}$ realizes an interpolation between the piece-wise linear map and the quadratic one. Actually as $\epsilon$ is different from zero the fine structure of the chaotic regime of all these maps are qualitatively the same. The main property of the piece-wise linear map is that all the periodic orbits are in principle calculable. Furthermore, as we shall see in the chaotic regime, there is no stable periodic orbit so that in some sense the chaotic regime is fully chaotic. There exists no window of parameters where stable periodic motions occur.

When $\mu \leqslant 1$ we have only one stable fixed point. Infinitely many unstable periodic orbits appear by pairs when $\mu>1$ and give use to chaotic behaviors. For $\mu=2$ this transformation is known as the famous Baker transformation, so that the chaotic regime is defined by $1 \leqslant \mu \leqslant 2$.

As an example, let us consider the appearance of period 3 orbits $f^{(3)}(x)=x$. The property used to do this calculation is that when a pair of periodic orbits 
appears the cifical point (0 in our case) is a periodic point.

$$
f_{\mu}^{(3)}(0)=0
$$

Give the equation to be sacisfied by $\mu$ to give use to period 3 motions

$$
f_{\mu}(0)=1 \quad f_{\mu}(1)=1-\mu \quad f \mu(1-\mu)=1+\mu(1-\mu)=0
$$

this gives $\mu^{2}=1+\mu$

or

$$
\mu=1+\frac{1}{\mu}=1+\frac{1}{1+\frac{1}{1+\ldots}}=\frac{1+\sqrt{5}}{2} \quad \text { (golden number) }
$$

Now it has been shown by Derrida, Gervois and Pomeau that the general algorithm to locate the parameter $\mu$ where it appears some pair of periodic orbit of period $N$ consists to expand $\mu$ in its own basis (aucoexpansion) and select the value of $\mu$ where this expansion has a finite length $N-1$.

$$
\mu=a_{0}+\frac{a_{1}}{\mu}+\cdots+\frac{a_{N-2}}{\mu^{N-2}} \quad \text { where } a_{i}=0 \text { or } 1 \text { only }
$$

The number between 1,2 which has a finite autoexpansion tends to be dense and of zero measure on $(1,2)$. Now the question is what happens when $\epsilon$ is no

longer zero? fe

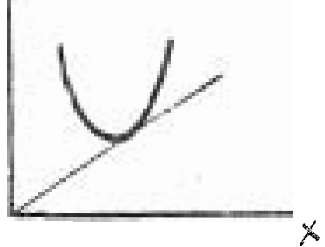

rather than

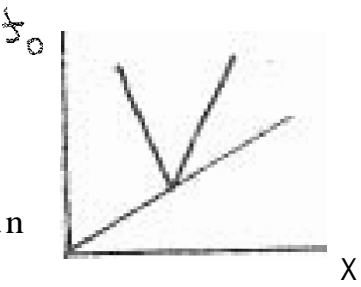

FIGURE 9

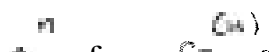

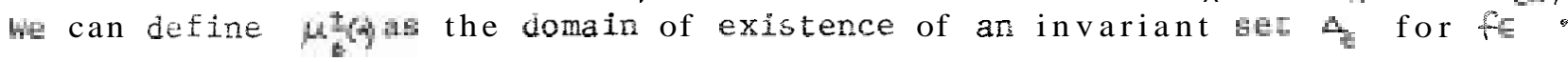
Let us draw an $\epsilon-\mu$ diagram for these periodic Sands $\varepsilon$,

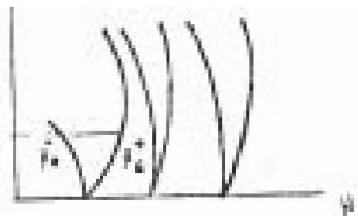

Now the point is that between $\mu_{f}^{-}(n)$ and $\mu_{e}^{*}(u)$ the map $f^{u} I_{\Delta_{n}}$ reproduces twe complete bifurcation story as the initial map. So that if we 100 a $a$ "periodic band" we have the following picture:

period doubling cascade chaotic regime with the rough period of the band

FIGURE 11 
The chaotic regime inside any periodic band has the same structure as the chaotic regime of the original map so that we have a nice self-similarity structure for the chaotic regime.

IV. Formal Analogy Between the Transition to Chaos and Phase Transition,

Let us consider here maps of the form $x^{\prime}=1-\mu|x| 8$

1) Critical exponents for various quantities

- Period of cycle in the regular regime
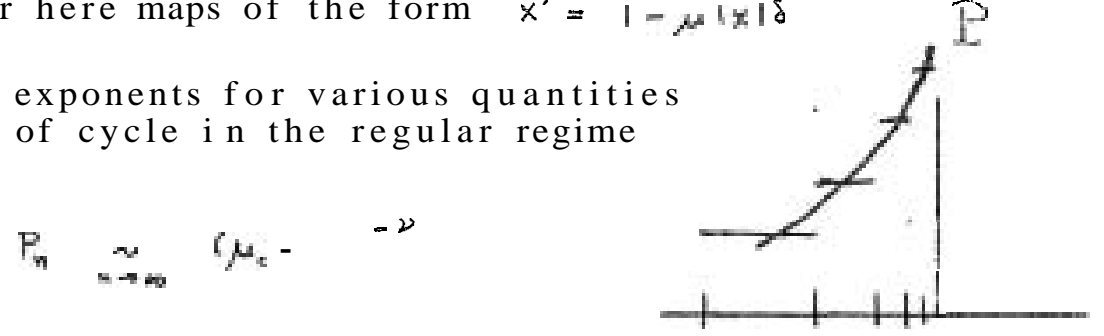

FIG URE 12

- Cross period of the dynamical behavior in the chaotic regime

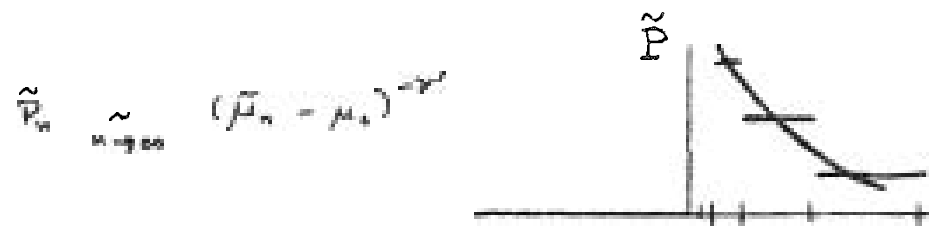

FIGURE 13

It appears that $y=y^{n}$ (scaling law) and depends only on $\delta$. This is a conjecture done after numerical experiments $(\nu \simeq 45)$ for quadratic maps

- Measure of the chaotic region

For $\mu<\mu_{\mathrm{a}}$ we have no chaotic region so that $m(c)=0(\mathrm{~m}(\mathrm{c})$ means measure or length of the support of $C$ ). For $\mu=\tilde{\mu}$, we have a strange attractor consisting in $2^{n}$ piece whose support is called $C_{n}$.

$$
m\left(C_{n}\right) \sim\left(\bar{\mu}_{m}-\mu_{n}\right)^{-1}
$$
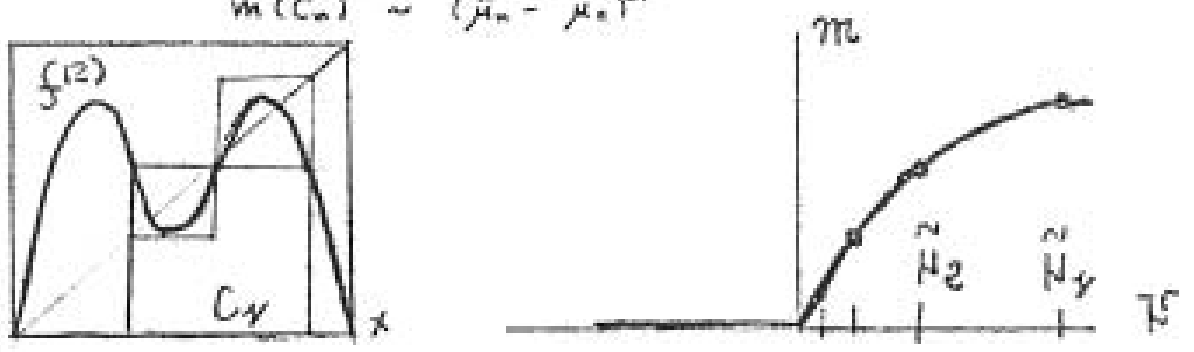

FIGURE 14

Numerical experiments suggest again that $\alpha$ is universal and depends only on

2) Self-similarity of the orbit at the transition point.

At the accumulation point of the period doubling cascade the orbit is aperiodic and supported by a Cantor set which is nothing else than $C_{\infty}$ ( $C_{n}$ has been previously defined as the support of strange attractors occurring a t $\mu=\tilde{\mu}_{n}$ ).

This Cantor set has remarkable self-similarity properties whose quantitative feature seems again to be universal.

3) Renormalization group

We shall recall here briefly the construction of the renormalization group which allows us to understand the origin of the critical exponents and to compute them.

In fact, there are many differences between this renormalization group and those used up to now in physics; here, we study deterministic dynamical systems. We are interested in the variation with some parameter of such quantities as the period of stable cycles. These variations are not 
continuous and arise for a discrete sequence of values of the parameter. This will impose an irreductibly discrete version of the renormalization procedure. Another fundamental differnece comes, as we shall show, from the existence of two generators for this renormalization group. This will generate infinitely many operations which would be necessary to describe the local scale invariance of the Cantor set.

The idea of renomalization is that, for $\mu=\mu_{\text {, and }} \mathrm{via}$ some simple transformation, the relation between $x_{i \rightarrow 2}$ and $x_{i}$ is very similar it@ that between $x_{i+1}$ and $x_{i} \quad$ This is suggested by the numerical evidence of a scale

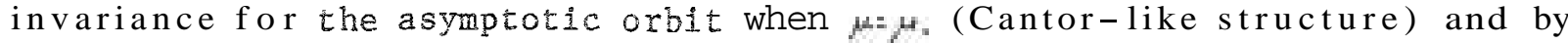
the evidence of a self-similar order for the iterates of a point on this orbit. Among the transformations we shall have to do, two are similar to those we know as classic renormalization schemes:

-we rename the variables $x_{i+2} \rightarrow x_{j+1} ;$

- we do a scaling on these variables $x_{i} \Rightarrow \xi_{i}$.

Then there is one more transformation:

We do a translation on variables $\xi x_{i}>\xi x_{i}+\eta$.

This leads to the study of the transformation

This may be written as

$$
f(x) \rightarrow f_{\xi, \eta}(x)=\frac{1}{\xi}[f \circ f(\xi x+\eta)-\eta] \text {. }
$$

where $h_{F,}$ is the affine map $h_{\xi y, \eta}(x)=\zeta x+\eta$.

Then

where

$$
\left.F_{F, 1}(x)=15 \quad 17-1\right]+f^{\prime}[f(\eta)] f^{\prime}(\eta) x+o\left(x^{2}\right),
$$

$$
f^{\prime}(x)=\frac{\partial f(x)}{\partial x},
$$

$\xi$ and $\eta$ are determined by the conditions

$$
\begin{aligned}
& \text { (a) } \eta=0 \text { or } \eta>0 \text { and } f(\eta)=0 \\
& \text { (b) } \xi=[f \circ f(\eta)-\eta] \text {, }
\end{aligned}
$$

(a) ensures the canceliation of the linear part of $f^{\prime}(x)$ and (b) implies $f_{\xi, \eta}[0]=1$

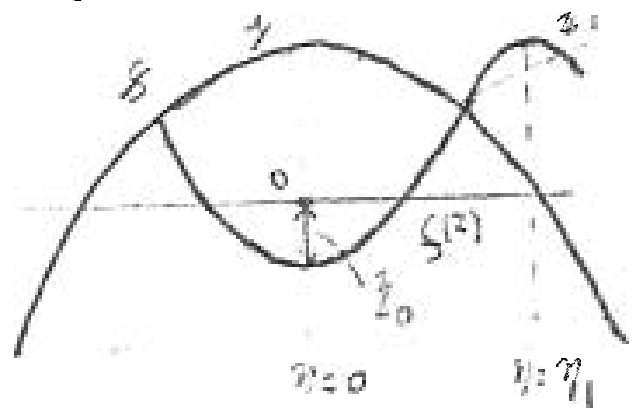

The two possible choices for n correspond to two distinct operations, denoted as $\ell_{1}$ and $\ell_{2}$ and which are the generacors of the renormalization group. The possibility of finding fixed points for these transformations and the study of their stability is crucial for the explanation of the criticality. This 
problem has been studied using truncated recursion formula and numerical studies, Some exact results have been obtained recently. The conjecture, at least when $f$ is analytic, is then: there exists a unique fixed point $f_{i}^{*}$ for the operation $R_{i}$ with $f_{i}^{*}(0) \neq 0, i=1,2$ such that the spectrum of the linearized operator $D R_{i} / f_{i}^{*}$ is inside the open unit disk except a unique positive eigenvalue $\lambda_{i}$ such that $\lambda_{1}=\lambda_{2}=\lambda$.

The picture is then the same as that in critical phenomena theory. Let us consider the stable manifold of e.g.8\%. In the set of functions $g, a$ sufficiently general one parameter path will get a critical point if it precisely cuts this manifold. Then, using usual techniques of renormalization groups, we get a relation between the critical exponent $\nu$ for the period and the eigenvalue $\lambda$.

$$
y=\ln 2 / \ln \lambda
$$

There is no easy way to compute the other exponent, due to the very existence of two generators for the renormalization group (the existence of a unique operation would lead to an exponent $\alpha=\ln \xi(\ln 2)$.

\section{Bifurcation and Chaos}

\section{Pierre Coullet}

We have discussed in this lecture in a rather general way the problem of competition between instabilities. Poincare's normal form theory has been used to derive standard equations describing the interaction for the modes near the marginal situation. As an example of such an equation, let us mention the Landau equation $\partial_{t} A=\mu A-\alpha \hat{A}^{3}$ which describes the self-interaction of an unstable mode which possesses a symmetry reflexion. Ir a similar way standard second order equations describe the competition between two instabilities.

In general, the structure of the nonlinear terms involved in these equations depends only on the linear problem in the marginal situations, We have focused our attention on a third order system which describes the interaction between three instabilities. We suggest that whatever be the coefficients of this equation (which depend on the starting physical problem) we have in the space of central parameter chatic situation as nesr as we have of the marginal situation. The possible route leading to chaotic behavior has been discussed. This kind of interaction arises quite naturally in triole convection problems. (see Ed Spiegel lecture), The result presented here cones from a joint work with A. Arneodo, E. Spiegel, C. Tresser, The lecture is based mainly on the following unpublished manuscript "Bifurcation and Chaos" (A. Arneodo, et. a1.)

\section{Introduction.}

In this paper, we are ipterested in physical systems described by a set of variables which can be arranged in two distinct groups: order variables $x_{i}$, $i=1, \ldots, p$, which are characterized by small linear growth rates at strongly linearly damped variables $y_{i}, i=1 \ldots, q(q \leqslant \infty)$. We assume the evolution equations in the form;

$$
\begin{aligned}
& \partial_{t} x=A_{\mu} x+F_{\mu}(x, y) \\
& \partial_{\Delta} y=B_{\mu} y+G_{\mu}(x, y)
\end{aligned}
$$


where $\mathrm{X}=\left(\mathrm{x}_{1}, \ldots, \mathrm{x}_{\mathrm{p}}\right)$ and $\mathrm{Y}=\left(\mathrm{y}_{1}, \ldots, \mathrm{y}_{\mathrm{q}}\right) ;$ the parameter $\mu=\left(\mu_{1}, \ldots, \mu_{p}\right)$ is such that, for $\mu_{1}=0\left(\mu_{4}=0\right.$ for all i) all the eigenvalues of the matrix $A_{0}$ are located on the imaginary axis; the eigenvalues of $B_{0}$ have finite negarive real parf. $F$ and $G \mu$ are purely nonlinear anaiytic functions which preserve $X=Y \stackrel{Y}{=} 0$ as an equilibrium solution of (1). If the respective linear scales of the variables $X$ and $Y$ are well separated, the dynamics of the system on large time ranges are essentially controlled by the order variables. In the asymptotic regime, the time dependence the fast relaxing variables follows the variations of the weakly uamped ones. This "slaved variables principle" (1) is closely related to the central manifold theory (Carr, 1979). Essentially, for $\mu$ small enough the dynamics desribed by (1) are contained in the p-dimensional system:

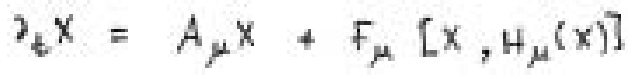

where

$$
Y=H_{\mu}(x)
$$

gives the dependence of the slaved variables upon the order variables. Generally, the computation of $H_{\mu}(X)$ can be only achieved up to any (arbitrarily high but) finite order, the order of truncation being chosen according to the information one weeds to extract from the problem at hand.

This dimensional reduction principle nas been mostly used to describe physical solutions where the increase of some external constraint gives rise to dissipative structures (Haken, 1979). However, even in systems which involve only a small number of order variables, one may observe very complicated dynamics and in particular chaotic behavior. Our attention be specially devoted to such interactions of a few order variables which can senerate so called "strange attractors". Our purpose is closely related co the theory of bifurcations of codimension one, two or three. (On this subject, see e.g., Arnold, 1980 or Guckenheimer, 1981). This dynamical version of catas"trophe cheory is a promising tool to study complex physical situations where several linear instabilities arise almost simultaneously (Holmes and Marsden, i978 anu Poincare, 1928).

This paper is organized as follows;

In section II, we use the apparatus of normal forms theory to derive standard equations which describe the dynamics of the interacting order variables. We thake a survey of these equations up to interactions of three order variable =

In section ILI, we briefly examine bifurcations of codinension one (self-interactions), and two (interactions of two order variables).

in section IV, we suggest the relevance of typical scenarios for the transition to cheos for some three instabilities problems (codimension three bifurcations). In section IV, the connection between normal forms techniques and amplitude txpansion methods is discussed in the context of fluid dynamics

\section{Normal Forms For The Interaction of Order Variables.}

\section{II.1 Reduction to Normal Form}

Foincare's normal forms theory (Arnold, 1980 and Poincare, 1928) points out what are the simplest equivalent forms of a given differential equation, near 
an equilibrium or a periodic solution. The reduction to these simplest forms (or normal forms) is done using a change of variables which is defined as a formal series in the deviation from the equilibrium or the periodic solution. In this section, these techniques are used to derive normal forms for the interaction of order variables. We consider the reduced system (2) rewritten as:

$$
\partial_{t} x=A_{\mu} x+F_{\mu}(x)
$$

where, from now on, $F_{\mu}(X)$ stands for $F_{\mu}\left[X, H_{\mu}(X)\right]$. The characteristic equation for the solution $X=0$ of (4) is given by:

$$
\operatorname{det}\left(A_{\mu}-. s\right)=0 \text {. }
$$

For $\mu \neq 0$, the zeros of the degree p characteristic polynomial:

$$
P_{\mu}(s)=\operatorname{det}\left(A_{\mu}-1-s\right)
$$

remain close to the imaginary axis. The structure of the nonlinear terms involved in the normal forms will depend only on the particular form $\mathrm{P}_{\mu}(\mathrm{s})$.

To proceed we introduce two formal series:

$$
\Phi_{\mu}(x)=\sum_{n \geqslant 2}^{P} \Phi_{\mu}^{(n)}(x) \text { and } R_{\mu}(x)=\sum_{n \geqslant 2}^{P} R_{\mu}^{(n)}(x) .
$$

where $\Phi_{\mu}^{(n)}$ and $R_{\mu}^{(n)}$ are homogeneous vectorial polynomials of degree $n$. Furthermore, we formally define a new variable $\mathrm{X}^{\prime}$ by:

$$
x=x^{\prime}+\Phi_{\mu}\left(x^{\prime}\right),
$$

designed to verify:

$$
\partial_{b} x^{\prime}=A_{\mu} x^{\prime}+R_{\mu}\left(x^{\prime}\right) .
$$

Inserting (8) into (4), we get:

$$
\partial_{t} x^{\prime}+\left(\partial_{6} x^{\prime} \cdot \partial_{x^{\prime}}\right) \frac{\delta_{\mu}}{\delta_{\mu}}=A_{\mu} x^{\prime}+A_{\mu} \sigma_{\mu}\left(x^{\prime}\right)+F_{\mu}\left[x^{\prime}+3_{\mu}\left(x^{\prime}\right)\right],
$$

where we use the notation:

$$
\left(\partial_{b} x^{\prime}+\partial_{x^{\prime}}\right) z_{\mu}=\sum_{i=1}^{p} \partial_{t} x_{i}^{\prime} \frac{\partial \Phi}{\partial x_{i}^{\prime}} \mu \text {. }
$$

With (9), (10) acquires the form:

$$
\begin{aligned}
R_{\mu}\left(x^{\prime}\right)+ & {\left.\left[A_{\mu} x^{\prime}+R_{\mu}\left(x^{\prime}\right)\right] \partial_{x},\right\} \Phi_{\mu}\left(x^{\prime}\right) } \\
= & A_{\nu} \xi_{\mu}\left(x^{\prime}\right)+F_{\mu}\left[x^{\prime}+\Phi_{\mu}\left(x^{\prime}\right)\right] .
\end{aligned}
$$

At order $n$ this yields the equation:

$$
L_{\mu} \bar{\sigma}_{\mu}^{(a)}\left(x^{\prime}\right)=\psi_{\mu}^{(a)}(x)-R_{\mu}^{(\alpha)}\left(x^{\prime}\right)
$$

where:

$$
L=\left(A_{\mu} x^{\prime}, \partial_{x}\right)-A_{\mu},
$$

is a linear operator on the space of homogeneous vectorial polynomials; at 

each order $\Psi_{\mu}(n)$ is a known function, depending on $\Phi_{\mu}(k)$ and $R_{\mu}(k)$ for
$k<n:$

$$
\psi_{\mu}^{(n)}\left(x^{\prime}\right)=\psi_{\mu}^{(n)}\left[x^{\prime}, \Phi_{\mu}^{(2)} \ldots \Phi_{\mu}^{(n-1)}, R_{\mu}^{(2)} \ldots R_{\mu}^{(n-1)}\right] .
$$

If $L$, is singular, i.e., if $L \mu$ has some zero eigenvalue, $R_{\mu}(\mathrm{n})$ is chosen in such a way that the right-hand side of (13) is orthogonal to the null space of the adjoint operator to $L_{\mu}$. This solvability condition for (13) selects particular nonlinear terms (the resonant ones) from (4), which cannot be removed by the change of variables (8). On the other hand, we can get rid of the nonresonant terms, which produce only harmonic effects as illustrated in Fig. 1.

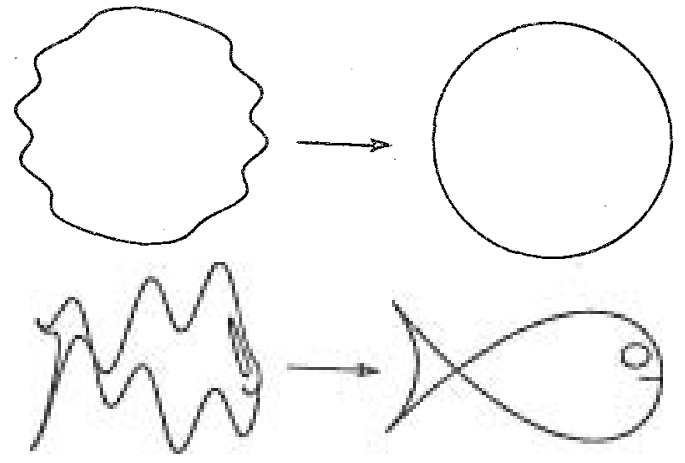

FIGURE 1. Typical Reductions to Norma1 Forms

$\Phi_{P}^{(n)}$ is then computed by solving (13). Indeed, there is some arbitrariness in the choice of $s_{\mu}$ and $R_{\mu}$ since $R_{\mu}$ can contain arbitrary nonresonant terms.

If $L_{\mu}$ is not singular, $\mathbb{K}_{\mu}$ can be chosen to be zero and we have, by the change of variables defined by (8) and (9), formally linearized the system (4). This is almost always the case when we deal with a hyperbolic equilibrium solution, i.e., when all $\mu$ are different from zero. For the problem at hand, we are not interested in such a choice; we rather want to keep from (4) the relevant nonlinear terms needed to produce nontrivial dynamical behavior in the neighborhood of the hyperbolic equilibrium which is likely to be unstable. These relevant terms are precisely those which are resonant for $\mu=0$

To summarize, we have formally reduced the initial problem (4) of the interaction of order variables to the desired normal form

$$
\partial_{b} x=A_{\mu} x+R_{\mu}(x)
$$

where now $\mathrm{R}_{\mu}(\mathrm{X})$ corresponds to the previous prescription.

\section{2 Normal Forms Describing the Interaction of One, Two and Three Order} Variables

Table I gives access to the general expression of the normal forms describing the interaction of order variables in turn of the sole knowledge of the characteristic polynomial $\mathrm{P}_{\mu}(\mathrm{s})$. Note that some symmetry properties may induce simplifications in the above expressions. For example, if the system which reduces to the normal form (1.a) is invariant under a reflection symmetry, then the corresponding $f(x)$ must be an odd function.

In this table we make use of the following notations: 


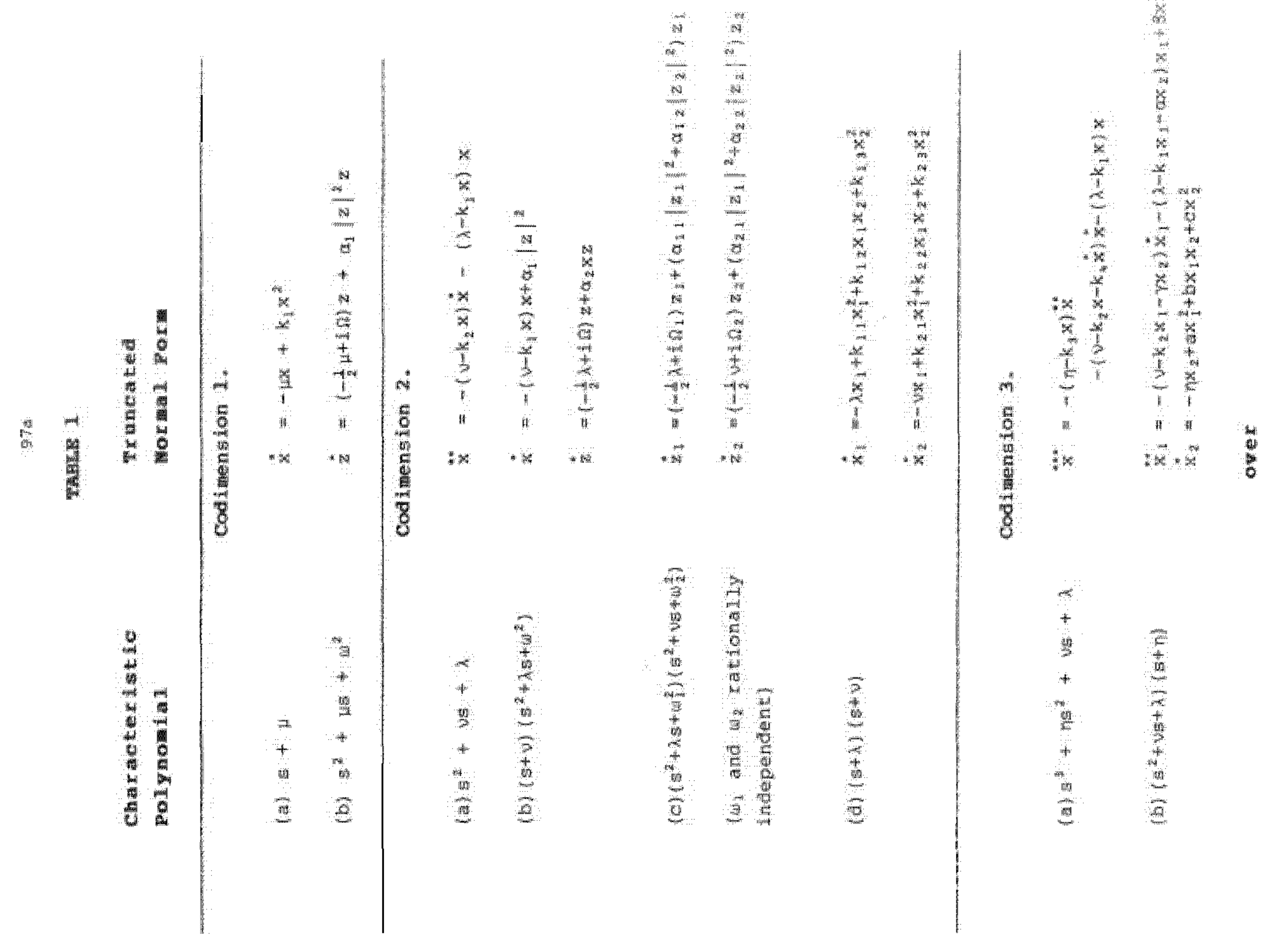


TABLE 1 Continued

(c) $\left(s^{2}+v s+\lambda\right)\left(s^{2}+\eta s+\omega^{2}\right)$

(d) $\left(s^{2}+\lambda s+w^{2}\right)\left(s^{2}+v s\right.$

$\left.+\omega_{2}^{2}\right)\left(s^{2}+n s+w_{3}^{2}\right)$

$\left(\omega_{1}, \omega_{2}\right.$ and $\omega_{3}$

rationally independent)

$$
\begin{gathered}
\ddot{x}=-\left(v-k_{2} x\right) \dot{x}-\left(\lambda-k_{1} x\right) x+\alpha|z|^{2} \\
\dot{z}=\left(-\frac{1}{2} n+1 \Omega\right) z+\beta x z \\
\dot{z}_{1}=\left(-\frac{1}{2} \lambda+i \Omega_{1}\right) z_{1}+\left(\left.\alpha_{1}|| z_{1}\right|^{2}+\right. \\
\left.\alpha_{12}\left|z_{2}\right|^{2}+\alpha_{13}\left|z_{3}\right|_{2}\right) z_{1} \\
\dot{z}_{2}=\left(-\frac{1}{2} \lambda+i \Omega_{2}\right) z_{2}+\left(\alpha_{21}\left|z_{1}\right|^{2}+\right. \\
\left.\alpha_{22}\left|z_{2}\right|^{2}+\alpha_{23}\left|z_{3}\right|^{2}\right) z_{2} \\
\dot{z}_{3}=\left(-\frac{1}{2} \lambda+i \Omega_{3}\right) z_{3}+\left(\alpha_{31}\left|z_{1}\right|^{2}+\right. \\
\left.\alpha_{32}\left|z_{2}\right|^{2}+\alpha_{3}\left|z_{3}\right|^{2}\right) z_{3}
\end{gathered}
$$

(e) $(s+\lambda)\left(s^{2}+u s+\omega_{1}^{2}\right)\left(s^{2}+n s+\omega_{2}^{2}\right) \quad \dot{x}=-\left(\lambda-k_{1} x\right) x+k_{2}\left|z_{1}\right|^{2}+k_{3}\left|z_{2}\right|^{2}$

( $w$, and $\omega_{2}$ rationally

$\dot{z}_{1}=\left(-\frac{1}{2} v+i \Omega_{1}\right) z_{1}+\alpha_{1} \times z_{1}$

independent)

$\dot{z}_{2}=\left(-\frac{1}{2} n+i \Omega_{2}\right) z_{2}+\alpha_{2} \times z_{2}$

(f) $(s+\lambda)(s+\nu)\left(s^{2}+n s+\omega^{2}\right)$

$$
\begin{aligned}
& \dot{x}_{1}=-\left(\lambda-k_{1} x_{1}\right) x_{1}+\alpha_{1}|z|^{2}+r_{1} x_{1} x_{2}+c_{1} x_{2}^{z} \\
& \dot{x}_{2}=-\left(v-k_{2} x_{1}\right) x_{2}+\alpha_{2}|z|^{2}+r_{2} x_{1} x_{2}+c_{2} x_{1}^{2} \\
& \dot{z}=\left(-\frac{1}{2} \eta+i \Omega\right) z+B_{1} x_{1} z+B_{2} x_{2} z
\end{aligned}
$$

(g) $(s+\lambda)(s+v)(s+n)$

$$
\begin{aligned}
\dot{x}_{1}= & -\lambda x_{1}+\alpha_{11} x_{1}^{2}+\alpha_{22} x_{2}^{2}+\alpha_{33} x_{3}^{2} \\
& +\alpha_{12} x_{2} x_{1}+\alpha_{31} x_{3} x_{1}+\alpha_{23} x_{2} x_{3} \\
\dot{x}_{2}= & -v x_{2}+\beta_{11} x_{1}^{2}+\beta_{22} x_{2}^{2}+\beta_{3} x_{3}^{2} \\
& +\beta_{12} x_{2} x_{1}+\beta_{31} x_{3} x_{1}+\beta_{2} x_{2} x_{3} \\
\dot{x}_{3}=- & n x_{9}+\gamma_{11} x_{1}^{2}+\gamma_{22} x_{2}^{2}+\gamma_{33} x_{3}^{2} \\
& +\gamma_{12} x_{2} x_{1}+\gamma_{31} x_{3} x_{1}+\gamma_{23} x_{2} x_{3}
\end{aligned}
$$

We thank J.Guckenheimer and E. Knobloch for pointing out some errors, 
- $x_{1}, x_{2} \ldots$ denote the real order variables while $z_{1}=p_{1} e^{i \theta_{1}}$ refer to the complex ones.

- $f, g, h, \ldots$ are arbitrary functions whose lowest order terms do not affect the linear problem.

- The symbols $\lambda, \mu, \nu, \eta$ have to be identified to the small parameters $\left(\mu_{1}\right)$ involved in $(1)$.

$$
\sqrt{\left(\omega_{1}^{2}-\mu_{1}^{2} / 4\right)}
$$

\subsection{Comments}

Subsections II.1 and II.2 report only formal considerations. Difficulties connected with small denominators arise if one tries to find an analytic change of variables reducing a given differential system to its normal form (Guckenheimer, 1981). However, one can always use polynomial changes of variables to bring the system to a form which coincides with its normal tom, up to terms of arbitrarily high degree. Since we are mainly interested in the behavior of the solution in the neighborhood of the origin and close to te degenerate situation $\mu=0$, it is likely that the first few terms of the formal series give a good description of the dynamics. Such a truncation may involve only the nonlinear terms of lowest order. As an illustration let us quote the case (1.a) in Table I with reflection symmetry: the equation w ich describes correctly this self-interaction is the Landau equation:

$$
\dot{\mathrm{x}}=-\mu x+2 x^{3}
$$

when the constant parameter a is different from zero. Sometimes, higher irder terms are needed as, e.g., in the case (2.b) if one intends to take into account the possibility of a tertiary instability corresponding to a bifurcation to a quasiperiodic solution (Langford and Siam, 1979, Guckenh Imer, 1979, and Holmes, 1980)

In any case, the original system can be considered as a perturbation or its truncated normal form and one can wonder about the connection between the dynamical behavior they display respectively (J. Guckenheimer, 19! '). A precise mathematical answer to this question is hopeless but this is of litele consequence for some practical purposes.

When complex variables are involved, one is faced (in nonresonant cases) with equations for the amplitudes which are not coupled to the equacions cor the phases. Then, when a quasiperiodic behavior is predicted, its results form a product of independent periodic behaviors. As a consequence the well-known frequency locking phenomenon (Iooss and Joseph, 1980) is missing in the description with truncated normal forms. Moreover, this lack of coupli g implies that the nontrivial aspects of the dynamics are described by a lower dimensional system. For example, the truncated normal form associated with the tridimensional system $(2 . b)$ reads:

$$
\begin{aligned}
& \dot{x}=-\nu x+a x^{2}+b z^{2} \\
& \dot{z}=(-x / 2+i \Omega) z+c z x
\end{aligned}
$$

where $a, b, c$ are constant parameters. With $z=\rho \mathrm{e}^{\mathrm{ie}}$, one gets a 
bidimensional system for $x$ and $p$

$$
\begin{aligned}
& x=-y x+a x^{2}+b \\
& \dot{p}=-(\lambda / 2) p+\operatorname{Re}(c) \cdot x
\end{aligned}
$$

the equation for the phase; being given by

$$
\dot{\theta}=\Omega+\operatorname{Im}(c) \cdot x \text {. }
$$

Of course, this reduction from three to two dimensions prevents the truncated normal form to exhibit chaotic behavior.

These remarks give evidences that the predictions on an explicit system, obtained using its truncated normal form, can be qualitatively false. However, at least for small enough, one can reasonably expect minor quantitative discrepancies between the actual dynamics and the predicted one (Arnold, 1980; p. 297.)

\section{Interactions of One or Two Order Variables.}

Generally, the nontrivial aspects of a csdimension p bifurcation problem are described by a $p$ dimensional differential system (Takens, 1974). Consequently, the study of the self-interaction of one order variable reduces to the study of a one dimensional real system, while for two interacting order variables the reduced system is bi-dimensional.

\section{III.1 Self Interaction of One Order Parameter: A Typical Example.}

Let us consider the classical Hopf bifurcation (Marsden and McCracken, 1976) whose truncated normal form is:

$$
\dot{z}=(-\mu / 2+i \Omega) z+a \cdot z \cdot|z|^{2}
$$

when the parameter a is different from zero. The standard analysis stands on the one dimensional equation for the amplitude:

$$
\dot{p}=-(\mu / a) p \text {. Re(a) } p^{a} \text {. }
$$

The nontrivial solution of (22) reads:

$$
p=\sqrt{\mu /[2 \operatorname{Re}(a)]} \text {, }
$$

and gives the radius of the invariant circle issued from the bifurcation.

The above analysis fully uses the one dimensional character of Hopf bifurcation. The following approach ignores this simplifying feature but offers the advantage to generalize to codimension two problems. With the scaling transformation

(21) becomes:

$$
z, \sqrt{\epsilon} \tilde{z}, \quad \mu=\epsilon \tilde{\mu}
$$

$$
z=i e z+\epsilon\left[-(\mu / 2) z+a z \cdot|z|^{2}\right]
$$

where the tildes have been omitted. In the limit $\epsilon=0$, (25) yields:

$$
\dot{z}=i \Omega_{z} .
$$


The phase portrait of this (linear) Bamiltonian system is shown in Fig. 2.

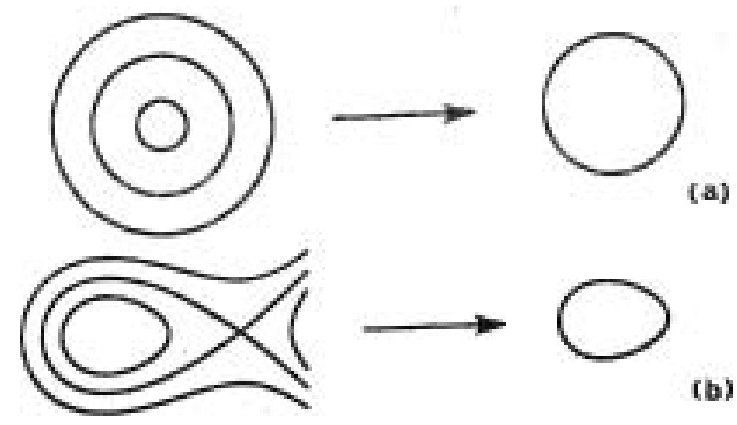

FIGURE 2. Illustrations of the variational principle:

a) in the case of Hopf bifurcation, the bifurcated orbit is selected from a linear system;

b) an the contrary, in a typical codimension two bifurcation (29), the selection is done on a nonlinear system.

The constants of motion of (26) are simply the radii of the invariant circles. The condition of existence for $€$ of a given circle of radius $p$ is obtained by solving the following variational equation (Arnold, 1980, and Guckenheimer, 1981):

$$
\frac{\partial}{\partial 6} \int_{D_{p}} \operatorname{div} \mathbb{V}_{c}=0
$$

where $D_{\beta}$ is the open disk of radius $p$ and $V_{\epsilon}$ is the planar vector field corresponding to (25). This leads to the equation:

$$
-\mu p+2 \operatorname{Re}(a) p^{3}=0
$$

which gives the same results as the one dimensional method.

\section{III.2 Interactions of Two Order Variables: A Typical Example}

In this subsection, we choose to discuss the codimension two problem (2.a) in Table I, which will be of central use in the next section to suggest the existence of chaotic behavior in some systems describing the interaction of three order variables. The corresponding truncated normal form is:

$$
\ddot{x}+(\nu+a x) \dot{x}+\lambda x+b x^{2}=0 \text {, }
$$

when the constants $a$ and $b$ are different from zero. With the following scaling transformation (Guckenheimer, 1981)

$$
t=\epsilon^{-1} \tilde{t}, \quad x=\epsilon^{2} \tilde{x}, \quad \lambda=\epsilon^{2} \tilde{\lambda}, \quad \nu=6^{2} \bar{\nu},
$$

(29) yields:

$$
\ddot{x}+e(\nu+a x) \dot{x}+\lambda x+b x^{2}=0
$$

where the tildes have been omitted. En the limit $\epsilon=0$, (31) becomes Hamiltonian with the Bamiltonian function:

$$
H(x, \hat{x})=\frac{x^{2}}{2}+\frac{\lambda x^{2}}{2}+\frac{b x^{3}}{3} .
$$



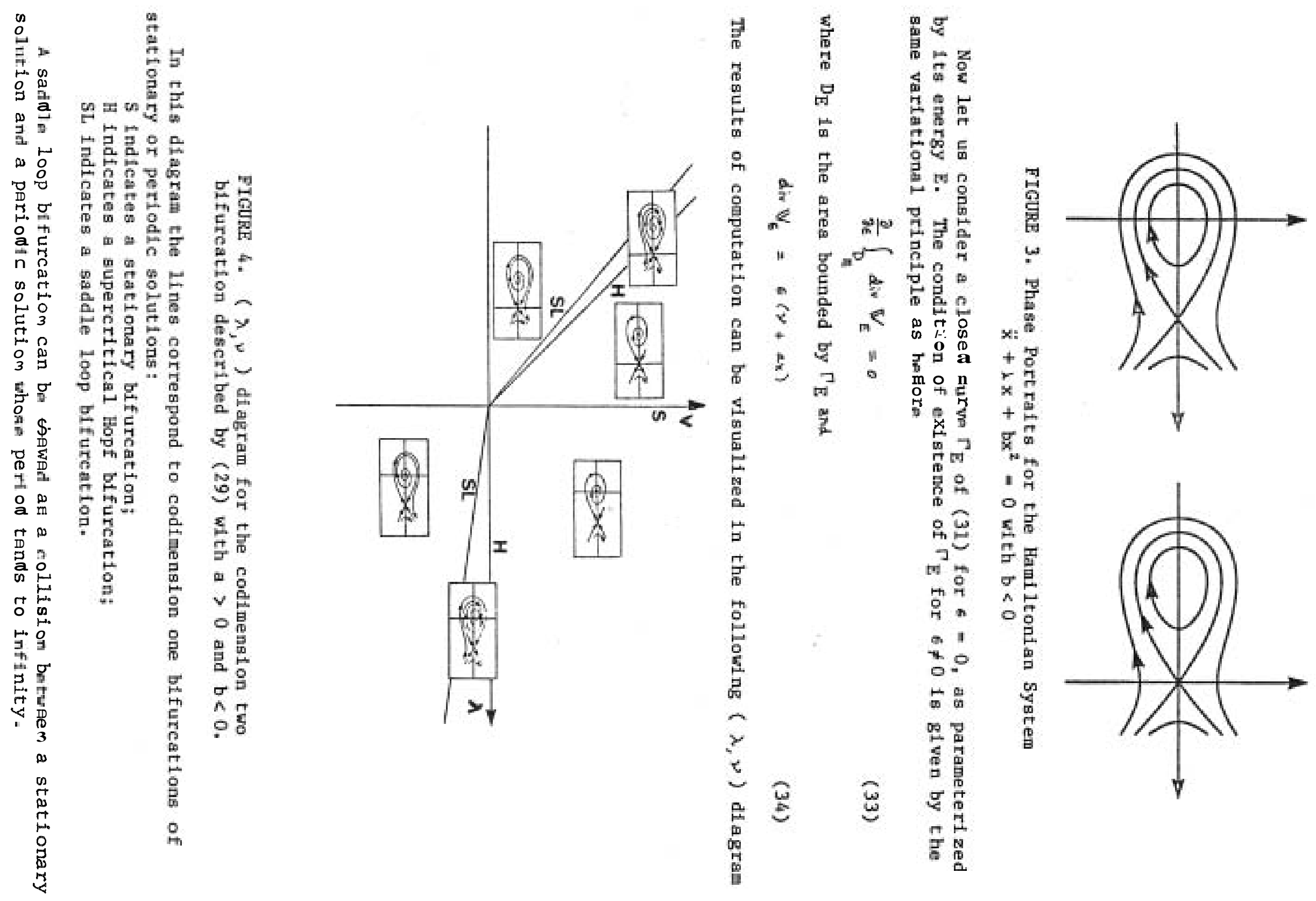

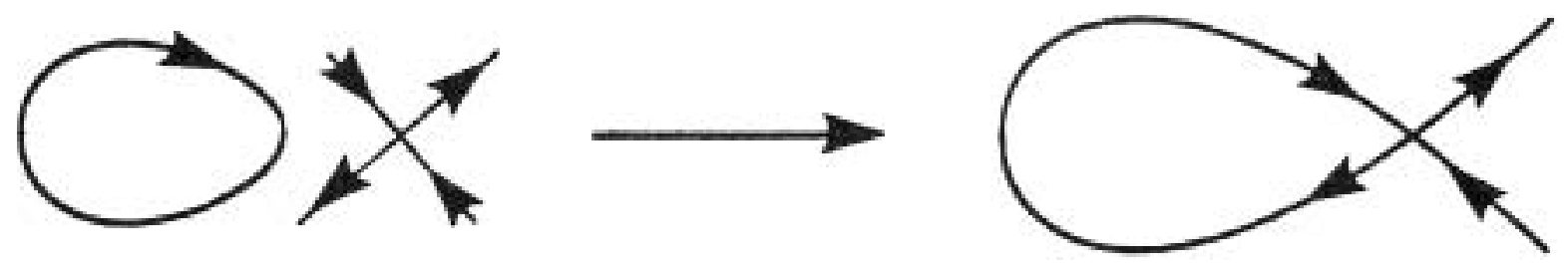

FIGURE 5

A Saddle Loop Bifurcation

Let us recall that the truncated normal form (29) applies to the general case (2.a). If a central symmetry is imposed, (29) must be replaced by a "Van der Pol-Duffing" equation

$$
\ddot{x}+\left(\nu+a x^{2}\right) \dot{x}+\lambda x+b x^{3}=0
$$

The conservative limit of (35) is the Duffing equation

$$
\ddot{x}+\lambda x+b x^{2}=0
$$

This situation is somewhat richer: the phase portraits of (36) are qualitatively different according to the sign of b.

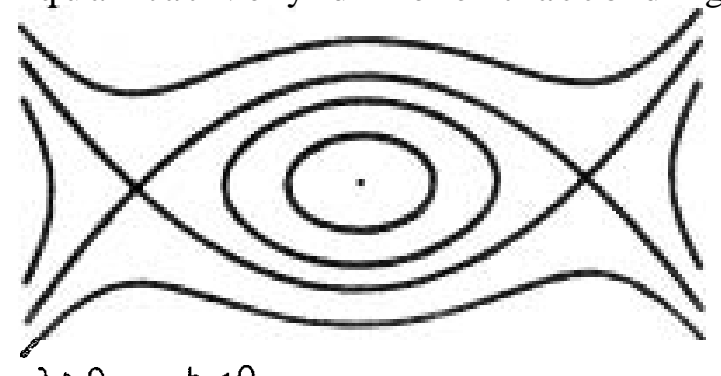

\section{FIGURE 6}

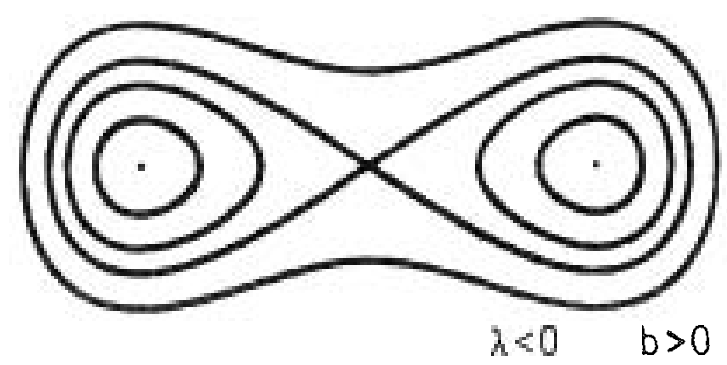

Typical Phase Portraits for the Duffing Equation

IV. 1. Interactions of Three Order Variables.

In this subsection, we suggest how chaotic behavior can occur in the typical co-dimension three bifurcation (3.a) in Table I, whose truncated normal form is

$$
\ddot{x}+(\eta+a x) \ddot{x}+(\nu+b x+c x) x+\lambda x+d x^{2}=0
$$

where a, b, c, d are constant parameters. This equation has two equilibrium solutions $x=0$ and $x=-\lambda / d$. The corresponding characteristic equations are

$$
s^{3}+\eta s^{2}, x s+\lambda=0
$$

for the null solution, and

$$
s^{1}+\left(\eta-\frac{a h}{d}\right) s^{2}+\left(\nu-\frac{b \lambda}{d}\right) s \rightarrow=0
$$

for the nontrivial solution.

We first study the bifurcations of the nontrivial solution. When $\eta>0, x>0$ and $\nu>\lambda\left(\frac{b}{d}-\frac{d}{d-a d}\right)$, (39) has only roots with negative real part so that the nontrivial solution is asymptotically stable in this parameters' range. 
For $y=\lambda\left(\frac{b}{d}-\frac{d}{\eta d-a^{\lambda}}\right)$ a pair of complex conjugate roots crosses the imaginary axis and the nontrivial equilibrium becomes unstable. This Hopf bifurcation gives rise to a periodic orbit. For $\lambda$ and $\nu$ small enough this periodic solution stands near the unstable trivial solution and a "collision" between these two solutions is likely to occur (Fig 5). This "collision" generates a homoclinic orbit (saddle loop bifurcation for the periodic s(lution) which can be used, in some cases to prove the existence of chaotic behavior. Em particular, if this collision arises when the trivial solution has the following property:

$$
\xi>-p>0
$$

where $\xi$ and $p \pm i w$ are the roots of the characteristic equation (38), then there exists chaotic behavior in the neighborhood of the unstable homoclinic orbit (Shil'nikov, 1965). Figure 7 suggests the occurrence of a horseshoe map (Smale, 1965) on a Poincare plane transverse to the homoclinic orbit. Such a homoclinic orbit arises quite naturally in one-parameter families of differential equations which exhibit a Hopf bifurcation close to another

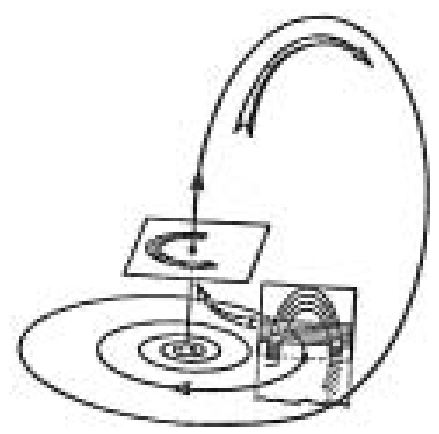

FIGURE 7

The Horseshoe Map Viewed as the Composition of a Local Motion near the Origin and a Nonlocal Motion along the Homoclinic Curve.

equilibrium point which satisfies the condition (40). A schematic evolution involving a supercritical Hopf bifurcation is illustrated in Fig. 8 where the shaded zone corresponds to a rather complicated sequence of events (bifurcations): one can numerically observe cascades of period-doubling bifurcations, intermittency phenomena, "strange attractoss",...
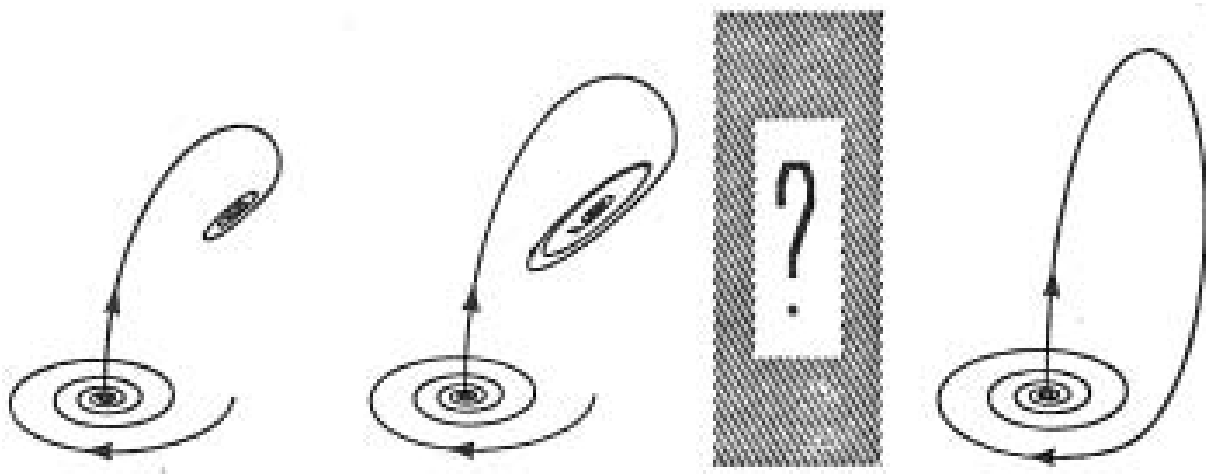

FIGURE 8

From Hopf to Saddle Loop Bifurcation.

In the remainder of this section, we will suggest the existence of such an homoclinic orbit for (37) with arbitrarily sma11 $\lambda, \nu, \eta$

For any fixed ? $-\eta$. 
and

$$
|\lambda| \sim|\nu| \gg \eta
$$

the slaved variables principle allows us to reduce (37) in an approximate way to a bi-dimensional system.

The slaved variable $x$ has a growth rate:

$$
s=-\eta+\frac{\nu}{\eta}-\frac{\lambda}{\eta^{2}},
$$

so that the dispersion relation for the two other variables is given by:

$$
s^{2}+\tilde{\nu} s+\tilde{\lambda}=0
$$

where $\tilde{\lambda}=\lambda / \lambda$ and $\tilde{\nu}=\nu / \eta-\lambda / \eta^{2}$. For $\ddot{\lambda}$ and $\tilde{\eta}$ small enough, the reduced bi-dimensional system yields the truncated normal form:

$$
\ddot{x}+(\ddot{\nu}+a x) \dot{x}+\tilde{\lambda} x+b x^{2}=0,
$$

where $a$ and $b$ are functions of the parameters a, b, $c$, d involved in ( 37 ). (44) is precisely the truncated normal form for the case (2.a) in Table I, which has been discussed in the previous section. As already mentioned, there exists, in the ( $\left.\tilde{x}_{,} \nabla\right)$ ) plane, a line SL starting from zero and which corresponds to a saddle loop bifurcation for a periodic solution, itself issued from a Hopf bifurcation of nontrivial equilibrium. This line SL furnishes an approximation of a curve $\Gamma$ in the plane $\eta_{-} \eta_{0}$ of the original (, $\eta$ ) parameters space; this curve $\Gamma$ in turn corresponds to a saddle loop bifurcation, but of somewhat more complicated nature since we now deal with the full three-dimensional problem.

The above dimensional reduction method ensures the existence of $\Gamma$, and allows us to compute it approximately, only for $\lambda$ and $\nu$ small enough* But it is likely that does exist for a much wider parameter's range, up to values such that the inequality (41) is no longer verified. This is confirmed by numerical computations of $\Gamma$ for explicit systems (a, b, $c, d$ fixed) (see subsection IV.2). It appears that the curve generally enters a region in the $(\lambda, \nu)$ diagram where the trivial solution possesses the desired property (40). If one follows, one observes qualitative changes in the saddle loop orbit as sketched in Fig. 9.
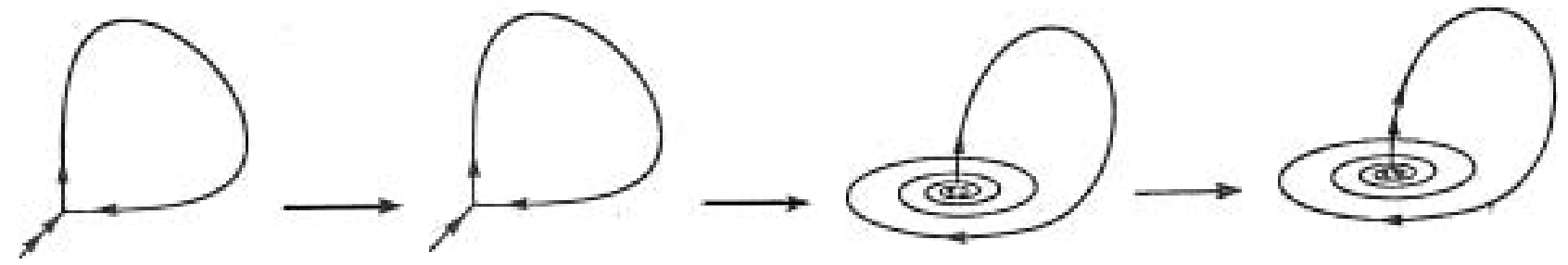

FIGURE 9. Evolution of the Homoclinic Orbit When One Moves Along $\Gamma$.

Let us remark that this analysis holds for $\lambda, \nu, \eta$ as small as we want, i n such a way that chaotic behavior may occur as close as we want to the neutral situation.

\section{IV.2 Numerical Illustration}

For the sake of simplicity we will consider the case $a=b=c=0, d=-1$ (Coullet, et.al., 1979). Then, the truncated normal form (37) reads: 


$$
\ddot{x}+\eta \ddot{x}+y \dot{x}+2 x-x^{2}=0 .
$$

Fig. 10 visualizes analytical and numerical results on a $(\lambda, \nu)$ diagram ( $\eta$ fixed).

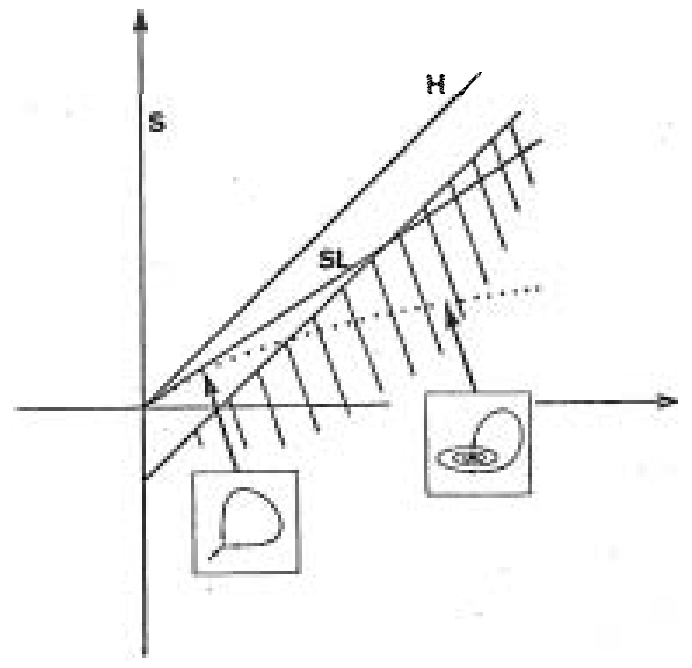

FIGURE 10. S corresponds to a stationary bifurcation, H to a Hopf bifurcation for the nontrivial equilibrium, $\Gamma$ to a saddle loop bifurcation and condition (40) is satisfied in the shaded area. These results are obtained with the full system (37). SL is an approximation to $\Gamma$ derived from the reduced system (44).

Under condition (41), (45) reduces to the bi-dimensional system

$$
\left.\ddot{x}+\left(1 \frac{\pi}{\eta}-\frac{\lambda}{\eta^{2}}\right)+\frac{2}{\eta^{2}} x\right) \dot{x}+\frac{\lambda}{\eta} x-\frac{1}{2} x^{2}=0
$$

Fig. 11 reports direct numerical simulations performed on (45). Keeping and fixed, we vary $\lambda$ i $n$ the negative half plane. We observe the following sequence of bifurcations: the Bopf bifurcation of the nontrivial equilibrium gives rise to a stable periodic orbit. Later on, this orbit loses its stability and a cascade of period doubling bifurcations seems to lead to chaotic behavior. As soon as such a complicated dynamic sets up, there is no more hope to follow precisely the sequence of bifurcations.

Globally, this evolution, as viewed on a typical Poincare map, looks like a transition from a Henon-like "strange attractor" (M. Henon, 1976) to a spiral type "strange attractor" whose structure reflects precisely the geometry of the homoclinic connections involved in this problem (A. Arneodo, et. al., 1981, and Arneodo, et. 21., 1981) (Fig. 12)。

\section{3 Comments}

The analysis presented in subsection IV.I applies to other systems. If one considers the case (3.a) in Table $\mathbf{I}$, when the equations are invariant under a central symmetry, the preceding method allows us to predict orbits as sketched in Fig. 13.

A simple differential model (Moore and Spiegel, 1966) of thermohaline convections (Turner, 1973) exhibits chaotic behavior interpretable in such a way. 


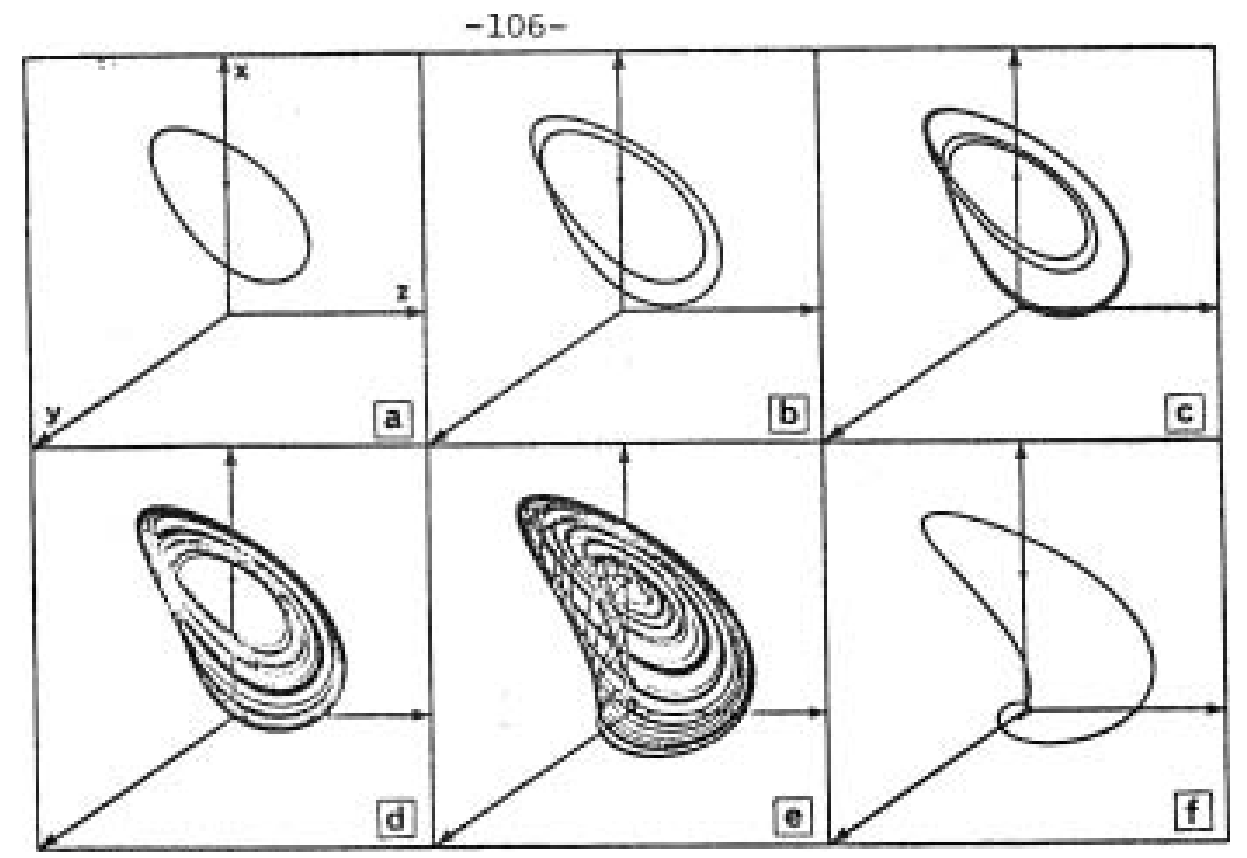

FIGURE 11. (a), (b), (c), (d), (e) represent attractors of system (37) obtained by direct simulations when increasing the parameter $d$. For a still larger value of $\lambda \quad$ one gets the unstable homoclinic orbit depicted in (f).
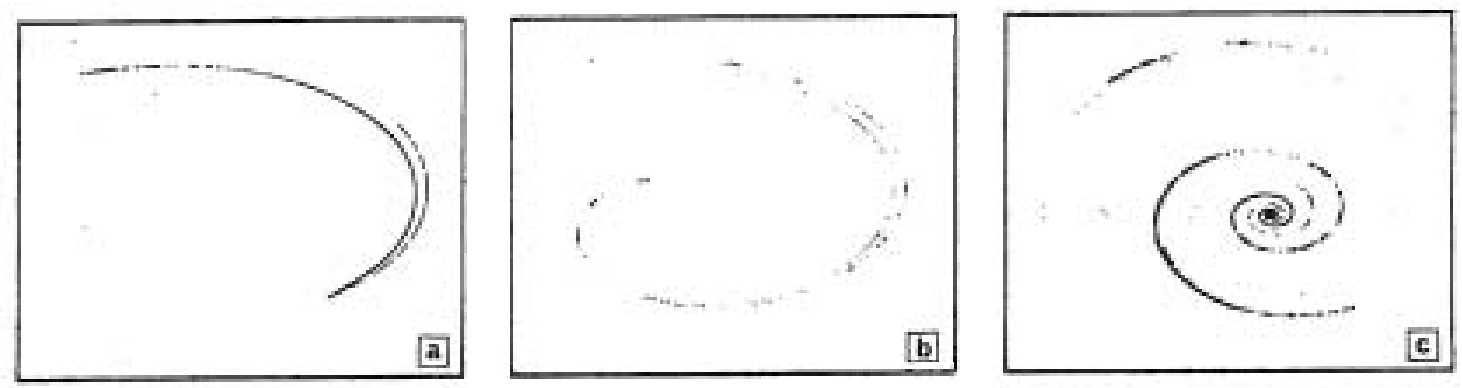

FIGURE 12. The Poincaré maps (a) and (b) correspond to Fig. 11 (d) and (e) respectively. The full double spiral (c) can only be obtained with a system invariant under a central symmetry. In all cases we have chosen $\mathrm{x}=$ cte as typical Poincaré plane.
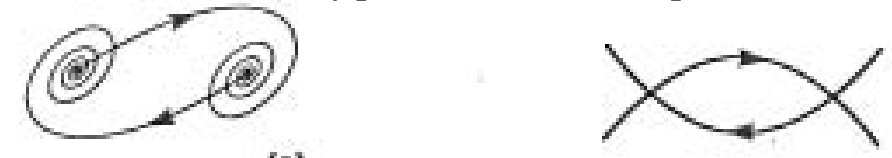

(a)

(a)
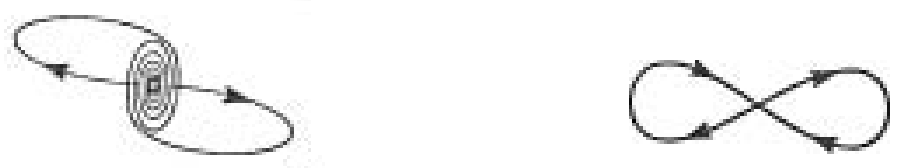

(b)

(b)

FIGURE 13. (a) and (b) represent two typical three-dimensional saddle loop connections for the case (3.a) with central symmetry. Their respective two-dimensional versions are shown in $\left(a^{\prime}\right)$ and $\left(b^{\prime}\right)$. 
It is also interesting to consider the case (3.b) with axial symmetry* Here, one can suggest the existence, for some arbitrarily small, $\eta$, of a pair of homoclinic orbits on Fig. 14. If these orbits are unstable, one is

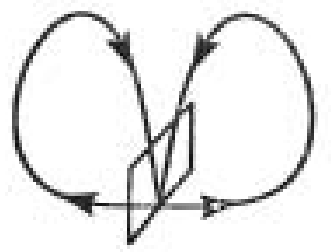

(a)

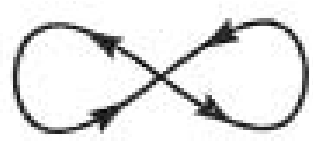

(a')

FIGURE 14. (a) represents a pair of homoclinic orbits for the case (3.b) with axial symmetry. (a') is the corresponding two-dimensional saddle loop.

likely to observe chaotic behavior of the Lorenz type (Lorenz, 1963, and Guckenieimer, 1976). On the contrary, if these homoclinic orbits are stable, a cascade of instabilities precedes the occurrence of chaos (Arneodo, et. al., 1981).

Cther scenarios for the transition to chaos can be encountered near degenerate singularities. For example, in the case (2.b) in Table I one can oet tori whose destruction leads to chaotic behavior (Langford, 1979 and Guchenueimer, 1979). Let us also mention the cascade of period doubling bifurcations for tori which is obtained with the truncated normal form coriespouding to case ( $3 . \tilde{f})$ (A. Arneodo, et. a1., in preparation).

\section{REFERENCES}

Arneodo, A., Couliet, P., and Tresser, C., 1981. To appear in Commun. Math.

Phys .

Arneodo, A., Coullet, P., and Tresser, C., 1981. To appear in J. Stat. Phys. Arneodo, A., Coullet, P., and Tresser, C., 1981. Phys. Lett., 81A, 197. Arneodo, A., Coullet, P., and Tresser, C., in preparation.

Arnold, V., 1980. Chapotres Supplementaires de la Theorie des Equations Diffentielles Ordinaires, MIR Moscou, 297.

Carr, J., 1979. Applications of Center Manifold Theory. Preprint, Brown University.

coullet, P., Tresser, C., and Arneodo, 1A., 1979. Phys. Lett., 72A, 268. Guckenueimer, J., 1976.

Guckenheimer, J.; 1979. On a Co-dimension Two Bifurcation. Preprint, Santa

Cruz .

Guckenheirier, J., 1981. Multiple Bifurcation Problems of Codimension Two.

Prtprint, Santa Cruz.

Guckenheiner, J. and Knobloch, E., 1981. Nonlinear Convection in a Rotating

Layer: Amplitude Expansion and Center Manifold. Preprint, Santa Cruz= Haken, H., 1979. Synergetics: An Introduction, Springer Verlag, New York. Henon, M., 1976. Commun. Math. Phys., 50, 69. 
Holmes, P. J., 1980. Annals of the New York Academy of Sciences, 357, 473. wolmes, P. J., and Marsien, J. E., 1978. Automatiqua, 14, 367.

Loess, G. and Josuph, D. D., 1980. Elementary Stability and Bifurcation

Theory, spranjer Verlag, New York.

Langford, W., 1979. Siait J. Appl. lath., 37, 22.

Lolenz, E., 1973. J. Atmos. Sci., 20, 130.

Marsden, J. E. and Micracken, K., 1976. Hopf Bifurcation and its

Aprlications, Springer Verlag, New York.

Moore, D. F. and Spiegel, E. A., 1966. Astrophys. J., 143, 871.

Poincare, H., 1928. These: Oeuvres Completes, vol I, 1879, Gauthier Villars, Paris.

Shil'nikov. L. P•, 1965. Soviet Meth. Dokl., 6, 163.

Smale, S., 1365. Differential and Combinational Topology, (Cairns S., Ed.)

Princeton University Press.

Takens, F., 1974. Pub. Math, I. H. E. S., 43, 47.

Turner, J. S•, 1973. Buoyancy Effects in Fluids, Cambridge University Press.

\section{SLOWLY VARYIHG WAVES AND CONVECTION:}

\section{Louis Howard}

Systems of partial differential equations describing physically interesting continuum phenomena not uncommonly have families of plane-wave solutions. There are perhaps three main types - 'pulses', 'transitions', and 'wavetrains'. In each of these the solutions are expressible as functions of a single variable, the 'phase' $\theta$, of the form $\theta=\sigma t-\underline{d} \cdot \underline{x}$ (or simply $\sigma-\alpha x$ if the coordinate system is properly oriented). In the wave-train case, with which we are tost 1 y concerned here, these functions are $2 \pi$-periodic functions of $\theta$, so that $r$ is the angular frequency and $\alpha$ the wave-number vector. In the other types we do not have periodic functions, but functions which tend to limits at $t$ oo. Usually in these cases, and sometimes also for wave-trains, it is more convenient to replace the phase by the position relative to the wave; $z=x-c t, t=\sigma / k$ is then the wave-speed). For pulses, the limits at $\pm \infty$ are tue same; for transitions, tuey are different. To investigate the possibility of such solutions, one introduces the assumption that the dependent variables depend only on (or $\xi$ ) and ckecks first that the resulting system of equations is consistent with this assumption, i.e., that the result is in fact an autononous system of ordinary differential equations; this will always be the case if the original system of partial differential equations is invariant uncer spatial and tempoial translations. Then one must look to see if the autonomous system, in fact, has one or more solutions of the required sort: a $2 \pi$-periodic solution for wave-tiains, a homoclinic orbit solution for pulses, or a heteroclinic orbit solution (joining two critical points) for t anstions. In particular cases there may be no such solutions, or solutions oiily for a special value of the wave-speed c (or of $\sigma$ and $\alpha$ ) or families of solulions de eliding on one or more pasmeters, in which case $\sigma$ and $\propto$ would In general ve depencient on these parameters. 
Examples of all three types appear in various aspects of the theory of (nonlinear) water waves. Pulse solutions are of special interest in models of nerve signal transmission and in many kinds of 'soliton' problems. The transition type occurs, for instance, in models of the structure of shock waves, explosion and flame front propagation, and the spread of an epidemic. Wave-train solutions occur also in certain types of reaction-diffusion systems, notably those involving oscillatory chemical reactions.

Sometimes the existence of plane-wave solutions can be demonstrated by explicit construction in terms of formulas, but this is relatively rare, especially in nonlinear problems. Other methods which have been successfully used include: bifurcation methods, particularly applicable for weakly nonlinear wave-trains or weak shocks; singular perturbation methods (e.g., the Flatto-Levinson theorem); topological methods, sometimes very powerful for existence, even with strong nonlinearity, but usually not very constructive; and numerical methods, good for quantitative purposes but harder to make into proofs.

When a family of plane wave solutions is known, or may reasonably be regarded as known because some relatively practical algorithm for its construction is available, it may be put to use as a 'center of perturbation " the theory of slowly-varying waves attempts to do this. It seeks to find approximations to more general solutions of the partial differential equations which are "close" to plane waves. In the wave-train context one may think of giving initial conditions which at each point are close to a particular member of one of the family of plane waves, but the 'particular one' varies slowly from point to point. One may reasonably expect that evolving from such initial conditions, at least for a considerable time, would be a solution which at any time is likewise locally close to certain members of the family of plane waves, but that the particular ones would also vary slowly with time. The goal then is to describe in simple and useful terms the slow temporal evolution of the spatially slowly varying approximate plane wave solution. These general ideas, which follow on from those behind the WKBJ method for linear problems, have been developed and applied by numerous authors, for instance Whitham and Benney: Whitham's book, "Linear and Nonlinear Waves", is a very good general reference especially for problems involving conservative systems such as water waves. A fairly general theory of slowly varying waves in the context of reaction-diffusion equations with oscillatory chemical kinetics, perhaps typical of dissipative systems, has been given in a paper "Slowly Varying Waves and Shock Structures in Reaction-Diffusion Equations" by L. N. Howard and N. Kopell (1977). This paper also considers the more precise mathematical formulation of such problems, and the present lecture was largely devoted to a sketch of this general theory in the case of such chemical waves.

To give a more precise definition of slowly varying waves, one may introduce the 'slow' space and time variables $X=\varepsilon x, T+\varsigma t$ where the small parameter $\varepsilon$ is the measure of 'slowness'. (The above is the scaling appropriate to chemical waves - in other problems the spatial and temporal scalings might have different powers of $\left.\varepsilon_{.}\right)$Then, a slowly varying wave is a family of (vector) functions (parameterized by $\varepsilon$ ) $F(\underline{x}, t, \epsilon)$ which has the representation

$$
F(x, t, \epsilon)=Y(\theta(z, t, \epsilon), X, T, \epsilon)
$$

where 
(1) For each fixed $\underline{X}, T$ and $\epsilon$ (sufficiently sma11) $Y(\theta, \underline{x}, T, \epsilon)$ is a periodic function of its first argument having least period $2 \pi$ "

(2) $Y(\theta, x, T, \epsilon)$ has an asymptotic expansion in $\varepsilon$, uniformly for the other arguments i n some compact set.

(3) If $\Theta(\underline{x}, T, \epsilon)$ is defined by $\varepsilon \theta(x, t, \epsilon)=\Theta(\underline{x}, T, \epsilon)$ then $\Theta$ has an asymptotic expansion in $\varepsilon$, similarly uniform.

(The second condition says that the fast variation of $Y$ with $\underline{x}$ and $t$ comes only from the dependence of $\theta$ on $\underline{x}$ and $t$ The third condition expresses the idea of local resemblance (for small $\varepsilon$ ) to a plane wave of frequency $\Theta_{\tau}$ and wave-number $-\Theta_{X}$, as one sees by expanding $\Theta$ in a Taylor series about any particular point $\underline{x}_{g}, T_{a}$.)

Now in the case of reaction-diffusion systems with oscillatory kinetics which are described by equations of the form $c_{t}=f(s)+K \nabla^{2} c$, for the concentration vector $c$, in which $f$ is such that the kinetic equations $c_{t} \equiv f(c)$ have a limit cycle solution - it turns out that there is a one-parameter family of plane-wave solutions, apart from a rotation or translation of coordinates.

These may be parameterized by the squared magnitude of the wave number vector: for each $\alpha^{2}$ in a certain range there is a frequency $\sigma=H\left(\alpha^{2}\right)$ and a \%wave--form' $Y_{\alpha^{2}}(\theta)$ (a $2 \pi$-periodic vector function) such that $c=y_{\alpha^{2}}(\sigma t-\alpha x)$ is a plane-wave solution to the reaction-diffusion equations. [Note the difference between this dissipative system and nonlinear water waves, where there is a two parameter family. Wave number and amplitude parameters can be independently prescribed, and the 'dispersion relation' has the form $\left.\sigma=H\left(\alpha^{\prime}, A\right)\right]$.

Hypothesizing the existence of a slowly-varying wave which is a solution of the reaction-diffusion equations (i.e., a family parameterized by $\varepsilon$ of functions $Y(\theta, X, T, \theta)$ and $\theta(x, T, \zeta)$ as described above, which give solutions), one can show that the lowest order term $\Theta^{\circ}(x, T)$ in $\theta=\theta^{\circ}+\theta^{\circ}+\ldots$ must satisfy the equation $\Theta_{T}^{0}=H\left(\Theta_{x}^{0}\right)$, and also $Y^{\prime}(\theta, x, T) \cdot y_{\theta_{R}^{*}}^{*}(\theta)$ (assuming a consistent normalization of the zero of phase). Thus to a first approximation the temporal evolution of a slowly varying solution is determined by solving the initial value problem for the Hamilton-Jacobi equation $\Theta_{;}^{*}=H_{(}\left(\Theta_{x^{*}}^{*}\right)$, which can be described quite completely and simply in terms of the characteristic curves (here actually straight lines) for this problem.

This description may, however, break down after a certain time, for the characteristics projected into the $\underline{X}, T$ space may well eventually intersect. This is not just a weakness of the method of characteristics, but indicates that the slow variation in space has, in certain places, become fast. The usefulness of the slowly varying wave description can, however, be extended beyond the time of intersection of characteristics by introducing the idea of shocks. Provided the position of the shocks is known, the Hamilton-Jacobi equation can still be used in the regions between them. But to find where the shocks are, or how they move, something else must be added: an analog of the Rankine-Hugoniot equations of gas-dynamics. This turns out to be simply continuity of the phase function $\theta^{\circ}$ (but not of its derivatives) across the shock, together with the 'entropy condition' that characteristics should converge on the shock, not diverge from it.

From the point of view of the large-scale description of the slowly varying wave theory, the shocks appear to be infinitely thin layers across which there are discontinuities in the local wave number and frequency, but on a smaller scale they have the structure of transition zones. To determine 
this structure one must, in principle, return to the full reaction-diffusion equations, with the simplification, however, that to a first approximation the slock may be regarded as plane and moving with a constant speed. Thus one gets a mathematical problem, the 'ideal shock', which can still be formulated in terms of ordinary differential equations. It turns out that the thickness of the shock zone depends upon the 'strength' of the shock, and for weak shocks, those with only a small jump in wave number across them, this thickness becomes large. This suggests that an extension of the slowly varying wave picture might be available which would include, not only the regions between shocks, but also the structure of the shocks themselves so long as they are weak. This turns out to be the case, at least to a first approximation, and (in terms of the original unscaled variables and i $n$ one space dimension) one obtains the equation

$$
\theta_{t}=H\left(\theta_{x}{ }^{3}\right)+K_{1} \nabla^{2} \theta
$$

where the diffusivity $K_{1}$, is determined from the full diffusivity matrix by a somewhat complicated formula involving the properties of the plave wave solutions. In general, it is a function of $\theta_{x}^{2}$ (for details see the paper by Howard and Kopell (1977). This equation should give a reasonable description not only between shocks (where the Lapacian term is negligible) but right through them so long as they are weak. The description essentially in terms of a single phase function $\theta$, however, cannot be maintained for sufficiently strong shocks.

Now convection, also a dissipative system, has an analog of the chemical plane waves: the two dimensional roll solutions. They also form a oneparameter family, apart from rotations and translations of coordinates, which may be parameterized by the wave number (in the unstable range of the linear theory). Since they do not propagate, the situation is somewhat different, but the analogy seems close enough that one may expect that the same general approach will be useful. It is true that the properties of the roll solutions have mostly been studied by amplitude expansions near the neutral curve, but effective numerical methods, relatively simple by contemporary standards, are available to calculate them for larger amplitude. It seems reasonable to regard the roll solutions as "known", and try to use them as centers of perturbation for a slowly varying wave theory which would be useful in studying the temporal evolution of 'imperfect ${ }^{8}$ patterns of convection. The theory of Newell and Whitehead (1969) is a step in this direction, but is only applicable near the critical Rayleigh number. This is because although there are really two independent parameters, the 'slowness' parameter $\varepsilon$ and the supercriticality $R-R_{c}$, for technical reasons Newe11 and Whitehead linked them by considering $R-R_{C}=O\left(\varepsilon^{2}\right.$ ) (so that $\varepsilon$ also measures the amplitude of the convective rolls). This approach permits the properties of the rolls to be brought in in the familiar way by amplitude expansions, but restricts the results to the neighborhood of $R_{C}$. What $I$ advocated in this lecture is the separation of these two parameters: the general idea of slowly varying waves depends only on the slowness parameter and not on the supercriticality. Since the properties of the rolls can be effectively determined without the assumption that $\mathbf{R}-R_{c}$ is small, it should be possible to develop a slowly varying wave theory also applicable when $R-R_{c}$ is not small, and in fact, most experiments on pattern evolution are done under such circumstances.

\section{REFERENCES}

Howard, L. N. and N. Kope11, 1977. App1. Math., 56, 95-145.

Newe11, and J. Whitehead, 1969. J.Fluid Mech., 38, 279-303. 
NONLINEAR OSCILLATIONS IN DOUBLY-DIFFUSIVE CONVECTION

Edgar Knobloch

Doubly-diffusive convection is characterized by a competition between a destabilizing temperature gradient and a stabilizing effect due to a stable solute gradient, rotation, or an imposed magnetic field. This competition has several important consequences. First, subcritical finite amplitude steady convection becomes possible. Second, if the stabilizing agent diffuses more slowly than the temperature, convection can set in as growing oscillations. Finally, the competition results in interesting and complicated dynamics already at small amplitudes, and these are accessible to perturbation methods.

The problem of magnetoconvection is typical. The two-dimensional Boussinesq problem with free boundaries, periodic in $x$, and an imposed vertical magnetic field is described by the dimensionless equations (Weiss, 1977)

$$
\begin{gathered}
\sigma^{-1}\left[\partial_{t} \nabla^{2} \psi+J\left(\psi, \nabla^{2} \psi\right)\right]=R \partial_{A} \theta+\zeta Q J\left(x, A, \nabla^{\prime} A\right)+\nabla^{4} \psi \\
\partial_{t} \Theta+J(\psi, \theta)=\partial_{t} \psi+\nabla^{2} \theta \\
\partial_{t} A+J(\psi, A)=\partial_{t} \psi+\zeta \nabla^{2} A
\end{gathered}
$$

where $\quad \sigma=\nu / k, \zeta=\nu / \kappa, R=g \alpha \Delta T k^{2} / k \nu, Q=B_{0}^{2} h^{2} / \mu+\rho_{0} \eta^{\nu}$.

The boundary conditions are

$$
\begin{aligned}
& \psi=\partial_{n}^{2} \psi=\theta=\partial_{2} A=0 \quad \text { on } z=0,1 \\
& \psi=\partial_{n}^{2} \psi=\partial_{x} \theta=A=0 \quad \text { on } x=0, \lambda .
\end{aligned}
$$

These are the least offensive boundary conditions; as a consequence the eigenfunctions of the linearized problem (about the conductive state $\Psi=\Theta=A$ $=0)$ are sines and cosines.

The linear stability theory is best summarized in terms of the normalized quantities

$$
r=\left(\pi^{2} / \lambda^{2} p^{3}\right) R, \quad q=\left(\pi^{2} / p^{2}\right) Q, \quad P=\pi^{2}\left(1+\lambda^{-1}\right) .
$$

If $\zeta<1$, and $q>q_{c}=\xi^{(1+\sigma) / \sigma(1-\zeta)}$, instability sets in as an oscillatory mode at $r=r^{(o)}$ prior to a positive (unstable) eigenvalue passing through zero at $r=r^{(e)}>r^{(o)}$. In an amplitude-Rayleigh number diagram a branch of oscillatory (steady) solutions bifurcates from $\mathrm{r}^{(\mathrm{o})}(\mathrm{r}(\mathrm{e}))$.

When $q$ is close to $q_{c}, r^{(0)}$ is close to $r^{(e)}$ and the oscillations have a long period. In this regime the interaction of oscillatory and steady convection can be studied analytically. The following method anngars to be the simplest. We define $\epsilon \ll$, by the relation $q=q_{*} \epsilon^{2}$. Then $\mathbf{r}^{(\mathbf{e})}-\mathbf{r}^{(0)}=$ $O\left(e^{2}\right)$, and we write $r=r_{c}+\mu \varepsilon^{2}$, where $r_{c}=(\sigma+\varepsilon) /(1-\varepsilon)$ and $\mu=o(1)$. Since the frequency of the linear oscillations is $O(E)$, we define a slow time $\tau=\epsilon \mathrm{pt}$. Finite amplitude perturbation expansion gives to $O\left(\epsilon^{2}\right)$, the representations

$$
\begin{aligned}
& \Psi=2(2 p)^{1 / 2}(\lambda / \pi) \epsilon a(\tau, \epsilon) \sin \pi x / \lambda \sin 2 z+\cdots \\
& \Theta=2(2 / p)^{1 / 2} \epsilon b(\tau, \epsilon) \cos \pi \times / \lambda \sin \pi z-(1 / \pi) \epsilon^{2} c(\tau, 6) \sin 2 \pi+\cdots \\
& A=2(2 / p)^{k / 2} \lambda \epsilon d(\tau, \epsilon) \sin \pi x / \lambda \cos \pi z+(\lambda / \pi) \epsilon^{2} e(\tau, \epsilon) \sin 2 \pi x / \lambda+\cdots
\end{aligned}
$$


From the equations of motion it therefore follows that -

$$
\begin{aligned}
& \sigma^{-1} \epsilon a^{\prime}=-a+r b-\epsilon_{q d}\left(1+(\epsilon-\sigma) \epsilon^{2} e\right)+o\left(\epsilon^{4}\right) \\
& \epsilon b^{\prime}=-b+a-\epsilon^{2} a c+o\left(\epsilon^{4}\right) \\
& \epsilon c^{\prime}=\omega(-c+a b)+o\left(\epsilon^{2}\right) \\
& \epsilon d^{\prime}=-f d+a-\epsilon^{2} a c+o\left(\epsilon^{4}\right) \\
& \epsilon e^{\prime}=-(4-\omega) \epsilon e+\omega a d+o\left(\epsilon^{2}\right) .
\end{aligned}
$$

Here the prime denotes differentiation with respect to $\tau$, and $\boldsymbol{\theta}=4 \boldsymbol{T}^{2} / \mathrm{p}$ It follows that $b(\tau, \epsilon)=a(\tau, \epsilon)+\epsilon g(\tau, \epsilon)$, where $g=O(1)$. We obtain an equation for $g(\tau, \epsilon)$ to any required order by iteration:

$$
g=-b^{\prime}-\epsilon a c+o\left(\epsilon^{3}\right)=-a^{\prime}-\epsilon g^{\prime}-\epsilon a c+o\left(\epsilon^{3}\right) e+c_{1}
$$

Hence

$$
b(\tau, 6)=a-\epsilon a^{\prime}+c^{2}\left(a^{\alpha}-a^{2}\right)-\epsilon^{3}\left(a^{\prime \prime}-\left(4+\frac{2}{6}\right) a^{2} a^{\prime}\right)+a\left(6^{4}\right)
$$

This, and similar expressions for d and e are then substituted into (la), resulting in an evolution equation for a $(\tau, \epsilon)$ of the form

where

$$
a^{m}-M a^{3}+M N a=\epsilon F(a)+O\left(6^{2}\right) \text {, }
$$

$$
\begin{aligned}
& M=-r_{2}^{(e)} r c /(1+\sigma+\varepsilon), \quad N=-(1-\mu) / r_{k}(e) \\
& F(a)=\sigma\left[\left(a^{2} a^{\prime}+D a^{0}+(1-\mu)^{\prime}\right] /\left(1+\sigma+a^{\prime}\right)\right.
\end{aligned}
$$

and $C, D$ are known constants. For $E \ll C_{1},(2)$ is solved in terms of a one parameter family of elliptic functions. In general, however, the energy of the corresponding oscillations, $E a \frac{1}{2} a^{2}-4 M a^{4}+\frac{1}{2} M N a^{2}$ changes on the superslow time scale $T=\epsilon \tau$ according to

$$
\frac{d E}{d T}=\frac{1}{p} \int_{0}^{P} d \tau a^{\prime} F(a)+o(\epsilon)
$$

where $P$ is the period of the oscillations. Only for those values of the modulus of the elliptic function satisfying $d E / d T=0$ is the energy constant and the oscillations periodic on the time scale $T$. This condition, evaluated in terms of the zeroth order solution, gives a relation between the modulus and the Rayleigh number $\mu$, and hence between the oscillation amplitude and $\mu$. The amplitude-Rayleigh number diagrams corresponding to subcritical ( $r_{b}^{(\ell)}<o$ ) and supercritical ( $r_{2}(e)>0$ ) steady convection are shown if Fig. 1; unstable portions of the solution branches are indicated by broken lines. Details can be found in Knobloch and Proctor (1981).

Since $E \ll 1$, (2) reduces to the normal form (Guckenheimer, 1981); Guckenheimer and Knobloch, 1981).

$$
a^{\prime \prime}=M a^{2}-\Gamma a^{2} a^{\prime}+\alpha a+\beta a^{\prime}
$$

where $M, \Gamma(>0)$ are known coefficients, and $\alpha, \beta$ are the unfolding parameters $\left(\alpha=\beta=0\right.$ when $\left.q=q_{c}\right)$. This is the Van $d$ er Pol-Duffing equation. The phase portraits for the two cases $\left(r_{2}^{(\omega)} \leqslant 0\right.$ ) as functions of $\alpha, \beta$ are shown in Fig. 2. The heavy line in the $\alpha, \beta$ plane corresponds to increasing $r$; Fig. 2 thus shows the succession of phase portraits that occurs as $r$ increases. In the subcritical case the branch of oscillations ends on the unstable steady branch with the oscillation period becoming infinite; in supercritical case it ends on the steady branch via a subcritical Hopf bifurcation. Note, however, that 


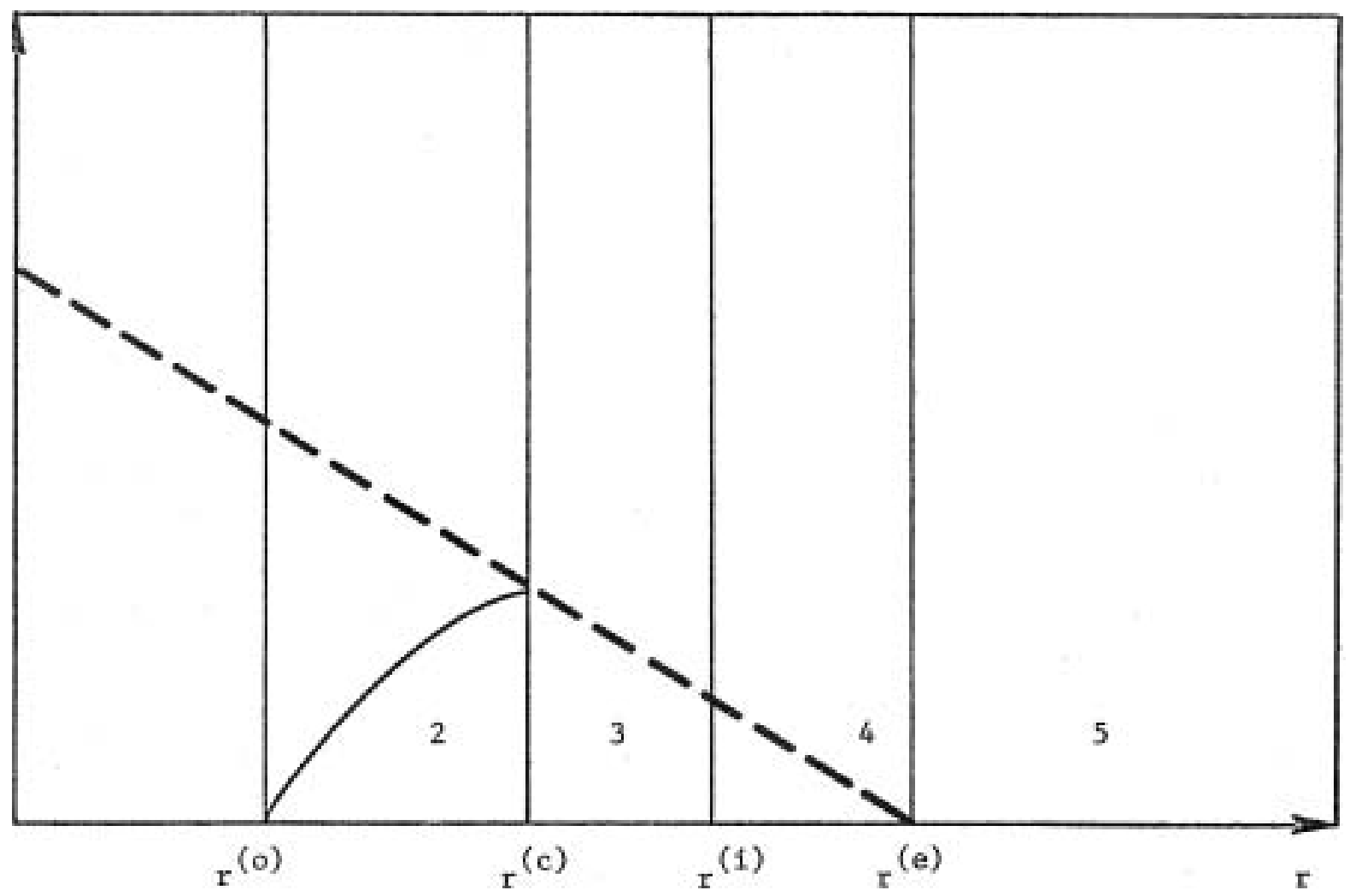

FIGURE 1: Case $r_{2}^{(e)}<0$.

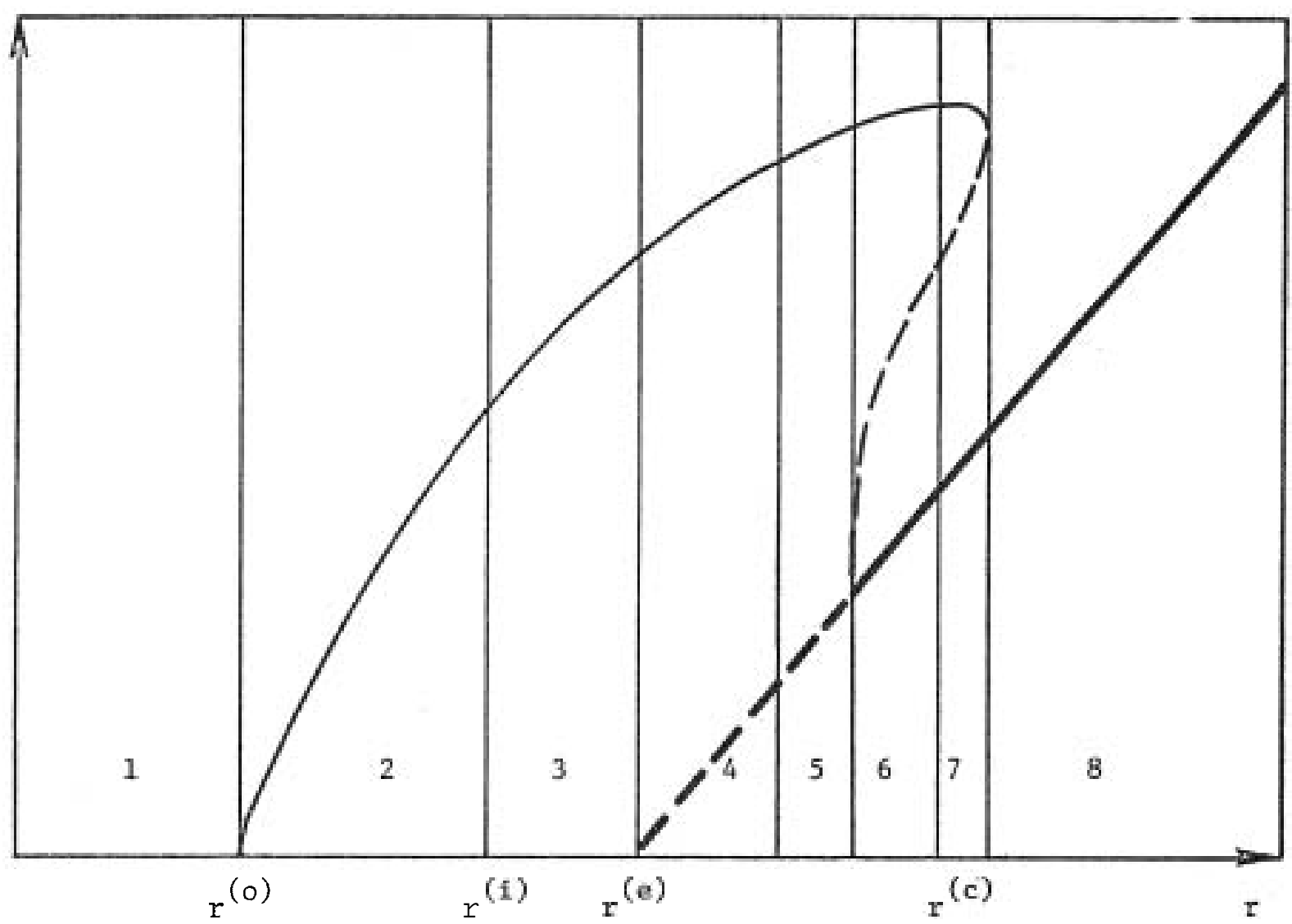

FIGURE 1: Case $\mathrm{r}_{2}^{(\mathrm{e})}>0$. 
- 115 -

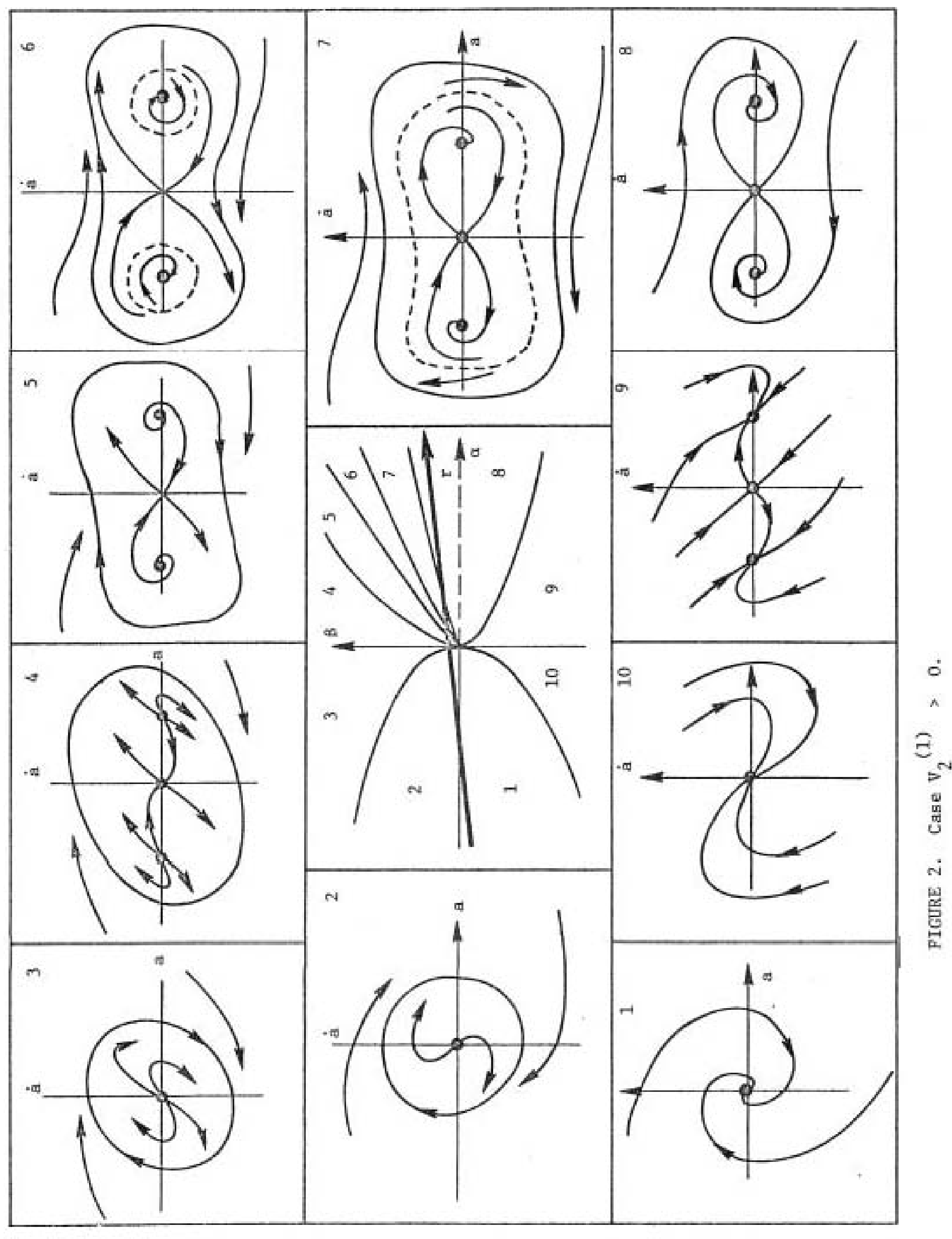




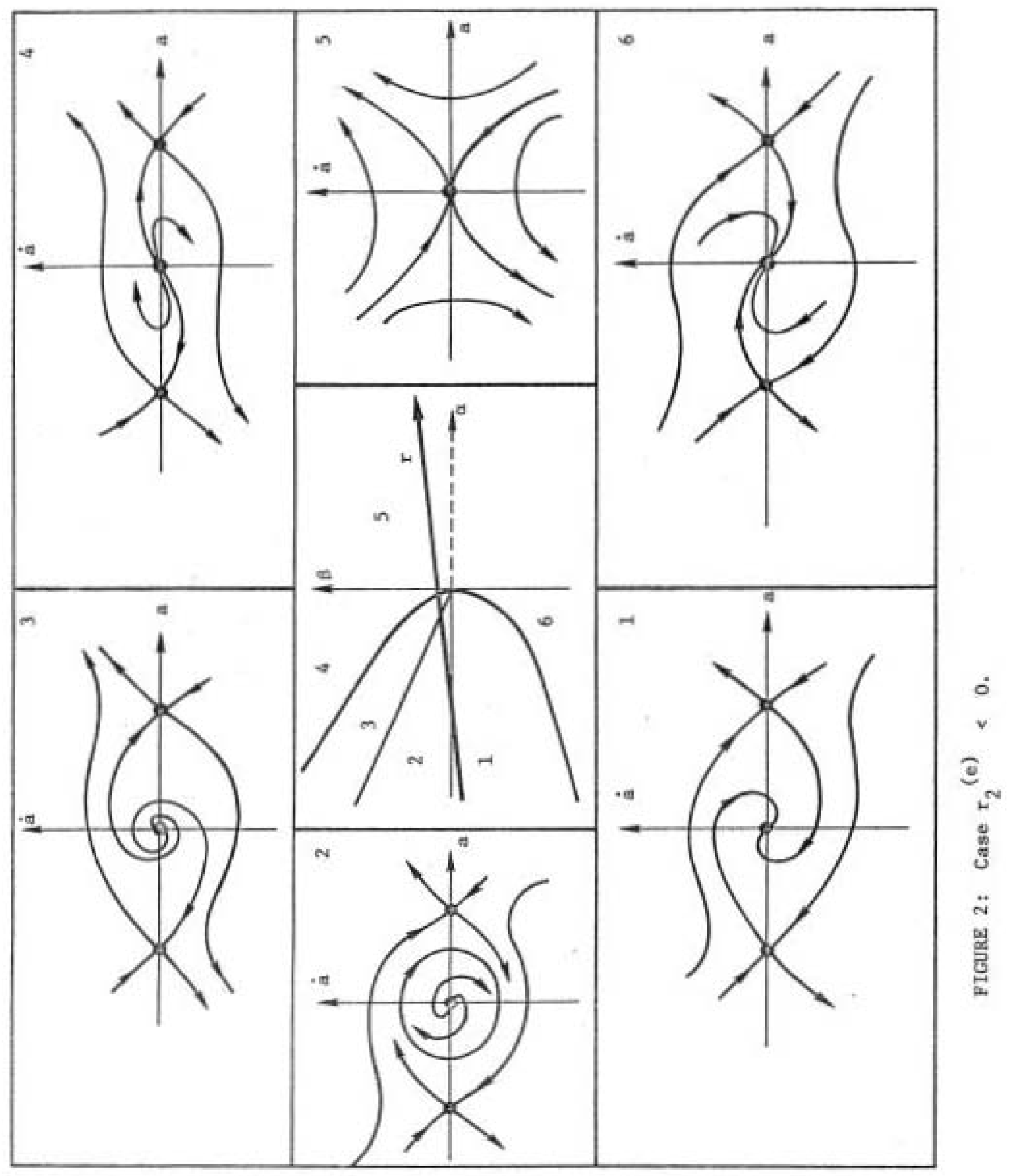


i n both cases heteroclinic limit cycles occur, and for these the condition (3) formally breaks down.

The form of equation (4) depends only on the codimension of the bifurcation ( 2 , i $n$ the present case), the reflectional symmetry of the underlying partial differential equations (and boundary conditions), and the requirement that $a=0$ remains a fixed point for a $11 \alpha, \beta$. Identical dynamics are therefore found in other doubly diffusive systems. The coefficients, however, depend on the specifics of the problem; for example, in thermohaline convection only $M>0$ is possible. Equation (4) can also be obtained by doing straightforward perturbation theory that yields

$$
a_{1}\left(\tau_{1} \epsilon\right)=a_{0}(\tau)+\epsilon a_{1}(\tau)+\cdots
$$

and obtaining coupled Landau-type equations for the amplitudes $a_{0}, a_{1} .$. and then reconstituting these equations to obtain an equation for a $(\tau, \epsilon)$. This method is described by Spiegel (1981).

When $E$ is not infinitesima1, (2) can be written approximately as

$$
a^{a}-a^{0}+\left(\bar{\varphi}-\bar{\Gamma} a^{2}\right) a^{\prime}+\left(\alpha+M a^{*}\right) a=0
$$

This equation is also known (Spiegel, 1981). In the present case it is expected that it gives a good description of the qualitative aspects of larger amplitude doubly-diffusive convection, particularly the period doubling cascade and strange attractors found in fifth order models (DaCosta et,al., 1981; Knobloch et. a1., 1981; Knobloch and Weiss, 1981; Weiss, 1981). Except for the large amplitude steady solutions, (5) is therefore a good model for moderate amplitude oscillatory double-diffusive convection.

In conclusion, we note that the present method can be applied to co-dimension 3 (and higher) bifurcations; the corresponding amplitude evolution equations will be of higher order, and will generically contain strange attractors. In such cases these strange attractors are solutions of the underlying partial differential equations. This is an exciting prospect.

\section{REFERENCES}

DaCosta, L. N., E. Knobloch, and N. O. Weiss, 1981. J.Fluid Mech. (in press).

Guckenheimer, J., 1981. Preprint and lectures at GFD 1981.

Guckenheimer, J. and E. Knobloch, 1981. J.Fluid Mech., (submitted).

Knobloch, E. and M.R. E. Proctor, 1981. J. Fluid Mech., (in press).

Knobloch, E. and N. O. Weiss, 1981. Phys, Lett. A. (submitted).

Knobloch, E., N. O. Weiss, and L. N. DaCosta, 1981. J.Fluid Mech. (in press).

Spiegel, E. A., 1981. Double Convection, Lecture \#8, GFD 1981.

Weiss, N. 0., 1977. In Problems of Stellar Convection, ed. E. A. Spiegel and J. P. Zahn, 176-187.

Weiss, N. O., 1981. Bifurcations in Double Diffusive Convection, Lecture \#2, GFD, 1981. 
WHO IS TURBULENCE?

Melvin E。 Stern

A general control theory is proposed (Stern, 1980) to select the realized stationary state wherever the equations of motion are densely nonunique (degenerate). For turbulent flow in a pipe the theory implies that the discharge is an extremum. Quantitative results (Stern, 1979), such as Von Karman's constant, emerge when this variational principle is combined with inequalities pertaining to the mean field. The control theory is also applied (Stern, 1979) to fully turbulent thermal convection, and a variational principle is obtained which is also consistent with measurements.

Current work includes application of the variational principle and the inequalities to double diffusive turbulence (Stern, 1981, in press) and to the turbulent Ekman boundary layer (Stern, i n preparation).

\section{REFERENCES}

Stern, Melvin E., 1979. Inequalities and variational principles i n turbulence, J. Fluid Mech., 91.

Stern, Melvin E., 1980. A variational principle for turbulent flow, Phy。Fluids, 23, (11), 2161-2169.

Stern, Melvin E., 1981. Inequalities and variational principles i n double diffusive turbulence, J. Fluid Mech., (in press).

Stern, Melvin E. Inequalities and variational principles for the turbulent Ekman layer, (in preparation).

\section{A NUMERICAL MEIHOD FOR SIMULATING ENVIRONMENTAL FLUID MECHANICS}

Rory Thompson

There was first some mention of interests of possible use to the summer fellows, before settling on the difficulties in numerically simulating flows with a sharp interface (as is often caused by turbulent mixing), and a possible cure.

An example was considered of a stratified lake under wind-stress. A well-mixed surface layer moves downwind and piles up, hence pushing down the pycnocline at the down-wind end of the lake. Therefore, a sharp, moving pycnocline is an inherent feature of the problem. We consider now only the advection of this front, neglecting any dissipation or turbulence. In this case, the mean, variance, and extrema of the density are preserved in the continuous problem, so should be i n the simulation. It was shown that a grid (Eulesian) scheme which preserves the mean and variance of the advected quantity will exhibit a "Gibbs phenomenon", that is, as the pycnocline moves down through the grid, the density behind the front must "overshoot"' Hence, a downward water-velocity at the base of the mixed layer causes the grid-layer there to become lighter than those above? The resultant convection makes hash 
of the simulation. If one puts i n a "convective adjustment" to simulate "sub-gridscale" convection, the mixed layer will become continually lighter, violating conservation of extrema.

The "Cloud-In-Cell" method is a Lagrangian scheme that uses extended particles ("clouds'" to carry the advected quantities (e.g., $p$ ). It differs from many Lagrangian schemes in using a grid to compute particle interactions. Instead of searching over all other particles in handling each particle [requiring $O(n)$ operations if there are $n$ points], one need only go through the list of particles once [O(n) operations] to form averages $\mathbb{P} 1$ on a grid - that is, to form a field. From these averages, one forms fields for the pressure, its gradients, and hence accelerations, etc. The velocity (and any time-derivative) fields are evaluated [again in $O(n)$ operations] at the particles, which are consequently moved.

In the lake, as the pycnocline moves across the grid, so do the particles, so the Cloud-In-Cell method has no difficulty with sharp fronts. In fact, if $\mathrm{d} p / \mathrm{dt}=0$, then each individual particle preserves its original $\mathrm{p}$, so clearly $\Sigma \rho, \Sigma \rho^{2}$, and extreme values are preserved, for sums over the particles: The grid field? will not have these conservations, though experiment shows near-conservation. The field $\bar{p}$ is in a sense ficticious, existing only for intercourse among the particles. One would hope the grid field would go to the desired continuous field as the grid size shrinks simultaneously with the particle number per grid box increasing, but I know of no proof, nor even of any work on such a proof.

In summary, the Cloud-In-Cell technique exploits the ability of Lagrangian particles to handle advection and the ability of a grid to easily handle linear terms -- at the costs of complicated and expensive interaction between the two systems.

\section{CONVECTION AND MAGNETIC FIELDS IN STARS}

Nigel O. Weiss

The sun is the only star on which magnetic structures can be resolved in detail and for which we have a record of magnetic activity extending over several centuries. Recent observations have, however, confirmed the presence of magnetic cycles in other stars with deep convective envelopes. These observations also make it possible to explore the relationship between magnetic activity and angular velocity of rotation in such stars. Solar magnetic fieids have a highly intermittent structure: flux emerging from the sun is confined to isolated tubes with strong magnetic fields and the magnetic pressure within them approaches the thermal pressure of the ambient gas (Parker, 1979). Model calculations, in which the induction equation

$$
\frac{\partial B}{\partial t}=\nabla \times(y \times B)+4 \nabla^{2} B
$$

is solved numerically, have demonstrated how isolated flux tubes can be formed by kinematic flux expulsion (e.g., Galloway and Weiss, 1981; Ga1loway and Proctor, 1981). Numerical experiments on nonlinear Boussinesq magnetoconvection (Galloway and Moore, 1979; Weiss, 1981) show that motion is excluded from regions with strong fields so that the flux tubes are almost stagnant. In a turbulent region one might expect magnetic flux to be separated from the motion with fields confined to ephemeral flux ropes (Meneguzzi, Frisch and Pouquet, 1981). In turbulent Boussinesq fluids the 
magnetic energy density is unlikely to be much greater than the kinetic energy density of the motion: compressible flow is needed to explain the strong fields at the surface of the sun.

In order to provide a model of the solar dynamo it is necessary to average over the intermittent field structures. This could be done if there were a separation of scales between the global magnetic field, the individual convective eddies and the small-scale turbulence; unfortunately this does not happen in the sun. Nevertheless, mean field dynamo models, with the induction equation recast in the form

$$
\frac{\partial B_{i}}{\partial t}=\epsilon_{i j k} \epsilon_{k i m} \frac{\partial}{\partial x_{j}}\left(u_{\ell} B_{m}\right)+\epsilon_{i j k} \frac{\partial}{\partial x_{j}}\left(\alpha_{k=1} B_{l}\right)+\tilde{\eta} \frac{\partial^{2}}{\partial x_{j}^{2}} B_{i},
$$

where $\tilde{\eta}$ is a turbulent diffusivity and $\alpha_{i j}=\alpha \delta_{i j}-\gamma_{k} \epsilon_{i j k}$ for isotropic turbulence, do reproduce the overall behavior of the solar cycle (Parker, 1979). The crucial $\alpha$-effect depends on nonmirror symmetric turbulence: $\propto$ is proportional to the mean helicity $\langle\underline{u}, \nabla \times y\rangle$, generated by the Coriolis force. More detailed models require a better physical picture of the dynamo process. It seems likely that, owing to a combination of flux expulsion and topological pumping, which can be represented by the velocity $\underset{\sim}{\gamma}$, magnetic flux is concentrated in a layer at the interface between stable and unstably stratified regions. This layer can become unstable by magnetic buoyancy, allowing flux tubes to escape to the surface and emerge as active regions (e.g., Spiegel and Weiss, 1980).

X-ray observations of other stars like the sun indicate that they are magnetically active and that their activity increases with the angular velocity $\Omega$ (Vaiana et. al., 1981). Measurements of $\mathrm{Ca}^{+}$emission (Vaughan et. al.,1981) confirm that the average field strength $\bar{B}$ depends on $\Omega$. Slowly rotating stars like the sun have similar magnetic cycles but rapid rotators behave differently (Vaughan, 1980). Edgar Knobloch and I suggest the following explanation for this transition. In relatively slowly rotating stars the parameter $\Omega \tau \sim \mathcal{L}$ (where $\tau$ is an eddy turnover time) and the Coriolis force produces helicity in large eddies at the base of the convective zone, so favoring dynamo action as described above; in rapidly rotating stars $\Omega \tau \gg l$ and the pattern of convection is dominated by the Proudman-Taylor constraint, leading to elongated cells with a strong reduction in helicity. The dynamo ceases to operate but the residual field, maintained by differential rotation, is still brought to the surface by magnetic buoyancy. Since the ohmic decay time is long, the poloidal field can survive without a dynamo. During its lifetime on the main sequence a star like the sun loses angular momentum owing to magnetic braking and magnetic cycles appear at some critical value of $\Omega$ when dynamo action first becomes possible.

\section{REFERENCES}

Galloway, D. J. and D. R. Moore, 1979. Geophys. Astrophys. Fluid Dyn. , 12, 73.

Galloway, D. J. and M. R. E. Proctor, 1981. Planetary and Stellar Magnetism, ed., A. M. Soward, Gordon and Breach (in press).

Galloway, D. J. and N. O. Weiss, 1981. Astrophys. J., 243, 945.

Meneguzzi, M., U. Frisch, and A Pouquet, 1981. Phys. Rev. Lett. (submitted).

Parker, E. N., 1979. Cosmical Magnetic Fields, Oxford. 
Spiege1, E. A. and N. O. Weiss, 1980. Nature, 287, 616.

Vaiana, G. S. and 15 others, 1981. Astrophys. J., 245, 163.

Vaughan, A. H., 1980. P.A.S.P., 92, 392.

Vaughan, A. H., S. L. Baliunas, F. Middelkoop, L. W. Hartman, D. Mihalas, R. W. Noyes, and G. W. Preston, 1981. Astrophys. J. (in press).

Weiss, N. 0., 1981. J. Fluid Mech., 108, 247, 273.

\section{A MEASUREMENT OF CONVECTION AT HIGH RAYLEIGH NUMBER}

Douglas Gough

The sun is a body which possesses an intensely turbulent convective envelope. Moreover, it is sufficiently close for precise measurements of the convection zone to have been made. This is a report of a conclusion drawn from these measurements.

Because the convecting region in the sun is compressible and deep, and is not contained within prescribed boundaries, one cannot describe even the gross properties of the motion in the simple terms appropriate to most Boussinesq studies. The heat $f l u x$ is provided by the thermonuclear reactions in the core of the sun, and one can estimate a characteristic Rayleigh number to be about $10^{30}$, evaluating diffusion coefficients at the midheight of the region. One could define a characteristic Nusselt number in a similar way, but it would not be of any immediately obvious value since the boundaries of the convectively unstable region would be in quite different places were convection not to take place. Indeed, the radius of the sun itself would be substantially greater than it is at present were a deity to prohibit the flow. Nevertheless, any measure of the efficacy of convection under such conditions is of considerable interest, especially, perhaps, because the Prandt 1 number, which is typically about $10^{-7}$, and the Rayleigh number are well beyond the range of terrestrial laboratory experiments.

Before describing the details of the measurements I should say something about the quantity that is measured. As in any laboratory experiment, the true conditions are idealized somewhat in order to render the interpretation of the observations tractable. The idealization of the solar laboratory is embodied in a set of partial differential equations that describe the evolution of a self-gravitating gaseous sphere. In its simplest form, which is all I shall consider here, the differential system contains three independent parameters: the initial abundances $Y$ and $Z$ of helium and heavy elements, and a measure $\alpha$ of the efficacy of convection. These parameters are to be determined by calibrating the theoretical solar model against observation.

The standard astronomical calibration is to adjust $Y, Z$ and $\propto$ to fit the present solar radius and luminosity. This provides a singly infinite sequence of acceptable models, which I label with $Y$. What is required now is an additional datum to determine which model in the sequence best represents the sun. This would fix $Y$, and hence or, and thus would measure the convection. 
I choose as my label the parameter $\mathrm{Y}$ in preference to the others because astronomers have prejudices concerning its value. These are based mainly on studies of the spectra and evolution of stars and on cosmological considerations. Indirectly the former suggests $Y \propto 0.25$ and the 1 atter sets a lower $1 \mathrm{imit}$ to $Y$ which is a 1 ittle greater than 0.2 , though I must hastily point out that the premises upon which this deduction is based are not firmly established.

Neutrinos produced in a side reaction in the sun's core have been measured by Davis and his collaborators (1979). The result should determine the central temperature of the sun, and should thus enable the solar calibration to be completed. Unfortunately, that calibration yields $\mathrm{Y}<0.2$, which contradicts astronomers' beliefs. Thus it has been customary to discount the neutrino calibration, and to regard the disparity between theory with plausible values of $Y$ and the neutrino measurements as an unresolved puzzle.

I consider now two fluid dynamical calibrations of the model. Both are seismological, and rely on comparing observed oscillation frequencies near $3 \mathrm{mHz}$ with the eigenfrequencies of normal acoustic modes of the sequence of models. The modes are characterized by their order $n$, which, roughly speaking, is the number of spherical nodal surfaces in the radial velocity eigenfunction, and the degree $\mathbf{1}$ and order $m$ of the tesseral harmonic that factors from the eigenfunction. The eigenfrequencies $\nu$ are independent of m. I shall consider two classes of modes: one has $1 \gg n$ and the other $n \gg 1$.

Modes with $1 \gg n$ are almost horizontally propagating sound waves. Because the sound speed increases with depth, slighly downward propagating waves are refracted back up. Upward propagating waves cannot pass through the almost isothermal atmosphere if their frequency is less than Lamb's acoustical cutoff frequency $\nu_{c}$ (this corresponds to the minimum acoustic frequency in the dispersion relation discussed by Spiegel in his lectures, and is about $5 \mathrm{mHz}$ in the solar atmosphere) and so the waves are trapped in a waveguide just beneath the surface of the sun. It is easy to demonstrate that modes with $\nu<\nu_{c}$ penetrate beyond the boundary layer at the top of the convection zone into the adiabatically stratified interior, and that their frequencies measure the entropy jump across that boundary layer. The dispersion relation has been measured by Deubner et. a1. (1979), who observed the spatial and temporal variation of spectrum line shifts. Comparison of theory with observation thus provides a direct calibration of the convective boundary layer, and hence, via the evolution calculation, determines $Y$. The result is $Y=0.25$ (Berthomieu et. al., 1980; Lubow et. al,, 1980)。

Modes with $n \gg 1$ propagate almost vertically. They penetrate right to the center of the sun and so provide a more direct measure of conditions in the interior. Their frequencies have been measured accurately by Grec et. al. (1980) who succeeded in obtaining a long continuous record by observing the sun from the South Pole. A least squares fit of the appropriate eigenfrequencies of the sequence of solar models to the observations yields $=0.25$

(Christensen-Dalsgaard et. al, 1981), in agreement with the previous calibration, and so provides indirect confirmation of the value of $\alpha$.

Though these preliminary results are in reasonable accord with astronomers' beliefs, there are small systematic differences between the theoretical eigenfrequencies and observation which may be difficult to explain without additional data. Preliminary theoretical helioseismological studies have revealed that modes with small 1 and $n$ may provide the answer, but these 
modes have not yet been unambiguously identified by observation.

\section{REFERENCES}

Berthomieu, G., A. J. Cooper, D. O. Gough, Y. Osaki, J. Provost, and A. Rocca, 1980. Nonradial and nonlinear stellar pulsation. (ed. H. A. Bill and W. A. Dziembowski, Springer, Heidelberg) p. 307.

Christensen-Dalsgaard, J。 and D. O. Gough, 1981. Astron. Astrophys. (in press).

Davis, R., 1979. Status and future of solar neutrino research (ed. G Friedlander, Brookhaven Nat. Lab.) 1,1 .

Deubner, F. L。, E. J. Rhodes, Jr॰, and R。K. Ulrich, 1979. Astron. Astrophys., 72,177 .

Grec, G., E. Fossat, and M. Pomerantz, 1980. Nature, 288, 541.

Lubow, S. H., E. H. Rhodes, Jr., and R. K. Ulrich, 1980. Nonradial and nonlinear stellar pulsation (ed.H.A. Hill and W. A. Dziembowski, Springer, Heidelberg) p. 300 .

\section{SOME OBSERVATIONS OF TURBULENT STRUCTURES I N LARGE SCALE FORCED CONVECTION}

\section{David Topham}

Studies of the Arctic Ocean heat budget suggest that a large proportion of the heat flux to atmosphere is transmitted through the relatively small proportion of the surface area which is made up of open water and ice less than $10 \mathrm{~cm}$ in thickness. These areas of open water may be more or less permanent features caused by upwelling of sensible heat from below, known as polynyas, or swiftly changing features such as leads. The former are irregular openings ranging in extent from a few hundred meters to many kilometers, while the latter tend to be linear features.

Several groups have mounted experiments to study the effect of such surface anomalies on their surroundings, both in the atmosphere and in the ocean. Typical conditions are atmospheric temperatures in the range -20 to $-40^{\circ} \mathrm{C}$ with wind speeds up to $10 \mathrm{~m} / \mathrm{sec}$. There is thus a violent change in surface conditions as the airflow passes from solid ice cover at close to atmospheric temperature out over the water surface at about $-1,8^{\circ} \mathrm{C}$. Previous investigations were limited to areas of open water with maximum fetches of $80 \mathrm{~m}$, with many of the most detailed measurements confined to fetches of $20 \mathrm{~m}$ or less.

The work briefly described here represents the combined efforts of three groups: The Frozen Sea Research Group of the Institute of Ocean Sciences, Sidney, B. C.; the Air-Sea Interaction Group of the Bedford Institute of Oceanography and the Boundary Layer Meteorology Group of the Canadian Atmospheric Environmental Service, Toronto. The aims were to measure the heat flux from an area of open water several hundred meters in extent under conditions of extreme temperature difference. 
The site chosen for study was a small polynya situated off the northern tip of Dundas Island, N. W. T., positioned as shown in Fig. 1., the point A marking the open water. Dundas Island is situated on a relatively shallow sill dividing two deeper channels, Wellington Channel to the east and Crozier Strait to the west. Tidal flows over this shallow sill exceed $1 / \mathrm{m} / \mathrm{sec}$. and this is sufficient to cause mechanical removal of newly formed ice. In addition, there are significant quantities of heat available in the water column, the near surface (10 m depth) water temperatures showing fluctuation. of about $0.10^{\circ} \mathrm{C}$, at tidal frequencies, with the lowest temperatures approaching the freezing point. It is possible that the large increases in water temperature which occur at the faster current conditions are caused by an upwelling process as the flow over the sill increases in speed. The combination of fast tidal currents and a supply of sensible heat are sufficient to keep the area open throughout the winter on a regular basis.

The following measurements were made in an effort to deduce the heat losses from the area and to provide both qualitative and quantitative descriptions of the dominant processes.

(1) Local fluctuating quantities within the growing internal boundary layer were measured using a 3 -axis sonic anemometer mounted at $4 \mathrm{~m}$ height. This instrument gives the three components of velocity and the local temperature fluctuations. In addition, an array of $4 \mathrm{fast}$ response temperature sensors could be mounted to examine spacial coherence in either the vertical or horizontal. The measurement of the fluctuating component of vertical velocity together with the temperature fluctuations enable the turbulent eddy flux at that height to be estimated.

(2) Mean velocity and temperature profiles were measured, both upstream and downstream with the intention of obtaining on overall turbulent heat loss. In practice such an integral method can only be interpreted in situations which are approximately two-dimensional.

(3) The fog forming above the surface of the water served as a natural flow tracer and photographs clearly revealed the large scale feature of the flow patterns.

The measurements have yet to be analyzed in detail and the small sample presented here is intended to give a qualitative picture of events. Fig. 2 shows the planview of the open water at a particular time; the details of the outline changed throughout the period of the experiment (6 weeks) but this general shape was retained. The larger black dots mark observation sites and the arrows denote wind direction for different measurement periods.

The shape is such that the overall flow pattern over and around the open water depends strongly on wind speed and direction. For the sites between points $C$ and $D$ and the indicated wind directions the flow was approximately two-dimensional and Fig. 3 shows typical profiles of mean wind speed and temperature. It Is to be noted that most of the change from the surface conditions takes place within $50 \mathrm{cms}$ of the surface. The highest point on the profile lay well within the internally developing boundary layer made visible by the fog patterns. That this area contains considerable vertical activity is shown by Fig. 4 which is a section of a time series of air temperature measured simultaneously at four heights. The high degree of vertical coherence suggests the passage of tall narrow elements past the measurement point. The tendency for the signals to take on a rectified appearance at the 


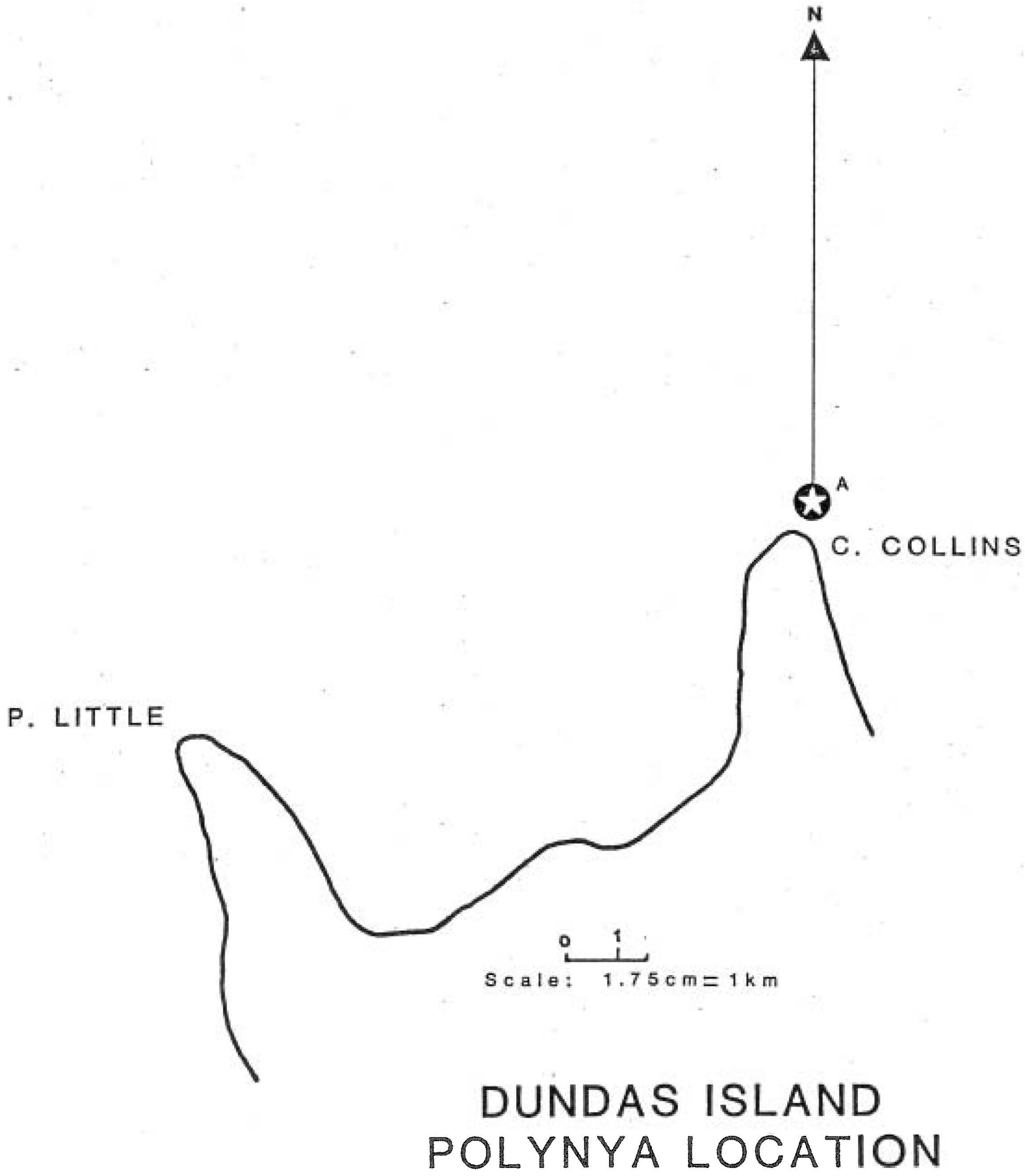

FIGURE 1 


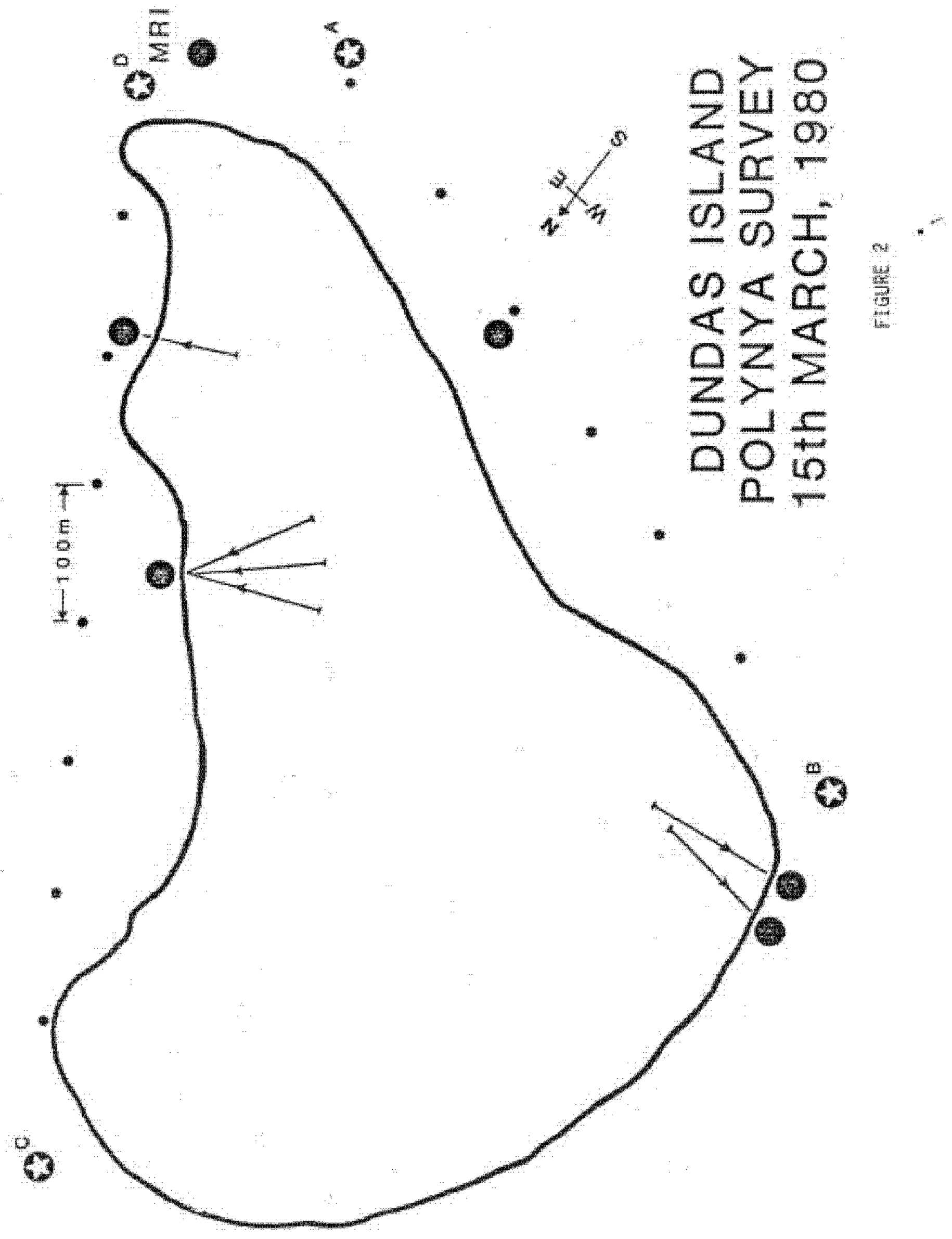




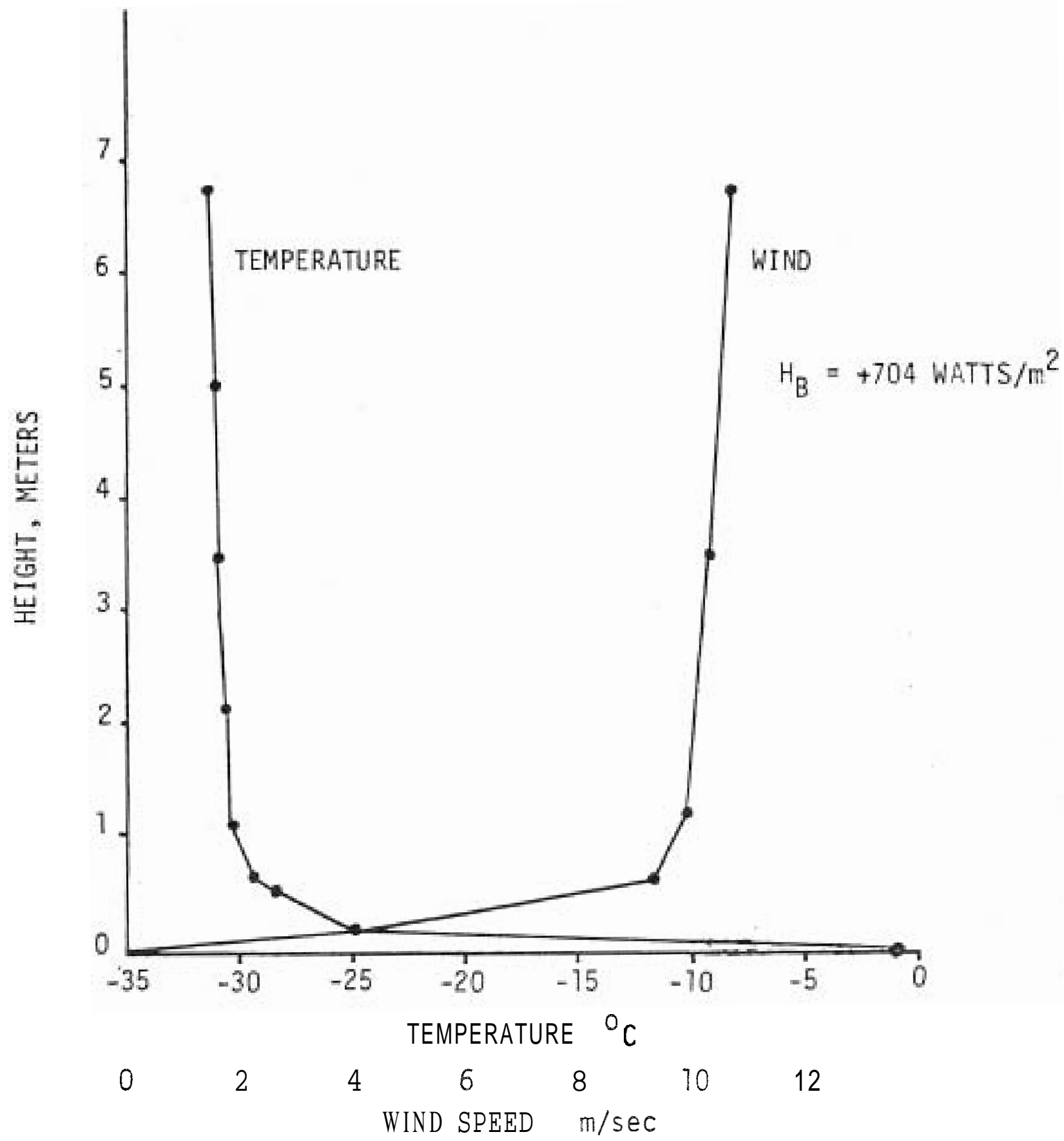

FIGURE 3

TEMPERATURE AND WIND PROFILES MARCH $17 \quad 12: 27$ GMT. 


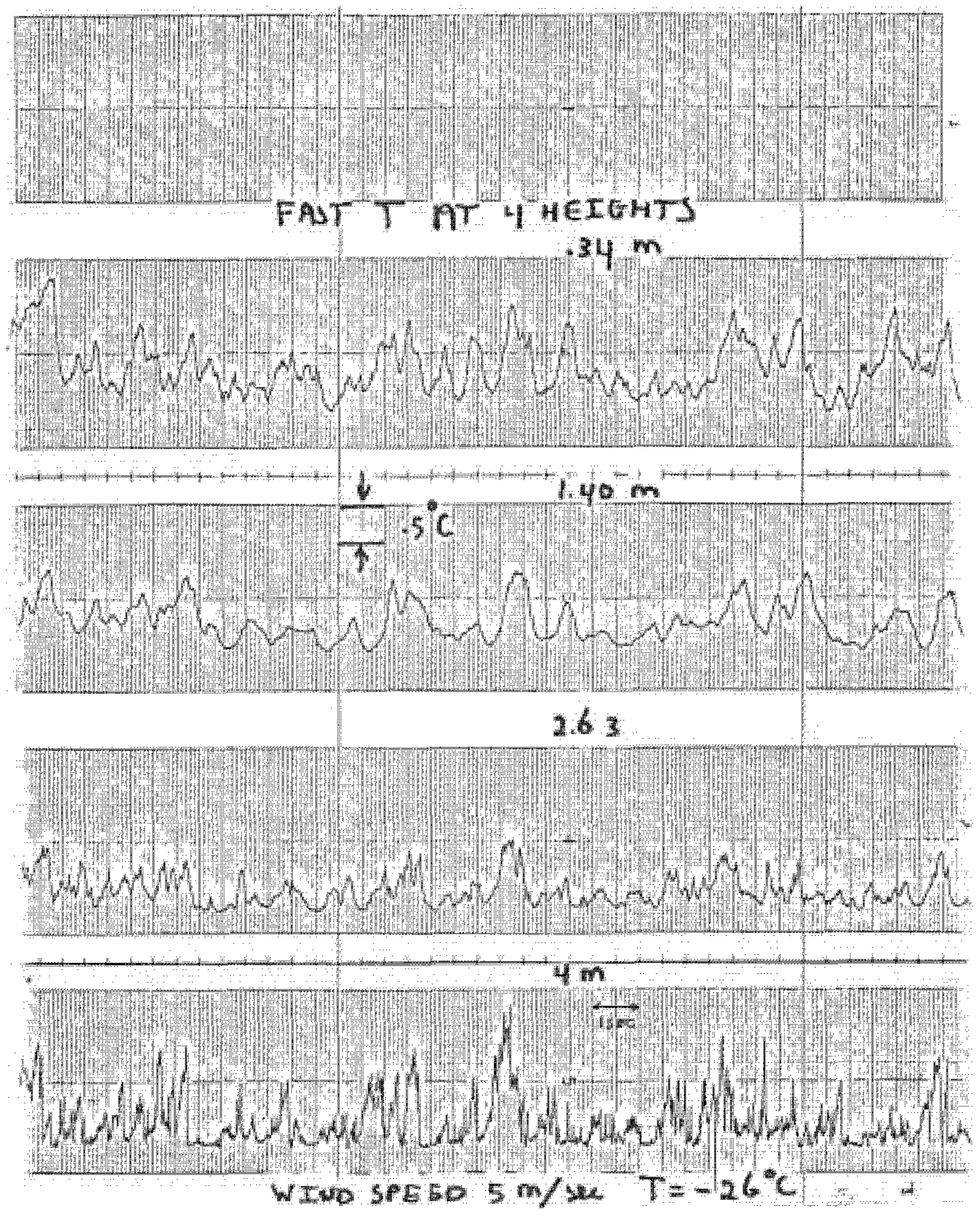

Figure 4 
higher levels indicates that the elements are moving through a constant temperature background, and indeed, the lowest temperatures correspond to that of the unmodified air. The vertical velocity measurements made at the 4 meter level confirm this picture of a layer of strong vertical interchange. These showed vertical velocities exceeding 1 meter/sec for both upwelling and downwelling flows. Thus the picture one obtains is of a thin layer within which the transport of heat and momentum are accompanied by shearing action and a much thicker layer in which heat is transported by long vertical plumes but accompanied by 1 ittle momentum transfer. Estimates of the Monin-Oboukhov length yield a surface layer of the order of 1 meter and the overall picture closely resembles an unstable atmospheric boundary layer. Fig. 5 illustrates typical structures outlined by the fog plumes. Photographs taken from above show the plumes to be aligned in linear features along the wind direction. These were particularly evident to an observer standing on the downwind edge when clear "open streets" could be seen across the whole width of the water. Observation suggested that the plumes arose from the shear layer at spacings roughly equal to the depth of the shear layer, but that later in their development a particular plume would grow at the expense of its neighbors, leaving an area 'drained ${ }^{p}$ of activity, into which a new generation of plumes would grow.

For winds aligned along the long axis of the polynya the overall flow patterns became highly three-dimensional, the heat input inducing convergence in the surrounding flow field. Fig. 6 shows the general flow features for winds in such a direction. The convection dominated region containing the largest plumes is now confined to a central region running along the wind axis with thinner boundary layers growing in from the edges under the influence of convergence induced by the main convective flows. At lower wind speeds the heated flow separates from the ground at the downstream edge, allowing an inflow at the base of the heated flow around all sides.

The three-dimensional aspects of the overall flow pattern induced by the isolated surface discontinuity suggests that caution should be exercised in deriving values of heat flux from standard formulas used in boundary layer meteorology.

The continued existence of such an area of open water under extremes of atmospheric conditions depends on a complex interaction of mechanical and thermal processes. A dominant feature is ice removal by the strong currents of the tidal flows. At slack tide a thin skin of ice several mm thick rapidly forms over the surface. As the tidal flows increase, skin friction causes this thin covering to be broken up and to collect in thick wedges against the downstream edge. As the currents increase further the leading edge of this consolidated soft ice is forced downward by the dynamic water pressure, eventually breaking off from the main edge and being swept away downstream.

The warmer the air temperature the larger the proportion of fresh ice removed, as the ice strength depends on its temperature. If there is an appreciable increase in atmospheric temperature the surrounding ice weakens to such an extent that the whole area of open water extends rapidly. Such a general opening of the ice cover was observed during the course of the experiment when the air temperature rose from close to the monthly average of $-30^{\circ} \mathrm{C}$ to $-7^{\circ} \mathrm{C}$ over the course of about a week.

Conversely, cold temperatures result in less ice being removed with consequent encroachment of the solid edges. If, however, conditions become so extreme that a continous sheet of ice formed at slack tide gains enough 
strength to persist over a complete tidal cycle, no free edges exist and ice removal by bending and fracture is prevented. The ice thickness is then determined by the thermal balance alone and once completely ice covered, such an area may not reopen, even though the air temperature returns to its former level or higher.

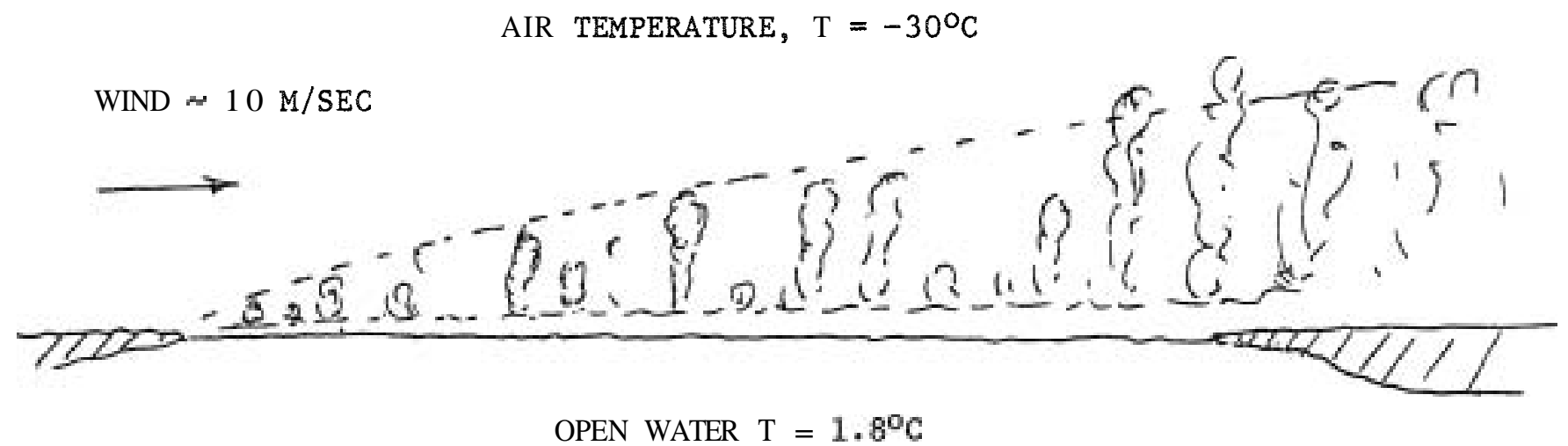

SIDE VIEW

WIND DIRECTION

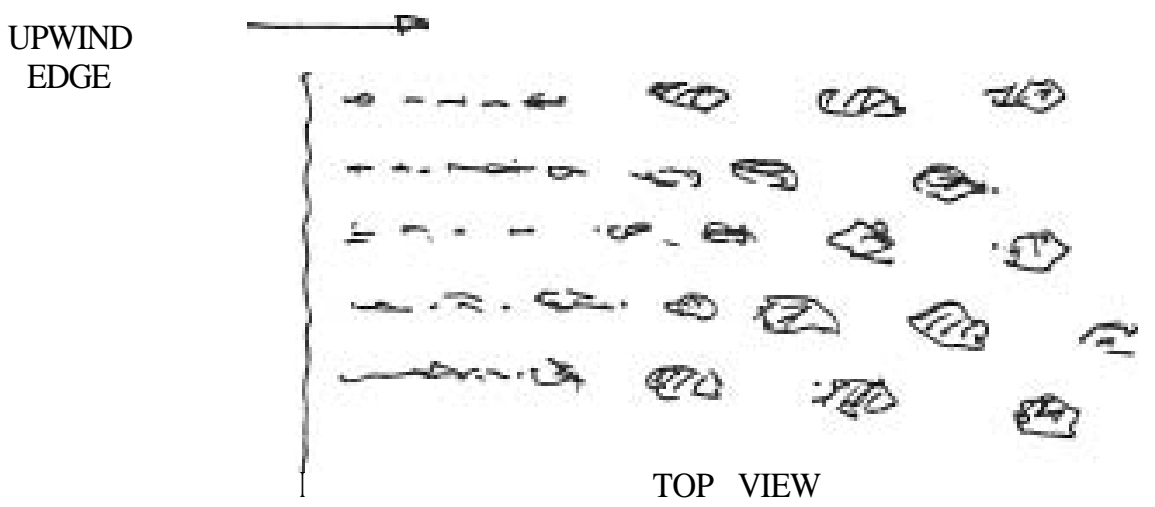

FIGURE 5. FOG PATTERNS OBSERVED IN 2-D FLOW WIND NORMAL TO LONG AXIS OF POLYNYA 

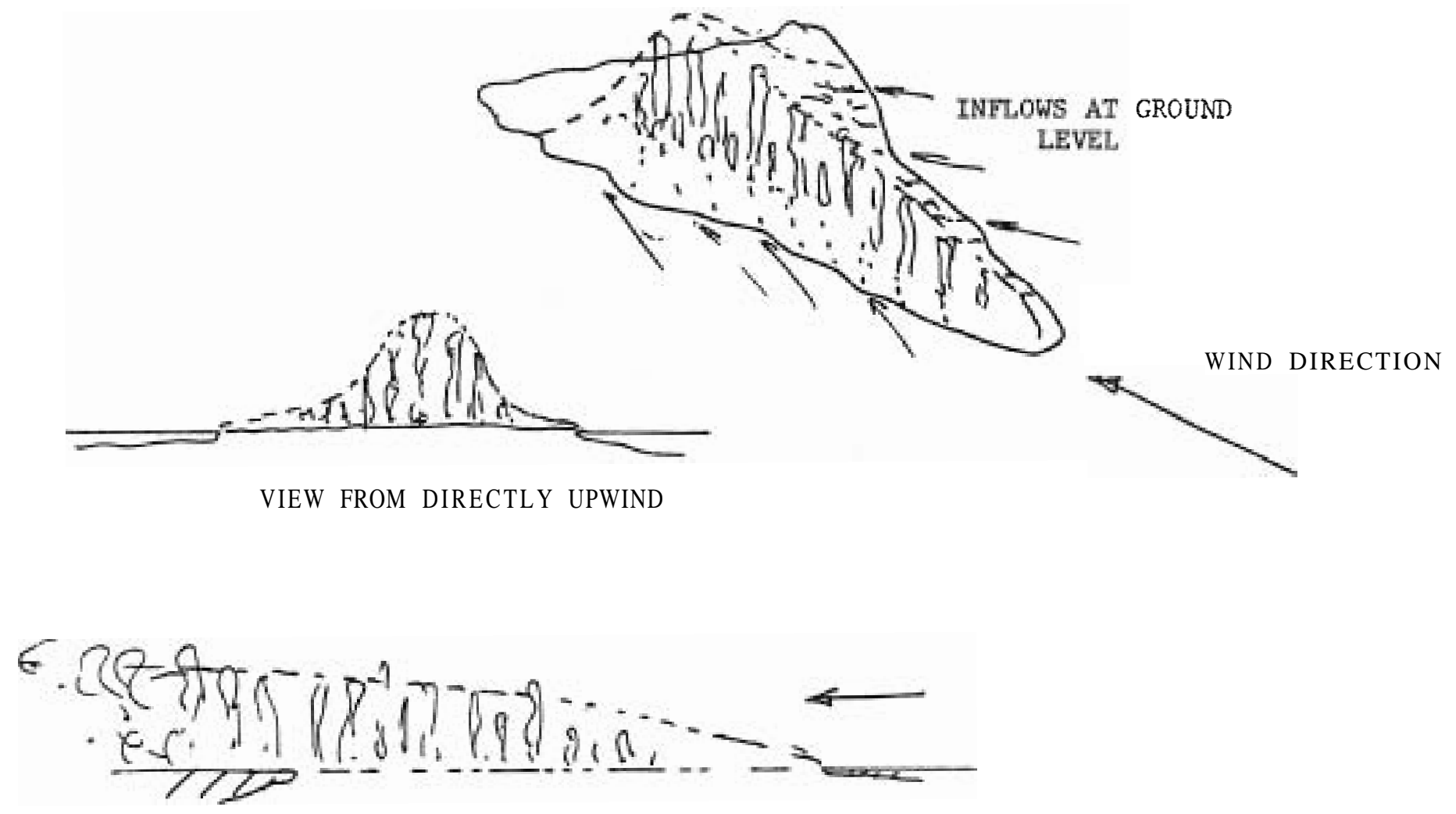

SIDE VIEW IN STRONG WINDS

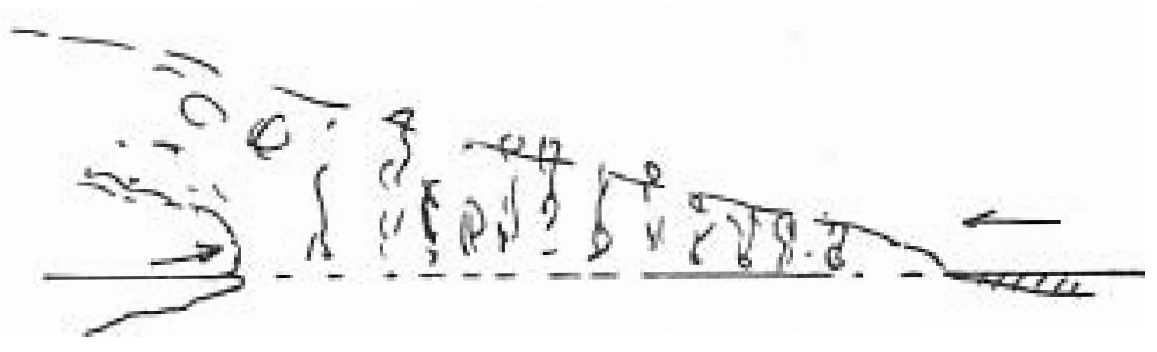

SIDE VIEW IN WEAKER WINDS

FIGURE 6. FOG PATTERNS IN 3-D FLOWS OBSERVED WITH WIND ALONG LONG AXIS OF POLYNYA 


\section{HIGH RAYLEIGH NUMBER CONVECTION MODEUED AS A TWO-FLUID SYSTEM, WITH APPLICATION TO CONVECTION IN THE ATMOSPHERIC BOUNDARY LAYER}

\section{Benoit Cushman-Rois in}

When the ground is heated by solar radiation or the sea surface is warmer than the overlying air, thermal instability sets in and convection mixing develops in the lower part of the atmosphere, forming the so-called atmospheric boundary layer. This convective region is characterized by a very high Rayleigh number. Moreover, it expands upward as long as the destabilizing surface heat flux is present. Numerous observation (Lenschow, et. al., 1980) reveal that: (i) the temperature is nearly homogeneous in the mixed layer (adiabatic gradient) and rapidly increases in an inversion layer which caps the convective region; (ii) the temperature variance is maximum at the surface, decays by about two orders of magnitude through the mixed layer, reaches a well-pronounced minimum somewhat below the inversion, and increases again in the inversion zone; (iii) the vertical convective heat flux decays linearly from its surface value, changes sign, reaches a minimum at the low edge of the inversion, and decays to zero in the inversion; (iv) the vertical-velocity variance and turbulent kinetic energy flux both exhibit a bulge near mid-height and virtually vanish in the inversion; and (v) the rate of dissipation is almost vertically uniform in the mixed layer and rapidly drops to zero in the inversion.

Transfer of heat, momentum, and other passive scalar such as moisture from the surface and throughout the overlying atmospheric bouindary layer is accomplished to a large extent by discrete convective elements called thermals (Lenschow and Stephens, 1980). Thermals can take the form of isolated volumes of buoyant fluid or of an elongated plume, which may extend vertically over a significant fraction of the mixed layer (Turner, 1973). Although other convective elements exist (e.g•, longitudinal rolls, dust devils, and small-scale eddies) thermals are the dominant mode of turbulent mixing in this highly convective system.

The journey of a typical thermal can be described as follows. The thermal leaves the surface, where it has been formed, with a large temperature excess (positive buoyancy). Under the action of gravity, it accelerates and rises through the mixed layer. As it rises, it gradually mixes with the downward return flow, and its buoyancy diminishes. The combined effects of friction and mixing force the thermal to reach a level of maximum vertical velocity. Beyond that level, the frictional force is greater than the buoyancy force, and the thermal, although still buoyant, decelerates. Later and higher, the thermal reaches the neutral level, where it is no longer buoyant. Because of its non-zero vertical velocity it overshoots that equilibrium level by inertia and starts to carry upward a temperature deficiency (negative buoyancy). In this part of the mixed layer, the heat flux is downward and thus opposite to the surface flux. Ultimately, as the temperature profile starts to curve, the thermal " negative buoyancy increases sharply, and its vertical velocity decreases. The thermal comes to rest, marking the top of the inversion zone, and begins a downward journey as a non-thermal or return flow.

This general description explains qualitatively the main features of the convective boundary layer as described above. It seems, therefore, natural to think of thermals and non-thermals (return flow) as two distinct fluids migrating up and down, carrying different properties (temperature, moisture, ...) and exchanging these properties by mixing. Such a two-fluid concept can 
indeed lead to a closed model capable of simulating the physics of the rmals and thus of reproducing the characteristics of the atmospheric boundary layer.

Let us denote by primes and double primes the properties of thermals and nonthermals, respectively. If $f$ represents the fraction of area occupied by thermals at any level (assumed to be constant with height), the fraction of area available to nonthermals is $(1-f)$, and one may define:

- the mean vertical velocity:

$$
\begin{aligned}
& \overline{\mathrm{w}}=f \mathrm{w}^{\prime}+(1-\mathrm{f}) \mathrm{w}^{\prime \prime} \\
& \overline{\mathrm{T}}=f \mathrm{~T}^{\prime}+(1-\mathrm{f}) \mathrm{T}^{\prime \prime} \\
& \mathrm{w}_{\mathrm{rms}}^{2}=f \mathrm{w}^{\prime 2}+(1-\mathrm{f}) \mathrm{w}^{\prime \prime 2} \\
& \mathrm{~T}_{\mathrm{rms}}^{2}=f \mathrm{~T}^{\prime 2}+(1-\mathrm{f}) \mathrm{T}^{\prime \prime 2} \\
& \overline{\mathrm{WT}}=f \mathrm{w}^{\prime} \mathrm{T}^{\prime}+\begin{array}{r}
(1-\mathrm{f}) \mathrm{w}^{\prime \prime} \mathrm{T}^{\prime \prime} \text { (no } \\
\text { conduction) }
\end{array}
\end{aligned}
$$$$
\text { - the mean temperature: }
$$$$
\text { - the vertical velocity variance: }
$$$$
\text { - the temperature variance: }
$$

- the vertical convective heat flux:

It can be shown easily from these simple relationships that:

$$
w_{\text {rms }}=\sqrt{f(1-f)}\left(w^{\prime}-w^{\prime \prime}\right), \quad T_{\text {rms }}=\sqrt{f(1-f)}\left(T^{\prime}-T^{\prime \prime}\right)
$$

(positive or negative)

and $\overline{w T}=\overline{w T}+w_{r m s} T_{r m s}$ (correlation coefficient equal to unity)

With these preliminaries, the governing equations can be written as (Cushman-Roisin, 1981):

$$
\begin{aligned}
& \bar{w}=0 \\
& \frac{\partial \bar{T}}{\partial t}=-\frac{\partial}{\partial r}\left(w_{r m s} T_{r m s}\right) \\
& \frac{\partial}{\partial z}\left(T+m T_{r m s}\right)=0 \\
& 3 m w_{r m s} \frac{\partial}{\partial} w_{r m s}=\alpha g T_{r a s}-\frac{D}{k h} \frac{w_{a}^{3}}{w_{r m s}+m h},
\end{aligned}
$$

and hold as long as horizontal variations and diffusion are negligible. The coefficient $m$ is a function of $f$ only $\left[m=(1-2 f) f^{-1 / 2}(1-f)^{-1 / 2 / 2}\right]$ and is of the order of unity. The first equation is a result of continuity and states that there is no mean vertical velocity. The second equation is the mean temperature equation (after the subtraction of the adiabatic gradient) and expresses the divergence of the vertical convective heat flux is the sole mechanism able to change the mean temperature. The third equation is the turbulent temperature equation. This diagnostic equation as well as the next one results from the assumption that turbulent motions are at all times in quasi-equilibrium with their environment. The last equation is the vertical momentum balance after its mean has been subtracted. It states that thermals acceleration is due to a buoyancy force ( $\alpha$ is the thermal expansion coefficient) and a frictional force (D i s a dimensionless dissipation coefficient of order one); $K$ is the von Karman constant, h the instantaneous mixed-layer thickness, $\dot{h}=\mathrm{dh} / \mathrm{dt}$ the rate of entrainment, and $w_{*}=$ $(\mathrm{KaghQ})^{1 / 3}$ the vertical-velocity scale derived from the surface heat flux, Q. 
When the mixed layer is well-developed, its mean temperature is almost vertically homogeneous and the inversion zone is thin. In that case, an approximate analytical solution is:

$$
\begin{aligned}
& w_{r m s}^{3}+3 / 2 \dot{h} w_{r m s}=u_{2}^{3}\left(1-\frac{z^{2}}{h^{2}}\right)+\frac{1-3}{k m} \quad w_{k}^{3} \frac{t}{h}\left(1-\frac{z}{h}\right), \\
& T_{r m s}=Q\left[1-2(1-D) \frac{z}{h}\right] /\left(w_{r m s}+m h \frac{z}{h}\right), \\
& \bar{T}=T_{0}+\frac{2(1-D)}{3-4 D} \Gamma h-m_{r m s},
\end{aligned}
$$

where $u_{*}$ is a friction velocity characterizing the turbulence level near the surface ${ }^{*}\left(u, c w_{*}\right)$ and $T_{\theta}+\Gamma z$ is the initial linear temperature profile. The rate of entrainment is given by

$$
\Gamma_{\text {hh }}=(3-4 \mathrm{D}) \mathrm{Q} \text {, }
$$

and the temperature jump across the inversion layer by

$$
\Delta \mathrm{T}=\frac{1-20}{3-4 D} \Gamma \mathrm{h},
$$

as long as $u_{3} \ll \quad$ and $h \ll w, \quad[$ Note that stability requires $\mathrm{D} \ll 0.5$, for $A T>0$.

Although this solution is implicit in wrms, it is not difficult to convince oneself that the resulting profiles exhibit the dynamic features of the atmospheric boundary layer. In particular, the level of maximum vertical-velocity variance ( $w^{2}$ r ) and of maximum turbulent kinetic energy flux $\left(1 / 2 \mathrm{ww}^{2}=\mathrm{mw}^{3} \mathrm{~m}\right)$ is $z=\mathrm{h} / 2$, and the neutral level where thermals ${ }^{\prime}$ buoyancy is zero $\left(T_{\text {rms }}=0\right)$ is $z=h / 2(1-D), i_{\bullet} e_{.}$, somewhere between $h / 2$ and $h$, since $0<D<0.5$.

Near the surface, in a layer of thickness of the order of the Monin-Obukhov length $\left[\ell=\left(u_{y} / w\right)^{3} h\right]$, the solution is similar and follows the $1 / 3$ power laws:

$$
\begin{aligned}
& T_{\text {rms }}=\frac{Q}{u_{3}}\left(1+\frac{1-D}{m k}\right) \eta^{-1 / 3} \\
& w_{\text {rms }} \approx u_{*}\left(1+\frac{1-D}{m k}\right) \eta^{-1 / 3}
\end{aligned}
$$

where $\eta=z / \ell=$

From the solution, one may also predict the inversion-layer thickness and the vertical-velocity variance at the base of the inversion:

$$
\delta_{h} \sim \mathbb{R}_{1}^{-3 / 4} h \text { and } \underset{\operatorname{rms}}{2} \sim \mathrm{w}_{*}{ }^{2} \quad \mathbb{R}^{-1 / 2}
$$

where $R_{i}$ is the Richardson number defined from the total instantaneous buoyancy in the mixed layer $\left(R_{\dot{1}}=\right.$ ศ gh $\left.\Delta \mathrm{T} / \mathrm{w}_{\rightarrow}{ }^{2}\right)$. In a study of turbulence and entrainment within the interfacial zone bounding a mixed layer, Long (1978) concluded the same expressions.

The numerous and excellent agreements between these results and observations, laboratory experiments, and numerical investigations strongly support the modelling by two interacting fluids as a theory of convective turbulence. The present model, however, is limited to a specific type of study, since it does not include molecular dissipation, lateral variations, wind turbulence, and internal gravity waves. 


\section{REFERENCES}

Cushman-Roisin, B., 1981. Penetrative convection in the upper ocean due to surface cooling. To appear in Geophys. Astrophys. Fluid Dyn

Lenschow, D. H., 3. C. Wyngaard, and W. T. Penne11, 1980. Mean-field and second-moment budgets in a baroclinic, convective boundary layer. J. Atmos. Sci., 37, 1313-1326.

Lenschow, D. H., and P. L. Stephens, 1980. The role of thermals in the convective boundary layer. Bound. Layer Meteor., 19, 509-532.

Long, R* R., 1978. The growth of the mixed layer in a turbulent stably stratified fluid. Geophys. Astrophys. Fluid Dyn., $\mathbf{1}, 1-12$.

Turner, J.S., 1973. Buoyancy effects in fluids. Cambridge Univ。 Press, $367 \mathrm{p}$.

\section{MEASUREMENTS OF LARGE SCALE FLOW IN A TURBULENT CONVECTING FLUID}

Ruby Krishnamurti

I would like to tell you about some convection experiments that L. N. Howard and I have started to further study the large scale flow which was found to be spontaneously generated at certain high Rayleigh numbers (Krishnamurti and Howard, 1981).

A schematic diagram of the apparatus is shown in Fig. 1. The convecting fluid occupies the annular region between two concentric right circular cylinders. The fluid is uniformly heated from below, cooled from above. In this configuration, we observe tilted transient plumes, (Fig. 2) with a flow in one direction around the cyclinder at the bottom and in the opposite direction at the top.

The purpose of the present experiment is to measure azimuthal velocitycomponent $u$ and vertical velocity-component w and to see if the Reynolds stress $\bar{W}$ (horizontally averaged) plays a significant role in balancing the viscous stress of the horizontally averaged large scale flow, $\nu \frac{\partial \pi}{a z}$

The procedure was to illuminate a cylindrical sheet of fluid (concentric with the cylindrical walls) by rapidly rotating a pencil of light from a 1-watt laser. Tracer particles in the fluid scatter this light, some of which is reflected from a conical mirror into the camera. The scheme is somewhat like a cylindrical version of an all-sky camera. However, since a pencil of light is used, a tracer particle is illuminated at known intervals, (each time the beam comes around, until the particle leaves the region of study).

Usually, we rotated the beam eleven times for one photographic exposure, but blocked off the light from entering the camera, on the ninth time. Thus we would get on photograph 8 bright dots followed by a dark spot, followed by 2 bright dots. From this we could deduce speed as well as direction of motion of the tracer particle. From each photograph, we were able to deduce on the order of 1000 vectors. 

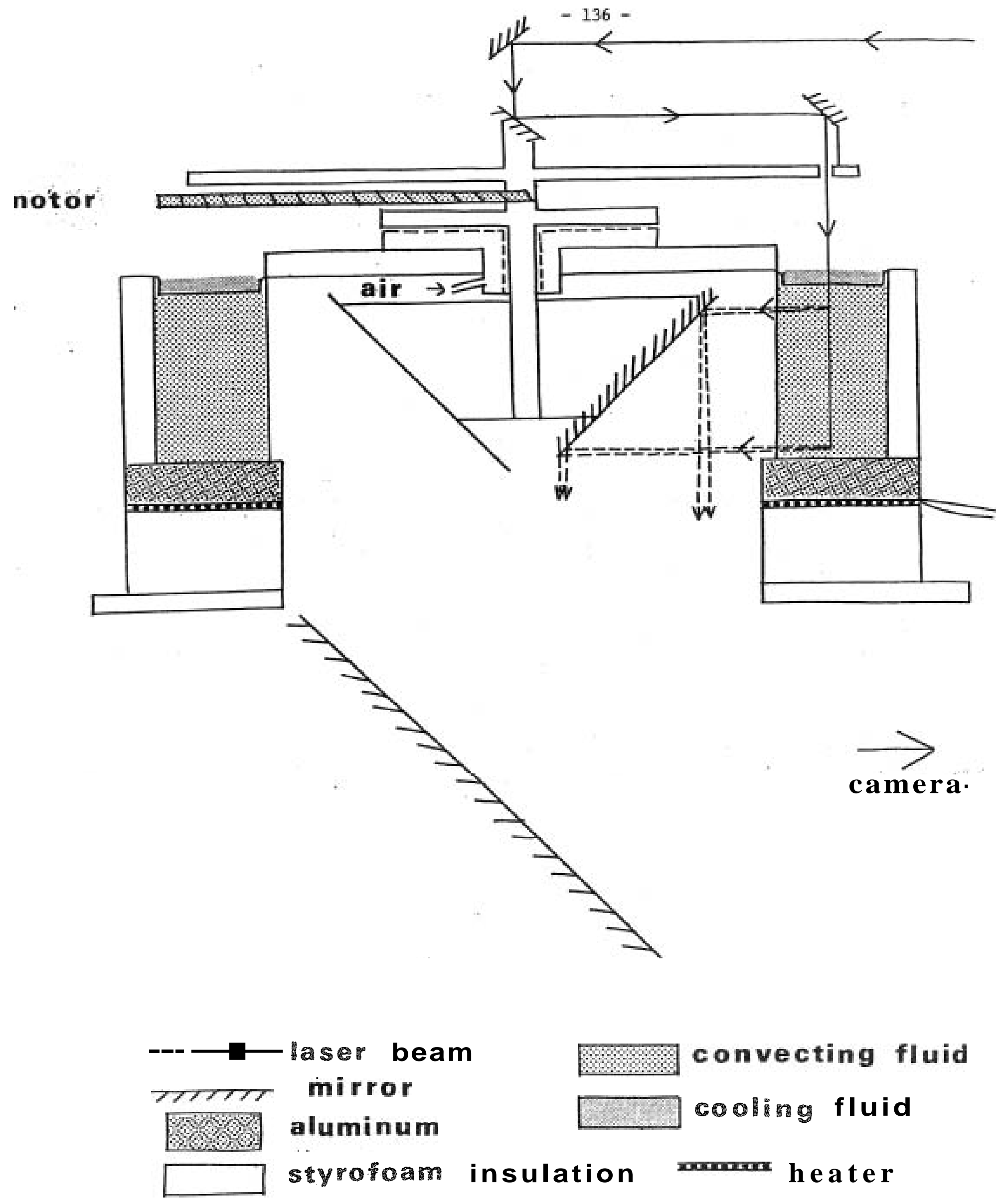

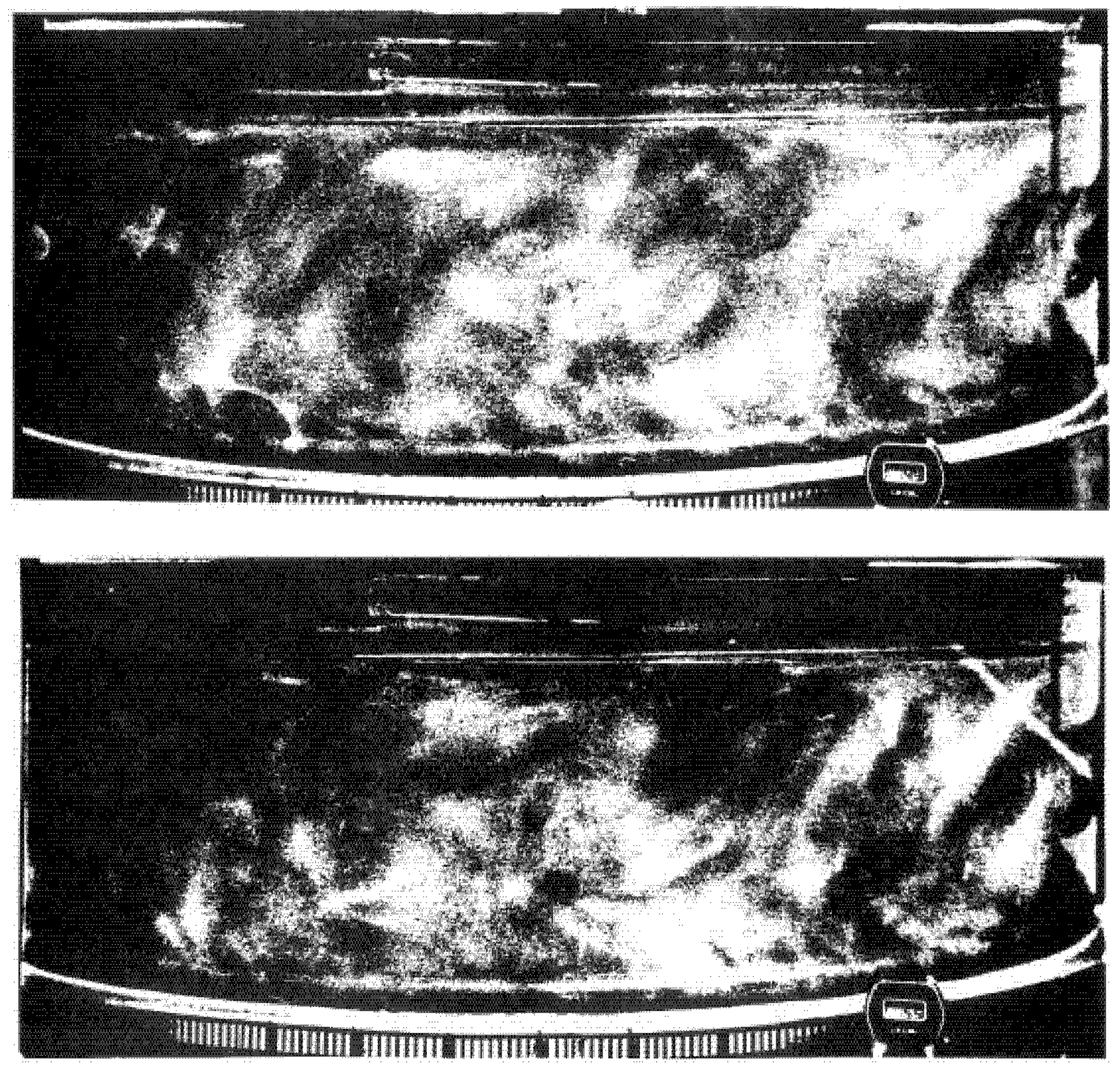

Figure 2: Tho side views of turbulent convection in an annular tank. The plumes tilt from lower left to upper right, and are transient. 


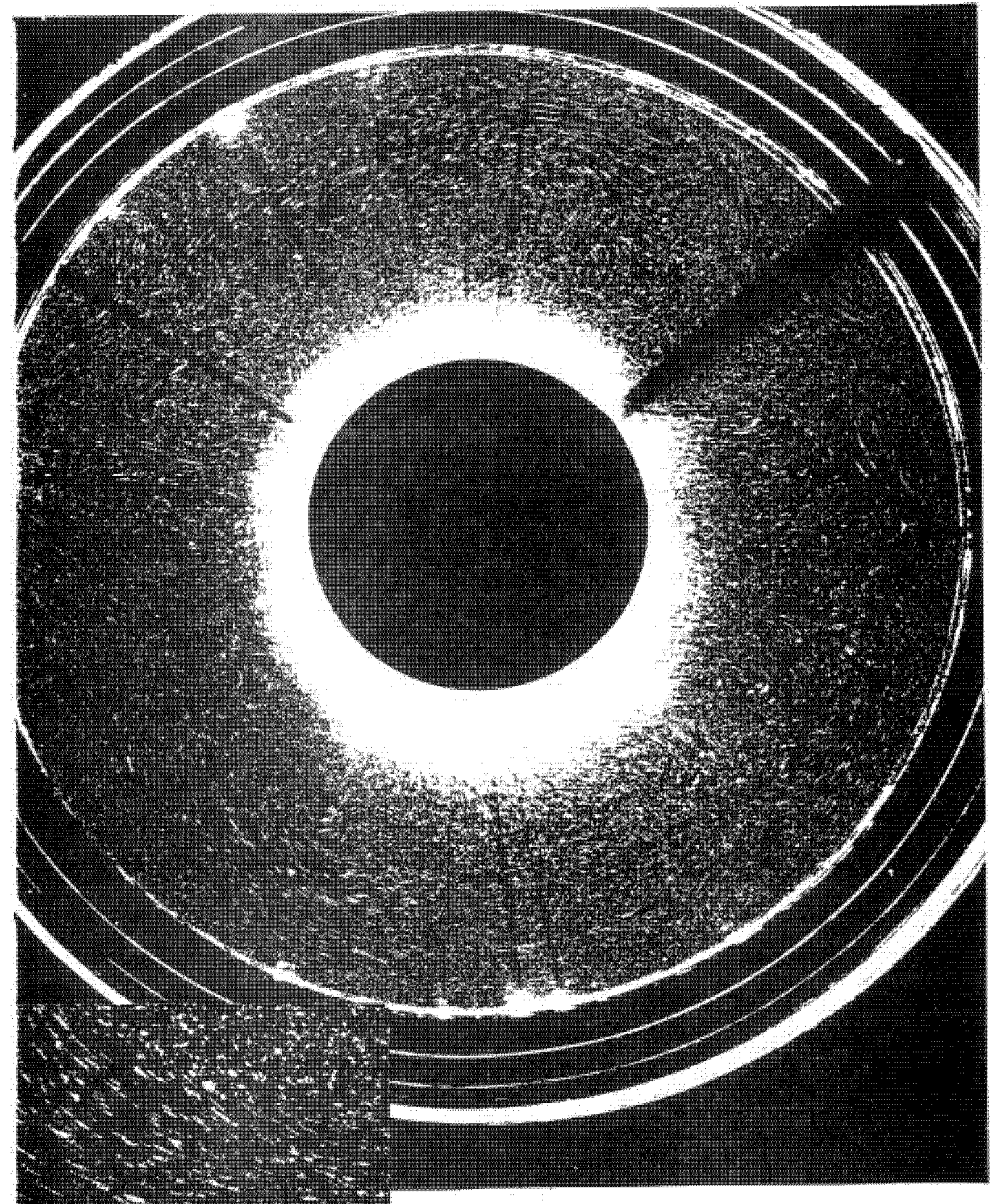

Frome

Euturement 


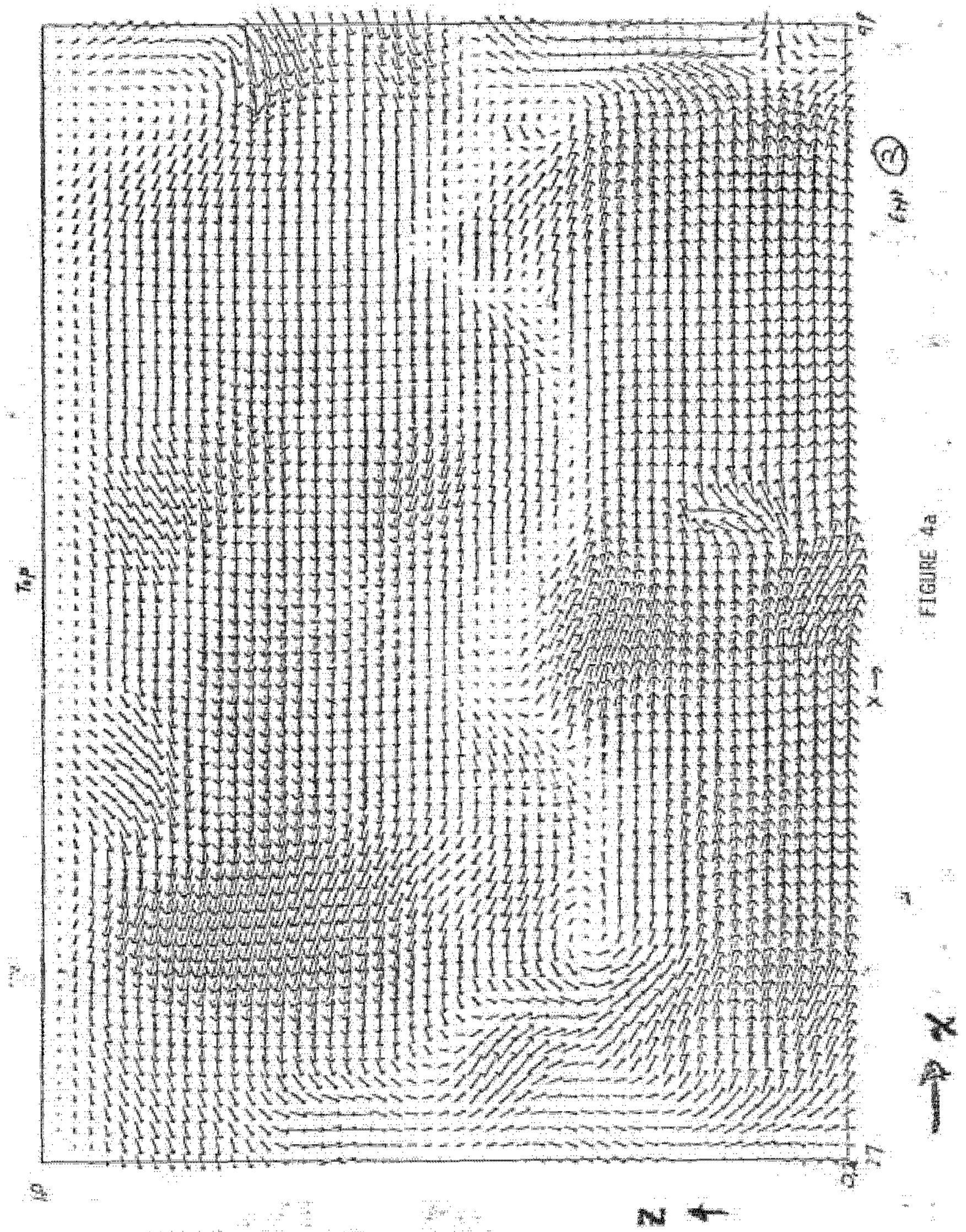




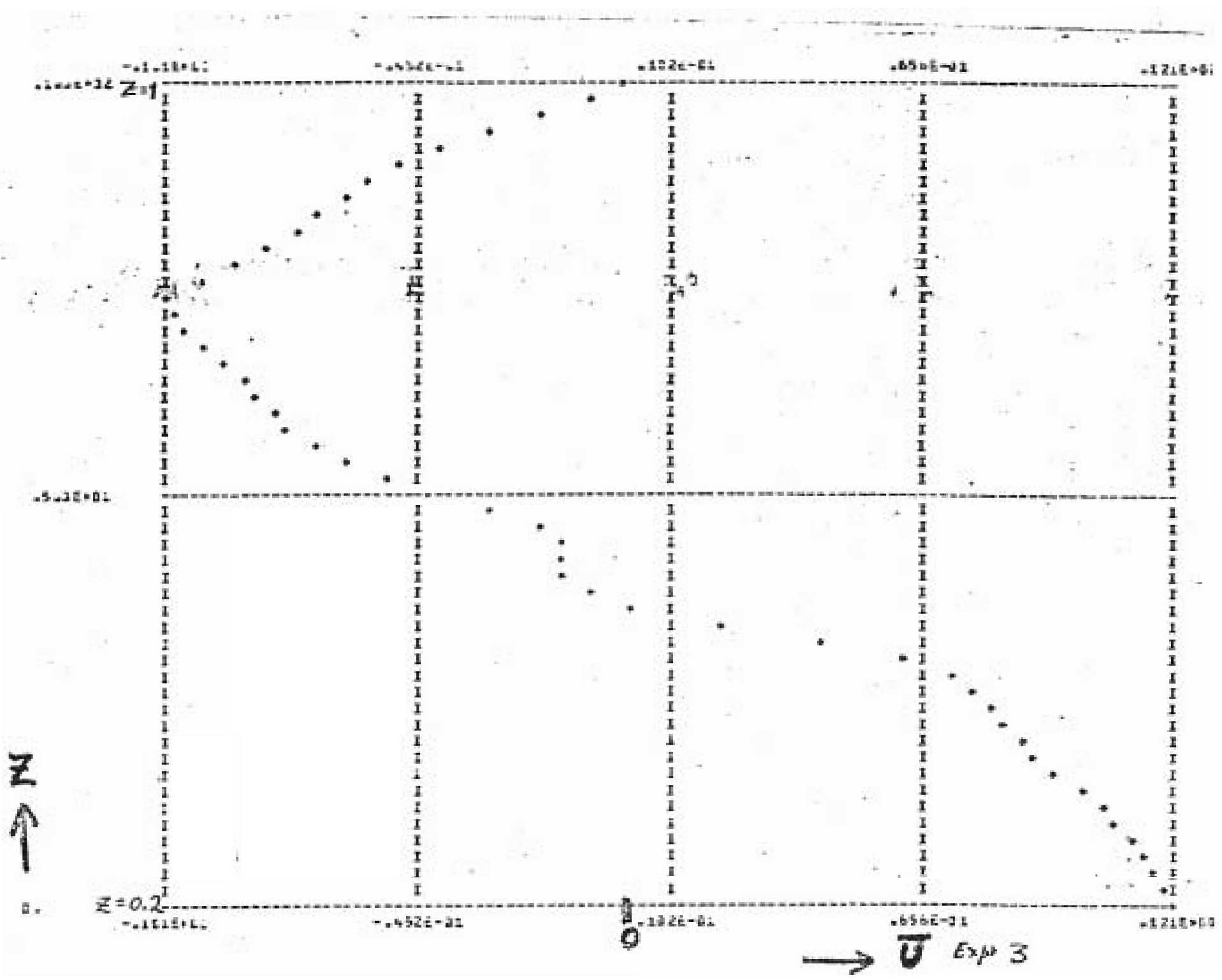

FIGURE $4 \mathrm{~b}$ 


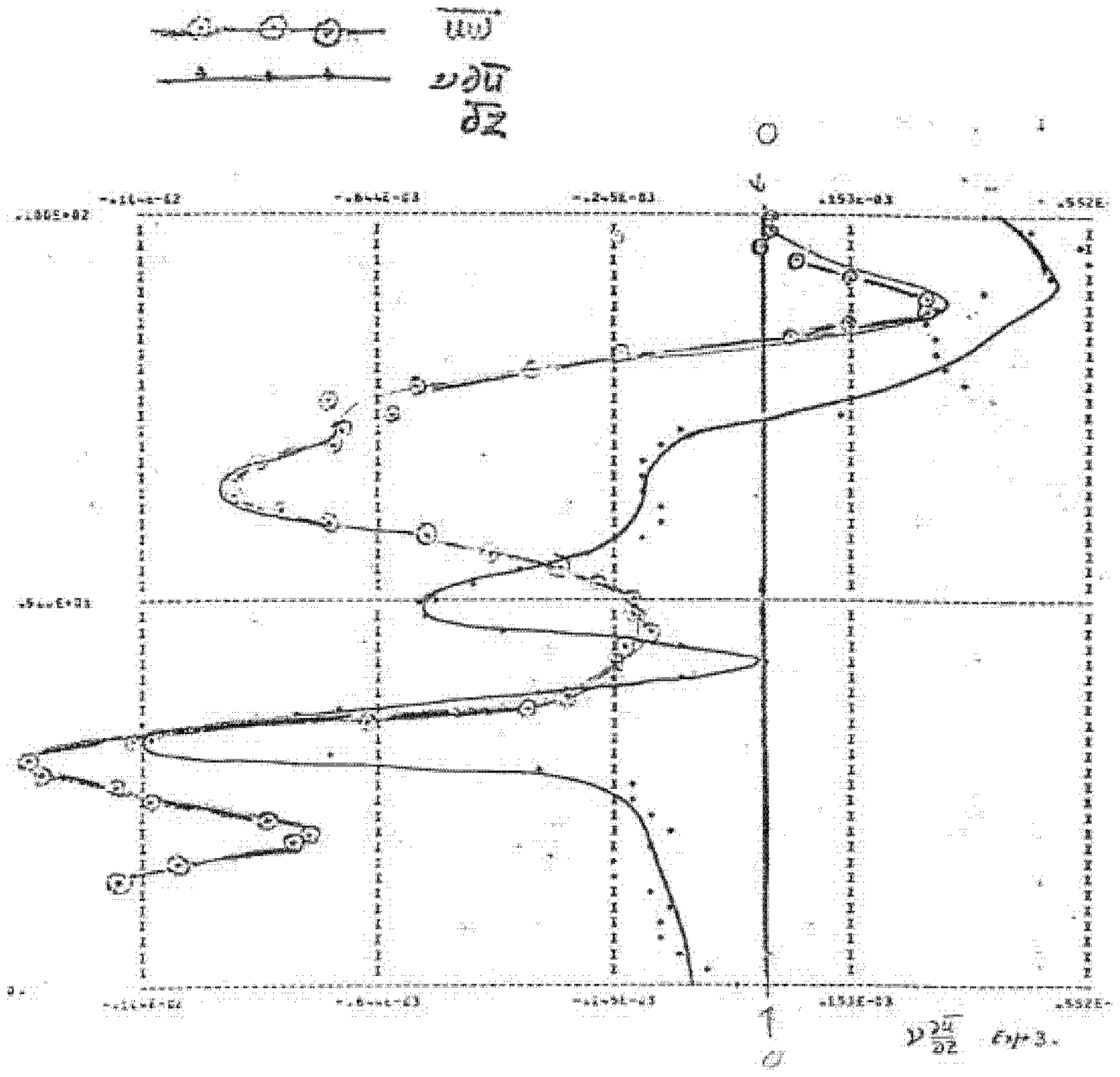

FIGURE $4 c$ 
One example of such a photograph is shown in Fig. 3. In this particular example, there were two cells each occupying $180^{\circ}$ of the annulus. The analysis shown below is for one of these cells. Enlarged versions of such photos were used, and particle positions were digitized. Since, in this method of photography the outer circle in Fig. 3 is at the top of the fluid layer while the inner circle is near the bottom, the data was rectified by mapping onto the $x-z$ plane, where $x$ is distance around the cylinder and $z$ is the vertical coordinate. Then an objective analysis was done to produce the flow vectors in Fig. 4a. Analysis of this data gives various quantities such a s

(i) spectral density as a function of height $z$ in the fluid

(ii) the horizontally averaged velocity u a a function of $z$

(i i i) the Reynolds stress uw。

An example of (ii) and (i i i) is shown in Pig. 4 b and $4 \mathrm{c}$.

\section{REFERENCE}

Krishnamurti, R* and L. N. Howard, 1981. Proceedings of the National Academy of Science, USA, 78, 1981-85.

\section{BIFURCATIONS I N DOUBLE DIFFUSIVE CONVECTION}

\section{Nigel Weiss}

Nonlinear convection can to some extent be modelled by truncated systems of coupled ordinary differential equations. The truncation is, however, dangerous and solutions of the model system have to be compared with solutions of the full partial differential equations. The Lorenz (1963) equations provide an obvious example: the bifurcation from the steady convecting solution i n the Lorenz system corresponds to a supercritical Hopf bifurcation i n the full problem, where strange behavior has not yet been found.

Fifth order models of double-diffusive convection can, however, provide an understanding of the bifurcation structure when convection sets in via a Hopf bifurcation when the Rayleigh number $r=r^{(0)}$, which precedes a simple bifurcation at $\mathbf{r}=\mathbf{r}^{(\mathrm{e})}$. Thermohaline convection, with an unstable thermal gradient can be represented by the system put forward by Veronis (1965), whose solutions are qualitatively similar to those for the full two-dimensional problem (Veronis, 1968; Huppert and Moore, 1976) provided R is not too large. The truncated system makes it possible to solve for finite amplitude steady solutions explicitly and to investigate their stability. Periodic solutions can be obtained numerically with sufficient accuracy. When convection sets in as overstable oscillations at $r^{(0)}$, the steady solution branch bifurcates from $r^{(e)}$ towards lower values of $r$ and stable steady solutions exist for $r_{3} r_{\min }$, where $r_{\min }<\mathrm{r}^{(0)}<\mathrm{r}^{(\mathrm{e})}($ DaCosta, Knobloch and Weiss, 1981). In simple cases the oscillatory branch terminates on the unstable portion of the steady branch, where the period becomes infinite. (In the limit $\mathrm{r}^{(\mathrm{O})}$ $r(e)$, solutions can be obtained analytically both for the full problem and for the truncated system (Knobloch and Proctor, 1981)). 
A similar treatment caw be applied to two-dimensional magnetoconvection (Knobloch, DaCosta and Weiss, 1981). In this case the steady branch may have zero, one or two turning points and behavior is correspondingly more complicated, though coesistent with solutions to the full problem (Weiss, 1981). When the steady branch bifurcates from $r(e)$ towards higher values of $r$ there may be $a$ hopf bifurcation from it to the oscillatory branch.

In both problems there can be further bifurcations from the oscillating branch. In two-dimensional the rmohaline convection Huppert and Moore found a bifurcation from symmetrical to asymmetrical oscillations, followed by a transition to aperiodic behavior. In the truncated model the bifurcation to asymmetry is at first followed by a bifurcation back to symmetry, after which the oscillatory branch is lost. For more extreme parameters there are further period-doubling bifurcations, forming a Feigenbaum sequence, followed by an inverse sequence of bifurcation at which the period is halved (Knobloch and Weiss, 1981). For yet more extreme parameters the complete sequence is followed briefly by a range in which solutions are aperiodic, suggesting that a strange attractor has appeared (DaCosta et. al., 1981). Further work is needed to determine the precise correspondence between the truncated model and the full equations.

\section{REFERENCES}

DaCosta, L. N., E. Knobloch, N. O. Weiss, 1981. J. Fluid Mech. 109, 25.

Huppert, H. E. and D. R. Moore, 1976. J.Fluid Mech. 78, 821.

Knobloch, E. and M. R. E. Proctor, 1981. J. Fluid Mech. 108, 291.

Knobloch, E. and N. O. Weiss, 1981. Phys. Lett. A., 85, 127.

Knobloch, E., N. 0. Weiss, and L. N. DaCosta, 1981. J. Fluid Mech., 113, 153.

Lorenz, E.N., 1963. J.Atmos。Sci. 20, 131.

Veronis, G., 1965. J. Marine Res. 23, 1.

Veronis, G., 1968. J.Fluid Mech. 34, 315.

Weiss, N. O., 1981. J.Fluid Mech. 108, 247.

\section{MODAL TRUNCATIONS}

\section{Philip Marcus}

Recently, there has been much interest in computing solutions to the nonlinear equations that govern thermal convection by using a Galerkin method in which the velocity and temperature fields are represented by a finite number of modes. In applying these truncated models to a convecting fluid in which the Rayleigh number is large, such as the convection zone of a star (Marcus 1979, 1980; Latour et. al., 1976; Toomre et. al., 1976), we should be somewhat cautious in taking too literally the exact pattern of the calculated velocity and temperature fields, However, the gross features of the computed flow such as the Nusselt number, kinetic energy spectrum, thermal variance spectrum, mean temperature gradient, central temperature, and size of the boundary layers 
may indeed be quite accurate and it is worthwhile to determine how sensitive these quantities are to the truncation.

In laboratory flows at more moderate Rayleigh numbers there have been recent measurements of the bifurcations as the Rayleigh number is increased. Gollub and Benson (1980) have carefully measured, as a function of Rayleigh number, the transitions from steady state to periodic, to one or more states of period doubling, quasiperiodicity or phase locking and then finally to nonperiodicity. In trying to explain these bifurcations theorists have performed modal calculations. Unfortunately, the number of bifurcations and types of bifurcations produced in the calculations strongly depend on how many modes are retained in the truncation. For example, in a fluid with Prandtl number of 10 , Lorenz (1963) has found there is one inverted bifurcation that takes the flow from a steady state to a strange attractor; whereas Curry (1978) for the same Prandtl number found that with a more extensive 14-component model the flow exhibits a normal bifurcation to periodic motion, followed by a bifurcation to period doubling. The flow then bifurcates to an attracting torus and finally changes to nonperiodic motion. Toomre, Gough and Spiegel (1977) and Marcus (1978) found the surprising result that if the vertical structure is finely resolved but only one Fourier mode is retained in the horizontal (single-mode theory) then there are no bifurcations. The fluid remains in a stable, steady-state regardless of Rayleigh number. For a Prandtl number of unity and 39-mode truncation, McLaughlin and Martin (1975) found four bifurcations in a fluid that initially was in a steady state with rolls aligned along the y axis: the first transition to periodic flow, the second to weakly nonperiodic motion, the third to a periodic state and the last to nonperiodic motion. When they reduced the number of modes in their calculation so that there were only three different wavelengths i n the y direction, they found that there was no final transition to nonperiodicity. These modal conclusions a 11 support Ruelle and Takens' (1971) assertion that after at most 4 normal bifurcations the solutions must be nonperiodic in time. However, it is important to know whether the bifurcations predicted by the modal equations are inherent to the full nonlinear equations that govern the convective motion or are a general property of the nonlinear, coupled autonomous equations that govern the finite modes of the truncation. If the truncated equations of motion do not have sufficient spatial resolution to mode 1 the physically important processes that occur in a convection fluid, then the bifurcations of the truncated equations may not be related in any qualitative way to the actual transitions observed in the laboratory.

We examine the Galerkin (including single-mode and Lorenz-type) equations for convection in a sphere to determine which physical processes are neglected when the equations of motion are truncated too severely. We test our conclusions by calculation solutions to the equations of motion for different values of the Rayleigh number and for different values of the limit of the horizontal spatial resolution. We show how the gross features of the flow, such as the mean temperature gradient, central temperature, boundary-layer thickness, kinetic energy and temperature variance spectra, and energy production rates are affected by truncation in the horizontal direction. We find that the transitions from steady-state to periodic, and then to aperiodic convection depend not only on Raylefgh number but also very strongly on the horizontal resolution of the calculation. All of our models are well resolved i n the vertical direction, so the transitions do not appear to be due to poorly resolved boundary layers. For a spherical Rayleigh number 100 times critical, our truncation with 168 modes produces a steady state. We find (holding the Rayleigh number and the resolution in the radial direction fixed) that, as we decrease the number of horizontal modes in the Galerkin expansion, 
there is a transition from steady-state convection to a solution that is periodic i n time. As the number of modes is decreased still further, the solutions become aperiodic. By computing how the energy spectra, convective flux and temperature gradient change as a function of the severity of truncation, we not only show how the gross features of the flow are affected by the truncation, but also provide a possible explanation for the time dependence of our solutions.

It is likely that a truncation is justified if the dissipative modes as well as those modes responsible for energy production and transport are included. An easy way, of course, to show that all of the physically important wavelengths are resolved is to repeat the calculation with an increased number of modes and have the solutions remain unchanged. We have predicted and numerically confirmed (for a spherical Rayleigh number $\sim 10$ times critical and a Prandtl number of 10) that a truncation with an insufficient number of horizontal modes will accurately predict the rate of energy production and will: (1) alter the kinetic and thermal spectra by increasing the amplitudes of the high-wavenumber modes; (2) make the mean temperature gradient more isothermal and thereby lower the central temperature; and (3) decrease the rate at which the temperature variance is produced in the fluid. We have further shown that, if the truncation is too severe, the thermal variance spectrum will become inverted, with the high-wavenumber dissipation modes having more energy than the low-wavenumber production modes. For a spherical Rayleigh number -10 times critical and a Prandtl number of 10 , the the rmal variance inversion does not destroy the time-independent property of the fluid but: a kinetic energy inversion does make the fluid time-dependent. We have also predicted and numerically confirmed that single-mode calculation produces artificially thin boundary layers (where the thickness is determined by the actual viscosity and not the eddy viscosity). These thin boundary layers are needed to dissipate the kinetic energy that is generated from the buoyancy. If the dissipative modes had been included i n the calculation, the kinetic energy would bave been lost primarily through a turbulent cascade and not in a viscous boundary layer.

Modal representation can be used to predict transition to time dependence i n convective flow if sufficient care is taken so that enough modes are included to resolve a 11 of the important length scales. Clever and Busse (1974) computed the bifurcation from steady-state rolls to time-dependent wavy rolls and have shown that their truncation is valid because the amplitudes of the velocity and temperature fluctuations are small. On the contrary, the transitions to aperiodicfty reported by Curry (1978) and McLaughlin and Martin (1975) occur at large amplitudes and the Kolmogorov lengths are smaller than the limits of resolutions of their truncations. Their sequences of transitions would be more credible if more modes had been included. Even with 168 modes in spherical convection, we find that when the flow changes to aperiodic the dissipative length are no longer resolvable and we cannot be certain that the transition is correct. Gollub and Benson (1980) have measured that the bifurcation to aperiodicity in plane-parallel convection with a Prandtl number of 2.5 occurs at a velocity of $\rightarrow 0.04 \mathrm{~cm} / \mathrm{s}$. Since the thermal diffusivity is $\sim 1.5 \times 10^{-3}$ and the horizontal dimensions of their cells are $\sim 3 \times 1.5 \mathrm{~cm}$, the thermal dissipation length is $-0.1 \mathrm{~cm}$. This means that we would require $25 \times 12$ horizontal modes to reslove the dissipative length scales. An optimist might argue that although the model calculations do not include the dissipative length scales they may still be qualitatively despite the fact that the bifurcations are not at the exactly predicted Rayleigh number. The pessimist might argue that, if a theorist were provided with an experimentally determined sequence of bifurcation, he could probable find a set of nonlinear 
autononous equations that qualitatively reproduced the sequence and then fin" a set of modes that correspond to his set of nonlinear equations. Our final caution is illustrated by considering the single-mode equations, which are a function of time and one spatial dimension. Although the single-mode equations do not correspond to any physical system, they are nonlinear and share many of the properties of actual nonlinear equations that govern convection. From our numerical experiments and those of Toomre et. al. (1977) it appears that the single-mode equations always admit at least one stable, steady-state solution for a 11 Rayleigh numbers. If we examine the transition to time dependence of these equations using a Galerkin expansion in the vertical coordinate we would arrive at some erroneous conclusion. With one vertical mode we obtain the Lorenz model that predicts a bifurcation to a strange attractor, which is incorrect. An important feature of the single-mode solution is the development of thin boundary layers which provide a place for kinetic energy to dissipate and whose thickness decreases with Rayleigh number. As the Lorenz model is supplemented with an increasing number of Fourier modes there will always be some Rayleigh number for which the Galerkin truncation can no longer resolve the boundary layers. We conjecture that any Galerkin truncation of the single-mode equation always produces an erroneous bifurcation to time dependence at the Rayleigh number at which the boundary layers become unresolvable.

\section{REFERENCES}

Clever, R. Pi. and F. H. Busse, 1974. Transition to time-dependent convection J. Fluid Mech. 66, 67-79.

Curry, J. H., 1978. A generalized Lorenz system. Commun. Math. Phys. 60, 193-204.

Gollub, J. P. and S.V. Benson, 1980. Time-dependent instabilities and the transition to turbulent convection. J.Fluid Mech.100, 449-470.

Latour, J., E. A. Spiegel, J. Tsomre, and J. P. Zahn, 1976. Stellar convection theory. I. The anelastic modal equations. Astrophys. J., 207, 233-243.

Lorenz, E. N., 1963. Deterministic nonperiodic flow. J.Atmos. Sci., 20, $130-141$.

McLaughlin, J. B, and P. C. Martin, 1975. Transition to turbulence in a statically stressed fluid system. Phys. Rev. A 12, 182-203.

Marcus, P. S., 1978. Nonlinear thermal convection in Boussinesq fluids and ideal gases with plane-parallel and spherical geometries. Ph.D. thesis. Ann Arbor, MI: University Microfilms.

Marcus, P. S., 1979. Stellar Convection. I. Modal equations in sphere and spherical shells. Astrophys. J., 231, 176-192.

Marcus, P.S., 1980. Stellar convection. III. Convection a t large Rayleigh numbers. Astrophys. J., 240, 203-217. 
Ruelle, D. and F. Takens, 1971. On the nature of turbulence. Commun. Math. Phys., 20, 167-192.

Toomre, J., J. P. Zahn, J. T. Latour, and E. A. Spiegel, 1976. Stellar convection theory. II. Single-mode study of the second convection zone i n an A-type star. Astrophys. J.2, 207, 545-563.

Toomre, J., D. 0. Gough, and E. A. Spiege1, 1977. Numerical solutions of single-mode convection equations. J. Fluid Mech., 79, 1-31.

\title{
TROUBLEMAKERS I N A FOURIER SERIES APPROACH TO TRANSITIONS
}

\author{
John Whitehead
}

High Prandtl number convection possesses a square flow pattern that is steady and is apparently stable to infinitesimal disturbances in that it has been observed to not break down i n experiments (Whitehead and Parsons, 1978). The square pattern appears to be unstable to finite amplitude disturbances, however, because a more chaotic (in time and space) spoke pattern of convection eats its way into the squares from the lateral boundaries. Experiments will be described here in which the breakup of the squares is initiated by dislocating one square in the middle of the apparatus with the use of a small, heated thermistor. Once a critical heating rate and time is exceeded, the squares cannot heal themselves even if the heater is removed, and the dislocation initiates a spoke cell which then eats into the squares and destroys them, resulting in the more chatic spoke pattern. It requires more heating to do this as the Rayleigh number is increased. Dislocations do not do this in some other regions of parameter space (Whitehead, 1976), and the conditions which must be fulfilled for dislocations to lead to chaos are not known.

\section{REFERENCES}

Whitehead, J.A., 1976. J. Fluid Mech., 75, 715-720.

Whitehead, J. A. and B. Parsons, 1978. Geophys. Astrophys. Fluid Dyn., 9, 201-217.

A DESCRIPTION OF THE LORENZ ATTRACTOR AT HIGH PRANDTL NUMBER

Andrew Fowler

The Lorenz equations

$$
\begin{aligned}
& \dot{x}=-r x+\sigma y \\
& \dot{y}=(r-z) x-y \\
& \dot{z}=x y-b z
\end{aligned}
$$


have the following bifurcation structure: for $0<r<1$, the origin $X=Y=Z=0$ is stable, for $r>E$ it is unstable and two nontrivial steady states $X=Y=$ $\pm \sqrt{b(r-1)} \quad z=r-1$, exist; these are stable for $I<r<r_{c}$, where

$$
r_{e}=\frac{\sigma(\sigma+b+3)}{\sigma-b-1}
$$

o r r $r_{c}$, a 11 three steady states are unstable, and Lorenz (1963) found numerically that the solutions oscillated irregularly on a 'surface' in phase space which is now called a strange attractor. He also found that a cartesian plot of successive pairs of maxima of $Z$ in a sequence $\left\{M_{n}\right\}$ obtained from numerical integration appeared to lie on a single curve with pronounced cusp, Knowledge of difference equations (e.g•, May, 1976) is sufficient to 'explain' the aperiodic behavior of the solutions, and indeed other facets of the solution behavior, such as intermittency (Manneville and Pomeau, 1980), period doubling (Robbins, 1979) successive transition between periodic and aperiodic regimes (Morioka and Shimizu, 1978), provided that an appropriate difference equation may be constructed directly from the differential system. This is the aim of the present work.

To attempt such a direct analysis, we need the help of an appropriate asymptotic regime. (Particularly, we might expect a difference equation for one variable to emerge from a relaxation-type of motion.) Robbins (1979) shows that a weakly dissipative 1 imit cycle emerges at high $r$, which thus precludes the chaotic motions we seek. Rather, motivated by (2), which shows that $r_{c} \rightarrow$ as $\sigma \rightarrow \infty$, we seek to analyze the $1 \mathrm{imit}$

by defining

$$
r \sim \sigma \gg 1, b \sim 1 \text {, }
$$

$$
\frac{r}{\sigma}=\rho \sim 1 ; \quad \bar{\gamma}=\frac{1}{b} \sim 1 ; \quad \delta=\frac{b}{\sigma} \ll 1,
$$

and studying the asymptotic form of the solutions as $6 \rightarrow 0$. To do so, we rescale the variables and time $\mathrm{T}$ by putting

$$
X=\sqrt{r \sigma} x, \quad Y=\sqrt{r \sigma} y, Z=r z, \quad t=\sigma T .
$$

so that

$$
\begin{aligned}
& \frac{d x}{d t}=-x+y \\
& \frac{d y}{d t}=p x(1-z)-\delta \bar{\gamma} y, \\
& d t=x y-\delta z .
\end{aligned}
$$

A complete analysis of these equations (as $\delta \rightarrow 0$ ) is provided elsewhere (Fowler and McGuinness, 1981): in essence, the behavior is as follows:

There is a fast time behavior for $t \sim 1$. To leading order, we neglect $0(6)$ in (5); there is then a first integral for y and $z$, and a substitution

$$
x=\sqrt{\frac{1}{\rho}} \dot{\phi}, \quad y=-\sqrt{k} \sin \phi, \quad 1-z=-\sqrt{\frac{k}{\rho}} \cos \phi
$$

leads to the damped pendulum equation

$$
\ddot{\phi}+\dot{\phi}+\sqrt{k \rho} \sin \phi=0
$$


whose solution $\phi \rightarrow 0$ exponentially as $t \rightarrow \infty$. For large $t$, the xy forcing in the $z$ equation switches off, and there is a slow recovery phase $t \sim 1 / \delta$ in which $z \approx M_{n} e^{-\delta t} \quad\left(M_{n} \equiv 1+\sqrt{K / p}\right)$; then $x, y$ are governed by the

linear equation

$$
\ddot{x}+\dot{x}+p x\left[M_{n} e^{-s t}-1\right]=0
$$

during the slow phase; this can be solved asymptotically or (fortuitously) exactly in terms of Bessel functions. Of the two independent solutions to (8) $\mathrm{x}_{1}$ and $\mathrm{x}_{2}$ say, $\mathrm{x}_{1}{ }^{\circ} 0$ as $\mathrm{St} \rightarrow \infty$, but $\mathrm{x}_{2} \rightarrow \infty$ as $\delta \mathrm{t} \rightarrow \infty$. Thus $x$ (and hence $y$ ) eventually grows again (from an exponentially small value) and another fast pulse is initiated. Generally, matching of the fast pulses gives a relation for the next maximum of $z$ (or $Z$ ), $M_{n+1}$, in terms of the previous maximum, $M_{n}$; this is analogous to Cole's (1968) procedure for the Van der Pol oscillator. However, for the particular choice of $M_{n}$ for which the coefficient of $x_{2}$ vanishes (or is small), the slow trajectory (almost) lies on the two dimensional stable manifold of the origin, and the succeeding maximum is very large (corresponding to a large value of $K$ ). Such trajectories signify cusps in the difference equation, which explains Lorenz's original cusp, and in fact produces a few more (see also Lorenz, 1979; Marzec and Spiegel, 1980; Fowler and McGuinness, 1981). An approximate description of the difference equation is given by

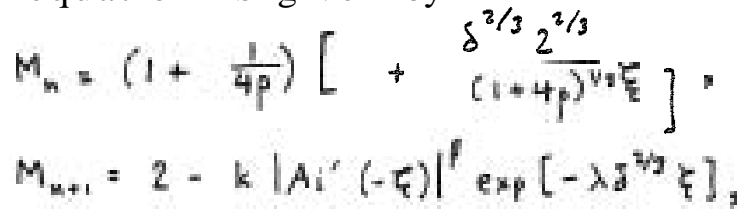

where $k, \lambda$ are $O(1)$ constants, and $\beta=O(6)$.

By examining the form of (9), one can obtain an explanation (and approximate location in parameter space) of such phenomena as periodic and aperiodic behavior, period doubling, intermittency and hysteresis.

Since the Lorenz equations were derived from a model of two dimensional Benard convection, it is pertinent to enquire whether an analysis such as the above will carry over to the full equation. This is under consideration at the time of writing: preliminary results are inconclusive; specifically, the distinguished limit Ra $\sim \sigma$ does not seem to admit a solution of the relaxation type discussed above and by Howard (1966), but an examination of $\mathrm{Ra} \gg \sigma \gg 1$ may be more relevant.

\section{ACKNOWLEDEMENIS}

I have benefited from conversations with John Guckenheimer, Ed Spiegel and Lou Howard. I would Pike to thank the Director of the GD summer program, Willem Malkus, for his hospitality and support during my stay at Woods Hole.

\section{REFERENCES}

Cole, J. D., 1968. Perturbation methods in applied mathematics. Blaisde11: Waltham, MA.

Fowler, A. C. and M. J. McGuinness, 1981. A description of the Lorenz attractor at high Prandtl number. (Submitted to Physica D) 
Howard, L. N., 1966. Convection at high Rayleigh number. In: Proc. Eleventh Int. Congress of Appl. Mech., Munich 1964, ed. H. Gortler, 1109-1115.

Lorenz, E. N.,1963. Deterministic nonperiodic flow. J. Atmos. Sci., 20, $130-141$.

Lorenz, E. N., 1979. On the prevalence of periodicity in simple systems. In: Global Analysis, ed. M. Grmela and J. E. Marsden, pp. 53-75, SpringerVerlag, Berlin.

Manneville, P. and Y. Pomeau, 1980. Different ways to turbulence in dissipative dynamica1 systems. Physica $1 \mathrm{D}, 219-226$.

Marzec, C. J. and E. A. Spiege1, 1980. Ordinary differential equations with strange attractors. SIAM J. App1. Math., 30, 403-421.

May, R. M., 1976. Simple mathematical models with very complicated dynamics. Nature, 261, 459-467.

Morioka, N. and T. Shimuzu, 1978. Transition between periodic and turbulent states in the Lorenz model. Phys. Lett., 66A, 447-449.

Robbins, K. A., 1979. Periodic solutions and bifurcation structure at high $\mathbb{B}$ i n the Lorenz model. SIAM 3. App1. Math., 36, 457-472.

\title{
ON THERMAL CONVECTION I N A FLUID WITH STRONGLY TEMPERATURE DEPENDENT VISCOSITY
}

\author{
Stephen Morris
}

When a fluid of infinite Prandtl number and strongly temperature-dependent viscosity convects i n a region with free-slip boundaries, two extremes arc possible. In the limit $R a \rightarrow \infty$ with the viscosity contrast $\nu_{e} / \nu_{h} \gg 1$ but fixed, we should expect the motion to resemble isoviscous convection, but if $\nu_{c} / \nu_{h} \rightarrow \infty$ with $R a \gg 1$ the motion resembles convection in the presence of a liquid to solid phase change. The two extremes can be seen in the numerical work of Lux and Sacks (1979), who present temperature and velocity profiles for convection driven by heating from below. As the Rayleigh number is increased with the viscosity contrast fixed, the vertical temperature profile approaches that for isoviscous convection as the fluid motion begins to penetrate the cold, stiff upper layer. At low Rayleigh numbers the motion is confined to the hottest part of the flow, and the temperature profile is very asymmetric.

Here we shall discuss two analytical models which show up the causes of this behavior. These analytical solutions are possible when the viscosity depends strongly upon temperature, for in that case the essential viscosity variations occur over a distancelmuch shorter than the length scale $\delta$ of the temperature field, see Morris (1981a). We shall describe the solutions for the case $\nu=A e^{-\gamma T}$ where $A$ and $\gamma$ are given constants.

(i) The two dimensional hot plume.

Suppose that the horizontal plane $z=0$ is impermeable and stress free, and that all of it except for the strip $|x| \leqslant d$ is at the undisturbed 
temperature of the fluid. The strip $|x| \leqslant d$ supplies a heat flux qo per unit length in the y direction. The virtue of this flow is that it is possible to get simple solutions which show the evolution of the plume from a flow dominated by the viscosity variations to one which is essentially isoviscous. As with all plume solutions It has the disadvantage that the ambient conditions are prescribed, so that the convection does not affect the environment.

There are two different contributions to the velocity field which maintains the thermal boundary layer of the plume. First, because the viscosity depends strongly upon temperature, it is most strongly reduced in a channel which straddles the centerplane of the plume and across which the temperature varies by an amount of order $\gamma-1$. The channel is thus much narrower than the thermal boundary layer if $\gamma \Delta \mathrm{T} \gg 1$ (sketch). The vertical volume flux will tend to be concentrated into this channel, for the fluid there is much less viscous than the surrounding fluid. This volume flux can

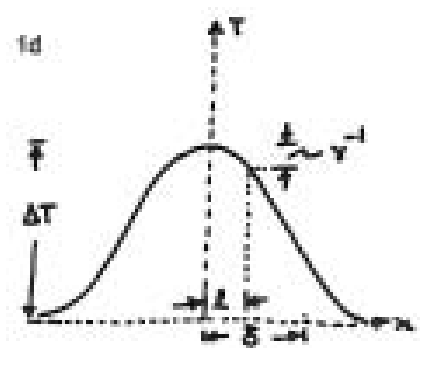

only be supplied by entrainment into the channel so that the external fluid

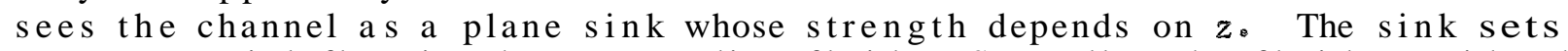
up a potential flow in the surrounding fluid. Secondly, the fluid outside the channel has a temperature difference of order AT applied to it and will thus see a shear stress applied to it at $x=0$. A vortical flow results, and in it the vertical velocity increases with $z$, for the longer a parcel of fluid has been rising, the longer it has experienced the buoyant force. On the other hand, the viscosity within the channel increases strongly with height, so that the potential flow should weaken with $z$. This suggests that the entrainment wind should be most important i m maintaining the plume close to the heat source, while the vorticalflow will take over this role for larger $z$.

A partial solution to this problem can be given by assuming the plume to be maintained by the entrainment wind. The flow which results has an analytical solution which can be obtained by using the fact that the channel is much thinner than the thermal boundary layer. It is then possible to estimate the vertical velocity in the external flow and hence to estimate the height at which the external flow takes over the maintenance of the plume. The details are given in Morris (1981b). I estimated that for reasonable values of the parameters in the problem the vertical velocity would be of order $10^{-6}$ to $10^{-5} \mathrm{~cm} / \mathrm{sec}$, that the central channel should be between 1 and $3 \mathrm{~km}$ wide, and that the vortical flow should control the thickness of the thermal boundary layer for distances $\geqslant 110 \mathrm{~km}$ above the source. If partial melting occurs within the plume, the last figure will be larger and the other two, smaller.

To give a more detailed analysis you must solve for the external flow and couple that solution to the one for the channel. That analysis is in progress. 
(i i) Convection in a narrow vertical slot.

It is fairly obvious how to give a self-consistent asymptotic analysis of the hot plume, for in that case the viscosity variations tend to concentrate the motion. It is less obvious how to analyze the cold plume, for the densest fluid is also the most resistant to deformation. The easiest cold flow to describe is that induced when a cold semi-infinite plate is placed vertically in an infinite fluid. Having solved that you can give a partial solution for the flow induced in a narrow vertical slot when its side walls are differentially heated. This problem has some difficulties in common with the corresponding isoviscous problem. W shall describe the partial solution first, and then the difficulties in their solution.

(i i a) Plow due to an isolated cold wall.

Consider the situation shown. There is a similarity solution to the governing equations. Let

$$
\psi=\frac{\alpha g \Delta T}{\nu_{\infty}} \cdot \delta^{3}(z) f(\xi) \text { and } T-T_{\infty}=-\Delta T h(\xi)
$$

where

$$
\Delta T=T_{\infty}-T_{1}>0, \quad \xi=\frac{x}{\delta} \text { and } \delta=\left(\frac{4}{3} \frac{k \nu_{t}}{\alpha g \Delta \cdot z^{3}}\right)^{1 / 4} \cdot z .
$$

Then the vertical momentum equation is

$$
\frac{1}{\sigma}\left\{\frac{2}{3}\left(f^{\prime}\right)^{2}-f f^{\prime \prime}\right\}=h(\xi)+\frac{d}{d \xi} e^{t h} f^{\prime \prime}
$$

where $t=\gamma \Delta \mathrm{T} \gg 1$, and $\sigma=\frac{2 b_{k}}{k}$. The energy equation is

$$
0=f h^{\prime}+h^{\prime \prime}
$$

where $f(0)=f^{\prime}(0)=f^{\prime}(-\infty)=h(-\infty)=h(0)-1=0$. We are interested in the case $\sigma \rightarrow$, and $t \gg 1$ and fixed. As i $n$ the corresponding isoviscous problem, the flow consists of a thermal boundary layer, in which the advection of momentum is negligible, and an external momentum boundary layer in which inertia returns the vertical velocity to zero from the plate.

Well into the cold boundary layer the fluid is so viscous that we expect heat to be transported by conduction alone so that $h$ is linear in $\xi$. Also, at $-\infty, h=0$. This gives the asymptotes shown. There must be a smooth transition from one asymptote to the other, and the temperature change across this 
corner region should be of order $\gamma^{-1}$, the e-folding temperature scale for

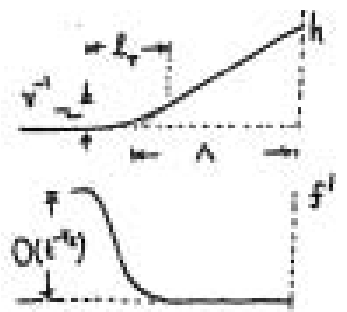

viscosity. Since the vertical velocity attains its maximum across this layer, it is a simple matter to scale the equations (1) and (2) with $\sigma m \infty$. We find that $\boldsymbol{L}_{\mathrm{T}} \mathrm{t}^{1 / 4}, \mathrm{~A} \sim \mathrm{t}^{5 / 4}$ and that $\mathrm{f}^{\prime}$ is of order $t^{-1 / 2}$ at the outer edge of the corner layer. The maximum vertical velocity is thus of order wo $=\frac{K}{d} R_{\gamma} 1 / 2$ where $R_{\gamma}=\frac{\alpha q \gamma d}{\gamma}$ while the heat flux, made dimensionless against $\rho C_{p} K \Delta T$ is $\frac{d}{\lambda}=R_{\gamma}^{1 / 4} \cdot t^{-1}$. Note that the Rayleigh number is based on the viscosity of the least viscous region and the temperature scale $\gamma$

When equations. (1) and (2) with $\sigma=\infty$ are rewritten in coordinates centered on the corner layer and using $\mathscr{L}_{\mathrm{T}}$ as the length scale and $t^{-1}$ as the temperature scale, there is no simplification in the differential equations for $\mathbf{t} \gg 1$. But because $\Lambda / \mathcal{L}_{\mathrm{T}}=O(t)$, the boundary conditions at $\zeta=0$ are now applied at $\rightleftharpoons$. After some juggling the boundary value problem can be solved numerically.

The analysis for the momentum boundary layer is the same as it is for the isoviscous problem. We find $\ell_{M} \sim \mathrm{q}^{1 / 2} \mathrm{t}^{1 / 4}$.

A related analysis of a variable viscosity flow has been given by Ockenden and Ockenden (1977).

(iib) Convection in a vertical slot with differentially heated walls. We can extend the solution (iia) to get an idea of the effects which the buoyant layer will have on its surroundings by studying convection in a narrow vertical slot. The floor and roof of the slot are perfectly insulated and stress free. Consider the case in which the flow in the slot is creeping flow with the temperature differences confined to thin thermal layers next to the boundaries of the slot. We shall suppose that the region outside the boundary layers is isothermal, although it is well known that this is not observed in practice (see section (iii)). With these assumptions a partial solution can be obtained, for we can solve for the hot and cold boundary layers in terms of the as yet unknown temperature differences across them. These temperature differences are fixed when the core temperature is known. It in turn is determined by the condition that the heat flux through the cold wall must equal that through the hot wall. The temperature profile then has the form shown in the sketch. The flow has two main parts. Near the cold wall there is a stagnant layer across which heat is transported by conduction; almost a 11 of the applied temperature difference $T_{h}-T_{c}$ occurs across this Payer. The temperature difference across the rest of the slot is of order $\gamma-1$. Here $\ell, \Lambda$ and $w$ have the orders of magnitude given in part (iia), with the panneter $t=\gamma\left(T_{h}-T_{C}\right)$. In particular, the heat flux through the cell is 


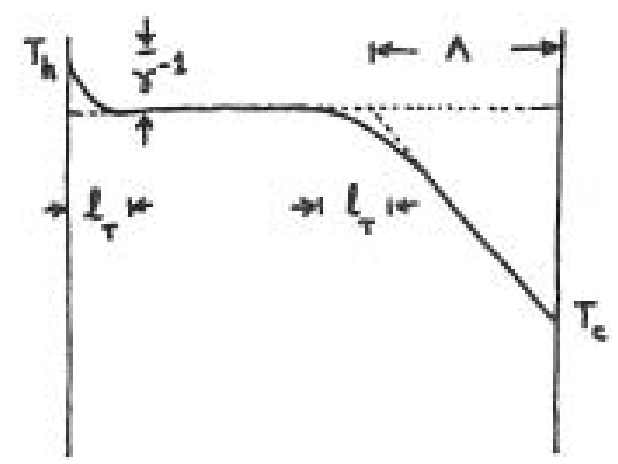

controlled by the Rayleigh number based on the viscosity of the least viscous region, and on the temperature difference across that region, for that is the only fluid which is convecting.

Having solved for the thermal boundary layer, you know the velocities at their outer edges and you can solve the lubrication equations to determine the velocity within the core. Because the slot is narrow, it seems reasonable to neglect the ends for points sufficiently far away from them (but see section (iii)); they can be replaced by the condition that there be no net mass flow up the slot, $\int \mathrm{dx} w=0$, which suffices to fix the vertical pressure gradient. The streamlines in the core then Rave the shape shown in the sketch; on the

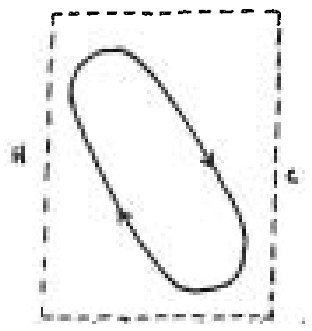

scale of the diagram the boundary layers occupy the dotted region. The orientation of the eddy is compatible with the idea that the boundary layers are maintained by entrainment.

So far the solution appears self-consistent, and gives a dramatic picture of the effects of strongly temperature-dependent viscosity on the temperature and velocity profiles. But we shall now show that this solution is at best quasi-steady, and that the core must slowly stratify. I have not yet done the underlying analysis and the arguments are thus more speculative.

(i i i) The development of stratification.

Elder (1965) noted that for aspect ratios greater than or of order one, the core of the cell is stably stratified, and that the vertical velocity is nonzero only in the boundary layers. Here is an explanation of why this should be so. A similar explanation has been given by Robinson (1967).

Consider first the case of convection driven by heating driven from below, nd look at the hot plume formed when the lower boundary layer detaches. Over he lower half of the cell, the plume entrains fluid from the isothermal core of the cell and there must be some way of cooling this fluid if the cell is not to stratify* in the sketch the dotted line marks the edge of the hot thermal layer, and the solid line is a streamline. Above the midplane of the cell, the two lines almost coincide. The lateral boundaries of the plume fan out near the 
upper surface of the cell. Sufficiently far from the centerline of the plume,

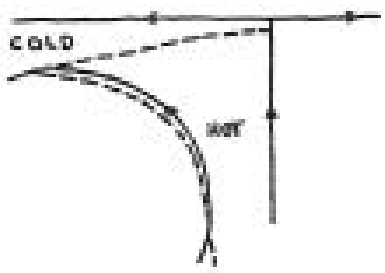

the outer edge of the cold horizontal boundary layer intersects the outer edge of the plume, and the absorption of the plume is complete. The mushroom of warm fluid is stable, because the heating is from below.

This is not so when the heating is from the side, and the mushroom (on its side this time) could exist only if the fluid in the core could exert enough stress to drag the buoyant fluid down. An order of magnitude estimate suggests that this is not the case, so that the warm fluid could be destabilized only by entering the cold vertical boundary layer directly (see sketch). Since that is a fairly slow process, warm fluid will slowly accumulate at the top of the slot. This of course is the beginning of the situation

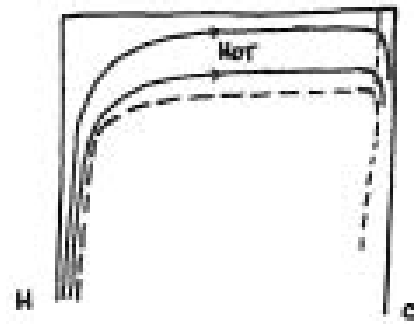

described by Elder; this upper, stably-stratified boundary layer must now grow i n thickness simply because all of the fluid entrained by the hot vertical boundary layer is added to it. What saves the solution (iib), for the purpose I have used it for, is that this stratification will apparently develop only over a time scale of order the width of the slot divided by the entrainment speed into the vertical boundary layer. The solution (iib) is thus quasisteady.

\section{(i v) Remarks.}

Convection in a slot can never show the return to isoviscous behavior which seems to be shown by a cell with stress-free boundaries. As the Rayleigh number of the slot is increased, the cold boundary layer becomes thinner but it is never easily deformable simply because of the presence of the rigid wall. That is obviously not the case when the boundaries are stress-free. I am at present formulating a simple model of this phenomenon. The idealized solution (iia) is a useful starting point.

\section{REFERENCES}

Elder, J.W., 1965. J. Fluid Mech., 23, 77-98.

Lux, R. and I. S. Sacks, 1979/80. Carnegie Inst. Wash. Year Book, 79, 512-520.

Morris, S., 1981a, 1981b (submitted to J.Fluid Mech.) 
Ockenden, H. and J. R. Ockenden, 1977. J. Fluid Mech., 83, 177-190.

Robinson, J. L., 1967. J.Fluid Mech., 30, 577-600.

\title{
A DISCUSSION OF COMPOSITIONAL CONVECTION
}

\section{David Loper}

Compositional convection occurs when a liquid mixture or alloy is cooled causing partial solidification. Examples of its occurrence include the casting of metallic alloys, freezing of sea water, subcrustal circulation of sea water near midocean ridges, magma chambers in the crust, the earth's core, other planetary interiors (particularly Saturn) and stellar interiors. Compositionally driven convection differs from themally driven convection in several respects. First, in a two-component system with two phases present, the equation of state for density may have $\partial \rho / \partial T>0$ rather than the normal $\Delta p / \partial T<0$ where $p$ is density and $T$ is temperature; in this case a fluid cooled from below is convectively unstable. Almost always the freezing interface is dendritic, forming a dendrite layer or "mushy zone" between the liquid and solid. Since material diffusion occurs only on very small scales, i.e., within the dendrite layer, the only way a parcel of fluid can change its composition is by passing through the dendrite layer. Hence, in a steady flow a 11 streamlines must pass through the dendrite layer. Calculations show that the inner-core boundary of the Earth is very likely to be dendritic and the dendrite layer is very thick, possibly extending to the center of the Earth.

\section{PERTINENT REFERENCES}

Copley, S. M., A. F. Giamei, S. M. Johnson and M. F. Hornbecker, 1972. The origin of freckles i unidirectionally solidified castings. Metall. Trans., $1,2193-2204$.

Fearn, D. R. and D. E. Loper, 1981. Compositional convection and stratification of the Earth's core. Nature, 289, 393-394.

Fearn, D. R*, D. E. Loper and P. H. Roberts, 1981. A new perspective on the structure of the Earth's inner core. Nature, 292, 232-233.

Loper, D. E. and P. H. Roberts, 2981. A study of conditions at the inner core boundary of the Earth. Phys. Earth Planet. Inter., 24, 302-307,

Loper, D. E. and P. H. Roberts, 1981. Compositional convection and the gravitationally powered dynamo. Manuscript.

\section{CHAOTIC BEHAVIOR OF MILDLY UNSTABLE BAROCLINIC WAVES}

\author{
Joseph Pedlosky
}

A review was given of the derivation of the amplitude evolution equation for a weakly unstable baroclinic wave on an $f-p l a n e$, whose amplitude $A(t)$ satisfies:

$$
\frac{d^{2} A}{d t^{2}}+\frac{3}{2} \gamma \frac{d A}{d t}-A+A \quad \int_{0}^{1} d y \sin 2 \ell y \frac{\partial^{2} \phi}{\partial y^{2}}=0
$$




$$
\frac{\partial}{\partial t}\left(\frac{\partial^{2} \Phi}{\partial y^{2}}-a^{2} \Phi\right)+\gamma \frac{\partial^{2} \Phi}{\partial y^{2}}=\left(\frac{d}{d t}|A|^{2}+2 \gamma|A|^{2}\right) \sin 2 \ell y
$$

where $\gamma$ is a nondimensional meascre of Ekman layer dissipation, a is the total wavenumber of the wave while $\Phi(y, t)$ is the stream function for the waveinduced correction to the mean thermal wind. \& must also satisfy

$$
\frac{\partial \Phi}{\partial y}=0 \quad \text { on } \mathrm{y}=0,1 \text {. }
$$

Numerical integrations of these amplitude equations showed a conplex dependence on $\gamma$. Simple limit cycle behavior for small $\gamma$ gave way, through a sequence of period doubling which satisfied the Feigenbaum relation (1978), to chatic behavior at moderate $\gamma$. At higher $\gamma$ steady solutions obtained.

When a weak $\beta=$-effect is added to the above model the coefficient of the linear term in the equation for A becomes complex. As $\boldsymbol{P}$ is increased from zero, the chaotic behavior is smoothed away and periodic halving occurs, until for quite small values of $\beta$ only steady solutions obtain. This latter result is a joint consequence of and Ekman dissipation.

\section{REFERENCES}

Feigenbaum, J. J., 1978. Quantitative universality for a class on nonlinear transformations. J. Statis. Phys., 19, 25-52.

Pedlosky, J., 1981. The effect of on the chaotic behavior of unstable baroclinic waves. J. Atmos. Sci., 38, 717-731.

and C. Frenzen, 1980. Chaotic and periodic behavior of finite amplitude baroclinic waves. J.Atmos. Sci., 37, 1177-1196.

\section{STABILITY BOUNDS ON THE AMPLITUDE AND STRUCTURE OF TURBULENT TRANSPORT PROCESSES}

\section{Willem V. R. Malkus}

In the spirit of optimum transport theory (L. N. Howard, 1972; F。 H. Busse, 1978), vector fields are considered which include the realized fluid flows and are compatible with an arbitrary number of constraints derived from the equations of motion.

Here, however, only the vector fields associated with the averaged transport equations are broadened beyond those permitted by the full equations. Among these vector fields one seeks to determine that subset which produces the most stable equilibration at any particular values of the Reynolds, Rayleigh, and Prandtl numbers. Hence the flows and temperature fields which are found are at least as stable as the realized flows and fields. Quantifying arguments based on marginal equilibration have been used frequently for turbulent flows driven by buoyancy-like forces (e.g., Barcilon et. al., (1979). However, it has been thought that turbulent shearing flows had very stable mean fields (e.g., Reynolds and Tiederman, 1967). Here we discuss the form of this theoretic proposal and first results for the case of parllel channel flow, exhibiting the sense in which the observed velocity profiles are marginally equilibrated against both two and three dimensional disturbances. 


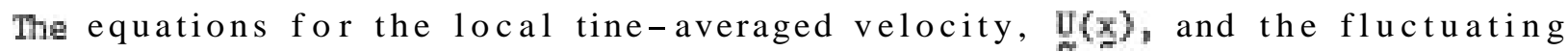
velocity, ur, in channel flow are

$$
\underline{U}+\nabla \underset{\sim}{U}+\underline{u} \cdot \nabla \underline{u}+\nabla \nabla / p-\nu \nabla^{\prime} \underline{u}=0
$$

and

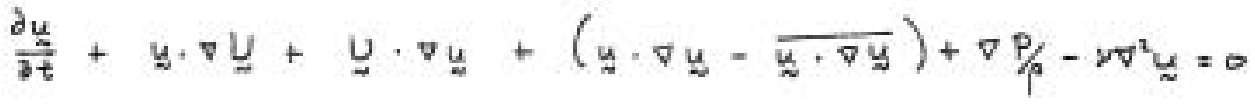

where the overbar indicates a local time average. By definition no unbounded on the average $\underset{\sim}{\mathbb{W}}$ i n equation (2). A typical disturbance decay, or at most achieve a finite amplitude equilibration. From equation (1),

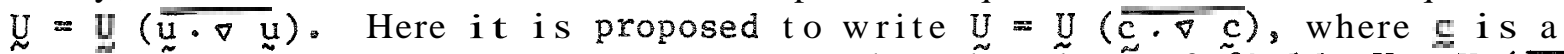

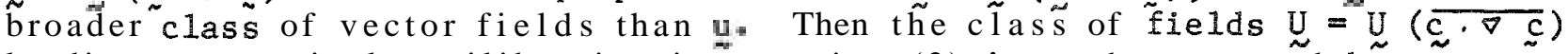
leading to marginal equilibration in equation (2) is to be narrowed by requirements relating $\underset{\sim}{\mathrm{u}} \mathrm{to} \underset{\sim}{\mathcal{c}}$. For example, if $\bar{\sim} \underset{\sim}{\underline{u}}$ is one dimensional then one may write

$$
\overline{\underline{u} \cdot \nabla \underline{u}}=\sum_{k=1+\infty}^{+\infty} u_{k} e^{i k \phi}
$$

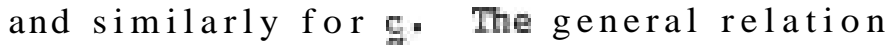

$$
\sum_{k=-\infty}^{\infty} k^{n} u_{k}=\sum_{k=-\infty}^{+\infty} k^{n} c_{k}
$$

is to be met, first for a few $n_{s}$ then in principal for a 11 no At any point in the application of constraits an idealized flow emerges. One is to determine its correctness by computing the change in average properties caused by the next constraint and, of course, comparison with experiment. The first step in this proposal is to find the most stable marginal equilibration free of any of the constraints (4) other than boundary conditions. In the language we became familiar with during our GFD 1981 sumer program, we seek that central manifold of the disturbance (2) which is of maximum codimensionality. Adjacent to that manifold is the realized chaotic flow. This first step quantifies mean fields for the problem, which may or may not be unique. The second step of the proposal is to apply one or more of the constraints (4) to the lowest order amplitude equations of the central manifold. This will determine the amplitude and structure of a 'model' $y$ field, whose moments can be compared to observation to test the adequacy of the constraints employed. Only results of first steps towards the parallel channel flow problem are reported here. Numerical computations utilizing an analytical-empirical class of velocity profiles proposed by Reynolds and Tiederman (1967) as a base, but adding zero-averaged downstream stresses determined by the three dimensional eigensolutions $y$, establish that this (generalized) $\underset{w}{\mathbb{W}}(z, x) 2$ is marginally stable for values of logarithmic slope and boundary intercept constant close to those observed. The downstream and cross-stream oscillations which lead to chis result indicate that this first theoretical ${ }_{*}$ is not unique.

\section{REFERENCES}

Barcilon, A., et.al,, 1979. J.Fluid Mech。, 94, 453.

Busse, F。H., 1978. Advance in App1. Mech., 18, 77.

Howard, L. N., 1972. Ann. Rev. Fluid Mech., 4, 473.

Reynolds, W. C. and 65. G. Tiederman, 1967. J.Fluid Mech., 27, 253. 


\title{
INVERSE METHODS FOR OCEANOGRAPHY
}

\author{
George Veronis
}

The principal constraint that is used with the inverse method for determining barotropic velocities in the ocean is the conservation of $m$ as for (M) suitable chosen layers in a closed-box region. When mass conservation is coupled with geostrophic balance for the baroclinic field velocities in a region with $\mathrm{N}$ pairs of hydrographic stations, a system of M linear equations is obtained for the $\mathrm{N}$ barotropic velocity components that are required to provide mass balance. In matrix form the system reads $A_{i j} b_{1}=C j$ where $A_{1 j}$ is the matrix of coefficients determined by the areas bounded above and below by the bounding density surface of $a$ layer and at the sides by the stations, $b_{i}$ is the barotropic velocity vector at station pair, $I$, and $\mathrm{C}_{j}$ is the transport imbalance for the layer, $\circ$ With $M \ll N$ the $M x N$ system is highly underdetermined.

The method of solution augments this system with an artificial $N$ X M system of overdetermined equations, $A_{j 1}^{T} d_{j}=e_{i}$, where $A_{j i}^{T}$ is the transpose of $A_{i} j$, to generate a symmetric coefficient matrix of size $(N+M) x$ $(N+M)$. The eigenvectors and eigenvalues of this augmented problem are then used to obtain a representation for the barotropic velocity, $b$, where the amplitudes of the eigenvectors are determined by the $C_{j}$. The formal solution makes no use of the null vectors of the space of the A matrix and in that sense the quantity $\sum_{i=1}^{N} b_{i}$ is minimized. The solution is sensitive to noise and other uncertainties in the quantities used to make up the components of the A matrix and of the $C$ vector.

A simple analysis of some schematic examples shows why the above underdetermined system is sensitive to noise. Basically, the layer thicknesses have only small deviations from a constant value and it is the small deviation which determines the solution. The largest deviation with real data nomally arises from bottom topography so topography should show up strongly in the horizontal structure of the barotropic velocity fleld. Results taken from a recent study by Wuasch and Grant (unpublished) show just such behavior.

Since the primary reason for the high sensitivity of the barotropic solution to noise, etc, is the poor horizontal structure of the layers, an attempt to use information other than the (nearly uniform thickness) density layers is suggested. A preliminary map of fields of potential vorticity and the Bernoulli function provides the desired structure. Use of these fields of greater structure is now being incorporated into the universe method.

\section{SLOW BOX-MODEL OSCILLATORS}

\section{Pierre Welander}

Well-known oscillators driven by differential heating have compressibility or inertia as critical effects: examples include the "glassblowers organ" (compressibility) and the Howard-Malkus "loop oscillator: (inertia), and various oscillators studied by astrophysicists (inertia and compressibility). There also exist oscillators which lack both compressibility and inertia, but with destabilizing diffusive effects. Two specific examples, studied by the author, are used as examples. The first example is the U-oscillator, obtained by connecting two open reservoirs by $a$ U-loop, and applying heating from below 
(Welander, 1957), see Fig. I. Once warm fluid starts up one of the branches,

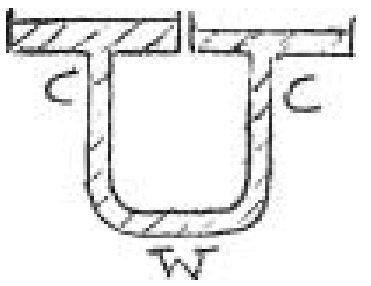

FIGURE 1

it creates a net buoyancy that keeps the motion going until arrested by the slowly growing opposing head due to level changes in the reservoirs. The system would stop in an unsymmetric position were it not for diffusive effects that tend to make the temperature distrfbution symmetric; once the flow reverses it keeps going in the same direction, etc. Typical is the strongly nonharmonic shape of the oscillation, and the critical dependence of the period on the diffusion. A simple theoretical model of third order, involving two well-mixed boxes can be constructed (not given here; after seeing the next system the reader may get the idea how to do it him/herself).

It is pointed out that the "salt-oscillator" by Martin (1970) has many similarities with the U-one (see Fig. 2).

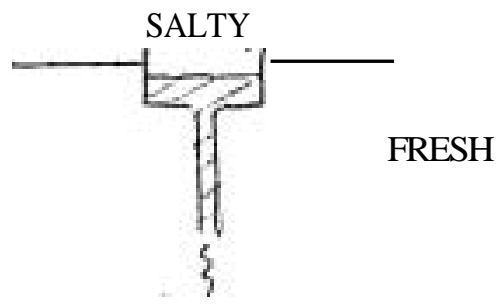

FIGURE 2

In the second oscillator considered, the existence of two diffusive timescales is important, like in well-known double-diffusive phenomena (saltfingers, etc:). Consider a well-mixed box of water warmed by a flux $k_{T}\left(T_{A}-T\right)$ and made salty by a flux $k_{S}\left(S_{A}-S\right)$. Generally $k_{T} \gg k_{S}$, and we assume this to hold. The box sits on top of a large reservoir with temperature $\mathrm{T}_{0}=0$, salinity $\mathrm{S}_{\mathrm{Q}}=0$ and (perturbation) density $P_{Q}=0$, with a false bottom allowing some turbulent exchange (see Fig. 3). The flux

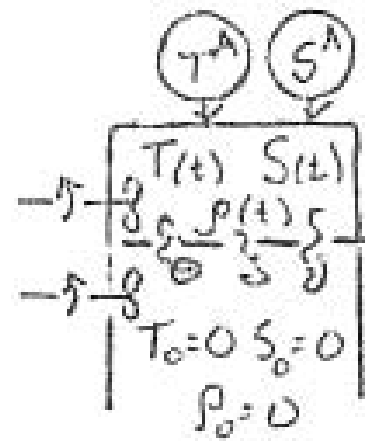

FIGURE 3 
from this reservoir to the upper box is $k(\rho)\left(T_{0}-T\right)$ for heat and $k(\rho)\left(S_{0}-S\right)$ for salinity, with $k(\rho)$ small if o 0 (statically stable), large if $F \geqslant 0$ (statically unstable). The equations are in one specific, simple example:

$$
\begin{aligned}
& \left.\dot{\mathrm{T}}=k_{\mathrm{T}}\left(\mathrm{T}_{\mathrm{A}}-\mathrm{T}\right)-k_{(\rho}\right) \mathrm{T} \\
& \left.\dot{\mathrm{S}}=\mathrm{k}_{\mathrm{S}}\left(\mathrm{S}_{\mathrm{A}}-\mathrm{S}\right)-k_{p}\right) \mathrm{S}
\end{aligned}
$$

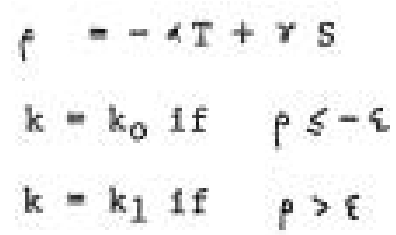

Here $k_{0}$ and $\varepsilon$ are small numbers, $k_{1}$ a large number. The parameters are chosen such that the steady state for the statically stable regime lies in the statically unstable regime, and vice versa, (see Fig. 4). Thus a steady state

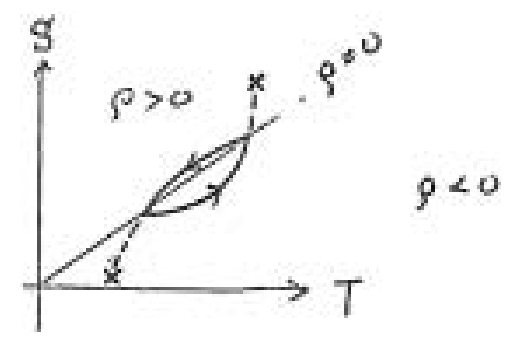

FIGURE 4

can never be reached. If $\varepsilon=0$, the solution goes into a vibration that increases its frequency forever; if $\varepsilon$ is finite but small self-sustained oscillations can be obtained. Again, an example of how a small parameter becomes decisive: Since the solution is exponential in each regime, the problem can be solved analytically, but the joining of the exponentials to get a full cycle becomes a little tricky. A problem recommended for students, therefore.

\section{REFERENCES}

Martin, S., 1970. Geophys Fluid Dyn*, 1, 143-160.

Welander, P., 1957. Note on the self-sustained oscillation of a simple thermal system. Tellus IX, 3, 419-420.

\section{LARGE AMPLITUDE CONVECTION I N POROUS MEDIA}

\section{John Booker}

Despite its importance in several geophysical contexts, strong thermal convection in a porous medium is poorly understood. Numerical work is plagued by instabilities even in the $2 \mathrm{D}$ case and experimental results are divergent. There have been several attempts to construct a boundary layer theory (i.e., Robinson and O'Sullivan, 1976) but all turn out to be inconsistent. Estimates of the exponent in the Nusselt number-Rayleigh number relation range over the remarkably wide range of $1 / 3$ to 1 . 
We have performed a simple experiment in which a Hele-Shaw analog of a porous medium is heated from below and cooled from above. At large Rayleigh number (20 times critical) the flow is stable and exhibits unmistakable boundary layer character: Each roll develops a stagnant core around which fluid circulates in a thin layer.

Our experiment suggests the boundary layer model shown in Figure 1.

The vorticity equation in a $2 D$ porous medium is

$$
\frac{\partial w}{\partial x}-\frac{\partial u}{\partial z}=R \frac{\partial \theta}{\partial x}
$$

where $R$ is the Rayleigh number and $y$ and $\theta$ are nondimensional velocity and temperature. (1) immediately implies that the stagnant core is vertically stratified and that the plume velocity

$$
w=R\left[\theta-\theta_{1}(v)\right]
$$

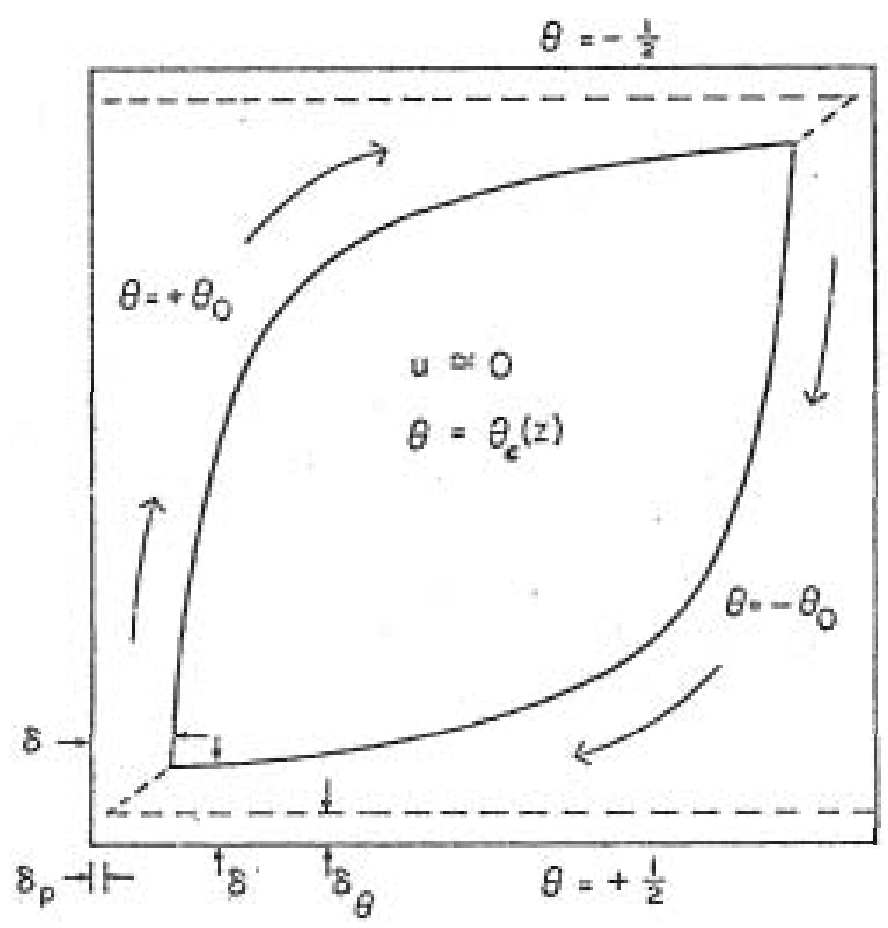

FIGURE 1

An analysis similar to Gill's (1966) for a thermal boundary layer on a vertical plate in a viscous fluid shows that the plume rises with constant temperature and without entraining fluid from the core, if the core has a stable linear stratification. Then (2) shows that $W$ decreases as the plume rises. The plume spreads and eventually flows over the top of the core where the temperature of the plume becomes the boundary condition which maintains the stratification of the core.

A top hat approximation for the plume temperature predicts that the stream lines in the plume are rectangular hyperbolas. This velocity field has a sinilarity solution for the thermal boundary layer on the horizontal boundarv which is independent of $x$ and has thickness $\delta_{\theta}=1 / \sqrt{R} \theta_{0}$ where $\theta_{0}$ is the plume temperature. It also turns out that the thickness of the transition

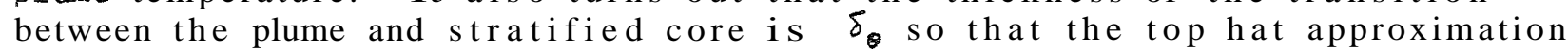


is consistent if $\delta_{0} \ll \delta$, the plume width. Conserving heat and mass flux through the corner where the horizontally spreading fluid turns into the rising plume gives $\delta=1 / 4 \pi \theta_{0} \sqrt{R \theta_{0}}$.

To close the problem and determine one needs to consider the details of the corner. Although it is virtually certain that similarity techniques will give an approximate solution that properly entrains the horizontal flow above the boundary layer and matches fluid input from the thermal boundary layer and output to the plume, we have not completed the details. I therefore use an empirical closure.

Displacement of a horizontal dye line at mid-depth corrected for the spreading streamlines (which are in good agreement with the expected hyperbolic form) gives $\quad w\left(z=y_{2}\right)=0.846 R^{0.80}$

Hence

$$
\begin{aligned}
& \theta_{0} \sim \delta \sim R^{-1 / 5} \\
& \delta_{0} \sim 1 / N-R^{-2 / 5} \ll 6
\end{aligned}
$$

where $N$ is the Nusselt number. Scaling of the corner physics confirms the liklihood of the $\quad-\theta_{a}$ conclusion.

Most of the problems in published heat transfer experiments occur because $\delta_{0}$ becomes comparable to the grain size. The only high $\mathbb{R}$ data without this difficulty are given by Combarnous (1970). His results are very close to the predicted $N \sim R^{2 / 5}$ relation.

\section{REFERENCES}

Combaronous, M. A., 1970. Rev. Gen. Thermique, 29, 1377.

Gill, A. E., 1966. The boundary layer regime for convection in a rectangular cavity, J.Fluid Mech., 26, 515.

Robinson, J. L. and M. J. O'Sullivan, 1976. A bcundary-layer model of flow in a porous medium at high Rayleigh number, J. Fluid Mech., 75, 459.

\section{PATTERN SELECTION I N RAYLEIGH-BENARD CONVECTION NEAR THRESHOLD}

Eric Siggia

The influence of internally generated vertical vorticity on the RayleighBenard problem has been quantitatively analyzed by means of conventional multiscale perturbation theory for free slip boundary conditions near onset. The competition between various roll patterns, all with the same basic orientation, was first analyzed by means of an amplitude expansion by Newel1 and Whitehead (1969). Their theory has the characteristic that the slowly varying amplitude, A, satisfies a first order equation in time whose right-hand side is derivable from a Liapunov functional. The system must then relax so as to minimize this potential.

To our surprise we found that for free-slip boundary conditions the Newe11Whitehead equation is incomplete even to lowest order in $\varepsilon=R / R_{c}-1$. Any curvature in the rolls generates a vertical vorticity that is independent of the vertical coordinate. The associated slowly varying horizontal velocity enters the equation for A convectively, thereby leading to a feedback onto the 
source of the vorticfty itself.

The secondary instabilities which nearly parallel rolls undergo are qualitatively modified by the inclusion of vertical vorticity. All wavelengths shorter than the most unstable ones are unstable to a version of skewed varicose instability first discussed by Busse and Clever (1979) for rigid boundaries, The zig-zag instability is suppressed for Prandtl number, $P 10$.

While no calculations have been done for rigid boundaries, it is plausible that the source of vertical vorticity is unchanged except for being a factor E $1 / 2$ smaller, since it must vanish on the top and bottom surfaces. The stability predictions of our model agree with exact numerical calculations of Busse and Clever (1979) for small P, which is where agreement would be expected expected.

The most interesting conceptual and perhaps calculational applications of our amplitude expansions may be to the onset of convection in a large box for $P \quad 1$. Since the dynamics of $A$ is no longer relaxational, a time dependent flow can persist even within the "Busse Balloon" if the pattern is not ideal, due either to the lateral boundaries or uncontrolled initial conditions.

Numerical experiments were done at $\quad=0.5$ and $\mathrm{P}=4$ which suggest that as the system size increases, a slightly scrambled pattern does not relax back to the stationary locally stable states we know to exist for these parameters or at least does so on a time scale that is much longer than a diffusive one,

\section{REFERENCES}

Busse. F. H. and R. M. Clever, 1979. J.Fluid Mech. 91. 319: R. M. Clever and F. H. Busse, J.Appl. Math, and Phys. (Z.A.M.P.) 29, 711 (1978). The second paper has stability diagrams for P 1.

Newe11, A C. and J.A. Whitehead, 1969. J.Fluid Mech. 38, 279.

Siggia, Eric D. and Annette Zippelius, 1981. Pattern selection in RayleighBernard convection near onset. Phys. Rev. Lett. 47, 835.

1981. Dynamics of defects in Rayleigh-Bernard convection. Physical Review A24.1036.

\section{WEAK TURBULENCE IN SMAUL ASPECT RATIO CELLS: THE CASE OF LIQUID HELIUM}

\section{Albert Libchaber}

\section{$\underline{\text { Introduction }}$}

We describe some experimeats on Rayleigh Benard convection where, through a strong truncation of the experimental situation (use of small aspect ratio cells), some direct comparison can be made between the various routes to turbulence observed $\mathbb{I}$ experiments and the theory of dynamical systems.

As a liquid, helium is an interesting ease. One can vary the Prandt number from about 0.4 to a few units. This is the range where the two competing nonlinear terms in the heat equation and the Navier Stokes equation overcome one another. At low $P$ the $V: \nabla v$ term is dominant and lead to the oscillatory instability, At high $P$ the $r \cdot \nabla T$ term is dominant and the 
skewed varicose instability sets in.

Also in liquid helium, given the large expansion coefficient and the small kinematic viscosity, experiments are performed with a small distance between top and bottom piates. This leads to somewhat higher dynamical frequencies and the data acquisition can be fast.

Finally, the low temperature techniques allow a very good stability and regulation of the experimental set up.

In experiments described here we use rectangular cells with two convecting rolls present. We present here two routes to chaos which have their analogy i n dynamical system theory: the period doubling bifurcation to turbulence (Collet and Eckmann, 1980; Echman, 1981; Feigenbaum, 1979) and the transition to turbulence though intermittency (Manneville and Pomeau, 1980). We refer the reader to other experimental work for a more general point of view of the experimental scene (Gollub and Benson, 1980; Ahlers and Walden, 1980; Dubois and Berge, 1980). In those scenarios we have always two oscillators present. In the first case they are locked, in the second case they are unlocked.

\section{The Period Doubling Bifurcation}

This transition has been well analyzed in mathematical terms for one dimensional maps of the interval (Collet and Eckmann, 1980). (Figure 1)

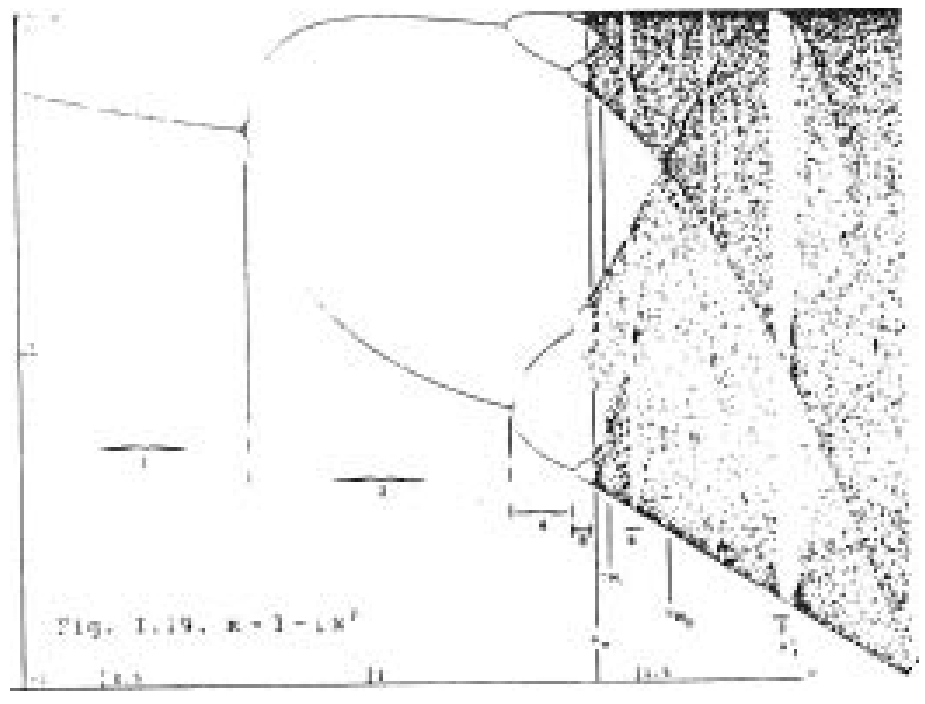

FIGURE 1

It is a continuous transition where, starting from an oscillatory state, as one increases the Rayleigh number a frequency f appears, first with zero amplitude and the amplitude grows continuously ${ }^{2}$ as the Rayleigh number increases. This elementary period doubling may reproduce itself infinitely as one increases the control parameter. The fundamental frequency is then indefinitely divided by two and goes to zero at the end of the process which is the beginning of a chaotic state. There is a scaling low in the transition $\frac{f}{2^{n}} \rightarrow \frac{f}{2^{m+n}}$ which reproduces itself in the next transition just changing the scale in Rayleigh number, the frequencies by a power of two, and reducing the amplitude of the new oscillator by a constant ratio. 

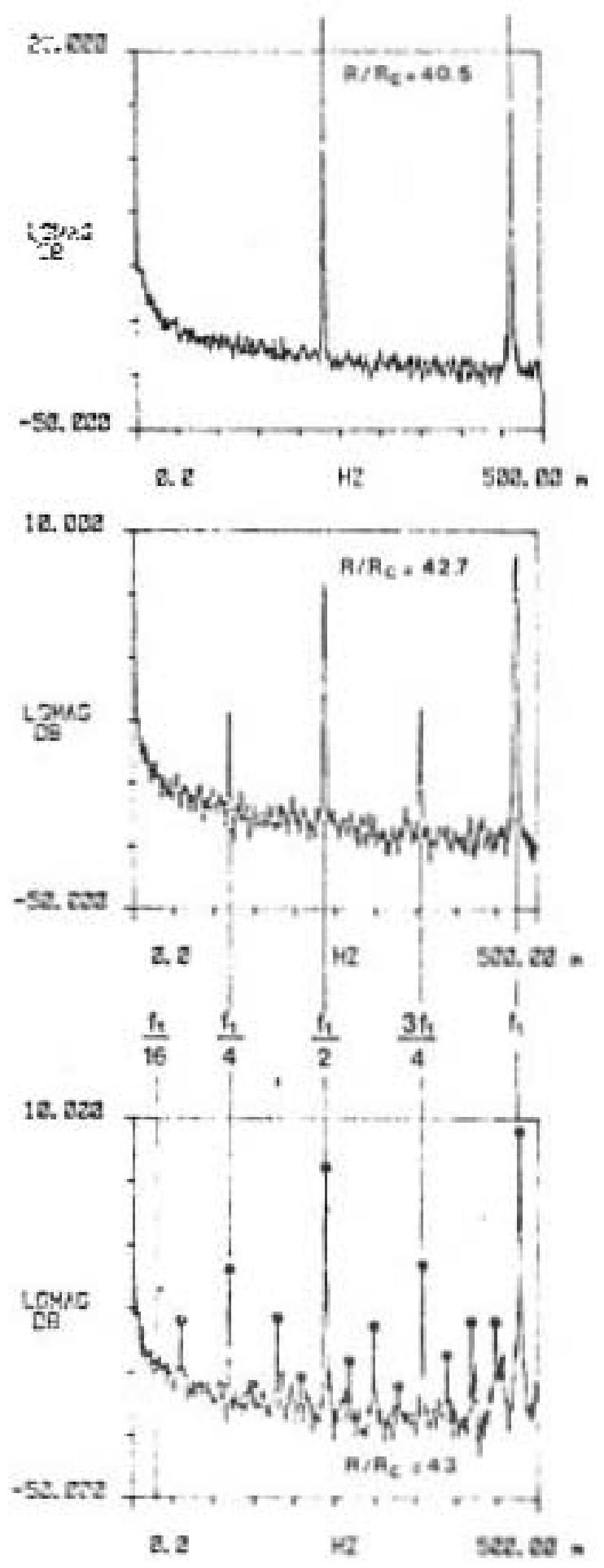

Figure 2 
We have performed the experiment in a rectangular cell of depth $1.25 \mathrm{~mm}$ and lateral dimensions $3 \mathrm{~mm}$ and $1.5 \mathrm{~mm}$, the $P$ nunber being $P=0.55$. The evolution of the period doubling cascade is shown on Figure 2 for increasing values of $R$.

Another prediction of the theory is that beyond the accumulation point, as one keeps increasing $R$, a mirror image of the cascade should exist (Figure 1 ). We have also observed this phenomena (Libchaber and Maurer, 1981).

\section{$\underline{\text { Transition to Turbulence through Incermittency }}$}

This transition has been analyzed theoretically by Manneville and Pomeau (1980). Whereas the preceding one has been associated with pitchfork bifurcation this one is associated with a saddle node bifurcation, i.e., the collision between a stable and unstable fixed point which then both disappear. But, beyond this value the system stays quasilaminar for a finite time and this
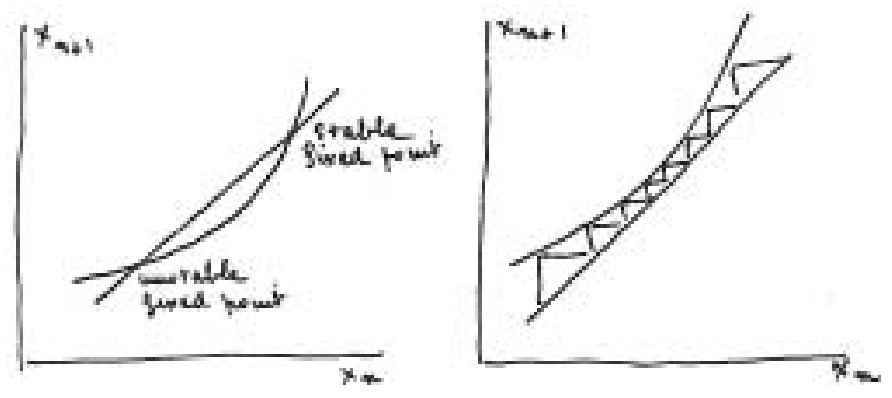

leads to intermittency. We observed the phenomena for a sample of depth 1.29 $\mathrm{mm}$ and lateral dimensions $3 \mathrm{~mm}$ and $1.5 \mathrm{~mm}, P=0.62$. We believe that the skewed varicose instability was present. The data are presented on Figure 3.

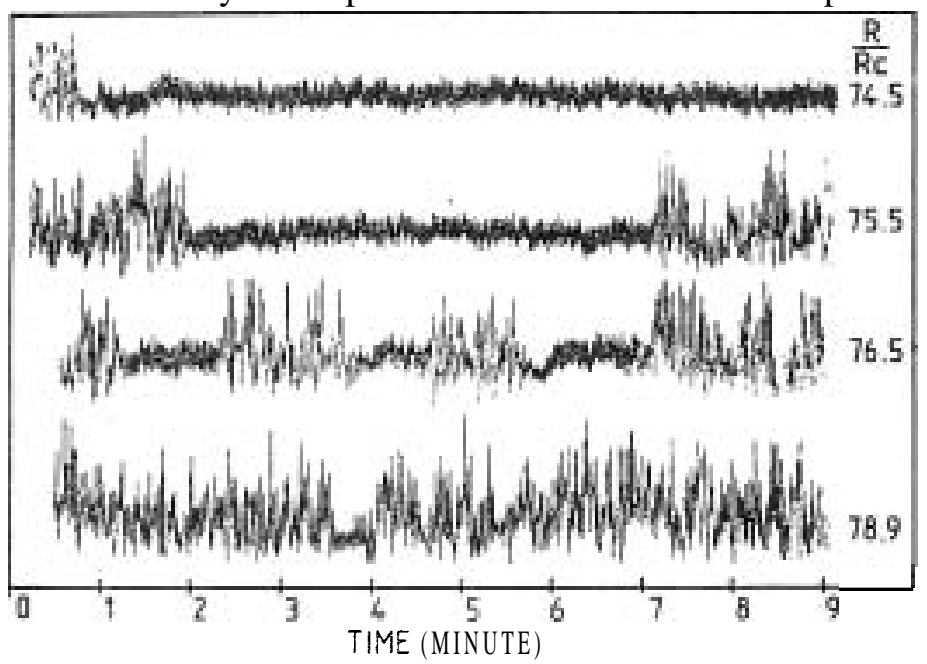

Fig. 3. - Direct time recordings of the intermittent transition to turbulence for a cell, a $=2.7, P r=0.62$. ( $R_{\mathrm{c}}$ Rayleigh number for the onset of convection). For $R / R_{\mathrm{c}}=74.5$, a burst of noise is seen on the left ; otherwise the signal, Fourier analysed, is a combination of two frequencies $f_{1}$ arid $f_{2}$ (Fig. 4).

For $\underline{R}=74$ the recordings present no bursts of noise and the Fourier spectrum hrdicates the presence of two unlocked oscillators. As $R / R_{c}$ increases bursts of noise become more and more frequent and for $R / R_{c}=79$ the recording is completely chaotic. If we define the onset at this transition at the value of the Rayleigh number corresponding to $R / R_{c}=74$ and $c$ all it $R_{i}$, we find that the laminar times diverge like $\tau=\frac{1}{(\mathrm{R}-\mathrm{R} i)^{3 / 2}}$. 


\section{REFERENCES}

Ahlers, G., and R. W. Walden, 1980. Phys. Rev. Lett., 44, 445.

Collet, J. and J. P. Eckmann, 1980. Iterated maps of the interval as dynamical systems, Birkhauer, Ed.

Eckmann, J. P. 1981. Roads to turbulence in dissipative dynamical systems, Review of Modern Physics.

Dubois, M. and P. Berge, 1980. J. Physique, 42, 167.

Feigenbaum, M. J., 1979. Phys. Lett., .74A, 375.

Gollub, J. P., and S. V. Benson, 1980. J.Fluid Mech., 100, 449.

Libchaber, A. and J. Maurer, 1978. J. Physique Lettres, 39, 369.

Libchaber, A., and J. Maurer, 1980. J. Physique Letters, 40, 419.

Libchaber, A. and J. Maurer, 1980. J. Physique Lettres, 41, 515.

Libchaber, A., and J. Maurer, 1980. J. Physique Co11. C3, 41, 51.

Libchaber, A. and J. Maurer, 1981. Proc. of the Nato School in nonlinear physics. Geilo, T. Riste, ed.

Manneville, P. and Y. Pomeau, 1980. Different ways to turbulence in dissipative dynamical systems, Physica $D, 219$

\section{CONVECTION AT VERY HIGH RAYLEIGH NURBERS}

Theodore D. Foster

As the Rayleigh number of a convecting layer of fluid is increased, the fluid motion becomes oscillatory and at very high Rayleigh numbers becomes intermittent. Howard (1964) examined this phenomenon and proposed that at very high Rayleigh numbers the buoyant boundary layer grows so rapidly that it becomes unstable while its thickness is still a small fraction of the depth of the entire fluid layer. The resulting convection rapidly destroys the buoyant boundary layer which then starts to reform by diffusion. We (Foster, 1971) have modelled this intermittent convection numerically and found that for flux Rayleigh numbers,

$$
R_{f}=\frac{\alpha g Q h^{4}}{k^{2} p c c}
$$

where $Q$ is the flux of heat through the boundary of the fluid layer, exceeding about 107 the convection became intermittent. The period of this

intermittency was found to be given by

$$
\tau_{f}=c\left(\frac{y \rho c}{\alpha g \theta}\right)^{\gamma_{3}}
$$

where $C=14$ for an infinite Prandtl number fluid.

We have recently performed experiments in a small (25 am cube) tank with a controlled heat flux at the bottom and insulated on the sides and top. At 
flux Rayleigh numbers from $10^{9}$ to $10^{11}$ the spectra of the temperature fluctuations in the boundary layer at the bottom showed most of the power was centered around the predicted periods with an inverse square root dependence on the heat flux.

We are now carrying out experiments in an insulated tank ( $3 \mathrm{~m}$ i $n$ diameter and $10 \mathrm{~m}$ deep) which is heated $10 \mathrm{to} 200 \mathrm{C}$ above ambient and then allowed to cool from the top. Flux Rayleigh numbers up to 1017 ere obtained. Initial results show that the spectra of temperature fluctuations in the top boundary layer (at the 1 em level) follow the predictions of the numerical model; however, spectra of the temperature fluctuation in the middle of the tank (at the 5 m level) are quite different and show highest power for periods an order of magnitude larger than at the top. Our preliminary explanation of $t h$ is shift in period is that it is due to the generation of a larger scale convection by interaction of the smaller scale convection as it moves away from the boundary.

\section{REFERENCES}

Foster, T. D., 1971. Intemittent convection. Geophys.Fluid Dyn.2, 201.

Howard, L. N, , 1964. Convection at high Rayleigh numbers, in Proceedings of the Eleventh International Congress of Applied Mechanics

\section{TURBULENT CONECTION}

John L. Lumley

We discuss fully developed convection, which we treat statistically; we do not examine the mechanism responsible for the apparent stochastic behavior. We are interested in predicting quantitatively the structure of convectively driven mixed layers including such practically important quantities as refractivity fluctuations which depend on water vapor and temperature fluctuations. We describe first the work done on the atmospheric surface mixed layer, and then its extension to include double diffusive effects.

The basic physical phenomenon (described in Lumley, et al, 1978) involves the modifications of turbulent transport by buoyancy. Effectively, the integral time scale of vertical motion may be much longer or shorter than that of horizontal motions. We may see this by considering a first order correction due to buoyant acceleration to a nonbuoyant field, leading to a diffusion coefficient of the form

$$
J\left(\overline{u_{i} u_{j}}+\alpha \beta_{j} \overline{v_{u}} J_{j}\right)
$$

where $v_{i}$ is fluctuating velocity, $\vartheta$ is temperature fluctuation, $\mathfrak{J}_{\vartheta}$ is a Lagrangian time scale for persistence of temperature-velocity correlations, and $\supset$ is the usual. Lagrangian integral time scale. This is the same form obtained by Deardorff $(1966,1372)$ from simple modelling of counter gradient transport. Notice that, near the inversion base, where $\overline{v w}<0$, this effectively suppresses the vertical transport, but substantially augments it near the ground.

Now we wish to develop a simple, mathematically consistent formulation embodying the above physics leading to a tractable problem. We have satis factory equations for second moments in homogeneous situations (Newman et al., 
1981). We need an expression for the third moment. We know that homogeneous turbulence without buoyancy is approximately Gaussian in the energy containing range. We postulate a turbulence that has a probability density that relaxes to Gaussian in the absence of inhomogeneity, buoyancy, and so forth, and that has at second order the accepted equations. find-grained relative to the inhomogeneity, This development is described In Lumley (1978). Briefly, this leads to an eddy-damped, quasi-Gaussian Markovion one-point form for the third moments, which are proportional to the gradients of the second moments with a matrix of coefficients all of the form of (1).

This form was applied by Zeman (1975), Zeman and Lumley (1976, 1979), Lumley e t al. (1978), and Zeman (1981) to the modelling of the atmospheric buoyancy-driven mixed layer. Equations for $\overline{u^{2}}, \overline{v^{2}}, \overline{w^{2}}, \overline{w \vartheta}, \ominus, \bar{\epsilon}, \overline{\sigma_{p}}$ were solved by simple-time-stepping. First-order mean quantities were well reproduced, of course, including inversion rise, as were second-order quantities. More significant, however, was the prediction of third-order quantities within experimentor error.

We now wish to extend these ideas to include double-diffusive effects, in particular the layered structure observed i n the Mediterranean outflow, where layers of turbulent buoyant convection are observed to alternate with layers of saltfingers. We observed that the salt-fingering regions are characterized by essentially one-dimensional turbulence in which the variables are perfectly correlated (Lumley, 1980). We postulated there, if the modelled terms were made to behave properly in the one-dimensional, perfectly-correlated limit, the salt-fingering phenomenon could be produced. In support of this, Lumley (1980) showed that these assumptions, applied to the second-moment equations, lead to the classical stability criterion for salt-fingering.

To obtain well-behaved models in the one-dimensional perfectly-correlated linit, the concept of realizability was extensively used: i.e., it was required that the time derivative of quantities which were required to remain non-negative, should itself vanish if the quantity in question vanished. This lead to required forms in this limit, which could be modified by the inclusion of an unknown function of the non-negative quantity. An example is the crossdissipation:

$$
\left(k_{T}+k_{s}\right) \overline{t_{j} s_{j}}=\overline{t s}\left(\bar{\epsilon}_{t} / \overline{t^{T}}+\overline{t_{s}} / \overline{s^{2}}\right)_{g}\left(1-\overline{t s}^{2} / \overline{t^{2}} \overline{s^{2}}, k_{s} / k_{t}\right)
$$

where $g(x, y)$ is unknown save for the requirement that $g(0, y)=1$ and probably that $g \geqslant 1$.

Similar considerations apply to the velocity field, where the quantity $F_{0}=\left(\overrightarrow{u_{1}^{2}} \overrightarrow{u_{1}^{2}} \overrightarrow{u_{s}^{2}}\right) / q^{b}$ (in principal axes) must remain non-negative. The various correlations have for the most part, known values in the one and two dimensional $1 \mathrm{imits}$ amd in the isotropic case, and it is necessary to construct interpolation forms dependent on $F_{0}$ connecting these cases.

This work i $\underline{\mathrm{s}}$ described_i $\mathrm{n}$ detail i n Zeman and Lumley (1981). There equations for $w^{2}, g^{2}, t w, s w^{2}, t^{2}, \overline{s t}, \overline{6}$ were solved with simplified forms for $\bar{\epsilon}_{t}$ and $\overline{\epsilon_{0}}$ (here $t$ is temperature fluctuation and $s$ the salinity fluctuation). A simplified form for the transport terms was used, with a single transport coefficient

$$
K=\frac{a_{3} J\left[\overline{w^{2}}+\zeta_{1}\left(J_{e t} \alpha \overline{E w}-J_{e s} p \overline{s w}\right)_{g}\right]}{1+\zeta_{t}\left(N_{T}^{2} \partial_{t} J_{e t}-N_{s}^{2} J_{t} J_{t s}\right)}
$$


which bears a clear family resemblance to (1). $J_{\epsilon s}$ and $J_{f s}$ are composite mechanical/thermal and mechanical/saline time scales and $N_{T}, N_{S}$ are the Brunt-Välsälä frequencies based on the temperature and salt gradients.

We computed (one-half) a symmetric region consisting of a salt-fingering layer between two convective regions. initially, the mean temperature and salinity profiles are specified, with a region of steep gradients where the fingering layer is expected to develop. The initial distribution of anistropy is uniform, at a value characterfstic of turbulent buoyant convection. Very quickly i n the region of steep gradients the motion becomes one-dimensional, indicating the development of a fingering layer. Predicted values of the salt/heat flux ratio, as a function of the buoyancy ratio, are quite similar to the measurements of Turner. The salt-sugar case was also computed. To obtain the correct ratios in both cases, it was necessary to introduce a weak dependence on Prandt 1 number in the equations.

\section{ACKNOWLDGEMENIS}

This work was supported in part by the U. S. Office of Naval Research under the following programs: Physical Oceanography (Code 481); Power (Code 473); and Fluid Dynamics (Code 438); and by the U. S. National Science Foundations, Division of Atmospheric Sciences under Grant No. ATM 79-22006.

\section{REFERENCES}

Deardorff, J. W., 1966. J, Atmos. Sci., 23, 503-506.

Deardorff, J.W., 1972. I. Geophysics Res., 77, 5604-5900.

Lumley, J. L., 1979. Computational modelling of turbulent flows. Applied Mech., 18, , 123-176.

Lumley, J. L., 1980. Some fundamental aspects of turbulence with implications i n geophysical flows. In Marine Turbulence, ed. J,C.J. Nihoul, 175-192, Amsterdam, Netherlands: Elsevier.

Lumley, J. L., O. Zeman, and J. Siess, 1978. The influence of buoyancy on turbulent transport. J. Fluid Mech., 84, 581-597.

Newman, G. R., B. Launder, and J. I. Lumley, 1981. Modelling the behavior of homogeneous scalar turbulence. J.Fluid Mech. (In press)

Zeman, 0., 1975. The dynamics of entrainment in planetary boundary layers: a study in turbulence modelling and parameterization, Ph.D. Thesis, Pennsylvania State University.

Zeman, 0., 1981. Progress in the modelling of planetary boundary layers. Ann. Rev. Fluid Mech., 13, 253-272.

Zeman, O. and J. L. Lumley, 1976. Modelling buoyancy-driven mixed layers. I. Atmos. Sci., 33, 1974-1988.

Zeman, 0. and J. L. Lumley, 1979. Buoyancy effects in entraining turbulent boundary layers; a second order closure study. In Turbulent Heat Flows $\mathbf{I}$, eds. Fs Durst, B. E. Launder, F. W. Schmidt, and J. H. Whitelaw, 295-302. Berlin/Heidelberg: Springer-Verlag. 
Zeman, 0 , and Jo Lo Lumley, 1981. Modeling of salt-fingering structures. S1bley School of Mechanical and Aerospace Engineering Report \#FDA-81-03. Ithaca, N.Y. Cornell University.

\title{
EXAYPLES OF REVERSE CASCADES IN TURBULENT PHENOMENA
}

\author{
Virten T. Zandahl
}

A number of different mechanisms are discussed whereby small-scale turbulent eddies nay induce large-scale motion and thus give rise to spectral transfer from higher to lower wave numbers. Weak nonlinear interaction between two wave components $K_{1}$ and $K_{2}$ will result in motion at a wave number $\mathrm{K}_{1}-\mathrm{K}_{2}$, as well as at a wave number $\mathrm{K}_{1}+\mathrm{K}_{2}$, Beading to such phenomena, as well as, e.g., subhamonic instability, Intermittent and spatially locahized small-scale motion, caused for example by local instability, will introduce motion sf a scale of the size of the region of instability. Small-scale intermittency is characteristic of strong nonlinear interaction processes and is an observed feature of fully developed turbulence. It is especially prevalent in the final process of transition to turbulence in a shear flow in which the onset of small-scale motion is extremely rapid making large spectral jumps both up and down the wave number range possible.

Yet another class of backward cascading mechanisms, which has so far been very little explored, is that in which the presence of the small scales can induce the transfer of kinetic energy from the mean flow to large-scale fluctuations, $\dot{i} . e .$, the small scale motion acts as a catalyst for the creation of the large scales. It has usually been assumed that the presence of the small scales adds an effective eddy viscosity to the flow, thus giving rise to additional dissipation for the large scales. In the lecture, a counter example from wind generation of water waves is presented. The model problem treated is that of detemining the wind induced growth rate of long water waves of infinitesimal amplitude in the presence of short waves of finite (but small) amplitude. Miles (1957, 1962) considered the problem for a single wave component and found that waves in the capillary regime grow the fastest. Growth rates calculated from his theory show generally good agreement with measured data for short waves, but the theory tends to underestimate the growth rate for long waves in the gravity wave regime. Interaction between the short and Iong waves is shown to occur both in the water and the air and give contributions to the growth rate of the long waves which are proportional to the mean square of the short-wave amplitude. The interaction in the air is found to be by far the strongest of the two. It arises because the modulation of the shortwave Reynolds' stresses due to the presence of the long-wave velocity field produces an added phase lag between the streamline at the edge of the air boundary layer and the surface wave deflection. Thereby, the pressure in phase with the long-wave slope increases and causes a corresponding increase in the momentum transfer from the air to the water. The numerical example presented, which is based on an inviscid model, demonstrates that this mechanism can give $a$ large contribution to the rate of growth of waves in the short gravity wave length regime, also when the amplitudes of the capillary waves are quite small. It is sumised from this example that small-scale motion i n a fully developed shear flow could have a similar catalytic effect on the largo scales. 


\section{STUDIES OF THE TRANSITION TO TURBULENT CONVECTION USING SCATTERED LASER LIGHT}

\section{J. P. Gollub}

We have studied convective phenomena in a large rectangular fluid layer using a laser Doppler scanning technique that permits the structure of the velocity field to be mapped in the horizontal plane. This mapping could be done in a time shorter than the characteristic times of velocity fluctuations near their onset, so that the space and time structure of one component of the velocity field could be recorded in $\overline{d i g}$ ital form. This approach is considerably more powerful than local probe measurements a t a fixed point. The experiments were performed in a $10 \times 15 \times 0.5 \mathrm{~cm}$ layer bounded by copper plates, using water at $70^{\circ} \mathrm{C}$ where its Prandtl number is 2.5 . The 1 ight scattering techniques, and experiments on layers of small aspect ratio showing multiple periodic flows, phase locking, and subharmonic bifurcations, have been thoroughly described elsewhere (Gollub and Benson, 1980; Gollub, Benson, and Steinman, 1980).

We found that stable flows do exist above $R_{c}$, but are strongly influenced by the lateral boundaries even for large layers. The rolls preferentially align with their axes perpendicular to the boundaries, causing the pattern to be splayed and to contain defects, where a roll of positive (or negative) vorticity ends. Stable flows are only reached after transients lasting for a day or so, a time comparable to the horizontal thermal diffusion time for this system. We identify stable flows by verifying that Doppler contour maps made several days apart can be superimposed; the roll boundaries move by no more than a few percent of the roll spacing, except very near the defects.

There are several distinct stable flows with different symmetries having the minimum number of defects, which is apparently two. On the other hand, patterns with many defects, which can be created by decreasing $R$ from $30 R_{c}$, are not stable, and continue to evolve very slowly even after a few days. In this respect, the behavior of the system when many defects are present resembles that of a glass.

The stable flows become unstable when the Rayleigh number is raised to $R_{1}{ }^{2} 5 R_{C}$. However, the fluctuations are sufficiently slow that their space and time structure could be followed in detail by repetitive laser Doppler imaging. We found that the rolls become unstable with respect to deformations which repetitively pinch off rolls to form new defects. Furthermore, this process leads to an increase in the average wave length of a factor of two. These properties head us to identify these fluctuations as arising from the skewed varicose instability of Busse and Clever (1979). The onset Rayleigh number is observed to be in quantitative agreement with their prediction at our Prandt 1 number and wave number.

Above $R_{1}$, the velocity field is not only time-dependent, but also noisy. The power spectrum $P(E)$ of the local velocity fluctuations is flat at low frequencies, and falls off as $f^{-n}$ at "high" frequencies (but less than $0.025 \mathrm{~Hz}$ ), where $n=4.1 \pm 0.4$, independent of $R$, for $5<R / R_{C}<10$. The linewidth $f_{\text {rms }}$ of the spectrum (square root of the second moment), whose inverse is a characteristic time-scale, is approximately linear in $R$ above $R_{1}$. However, frms may be finite just above $R_{1}$. Beginning at about $9 R_{c}$, intermittent oscillations appear in the local velocity records, and these are superimposed on the slower fluctuations already described. The 
spectrum then shows a bread peak at about $0.04 \mathrm{~Hz}$ which is initially rather well separated from the $f^{-4}$ falloff associated with the structural

deformations. This peak results from the oscillatory instability of Busse and Clever (1979), and has also been noted in other experiments. These two prominent spectral features associated with the transition to turbulence gradually merge as $\mathbb{R}$ is increased, hut remain distinguishable even at $R=$ SOR $\mathrm{e}^{\text {* }}$

En order to obtain a quantitative measure of the strength of the fluctuations we applied a line source of heat parallel to the short side of the cell using a teflon coated wire located $0.6 \mathrm{~mm}$ above the lower plate and $3 \mathrm{~cm}$ from the end of the cell. We found that this local heating could suppress the fluctuations. The area $A$ under the low frequency portion of the spectrum $P(f)$ varies exponentially with $h$, the heat input: $\mathbf{A}=A_{\mathrm{o}} \exp \left(-\mathrm{h} / \mathrm{h}_{\mathrm{o}}\right)$, where the attenuation constant $\mathrm{h}_{\mathrm{o}}$ is $14 \%$ of the heat flux carried per wave length at $15 R_{C}$. We interpret this observation to mean that the fluctuations are relatively robust.

Thus, the following picture of the transition to turbulent convection in large aspect ratio layers (at moderate to low Prandtl numbers) emerges: Stable flows exist above $\mathbb{R}_{c}$, but they contain defects because the rolls align perpendicular to all lateral boundaries. Flows with many defects are not stable. The flows always become time-dependent (and noisy) above $R_{1}$, a threshold that we identify with the skewed varicose instability. The oscillatory instability is a later identifiable discrete step in the transition process. Further documentation of this work is in press (Gollub and Steinman, 1981).

\section{ACKNOWLEDGEMENT}

This work was supported by the National Science Foundation.

\section{REFERENCES}

Busse, F. H. and R. M, Clever, 1979. Instabilities of Convection Rolls in a Fluid of Moderate Prandtl Number. J. Fluid Mech., 91, 319.

Gollub, J. P. and S. V. Benson, 1980. Many Routes to Turbulent Convection. J. Fluid Mech., 100, 449.

Gollub, J. P., S. V. Benson and J. Steinman, 1980. A Subharmonic Route to Turbulent Convection. Ann. N. Y. Acad. Sci.3 357, 22.

Gollub, J. P. and J. F. Steinman, 1981. Doppler Imaging of the Onset of Turbulent Convection. Physical Review Letters, 47, 505.

STATISTICAL PROPERTIES OF MAPPINGS BY THE CHARACTERISTIC FUNCTION FORMALISM

$$
\text { J. D. Meiss }
$$

Numerical experiments on two dimensional, area preserving mappings have shown that motion which is formally deterministic can nonetheless have properties characteristic of a random process (Chirikov, 1979). For example, the Chirikov-Taylor or standard map:

$$
\begin{aligned}
& P^{\prime}=P+P \sin x \\
& x^{\prime}=x+P^{\prime}
\end{aligned}
$$


exhibits momentum diffusion, $D$, wh th a value given approximately by assuming that $x$ is completely random (when $E \gg 1$ ).

Recently a series has been obtained for this diffusion coefficient by using the Fourier transformed version of the map (Rechester and White, 1980; Rechester, Rosenbluth and White, 1981). We have developed an extension of this procedure which permites computation of any statistical property of the map (Cary, Meiss and Bhattacharjee, 1981). The primary objects of our technique are the characteristic functions

$$
\chi_{k}\left(m_{0}, m_{1}, \ldots, m_{k}\right)=\left\langle\exp \left[i \sum_{j=1}^{k} m_{j} x_{j}\right]\right\rangle
$$

where the $x_{j}$ are functions of initial conditions $\left(x_{0}, p_{0}\right)$ through the map, and the brackets indicate a time average along an orbit or an average over initial conditions.

The characteristic functions are Fourier transforms of joint probability distributions - and therefore contain a 11 statistical information. In particular, two point correlation functions $C_{j}$ for tine difference $j$ are obtained directly from $x_{j}(m, 0, \ldots, 0, n)$. The diffusion coefficient is i $n$ turn given by suming $C_{j}$ over $j$.

Explicit formulae for $\chi_{\overline{1}}$ are obtained by repeatedly substituting for $x_{j}$ in (2) in terms of $x_{j-1} a d x_{j-2}$ as given by the map (Cary and Meiss, 1981).

As an example, we calculate the diffusion for the sawtooth map (Cary and Meiss, 1981).

$$
\begin{aligned}
& P^{\prime}=a+c S(x) \\
& x^{\prime}=x+p^{\prime}
\end{aligned}
$$

where $S(x)=x$ for $-\pi<x<\pi$ and $S(x+2 \pi)=S(x)$.

We show that when $C$ is an integer, the series for $D$ truncates, and therefore we obtain exact results:

$$
D=\left\{\begin{array}{cl}
\frac{(\pi 0)^{2}}{6} & |\varepsilon+2|>2, \varepsilon=1 \text { nteger; or } \varepsilon=-4 \\
0 & \varepsilon=-2,-1,0 \\
0 & \varepsilon=-3
\end{array}\right.
$$

The infinite value at $\varepsilon=\mathbf{- 3}$ is due to the presence sf a stable "accelerator mode". These results show that a deterministic motion can, in fact, be rigorously diffusive.

The same techniques are also applicable to dissipative mappings and can be used to obtain the statistical properties sf strange attractors (Jensen and Oberman, 1981). Furthermore, there has been some application of the method to flows by discretizing the flow to construct a map and then Setting the time step go to zero (Jensen, 1981; Abarbanel and Crawford, 1981). 


\section{REFERENCES}

Abarbane1, H, and Jo Crawford, 1981. Lawrence Berkeley Laboratory Report, LBL -11887.

Cary, Jo, J. Meiss and A. Bhattacharjee, 1981. Phys. Rev. A23, 2744.

Cary, Jo and Jo Meiss, 1981. Phys. Rev. A, Nov,

Chirikov, B., 1979. Phys.Reports, 52, 263.

Jensen, R., 1981. J.Stat. Phys., 25, 183.

Jensen, R. and C. Oberman, 1981. Phys. Rev. Lett., 40, 1547; and Princeton Plasma Physics Laboratory Report PPPL-1780, 1981.

Rechester, A. and R. White, 1980. Phys. Rev. Lett. 44, 1586.

Rechester, A., M. Rosenbluth and R. White, 1981. Phys. Rev. A23, 2664. 


\title{
LECTURES OF FELLOWS
}

\section{FIXED FLUX PENETRATIVE CONVECTION}

\author{
Anthony J. Roberts
}

Naturally occuring convection rarely occurs between two plates as most theories and experiments presume. Far more often the convection is bounded vertically by a stably stratified region. Examples are the atmosphere when the sun heating the ground initiates convection into the stably stratified air above, while stellar convection zones are bounded by stable regions where the mean free path of a photon has lowered the static temperature gradient below critica1. To model the situation where a stable region lies over a convectively unstable region Malkus (1960) suggested an ice-water experiment where water is contained vertically between two boundaries, the lower one being held at a temperature near freezing and the upper boundary being held at a temperature greater than $4{ }^{\circ} \mathrm{C}$. Because water has a density maxima at $4{ }^{\circ} \mathrm{C}$ we have the situation, displayed in Figure 1 , of a gravitationally unstable region lying underneath a stably stratified region.

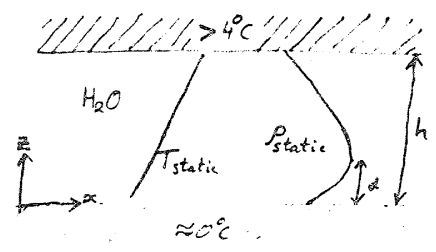

FIGURE 1. Configuration of an Ice-Water Experiment

This experiment has been carried out by Furumoto and Rooth (1961), Townsend (1964) and Myrup et al. (1970), while numerical experiments in two dimensions are described by Musman (1968) and Moore and Weiss (1973). Veronis (1963) analyzed the marginal stability using a vertically truncated spectral method. Briefly the above authors found that the convection is subcritical and usually occurs in a three-dimensional pattern of narrow rising plumes that can penetrate into the stable region typically to a height about twice that of the original unstable region. For Rayleigh numbers much bigger than critical the convection excites gravity waves in the stable layer above the convection. However, it is possible that these waves are excited by a different mechanism that is discussed by Cattaneo in this volume.

Most of the above workers have concentrated on fixed temperature boundary conditions. Here I will be concerned with the theory of large horizontal scale convection that can occur using fixed flux boundary conditions. In section 2 the marginal stability criterion is derived, and also an approximation to the exact solution that can be used to calculate the stability curve for arbitrary boundary conditions for moderate large wave numbers. Section 3 looks at a nonlinear evolution equation near the critical Rayleigh number which displays some of the behavior described from the experiments. The correction to the evolution equation obtained at the next order is used to derive a reconstituted equation that we hope is of wider applicability.

\section{Equations}

We consider the idealized situation as shown in Figure 1 where a fluid, infinite in horizontal extent, is confined between two rigid ("sticky") plates 
upon which fixed flux boundary conditions are now applied. The idealized equation of state for the fluid is

$$
p=p_{0}\left[1-\alpha\left(T-T_{0}\right)^{2}\right]
$$

where $\beta_{0} \propto$ and $T_{0}$ are absolute constants of the fluid, in water the values $p_{0}=1 \mathrm{Mgm} / \mathrm{m}^{3}, \alpha=8.0 \times 10^{-6} \mathrm{~K}^{-2}$ and $\mathrm{T}_{0}=3.98^{\circ} \mathrm{C}$ give a reasonably accurate prescription. Pose the temperature distribution

$$
T=\theta+\beta(z-d)+T_{0}
$$

where $\beta(z-d)+T_{g}$ is the static temperature distribution and $\theta(x, y, z, t)$ is the difference caused by the fluids motion. Thus, $z=d$ corresponds to the vertical position of the static density maximum, hence for $0<z<d$ the fluid is gravitationally unstable while for $d<z<0$ it is stably stratified.

On applying the Boussinesq approximation the equations of motion become

$$
\begin{aligned}
& \frac{D u}{D t}=-\nabla\left(\frac{\tilde{p}}{\rho_{0}}\right)+\alpha g[2 \beta(\alpha-d)+\theta] \theta \underline{\underline{z}}+\nu \nabla^{2} \underline{\mu} \\
& \frac{\partial \theta}{\partial t}=-\beta w+k \nabla^{2} \theta \\
& \underset{\sim}{\nabla} \cdot \underset{\sim}{*}=0 \\
& \theta_{3}=k=v=w \text { on } z+q h \text {. }
\end{aligned}
$$

These equations are identical to those used for fluids with linear density dependence on temperature except that the buoyancy term in the momentum equation is here quadratic in $\theta$ and the linear part has a $z$ dependent coefficient. In this work our attention is restricted to two-dimensional flow and so we introduce the stream function $\Psi$ ' such that

$$
=\left(-\Psi_{2}, 0, \Psi_{x}\right) \text {. }
$$

The equations can be nondimensionalized by scaling the variables with respect to the reference length $d$, the reference time $d^{2 / k}$ and the reference temperature $I$.

Upon substituting the nondimensional quantities defined by

$$
(\hat{x}, \hat{z}, \hat{h})=\frac{1}{d}(x, z, h), \quad \hat{t}=\frac{k}{d^{2}} t, \quad=\beta d \hat{\theta}, \Psi=k \hat{\psi},
$$

the nonlinear equations become (after dropping the $\mathrm{A}$ ' $s$ )

$$
\begin{aligned}
& \theta_{t}+\frac{\partial(\psi, \theta)}{\partial(x, t)}=-\psi_{n}+\nabla^{2} \theta \\
& \frac{1}{\sigma}\left[\nabla^{2} \psi_{t}+\frac{\partial\left(\psi, \nabla^{2} \psi\right)}{\partial\left(x_{t}, t\right)}\right]=R(z-1+\theta) \theta_{n}+\nabla^{4} \psi \\
& \theta_{t}=\psi_{z}=\psi=0 \text { on } z=\theta, h .
\end{aligned}
$$

where the Prandt number $\sigma=p / K$ and the Rayleigh number of the flow is

$$
R=\frac{2 \alpha g \beta^{2} \alpha^{5}}{K \nu}
$$

The above Rayleigh number has exactly the same form as its normal definition, the factor $2 \alpha \beta_{\alpha}$ is just the density gradient at the lower boundary.

Note that hy integrating the equation over the whole fluid and using the boundary conditions, it is easy to show that $\frac{\partial}{\partial t}\langle\theta\rangle=0$ where \langle\rangle denote as usual 
the whole volume integral, Thus $\langle\theta\rangle$ must be a constant, without loss of generality taken to be zero, and this provides an extra condition needed to make the solutions of (4) unique.

\section{Linear Marginal Stability}

For a 11 values of the parameters $\mathbb{R}_{\Omega} h_{8}$ and $\sigma$ there exists a simple exact solution to the set of equations (4), that of no motion and no temperature gradient, that is

$$
\theta=\psi=0, \forall x, z, t
$$

However, for some values of the parameters the above solution is unstable to linear perturbations. The alm of this section is to calculate the critical values of the parameters ac which the above solution loses its stability.

Take the linear part of equations (4) and separate the $x, z$ and $t$ dependencies by assuming a form

$$
\begin{aligned}
& \theta \rightarrow e^{\eta t+i \alpha x} \theta(z), \\
& \psi \rightarrow-i a e^{\eta t+i a x} \psi(\gamma) .
\end{aligned}
$$

The condition on $\mathbb{R}$ and $\sigma$ as functions of $h$ and a for marginal stability is that $(n)=0$. However, we make the assumption, which appears to be consistent, that $n w 11$ be real and hence the criterion is just $\eta=0$. Thus we get the following eigenvalue problem for the critical Rayleigh number $R_{c}$ as a function of horizontal wave number a and the Payer depth $h$,

$$
\begin{aligned}
& \left(D^{2}-a^{2}\right) \theta=a^{2} \psi \\
& \left(D^{2}-a^{2}\right)^{2} \psi=R_{c}(z-1) \theta \\
& D \theta=\psi=D \psi=0 \quad \text { on }=0, h,
\end{aligned}
$$

where $D$ denotes $\frac{d}{d z} \quad$ As usual the Prandt 1 number does not appear in this stability problem. For some purposes it is convenient to combine the above equations and just consider one equation for $\theta$, namely

$$
\begin{aligned}
& \left(D^{2}-a^{2}\right)^{3} \theta=a^{2} R_{c}(b-1) \theta \\
& D \theta=\left(D^{2}-a^{2}\right)_{\theta}=D^{2} \theta=0 \quad \text { at } z=0, h .
\end{aligned}
$$

The general solution of the above equation (excluding the boundary conditions) can be written down in terms of the functions

as

$$
\Phi_{\omega}(\zeta ; p)=\int_{C_{m}} \exp \left[p\left(-\frac{1}{7} \omega^{7}+\frac{3}{5} \omega^{5}-\omega^{3}+\omega+\zeta \omega\right)\right] d \omega,
$$

$$
\theta=\sum_{m} A_{m} \Phi_{m}\left(\frac{R_{e}}{a^{4}}(2-1) ; \frac{a^{5}}{R_{s}}\right),
$$

where the end points of the integration contours $C_{m}$ are chosen to be two of the seven complex seventh roots of tinfinity. The solution (8) is obtained by taking the Fourier (or Leplace) transform of equation (7) and solving that. The integral in (8) is then the inverse transform. Observe that $\delta_{m}$ satisfies the differential equation 


$$
\left(\frac{1}{\rho^{1}} \frac{d^{*}}{d \xi^{2}}-1\right)^{3} \Phi-\xi \Phi=0,
$$

which is the cannonical differential equation for a 11 linear problems in penetrative convection, in the same sense that the Alry functions are cannonical functions for second order differential equations with a turning point. The integral in (8) can be approximately evaluated by using the method of steepest descent for large $\beta$. This is, In fact, equivalent to using the WKB method to solve equation (9) except that the integral representation automatically gives the connection across the various singularities in the approximations. We now define six linearly independent basis functions $\Phi_{w_{*}}$ as

$$
\begin{aligned}
& D_{1}=\frac{i}{2} \int_{e^{i a 1 / 7}}^{e^{-i 4 \pi / \infty}} \cdots d w
\end{aligned}
$$

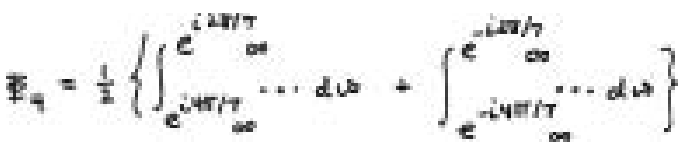

$$
\begin{aligned}
& \Phi_{1}=i \int_{e^{i \omega \in / \pi}}^{e^{-i b \pi / \pi} \infty} \cdots d \omega
\end{aligned}
$$

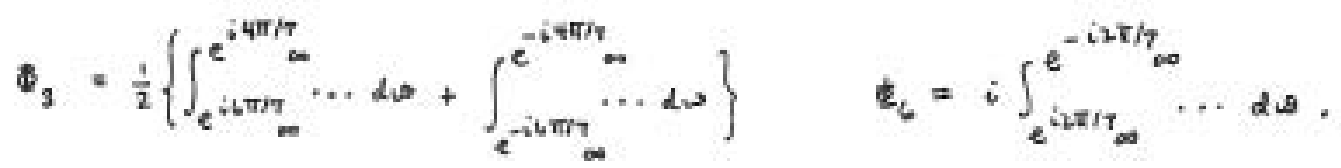

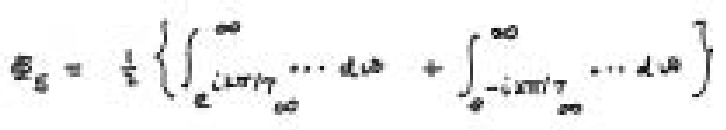

Using these definitions steepest descent approximations of the above integrals displays a 11 the different asymptotic behaviors. For negative $\Phi_{1}$ and $\Phi_{4}$ oscillate, algebraically decay and are out of phase; $\mathbb{2}_{2}$ and ${ }_{3}$ oscillate, exponentially grow and are out of phase; and $\Phi_{5}$ and $E_{6}$ oscillate, exponentially decay and are out of phase. For postive $\$$ : $\sigma_{1}$ and oscillate out of phase and decay exponentially; $\mathbb{F}_{2}$ has monotonic exponential decay;

$\Phi_{5} \quad$ is monotonic exponential increasing; $\Phi_{4}$ and $\vec{b}_{6}$ oscillate out of phase and grow exponentially.

Knowing these functions we could solve the linear stability problem (7) exactly for arbitrary boundary conditions. Knowing the asymptotic form of the functions we can solve equation (7) for moderate to Large horizontal wave number. However, here we are primariPy interested in possible large horizontal scales and so leave the above problems for later study.

To look the marginal stability of long wave lengths we expand the unknowns i $n$ powers of $a^{2}, \forall i z$

$$
\begin{aligned}
& R_{e}=R_{c_{0}}+a^{2} R_{c_{2}}+\ldots, \\
& e=\theta_{1}(z)+a^{2} \theta_{2}(z)+\ldots, \\
& \psi=\psi_{g}(z)+a^{2} \psi_{2}(z)+\ldots .
\end{aligned}
$$

Substituting these into the eigenvalue problem (6) and equating like powers in $a^{2}$ we obtain the following set of recursive equations 


$$
\begin{aligned}
& D^{2} \theta_{n}=\theta_{n-2}+\psi_{n-2}, \quad D \theta_{n}=0 \text { at } \quad z=0, n
\end{aligned}
$$

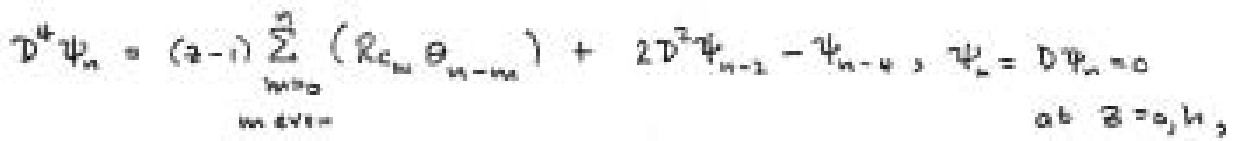

where quantities with negative subscripts are defined to be zero.

At any order $n$ we solve the above equations by first choosing $R_{n-2}$ such that equation (10) for $\theta_{\mathrm{s}}$ can be solved. This solvability condition is just

$$
\int_{a}^{b} \theta_{n-3}+\psi_{n-2} d z=0 \text {, }
$$

as can be seen by integrating equation (10). We then solve the problem for $\theta_{n}$ followed by solving equation (11) for $\psi_{n}$. The zeroth order solution is

$$
\begin{aligned}
& \theta_{0}=1 \\
& \psi_{0}=\frac{R_{\varepsilon_{0}}}{5 !} z^{2}(h-5)^{2}(a+2 h-5) .
\end{aligned}
$$

The solvability condition at the next order gives Re to be

$$
R_{c_{0}}=\frac{6 !}{h^{H}(1-h / h)}
$$

There are a number of features to note about this solution. The critical Rayleigh number has the correct asymptotic form as the layer depth $h$ becomes small, where this problem reduces to the linear temperature dependence fixed flux problem. The most interesting feature is that $R_{c_{e}} \rightarrow+$ as $h \rightarrow 2^{-}$ and hence very long horizonal scales are only feasible for $h<2$, for larger $h$ the motion must have a finite horizontal extent (at least linearly). It is intersting to note that the negative values of $R_{a}$ occurring for $h>2$ are physical if the density expansion coefficient $\Rightarrow$ is negative in which case we have the reverse situation of a gravitationally unstable region lying above a stable region. Also observe that for $5 / 3<\mathrm{h}<2$ there exists a counter cell at the top of the fluid, in the region $(5-2 \mathrm{~h})<z<\mathrm{h}$. For stress free boundary conditions the above results stay much the same.

Equations (10) and (11) were solved for the next two orders and involved calculating polynomials in $z$ of up to the 19th degree with coefficients that are rational functions of $h$. Higher order $\theta_{n}$ 's are chosen to be orthogonal to

$\theta_{0}$ The derived formulas for $R_{c_{y}}$ and $R_{e_{4}}$ are

$$
\begin{aligned}
R_{c_{2}}= & \frac{30\left(211 h^{2}-884 h+114\right)}{1001 h^{2}(1-h / 2)^{2}} \\
R_{c_{4}}= & \left(219091383 h^{4}-1713638342 h^{3}+5073065592 h^{2}-6718254480 h\right. \\
& \quad+3559427240) / 25 \times 9170594(1-h / 2)^{5} .
\end{aligned}
$$

observe that our expansion is not really in a but in ha/(1-h/2). The above coefficients have a fairly simple behavior. $R_{\xi_{\mathrm{j}}}$ is positive for $0<\mathrm{h}<1.6492$ 
and negative for $1.6492<h<2$, while $R_{c_{4}}$ is positive for a 11 st $h<2$. Thus we conclude that the critical Rayleigh number versus horizontal wave number curve looks like Figure 2 . For $<1.6492$ long horizontal scales are

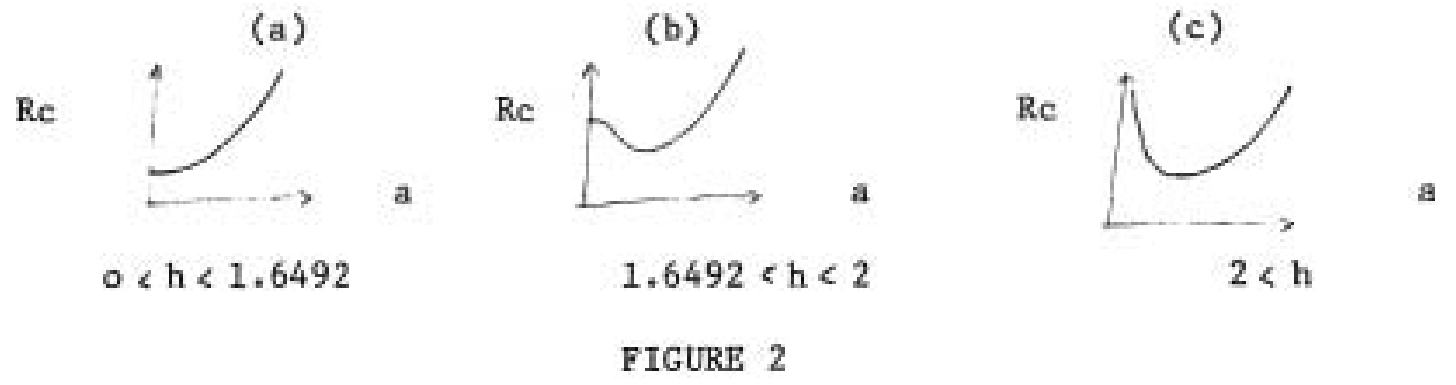

preferred, as h crosses 1.6492 there is a bifurcation of the preferred wave number to finite values, while for $h>2$ only finite wave numbers are allowed. It is interesting to note that the critical h of 1.6492 is just 0.017 below the $\mathrm{h}$ at which a counter cell first appears:

\section{Nonlinear Evolution Equations}

We now turn to the behavior of nonlinear disturbances for Rayleigh numbers near critical, in particular we are interested in the subcriticality of the instability. We make a long horizontal scale assumption which, as was seen in the previous section, restricts us to considering layer depths $\mathrm{h}<2$. The following should be compared to the work in Depassier and Spiege1 (1981) which treats, in a similar manner, the effects on the motion of departures from strict Boussinesq convection.

We assume that the motion takes place over long times on long horizontal scales by introducing the scaling

$$
\xi=\epsilon x, \quad \tau=\epsilon^{4} s,
$$

where $\epsilon$ is a smail parameter. We then expand the unknowns in powers of $\epsilon^{2}$,

$$
\begin{aligned}
& R=R_{0}+\epsilon^{2} R_{2}+\cdots \\
& \theta=\epsilon^{2} \theta_{0}+\theta^{4} \theta_{2}+\cdots \\
& \psi=\epsilon^{3} \psi_{0}+\epsilon^{5} \psi_{2}+\cdots .
\end{aligned}
$$

The leading orderin $\theta$ and $\psi$ is the two powers of $\epsilon$ smaller than is normally used. This has to be done in this problem to ensure that the leading order evolution equation has fourth order derivative in $\xi$ Substituting the above expansions into the governing equations (43 we obtain the following set of recursive equations

$$
\begin{aligned}
& D^{2} \theta_{n}=\psi_{n-2 \xi}-\theta_{n-2 \xi \xi}+\theta_{n-4 v}+\sum_{m=14}^{n} \frac{8\left(\psi z--4, \theta_{n-m}\right)}{\partial(\xi, z)}, D \theta_{n}=0 \text { at } z=0, n
\end{aligned}
$$

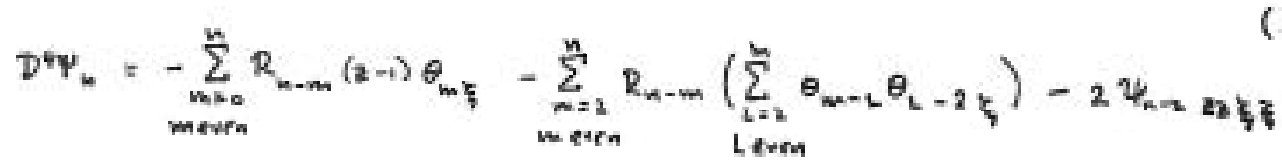

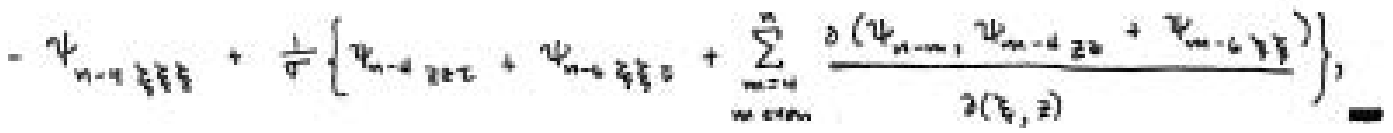


where quantities with negative subscripts are defined to be zero. The above set of equations are solved i a similar manner to marginal stability calculation but with modifications to allow for the nonlinearities and the dependencies on $\$$ and $\tau$ *

The zeroth order solution $1 s$

$$
\begin{aligned}
& \theta_{0}(\xi, z, z)=f_{0}(\xi, t), \\
& \psi_{0}=-R_{0} f_{0} P_{0}(z), \quad P_{0}(z)=\frac{1}{5 !} z^{2}(h-t)^{2}(z+2 h-5),
\end{aligned}
$$

where $f_{0}$ is, as yet, an arbitrary function. At the second and higher orders there is solvability condition from the $\theta$ equation that the integral of the right-hand side from $z=0$ to h must be zero. At second order this gives an equation for $R_{0}$ which is, as before,

$$
R_{0}=\frac{6 !}{h^{4}(1-h / 2)}
$$

At the fourth and higher orders the solvability condition gives evolution equations for the functions, introduced at the previous order in the expression for $\theta_{m}$ The fourth order solvability condition gives the equation

$$
-P_{0} f_{0 \tau}+P_{1} f_{0 \xi \xi \xi \xi}-P_{0} \gamma_{2} f_{0} \xi_{\xi}-\frac{1}{2} P_{2}\left(f_{0}^{2}\right)_{5 \xi}=0 \text {, }
$$

where $\nu_{2}=R_{2} / R_{0}$ and the $P_{1}$ 's are functions of h given in the appendix.

The above equation can be written, together with periodic boundary conditions, as

$$
f_{0 \sigma}+k f_{0 k \xi \xi \xi}+v_{1} f_{0 \xi \xi}-2\left(f_{0}^{\pi}\right)_{5 \xi}=0, f_{0 \xi}=f_{0 \xi \xi \xi}=0 \text { at } \xi=0,2 \pi / k \text {, }
$$

where

$$
K(h)=R_{c_{2}}(h) / R c_{0}(h), \quad \lambda(h)=\frac{1}{2(1-n / 2)},
$$

and $k$ is some horizontal wave number in the long scale. This equation is similar to the equation that Chapman and Proctor (1980) found for strict Boussinesq fixed flux convection, but it omits the term ( $f_{0}^{2}$, ) and instead contains the term ( $\left.f_{e}^{*}\right)_{\text {t }}$ which Depassier and Spiegel (1981) found to be present when nonBoussinesq effects are considered. Note that the condition $\langle\theta\rangle=0$ is satisfied if we require that $\bar{f}_{\theta}=0$ is satisfied.

We now wish to investigate the solutions of equation (16). First, note that there does not appear to be a simple Liapunov function for the system. To look at the time evolution we consider that part of the parameter ( $r_{2}, k$ space where the behavior of $f_{0}$ Is near linear and can be described by an expansion in some small parameter $\delta$. Substituting the expansion

$$
\begin{aligned}
& s=\delta^{2} \tau \\
& r_{2}=r_{20} 3 \delta^{2} r_{21} 6 \ldots \\
& f_{0}=\delta F_{1}+\delta^{2} r_{2}+\cdots,
\end{aligned}
$$

into equation (16) we find that

$$
F_{1}=A_{1}(s) \cos \left(h \frac{\pi}{5}\right)
$$


and $\quad r_{20}=k k^{2}$

which gives the region in the parameter space near which this analysis is valid. At the third order we find a solvability condition which gives the Landau equation for $A_{1}$ to be

$$
\dot{A}_{1}=r_{22} k^{2} A_{1}+\frac{1}{24(1-n / 2)^{2} \times} A_{1}^{3} \text {. }
$$

From this equation we can easily see the expected results. For $r_{2}$ less than the critical $r_{2,0}$ the no motion solution $A_{1}=0$ is stable, while for $r_{2}>r_{2,0}$ it is unstable. Also, if $h$ is 1 ess than $1.6492, k \geqslant 0$ and for all wave numbers $k$ there is the unstable subcritical finite amplitude solution

$$
A_{1}^{2}=-24 r_{22} k^{2}(1-h / 2)^{2} k,
$$

while for $1.6492<\mathrm{h}<2$ there is a stable supercritical finite amplitude solution.

It is possible to find an exact steady solution to equation (16) in terns of squares of the Jacobian elliptic functions. Substituting

$$
f_{0}=B+A C n^{2}\left(\frac{k K}{\pi} \xi \mid m\right)
$$

into equation (16) and requiring $\mathcal{F}_{0}=0$ we find, in terms of the elliptic parameter $m$, that

$$
\begin{aligned}
& r_{2}=\frac{4}{\pi^{2}} k^{2} k\{1+m-3(1-E / k)\} k^{2} \\
& A=-\frac{12}{\pi^{2}} k^{2}(1-h / 2) k m k^{2} \\
& B=\frac{12}{\pi^{2}} k^{2}(1-h / 2) k\{m-(1-E / k)\} k^{2}
\end{aligned}
$$

where $k$ and E are the complete elliptic integrals of the first and second kind respectively. Plotted in Figure 3 is the curve relating $A^{2}$ and $r_{2}$.

Taking a small. $\mathrm{m}$

(a) $\mathrm{h}<1.6492$

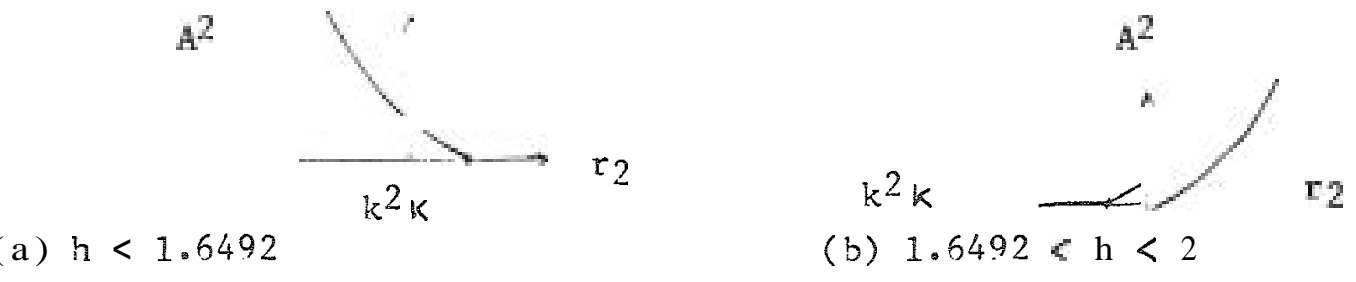

FIGURE 3 .

approximation to the solution (19) we find that

$$
A^{2}=96 k^{2}(1-h / 2)^{2} k \cdot\left(k^{2} k-r_{2}\right) \text {, small } A \text { and }\left(k^{2} k-r_{2}\right) \text {; and }
$$

taking approximations for m close to 1 we find that

$$
A^{2} * 9(1-h / 2)^{2} r_{2}^{2} \text {, large } A \text { and } r_{2} \text {. }
$$

For m near 1 (and $h<1.6492$ ) a sketch of the velocities and temperature structure in the horizontal is given in Figure 4. From the figure it can be seen that the theoretical solution, even though it is an unstable one, agrees qualitatively with the experimental observation (with fixed temperature boundary conditions) that the vertical motion of the fluid occurs in a 
localized plume. This vertical motion can be understood easily by considering that a wide ascending cold plume will create in the mean a more unstable density gradient in the center, will then tend to accelerate further. on the other hand a wide descending region of warm fluid creates a local density distribution that is more stable and so the downard motion is confined to the sides of the upward moving fluid.

h

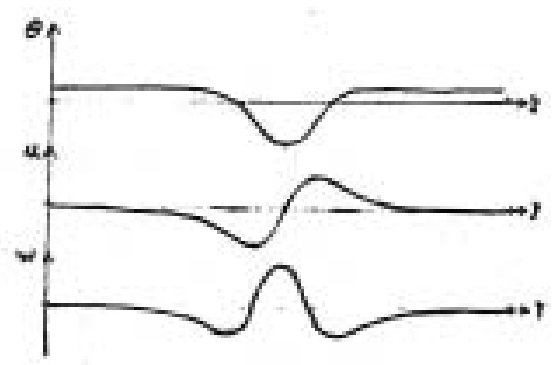

FIGURE 4

However, there is one glaring problem in the above exact solution; it is infinitely subcritical! Unlike the general ease discussed by Depassier and Spiegel (1981), who found a Power bound to the subcriticality, we have that for any Rayleigh number less than critical the theory predicts the existence of a finite amplitude solution; this appears to be clearly unphysical. In an attempt to resolve this difficulty we look at a reconstituted evolution equation.

To this end we derive the evolution equation for $f_{2}$ from the solvability condition at the sixth order, it Is

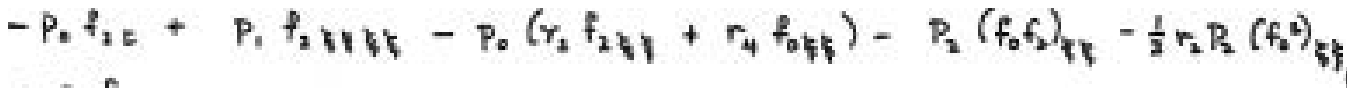

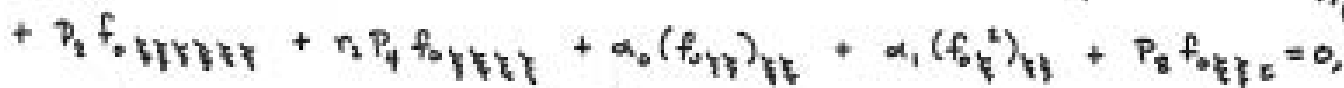

where

$$
\begin{aligned}
& \alpha_{0}=2 P_{5}-P_{6}, \\
& \alpha_{1}=\frac{1}{2}\left(6 P_{5}+P_{7}-q_{0}\right),
\end{aligned}
$$

and the $P_{h}{ }^{\prime} s$ and $q_{0}$ are defined in the appendix. To form the reconstituted equation we add equation (15) to $\epsilon^{2}$ time equation (20) after first removing derivatives of $f_{0}$ in $\xi$ higher than fourth by substituting from equation (15). The equation is then written solely in $f=f_{0}+\epsilon_{2}$

and $r=r_{3}+6^{2} r_{4} \quad$ adding higher order terms where necessary; this is the reconstituted equation. It is convenient to remove the explicit $\boldsymbol{E}$ dependence and write it in terms of the original variables , $\alpha, t$ and $\mathbb{R}$ togive

$$
\begin{aligned}
& -P_{0} \theta_{4}+\alpha_{4} \theta_{x x t}+\left(P_{1}+\alpha_{2} \rho\right) \theta_{x N x s}-P_{0} \rho \theta_{x x}+\frac{1}{2} \alpha_{2}(\theta)_{x x x=x}^{2} \\
& -\frac{1}{2} P_{2}(1+p)(\theta)_{x x}+\alpha_{0}\left(\theta \theta_{x a}\right)_{x x}+\alpha_{1}\left(\theta_{x}{ }^{2}\right)_{x x}=0
\end{aligned}
$$

where

$$
p=\frac{R}{R_{0}}-1,
$$


not to be confused with the density, and

$$
\alpha_{2}=\frac{1}{P_{1}}\left(P_{1} P_{4}+P_{0} P_{3}\right), \alpha_{2}=\frac{P_{2} P_{2}}{P_{1}}, \quad \alpha_{4}=\left(P_{1} P_{8}+P_{0} P_{3}\right) / P_{1} .
$$

Note that the $\theta(x, t)$ in equation $(21)$ is not the same as the temperature perturbation $\theta(x, z, t)$, they are related, correct to order $\theta^{t}$ by

$$
\theta(x, z, t)=\theta(x, t)+Q_{0}(z) \theta_{x x}(x, t),
$$

where $Q_{0}(z)$ is some calculable polynomial in $z$ of seventh degree.

We now investigate the solutions of equation (21). Treating it exactly like a full convection problem we first find the critical Rayleigh number at which the purely conductive solution $\theta=0$ loses its stability. Unlike the full convection problem that it approximates in this problem we can find exactly. Assuming $p$ and $x$ to be $0(1), \theta$ small and proportional to $e^{1 a x}$ we find

$$
P_{c}=\frac{-P_{1} a^{2}}{P_{0}+d_{2} a^{2}}=\frac{\left(R c_{2} / R c_{0}\right) a^{2}}{1-\left(R c_{4} / R c_{2}\right) a^{2}}
$$

This marginal stability curve is sketched in Figure 5. These curves must be quantitatively correct for small a and $p_{c}$, for larger a and $p_{c}$ there is an interesting qualitative agreement with parts of Figure $\mathbf{2}$.

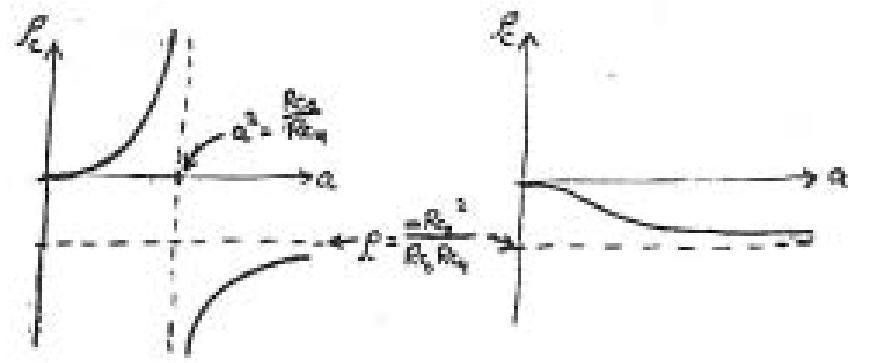

(a) $\mathrm{h}<1.6492$

(b) $1.6492<\mathrm{h} \leqslant 2$

FIGURE 5

To find the steady solutions to the reconstituted equation (21) we can integrate twice directly. Because the independent variable $x$ does not appear explicitly in the equation we then make the substitution $\phi(0) \cong(\mathrm{d} / \mathrm{d} z)^{2}$ to give the first order equation

$$
\left[P_{1}+p \alpha_{2}+\left(\alpha_{2}+\alpha_{1}\right) \theta\right] \frac{d b}{d \theta}+2\left(\alpha_{1}+\alpha_{3}\right) \phi=A+2 p \cdot \rho \theta+P_{2}(1+\rho) \theta^{2},
$$

where $\mathrm{W}$ i s an arbitrary constant of integration. The above equation can be solved exactly to give the following first order differential equation for $(x)$

$$
\left(\frac{d \theta}{d x}\right)^{2}=b \theta+c \theta^{2}+A+B\left(\theta+\frac{p_{1}+\alpha_{2} p}{\alpha_{1}+\alpha_{2}}\right)^{-2\left(\alpha_{1}+\alpha_{3}\right) /\left(\alpha_{0}+\alpha_{1}\right)},
$$


where $A$ and $B$ are integration constants while $b$ and $C$ are given by

$$
\begin{aligned}
c= & \frac{1}{3} P_{3}(1+\beta), \\
& \alpha_{0}+\alpha_{1}+2 \alpha_{1} \\
b= & 2 P_{0} p-P_{0}(1+p)\left(P_{1}+\alpha_{2} \rho\right) /\left(\alpha_{0}+\alpha_{1}+2 \alpha_{3}\right) \\
& \left(\alpha_{0}+2 \alpha_{1}+2 \alpha_{1}\right)
\end{aligned}
$$

Note that the Prande 1 number only appears in this problem through the coefficient $\alpha_{1}$. Unfortunately lack of time prevented my obtaining the solution of the above equation.

\section{Remarks}

We have considered an idealized problem in penetrative convection and have calculated exactly the coefficients in the perturbation expansions based near the critical Rayleigh number. This has been possible by the simplifications ensuing from the long horizontal scale assumption allowed by the fixed flux boundary condition. However, we have also derived an integral representation of the exact solution to the linear problem for arbitrary boundary conditions. This solution should be of use $\mathfrak{i n}$ more general linear penetrative convecting problems as well. as serving as a foundation for the general nonlinear theory.

For long horizontal scales we have discovered that the penetration has an absolute upper limit of $h=2$ (true for various velocity boundary conditions). For rigid boundary conditions a finite wave number is preferred when $h$ is bigger than 1.6492 which is thus the true upper limit of penetration for rigid boundaries.

The nonlinear analysis confirms once more the subcriticality of penetrative convection and agrees qualitatively with experiments. However, the finite amplitude solution does not "turn around" and become the stable subcritical branch. In the start of an attempt to calculate this branch we have derived the reconstituted equation and integrated it to obtain a fairly simple first order equation which will have to be integrated numerically i n further work.

I wish to thank Prof. E. A. Spiegel for proposing this problem and providing many illuminating discussions at a 11 stages of this work. Also I thank Dr. W. Young for suggesting that I look for an integral solution and for his many other comments. Finally, I wish to thank Barbara, my wife, for drawing my graphs and Prof. W. Malkus and the Woods Hole Oceanographic Institution for running the sumet Geophysical Fluid Dynamics program and inviting me to participate.

\section{APPENDIX}

Listed here are the various coefficients that appear in the evolution equations.

$$
\begin{aligned}
& D_{0}=-\frac{h}{R c_{0}}=\frac{h^{5}(h-2)}{1440} \\
& P_{1}=\frac{h R c_{2}}{R c_{0}^{2}}=\frac{h^{7}\left(211 h^{2}-814 h+816\right)}{8648640(2-h)},
\end{aligned}
$$




$$
\begin{aligned}
& P_{2}=\frac{h^{5}}{720} \\
& P_{3}=\frac{h^{9}\left(14232703 h^{3}-71651076 h^{2}+116179965 h-61749050\right)}{6453009763200(2-h)^{2}} \\
& P_{4}=\frac{h^{7}\left(-443 h^{2}+754 h+104\right)}{17297280(2-h)} \\
& P_{S}=\frac{-h^{7}(7 h+2)}{443520(2-h)} \\
& R=\frac{-h^{\prime}}{66521} \\
& P_{7}=\frac{h^{7}}{47520} \frac{1}{\sigma}+\frac{43 h^{7}}{277200} \\
& P_{e}=\frac{h^{7}(2-h)}{60+80} \frac{1}{\sigma}+\frac{h^{7}(4-6 h)}{120960} \\
& \alpha_{0}=-\frac{h^{7}(31 h+4)}{666280(2-h)} \\
& d_{1}=\frac{h^{7}}{95040} \frac{1}{\sigma}+\frac{h^{7}(101-133 h)}{110000(2-h)} \\
& \begin{array}{c}
\alpha_{2}=\frac{1 \hat{R}_{c_{4}}}{R_{c_{1}} R_{c_{2}}}-h^{7}\left(219091383 h^{4}-i-13 r_{6} 98342 h^{t}+5073065582 h^{2}-6717154470 h\right. \\
+335942730) /\left(27935107200(2-h)\left(211 h^{2}-824 h-884\right)\right)
\end{array} \\
& \alpha_{3}=\frac{P_{2} P_{3}}{P_{1}}=\frac{h^{7}\left(14232703 h^{2}-71651476 h^{2}+116179965 h-61749050\right)}{537313600(2-h)\left(211 h^{2}-814 h+814\right)} \\
& \alpha_{4}=\frac{h^{7}(2-h)}{60470} \frac{1}{\sigma}+\frac{h^{7}\left(-47207481 h^{3}+236217112 h^{3}-373691270 h+186315140\right)}{2148854400\left(211 h^{2}-814 h+984\right)} \\
& q_{0}=\frac{h^{7}}{100200} \text {. }
\end{aligned}
$$

\section{REFERENCES}

Chapman , and M. Proctor, 1980. Nonlinear Meyle1 chi-Henerc convection between poorly conducting boundaries. $\dot{x}$. Fluid dech., 107.

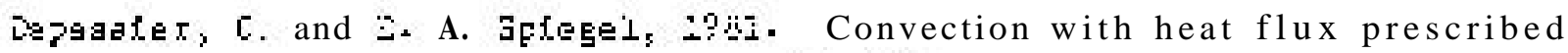
on the boundaries of the system. 2. The effect of temperature dependence of material properties. Prizirint. 
Furumoto and Rooth, 1961. Geophysical Fluid Dynamics. Woods Hole Oceanographic Institution Report.

Malkus, W.V.R., 1960. In Aerodynamical Phenomena in Stellar Atmospheres (ed. R. N. Thomas), p. 346. Bologna: Zaniche11.

Moore and Weiss, 1973. Nonlineat penetrative convection. J. Fluid Mech., 61, $553-581$.

Musman, 1968. J. Fluid Mech. 31, 343.

Myrup, Gross, Hoo and Codard, 1970. Weather, 25, 150.

Townsend, 1964. Quart, J. Roy. Met. Soc., 90, 248.

Veronis, Go, 1963. Penetrative convection. Astrophy. J。, 137, 641.

\section{AN INSTABILITY OF GRAVITY WAVES}

Fausto Cattaneo

\section{$\underline{\text { ABSTRACT }}$}

The motion of $a$ thin horizontal layer of inviscid fluid is studied. Linear analysis shows that for certain thermal boundary conditions long gravity waves are unstable provided the fluid is stably stratified. In the case of perfectly conducting boundaries a variational calculation suggests that for a class of temperature dependent thermal diffusivities a similar result applies.

Motion of small but finite amplitude of these waves is studied and a Landau type equation is derived.

\section{INTRODUCT ION}

Oscillatory motion is observed in a large number of situations of geophysical and astrophysical interest. Some oscillations are driven by other types of motion, like turbulence; other arise spontaneously as instabilities. It is natural to ask in what sense are these instabilities a generic phenomenon.

In doubly diffusive convection (see Spiegel lectures, this volume) the presence of potential energy stored in the salinity gradient or in the magnetic field produces restoring forces that cause the fluid to develop oscillatory instabilities. In general we expect that most mechanisms, capable of storing and retrieving potential energy, will lead to overstability.

Another source of energy for the motion is the heat flux going through the fluid. An astrophysical example is the $\kappa$ mechanism. The fluid possesses $a$ temperature dependent thermal diffusivity and this allows it to reduce the heat flux and convert it into kinetic energy.

In this gaper we investigate two cases where the conversion of heat flux into kinetic energy could produce oscillatory motion. In Section 1 we study the effect of imperfect boundary conditions on the stability of the static solution. It is found, by a linear analysis, that in a stably stratified fluid gravity waves become unstable. 
In section 2 we investigate the effects of variable thermal diffusivity on the stability of a fluid between perfectly conducting boundaries. A variational calculation suggests that, for a class of fluids with temperature dependent diffusivity, gravity waves are again unstable. In the last section we return to the scenario of section $\mathbf{1}$ and consider motion of small but finite amplitude. An asymptotic calculation gives that the amplitude and phase of the oscillation obeys a Landau type equation. This correctly describes a Hopf bifurcation of gravity modes from the statlc solution.

\section{Section 1}

We recall some elementary results of linear theory. In a stably stratified fluid (entropy decreasing upward) with constant heat flux prescribed on the boundary the static state is stable. Disturbances such as gravity waves decay exponentially with time, at a rate proportional to the wave number, so that the only marginal mode is a wave of infinite wave length. We want to study whether this result is susceptible to small changes in these ideal boundary conditions and in particular if there are cases where the static state becomes unstable.

We consider a horizontal layer of inviscid fluid of thickness $d$, we assume that the motion is two dimensional and described by the Boussinesq equations. We also let the thermal diffusivity $k$ depend on temperature. If $z$ increases upward, $g$ and $c_{p}$ have their standard meaning and $\psi$ is the stream function then the static solution is described by

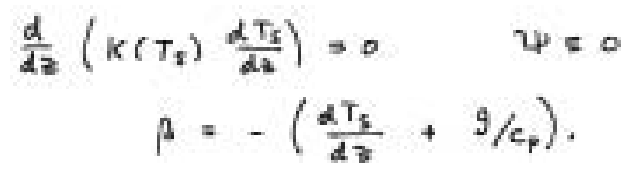

As we have said linear theory shows that for $\beta<0$ and fixed flux $T=T_{s}(z)$, $\Psi$ a 0 is stable. We now alter the boundary conditions slightly. We choose d as the unit of length, $1 \beta ! d$ as the unit of temperature and $d^{2} / k_{o}$ as the unit of time. ( $k_{g}$ is the static value of $k$ at some reference point in the fluid.) We consider infinitesimal perturbations of the static solution and assume that the motions described, at least initially, by the linearized equations. In dimensional form these are:

$$
\begin{aligned}
& T=T_{s}+\theta \\
& \nabla^{2} \psi_{t}=\wedge \theta_{x} \\
& \theta_{t}-\beta \psi_{x}=\nabla \cdot[k(T) \nabla(T)]
\end{aligned}
$$

where $\wedge$ is an inviscid version of the Rayleigh number and is given by:

$$
\Lambda=\frac{9 d \frac{d^{4}}{R_{0}}}{1}
$$

( $\alpha$ is the coefficient of themal expansion). We expand the RHS of (2) retaining linear terms only; we get

$$
\left.\left.\nabla[K(T) \nabla T] x \quad K_{T}\right|_{T_{s}} \frac{d T_{s}}{d z} \theta \hat{z}\right)_{z}+K\left(T_{s}\right) \theta_{x x}
$$


Equations (1), (2), (3) are linear and separable; thus we seek solutions of the form:

$$
\left(\begin{array}{l}
\psi \\
\theta
\end{array}\right)=e^{\sigma t}\left(\begin{array}{l}
\psi(\sigma) \sin a x \\
\sigma(\xi) \cos a x
\end{array}\right)
$$

Substituting (4) into equations we obtain:

$$
\begin{aligned}
& \sigma\left(D^{2}-a^{2}\right) \psi=-\wedge a \theta \\
& \sigma \theta-p a \psi=D(K D \theta+\theta D K)-a^{2} k \theta \\
& K(z)=k\left(T_{s}(\theta)\right), D K(z)=\left.k_{T}(T)\right|_{T_{s}} \frac{d T_{s}(z)}{d z}, D=\frac{d}{d z} .
\end{aligned}
$$

We now make precise what we mean by slightly changing the boundary conditions: we assume that the static heat flux is prescribed on the boundary and demand the perturbations to this flux to be of the order of some small parameter $\varepsilon^{2}$. This can be expressed as

$$
(K D \theta+\theta D K)=\epsilon^{2} q^{I} \theta \quad z=0,1 \quad 0<\epsilon \ll 1
$$

where $\mathrm{q}^{+}$and $\mathrm{q}^{-}$are constants measuring the relative weight of the perturbation flux to the perturbation temperature $(q+$ and $q-$ are related to the Biot number). We also have that for rigid walls and inviscid fluid $\psi=0$ at $\mathrm{z}=0,1$. Treatment of fixed flux by Spiegel (see lecture) shows that the equations are amenable to a long wave length approximation; accordingly we let

$$
\partial_{x} \rightarrow \in \partial_{x}, \quad \partial_{t} \rightarrow \epsilon^{2} \partial_{t}, \quad \psi \rightarrow \epsilon \psi, \quad \Lambda \rightarrow \epsilon^{2} \Lambda
$$

The scaled equations become:

$$
\begin{aligned}
& D^{2} \psi+\frac{\Lambda a}{\sigma} \theta=6^{2} \alpha^{2} \psi \\
& D(K D \theta+\theta D)=6^{2}\left(\sigma \theta+a^{2} K \theta-a \beta \psi\right)
\end{aligned}
$$

We assume that $\Psi$ and have an asymptotic expansion in $\Theta$ and write :

$$
\begin{aligned}
& \psi=\psi_{0}+\epsilon^{2} \psi_{2}+\cdots \\
& \theta=\theta_{0}+\epsilon^{2} \theta_{2}+\cdots
\end{aligned}
$$

We solve for the zeroth order

$$
\begin{aligned}
& D\left(k>\theta_{0}+\theta_{0} D K\right)=0 \\
& D^{2} \phi=\theta_{0}, \quad \Psi_{0}=\frac{-\lambda_{a}}{\sigma} \phi
\end{aligned}
$$


Equation (11), together with zeroth order boundary conditions give

$$
\theta_{0}=\frac{1}{k(z)}
$$

Order of $\epsilon^{2}$ gives

$$
D\left(k \theta_{2}+\theta_{2} D K\right)=\frac{\sigma}{k}+a^{2}+\frac{\Lambda a^{2}}{\sigma} \phi
$$

with B.C.'s $\left(x \theta_{2}+\theta_{2} D k\right)=q^{T} \frac{1}{k} \quad z=0,1$

Integrating (14) from top to bottom and using (15) we obtain an equation for $\sigma$.

$$
\sigma^{2}\left\langle\frac{1}{k}\right\rangle+\sigma\left(a^{2}-\delta Q\right)+a^{2} \wedge\left[\frac{e T^{2}}{2}-F\left\langle(D \phi)^{2}\right\rangle\right]=0
$$

where pointy brackets denote integration from 0 to 1 and

$$
\begin{aligned}
& d Q=q^{+} k(1)-q^{-k(0)} \\
& -I^{2}=\left\langle 3(a-1) \frac{1}{k}\right\rangle<0
\end{aligned}
$$

F and $\mathrm{c}$ are dimensionless constants related to the static temperature distribution. For $\beta<0$ and a layer heated from below we have

$$
\beta=\frac{E}{K}-c
$$

We see from (16) that the presence of $\delta$ alters the stability of the static solution. In particular for $\oint Q>0$ there is a marginal mode of finite wave length $\lambda_{s}=\frac{3 \pi}{a_{s}} \quad$ and all the modes of larger wave length are overstable.

We can specialize our result to the case of constant diffusivity: $K(z)=$ $\mathrm{K}_{0}$ constant. Then for $\mathrm{q}^{+-} \mathrm{q}^{-}=\Delta \mathrm{q}, \beta_{0}<0$

$$
\sigma=\frac{k_{p}}{2}\left\{\left(\frac{\Delta q}{k_{o}}-a^{2}\right) \pm \sqrt{\left(a^{2}-\frac{\Delta q}{k_{p}}\right)^{2}-\frac{\beta_{0} a^{2} \wedge}{\sigma}}\right\}
$$

which shows that a 11 modes of wave length greater than $\lambda_{c}\left(\lambda_{c}^{2}=\frac{2 \pi K_{0}}{\Delta q}\right)$ are unstable. So, if we consider a single mode of given wave number, we find that it becomes overstable as $\Delta q$ is increased.

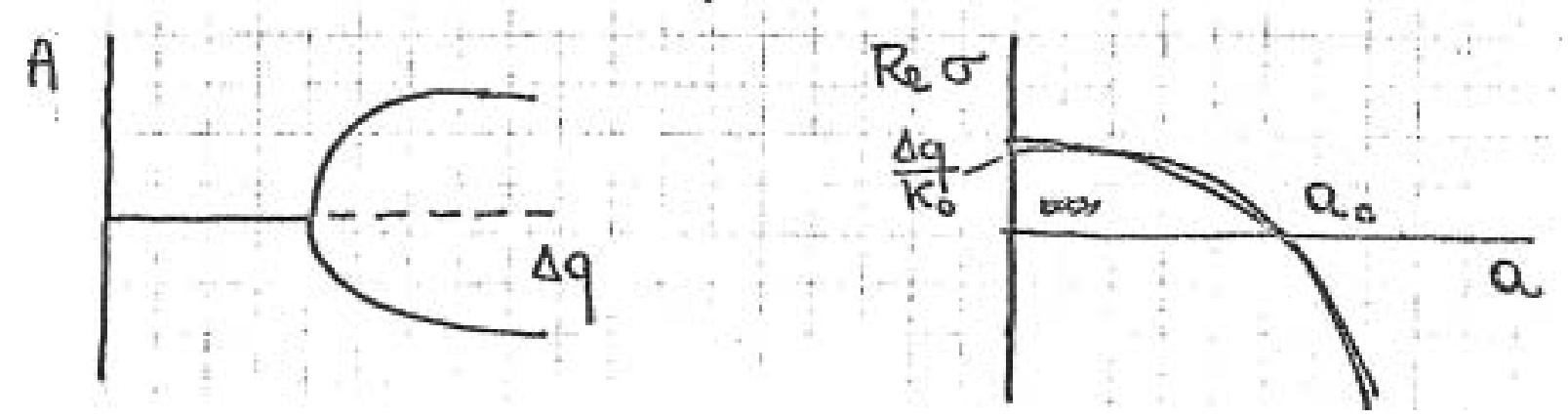

Hopf Bifurcation Diagram For Mode, of Wave Number a

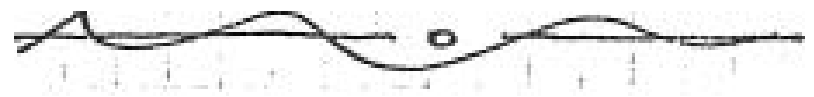


We now briefly reconsider the thermal boundary conditions used so far and mention their physical meaning. The point is best made by considering equation (18). The stability criterion is derived assuming $04>0$. This can only be achieved if at least one of the boundaries behaves like a heat pump, For a system obeying Newton law of cooling $a q<0$ and the above analysis does not apply.

$\underline{\text { Section } 2}$

We now turn to the case of perfectly conducting boundaries. Again linear theory shows that for $k=k_{g}$ and ${ }^{\circ}$ the static solution is stable and disturbances decay exponentially with time. We ask whether this result remains true when $K$ is allowed to vary with temperature. We cannot adopt the same technique as before, so we seek a variational equation for $\sigma$. We define the following euclidean space and linear operators:

$$
\begin{aligned}
& V=\left\{f(t) i f_{i} \in C^{2}(0,1), \quad f_{i}(0)=f_{i}(D)=0\right\} \\
& L=\left(\begin{array}{cc}
\left(D^{2}-a^{2}\right) & 0 \\
0 & 1
\end{array}\right) \quad M=\left(\begin{array}{cc}
0 & \wedge a \\
-p_{a} & a^{2}-D(K D+a k)
\end{array}\right)
\end{aligned}
$$

with inner product $(u, v) \equiv \int_{0}^{1} u^{\pi} v \alpha z \quad u, v \in v_{x} v$

Then equation (5) and (6) of section 1 are equivalent to

$$
\left(\sigma L+N_{i}\right) V=0 \quad \vee 6 \vee \times V
$$

and if

$$
(u, 4 v) \sigma=-(u, M v)
$$

Then it is easy to show that $\sigma$ is stationary with respect to independent variations of $u_{2} v$ provided that these satisfy:

$$
\begin{aligned}
& (\sigma \mathbb{L}+M) u=0 \\
& \left(\sigma^{*} \mathbb{L}^{+}+M^{+}\right) v=0
\end{aligned}
$$

where $\mathbb{L}^{+}=\mathbb{L} \quad$ and

$$
\left.N a\right|^{t}=\left(\begin{array}{cc}
0 & -\beta a \\
\wedge a & a^{2}-k D^{2}
\end{array}\right)
$$

We now have $u^{*}=\left(\begin{array}{c}\hat{\Psi} \\ \tilde{\theta}\end{array}\right) \quad \forall=\left(\begin{array}{l}\psi \\ \theta\end{array}\right) \quad$, so we choose dual functions $\tilde{\theta}_{\tilde{\theta} \boldsymbol{\theta}} \mathrm{v}_{j}$ solve for $\Psi \quad$ and substitute into (3) thus obtaining an estimate for $\sigma$.

We choose $\theta=\tilde{\theta}$ and as before we let

$$
\left(D^{3}-a^{2}\right) \phi=0
$$

Then $\psi$ and $\tilde{\psi}$ are given by

$$
\begin{aligned}
& \psi=-\frac{\Lambda_{a}}{\sigma} \phi \\
& \tilde{\psi}=\frac{\rho_{a}}{\sigma} \phi
\end{aligned}
$$


Substituting (7) into (3) we get:

$$
\begin{gathered}
\sigma^{2}\left\langle\theta^{2}\right\rangle+\sigma\left[a^{2}\left\langle\theta^{2}\right\rangle-\langle k \theta D \theta\rangle\right]-a^{2} \Gamma\left(3\left\langle\langle\phi \phi)^{2}\right\rangle+a^{2}\left\langle\phi^{2}\right\rangle\right)=0 \\
\Gamma=-\beta \lambda
\end{gathered}
$$

Again we see that if $\langle K \theta D \theta\rangle\rangle$ o there is a finite band of wave numbers which become unstable just as in section 1 and the same conclusions apply.

\section{$\underline{\text { Section } 3}$}

We continue by studying the finite amplitude development of the instability described i n section 1 . We consider a mode near $a_{c}$ and follow its nonlinear evolution. The equations are:

$$
\begin{aligned}
& \nabla^{2} \psi_{t}+\Lambda \theta_{k}+J\left(\nabla^{2} \psi_{i} \psi\right)=0 \\
& \theta_{t}+\beta \psi_{k}+J(\theta, \psi)=\nabla^{2} \theta \\
& k=\text { const } \quad \beta=-1 .
\end{aligned}
$$

It is convenient to distinguish between integrals of even and odd functions so we change the domain slightly. The boundary conditions are

$$
\theta_{z}+\epsilon^{2} q^{t} \theta=0 \quad \psi=0 \quad \text { zt } z= \pm 1 / 2 .
$$

Again, we consider small amplitude motion on large horizontal scales. Thus we let:

$$
\partial_{x} \rightarrow c \partial_{x}, \quad \rightarrow \in \theta, \quad \psi \rightarrow 6 \psi, \quad \partial_{t} \rightarrow \in \partial_{t}+\epsilon^{3} \partial_{k}
$$

Notice that we have introduced a fast time $t$ and a slow time $\mathbf{s}$; this is $O\left(e^{3}\right)$ and not $O\left(6^{2}\right)$. If the latter choice is made, the nonlinear terms do not contribute to the amplitude equation which turns out to be linear. The scaled equations are

$$
\begin{aligned}
& \theta_{z 2}=\epsilon\left[\omega \theta_{t}+\psi_{t}\right]+\epsilon^{2}\left[J(\theta, \psi)-\theta_{x x}\right]+\epsilon^{3} \theta_{5} \\
& \omega \psi_{2 E t}+\Lambda \theta_{x}=\epsilon J\left(\psi, \psi_{\theta t}\right)-\epsilon^{2} \psi_{22 s}-\epsilon^{2} w \psi_{\lambda x t}+O\left(\epsilon^{2}\right)
\end{aligned}
$$

We now express the variables as asymptotic series in

$$
\begin{aligned}
& \theta=\theta^{(0)}+\epsilon \theta^{(1)}, \cdots \\
& \psi=\psi^{(0)}+\epsilon \psi^{(1)}+\cdots \\
& v=1+\omega^{(1)}+\cdots \\
& q_{t}=q_{t}^{(0)}+\epsilon q_{1}^{(1)}+\cdots
\end{aligned}
$$


We substitute (6) in (4) and (5) and obtain to zeroth order

$$
\begin{aligned}
& \theta^{(\theta)}=\Phi(x, t, 5) \\
& \psi^{(t)}=\Psi(x, t, s) R(z) \\
& \Psi_{t}+\wedge \theta_{x}=0
\end{aligned}
$$

where $\mathrm{P}$ is a polynomial in $\mathrm{z}$ satisfying:

$$
\mathrm{P}^{\prime \prime}(z)=1, \quad \mathrm{P}( \pm 1 / 2)=0 \text {. }
$$

We substitute ( 7$),(\nabla)$, in equation (4) and to $O(\epsilon)$ we obtain:

$$
\theta_{z 2}^{(1)}=\Phi_{t}+\beta P(z) \Psi(x) \quad \theta_{z}^{(1)}( \pm 1 / 2)=0
$$

which when integrated from $-1 / 2$ to $1 / 2$ gives:

$$
\theta_{t}+\beta p \Psi_{x}=0
$$

where $p=\langle P\rangle$. Equation (11) and (9) describe the fast time behavior of the waves; these satisfy:

$$
\Psi_{t t}-\beta \wedge_{p} \Psi_{x x}=0
$$

which is a standard wave equation describing a wave travelling at speed $c$; $c^{2}=\Lambda \beta$ p. We write the solution to (12) as

$$
\begin{aligned}
& \Psi(x, t, s)=A(s) \cos (\Omega t-k x)+B(s) \sin (\Omega t-k x) \\
& \Omega^{2}=\Lambda \beta p k^{2} \quad c^{2}=\Lambda \beta p \quad \theta=\frac{1}{c} \Psi .
\end{aligned}
$$

We can then solve for $\psi^{(1)}$ and $\theta^{(1)}$ explicitly.

$$
\begin{aligned}
& \theta^{(1)}=\beta \Psi_{n} Q(z)+\omega^{\infty}(x, t, s) \\
& \psi^{(1)}=\Gamma(x, t, s) P(z)-\Phi(x, t, s) M(z)
\end{aligned}
$$

Q, Mare polynomials in $z, \omega^{0)}$ is an arbitrary function and $\Gamma$ and are known functions of $\Psi, Q, \oplus^{(1)}$ (see appendix for details).

The next orderin gives

$$
\theta_{z z}^{(2)}=\theta_{t}^{(1)}+\beta \psi_{x}^{(1)}+J\left(\theta^{(\omega)}, \psi^{(\theta)}\right)-\theta_{x k}^{(\omega)}+\omega^{(1)} \theta_{t}^{(0)}
$$




$$
\theta^{(x)}=-i^{2} \theta^{(0)} \quad z+ \pm 1 / 2
$$

Integration from top to bottom and substitution for $\theta^{(1)} \psi^{(0)}, \psi^{(a)}$, $\theta^{(0)}$, gives

$$
\beta q \bar{\Psi}_{x t}+\Delta q^{(0)} \oplus+\beta p \tilde{\Gamma}_{v}-\Phi_{\lambda x}+\theta^{(1)} \Phi_{t}=-\left[\Phi_{t}^{(1)}-\beta \wedge_{p} \Xi_{x x}\right]
$$

Requirement that the RHS be nonresonant gives a wave equation for $\Theta^{\omega}$,

$$
\theta_{t}^{(1)}-\beta \wedge p \theta_{x x}=0
$$

The LHS of (19) is a homogeneous equation for $\mathbf{A}$ and $B$. The requirement that the deterninant vanishes gives the marginality conditin for $k$.

$$
\omega^{(1)}=0 \quad k^{2}(p(p+1) \wedge-1)=\Delta i^{(0)}
$$

where $q=\langle Q(z)\rangle$.

Again $\psi^{(2)}$ and $\theta^{(2)}$ can be found explicitly in terms of known quantities and polynomial in $z$. $\theta^{(t)}$ involves a new arbitrary function $\theta^{\alpha}(x, t, s)$.

The next order gives:

$$
\begin{aligned}
\theta_{z: 1}^{(3)} & =\left(\theta_{t}(2)+\beta \psi_{x}^{(2)}\right)+\omega^{(2)} \theta_{t}^{(0)}+J\left(\theta^{(0)}, \psi^{(n)}\right) \\
& +J\left(\theta^{(1)}, \psi^{(0)}\right)+\theta_{s}^{(0)}-\theta_{x z}^{(1)}
\end{aligned}
$$

with B.C.'s

$$
\theta_{z}^{(3)}=-q_{ \pm}^{(0)} \theta^{(1)}-q_{t}^{(1)} \theta^{(0)}
$$

We see that the slow time has appeared explicitly in (22) (it is also present in $\psi^{(2)}$ ). Integrating (22) from $-1 / 2$ to $+1 / 2$ and using (23) gives an equation for $\Theta_{s}$ in terms of its fast time and space derivatives. The only nonlinear coupling contributing to this equation is

$$
\left(\Phi \psi_{x}\right) \quad \text { where } \quad \Phi_{t}=\psi \psi_{x}
$$

We can then project onto sin $(\Omega t-k x)$ and $\cos (\Omega t-k x)$ to get an equation for $A$ and $B$. Namely:

$$
\begin{aligned}
& \alpha \dot{A}=\Delta q^{(1)} A+a_{1} B-a_{2}\left(B^{3}+A^{2} B+B^{2} A\right) \\
& \delta \dot{B}=-\Delta q^{(1)} B+d_{1} A-d_{2}\left(A^{3}-A^{2} B-B^{2} A\right)
\end{aligned}
$$

Or in terms of a complex variable $z=R e^{i \phi}, A=R \cos \phi, B=R \sin \phi$

$$
\dot{R}=R\left[\frac{(\alpha-\delta) \Delta q^{(n)}+(\alpha+\delta) \alpha_{1}}{\left(\alpha^{2}+\delta^{2}\right)}\right]-\frac{\alpha^{2} \alpha_{2}}{\left(\alpha^{2}+\delta^{2}\right)} R^{3}
$$




$$
\dot{\phi}=\frac{\delta}{\alpha}\left[\frac{(\alpha-\delta) \Delta q^{(1)}+(\alpha+\delta) \alpha_{1}}{\left(\alpha^{2}+\delta^{2}\right)}\right]+\left(\Delta q^{(1)}-\alpha_{1}\right)-\frac{\delta \alpha_{2}}{\left(\alpha^{2}+\beta^{2}\right)} R^{2}
$$

which we recognize as a Landau equation for a Hopf bifurcation The constant term in (26) represents the frequency renormalization. (25) and (26)

describes the growth and nonlinear saturation of an oscillatory mode which is a distance $\in \Delta q^{(1)}$ away from the marginal mode given by (21).

\section{CONCLUSION}

We see that boundary conditions and the thermal properties of the fluid, for instance, its thermal diffusivity are crucial in determining the stability of the static solution. Growing oscillations can occur in fluid even though these are stably stratified. The mechanisms described in the previous sections could provide a different outlook for the study of oscillatory motion observed, for example, in the sun and in certain situations with liquid metals.

The boundary conditions used i n sections 1 and 3 are correct for steady convection between poorly conducting walls but need to be revised for time dependent motion. A more realistic situation, and one probably ameanable to experiment, would be to constrain the fluid between two slabs of finite conductivity and 'prescribe the boundary conditions on these slabs. In this case the value of the Biot number $(q+$ and $q-i n$ the notation of sections 1 and 3 ) depends on time and the problem is somewhat richer.

Another interesting possibility would be to study in details the interaction between imperfect boundaries and temperature dependent conductivity. This suggests the possibility of two simultaneous instabilities leading to a co-dimension two bifurcation from the static solution.

\section{ACKNOWLEDGEMENT}

I would like to thank Prof. Spiegel for suggesting this project to me and for his constant advice and supervision.

\section{REFERENCES}

Chandrasekar, Subrshmanyan, 1910. Hydrodynamics and hydromagnetic stability, (The int. ser. of monographs on physics), 625 pp., Oxford.

Spiege1, Edward, A., 1981. Lectures of this series. 
APPENDIX

otes

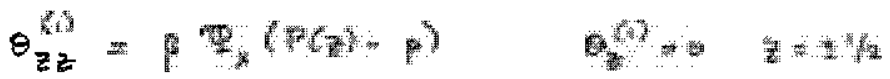

$$
\begin{aligned}
& \Rightarrow \theta^{n}=\beta_{x} a(\alpha)+\phi^{m}\left(w_{i}\right)
\end{aligned}
$$

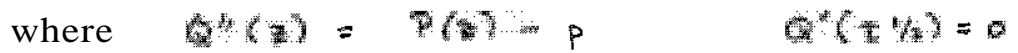

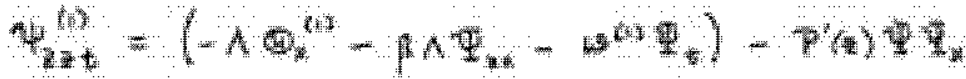

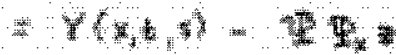

$$
\begin{aligned}
& 4^{h}=0 \quad+1 h
\end{aligned}
$$

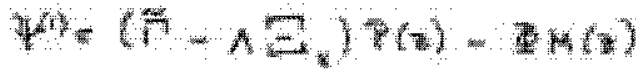

where $\Gamma=\gamma(\alpha, n) \Gamma=\tilde{\Gamma}-\Lambda \Xi$

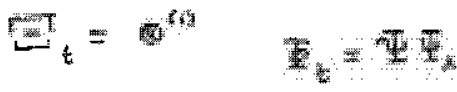

$$
\begin{aligned}
& M(z)-M( \pm 1 / 2)=0
\end{aligned}
$$

0,1

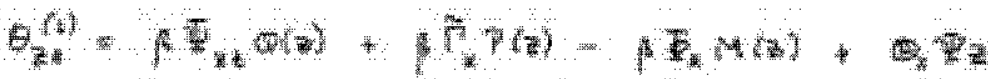

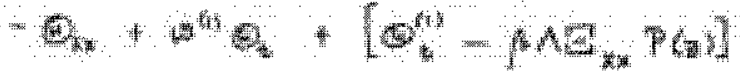

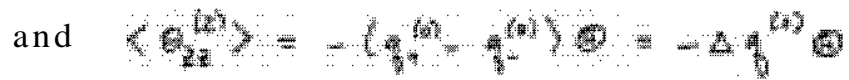


Integration gives Eqn. (19) and (20).

$$
\begin{aligned}
& \beta q q_{x t}+\Delta q^{(0)} \theta+\beta p \tilde{\Gamma}-\Theta_{p x}+\omega^{(0)} \Phi_{p}=-\left[\oplus_{t}^{(1)}-\beta \wedge_{p} \Xi_{x x}\right] \\
& \Theta_{40}^{(1)}-\beta \Lambda p \Theta_{x=0}=0 \\
& q=\langle Q(\nabla)\rangle
\end{aligned}
$$

but $c \oplus=\Psi=A \cos (\Omega t-k x)+B \sin (\Omega t-k x)$.

Which gives:

$$
\begin{aligned}
& A\left[\beta j k \Omega+\frac{\beta^{2} p \wedge k^{3}}{\Omega}-\frac{\Delta q^{(0)}}{c}-\frac{k^{2}}{c}\right]-B\left[k \beta p+\Omega\left(\varepsilon^{(a)}\right]=0\right. \\
& A\left[k \beta p \omega^{(1)}+\frac{\Omega_{(0)}^{(0)}}{c}\right]+B\left[\beta q k \Omega+\frac{\beta^{2} \Lambda p k^{3}}{\Omega}-\frac{\Delta q^{(0)}}{c}-\frac{k^{2}}{c}\right]=0 \\
& \Rightarrow \omega^{(1)}=0 \text { and } k^{2}(p(q+1) \wedge-1)=\Delta q^{(0)} \text {. } \\
& \begin{aligned}
\theta^{(t)}=\beta \Psi_{x t} \tilde{Q}(z)+\left[\Theta_{t}^{(1)} P(z)-\beta \Lambda E_{x x} \tilde{P}(z)\right]+\beta \tilde{\Gamma}_{x} \tilde{P}(z)-\beta \Phi_{x} \tilde{\mu} \\
+\Phi Q_{x} \frac{z^{3}}{b}-\Theta_{x x} P(z)+\Theta^{(a)}(x, t, s)
\end{aligned}
\end{aligned}
$$

where $\tilde{Q}^{\prime \prime}(z)=Q(z) \quad \tilde{Q}( \pm h)=0$

$$
\begin{array}{ll}
\tilde{M}^{\prime \prime}(z)=M(z) & \tilde{M}(\tau / h)=0 \\
\tilde{p}^{\prime \prime}(z)=P(z) & \tilde{p}( \pm / / 2)=0
\end{array}
$$




$$
\begin{aligned}
& \Psi_{t}^{(2)}=\left[-\omega^{(v)} \Psi_{t}-\wedge \Theta_{x}^{(t)}-\Psi\left(\tilde{r}_{x}-\wedge \Xi_{x x}\right)-\Psi_{s}\right] P(\varepsilon) \\
& +\left[-q \Phi_{x}+\left(\wedge \Xi_{x}-\tilde{\Gamma}\right) \Phi_{x}\right] \frac{z^{3}}{6} \\
& +\left[-\Lambda \Theta_{x t}^{(1)}+\Lambda \Phi_{x x x}-\Psi_{x} \Phi-\Psi_{x x t}\right] \tilde{P}(z)+\Phi \Psi_{k} \tilde{M}(s) \\
& +\left[-\Lambda \Psi \otimes_{x x}\right] N(z)+\left[\beta \wedge^{2} \Xi_{x \times x}-\beta \wedge \tilde{\Gamma}_{x x}\right] \widetilde{P}(z) \\
& +\left[\beta \wedge \Phi_{x k}\right] \tilde{M}(z)+\left[-\beta \wedge \tilde{\Psi}_{x x t}\right] \approx \tilde{Q}(z)
\end{aligned}
$$

where $\quad \tilde{M}^{*}(z)=\tilde{M}(z) \quad \tilde{M}( \pm / z)=0$ etc.

$\underline{0\left(\epsilon^{3}\right)}$

$$
\begin{aligned}
& \theta_{z z}^{(n)}=\left(\theta_{t}^{(2)}+\beta \psi_{x}^{(2)}\right)+\omega^{(2)} \theta_{t}^{(0)}+J\left(\theta^{(0)}, \psi^{(1)}\right)+J\left(\theta^{(1)}, \psi^{(0)}\right)+\theta_{s}^{(0)}-\theta_{x x}^{(1)} \\
& \theta_{z}^{(s)}=-q_{1}^{(0)} \theta^{(1)}-q_{ \pm}^{(1)} \theta^{(0)}
\end{aligned}
$$

Integrating from $-1 / 2$ to $1 / 2$ and projecting onto $\sin (\Omega t-k x)$ and $\cos (\Omega t-k x)$ we get the coefficients $\alpha_{2} \delta, \alpha_{1}, \alpha_{2}$

$$
\begin{aligned}
\alpha= & \left(\frac{k p}{\Omega}-\frac{1}{c}\right) \\
\delta= & \left(\frac{k p}{\Omega}+\frac{1}{c}\right) \\
\alpha_{1}= & -\left(k \Omega^{2} \tilde{q}+k^{3} \tilde{p} \Lambda+\frac{k^{2}}{\Omega} \frac{p}{c}+\frac{k^{4} \Lambda \tilde{p}}{c \Omega}-k^{3} \tilde{p}+\frac{k^{5}}{\Omega^{2}} \widetilde{p} \Lambda^{2}\right. \\
& \left.+k^{3} \wedge \tilde{q}-k^{3} q-k \Delta Q\right) \\
d_{2}= & \left(\frac{\tilde{m} r k^{3}}{\Omega}+\frac{m^{\prime} k^{2}}{c}+\frac{k^{3} \tilde{p}}{\Omega}\right) \frac{k^{2}}{g \Omega}
\end{aligned}
$$

where $\tilde{q}=\langle\tilde{Q}\rangle \quad \tilde{p}=\langle\vec{p}\rangle \quad \tilde{m^{\prime}}=\left\langle\tilde{m}^{\prime}\right\rangle \quad$ etc. 


\title{
DOUBLE DIFFUSION IN A LONG BOX
}

\author{
Chriscopher Bretherton
}

\section{$\underline{\text { ABSTRACT }}$}

We consider the generic problem of $2 \mathrm{D}$ thermohaline convection in a stress-free box of infinite horizontal extent, and derive equations for the spatially modulated roll amplitude in weak convection. Near the co-dimension two bifurcation, a 11 uniform roll patterns are unstable to nonlinear waves which suck energy into right and left going roll packets. If, in this 1imit, we go close to the Hopf bifurcation, these waves may be solitons whose amplitude and velocity slowly are forced by dissipation to specific values. Thus there is significant horizontal transport of energy on long scales by the waves.

\section{INTRODUCTION}

In recent years, there has been renewed interest in the spatial structure of large aspect ratio convection. Experiments (Gollub, 1981; Libchaber, 1981) have shown that for most initial conditions, even in weak convection, that no steady state is achieved, and instead persistent low frequency noise is observed due to the slow rearrangement of roll patterns.

At the same time, there has been substantial work done on dynamically degenerate systems between highly confining boundaries in which two or more instability mechanisms are present (Guckenheimer, 1981; Arneodo, et a1., in press). Depending on the relative strengths of the instabilities, one of a wide variety of behaviors may be seen. These reflect the behavior even when parameters of the system (such as heat input) are changed drastically, so they give a good qualitative feel for what is happening over a wide range of parameters. The competing influence of a few instabilities can be crystallized into a small number of normal forms whose bifurcation structure is known.

However, this approach has not been successfully applied to the geophysically important case of a horizontally unconfined fluid. In this case, dissipation and instability mechanisms can be nearly balanced everywhere by a myriad of convective patterns, while the small remaining energy goes into modifying the pattern. I would like to show that this spatial degree of freedom can drastically change the dynamics of a system with two nearly marginal instabilities so as to cause nonlinear waves which can transport substantial amounts of energy horizontally.

The situation we will study is the mohaline convection in a long, twodimensional box with stabilizing salt gradient counteracted by a destabilizing heat gradient between stress-free boundaries. Depending on the two gradients, two instabilities may act to release the potential energy of the hot bottom. There is a direct instability in which the fluid is unstably stratified, and hot fluid moves up faster than its heat can diffuse away, and an oscillating instability in which the same hot blob diffuses away its heat too fast and is forced downward by its salinity. Since it is now cold it descends Easter than it rose, leading to an overstability. Turner (1973) contains a good review of the physics.

The bifurcation diagram for rolls which are constrained to have a given wave length $\lambda$ (with $a=2 \pi d / \lambda$ where $d$ is the container height) is shown in Figure 1. At the point $P$ in the diagram both the direct instability and 
the overstability are neutrally damped, and the region in $r_{3}$ around $P$ reflects in microcosm all the behavior shown in the diagram.

If we relax the constraint on $\lambda$ and instead allow an infinite box in which a 11 roll wave lengths are permitted, we see three new effects. Firstly, there is a most unstable wave number with ${ }_{c}=v_{2}\left(\frac{\lambda}{d}=2^{3 / 2}\right)$. Imagine that we are at a point $Q$ near where this wave length becomes unstable. Nearby wave lengths are more stable, and if we draw their behavior on Figure 1 for $\chi_{-}=\mathrm{d}_{\mathrm{c}}$, we see it falls on the line $\chi$. Each wave number $\propto$ corresponds to a point $\left(r_{\mathrm{T}}, r_{\mathrm{z}}\right)$, where

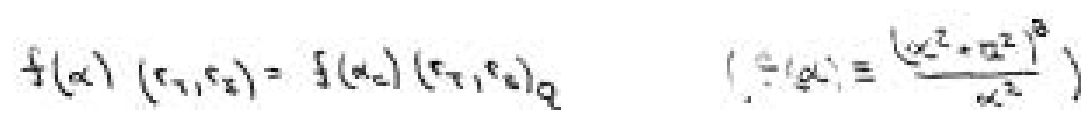

The convection may thus be trying to achieve disparate behaviors in regions along the box where the local wave number is different, mixing up the bifurcation picture of Figure 1 .

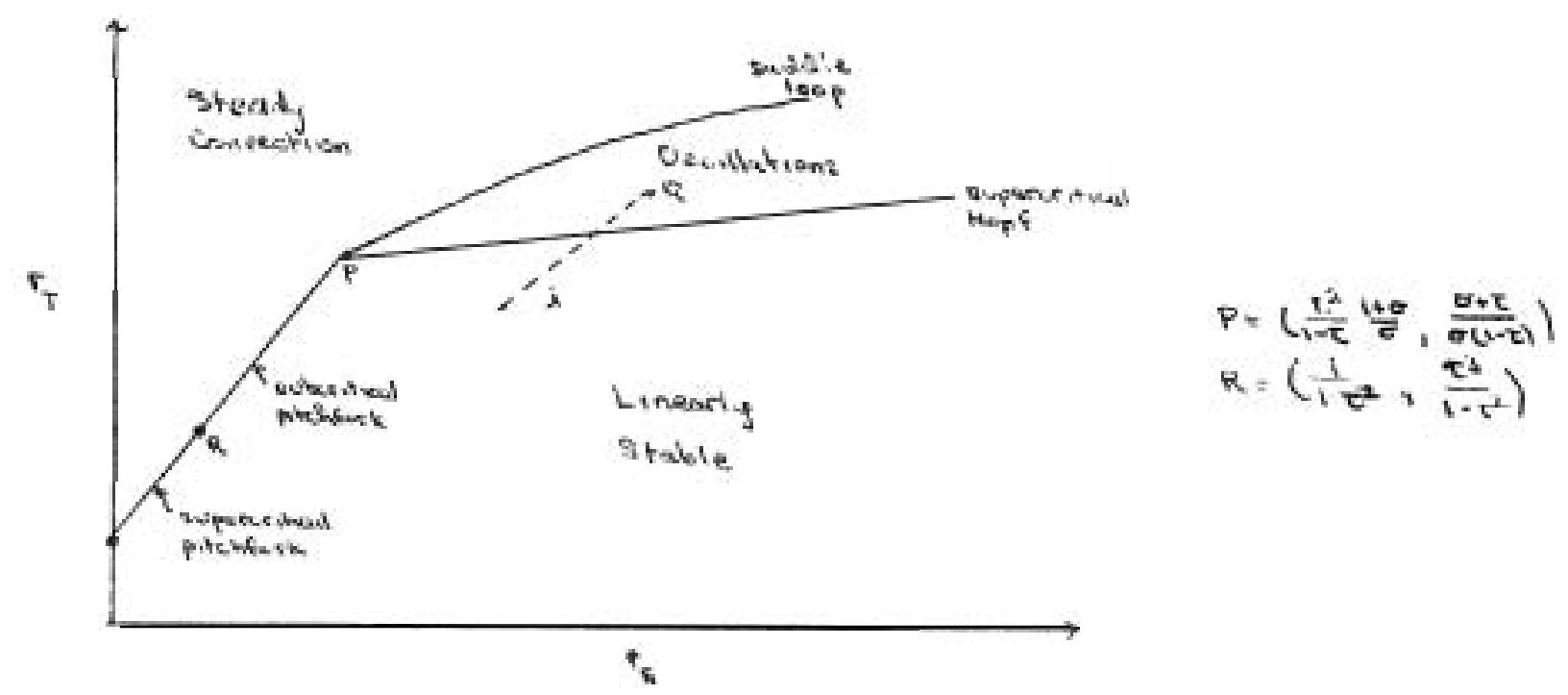

FIGURE 1. The bifurcation diagram for thermohaline convection. The parameters $r_{T}$ and $r_{s}$ are defined in terms of the Rayleigh numbers and the roll. wave number $x$ by $\quad\left(R_{i}, w_{s}\right)=f(s)\left(t_{\tau}, s_{s}\right)$

$P$ is the co-dimension two bifurcation point, while $R$ is the point at which the bifurcation to steady convection reverses to subcritical. If we fix $R_{T}$ and $R_{S}$ while varying,,${ }^{T}$ and $r_{5}$ move up along the line $\lambda$, reaching a maximum at the $x$ minimizing $f(x), \alpha_{4}, \frac{c}{\alpha_{2}}$ and then they descend back along $\ell$.

Second, and just as important, the character of a Hopf bifurcation changes in a long box. In a small container, we usually think of the oscillating instability as a sloshing of fluid back and forth, effectively a standing wave. In a long box, however, we must now think of two travelling roll waves rapidly moving to the right or left (Figure 2). 

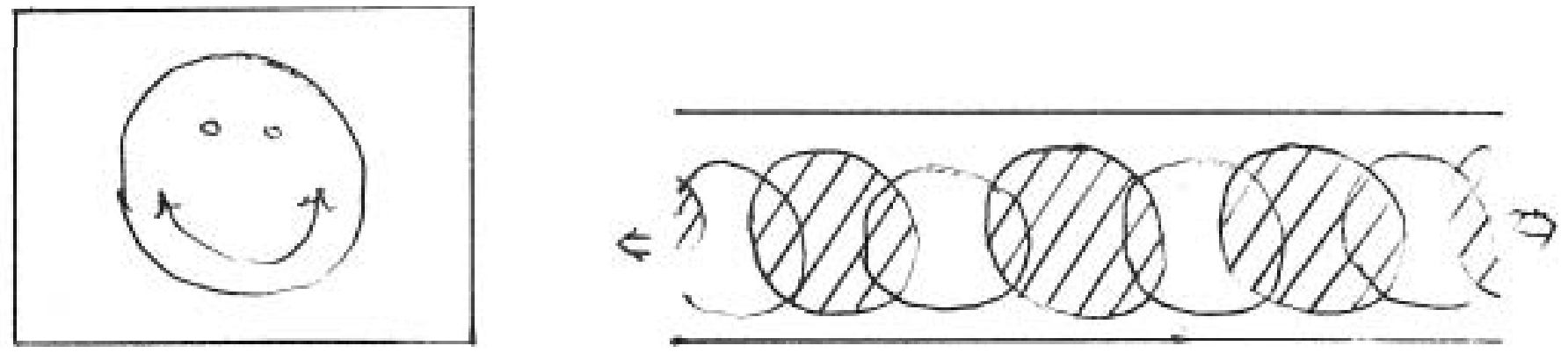

FIGURE 2. a) In a narrow box the Hopf bifurcation leads to a sloshing of fluid back and forth around the roll. The two small perturbations are not there.

b) In a long box we get a right and left going roll wave. If they are of equal amplitude there will be the standing wave of a), but this is not generally the case.

Each wave has its own amplitude, and even if initially the two waves have nearly equal amplitude, so that the behavior is similar to a standing wave, nonlinear damping causes the larger wave to swallow the smaller. We have at each point rapid transport of energy horizontally in whichever direction the dominant wave is going.

Thirdly, nonlinear effects play a profound new role in the dynamics. In addition to being an amplitude-dependent damping, nonlinearities act to produce instabilities of the Hopf waves which cause their amplitude to modulate, sucking in energy to feed locally stronger convection. No longer is any single wave number preferred; instead a time-dependent combination of a 11 the unstable wave numbers can be expected, leading to low frequency noise.

\section{The Amplitude Equations}

So much for philosophy; let us proceed. In this section I will derive amplitude equations for the sin a and $\sin 2 x z$ normal modes of the velocity, temperature, and salinity, using a combination of modal truncation (Veronis, 1965; Knobloch and Proctor, 1981) and slow spatial amplitude variation (Newe11 and Whitehead, 1968) to get equations which recover the small amplitude behavior of the system. We will specialize to the direct, Hopf, and then the co-dimension two bifurcation.

\subsection{Equations of Motion and Scaling}

If we scale our problem with the box height $d$, the thermal diffusion time $A^{2} / \mathrm{x}$, and the temperature and salinity difference $\Delta T, \Delta S$, and assume we have a Boussinesq fluid, we can introduce a streamfunction $\psi$ which determines the nondfmensional velocity $v=\left(-\psi_{z}, \psi_{*}\right)$, and perturbations

$9 \quad \bar{z}$ of the temperature and salinity from the conduction state. In these three variables, the equations of momentum, heat, and salt advection are

$$
\begin{aligned}
& \frac{1}{\tau}\left\{7^{2} \psi_{t}+J\left(\psi, \nabla^{2} \psi\right)\right\}=R_{\tau} 0_{x}-R_{s} \Sigma_{x}+\nabla+\psi \\
& \epsilon_{*}+J(\psi, \theta)=\psi_{*} * \nabla^{2} \theta \\
& \text { (III) } \quad \Sigma_{t}+J(4, \Sigma)=\psi_{x}+\tau \nabla^{2} \Sigma
\end{aligned}
$$


where

$$
\begin{aligned}
& R_{T}=\frac{q x \Delta T d^{2}}{E K_{T}} \quad R_{2}=\frac{9 B B_{2} d^{3}}{2 \pi T} \\
& r=\frac{v}{x_{i}} \\
& \tau=\frac{x_{3}}{x_{T}}
\end{aligned}
$$

The boundary conditions are that , $\sum \sum$ are bounded as $(\times 1-i$ and that the top and bottom plates are stress free, isothermal, and isosaline:

$$
\theta=\Sigma=\psi=\Psi_{z z}=0 \quad \text { on } z=0,1
$$

The geometry is shown in Figure 3.

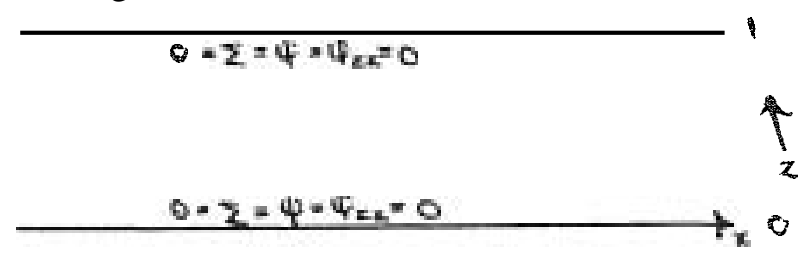

FIGURE 3. The Geometry of the Model

Now, we will rescale all variables so that they are small, and Fourier expand $\psi, \theta, \Sigma$ We will assume the supercriticality is $0\left(E^{2}\right)$. so the sin $2 \pi$ modes are $O(\varepsilon)$ and can have a local horizontal wave number with $O(\varepsilon)$ of $\alpha_{c}$ and thus can be represented as a slow spatial modulation on a carrier wave $e^{i x x}$ Only the first two vertical harmonics are kept. We now require some notation.

Wave number

Squared total wave number

Reduced Rayleigh numbers

Rayleigh numbers combinations

Long space scale

Time (scaled for convenience)

$x$ derivative of $e^{-m \pi}$ mode

Laplacian correction

Useful constant

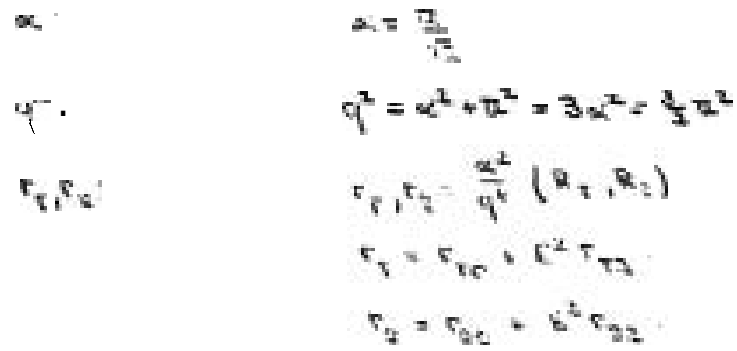

$C_{\ln n}$

$\eta_{\operatorname{man}}=r_{\mathrm{m} m}-\frac{1}{c^{4}} 5_{2 m}$

$x_{4}=t x$

$\Rightarrow=q t$

$D_{n}=k n+23$.

l. $E A=\frac{1}{T^{2}}\left(0_{1}^{2}-\pi^{2}\right)$

$\Delta=\frac{2 i x}{q^{2}} \partial_{x}+\frac{\varepsilon}{q^{2}} \partial^{2} x$.

$\infty$ 
Modal expansions $a, a, b, c, d, e$

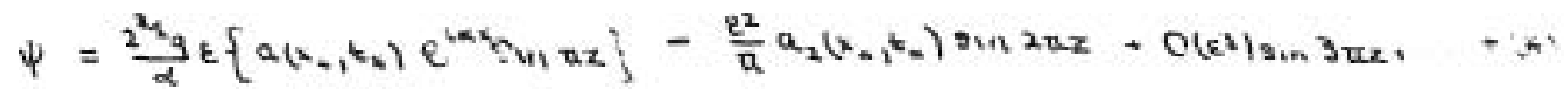

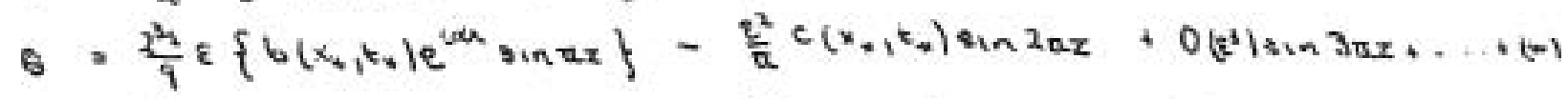

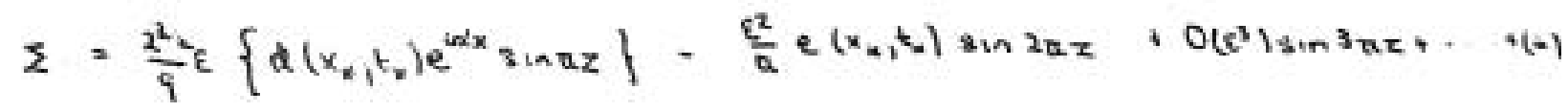

(where "+(*)" means to add the complex conjugate).

For the mean fields $A_{2}, C, E$ :

$$
\begin{aligned}
\varepsilon A_{2} & =a_{2}, u_{2}^{*} \\
c & =c+c \\
E & =e+e^{*}
\end{aligned}
$$

The method is patterned after Knobloch and Proctor, 1981). We Fourier expand the thermohaline equations. To a given order in $\boldsymbol{t}$, we need only keep a few modes to find the evolution of the fields to that order. In particular, to find all fields through $O($ ) requires only sin az and sin2 az modes, so we "truncate" the Fourier expansions at that order to obtain modal evolution equations. The reader uninterested in the details should skip to the final equations $(1.2 .1-5)$ of this section.

Equation $I, e^{\text {vex }} \sin$ \& $z$ mode

$$
\begin{aligned}
& \nabla^{2} \psi_{0}=\quad \frac{2^{2} x q}{a^{2}}=\left(0_{1}^{2}-a^{2}\right) q^{2} a
\end{aligned}
$$

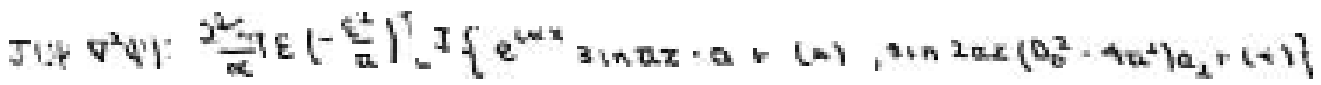

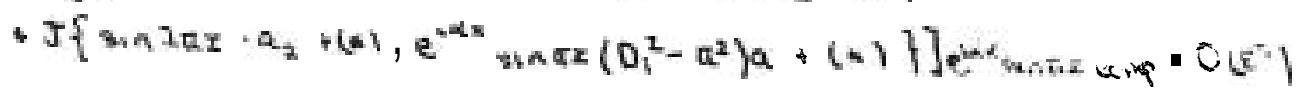

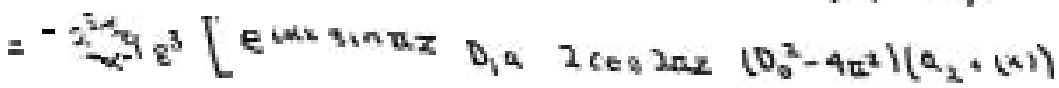

$$
\begin{aligned}
& \text { - e } \cos a z \text { a } \operatorname{ain} 2 \pi z\left(0_{0}^{2}-4 a^{2}\right)\left(0, a_{2}+(-1)\right.
\end{aligned}
$$

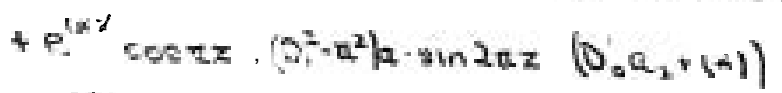

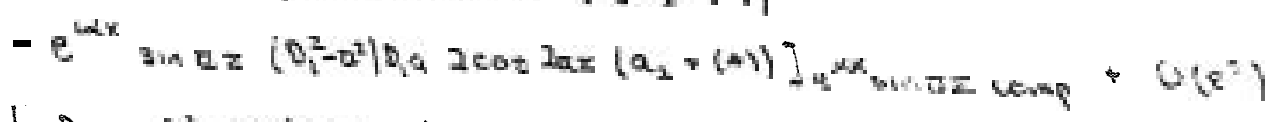

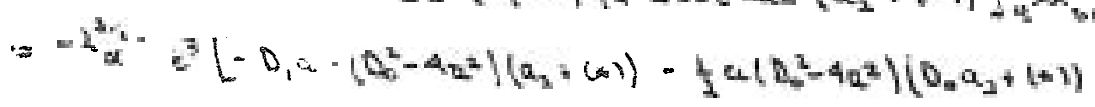

$$
\begin{aligned}
& \left.\frac{1}{2}\left(D_{1}^{2}-\pi^{2}\right) a\left[D_{0} a_{2}+(+)\right]+\left(D_{1}^{2}-\pi^{2}\right) D_{1} a\left(a_{2}+(+)\right)\right]+0\left(a^{2}\right)
\end{aligned}
$$

Now $a_{2}=O(c)$, $D_{s}+t a$, so that we can eliminate many of these terms:

$J\left(\psi, \nabla^{2} \psi\right)=-\frac{2^{2}-4}{2} \varepsilon^{3}\left[\left(0_{1}^{2}+3 \pi^{3}\right) 0 p\left(a_{2}+a_{2}-1\right]-D\left(e^{7}\right)\right.$

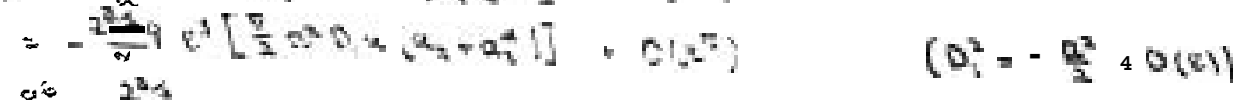

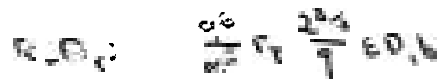

$-R \Sigma_{2} \quad-\frac{q^{6}}{s^{2}}+\frac{2^{2}-1}{4} \in D_{2} d$

$\nabla^{2} \psi \cdot \quad-\bar{\alpha} \cdot\left(0^{2}-\pi^{2}\right)^{2} \alpha$ 


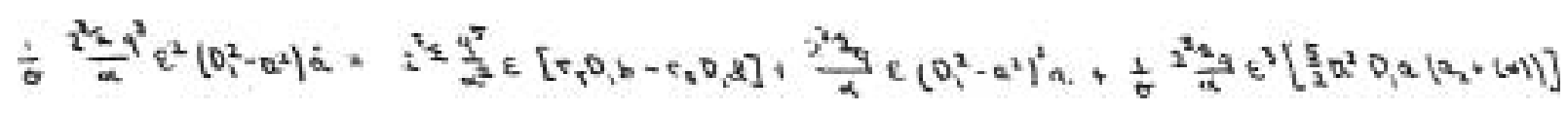

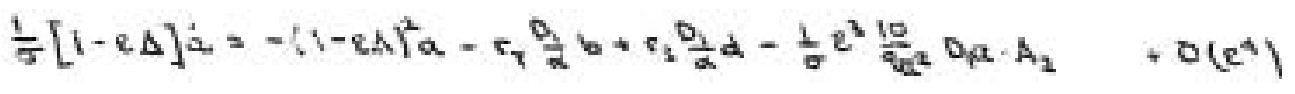

$\underline{\text { Equation I, sin tux mode }}$

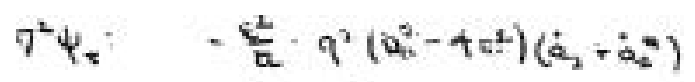

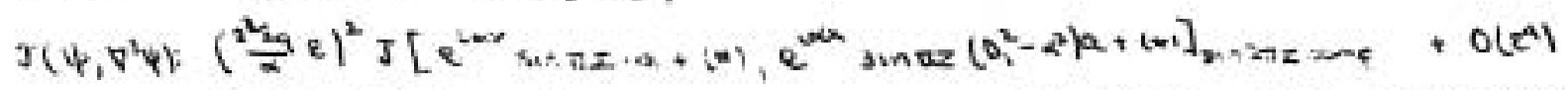

$$
\begin{aligned}
& =\frac{8 y}{2} E^{2} a\left[\frac{1}{2}\left(0, a\left(D_{-1}^{2}-a^{2}\right) a^{2}+\theta_{-1} a^{2}\left(D_{1}^{2}-a^{2}\right) a-a\left(D_{-1}^{2}-a^{2}\right) \theta_{-1} a^{2}-a^{2}\left(D_{1}^{2}-z^{2}\right) D_{1} a\right)\right] \cdot O\left(c^{2}\right)
\end{aligned}
$$

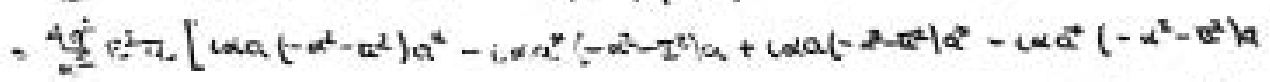

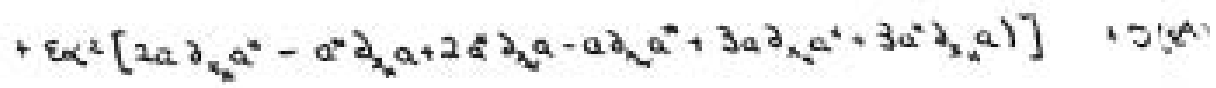

$$
\begin{aligned}
& =16 \pi^{2} a^{2} \varepsilon^{3}\left[a a^{2} x^{*}-\alpha x^{2} a\right]+O\left(x^{4}\right)
\end{aligned}
$$

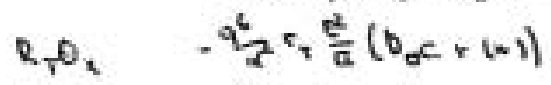

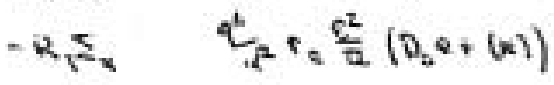

$$
\begin{aligned}
& \nabla \varphi \quad-\frac{c^{2}}{a}\left(D_{0}^{2}-4 z^{2}\right)^{2}\left(a_{a}+(\alpha)\right)
\end{aligned}
$$

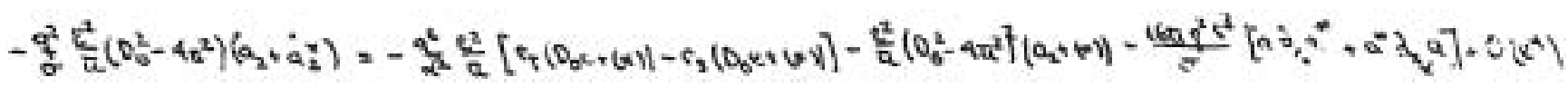

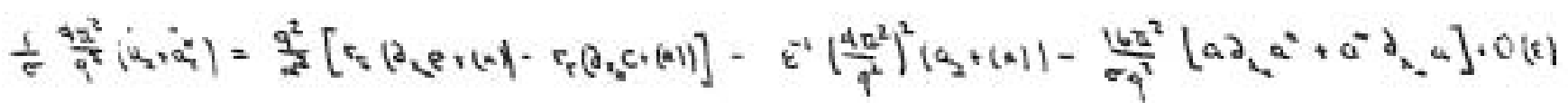

$$
\begin{aligned}
& \in A_{2}=a_{2} * a_{2}
\end{aligned}
$$

Using $q^{2} / \alpha^{2}=3$, we see

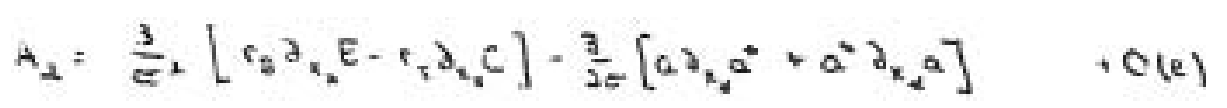

Equation II, $\mathrm{Z}^{\mathrm{ias}} \sin$ az mode 


$$
\begin{aligned}
& \nabla_{t} \theta: \quad \frac{2 \pi / 3}{2} x^{2}=i
\end{aligned}
$$

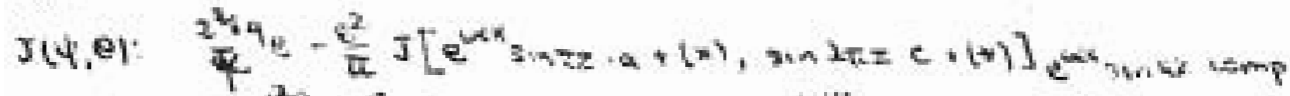

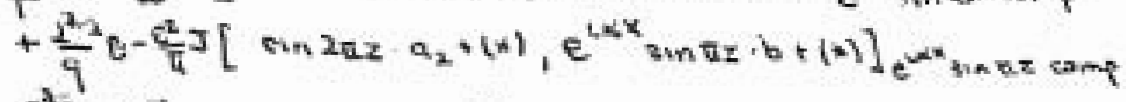

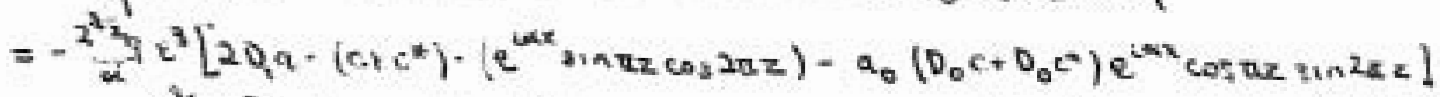

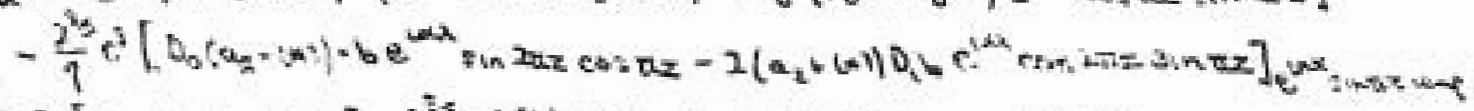

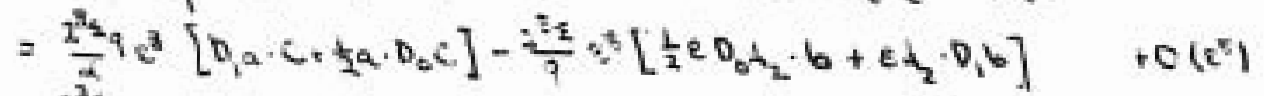

$$
\begin{aligned}
& \text { Y. } \quad \frac{2 \pi}{2}=0, a \\
& \nabla 20 \quad \frac{z^{2}+2}{a}\left(a^{2}-a^{2}\right) b
\end{aligned}
$$

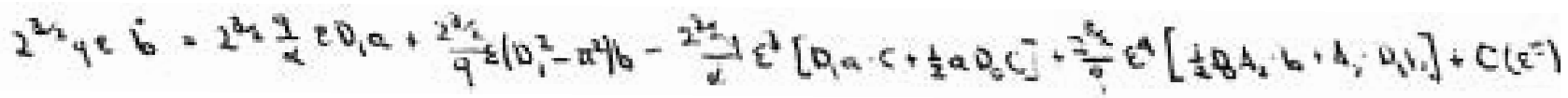

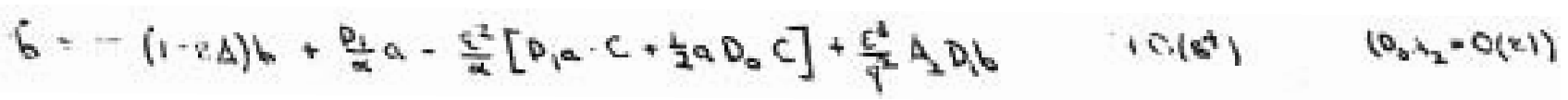

Equation II, sin $2 \pi z$ mode

20: $\quad-\frac{c^{2}}{a^{2}} q^{2}(\varepsilon+i-1)$

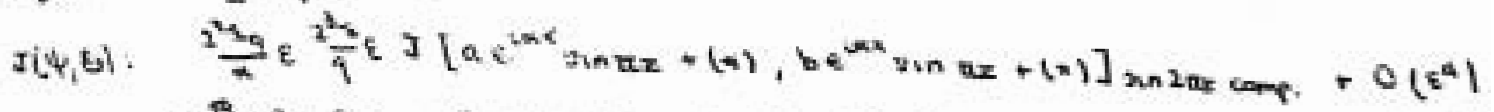

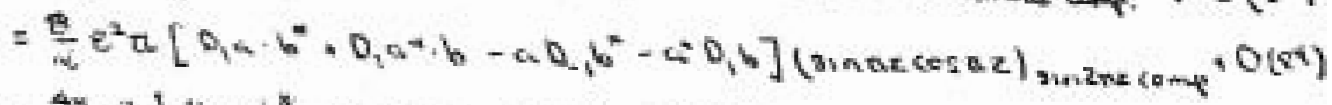

$$
\begin{aligned}
& =\frac{4 x}{2} \varepsilon^{2}\left[0, a+4^{*}+0, a^{*} \cdot b-a, 0, b^{*}-a^{2}-0, b\right] \\
& \partial_{1} \psi:-\frac{c^{2}}{a} D_{0}\left(u_{2}+(x)\right) \\
& \nabla 20 \quad-\frac{t^{2}}{a}\left(D_{0}^{2}-4 a^{2}\right)(c+c) \\
& \left.i=\pi-c=+\left[D, a-b^{2}+D_{-1} a^{2} \cdot b-a D, b^{2}-a^{4} D, b\right]\right\}+O\left(E^{2}\right)
\end{aligned}
$$

Equation III gives equations exactly analogous to the above two, except with $=$ in front of the diffusion terms. Collecting all our equations, we
get

The Modulated System

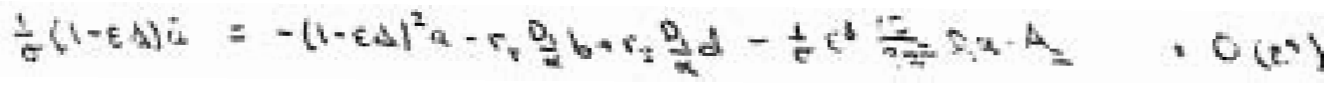

$$
\begin{aligned}
& \left.\dot{h}=-(1-2 \Delta) b+\frac{D_{1}}{2} a-\frac{b^{2}}{2} \mid 0 \cdot a \cdot c+\frac{1}{2} D_{a} a \cdot c\right]+\frac{b^{3}}{a^{2}} D_{1} b a_{2}+O\left(e^{4}\right)
\end{aligned}
$$

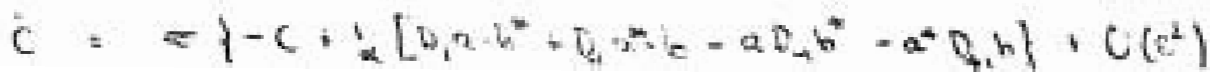


where

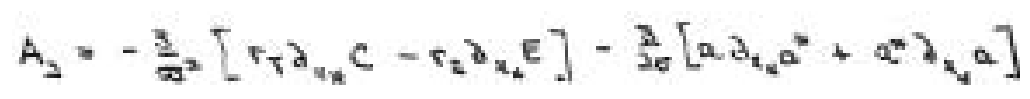

\subsection{Scalings of the Modulated System}

Depending on how we scale the time dependence of the modal amplitudes, we recover all of the small amplitude behavior of thermohaline convection. In terms of a rescaled $\mathrm{time} t^{\prime}$,

$$
\begin{array}{ll}
t^{\prime \prime}=t^{2} t & \text { Pitehfork bifurcation } \\
t^{\prime}=t & \text { Hopf bifurcation } \\
t^{\prime}=t & \text { Co-dimension two bifurcation }
\end{array}
$$

In each of these successively more complex eases, we eliminate a 11 the modal amplitudes but "a" to a given power of $\varepsilon$, following (Knobloch and Proctor, 1981). We show the so-dimension one amplitude equations break down as the second instability is approached and then look at co-dimension two itself. The reader who is bored by algebra need only look at the equations and discussion after $(1.3 .2),(1.3 .9-10)$, and $(1.4 .8)$.

$t=O\left(E^{2}\right):$ The Direct Bifurcation

Suppose the mode amplitudes depend only on the very slow time $\mathrm{T}=\varepsilon^{2} t$. Then $O(1)$ the modulated equations imply

$$
\begin{aligned}
& b=i a+k g \\
& d=i \frac{a}{r} \cdot k h \\
& c=4 a a^{*} \cdot e k \\
& E=\frac{4 a a^{2}}{r^{2}} \cdot 2 k
\end{aligned}
$$

At $O(\mathcal{C})$, we find the form of the $O(E)$ corrections $g, h, k, 1$ :

$$
\begin{aligned}
& (1.2 .2) \Rightarrow 3=\frac{1}{3} u_{x}+e C \\
& (1.2 .4) \Rightarrow n=\frac{1}{3} \angle \tau=x+E H
\end{aligned}
$$

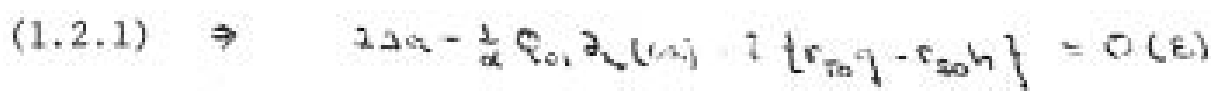

Thus

$$
\left.-\frac{2}{20}-\frac{2}{8 x_{0}} R_{01}\right) u_{x}=O(c)
$$

so

$$
r_{T_{0}}-\frac{r_{v s}}{\tau}=Q_{0 v}=1
$$

This gives the neutral linear stability line for the direct instability. We could determine $k, \mathcal{L}$ but this proves unnecessary. The $O\left(k^{I}\right)$ balance now gives equations for $G$ and $H$. 
$(1.2 .2) \Rightarrow$

$$
\begin{aligned}
& c a_{T}=-C+\frac{7 y}{\partial \alpha} \partial_{x}\left(\frac{1}{3 \alpha} a_{x}\right)+\frac{1}{q^{2}} \partial_{x}^{2}(i a)-c a\left(4 a a^{*}\right) \\
& c=-c a_{T}+i a_{x x}\left\{\frac{2}{2 \alpha^{2}}+\frac{1}{3 \alpha^{2}}\right\}-i\left(4 \alpha^{2} a^{*}\right)
\end{aligned}
$$

Similarly

$(1.2 .4) \Rightarrow$

$$
H=-\operatorname{arc} / \tau^{2}+\operatorname{lan} x / \tau\left\{\frac{2}{\tau^{2}}+\frac{1}{3 x^{2}}\right\}-i\left(\frac{4 a^{2} a^{*}}{v^{2}}\right)
$$

$80(1.2 .1) \Rightarrow$

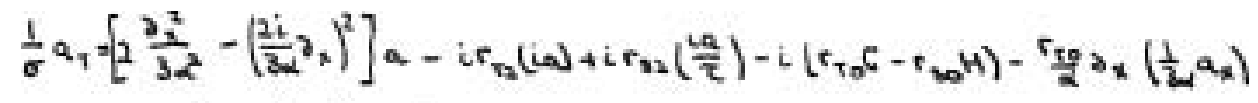

$$
\begin{aligned}
& +\frac{c_{30} z_{x}}{\alpha}\left(\frac{1}{8 \alpha \tau} a_{x}\right)+O(c) \\
& \frac{1}{\sigma} a_{\gamma}=\left(\frac{10}{q_{\alpha}^{2}}-\frac{1}{3 \alpha^{2}} Q_{01}\right) a_{x x}+Q_{21} a^{2}-\left[Q_{02} a_{7}-\frac{5}{9 x^{2}} a_{x x}+4 Q_{01} a^{2} a^{*}\right] \\
& {\left[\frac{1}{6}+Q_{02}\right] a_{\tau}=\frac{4}{3 \alpha^{2}} a_{x x}+Q_{21} a-4 Q_{03} \alpha^{2} \alpha^{4}}
\end{aligned}
$$

Refer to Figure 1. Along the pitchfork bifurcation below $Q_{2} \frac{1}{\sigma}+Q_{02}>0$ and $Q_{0}-0$, and so the bifurcation is supercritical. The equation relaxes into a time independent equilibrium state dependent on the initial conditions, i n which diffusion, which tries to make "a" uniform, becomes weak enough to be counteracted by the nonlinear term, which pulls "a" out toward

$$
|a|^{2}=\left(\frac{1}{0}+Q_{02}\right) / 4 Q_{03}
$$

Above $Q_{1} \frac{1}{\sigma}+Q_{0}>0$ but $Q_{0}<0$ so the bifurcation becomes subcritical. Every initial condition relaxes to $a=0$ or blows up, because if "a" starts growing the $\mathrm{a}^{2} \mathrm{a}^{*}$ term augments its growth as it becomes larger. The only nonzero equilibria are unstable. Near the co-dimension two bifurcation $P, \frac{1}{\sigma} * Q_{0 a}$ becomes very small, so the relaxational effects begin to act much faster due to the ability of the nearly marginal wave-like modes to propagate information more rapidly. Above $\mathbb{P}$ the equation breaks down due to negative diffusion, since it no longer represents a real bifurcation.

$\underline{t}=\mathbf{O}(1):$ The Hopf Bifurcation

The Hopf bifurcation is much more complicated than the direct bifurcation due to the two dispersive nonlinear wave trains in the problem. We must write

$$
a=a_{1}\left(x_{0}, \tau\right) e^{u, t}+a_{-}\left(x_{*}, \tau\right) e^{-1+a t}
$$

where $\tau=\varepsilon t$, not to be confused with the diffusivity ratio. This corresponds to

$$
\psi \sim a_{t}\left(x_{0}, \tau\right) e^{4\left(\alpha x+\omega_{0} t\right)}+\alpha_{-}\left(x_{x}, \tau\right) e^{t\left(\alpha x \cdot \omega_{0} t\right)}
$$


two travelling waves in $\psi$, with slowly varying amplitudes. In general our equations will be structurally stable after considering terms at $O\left(\varepsilon^{2}\right)$ in the modal equations, so we solve for $a_{ \pm}$to that order. Further terms do not alter the qualitative bifurcation structure.

The $O(1)$ terms merely determine $\omega_{s}$, as to this order we have a linear set of homogeneous equations for $a, b, c$ depending on $\omega_{0}$ :

so

$$
\operatorname{det}\left|\begin{array}{ccc}
\frac{\omega_{t}}{d}+1 & \omega_{v 0} & -i s_{v_{0}} \\
-i & \omega_{0}+1 & 0 \\
-i & 0 & \omega_{0}+t
\end{array}\right|=0
$$

$$
\omega_{0}^{s}-i \omega_{0}^{2}\{1+\sigma+\tau\}-\omega_{0}\{\sigma+\tau+\sigma \tau\}+i \sigma \tau+\omega_{0}\left(\sigma \tau_{\tau 0}\right)-i \sigma \tau e_{\tau 0}-\omega_{0}\left[\sigma \tau_{\nu 0}\right]+\sigma c_{0} i=0
$$

Require $\omega_{0}$ is a real frequency and separate real and imaginary parts:

$$
(1.3 .2 a)
$$

$$
\omega_{0}^{2}\left[1+\sigma_{1} \tau\right]=\sigma \tau\left[1-\left(\tau_{0}{ }^{-} \mathrm{se} / \tau\right)\right]
$$

and

$$
\omega_{0}^{2}-(\sigma+\tau+\sigma \tau)+\sigma\left(\varepsilon+\tau_{0}-\varepsilon_{s 0}\right)=0
$$

so from $(1.3 .2)$

$(1.3 .3)$

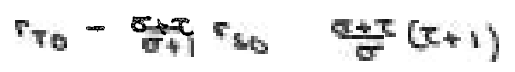

which defines the Nopf bifurcation. Note $\omega_{0} \rightarrow 0$ as $5_{T 0}-{ }^{5} \rightarrow 1$, near the co-dimension two point $P$. To this order we have

$$
\begin{aligned}
& b_{x}=\frac{6 a_{2}}{1+6 \omega_{0}}+\varepsilon g_{x} \\
& d_{t}=\frac{\operatorname{co}_{t}}{\tau_{2} \omega_{0}}+e h_{t}
\end{aligned}
$$

The mean fields $C$ and $E$ must be expanded

$$
\begin{aligned}
& C=C_{2}(x, \tau) e^{2 \ln t}+C_{0}(x, \tau)+C_{-2}(x, \tau) e^{-21 \omega_{0} t} \\
& E=E_{2}(x, \tau) e^{2 \operatorname{lng} t}+E_{0}(x, \tau)+E_{-2}(x, \tau) e^{-21 \omega_{0} t}
\end{aligned}
$$

From (1.2.3) and $(1.2 .5)$ we see

$$
\begin{aligned}
& c_{0}=\frac{4}{1+\omega_{0}^{2}}\left\{\left|a_{1}\right|^{2}+\left|a_{-}\right|^{2}\right\}+O(\varepsilon) \\
& E_{0}=\frac{4}{t^{2}+\omega_{0}^{2}}\left\{\left|a_{4}\right|^{2}+\left|a_{0}\right|^{2}\right\}+O(\varepsilon) \\
& c_{ \pm 2}=\frac{4 \omega_{0}}{\left(1+\omega_{0}\right)\left(\cos =2 \omega_{0}\right)} a_{4}^{*} a_{2} \quad+O(\varepsilon) \\
& E_{ \pm 2}=\frac{4 \infty}{\left(\tau \neq \omega \omega_{0}\right)\left(\tau \omega \pi+2 \omega 0_{0}\right)} a_{x+1}^{*}+O(\varepsilon)
\end{aligned}
$$

Now we look at $O(E)$ terms to find $g_{ \pm}$and $h_{ \pm}$ 
$-211-$

From $(1.2 .2)$

$$
\begin{aligned}
& \frac{\omega a_{s t}}{1 \pm \omega \omega_{0}} \pm \omega \omega_{0} g_{1}=\frac{2 i}{3 \alpha} \frac{\omega a_{2 x}}{1 \pm i \omega_{0}}-g_{ \pm}+\frac{1}{\alpha} a_{2 x} \\
& g_{1}=\frac{1}{2} a_{2 x}\left\{\frac{1}{1+\omega_{0}}-\frac{3}{3} \frac{1}{\left(1+\omega_{0}\right)^{2}}\right\}-\frac{1 a_{21}}{\left(1+\omega_{0}\right)^{2}}+2 C
\end{aligned}
$$

Similarly from $(1.2 .4)$

$$
h_{t}=\frac{1}{\alpha} a_{2 x}\left\{\frac{1}{\tau=\omega_{0}}-\frac{2}{3} \frac{1}{\left(\tau=\omega_{0}\right)^{2}}\right\}-\frac{\left(a_{2 \pi}\right.}{\left(\tau+\omega_{0}\right)^{2}}+\tau H
$$

To find $\boldsymbol{C}_{ \pm}$and $\mathrm{H}_{*}$ we proceed to $\mathrm{O}\left(\mathrm{\varepsilon}^{2}\right)$ From $(1.2 .2)$

$$
\begin{aligned}
& =\omega_{0} C_{t}+g=\tau=\frac{1}{3 \omega^{2}} \frac{i A_{2 x x}}{1+\omega_{0}}+\frac{3 i}{3 \alpha} g_{ \pm x}-C_{t}-i\left\{A_{5} C_{0}+A_{x} C_{t 2}\right\}+O(c) \\
& G_{ \pm}=-\frac{g \pm \tau}{1+1 \omega_{0}}+\frac{i}{3 \alpha^{2}} \frac{a_{2 \times x}}{\left(1 \pm+0_{0}\right)^{2}}+\frac{2 i}{3 \alpha} \frac{g_{x x}}{1+\omega_{0}}-\frac{i}{1+\omega_{0}}\left\{A_{1} C_{0}+A_{x} C_{22}\right\}+O(\varepsilon)
\end{aligned}
$$

Similarly from $(1.2 .4)$

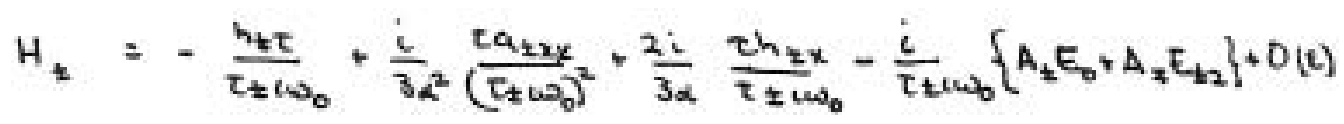

Now we substitute the expressions for b in terms of $a, g, G$, and $d$ in terms of $a, h, H$ into $(1.2 .1)$, keeping terms through $O\left(\varepsilon^{2}\right)$. Define

$$
R_{m n}^{(k)}=\frac{r_{+m}}{\left(1+\omega_{0}\right)^{n}}-\frac{\tau^{k} r_{3 m}}{\left(\tau=\omega_{0}\right)^{n}}
$$

We see

$$
\begin{aligned}
& (1-\varepsilon \Delta)\left[t \frac{c \omega_{2}}{\sigma} a_{2}+\frac{\varepsilon}{\sigma} a_{2 \tau}\right]=-(1-c \Delta)^{2} a_{t}-\left[r_{r_{0}} b_{2}-r_{\varepsilon_{0}} \alpha_{t}\right]-\frac{\varepsilon}{\alpha}\left[r_{20} b_{t}-r_{20} d_{t}\right] x
\end{aligned}
$$

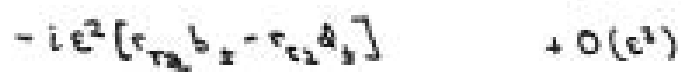

It pays to work in stages, going down successive powers of $\varepsilon$. In fact, reducing the $O(1)$ terms to functions of "a" alone,

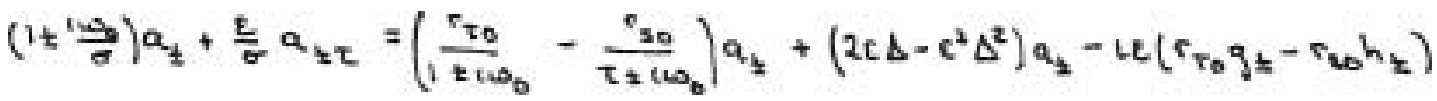

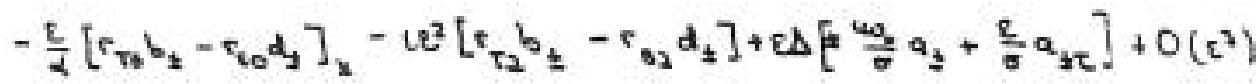


Due to the linear dispersion the $O(1)$ terms cancel. By use of the linear frequency relations $(1.3 .2 a-b)$ we see

$$
\begin{aligned}
& \frac{r 10}{1+\omega_{0}^{2}}-\frac{r_{00}}{\tau^{2}+\omega_{0}^{2}}=-\frac{1}{\sigma} \\
& \frac{r 10}{1+\omega_{0}^{2}}-\frac{\tau+10}{\tau^{2}+\omega_{0}^{2}}=1
\end{aligned}
$$

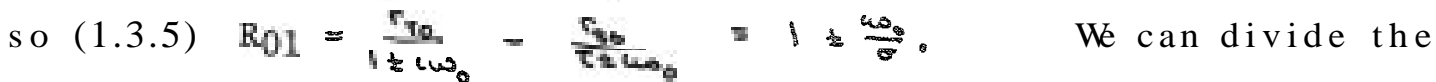
equation for "a" by $\varepsilon$ and reduce the $O(\varepsilon)$ terms.

$$
\begin{aligned}
& \frac{1}{\sigma} a_{t \tau}=\frac{4 i}{3 \alpha} a_{2 x}+\frac{2 \varepsilon}{3 \alpha^{2}} a_{t \times x}-\varepsilon \Delta^{2} a_{t}-2\left\{\tau_{00} a_{2}-r_{00} h_{t}\right\}-\frac{i}{\alpha} a_{t=x}\left\{\frac{T_{0}}{1+6 \omega_{0}}-\frac{r_{00}}{\tau=6 \omega_{0}}\right\} \\
& +\left(=\frac{\log }{\sigma}\right) \Delta a_{2}+\frac{\varepsilon}{\sigma} \Delta a_{2 \tau}-\frac{\varepsilon}{\alpha}\left(r_{\tau_{0}} g_{t}-r_{30} h_{1}\right)_{x}-L_{\varepsilon}\left(c_{r_{2}} b_{2}-c_{v_{2}} d_{t}\right)+O\left(\varepsilon^{2}\right)
\end{aligned}
$$

Now

$$
r_{T_{0}} g_{2}-r_{50} h_{t}=\frac{1}{\alpha} a_{5} k\left\{R_{0_{1}}-\frac{2}{3} R_{02}^{[1]}\right\}-i R_{02} a_{t}+\varepsilon\left\{r_{t_{0}} c_{2}-r_{s_{0}} H_{t}\right\}
$$

but

$$
R_{02}^{(1)}=R_{01} \mp \omega_{20} R_{B 2}
$$

so

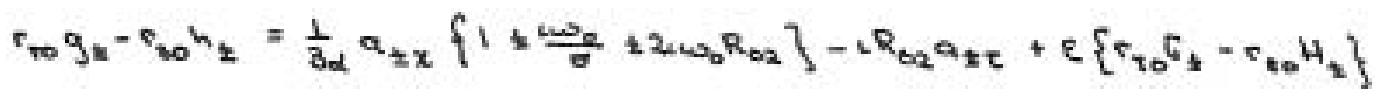

and thus our "a" equation reads

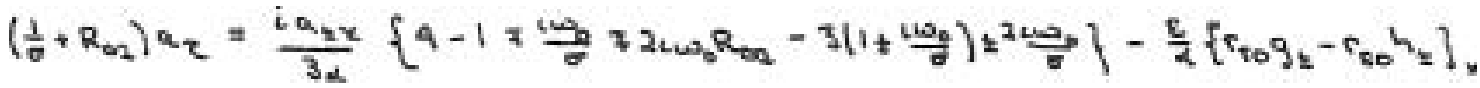

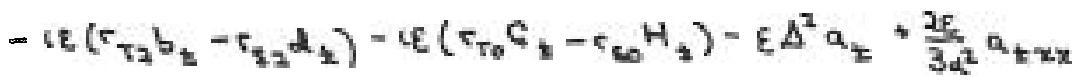

$$
\begin{aligned}
& \text { t. }
\end{aligned}
$$

or, combining the $O(\mathbb{1})$ terms while expanding the $O(\varepsilon)$ terms,

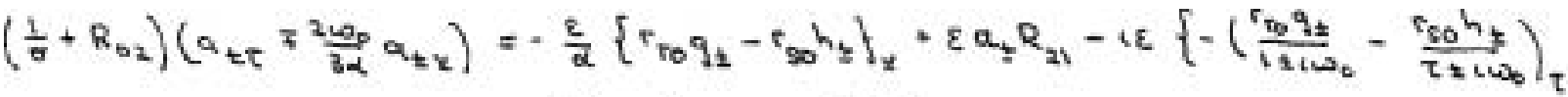

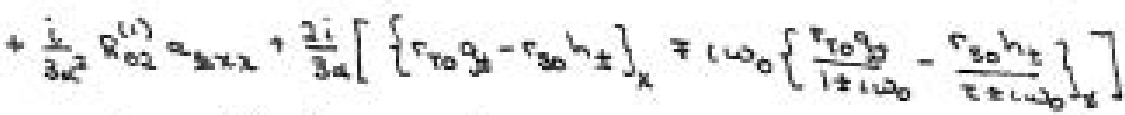

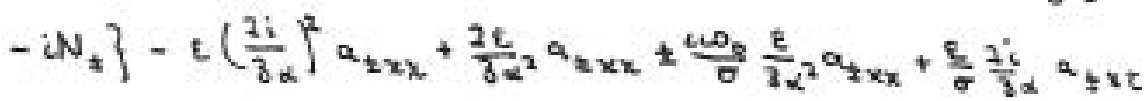


$-213-$

$\mathrm{N}$ combines the nonlinear terms

$(1,3,7) \quad N_{2}=\frac{C_{T 0}}{1+\omega_{0}}\left\{A_{t} C_{0}+A_{7} C_{22}\right\}-\frac{C_{0}}{\tau_{2 \omega_{0}}}\left\{A_{2} E_{0}+A_{x} E_{t_{2}}\right\}$

Now, we remember $(1.3 .6)$ and write

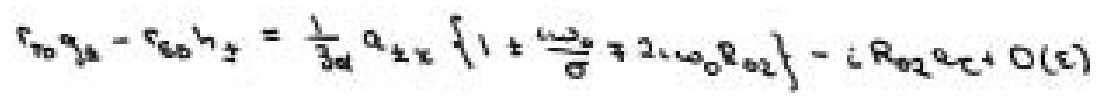

However, $(1.3 .8)$

$$
a_{x t}=\frac{2 b_{0}}{3 \alpha} a_{b x}=O(c)
$$

so

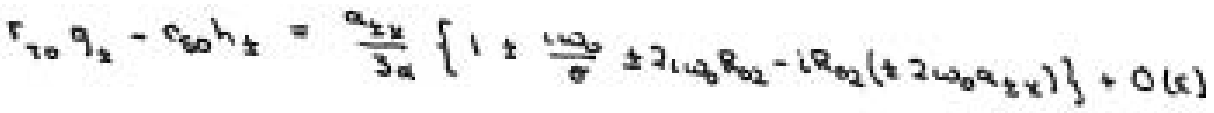

$$
\begin{aligned}
& =\frac{a_{2 x}}{3 x}\left\{1=\frac{n_{0}}{0}\right\}+O(8)
\end{aligned}
$$

and

$$
\frac{\varepsilon}{6} \frac{2 L}{3 a x} a_{x x}= \pm \frac{4 w_{0}}{9 x^{2} y} a_{2 x z}+O(\varepsilon)
$$

and

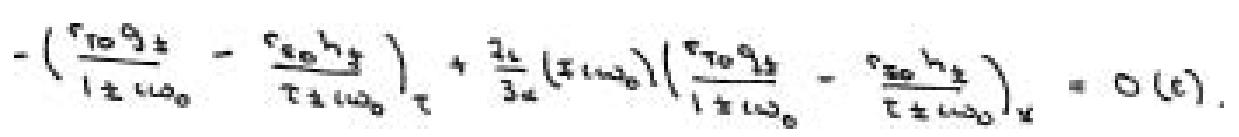

Our equation for "a" simplifies to

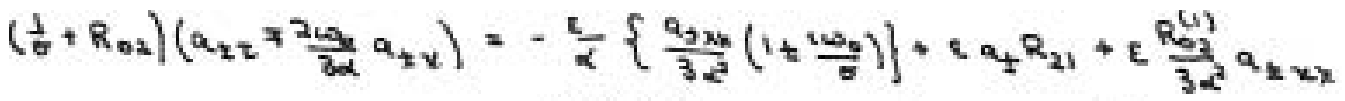

$$
\begin{aligned}
& +\frac{2 \varepsilon}{3 \alpha}\left\{\frac{a_{2 x x}}{3 \alpha}\left(1+\frac{\omega_{0}}{\sigma}\right)\right\}-\varepsilon N_{z}+\frac{4 c}{a_{\alpha^{2}}} a_{2 \pi x}+\frac{7 L}{3 \alpha^{2}} a_{ \pm x x}
\end{aligned}
$$

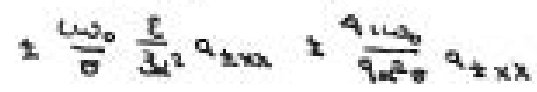

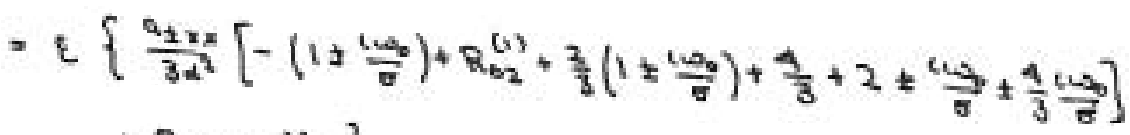

$$
\begin{aligned}
& \left.+R_{21} a_{2}-N_{2}\right\} \\
& =e\left\{\frac{a_{t x x}}{3 x^{2}}\left\{4+\omega_{0}\left[\begin{array}{ll}
\frac{3}{v} & R_{02}
\end{array}\right]\right\}+R_{21} a_{2}-N_{*}\right.
\end{aligned}
$$

Thus we can write $(1.3 .9)$

$$
a_{2 \tau}=\frac{2 \omega_{0}}{3 d_{1}} a_{2 x}=c F_{x}\left(a_{2}\right)+O\left(\tau^{2}\right)
$$


where

$$
\begin{aligned}
& Q_{1}=Q_{1} \neq L Q_{2}=\frac{1}{6}+R_{O_{2}} \\
& \eta_{2}=\eta_{1}+\eta_{2}=R_{21} / Q_{1} \\
& \mu_{2}=\mu_{1}=c \mu_{2}=\frac{1}{R_{2}}\left\{\frac{4}{3 \alpha^{2}}=\frac{\mu_{0}}{\alpha^{2}}\left\{\frac{1}{\sigma}-\frac{1}{3} R_{02}=\right\}\right. \\
& m_{6}=m_{1} 2 m_{2}=\frac{1}{Q_{2}}\left\{\frac{r_{0}}{1+\omega_{0}}\left(\frac{4}{1+\omega_{0}^{2}}\right)-\frac{r_{20}}{\tau \varepsilon \omega_{0}}\left(\frac{4}{\tau^{2}+\omega_{0}^{2}}\right)\right\}
\end{aligned}
$$

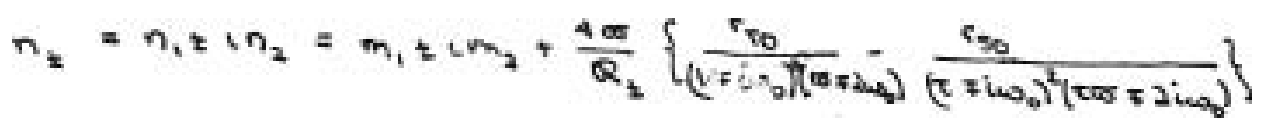

(1.3.9) and (1.3.10) constitute the relaxational equations for the Hopf bifurcation. There are three complications beyond the Van-der-Pol equation we found for the pitchfork bifurcation. Firstly, the equations mix orders in $B$. To leading order they are linear uncoupled equations for the amplitudes a $a_{\ddagger}$ the carrier waves. Now the amplitudes are propagated along with group velocities $10 \%$ which are $\pm^{200 / 3 x}$ However, 0 at the critical wave number so the dissipative effects act only at next order.

Secondly, at this next order they are two coupled equations. No longer does one amplitude equation suffice to describe the dynamics, since each wave feels the other wave's average amplitude as a damping,

Thirdly, the coefficients of $F(a)$ are complex. The real parts represent damping and instability terms, but these same terms also contribute frequency shifts. In the 1 imit $w_{s} \rightarrow 0$, when we approach the co-dimension two bifurcation,

$$
R_{t}=\frac{1}{\sigma}+R_{022} \rightarrow \frac{1}{\sigma}+\frac{s_{v_{0}}}{\left(1+\omega_{b}\right)^{2}}-\frac{s_{00}}{\left(T+\omega_{0}\right)^{2}} \rightarrow \frac{1}{\sigma}+Q_{02}+1 \omega_{0}\left(-2 Q_{03}\right) \cdot 0\left(\omega_{0}^{2}\right)
$$

Now, in fact, $\partial " Q_{O_{2}}=0$ at the co-dimension two bifurcation, and is $O\left(\omega_{0}^{2}\right)$ forfinite $\omega_{0}, 50$

Similarly, we see

$$
\begin{aligned}
& Q_{+} \rightarrow \pi \operatorname{lin}\left(-2 Q_{a}\right) \\
& \eta_{2} \rightarrow-\frac{1}{42 n_{0}} \frac{Q_{23}}{Q_{03}} \\
& \mu_{x} \rightarrow-\frac{4}{\operatorname{lin}_{0}} \frac{1}{3 \alpha^{2}\left(-Q_{03}\right)} \\
& m=\rightarrow-\frac{4}{62+4} \\
& n_{1} \rightarrow-\frac{4}{12 \omega_{0}}
\end{aligned}
$$

so a 11 the coefficients become purely imaginary and the equation becomes, evt $\mathbb{1}$ to $O(\varepsilon)$, conservative. We will explore a limit in the equations near the co-dimension two bifurcation which allow exploration of this case to next order, and will, in fact, produce slowly varying solitons. 
I have not thoroughly explored this equation, which is probably typical of a Hopf bifurcation in which horizontal waves are allowed. Some interesting questions are: Is the state $a_{+}=\mathrm{a}-\mathrm{stable?}$ Is the state of spatially uniform amplitudes stable? Many of the results I discuss in the co-dimension two case may, in fact, be primarily associated with the Hopf bifurcation, although ironically they are easier to study when $\omega_{\text {。 }}$ is small and we are near the eo-dimension two bifurcation.

\subsection{The Equations in Co-dimension Two}

If we let $t_{\infty}=\varepsilon t$ be the fastest time in the problem and solve for the modal amplitude, we recover the co-dimension two bifurcation. As the above discussion hints, we must go to $O\left(\varepsilon^{3}\right)$ to get a structurally stable result.

$\underline{\text { Dominant Balance }}$

$$
\begin{aligned}
& -a-4 s_{20} b+c s_{50} d=0 \\
& \text { ca-b }=0 \\
& \text { ca-rd }=0 \\
& -c+2 i\left(a b^{*}-a^{*} b\right)=0 \\
& -\tau E+2 i\left(a d^{2}-a^{*} d\right)=0 \\
& b=c a+\varepsilon g \\
& d=t \frac{a}{\tau}+e h \\
& c=4 a a^{2}+2 k \\
& E=\frac{4 a Q^{*}}{t^{2}}+e l
\end{aligned}
$$$$
\text { ( }
$$

and, using ${ }^{8}-\frac{c_{0}}{T^{2}}=-$, and dropping the stars on $x, t$, we see

$$
A_{2}=-\frac{3}{\sigma^{2}}\left\{t^{2}-\frac{2}{\tau}\right\} z_{x}\left(4 a a^{*}\right)-\frac{3}{40} \partial_{x}\left(a a^{*}\right)=\frac{1}{\sigma}\left(2 a^{*}\right)_{x}\left\{\frac{3.4}{\left(\frac{8}{3}\right)^{2}}-\frac{3}{4}\right\}+O(L)
$$

$(1.4 .1) \quad A_{2}=\frac{15}{160}\left(a 0^{*}\right)_{x}+O(\varepsilon)$

$\underline{\text { Iterate Equation for } b}$

$$
\begin{aligned}
& 1 \varepsilon \dot{a}+\varepsilon^{2} \dot{g}=-(1-\varepsilon \Delta)(1 a+\varepsilon g)+\frac{D_{1} a}{\alpha} a-\frac{\varepsilon^{2}}{\alpha}\left[D_{1} a\left(4 a a^{2}+\varepsilon k\right)+\frac{1}{2} a c\left[\left(B a a^{2}\right)+O(\varepsilon)\right]+\frac{\varepsilon^{2}}{q^{2}} L(L a) \lambda_{2}+O\left(\varepsilon^{4}\right)\right. \\
& g=-i a+\frac{1}{\alpha} a,+u a+\varepsilon[\Delta-\partial,] g-\frac{2}{\alpha}\left[0, a \cdot 4 a a^{2}+c \alpha \varepsilon a k+2 \varepsilon a\left(a \alpha^{*}\right)_{x}\right]-\frac{c^{3}}{q^{2}} \alpha a+a+0\left(c^{3}\right)
\end{aligned}
$$




$$
\begin{aligned}
& g=-i a+\frac{1}{\alpha} a_{x}+i \Delta a-\frac{\varepsilon}{\alpha}\left[0, a \cdot 4 a a^{x}\right]-\frac{e^{2}}{\alpha}\left[\left(\alpha a k+2 a\left(a a^{2}\right)_{x}\right]-\frac{e^{2}}{q^{2}}<a A_{2}\right. \\
& +2\left(\Delta-\partial_{t}\right)\left(-i a+\frac{1}{\alpha} a_{x}+i \Delta a-\frac{\varepsilon}{\alpha} D_{1} a\left(4 a a^{x}\right)\right)+e^{2}\left[\Delta-\partial c^{2}\right]^{2}\left(-c a+\frac{1}{\alpha} a_{x}+i \Delta a\right)+0\left(c^{3}\right) \\
& g=-i \dot{a}+\frac{1}{\alpha} a_{x}+i \Delta a+c\left[-\frac{\Delta D_{1}}{\alpha} a^{2} a^{*}+\left(\Delta-\partial_{2}\right)\left(-i a+\frac{1}{\alpha} a_{n}+i \Delta a\right)\right] \\
& +e^{2}\left[-\frac{2}{\alpha} a\left(a a^{*}\right)_{x}-c a k-\left(\Delta-\partial_{t}\right)\left(4\left(a\left(a a^{*}\right)\right)+\left(\Delta-\partial_{t}\right)^{2}\left(-c a+\frac{1}{\alpha} a_{x}+c \Delta a\right)-\frac{\alpha}{q} a \lambda_{2}\right]\right. \\
& +O\left(e^{8}\right)
\end{aligned}
$$

Similarly from $(1.1 .4)$

$(1.4 .3)$

$$
\begin{aligned}
& n=-\frac{c a_{2}}{\tau}+\frac{1}{\tau} a_{1}+\pi \Delta a+\varepsilon\left[-\frac{4 D_{1}}{\tau_{\alpha}} a^{2} a^{2}+\left(\Delta-\frac{1}{\tau} \partial_{6}\right)\left(-\frac{i a_{2}}{\tau^{2}}+\frac{1}{\tau \alpha} a_{3}+\frac{i}{\tau} \Delta a\right)\right] \\
& +c^{2}\left[-\frac{2}{\alpha \tau^{3}} a\left(a a^{3}\right)_{x}-\frac{1 a}{\tau} l-\left(\Delta-\frac{L}{\tau} \partial_{4}\right)\left(4 i \frac{a^{2} a^{*}}{\tau^{3}}\right)+\left(\Delta-\frac{1}{\tau} \partial_{t}\right)^{2}\left(-\frac{L a}{\tau^{2}}+\frac{a_{x}}{\tau \alpha}+\frac{\Delta a}{\tau}\right)\right. \\
& \left.-\frac{\alpha}{\tau^{2} q^{2}}+A_{2}\right]+O\left(e^{2}\right)
\end{aligned}
$$

where $k$ and $\ell$ are determined from (1.1.3) and (1.1.5)

$(1.4 .4)$

$$
\begin{aligned}
& \varepsilon\left(4 a a^{*}\right)=\sigma\left(-e k+\frac{1}{\alpha}\left(\varepsilon a b^{*}+\varepsilon a_{x}^{*} b-\varepsilon a b_{x}^{*}-\varepsilon a^{*} b_{x}\right)+\frac{2 \alpha}{\alpha} \varepsilon\left[a q^{*}-a g^{*}+a g^{*}-a^{*} g\right]+Q c^{*}\right)
\end{aligned}
$$

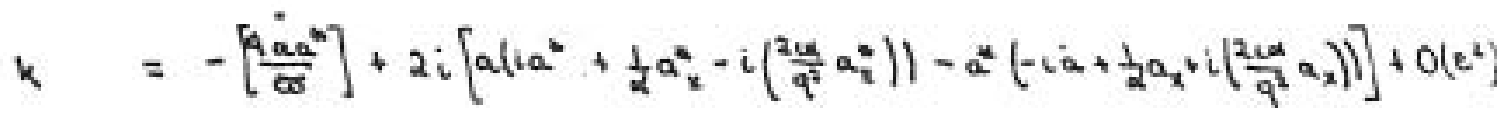

$$
\begin{aligned}
& k=-\left(2+\frac{4}{\infty}\right)\left(a+a^{*}\right)-2 i\left[\frac{1}{2}-\frac{2 a}{q^{2}}\right]\left(2 a_{x}-a a_{x}^{*}\right)+O(\varepsilon) \\
& k=-\frac{7}{2}\left(\dot{a}^{*}\right)-\frac{2 i}{2} \alpha^{*}\left(a^{n} a_{x}-a a_{x}^{*}\right)+o(z)
\end{aligned}
$$

Similarly

(1.4.5)

$$
\text { l } \quad-\frac{z}{2} \frac{\left(a a^{*}\right)}{\tau^{\gamma}}-\frac{2 i}{34} c_{0}\left(a^{*} a_{x}-a a_{x}^{*}\right)+O(\varepsilon)
$$

Equations (1.4.2) (with $k$ as above) and (1.4.3) (with $k$ as above) now can be be substituted into (1.1.1) to find the Duffing equation for a. Write (1.1.1) in its component terms, dividing out a factor $\mathrm{g}$

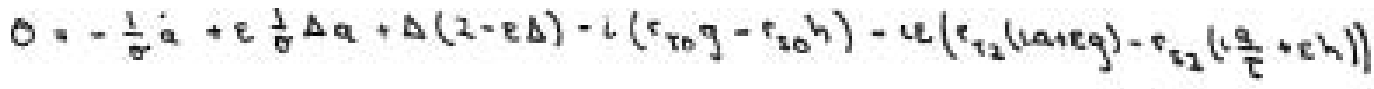

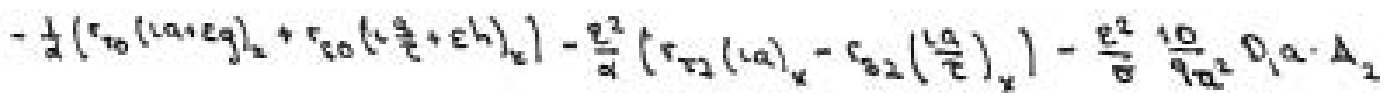


Expand the component $=c$ sues in powers of $\varepsilon$. Factors like $a, g, i=\frac{2}{\partial t}$

are not expanded, but it is assumed that they are $0(1)$ with posatbia of b,$O\left(e^{2}\right)$,. dependence as well:

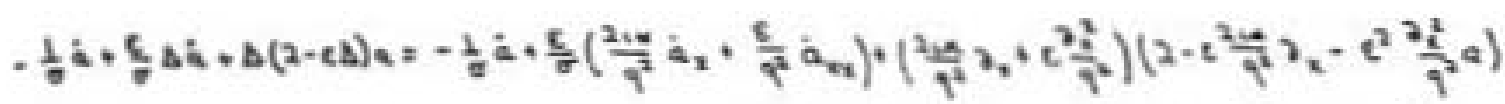

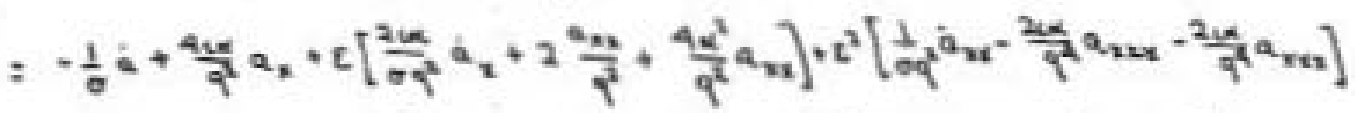

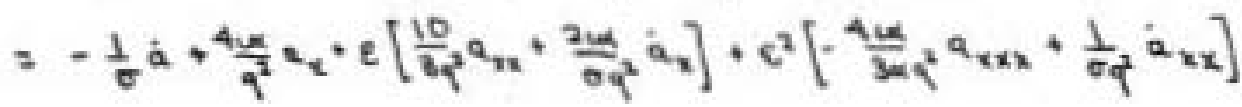

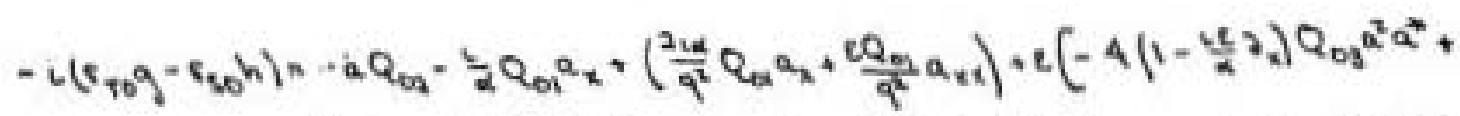

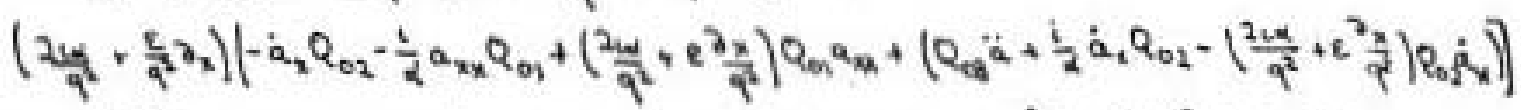

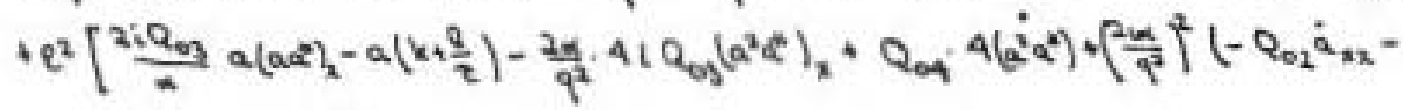

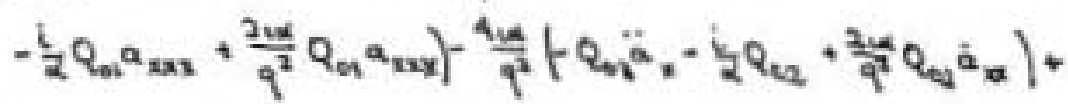

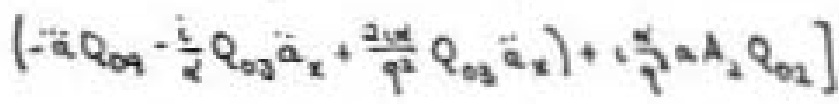

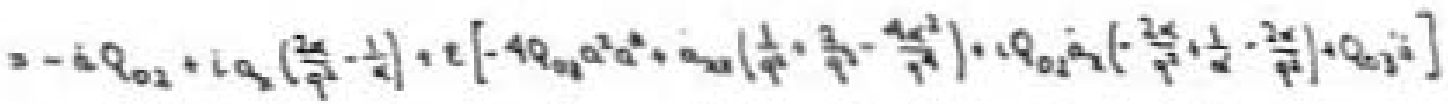

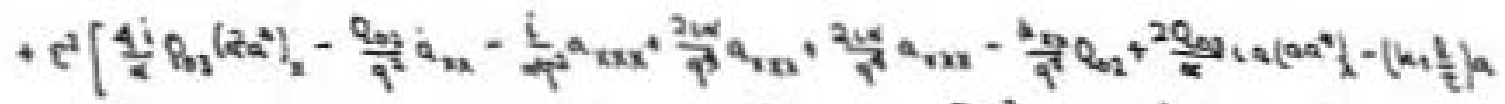

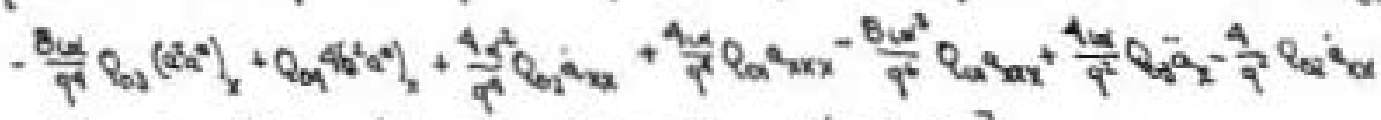

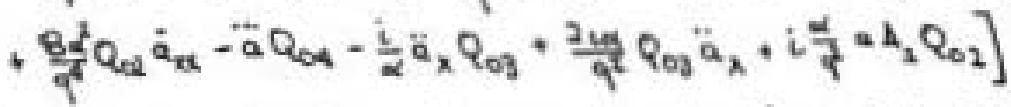

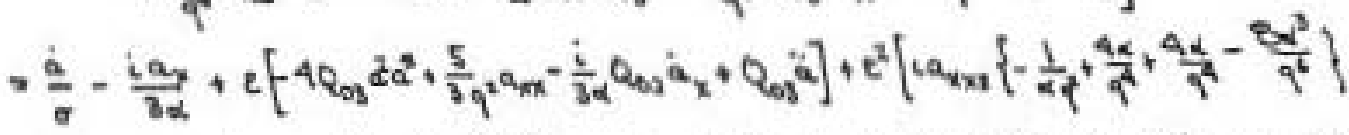

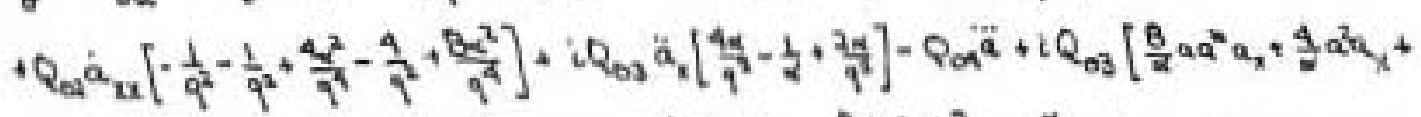

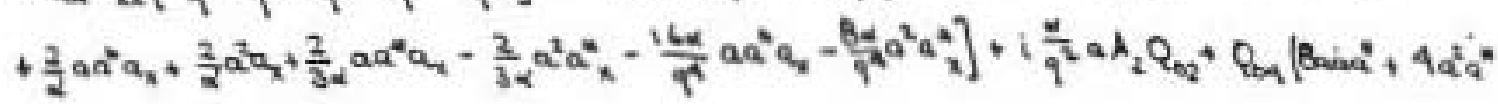

$$
\begin{aligned}
& \left.\left.-\dddot{a}+\frac{z}{2} a\left(a^{*}\right)\right)\right] \quad+O\left(c^{2}\right)
\end{aligned}
$$

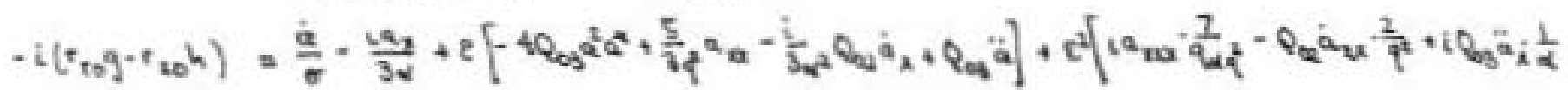

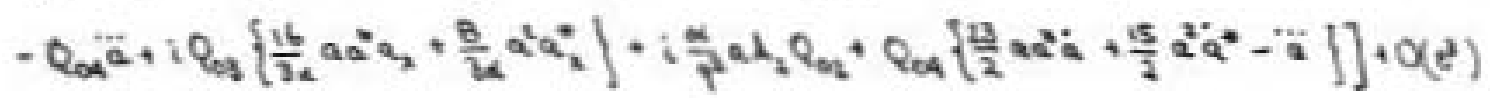

$$
\begin{aligned}
& -i e\left(r_{t_{2}}(t a+2 g)-r_{32}\left(t \frac{q}{t}+\varepsilon h\right)\right)=e Q_{21} a-i \varepsilon^{2}\left(r_{t_{2}} g-r_{22} h\right) \\
& =e Q_{21} a^{2}+e^{2}\left[-Q_{22} a-\frac{a_{2}}{B_{\alpha 2}} Q_{21}\right]+O\left(\varepsilon^{2}\right)
\end{aligned}
$$


Add the boxed terms to get the final equation:

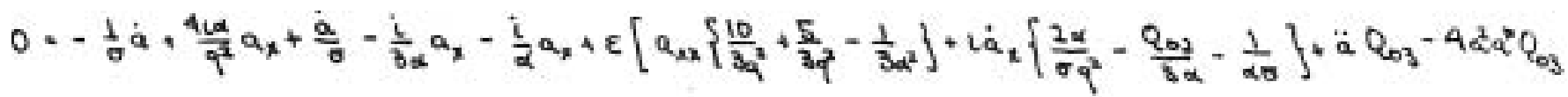

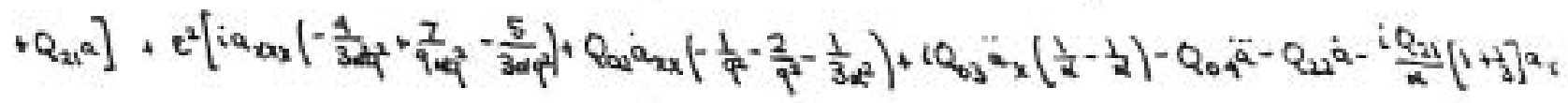

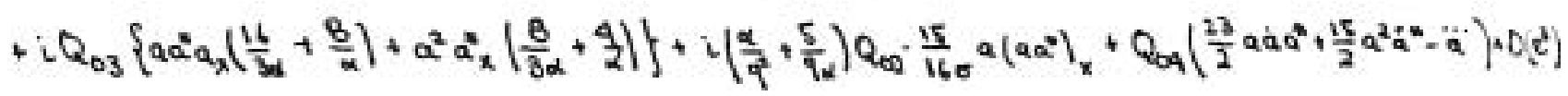

The zeroth order cancels, and the a, term (since $Q_{02}=-\frac{1}{\sigma}$ ) is zero, so dividing by $\varepsilon$ :

$$
Q_{03} \ddot{\alpha}+\frac{4}{3 \alpha^{2}} a_{x y}+Q_{21} a-4 a^{2} a^{2} Q_{03}=e F(a)
$$

where

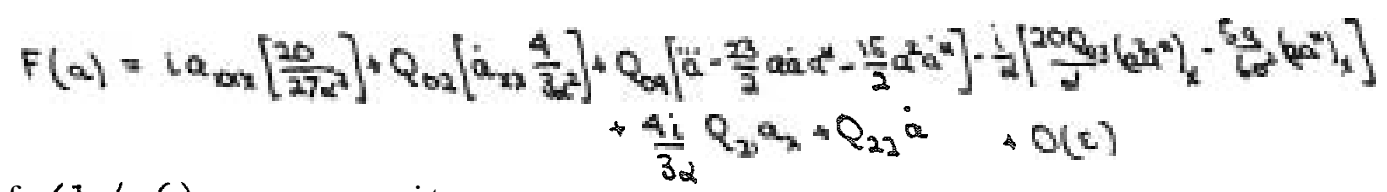

Upon use of $(1.4 .6)$ we can write

$$
\dddot{a}=4\left(a^{2} a^{2}\right)-\frac{Q_{23}}{Q_{03}} \dot{a}-\frac{a}{32 Q_{03}} a_{x x}
$$

so

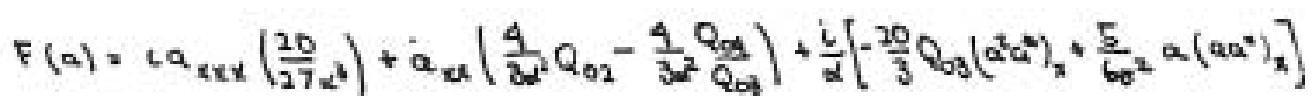

$$
\begin{aligned}
& -\frac{7}{2} Q_{B 4} a\left(\dot{c o s}^{\circ}\right)+\operatorname{co}_{1} \frac{q}{3 \alpha} Q_{21}+\left[Q_{22}-\frac{Q_{21}}{Q_{03}} Q_{0 A}\right] \dot{a}
\end{aligned}
$$

$(1.4 .8)$

where

We find:

$$
\begin{aligned}
& \ddot{a}-a_{\mathrm{xx}}+\gamma a-a^{2} a^{y}=e f(a)+Q\left(e^{2}\right) \\
& f(a)=-i c_{1} a_{x x x}+c_{2} \dot{a}_{x x}-i\left[c_{3}\left(a^{2} \alpha^{*}\right)_{x}+c_{4} a\left(a a^{*}\right)_{x}\right]-c_{5} a\left(a_{a}^{*}\right)+c c_{1}^{8} a_{x}+\lambda \dot{a}
\end{aligned}
$$

$$
\begin{aligned}
& \gamma=\frac{Q_{23}}{Q_{03}} \quad \lambda=\frac{Q_{23}}{Q_{03}}-\gamma \frac{Q_{03}}{Q_{03}} \\
& c_{1}=\frac{5}{18}\left(-Q_{02}\right)^{\frac{1}{2}}>0 \\
& c_{4}=\frac{5}{16 \sigma^{2}}\left(-\frac{1}{3 Q_{05}}\right)^{2}>0 \\
& c_{2}=\frac{Q_{\mathrm{OA}_{3}}}{Q_{\mathrm{OB}}}-Q_{\mathrm{O}_{2}}>0 \\
& c_{5}=\frac{7}{8} \frac{Q 04}{004}>0 \\
& c_{3}=\frac{5}{16 \sigma^{2}}\left(-\frac{1}{3 Q_{n 3}}\right)^{\frac{1}{2}}>0 \\
& c_{6}=\frac{2}{3}\left(-3 Q_{03}\right)^{1}>0
\end{aligned}
$$


We remember

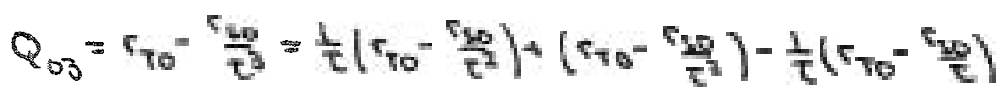

so

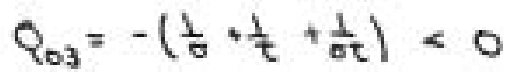

similarly

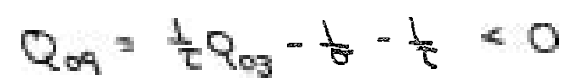

and

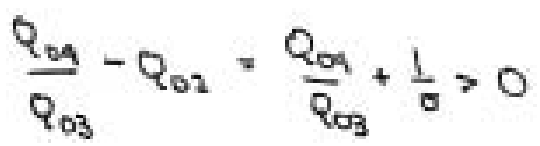

This gives us the signs of a 11 the $\mathrm{c}_{\mathbf{i}}{ }^{9} \mathrm{~s}$. Note that $c_{1}-\mathrm{c}_{6}$ depend only on $a, t$ and not on $s_{s_{2}}, r_{s_{2}}$. The dependence on $v_{v_{2}}, \nabla_{s_{3}}$ is seen only in the linear terms through 8 and $\lambda$.

All of the linear terms in (13) have been checked against a direct Fourier analysis of the original p.d.e s. All the purely time dependent terms have been checked against (Knobloch and Proctor, 1981). The calculation of the conservative terms was checked against an amplitude expansion. Thus, I have some confidence in the correctness of the equation (14); only $c_{3}$ and $c_{4}$ cannot be checked at all.

\section{Waves in the Co-dimension Two Equation}

In the second half of this report, we will go in more depthinto the solutions of the codimension two amplitude equation. The reason that we look at this equation, which of the three amplitude equations is the one with the smallest region of validity and contains the most complex physics is threefold.

First, it is a canonical equation. It is the simplest equation which summarized both physical instabilities in a large aspect ratio context. As such, it has a qualitative validity far beyond its formal limit, The only important effect it misses is the stabilization of the subcritical direct instability.

Perhaps more fascinating is the structure of the equation -- a nonlinear wave equation in a strongly dissipative medium. The waves themselves are a balance of forcing by buoyancy and dissipation by thermal and viscous diffusion. The exact balance between the effects detemines the average wave energy. But in our equation the time scale of wave motion is fast compared to energetic changes, which is very handy for analysis.

Lastly, the author found its logical precursor, the equation for the Hopf bifurcation, while investigating co-dimension two, and therefore has not had time to thoroughly investigate it. 
$\underline{2.1}$ The Structure of the Amplitude Equation

First, I would like to rationalize the equation (1.4.8). For convenience we write it again:

$(2.1 .1)$

$$
a_{t x}-a_{x x}+8 a-a^{2} a^{*}=\varepsilon F(a)
$$

$$
F(a)=-i c_{1} a_{x \times x}+c_{2} a_{x \times t}-i\left[c_{3}\left(a^{2} a^{4}\right)_{x}+c_{4} a\left(a a^{*} x_{x}\right]-c_{5} a\left(a a^{*}\right)_{t}+i c_{6} x_{a x}+\lambda a_{2}\right.
$$

$c_{1}-c_{6}$ are positive constant depending on $\sigma, \tau \quad$ The parameters $\gamma$, $r s$, the $O\left(\varepsilon^{2}\right)$ Rayleigh number corrections.

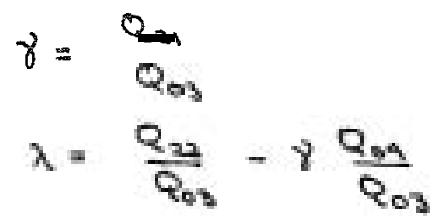

By changing and $\lambda$ we can change the qualitative behavior of the equation. Let's first ascribe a geometrical meaning to them. Look at the bifurcation diagram for $x$-independent solutions (waves at the most unstable wave number), redrawn in Figure 4. Instead of using $r_{T}$ and $r_{S}$ as coordinates of the diagram we can use $\gamma, \lambda$, which are linear combinations of $r_{\mathrm{T}}$ and $r_{\mathrm{S}}$. If we do so, $=\lambda=0$ is the co-dimension two bifurcation, $\gamma=0$ is the pitchfork bifurcation, and $\lambda=0$ is the Hopf bifurcation. We have thus found the natural unfolding parameters for the bifurcation.

Now, let's try to understand (2.1.1) physically. With no $x$-dependence, (2.1.1) with $\gamma>0$ is a soft spring. The restoring force is buoyancy. Over half of the layer depth the mean field forced by the oscillating roll makes conditions locally more favorable for a direct instability and strongly reduces the restoring force, decreasing the spring constant at large

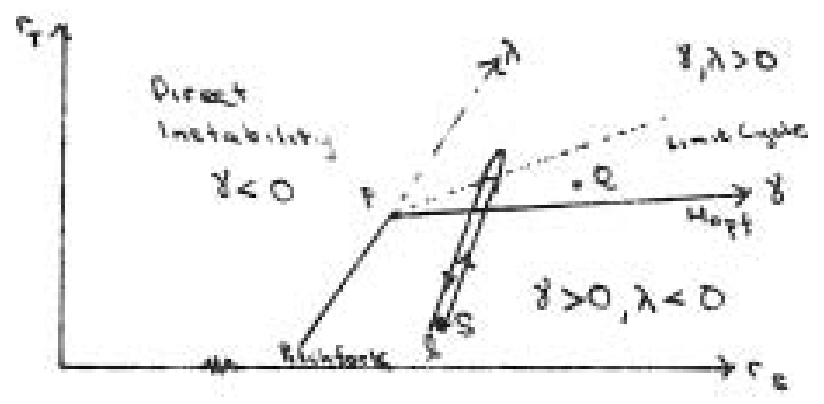

FIGURE 4. The local structure of the bifurcation of rolls of the most unstable wave number. The unfolding coordinates $\forall, \lambda$ are shown. As the roll wave number is changed the position on the diagram moves along the line $\ell$. Point $Q$, where $\gamma=O(1), \lambda=O\left(\mathrm{e}^{2}\right)$, is where slowly varying solitons will be investigated. 
amplitude. Letting the amplitude become $x$-dependent has two effects. Dissipation acts more strongly on rolls not at the most unstable wave number, so rapidly varying "a" increases the dissipation with terms like -ic $6{ }^{a x x}$ in the small forcing $\& F(a)$. But more directly, the effective spring constant depends on the roll wave number, so changes in the $x$-dependence of a cause the oscillations at different places to try to proceed at different rates, causing one to lag behind the other and change the effective wave number, propagating the new oscillation frequency along in $x$. This is the essential wave generating mechanism. I would like to indicate quantitatively how the wave number dependence of the oscillation frequency comes about.

Suppose that for some $x$

\section{$(2.1 .4)$}

so 1 ocally

$(2.1 .5)$

$$
a_{x x} \mid a=-k^{2}(x)
$$

The variable $x$ was a long space scale $x_{*}$ in the original thermohaline equations, and

$$
x_{*}=\frac{\left(-3 Q_{03}\right)^{\frac{1}{2}}}{2}+e_{x}
$$

in the original $x$, scaled by the layer depth. Thus with $a=e^{c k}$

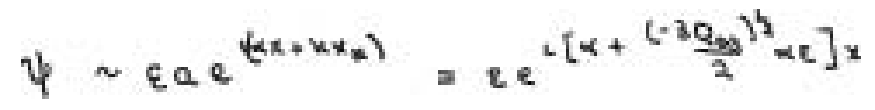

and so describes a roll of slightly different wave number $\alpha^{\prime}=\alpha\left[1+\frac{\left(-3 Q_{a}\right)^{\prime}}{2}\right]$ Mow as discussed in the introduction, the bifurcation diagram varies with roll wave number, because the parameters ${ }^{T}$ and $x_{S}$ vary with $\alpha^{\prime \prime}$ "

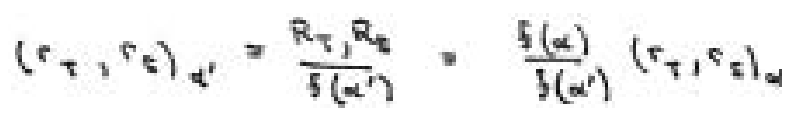

$$
=\left(r_{\tau_{1}} r_{2}\right)_{\alpha}\left[1-\frac{1}{2} \frac{f^{\prime \prime}(\alpha)}{f(\alpha)}\left(\alpha^{\prime}-\alpha\right)^{2}-\frac{1}{6} \frac{f^{n n}(\alpha)}{f(\alpha)}\left(\alpha^{\prime}-\alpha\right)^{3} \ldots\right]
$$

where

$$
f\left(\alpha^{\prime}\right)=\frac{\left(a^{2}+\alpha^{2}\right)^{3}}{\alpha^{\prime 2}}
$$

so since $\alpha=\frac{\pi}{\sqrt{3}}$

$$
f(\alpha)=\frac{27}{4} \pi^{4} ; f^{\prime \prime}(\alpha)=36 \pi^{2}
$$

Refer now to the bifurcation diagram of Figure 3. If $\left(r_{T}, r_{S}\right)$ lies at sone point $Q,\left(r_{T}, r_{S}\right) *$, Pies on the line connecting $Q$ to $r_{T}=0$, $r_{S}=0$, callit $\&$ Knowing $\alpha^{\prime}$ we find 


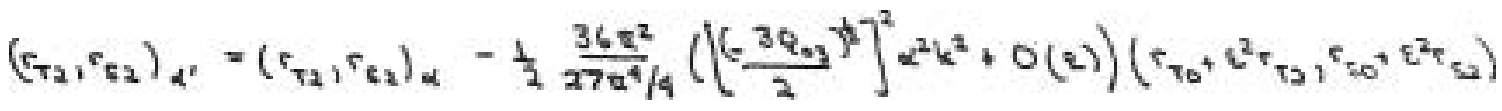

$$
\begin{aligned}
& =\left(c_{T_{2}}, s_{52}\right)_{\alpha}+Q_{03}\left(s_{10}, s_{30}\right)_{\alpha} k^{2}
\end{aligned}
$$

Thus the effective linear spring constant $y=\frac{Q_{21}}{Q_{03}}$ is

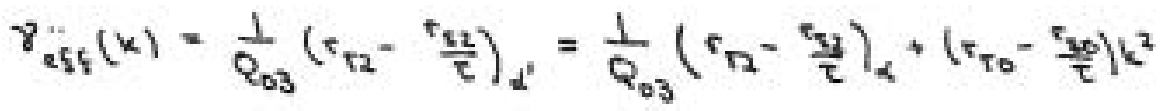

$$
\begin{aligned}
& =\gamma+k^{2} \\
& \gamma_{\text {efs }}(x)=\gamma-\frac{a_{x x}}{a}
\end{aligned}
$$

just what comes out of the co-dimension two equation! At every point, a is responding with its local effective roll wave number. Different wave numbers have different frequencies, so phase lags, which cause wave propagation develop. All the linear terms in $E$ F(a) can be viewed in this same way as wave number dependent damping. At this order the skewness of f(a) becomes important and introduces terms such -ic gaxxx which break the $\mathrm{x}, \mathrm{x}$ symetry in the equation for a.

\subsection{Stability of Wave Solutions}

In order to take advantage of the smallness of the dissipative terms of the equation, we must find solutions or integrals of solutions which vary in a known way over fast time scales, during which the forcing has not had time to act. So first of a 11 we look at the cubic Klein-Gordon equation without the

\& $F(a)$ term with an eye to finding conservation laws and stable explicit solutions.

Two conservation laws can be found. They are of little help without knowing more about the solutions, but can provide constraints on the slow variation of parameters which describe known solutions. Multiply (2.1.1) by $a^{*} T$ and add its conjugate. Integrate with respect to $x$. If we define a horizontal average

$$
\bar{f}=\lim _{h \rightarrow \infty} \frac{1}{2 l} \int_{-b}^{h} f(x, 2) d x
$$

then we get an "energy" integral

$$
\text { (2.2.1) } \quad \frac{d}{d c} \overline{H(x, r)}=\overline{\varepsilon\left(a_{c}^{*} F(a)+a_{\tau} F^{*}\left(a^{*}\right)\right)}
$$

where

$$
H(x, \tau)=\frac{1}{2}\left|a_{2}\right|^{2}+\frac{1}{2}\left|a_{x}\right|^{2}+\frac{\gamma}{2}|a|^{2}-\frac{1}{4}|a|^{4}
$$


We can multiply $(2.1 .1)$ by $a_{*}^{*}$ and add its conjugate. Averaging this gives

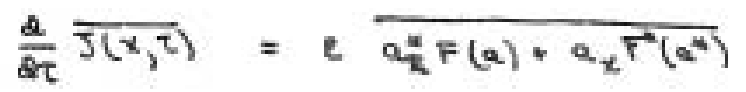

where

$$
J(x, \tau)=a_{x}^{*} a_{z}+a_{x} a_{z}^{2}
$$

$\mathbf{J}$ is an average wave momentum flux. There are no other conserved quantities on the fast time scale.

The study of periodic solutions of the cubic Klein-Gordon (CKG) equation which forms the $O(1)$ behavior of $(2.1 .1)$ is more revealing. We look for travelling wave solutions $a(x-c t)$ of the CKG. There are two types of waves. Waves with $c<1$ (space-like waves) are violently unstable. Waves of $c>1$ are unstable to modulations which smear their wave number out. All uniform roll patterns fall in this latter category and develop modulations on a wave length inversely proportional to their amplitude.

First we examine space-like waves. Consider a wave travelling along at speed $c$ with $c^{2}<1$. Make a Lorentz transformation into the rest frame of the wave.

$$
\left(\begin{array}{l}
y \\
s
\end{array}\right)=\frac{1}{\left(1-c^{2}\right)^{2}}\left(\begin{array}{cc}
1 & -c \\
-c & 1
\end{array}\right)\left(\begin{array}{l}
x \\
\tau
\end{array}\right)
$$

This transformation leaves the wave operator invariant, so

$$
a_{s s}-a_{g s}+8 a-a^{2} a^{*}=0
$$

If $\varphi_{\mathbf{P}}=\varphi_{\mathbf{p}}(\zeta)$ is a travelling wave of speed $c$, then we can write

$$
\varphi_{p}=r e^{L \theta}
$$

Then

$$
\begin{aligned}
r^{2} \theta_{y} & =0 \\
r_{s t}+\frac{\partial v}{\partial r} & =0
\end{aligned}
$$

where

$$
V(r)=\frac{a^{2}}{2 r^{2}}-\frac{r}{2} r^{2}+\frac{1}{4} r^{4}
$$

(Figure 5a,b). Thus

$$
\frac{1}{2} r^{2}+V(r)=E
$$

and we have a two parameter class of travelling waves dependent on $\mathbf{E}, \mathbf{A}$. By letting $r^{2}=s$ we can solve for $r$ in terms of elliptic functions. However, while $\mathbf{r}$ is periodic, 6 will in general not be, unless the phase changes by some rational fraction of $2 \pi$ for each cycle of $r$. There are two interesting limits of these waves. One is the case of $l=0$, the case of purely amplitude modulated waves of the most unstable wave length. The other case is that of $r=$ constant waves, which represent rolls of uniform amplitude 

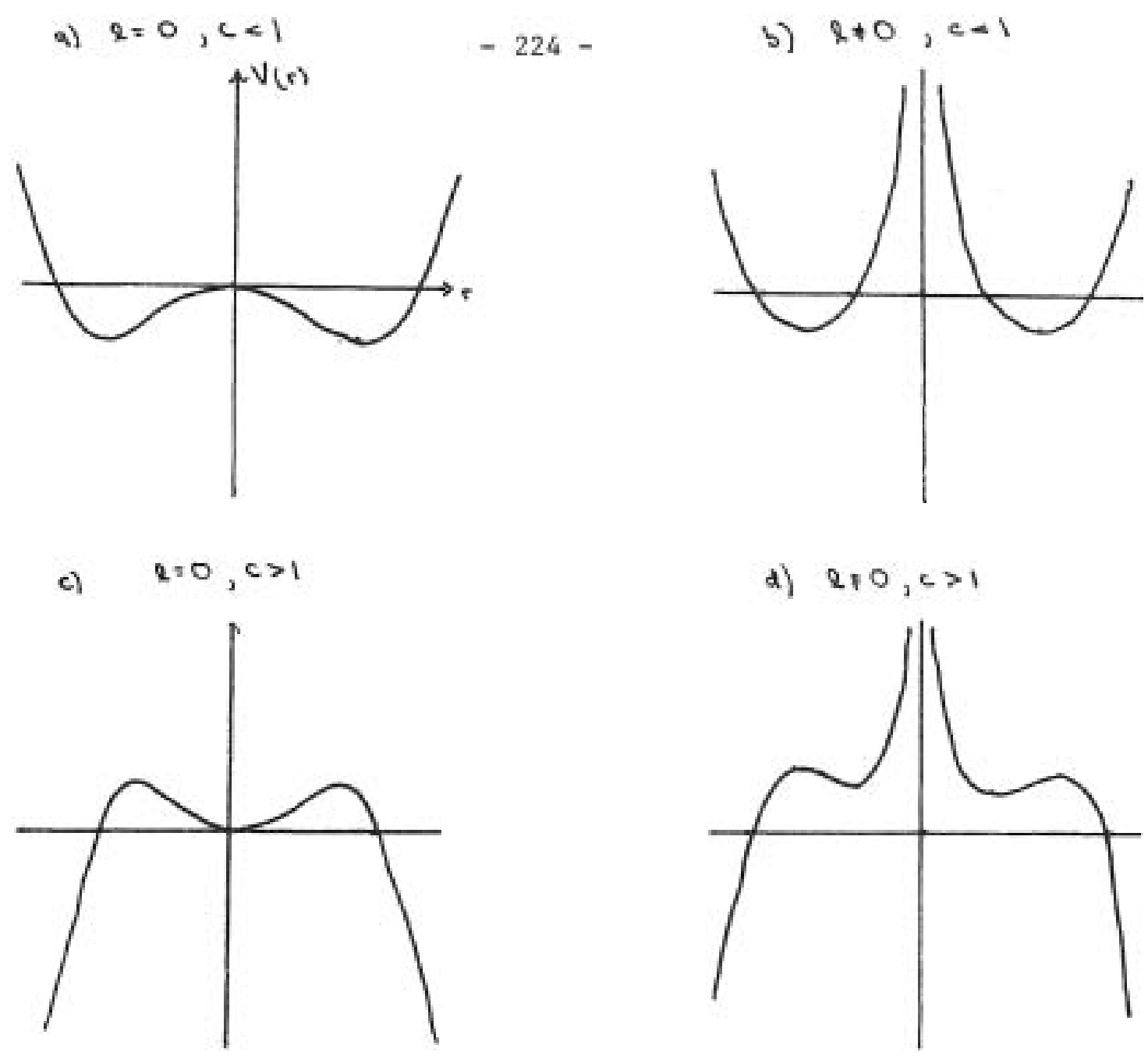

FIGURE 5. Potentials for Travelling Waves of the Cubic Klein Gordon Equation $(\mathcal{\gamma}>0)$

but a different wave length translating along at speed $\mathrm{c}$ in the original coordinates. Both of these solutions have perturbations which grow exponentially and thus are not physically realized. This happens because these solutions walk down a razor-blade between the destabilizing effect of the direct instability, which causes "a" to run away to infinity, and the stabilization of their high wave number, which makes each region in $x$ try to run in a different direction from its neighbors. Any perturbation which can neutralize this second effect will lead to instability of the wave.

First we examine the $\ell=0$ case and look for solutions

$$
a(s, s)=e^{\sigma s} \psi(s) \cdot \varphi_{p}(\varphi)
$$

i n which $\psi$ is a small perturbaticn so the CKG equation can be linearized. There is one subtlety. One must argue that stability of a solution bounded in $J$ when $s$ is increased is equivalent to stability of a solution bounded in $x$ with $t$ fixed. This can be argued by turning the equation into an integral equation and integrating it along characteristic coordinates $r_{1} r_{2}=\frac{1}{2}(\xi \pm \tau)$ If any initial condition on $x$ remains polynomial-bounded i $\mathrm{x}$ for all time, then any initial condition on $I$ at $\tau=0$ can be integrated back to give a 
polynomial-bounded initial condition on $x$ at $\tau=0$. If the $\tau=0$ condition blows up exponentially we have a contradiction to the assumption of stability i n $\mathbf{~}$.

Without loss of generality, $\varphi_{p}$ may be taken as real, and we look at real perturbations $\psi$. Then we get an eigenvalue problem for $\sigma$ :

$$
\psi_{\text {gy }}-\sigma^{2} \varphi=\left\{\gamma-3 \varphi_{p}^{2}(\gamma)\right\} \psi \quad(|\psi|<\infty \text { as }|y| \rightarrow \infty)
$$

This is a Sturm-Liouville problem with a periodic potential, and so has an increasing set of eigenvalues $\rightleftharpoons-\sigma_{n}^{2}$ with eigenvectors with $n$

zero-crossings per period of $\varphi_{p}^{2}(\xi) \quad(n=0,1,2, \cdots)$ However, one solution of (2.2.4) is known. Since a solution $\varphi_{\phi}(\zeta)$ can be moved a distance $\delta \xi$ and still he a solution of the CKG,

$$
\psi=\frac{\partial \varphi_{\rho}}{\partial \gamma}, \sigma=0
$$

is a solution of $(2.2 .4)$. This solution has two zero-crossings per period of $\varphi_{p}{ }^{2}(\xi)$ if the energy $E$ of the periodic solution is less than zero, so (referring to Figure 4a) a period of $\varphi^{2}$ is the sane as a period of $\varphi_{\text {p }}$ " and one zero crossing for $E>0$, when $\Phi_{\varphi_{p}}^{2}$ has half the period of $\varphi_{p}$, $\varphi_{\text {In }}$ any case we may argue that there is $\lambda_{0}=-\sigma_{0}^{2}<0$ with an eigenvector with no zero crossings, and thus there is a solution of $(2.2 .4)$ with $a>0$ which grows exponentially.

There is one exceptional case. At $E=0$ the potential (2.2.3) allows a solitary wave with

$$
\frac{d \varphi_{p}}{d g}=\varphi_{p}\left(8-\frac{1}{2} \varphi_{p}^{2}\right)^{\frac{1}{2}}
$$

which can be solved to give

$$
\varphi_{p}=(2 y)^{\frac{1}{2}} \operatorname{sech} \gamma^{\frac{1}{2}}+\frac{p}{2}
$$

However, in this case the stability equation

$$
\psi_{\pi}-\sigma^{2} \psi=\left(\gamma-6 \gamma \operatorname{sech}^{2} \gamma^{\frac{1}{2}} g\right) \psi
$$

has a solution

$$
\sigma=(38)^{\frac{1}{2}} \quad, \psi=\operatorname{sech}^{2} \gamma \frac{1}{2} 4
$$

so this solitary wave is also unstable, and thus the entire class of solutions with $c<1, \quad \ell=0$ is unstable.

We can also show solutions which represent modulation purely of the phase, the $\mathbf{r}=$ constant solutions, are unstable. These solutions are

$$
\varphi_{p}(\rho)=r e^{\lg \rho}
$$

where

$$
r^{2}=\beta^{2}+\gamma^{2}
$$


The linear stability equation

$$
\sigma^{2} \psi-\psi_{9 g}+\gamma \psi-\phi_{p}^{2} \psi^{n}-2\left|\phi_{p}\right|^{2} \psi=0
$$

has a solution

$$
\begin{aligned}
& =2^{\frac{1}{2}}\left(\rho^{2}+\gamma\right)^{\frac{1}{2}} \\
& \psi=e^{i \beta \gamma}
\end{aligned}
$$

which represents a slight uniform growth of the rolls, causing the subcritical direct instability to al low further growth. Therefore, since these two limits have instabilities in their periodic solutions, it seems highly likely all of the travelling waves with wave speed less than one are violently unstable.

So we turn to waves of speed greater than one in our search for a stable wave train. These waves, it will turn out, are also unstable, now to sideband instabilities in which the nonlinearity spreads the basic wave numbers into a band of wave numbers, turning the uniform wave train into a modulated one.

All waves with $\mathrm{c}>1$ are "time-like" i $n$ the sense that we can make a Lorentz transformation to a frame in which the wave is purely time dependent. Make such a transformation

$$
\left(\begin{array}{l}
y \\
g
\end{array}\right)=\left(\begin{array}{cc}
1 & -\frac{1}{c} \\
-\frac{1}{2} & 1
\end{array}\right)\left(\begin{array}{l}
x \\
\tau
\end{array}\right)
$$

Since $\frac{1}{c}$ is less than one, the CKG remains invariant. The variable $\mathbf{s}=\mathbf{t}-\frac{\mathrm{x}}{\mathrm{c}}$ travels with the wave, so a wave of speed " $\mathrm{c}$ " is of the form $\varphi_{p}(\mathrm{~s})$, where

$$
\varphi_{P}+\gamma \varphi_{P}-\varphi_{P}\left|\varphi_{P}\right|^{2}=0
$$

As before, let $\varphi_{r}=r e^{c \theta}$. Then

$$
\begin{aligned}
& r^{2} \theta_{s}=q \\
& r_{s s}+\frac{\partial v}{\partial r}=0
\end{aligned}
$$

where now

$(2.2 .5)$

$$
V(r)=\frac{r^{2}}{2 r^{2}}+\gamma \frac{r^{2}}{2}-\frac{r^{4}}{4}
$$

and $V(r)$ is shown (for $\gamma>0$ ) in Figure 5c,d. For a typical wave, with small "angular momentum", a plot of $r$ and 3 is shown i $n$ Figure 6. Again an exact solution in elliptic functions is possible but not edifying.

Physically, what happens to a uniform wave train is as follows: Imagine that somehow the amplitude of "a" is increased at one spot. Locally "a" behaves as if it were on a soft spring, so "a" gets delayed at this point. This piles up waves behind the point while they get sparser ahead, which pumps the energy of the faster moving higher wave number waves into the area faster than the slow wave number, more sluggish waves ahead can pull it out. The net energy influx further increases the local amplitude of "a". 

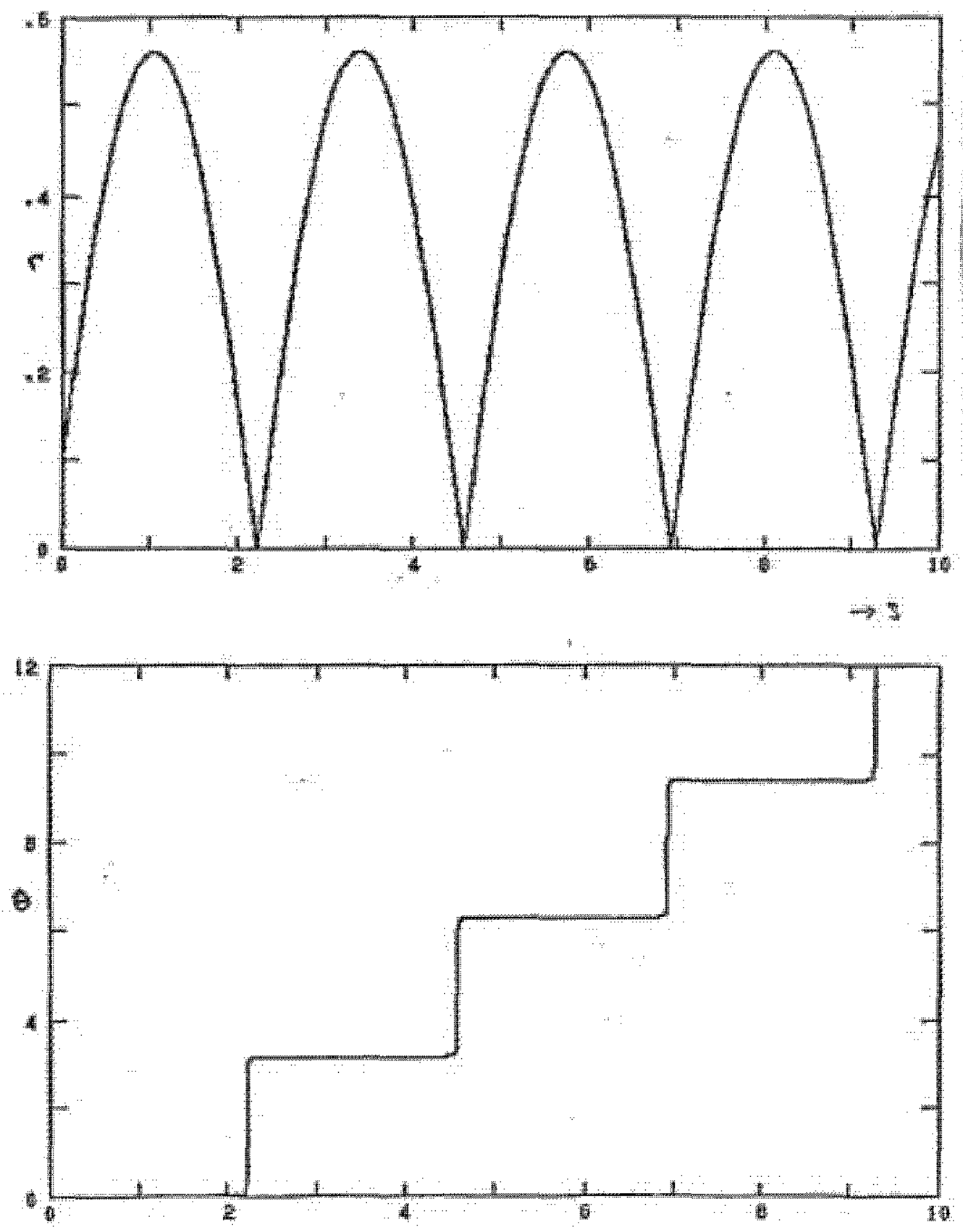

Figure 6. A typical solution of $(2.2 .5)$ for small $2,40(0)$ Rapid jumps in the phase occur as the trajectory flies by the origin. 
Mathematically, the analysis proceeds by the method of Chapter 14 of (Whitham, 1973). In Chapters 14-15 he works out the msdulational instability of the $l=0$ waves of the equation using a slowly varying Lagrangian (this corresponds to $\sigma=-1 / 4$ in his formulation). Because the angular dependence of causes its amplitude to be periodic but its phase to be quasiperiodic, the method does not easily extend to $\ell \neq 0$, except in the case of circular orbits with constant $r$ and

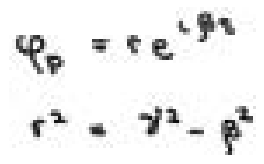

Since the instability involves waves of different speeds, we remember that $\beta^{2} \pm \omega^{2}-k^{2}$ i $n$ the original coordinates $x$ and $t$, so

$$
\omega^{2}=\gamma^{2}+k^{2}-t^{2}
$$

Again, larger " $r$ " decreases the frequency, while larger $k$ increases the energy transport velocity $\omega^{\prime}(k)$, so again the sidebands grow.

The end result of such an instability is a modulated wave train (Figure 7) in which the carrier wave moves at different speed from the envelope, Unless "a" is small, both carrier and envelope have comparable wave lengths, impeding analysis. If "a" is small, due to very small supercriticality, the carrier
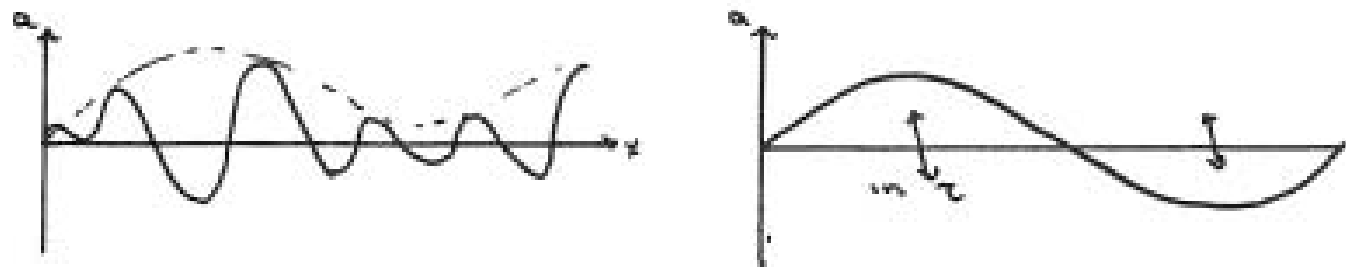

FIGURE 7. The end result of modulational instablity on a wave of speed is a modulated wave train. When seen in the frame in which the carrier wave is a temporal oscillation, the picutre of (6) results.

wave becomes a sinusoidal oscillation in s modulated by an envelope of long wave length in $\mathcal{S}$. In particular, the envelope can be a solitary wave. The separation of scales allows further analytic work, either by an extension of the averaged Lagrangian approach given by section 15.5 of (Whitham, 1973) for the $l=0$ case or a direct perturbation expansion. The latter approach has the advantage of allowing us to include the damping terms and the effects of a 11 modes $(\ell=0$ and $\ell \neq 0)$ and ean be done very neatly in this case.

\subsection{Slowly Varying Solitons near the Hopf Bifurcation}

Both the modulational equations of 2.2 and the conservative equations we found in the $1 \mathrm{imit}$ of $\omega_{2} \rightarrow 0$ of $\mathbf{L . 3 . 9}$ point to a very interesting limit in which analytic solution of the co-dimension two equations is possible. By making $\lambda=O\left(\varepsilon^{2}\right)$, point $Q$ in Figure 4, we move very close to the Hopf bifurcation, forcing us to rescale $" a "=O(C)$ and $x=0(/ e)$ since now only very long waves will not be damped under this scaling

$$
x<2 x ; \lambda \leftarrow \varepsilon^{2} \lambda ; a \leqslant \varepsilon a
$$


the codimension two equations become:

$$
a_{t z}+\gamma_{a}=\varepsilon^{2}\left[a_{x x}+a^{2} a^{*}+i c_{6} a_{x}\right]+e^{3}\left[\lambda_{1} a_{\varepsilon}-c_{5} a\left(a a^{*} L_{c}+c_{2} \varepsilon_{x x}\right]+O\left(\varepsilon^{2}\right)\right.
$$

In deriving the equation (2.1.1) we neglected terms of $O\left(e^{2}\right)$, so we might question the validity of (2.3.1). However, the terms we neglected are frequency corrections and damping-exciting terms. The latter are ( (supercriticality $\left.)^{2}\right)=O\left(e^{b}\right)$ and the former are not of qualitative importance. We will examine $(2.3 .1)$ with $\gamma$ fixed, which constrains ${ } T_{2}$ and $r_{S_{2}}$ to lie on a line; writing $\gamma=\gamma_{0}+e^{2} \gamma_{2}$ again only introduces frequency corrections to the solution and does not affect the qualitative behavior. Write, to take care of the leading behavior

$$
a=a_{+}(x, 3, T) e^{u \Delta \tau}+a(x, s, T) e^{-t \omega \tau}+e^{2} a_{43}(x, s, \tau) e^{3 \omega \tau}+\varepsilon^{2} a_{-3}(x, s, \tau) e^{-3 \cos t}
$$

where

$$
a_{t}=A_{t}+2 A_{1}+\cdots \quad, \omega=8 \frac{1}{2}
$$

and $s=2 \omega_{0} e^{2} \tau, T=2 c^{3} \tau$. are slow times scaled in a convenient way. At $O\left(\varepsilon^{2}\right)$ we recover

$$
\begin{aligned}
& i\left[A_{+5}+c_{6} 8 A_{+2}\right]=A_{+2 x}+A_{+}\left\{2\left|A_{-}\right|^{2}+\left|A_{+}\right|^{2}\right\} \\
& -i\left[A_{-5}-c_{6} 8 A_{-x}\right]=A_{-x x}+A_{-}\left\{2\left|A_{+}\right|^{2}+\left|A_{-}\right|^{2}\right\}
\end{aligned}
$$

We have two nonlinear Schrodinger equations coupled by their amplitudes. If one takes the $\omega \rightarrow 01 \mathrm{imit}$ of the Hopf bifurcation and appropriately rescales, the equations can alsa be recovered from it.

To usefully go to $O\left(\varepsilon^{3}\right)$ it will help to have solutions of (2.3.2). The easiest case, which proves to be very surprising, is to insist $A_{\xi}$ be independent of $x$, so we have two uniform waves of the most unstable wave number travelling through one another as in Figure $2 b$. Then

$$
\pm L A_{15}=A_{ \pm}\left\{2\left|A_{7}\right|^{2}+\left|A_{t}\right|^{2}\right\}
$$

whence, if we write

$$
\begin{aligned}
& A_{+}=p e^{-\cos s} \\
& A_{-}=q e^{\log t}
\end{aligned}
$$

we find solutions when

$$
\begin{aligned}
& >=2 q^{2}+p^{2} \\
& \beta=2 p^{2}+q^{2}
\end{aligned}
$$


Now we must go to $o\left(\varepsilon^{3}\right)$. We let $p$ and $q$ be functions of the very slow time $T=2 \varepsilon^{3} t$ and find, by matching coefficients of $e^{2 n t}$ that

$$
\begin{aligned}
\pm L A_{ \pm 1 S} & -\left[2\left|A_{4}\right|^{2}+2\left|A_{-}\right|^{2}\right] A_{12}-A_{ \pm}^{2} A_{15}^{*}-2 A_{2} A_{7}^{*} A_{17}-2 A_{4} A_{-} A_{4}^{*} \\
& = \pm \omega \omega\left\{\lambda_{1} A_{1}-2 C_{5} A_{2}\left|A_{7}\right|^{2}-A_{27}\right\}
\end{aligned}
$$

It is convenient to work with a four-vector

$$
\stackrel{A}{\sim}=\left(\begin{array}{l}
A_{+} \\
A_{+}^{*} \\
A_{-} \\
A_{-}^{*}
\end{array}\right)
$$

In terms of $\mathbf{A}$ the above equation can be written

$$
\left\llcorner A_{1}=\operatorname{RHS}\left[A_{2}\right]\right.
$$

where

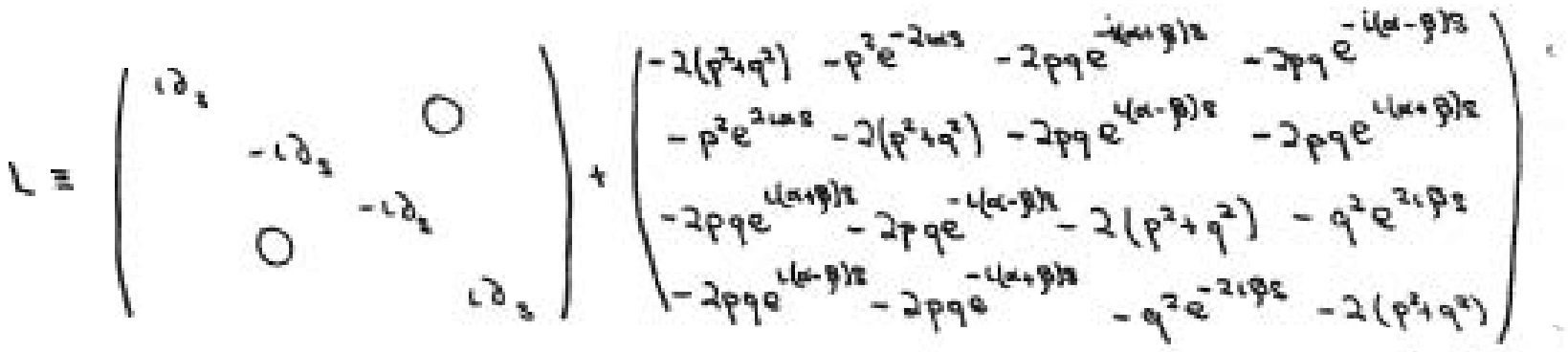

and

$$
\operatorname{RHS}\left[A_{\sim}\right]=\omega_{0}\left\{-\left[\begin{array}{c}
i A_{* 7} \\
-i A_{* T}^{*} \\
-i A_{-T} \\
i A_{-T}^{*}
\end{array}\right]+\lambda_{1}\left[\begin{array}{c}
i A_{4} \\
-i A_{4}^{*} \\
-i A_{-} \\
i A_{*}^{*}
\end{array}\right]-2 \varepsilon_{3}\left[\begin{array}{c}
i A_{4}\left|A_{-}\right|^{2} \\
-i A_{+}^{*}\left|A_{-}\right|^{2} \\
-i A_{-}\left|A_{4}\right|^{2} \\
i A_{-}\left|A_{4}\right|^{2}
\end{array}\right]\right\}
$$

The operator $L$ is self-adjoint with respect to a time averaging inner product

$$
\langle\underset{2}{A}, \underline{B}\rangle=\lim _{T \rightarrow T^{n}} \frac{1}{T} \int_{0}^{T} A^{T} B d s
$$

The dagger transposes and conjugates its vector, and the time $\mathrm{T}$ is assumed to be small compared with a unit of the long time $T$. Since L is self-adjoint we can find solvability conditions using the homogeneous solutions of $\mathrm{L}$. Remember $L$ is the variation of the nonlinear Schrodinger equation, so the difference of any two very close solutions to (2.3.3) is a solution $A_{h}$ of $\mathrm{LA}_{\mathrm{h}}=0$. The most general solution to 2.3 .3 is

$$
\begin{aligned}
& A_{+}=p e^{-i\left[\left(p^{2}+2 p^{2}\right) s+\phi_{1}\right]} \\
& A_{*}=q e^{\left.i\left(q^{2}+2 p^{2}\right) c+\phi_{2}\right]}
\end{aligned}
$$


Since $A_{+}$and A- couple only through their amplitudes each phase can be varied independently, so we find two homogeneous solutions

$$
\sim_{h}^{*}=\left[\begin{array}{c}
-i p e^{-i n s} \\
i p e^{\cos s} \\
0 \\
0
\end{array}\right] \quad A_{h}^{-}=\left[\begin{array}{c}
0 \\
0 \\
i q e^{-i n s} \\
-i q e^{-i n s}
\end{array}\right]
$$

The condition that $A_{1}$ remain bounded, so boundary terms in an integration by parts are negligible, is that

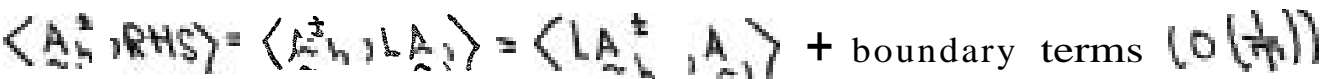

$$
\begin{aligned}
& =0
\end{aligned}
$$

which gives two solvability conditions

$$
\begin{aligned}
& p_{2}=\lambda_{1} p-2 c_{5} p q^{2} \\
& q_{8}=\lambda_{1} q-2 c_{5} q p^{2}
\end{aligned}
$$

The two waves couple only to each other: In fact, the phase plane diagram (Figure 8) shows that one wave always swallows the other and grows exponentially unless $p=q$ initially. This contrasts with the perfectly well behaved behavior in a finite box, in which reflection of waves off the wall keeps the amplitudes from growing indefinitely by changing $p^{8} s$ to $q^{8} s$, which can now damp $p^{i} s$.

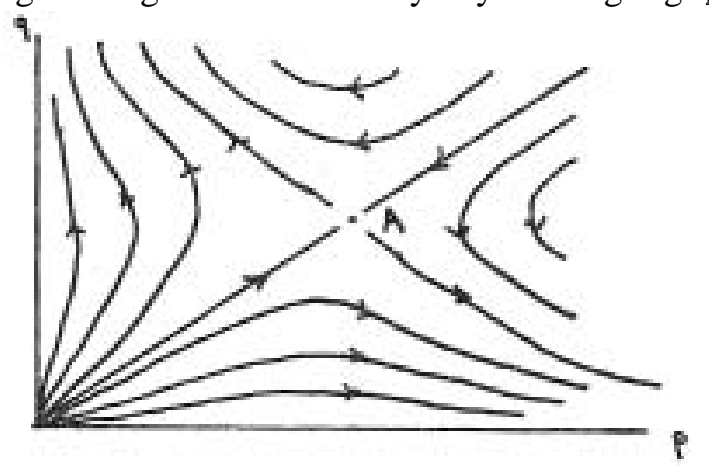

$$
A=\left(\left(\frac{\lambda_{2}}{2 c_{5}}\right)^{\frac{1}{2}},\left(\frac{\lambda_{3}}{2 c_{5}}\right)^{\frac{1}{2}}\right)
$$

FIGURE 8. The bifurcation diagram for equations (2.3.5). $p$ and $q$ are the slowly varying amplitudes of the travelling waves.

This behavior is very disturbing, since the bifurcation is supercritical in a finite box. I will now argue it is misleading, because the states $\mathrm{p}=0$ or $\mathrm{q}=0$ can easily be shown to be modulationally unstable, since the equations (2.3.2) reduce to a single nonlinear Schrodinger equation in those limits. Instead of a uniform state the equations break up into solitons and radiation. Thus, what we should really study is a solitary wave solution to see how it evolves.

Let us, however, take the suggestion of Figure 7 and study a solution in which one wave is very small, while the other is a soliton. Thus at $O\left(\varepsilon^{2}\right)$

$$
\begin{aligned}
& A_{t}=0 \\
& i\left(A_{-s}+c_{6} A_{-x}\right)+A_{-x x}+A_{-}\left|A_{-}\right|^{2}=0
\end{aligned}
$$


Look for solutions

$$
A_{-}=e^{i(2 s-r 8)} v(s) \quad, s=x-v t
$$

Then if we let

$$
\begin{aligned}
& R=\frac{1}{2}\left(U-c_{6} \gamma\right) \\
& r=\frac{1}{4}\left(U-c_{6} \gamma\right)^{2}-\alpha^{2}
\end{aligned}
$$

we find

$$
\begin{aligned}
& v^{\prime \prime}-x^{2} v+v^{2}=0 \\
& v(g)=2^{\prime} x \operatorname{sech} \alpha g
\end{aligned}
$$

"v" can be shown to be neutrally stable to small perturbations. In a similar way as i $n$ the $x$-independent case we construct the third order problem in the rest frame of the soliton:

$$
L A_{1}=\text { RHS }
$$

where now

$$
R H S=-\omega \omega \lambda_{1}\left[\begin{array}{c}
0 \\
6 \\
-A_{-} \\
A^{*}
\end{array}\right]-\omega \omega c_{2}\left[\begin{array}{c}
0 \\
0 \\
-A_{-13} \\
+A_{-11}^{-11}
\end{array}\right]-2 \omega_{0}\left(\frac{1}{2}\right)\left[\begin{array}{c}
0 \\
0 \\
A_{-\tau} \\
-A_{-1}^{-1}
\end{array}\right]
$$

and

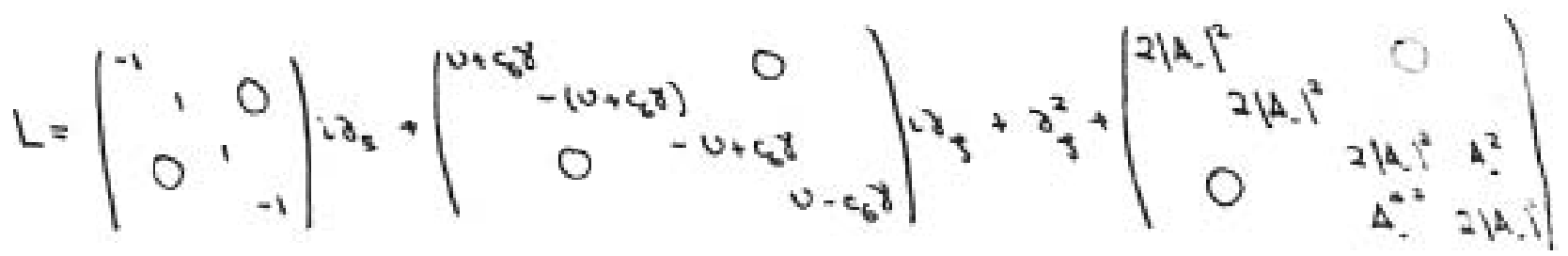

Note that the problem is block diagonal. The dissipative terms do not enter the equation for $A_{1+}$ because we have assumed $A_{0}+=0$. A long way from the soliton they cause $A_{1}+$ to grow away from zero, but only on an $O\left(e^{q}\right)$ time scale, too slow to affect the equilibration of the soliton. In fact

$$
i\left(A_{1+8}-c_{6} \gamma A_{1+2}\right)=A_{14 x x}+2\left|A_{-}\right|^{2} A_{16}
$$

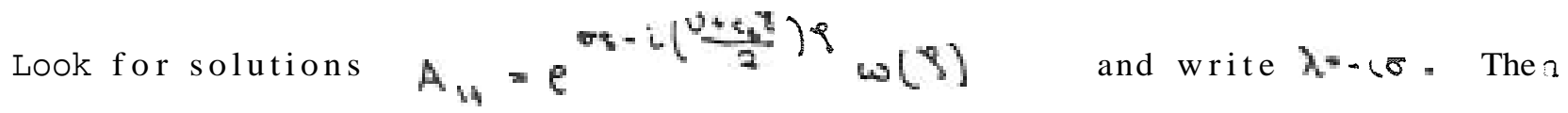

$$
\omega_{\text {ge }}+\left\{\lambda+\left(\frac{0+8 y^{2}}{2}\right)^{2}+4 \alpha^{2} \operatorname{sech}^{2} \alpha\right\} \omega=0
$$

There is a continuous spectrum with $\lambda$ real and greater than $\left(\frac{3+c x}{2}\right)^{2}$ and no discrete spectrum, so the growth rate $\sigma$ must be imaginary and ${ }^{2} A_{1+}$ remains bounded on the fast time. 
The equations for $A_{1-}$ can be written

$$
\mathcal{L}\left[\begin{array}{l}
A_{1-} \\
A_{1-}^{*}
\end{array}\right]=\text { RHS }
$$

$\mathcal{L}$ is the lower right $2 \times 2$ block of the matrix operator L, while RHS 34 is the bottom two columns of RHS. $\mathcal{Z}$ is self-adjoint with respect to an inner product

$$
\langle\xi, g\rangle=\lim _{T \rightarrow x_{-\infty}} \int_{-\infty}^{\infty} d x \int_{0}^{\pi} d s f^{t}(x, 2, T) g(x, s, T)
$$

so we can again make use of solutions of $\mathcal{I} \hat{A}_{h}=0$ derived by varying the soliton solution in its most general form

$$
\text { A. }(\xi, \tau)=e^{1\left(R g-\operatorname{ss}-\phi_{0}\right)} 2^{\frac{1}{2} \alpha \operatorname{sech} \alpha\left(\xi-y_{0}\right)}
$$

with respect to $\phi_{s}$ and $Y_{0}$. This gives us homogeneous solutions

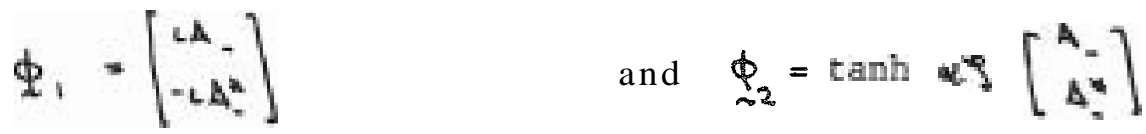

The two solvability conditions are

$$
\left\langle\phi_{,}, R H S\right\rangle=0
$$

or

$$
\lambda_{1} \sqrt{\left.A_{-}\right|^{2}}+c_{2} \overline{A_{-}^{*} \Delta_{-T S}+A_{-} A_{-v 1}^{\lambda}}-\frac{\partial}{\partial \tau} \overline{\left|A_{-}\right|^{2}}=0
$$

and

$$
\left\langle\phi_{2}, R+1 S_{34}\right\rangle=0
$$

$$
\begin{aligned}
\overline{\lambda_{1} \tanh \alpha g\left(\left(\left|A_{1}\right|^{2}-i\left|A_{1}\right|^{2}\right)\right.} & +c_{2} \overline{\tanh \alpha g\left(i\left[A_{-}^{*} A_{35}-A_{-} A_{-15}^{*}\right]\right)} \\
& -i \overline{\left.\left(A_{-}^{*} A_{-T}-A_{-} A_{-7}^{*}\right) \tanh \alpha\right]}=0
\end{aligned}
$$

From these conditions we arrive at the evolution equations for the amplitude and a velocity parameter, $R=\frac{U-c^{T}}{a}$

$$
\begin{aligned}
& \alpha_{T}=\left(\lambda_{1}-R^{2}\right) \alpha-\frac{1}{3} \alpha^{3} \\
& R_{T}=-\frac{8}{3} c_{2} \alpha^{2} R
\end{aligned}
$$

These equations give the slow variation of the soliton parameters. Their phase portrait (Figure 9) shows they converge on a fixed point $4=\left(3 \lambda_{3}\right)_{2}^{\frac{1}{2}}$ $\mathrm{R}=0$. All solitons will eventually attain the same amplitude and velocity. A train of solitons eventually turns into a uniform train of modulations which transport energy at a speed $U=c_{6} \gamma^{\prime}$. 
Note that no matter how wide the initial soliton it packs together. is a reassuring sign that the spatially independent solution is not the preferred mode, and our amplitude runaway has been prohibited.

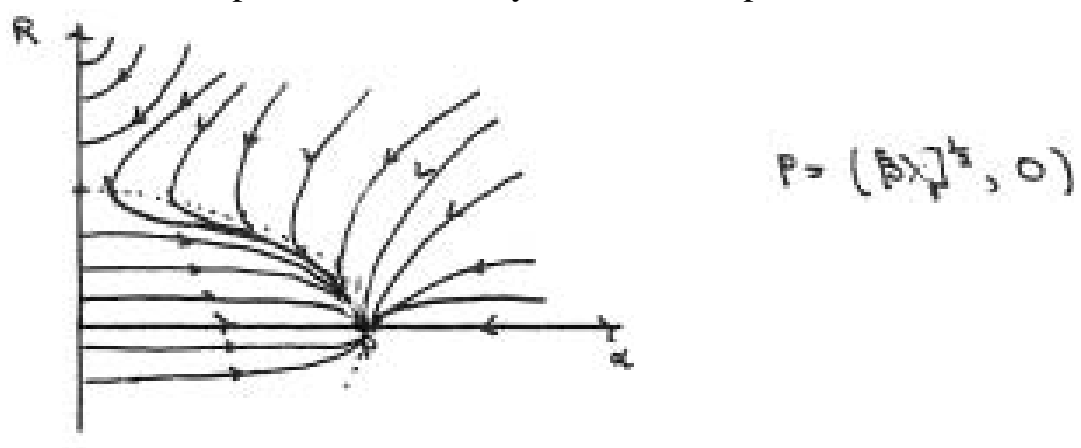

FIGURE 9. The phase plane diagram showing the slow evolution at solitons. All solutions converge on a fixed amplitude determined by the superciticality and a velocity determined by their basic oscillation frequency.

\subsection{Conclusions}

We have seen wave dynamics are very important in a large aspect ratio box when there is a wave-like instability that is activated. Uniform wave trains break into wave packets which suck energy from their surroundings and quite rapidly move it around.

There are many more investigations to be made in this model problem. First, we would like to look at larger amplitude dynamics to see what takes the place of the oscillating solitons of (2.3) and how it evolves. It would pay to look at the interaction of right and left going waves to see if the soliton collision behavior of the nonlinear Sehrodinger solitons of 2.3 is preserved. Most important is a statistical mechanics of the equations. What is the soliton (or other coherent structure) "density" and do the solitons, or radiation, account for most of the energy of the system? From such an approach we could look at the horizontal mixing that such moving waves must produce.

However, now the most important test is an experimental realization. The instability of a uniform roll pattern and the resultant waves should be detectable. But even without such verification, the model wave equation derived represents intriguing new phenomena caused by the interaction of dissipation, forcing, and waves in a nonlinear system.

\section{ACKNOWLEDGEMENIS}

I would like to thank Edward Spiegel for his untiring help and suggestions, Fausto Cattaneo for providing enough Pernot to drown my worries, and Willem Malkus for making it all possible.

\section{REFERENCES}

Arneodo, A., P. Coullet, E. A. Spiegel and C. Tressier, 1981. In preparation.

Gollub, J. P. and J. F. Steinman,, , 1981. Doppler imaging of the onset of turbulent convection. Phys. Rev. Lett. (In press) 
Guckenheimer, J॰s 1981. Multiple bifurcation of co-dimension two. Preprint.

Huppert, H. E. and T. S. Turner, 1981. Double diffusive convection. J.F.M., $106,299-329$.

Knobloch, E. and M.R.E. Proctor, 1981. Nonlinear periodic convection in double diffusive systems. J.F.M. (En press).

Libchaber, A. and J. Maurer, 1981. A Rayleigh-Benard experiment: Helium in a small box. Preprint.

Newe11, A. C. and J. A. Whitehead, 1968. Finite bandwidth, finite amplitude convection. J.F.M.9, 38, 279-303.

Turner, J. S., 1973. Buoyancy effects in fluids, Ch. 8. Cambridge University Press.

Vernonis, G., 1965. On finite amplitude instability in thermohaline convection. J.Mar. Res., 23, 1-17.

Whitham, G. B., 1974. Linear and nonlinear waves. John Wiley and Sons.

ON HORZONTAL ADVECTION IN RAYLEIGH-BENARD CONVECTION

Satoru Xonda

ABSTRACT

Simple experiments on horizontal advection of particles in Rayleigh-Benard convection were executed. Supposing that this phenomenon is a diffusion-like one as suggested by Malkus (1959), it is found that the mean square of the distance from the initial position $x^{2}$ is expressed as,

where $\mathrm{K}$ is the themometric diffusivity of fluid, Ra is the Rayleigh number and $\mathbf{t}$ is time. Possible implications of this result with regard to the mantle convection are discussed. This shows that effective horizontal diffusion constant is about $2 \times 10^{-3} \mathrm{KRa} 1 / 2$.

\section{INTRODUCTION}

Convection is undoubtedly an effective mixer of the interior of a fluid. For example, it is known that, at high Rayleigh number, the temperature within the convecting fluid is almost the same everywhere except in thin boundary layers. It is generally believed that convection can break an initial inhomogeneity and make a uniform fluid within a short time. However, there is much evidence that a slight density change caused by a compositonal difference produces a layered structure in the convection (e.g., Turner, 1974; Richter and McKenzie, 1981). Also, even if the fluid has a constant physical property, the horizontal advection may be small compared to the vertical one because of the existence of cell boundaries. If the flow is strictly laminar, there is no horizontal advection. To answer this problem, we have done simple experiments in which we observed the horizontal movement of particles in the Rayleigh-Benard convection. We will firstly report the experimental procedure and the results. Secondly, we will discuss an implication of results. 


\section{Experimental Procedure}

The apparatus used in this study is shown in Figure 1. This is the same one as Whitehead and Parsons (1978). The detailed description will be found in their paper. We used Dow Corning 200 silicone oil as a working medium.

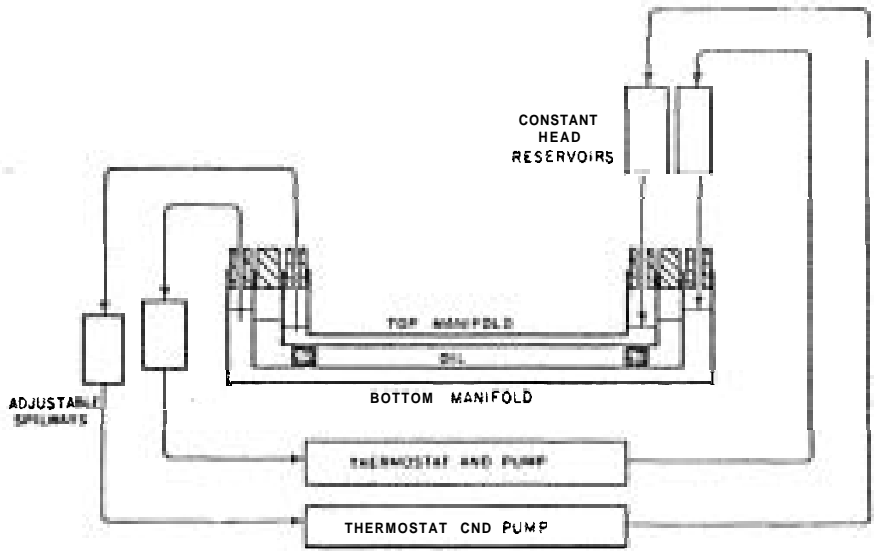

FIGURE 1. Sketch of the Experimental Apparatus.

The physical properties of the oil are: kinematic viscosity, $\nu=10 \mathrm{~cm}^{2} / \mathrm{sec}$, thermal diffusivity $k=1.16 \times 10^{-3} \mathrm{~cm}^{2} / \mathrm{sec}$, thermal expansion coefficient $\alpha=9.6 \times 10^{-4} 1 /{ }^{\circ} \mathrm{C}$ and Prandtl number $P_{Y}=8.6 \times 10^{3}$. Rayleigh number $R a$ is defined by

$$
R_{a}=\frac{g \alpha \Delta T d^{3}}{\nu k}
$$

where $g$ is the gravitational acceleration, $\Delta \mathrm{T}$ is a temperature difference between the boundaries and dis the depth of the fluid. In this study, Ra is chosen to be $1.8 \times 10^{4}, 3.0 \times 10^{5}$ and $5.5 \times 10^{5}$. Spacing d is selected to be $3 \mathrm{~cm}$ i $\mathrm{n}$ the first case and $7.7 \mathrm{~cm}$ in other cases. Selection of a neutrally buoyant particle, which we used as tracers, is the most difficult problem in our study. We made them by mixing methylalcohol with water and dye. By this method, we could make fairly buoyant particles with a large radius. However, we found that some of the particles sank down after about 6 to 8 hours run because of the small solubility of the alcohol to the silicone oil. All the experiments were done with an uncontrolled initial flow. After the apparatus had been held at the desired temperature for at least $\boldsymbol{a}$ few hours, particles were introduced into the fluid. Observations were made by a shadowgraph technique with time lapse movies. Movies were taken at the rate of about 6-8 sec/frame with 4000 frames, so that observations of about 7 to 10 hours were possible. Movements of particles were monitored by reading the positions from the movie after the end of experiments. The positions were defined by the arbitrary selected $x-y$ coordinate which was fixed to the screen.

\section{$\underline{\text { RESULTS }}$}

Obtained results are summarized in Table $I$ and several examples of particle motion relative to the fixed coordinate are shown in Figures 2 and 3. The general flow patterns are spoke-like flow except for the case of Ra $=$ $1.4 \times 10^{4}$ which we will describe later. The particle motion is dominated by a periodic motion as was inferred easily. Occasionally particles go from the 


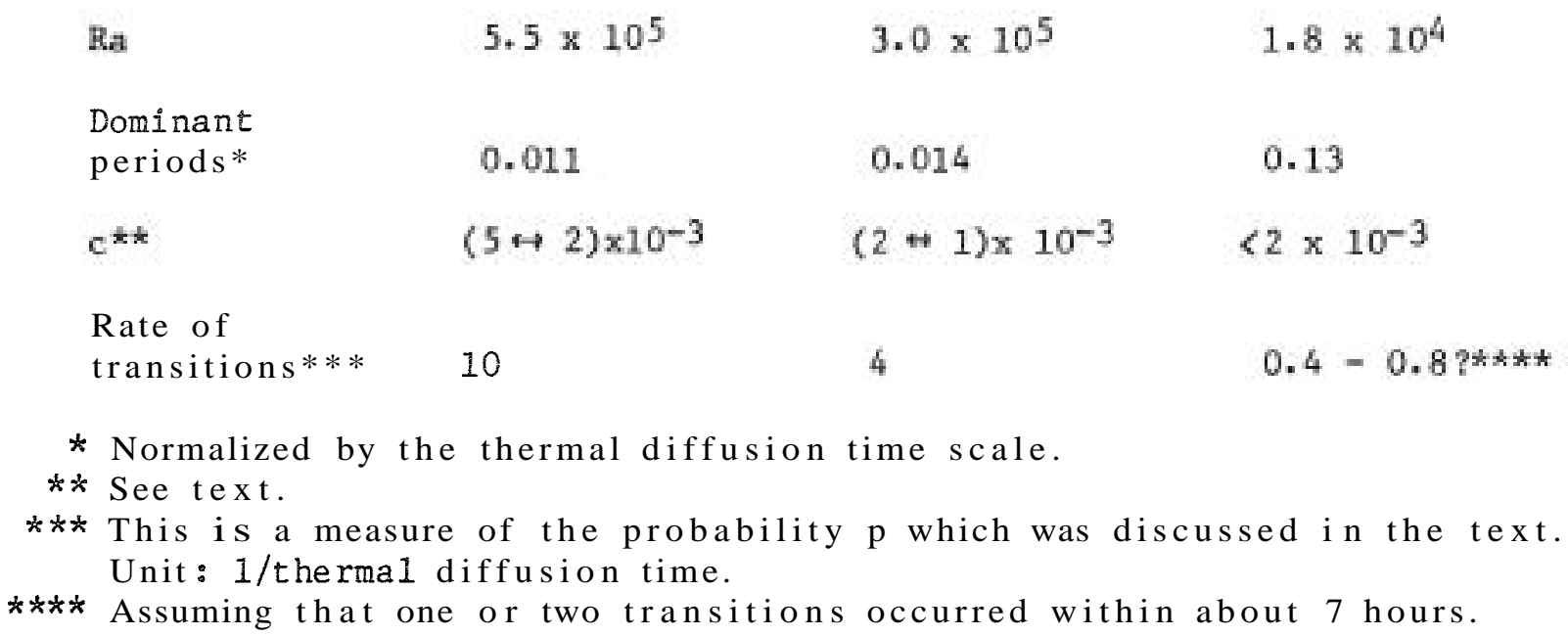

TABLE 1. Summary of Experimental Results.

cell in which they have existed into another one (we will call this phenomenon 'transition'). The typical transition pattern is shown in Figure 4a. Sometimes particles fail the transition and will go back to the nearly same position of Figure 4. Some transitions cause a drastic change of $X$ (which represents the distance from the initial position). The frequency of the transition is estimated from the movie and shown in Table 1. Dominant periods which are clearly related to the turnover time of convection are calculated by Figures 2 and 3 and by the direct measuring of movies. These values are in agreement with the results of Whitehead and Parsons (1978). Now, we will explain ' $c$ ' i n Table 1. Malkus (1959) predicted that the mean square of $X$ is a function of the Rayleigh number and time shown as follows:

$$
\overline{x^{2}}=c k R_{a}{ }^{h} t
$$

This is derived as follows: It is easily shown that, in the case of onedimensional random walk process, the mean square of the deviation from the origin is proportional to the frequency $n$ of changing direction.

$$
\overrightarrow{\mathrm{x}^{2}} \propto \mathrm{nd}^{2}
$$

If the horizontal advection in the Rayleigh-Benard convection can be regarded as a random walk process, $n$ must be proportional to $\frac{t}{d / v}=\frac{x t}{d}$ where v

is the representative velocity of the convection. Malkus (1954) found experimentally that the mean square velocity is proportional to the Rayleigh number, that is

$$
\overline{v^{2}} \sim R_{a}\left(\frac{k}{d}\right)^{2}
$$

Thus, he obtained (1) above.

We must comment on a physical meaning of $c$. Probably, c includes the information of probability of transitions and the geometry of convection. The probability $p$ will be defined as the ratio of frequency of transitions to the total frequency of meeting the boundaries. The geometry of convection will affect the horizontal advection too (see later section). So c does not show a 


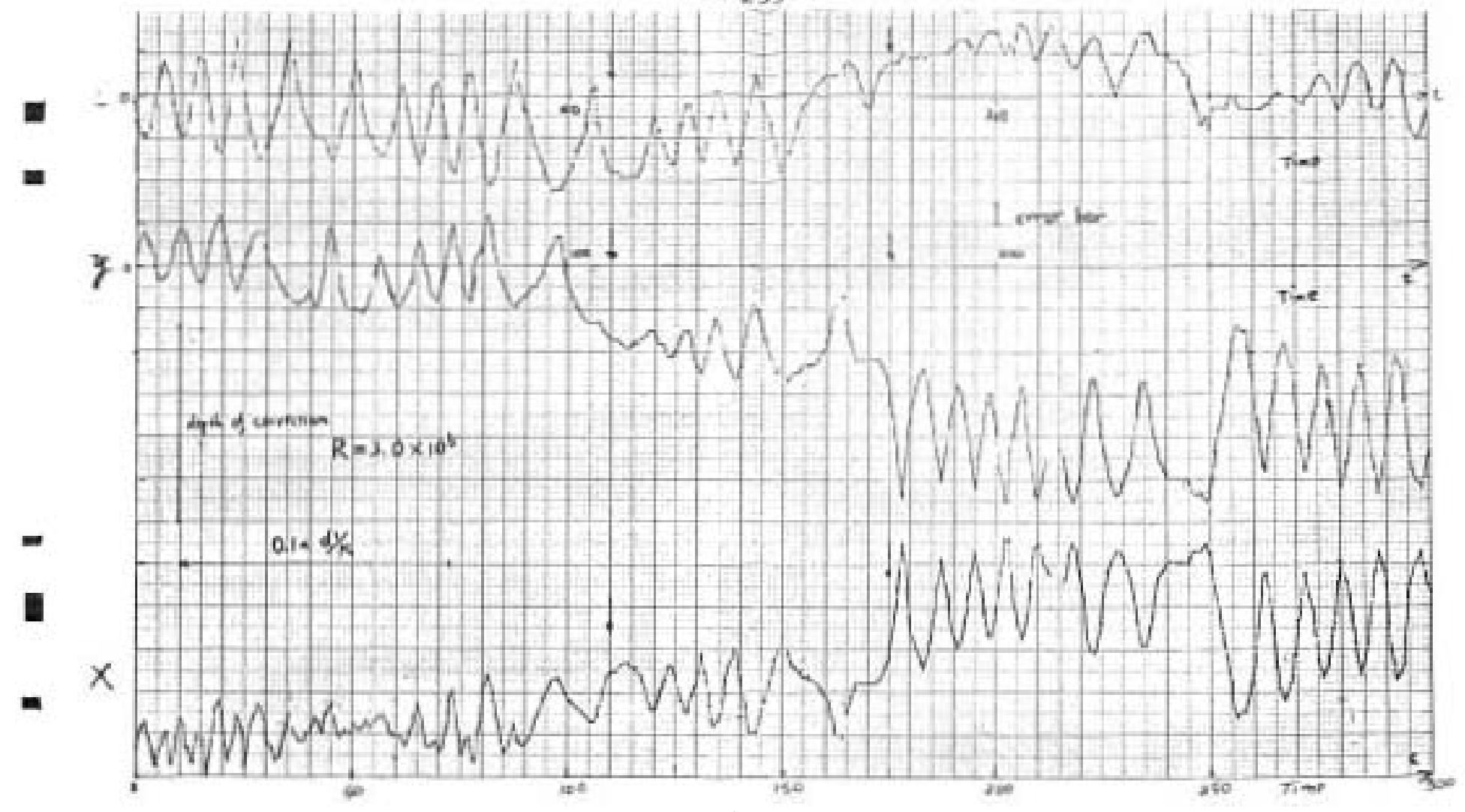

Figure $3 a$

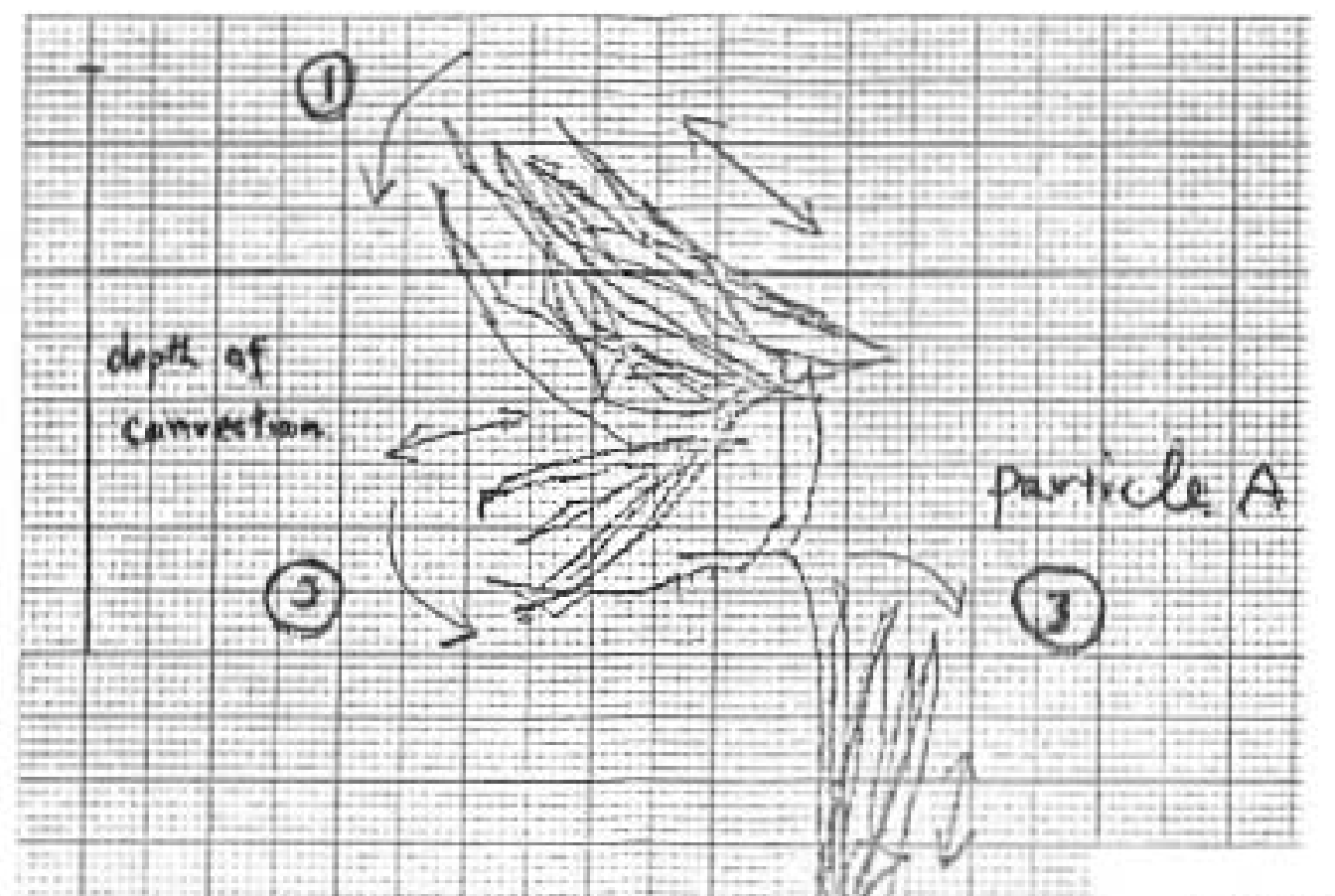


number but a process. This implies that c may be dependent on Ra. In our experiments, $x^{2}$ was calculated by the mean of five particles for the case of $\mathrm{Ra}=5.5 \times 10^{5}$ and four particles for the case of $\mathrm{Ra}=3.0 \times 10^{5}$. In reality, because the periodic motion in a cell is the most dominant part compared to the other slowly varying part, we choose $X$ as the nearest position to the initial position within almost a period, Accordingly, $t$ has the uncertainty of about one period. $X-t$ relations of each particle and $X^{2}-t$ relations of the case $\operatorname{Ra}=5.5 \times 10^{5}$ and $3.0 \times 10^{5}$ are shown in Figures 5 and $6 . \quad A$ calculation of c was made by using Figure 6 and values obtained are listed in Table 1. However, because of small numbers of samples, these values may have some uncertainties. To check the dependence of the number of sampling, we tried to test by removing the samples one by one. These results are also shown in Figure 6. According to these tests, we inferred that $c$ is about 1 to $5 \times 10^{-3}$, but we think that more elaborated experiments must be needed. We must give some comments on the case of $\mathrm{Ra}=1.8 \times 10^{4} \mathrm{in}$ which we estimated c by a different method. It is known that the roll type convection is stable until about $2 \times 10^{4}$ (Busse and Whitehead, 1974). In this case, we also obtained the stable roll-like convection with wave number of about $\mathbf{3 . 7}$ normalized by depth $d$. It is found that there is essentially no advection in the direction of the axes of rolls. Also, there are seldom transition of particles between rolls, but a few particles change position from one cell to another. So we estimated that $\overline{X^{2}}$ is almost the square of one cell size within about 7 hours of test run. This estimate may give an upper limit of c and we obtained as $2 \times 10^{3}$ which is in fairly good agreement with another one. As a conclusion of our experiments, c is about $2 \times 10^{-3}$ within factor of two, assuming that equation (1) is valid and $c$ is constant. However, note that, in this calculation, there is a basic assumption that the advection of particles in Rayleigh-Benard convection can be explained as a diffusion-like phenomenon. It is notable that the rate of transition seems to be proportional to Rayleigh number instead of $\mathrm{Ra}^{1 / 2}$ and that there is a tendency of $\mathrm{c}$ to increase with Ra.

\section{DISCUSSION AND IMPLICATION OF RESULTS WITH REGARD TO THE MANTLE CONVECTION}

The horizontal advection of particles in the convection may be dominated by four processes.

(1) quasiperiodic motion in a cell

(2) transition from one cell to another

(3) slowly deforming cell boundaries

(4) rapid oscillations

(1): This motion may be categorized into two parts; the motion which is clearly related to the turnover time of the convection and the slowly varying motion which is almost perpendicular to the former. Generally, transitions occur when the particle moves almost along with the boundary. So the probability of the transition may be restricted by the second type of the motion. The relation between two types of the motion is not clear at present because of small amounts of data sets.

(2): It is noteworthy that, even if the transition occurs, it does not imply the change of $X$. For example, see Figure 5. From this figure, we will find that the number of transition is not proportional to $X$. The geometry of the convection is certainly an important factor which controls the horizontal advection. Whitehead and Parsons (1978) reported that, at high Rayleigh number, a square pattern of the convection is stable. Because of its regularity, the horizontal advection in the squared pattern may be smaller 


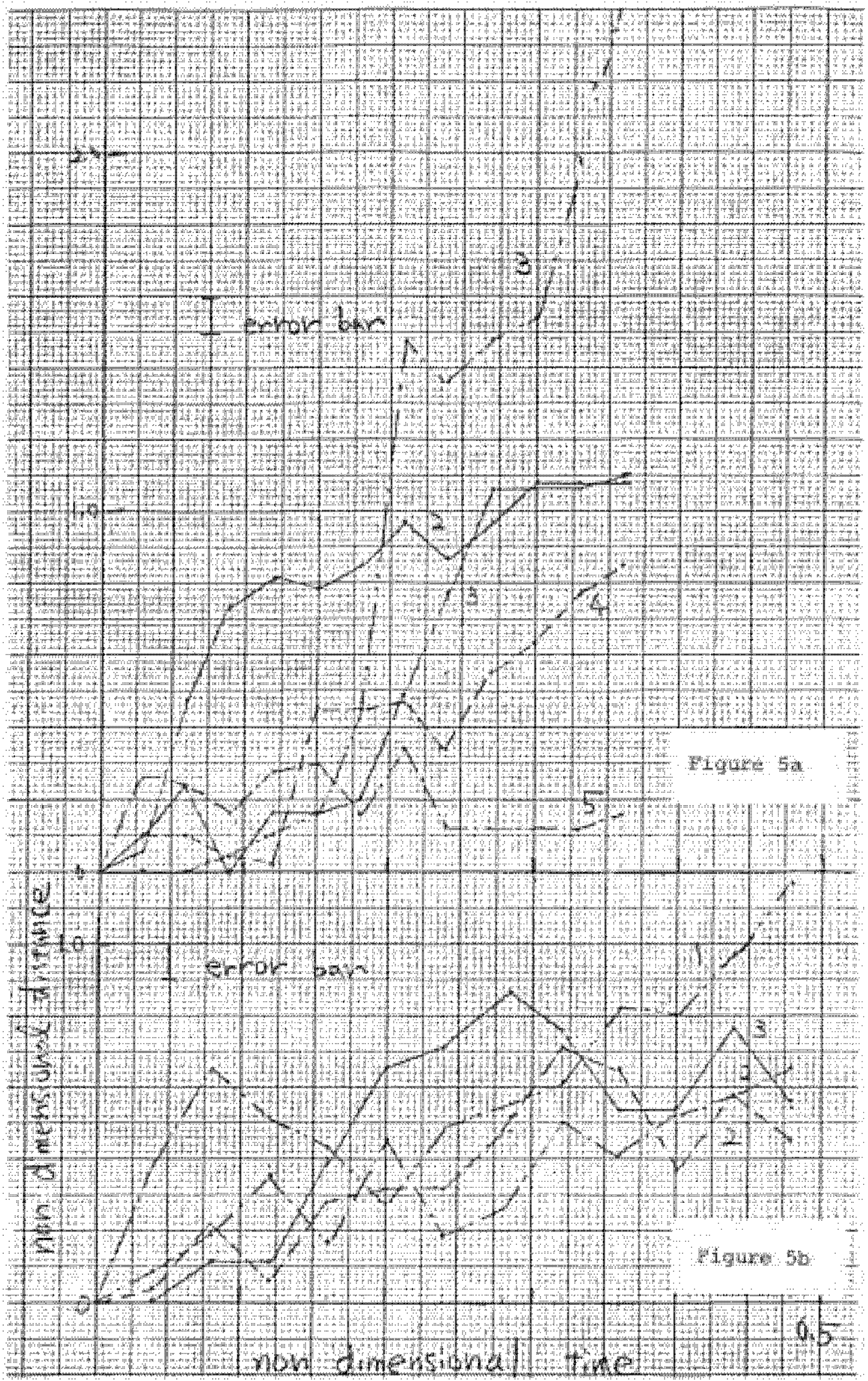




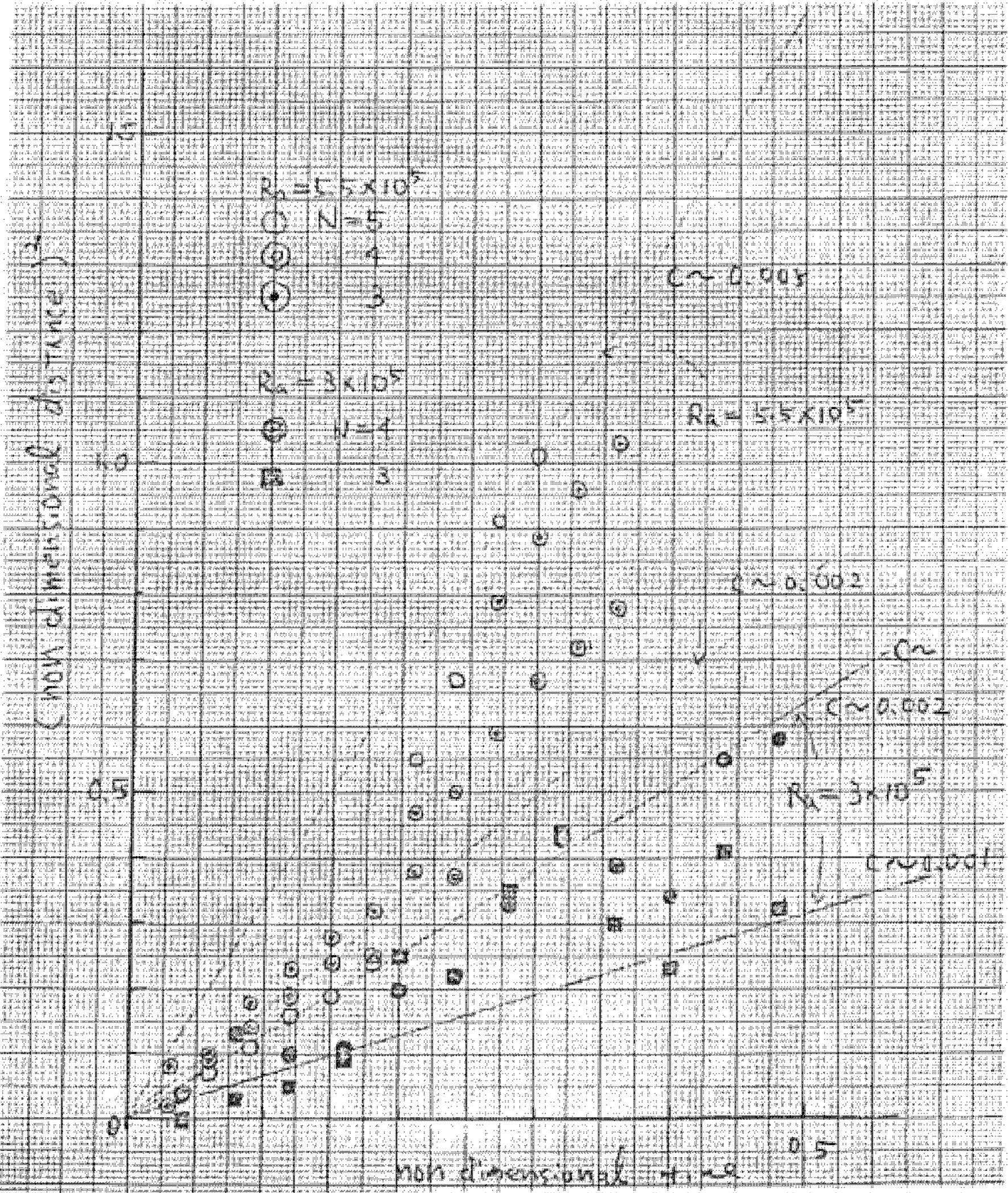


than i n the spokes pattern that we have studied. Even in our study, we found that the advection along the axis of rolls is almost zero. That implies the importance of the flow pattern. We must do more detailed experiments about this subject.

(3): (This may be related to (2)). Slowly deforming cell boundary was reported by Krishnamurt1 (1970) and Whitehead and Parsons (1978).

Krishnamurti (1970) reported that the $r$ ats of the slowly defoming boundaries is about an order of the themal diffusion time scale. Unfortunately, i $n$ our study, we could execute experiments only within a half of the thermal diffusion time. So it is not clear what relation exists between the slowly deforming cell boundaries and horizontal advection.

(4): It is well known that the convection begins to oscillate when the Rayleigh number exceeds about 105 at high Prandtl number (Krishnamurti, 1970; Whitehead and Parsons, 1978). En our experiments, oscillations begin clearly at $\mathrm{Ra}=5.5 \times 10^{4}$. It seems that this oscillation does not have $a$ major effect on the particle trajectory, but it is still uncertain.

Now, we will consider the possible implications of our results with regard to mantle convection. Recent development in the field of the geochemistry provides an evidence of the chemical heterogeneity in the mantle. Schilling (1973) proposed the two reservoir model of basalt source regions (One is the source region which can produce the Mid Oceanic Ridge Basalt and the other is the one which can produce the Oceanic Island Basalt or 'hot spot' magma) by the analysis of the chemical variation of the rocks observed between the Iceland and Mid Atlantic Ridge. Recently, Wasserburg and De Paolo (1979) proposed a chemically layered mantle based on the study of NC and Sr. Based on the simple mass conservation calculation of the various radiogenic isotopes, O'Nions et al. (1979) and Jacobsen and Wasserburg (1979) showed that only the small fraction of the mantle is associated with the creation of the continental crust. These studies imply that the chemically isolated reservoirs have existed for a long tine through the earth history. Because the diffusion constant of the isotopes is very small (order of $10^{-5}$ to 10-15 c.g*s.; see Hofmann and Hart, 1978), solid-state mantle convection of which existence is evident is considered to be a main disturber of the mantle. There arises a few recent works on this problem. For example, Richter and Ribe (1975) pointed out that the convection deforms the initial vertical inhomogeneity into thin spiral sheets, based on two dimensional convection models. Honda (1981b) considered the same problem by including the effect of rhe heat generation, and suggested the importance of the distribution of heat producing elements. On the viewpoint of the layered strncture of the earth (Wasserburg and De Paolo, 1979; Anderson, 1979; Richter and McKenzie (1981) and Honda (1981a) studied the layered convection. Richter and Mckenzie (1981) found by experiments that the layering of the convectioc breaks down when the density difference between the two flulds is almost the same as that produced by the themal perturbation. However, note that these studies axe a 11 concentrated on the vertical nixing.

It is well known that MORBs have fairly uniform isotope ratios of Sr (Hofmenn and Mart, 1978). There are a number of mechanisms which could mix the MORB forming materials. The characteristic mixing length of molecular diffusfon can be estimated as Dt where D is a diffusion constant. Supposing that the homogenization of MORBs is done only by the diffusion throughout the whole earth history, we can get the characteristic length of about $12 \mathrm{~cm}$ to $12 \mathrm{~km}$ which is very small compared to the characteristic length of the Mid Ocean Ridge (about $10,000 \mathrm{~km}$ ?). Our preliminary experiments show 
that the horizontal transport of particles in the Rayleigh-Bernard convection is represented as:

$$
\overline{x^{2}}=2 \times 10^{-3} \times R_{a}^{1 / 2} t
$$

assuming that horizontal transport phenomena can be regarded as a diffusion. (In the following discussions, we will assume that equation (2) is applicable to large Rayleigh number convection.) The magnitude of the Rayleigh number of the mantle convection is uncertain mainly because of the viscosity and depth extent of the convection. If the convection is assumed to be confined within the upper mantle, Ra is about 106 (Mckenzie et al., 1974). On the contrary, Ra is about 107, if the convection is whole mantle type. These estimates show that the characteristic length of the horizontal mixing by the RayleighBenard convection throughout the whole history of the earth is about $920 \mathrm{~km}$ $\left(\operatorname{Ra}=10^{6}\right)$ or $1200 \mathrm{~km}\left(\operatorname{Ra}=10^{7}\right)$. These values are still an order of magnitude smaller than the characteristic length of the Mid Oceanic Ridge. We must seek another alternative to explain the homogeneity of MORB. There are many differences between the mantle convection and the simple Rayleigh-Benard convection. Probably, main differences are:

(1) Mantle convection is, more or less, heated within.

(2) The viscosity of the mantle may vary considerably because of its temperature and pressure dependence.

(3) Mantle convection may be affected by the shear flow caused by the plate motion.

(4) A possibility of the difference in the turbulent process between our experiments and high Rayleigh number convection.

Mckenzie et a1. (1974) suggested by the simple two-dimensional calcu ations that the convection with the internal heat source appropriate to the mantle is time dependent. Probably, time dependent nature of the convect lon will greatly affect the horizontal advection. Effects of variable viscosity within the mantle on the horizontal advection are not clear. Yuen and Peltier (1980) suggested that the lower thermal boundary layer becomes unstable a id produces an upwelling because of the temperature pressure dependent visco ity. This may affect the homogenization. However, for the present time, we hi no image of the effect of the variable viscosity. The effect of the shear produced by the plate movement was studied by Richter (1973) and Richter and Parsons (1975). They showed that small scale convection of which axes a $1 \mathrm{gn}$ with the direction of the shear appears. This may give great effects on he horizontal advection.

The plate kinematics demands that ridges are not fixed to the mantle. Moving ridges and the large scale horizontal motions associated with platt tectonics are probably good mixers of the upper mantle. Krishnamurti and Howard (1981) showed that, over the Rayleigh number of about 106, the las se horizontal scale motion with tilted plumes appears. Although their experiments were done with small Prandtl number $\left(\mathrm{P}, \leqslant 10^{3}\right)$, this may occur in the mantle. These 'phase transition' may change c value by about an order. In any case large scale tectonic motions would cause large values of c in $\mathbb{A}$ similar way. Indeed, as described before, c seems to increase with $R_{a}$. There is one other trivial solution, that is, mantle is uniform at the stage of the early history of the earth. Indeed, there are many workers who considered that the earth was once totally melted because of the great accretionary energy and, as a result, the earth had become chemically stratified (Anderson, 1979). 


\section{CONOUUSIONS}

It is shown that the horizontal advection in Rayleigh-Benard convection may be expressed as follows by assuming that this phenomenon is a diffusionlike process.

$$
\overline{x^{2}} \approx 2 \times 10^{-3} \times R_{0}{ }^{4} t \quad\left(2 \times 10^{6} \leqslant R_{0} \leqq 6 \times 10^{5}\right)
$$

This shows the inefficient lateral mixing by small scale convection in te mantle compared to the vertical one. However, we think that experiments were still not extensive enough, and we must do the more detailed and systemallic experiments. We believe that such experiments will reveal the complex process of the convection by a different angle. In any event, it appears that snall scale mantle convection may not adequately mix the upper mantle.

\section{ACKNOWLDDEMENIS}

I would like to express my sincere thanks to Dr. J. A. Whitehead who suggested this problem and provided his experimental apparatus to me. I am grateful to Drs. Malkus, Veronis and Stern who discussed this topic. Drs. Malkus and Whitehead read the manuscript critically and pointed out the physical meaning of $c$. I also would like to thank Robert Frazel who helped me technically throughout the whole experiment. Especially he gave me the idea of particles used in this study. The Woods Mole Oceanographic Institution gave me an opportunity to attend the Geophysical Fluid Dynamics Summer Program through support from the Center for the Analysis of Marine System and is greatly thanked.

\section{APPENDIX}

Recently, Roberts (1979) analyzed ehe fast viscous Benard convection, and proposed the relation between the velocity and Rayleigh number as follows.

$$
\begin{array}{ll}
k \propto \frac{k}{d} R_{a}^{2 / 3} & \text { (in the case of free-free boundari } s) \\
u \propto \frac{k}{d} \mathrm{Ra}^{3 / 5} & \text { (in the case of rigid-rigid boundsies) }
\end{array}
$$

To check the velocity dependence on Rayleigh number, we tried to calculat $\overline{v^{2}}$. Results are shown in Figure 7. Also c value was calculated by adopting above relations, and results are shown in Table 2 and Figure 8 . Probably it is still premature to select above relations. Discussions described in the latter half of this paper is still valid, even if we choose another relation between the velocity and Rayleigh number.

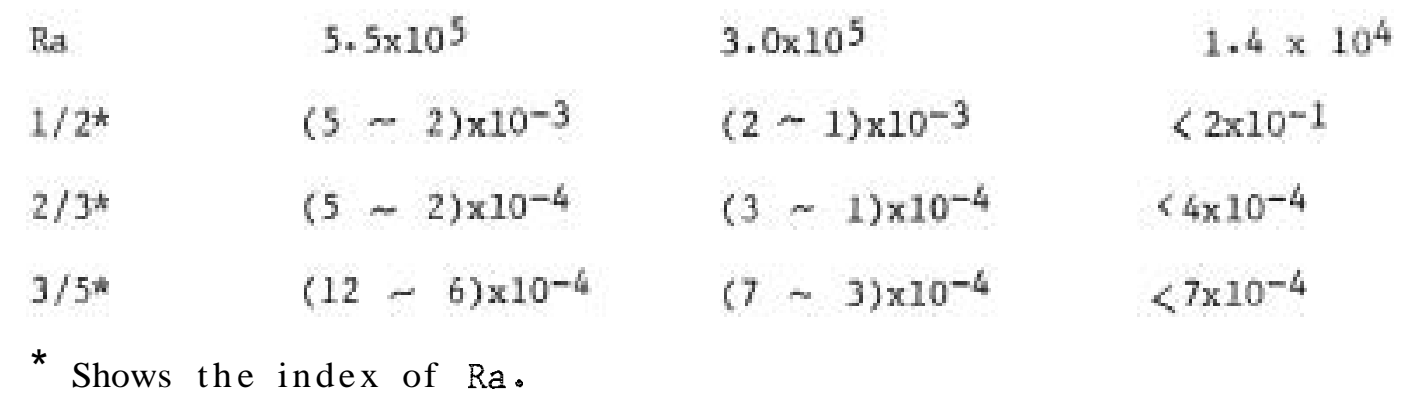

TABLE 2. 

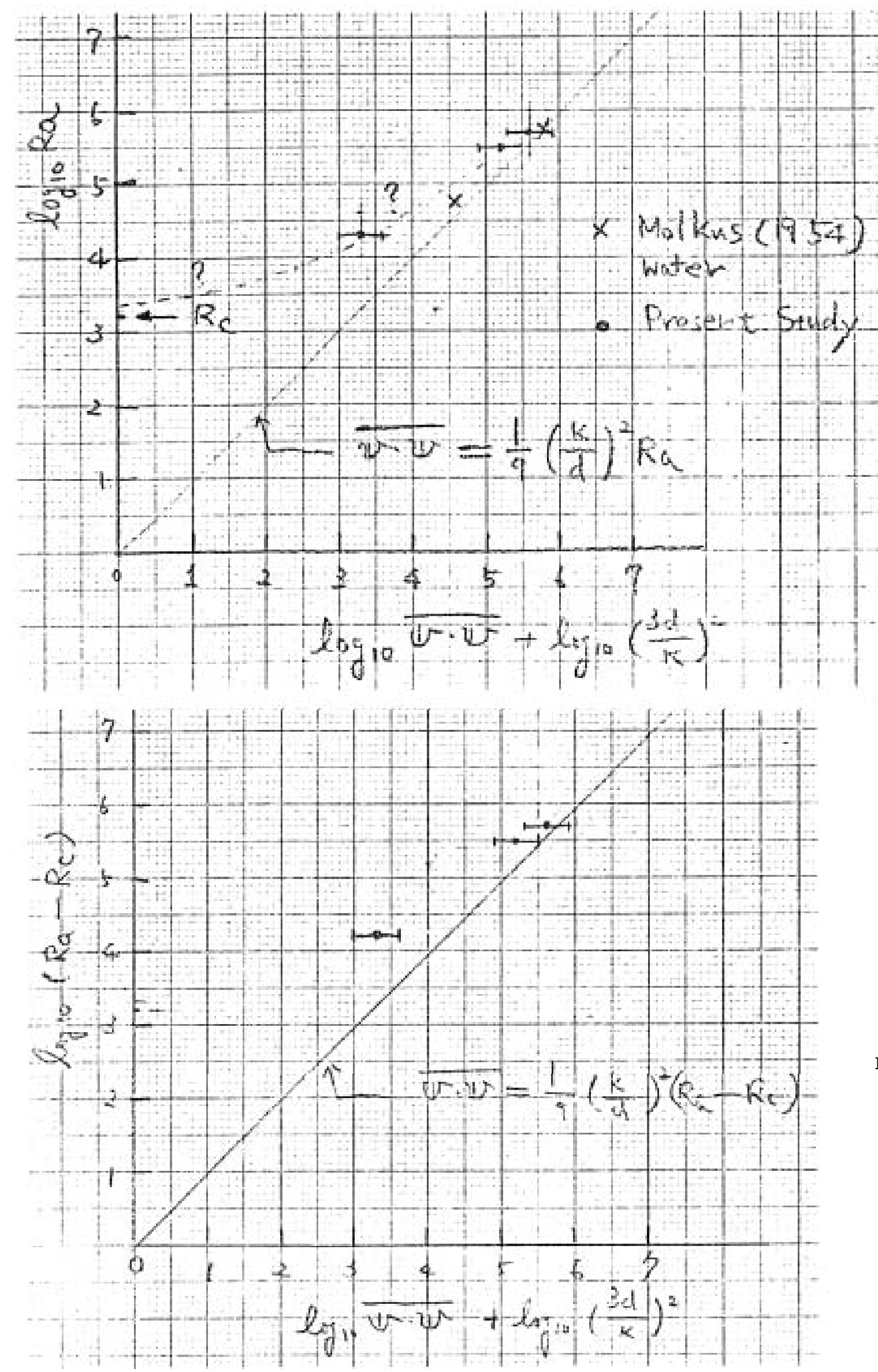


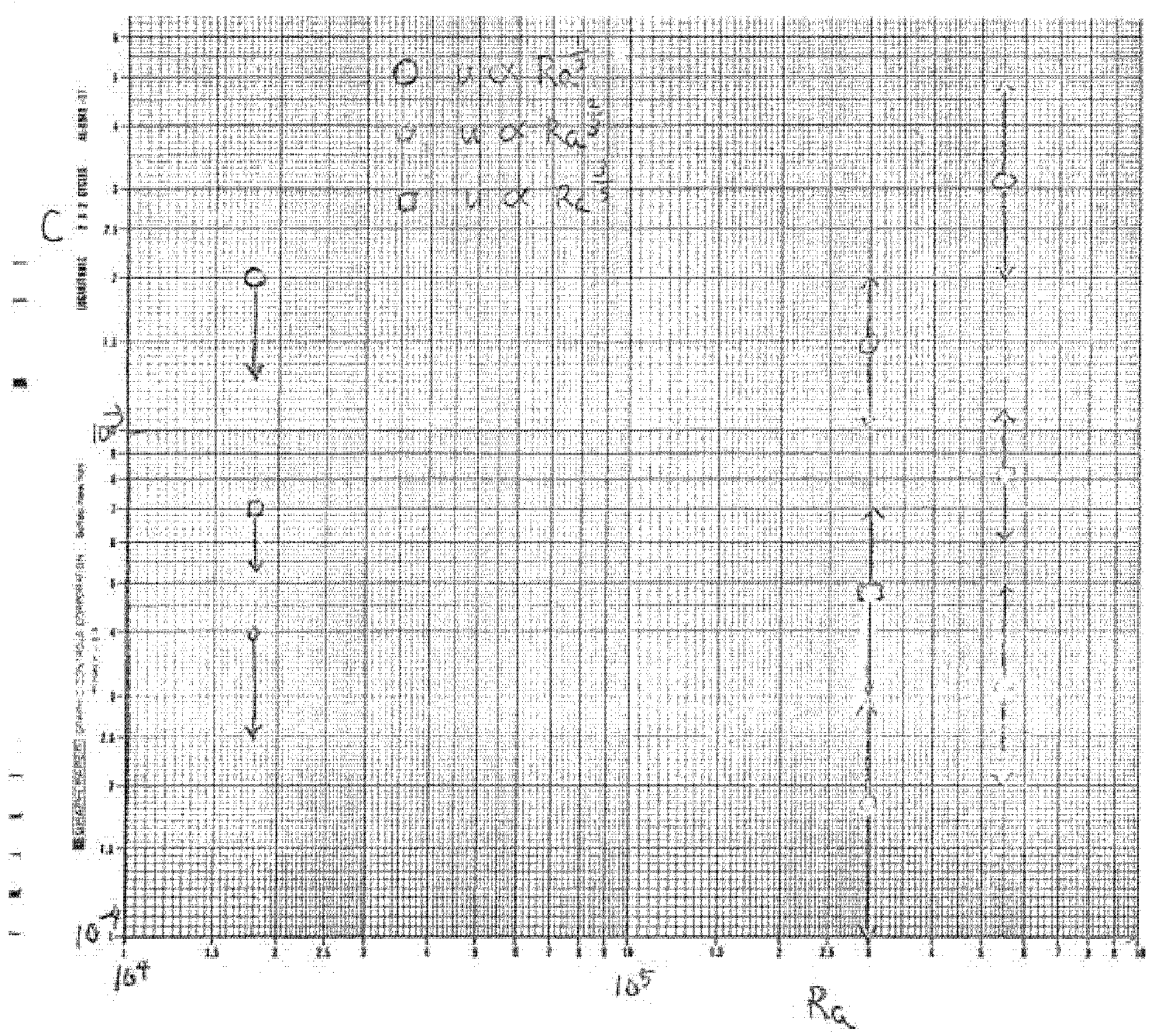

Figure 8 


\section{REFERENCES}

Anderson, D. L., 1979. Chemical stratification of the mantle, I. Geophys. Res. 86, 6297-6298.

Busse, $F_{0} H_{*}$ and JoA. Whitehead, 1971. Instabilities of convection rolls in high Prandt 1 number fluids. I. Fluid Mech., 47, 305-320.

Hofmann, A* W。 and S. R. Hart, 1978. An assessment of 1 ocal and regional isotopic equilibrium in the mantle. Earth Planet. Sci. Lett., 38, 44-62.

Honda, S., 1981a. Numerical analysis of layered convection. Preprint.

Honda, S., 1981b. On the dynamical stability of the heat producing element distribution. Preprint.

Jacobsen, S. B, and G. J. Wasserburg, 1979. The mean age of mantle and crustal reservoirs. Jo Geophys. Res., \$4, 7411-7427.

Krishnamurti, R., 1970. On the transition to turbulent convection. Part II. The transition to time-dependent flow. J.Fluid Mech., 42, 309-320.

Krishnamurti, R. and L.N. Howard, 1981. Large-scale flow generation i n turbulent convection. Proc. Nat1. Acad. Sci., USA, 78, 1981-1985.

Malkus, W.V.R., 1954. The heat transport and spectrum of thermal turbulence. Proc. Roy. Soc. A, 225, 196-212.

Malkus, W.V.R., 1959. Worizontal diffusion due to turbulent convection. Oxford Fluid Dynamnics Conference.

Mckenzie, D. P., J. M. Roberts and N. O. Weiss, 1974. Convection in earth ${ }^{9}$ s mantle: towards a numerical simulation. Jo Fluid Mech。, 62, 465-538.

O'Nions, R. K., N. M. Evensen and P. J. Hamilton. Geochemical modeling of mantle differentiation and crustal growth. J. Geophys. Res., 84 , 6091-6101.

Richter, F. M., 1973. Convection and the large-scale circulation of the mantle. J. Geophys. Res., 78, 8735-8745.

Richter, F. M. and D. P. McKenzie, 1981. On some consequences and possible causes of layered mantle convection. J. Ceophys. Res., 86, 6133-6142.

Richter, F. M. and B. Parsons, 1975. The interaction of two scales of convection in the mantle. Je Geophys. Res.: 80, 2529-2541.

Richter, F. M. and N.M. Ribe, 1979. On the importance of advection in determining the local isotopic composition of the mantle. Earth Planet. $\underline{\text { Sci. Lett }}, \underline{43}, 212-222$.

Roberts, G. O., 1979. Fast viscous Benard convection. Geophys. Astrophys. Fluid Dynamics, 12, 235-272.

Schilling, J. G., 1973. Iceland mantle plume: Geochemical study of Reykjanes ridge. Nature, 242, 565-571. 
Turner, J. S., 1974. Double-diffusive phenomena. Ann. Rev. Fluid Mech., 6 , $37-56$.

Wasserburg, G., Jo and De Paolo, 1979, Models of earth structure inferred from neodymium and strontium isotopic abundances. Proc. Nat1. Acad. Sci., LSA, Z6, 3594-3598.

Whitehead, J. A. and Be Parsons, 1978. Observations of convection at Rayleigh numbers up to 760,000 in a fluid with large Prandtl number. Geophys. Astrophys. Fluid Dynamics, 2, 201-217.

Yuen, D. A. and $W, R$. Peltier, 1980. Mantle plumes and the thermal stability of the "D" layer* Geophys. Res. Lett., 1, 625-628.

\section{SPATIALLY VARYING PLAN-FORMS: PERPENDICULAR SYSTEMS OF ROLLS}

\section{Evan Fishbein}

\section{INTRODUCTION}

Of the plan-forms which have been studied in Raylefgh-Benard convection, most are periodic structures. Examples of these are the rolls and hexagons discussed by Chandrasekhar (1961).

These plan-forms are idealized arrangements, which in physical systems are observed only over short distances. In solid state physics defects are seen which disrupt the large scale order of the crystal lattice. Corresponding defects, at least visually, are observed in convection plan-forms. Three types of these plan-form defects are pictured in the following diagram:

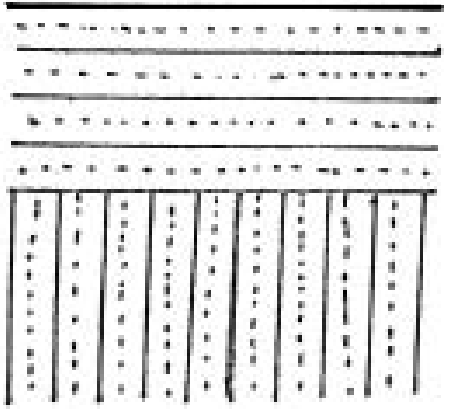

Grain Boundary

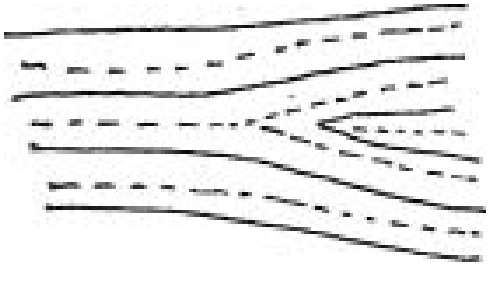

Dislocation

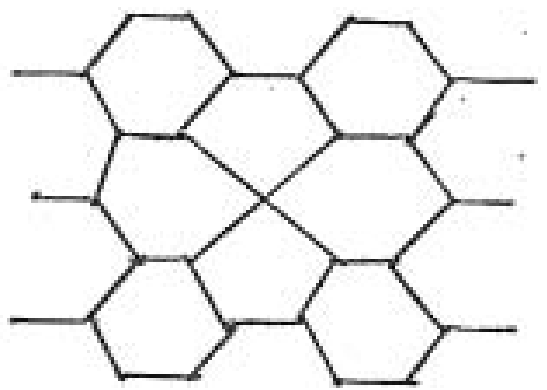

Point defect

There has been little study of defects in convection plan-forms. Siggia and Zippelius (1981) have described dislocations analytically and numerically. In this paper some analytic calculations and experimental observations will be discussed on the evolution of a grain boundary (henceforth abbreviated as bourdary) separating two regions in which the plan-form is almost periodic,

The boundary is straight and infinite in extent. In one region the rolls have axes parallel to the boundary, while in the other they are perpendicular. In either region, away from the boundary, the solution is equivalent to the one given by Malkus and Veronis (1958) for rolls in an infinite layer. 
Several questions which will be addressed are:

i) Is the boundary stable?

i i) If it is not, is one region preferred?

i i i) How wide is the boundary in terms of how quickly do the amplitudes decay?

iv) If it is unstable, what is the nature of the instability?

\section{Analytical Results}

Consider an infinite layer of high Prandtl number Boussinesq fluid heated from below and having the previously described plan-form imposed at the onset of convection. A section of the boundary is shown below. The $X$ axis is along the boundary, the $Y$ axis is perpendicular to it in the horizontal plane, and the $Z$ axis is anti-parallel to gravity. The origin is chosen so as to make the temperature-perturbation field an odd function in $\mathrm{X}$. The boundary conditions are free-free.
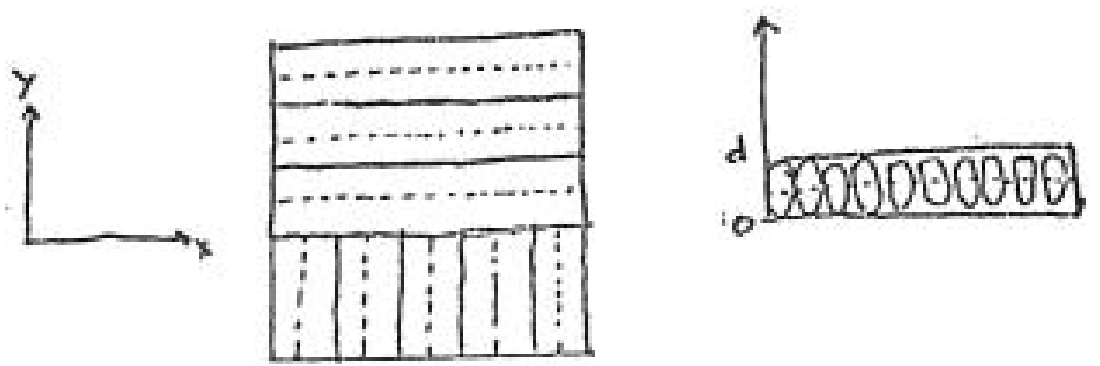

The dimensionless equations are

$$
\begin{aligned}
& u=(u \hat{x}, v \hat{y}, w \hat{z}) \\
& \nabla \cdot u=0 \\
& R\left(\nabla \partial_{t} \theta-\nabla^{2} \theta \hat{z}\right)=\nabla^{\mu} \underline{u} \\
& \partial_{4} \nabla^{4} \theta-\nabla^{6} \theta+R\left(\partial_{\lambda x}^{2}+\partial_{Y y}^{2}\right)=-\nabla^{4}(\mu \cdot \nabla \theta) \\
& R=\frac{g o \Delta T_{\alpha^{3}}}{k \nu}
\end{aligned}
$$

and have dimensions

$$
\begin{array}{ll}
\text { length } & x_{d}=d x \\
\text { velocity } & w_{d}=\frac{k}{d} \stackrel{u}{\sim} \\
\text { time } & t_{d}=\frac{d^{2}}{t} \\
\text { temperature } & T_{d}=T_{k}-\frac{\Delta I_{z}}{d}+\Delta T \theta
\end{array}
$$

where $K, \nu, \infty$ are the thermal diffusivity, kinematic viscosity and expansion coefficients of the fluid, and $g$ is the gravitational acceleration. 
Performing a modal expansion, where both regions have rolls with wave number $\alpha=\pi / \beta$, (the neutrally stable wave number at the critical Rayleigh number $R_{c}=\frac{27 r_{4}}{4}$, the Rayleigh number, velocity and temperature perturbation are

$$
\begin{aligned}
& R=R+e^{k} R_{k} \\
& u=\epsilon^{k} U_{k}^{m+r} \text { sn nax csriz } \\
& v=\epsilon^{k} V_{k}^{m} \text { es ndx su ret } \\
& w=6^{k} W_{k}^{n r} \text { es nore sis rigz } \\
& \theta=\epsilon^{k} \theta_{k}^{n} \text { cs nax sn rit }
\end{aligned}
$$

The indices $K, n$ and $r$ are summed over the ranges

$$
\begin{aligned}
& 1 \leqslant k \leqslant \infty \\
& 0 \leqslant n \leqslant \infty \\
& 1 \leqslant r \leqslant \infty
\end{aligned}
$$

The amplitudes $\cup_{k}^{m_{2} r}, \quad v_{k}^{n_{k} r} \quad W_{k}^{n_{2} r}$ and $\theta_{k}^{k_{2} r}$ are slowly varying functions of $\tau=\epsilon^{2} t, Y=y$, as well as the fast parameter $y$. To order $\epsilon$ the perpendicular rolls are given by

$$
U_{1}^{\prime \prime}(\tau, Y) \quad W_{1}^{\prime \prime}(\tau, Y) \quad \theta_{1}^{\prime 2 t}(\tau, Y)
$$

with

$$
\lim _{Y \rightarrow \infty} \theta_{1}^{\prime \prime}=0 \quad \lim _{Y \rightarrow-\infty} \theta_{1}^{1, Y}=1
$$

while the parallel rolls are characterized by

with

$$
\begin{aligned}
& v_{1}^{0, \prime}(Y, \tau, Y) \quad W_{1}^{0,1}(Y, \tau, y) \quad \theta_{1}^{0 \prime}(Y, \tau, y) \\
& \theta_{1}^{0,}=\Psi_{1}^{0,}(\tau, Y) \sin \alpha y+\Phi_{1}^{9}(\tau, Y) c s=1 Y
\end{aligned}
$$

$$
\lim _{y \rightarrow \infty}\left(\Phi_{1}^{s}\right)^{2}+\left(\Phi_{1}^{0,1}\right)^{2}=1 \quad \lim _{Y \rightarrow-\infty}\left(\Psi_{1}^{0,1}\right)^{2}+\left(\Phi_{1}^{0}+\right)^{2}=0
$$

Substituting (3) into (I), one obtains

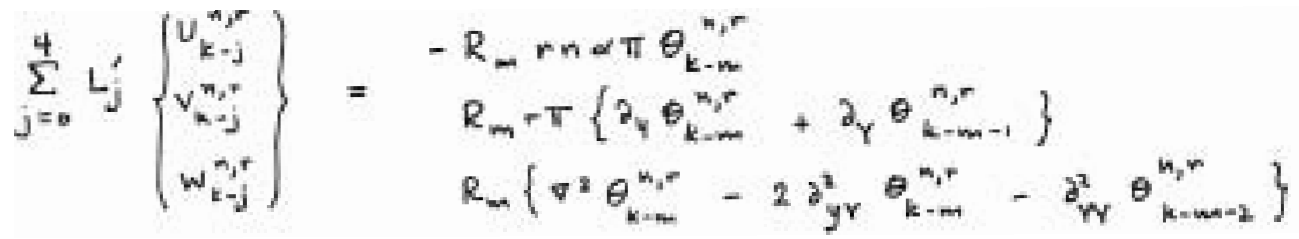


where

$$
\begin{aligned}
& \nabla^{2}=\partial_{Y Y}^{2}-k^{2} \\
& L_{0}^{\prime}=\nabla^{4} \\
& L_{1}^{\prime}=4 \nabla^{4} \partial_{y Y}^{2}
\end{aligned}
$$

and $L_{2}^{\prime}, L_{3}^{\prime}$ and $L_{y}^{\prime}$ are more complicated operators containing $\partial_{y}, \partial_{Y}$ and

$k$. The right-hand side of (6) has an implied sum of over the even integers from 0 to $\infty$, but only terms when $m=0$ or 2 enter into the calculation. Terms containing $\partial$ to any power do not enter into the calculation, hence on the left only $L_{o}$ is $\Upsilon_{\text {used. }}$

The energy equation is

$$
\sum_{j=0}^{b} L_{j} \theta_{1-j}^{n_{j}}-\sum_{j=0}^{+} L_{j}^{\prime} N_{L-j}^{n, r}=\sum_{j=7}^{\infty}\left(R_{j-2} \partial_{\psi \psi}^{2}+2 R_{j-1} \partial_{y Y}^{2}+R_{j} \nabla^{2}\right) \theta_{1-j}^{n, \gamma}
$$

where

$$
\begin{aligned}
& L_{0}=-\nabla^{6}+R_{0} \nabla^{2} \\
& L_{2}=\nabla^{4} \partial_{\tau}+R_{2} \nabla^{2}+\left(R_{0}-15 \partial_{Y \partial Y y}^{4}+18 k^{2} \partial_{y y}^{2}-3 k^{4}\right) \partial_{Y Y}^{2} \\
& L_{4}=6\left(\partial_{Y y}^{2}-k^{2}\right) \partial_{Y Y v}^{3}+R_{2} \partial_{Y Y}^{2}+R_{4} \nabla^{2}-\left(15 \partial_{Y y}^{2}-3 k^{2}\right) \partial_{Y Y Y Y}^{4}
\end{aligned}
$$

The operators $L_{1}, L_{3}, L_{4}, L_{5}, L_{6}$ do not enter the calculation, nor do the terms on the right-hand side of (7). The terms $N_{4}^{m}$ ar a obtained by expanding $\underset{\sim}{\sim} \nabla$ in a. Fourier series. Each of these terms is a series of all products of velocity and temperature-perturbation gradient which are resonant with $\cos$ mox sin $r \pi$ and of order $\tau^{\ell}$. The terms of the series can be obtained from the table below.

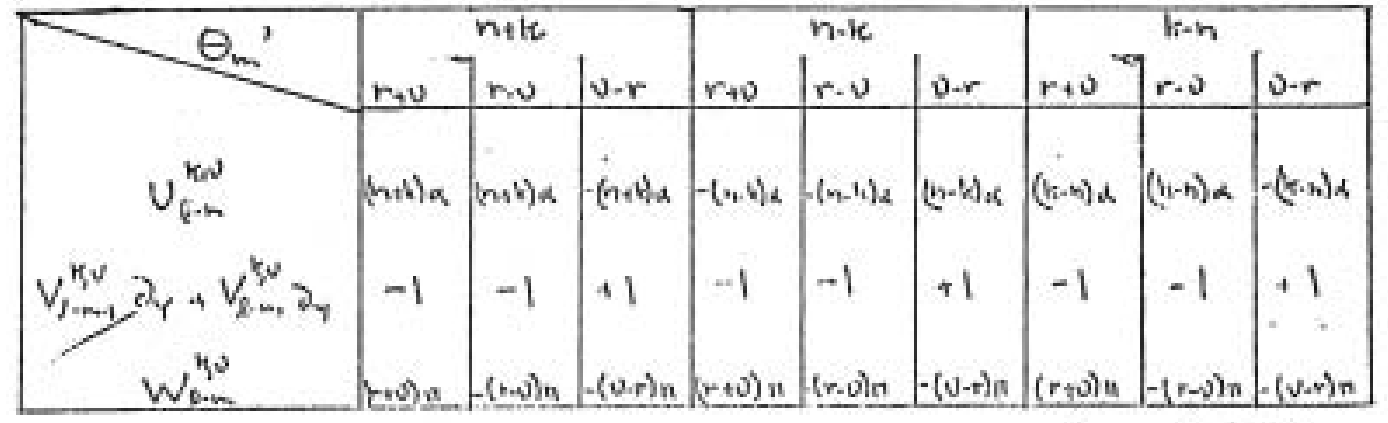

(not used when $n=0$ )

As an example, contribution, to $N_{1}^{1,2}$ arising from $u_{1}^{0,1}, e_{1}^{1,1}$ are obtained from column $2(n+k, r-\nu)$ when $h=1, k=0, r=2, \nu=1$ and column, 5 $(\mathrm{n}-\mathrm{k}, \mathrm{r}-\nu)$ when $\mathrm{h}=1, \mathrm{k}=0, \mathrm{r}=2, \quad \gamma=1$ giving $-2 \partial_{y} \theta_{1}^{\prime}, v_{1}^{\prime \prime}-2 \pi \theta_{1}^{\prime}, w_{1}^{\prime,}$, Similarly the contribution from 4,2 is obtained from column 5 when

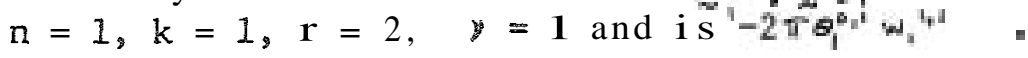

To obtain time dependence and diffusion in the order $\epsilon^{<}$amplitude requires that the $\epsilon^{2}$ amplitudes be evaluated and some of the $\epsilon^{3}$ equations. The non-trivial equations to order $\epsilon$ obtained from (6) and (7) in terms of $\Phi_{,}^{\infty, \eta}, \delta_{,}^{\theta_{1},}, \theta_{i}^{\prime}$ 
are

$$
\begin{aligned}
& V_{1}^{* \prime \prime}=\frac{4 \alpha R_{0}}{9 z^{3}}\left(B_{1}^{* 1} \operatorname{cs} \alpha y \quad \Phi_{1}^{* 1} \sin \alpha_{y}\right) \\
& W_{1}^{\prime \prime \prime}=\frac{2 R_{E}}{q \pi^{2}}\left(\Phi_{1}^{91} \sin \alpha y+\Phi_{1}^{\prime \prime} \operatorname{cs} \alpha y\right) \\
& U_{1}^{\prime \prime \prime}=\frac{-4 a R \alpha}{4 \pi^{3}} \theta_{1}^{\prime \prime \prime} \\
& W_{1}^{b^{\prime} t}=\frac{2 R_{a}}{9 \tau^{2}} Q_{1}^{l_{1} t}
\end{aligned}
$$

The $\epsilon^{2}$ equations are

$$
\begin{aligned}
& \theta_{2}^{\theta_{1} 2}=\frac{-R_{a}}{36 \pi^{3}}\left\{\left(\theta_{1}^{\theta_{1}}\right)^{2}+\left(\Phi_{1}^{\theta_{1}}\right)^{2}+\left(\Phi_{1}, \theta^{2}\right\}\right. \\
& \theta_{2}^{1,22}=\frac{R_{a}}{473 \times 9 \pi^{3}}\left\{E_{1}^{0,1} \sin a y+\Phi_{1}^{0,1} \mathrm{cr} a y\right\} \theta_{1}^{1,1} \\
& U_{1}^{1,2}=\frac{6 R_{a}}{473 d}\left\{Q_{1}^{0,1} \text { eray }-\Phi_{1}^{0,1} \sin a_{y}\right\} \theta_{1}^{1,1}
\end{aligned}
$$

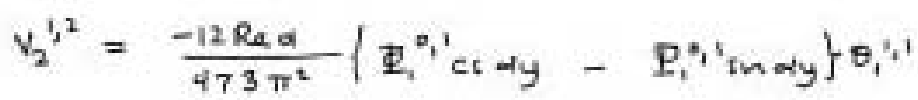

$$
\begin{aligned}
& W_{1}^{1,2}=\frac{-6 R a}{472 \pi}\left\{\Phi_{1}^{0,1} \text { inay }+\Phi_{1}^{*, 1} \operatorname{cs} \alpha y\right\} \theta_{1}^{1,1}
\end{aligned}
$$

and the $\epsilon^{3}$ equations of interest are

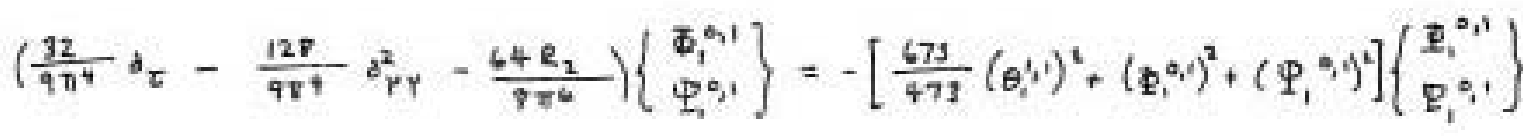

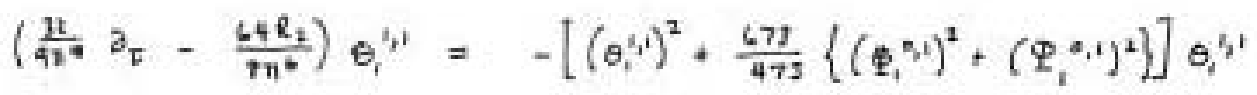

The above two equations describe the lowest order perturbation on one system of rolls from the other. The first new resonance terms, which are not present without the boundary are the $\left\{f_{2}^{1,2}\right.$ terms which describe three dimensional flow.

The amplitudes appearing $1 \mathrm{n}(10)$ are distinguishable without the indices, which are now removed. The equacions are symmetric in $\Phi$ and $\Psi$ in the sense that by an appropriate shift of the orfgin along $\hat{y}$, efther variable can be eliminated. Eliminating $\Phi,(4)$ and (5) give $R_{2}=\frac{3 \pi^{2}}{64}$. Time independent solutions to these equations were obtained by $L$. Howard. Trajectories in phase space which contain the point $\Phi=0, \theta=1, \psi--\infty$, 11e on the ellipsoidal cylinder

$$
\begin{array}{r}
\theta_{-\infty}^{2}=1-\gamma^{2} \phi_{-\infty}^{2} \\
\gamma^{2}=\frac{(7)}{473}
\end{array}
$$


and satisfy the equation

$$
\varphi^{\prime \prime}=-\left(\gamma^{4}-1\right) \varphi^{3}+\left(\gamma^{2}-1\right) \varphi
$$

Multiplying by $\Phi^{\prime}$, integrating so as to satisfy (5) taking the square root and integrating over the appropriate branch, the solution is

$$
\begin{aligned}
& \Phi_{-\infty}=\sqrt{\frac{2}{1+\gamma^{2}}} \operatorname{sech}\left\{\sqrt{\frac{\gamma^{2}-1}{2}} \frac{2 \pi}{\gamma}\left(Y_{-\infty}-\gamma\right)\right\} \\
& \theta_{-\infty}=\left\{\frac{1-\gamma^{2}}{1+\gamma^{2}}+\frac{2 \gamma^{2}}{1+\gamma^{2}} \tanh ^{2}\left[\sqrt{\frac{\gamma^{2}-1}{2}} \frac{2 \pi}{\gamma}\left(Y_{-\infty}-\gamma\right)\right\}^{1 / 2}\right.
\end{aligned}
$$

Similarly, solutions which contain $\Psi=1, \quad \theta=0$ lie on the plane

$$
\theta_{+\infty}=0
$$

and satisfy

$$
\psi^{\prime}=1-\psi^{2}
$$

giving

$$
\psi_{+\infty}=\tanh \left[\frac{3 \pi^{2}}{16}\left(Y-Y_{\infty}\right)\right]
$$

The constants, $Y_{-\infty}$ and $Y_{+\infty}$ are chosen so that

or

$$
\Psi_{-\infty}\left(\gamma_{0}\right)=\Phi_{-\infty}\left(\gamma_{0}\right)=\gamma^{-1}
$$

$$
\begin{aligned}
& Y_{-\infty}=Y_{0}+\frac{8}{3 \pi^{2}} \sqrt{\frac{2}{\gamma^{2} j}} \operatorname{sech}^{-1} \sqrt{\frac{1+\gamma^{2}}{2 \gamma^{2}}} \approx Y_{0}+.24 \\
& Y_{+\infty}=Y_{0}-\frac{\pi}{3 \pi^{2}} \tanh ^{-1} \gamma^{-1}=Y_{0}=.637
\end{aligned}
$$

The solutions, shown below

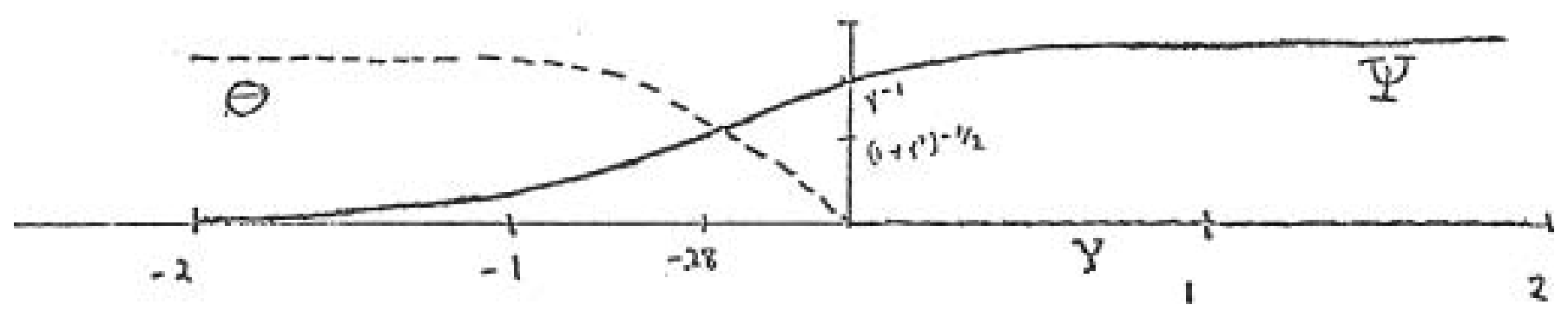

They are infinitely differentiable everywhere, except at $Y_{s}$ where $\theta$ is not and $\mathbb{Q}$ is only twice so. While this does not create a problem in the $6^{3}$ equation, in the $\epsilon^{5}$ equations, the term $\partial_{y y y y} \theta$ (contained in $L_{4} \theta$ ) is unbounded, which implies that the perturbation series does not converse at $\mathrm{Y}_{0}$. This problem is avoided by allowing $\theta$ to be a function of an inte:mediate length scale $y=E^{1 / 2} y$ " The operators $L_{i}$, $L_{i}^{\prime}$ are transformed by

$$
\partial_{Y} \rightarrow \partial_{Y}+e^{-1 / 2} \partial_{y}
$$


so that the diffusion term in the old $L_{4}$,

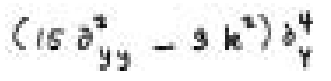

introduces

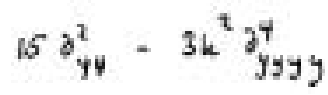

into the new L 2 giving the Newe11 and Whitehead (1969) equation

$$
\left\{\partial \tau+\frac{2}{\pi^{2}} \partial_{y y y y}^{4}-\frac{9 \pi^{4}}{22}\right\} \theta=-\frac{9 \pi^{4}}{32}\left[\gamma^{2} \psi^{2}+\theta^{2}\right] \Psi
$$

By using (10) a solution could be found. Since this was the first term of a perturbation series, the series diverges at $Y_{0}$. In the neighborhood of $Y_{0}$, a boundary layer convection, using the Newell-Whitehead equations could have been performed to smooth out the kink. One still can conclude that a stationary transition zone (boundary) with thickness $\frac{2 d}{\epsilon}$ exists, although its stability has not been tested.

$\underline{\text { Experimental Observations }}$

Some experiments on the evolution of a grain boundary were performed in a high Prandtl number fluid. The procedure is described in detail in Chen and Whitehead (1968). Briefly, a small lateral temperature field of the desired pattern is induced on the surface of the fluid layer with a heat lamp while the fluid is stably stratified. The mean horizontal temperature field is then changed to make the fluid unstable. When convection begins, the cold areas tend to form upwelling zones, while the warm become downwelling zones. The induced temperature field is then allowed to relax (the heat lamp is turned off) and the pattern evolves naturally. By the nature of the inducing procedure, the induced field determines the location and orientation of the roll axes, but not the amplitude of convection. Because of this, the structure of the boundary does not correspond to the structure found i $n$ the analytic solution, but is narrower.

The set-up is shown in Figure la during the inducement phase. The inducing pattern rests on top of the tank. The shadowgraph technique is used to visualize the flow; one of the mirrors used to bend the light path is seen above the tank. The fluid was Dow-Corning 200 silicon oil, which has a Prandtl number of 8,600 and a viscosity of 2 stokes. The rolls in both regions initially had wave number $\varnothing=3.11$, (the marginally stable mode at the critical Rayleigh number for fixed-fixed isothermal boundary conditions, $\mathrm{R}_{\mathrm{C}}=1708$ ) the tank was $91 \mathrm{~cm}$ long parallel to the boundary and $89 \mathrm{~cm}$ long perpendicular to it. The depth of the layer was $3 \mathrm{~cm}$, giving a thermal diffusion time of about 2.2 hours. The 'temperature at the top and bottom of the layer were controlled by water baths separated from the fluid layer by plate glass approximately $.64 \mathrm{~cm}$ thick. At $\mathrm{R}=10,000$ about $20 \%$ of the temperature difference between baths was across the glass, hence the top and bottom surfaces were not exactly isothermal.

Three runs, two at $\mathrm{R}=17,600$ and the other at $\mathrm{R}=9,000$ were performed. The evolution of one of the runs at $\mathrm{R}=17,600$ is pictured in Figure Ib-d. Five general observations can be made based on all three runs. 


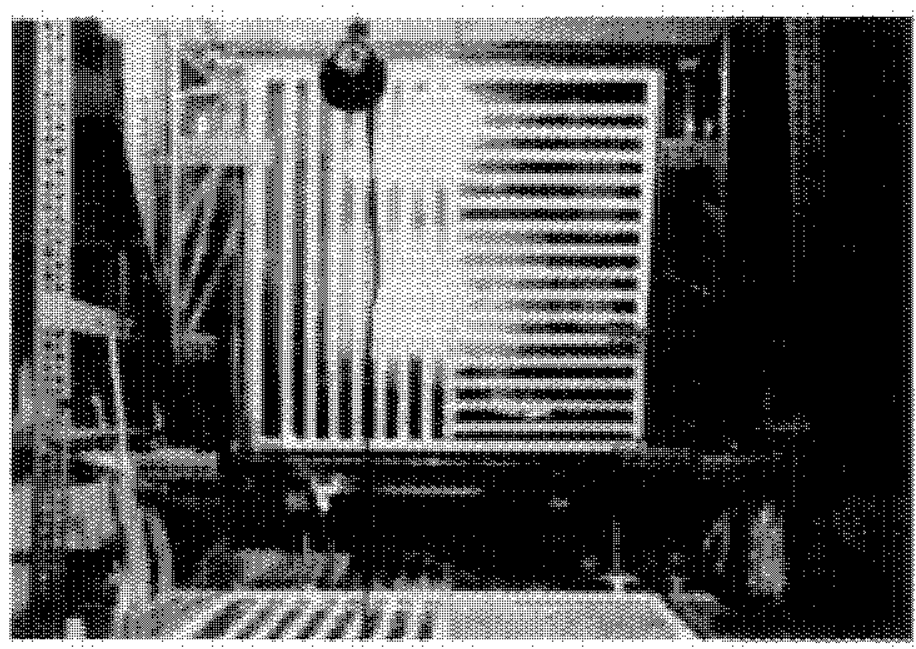

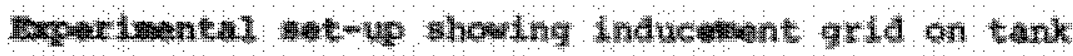

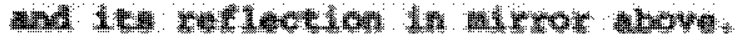

19ume 1 a

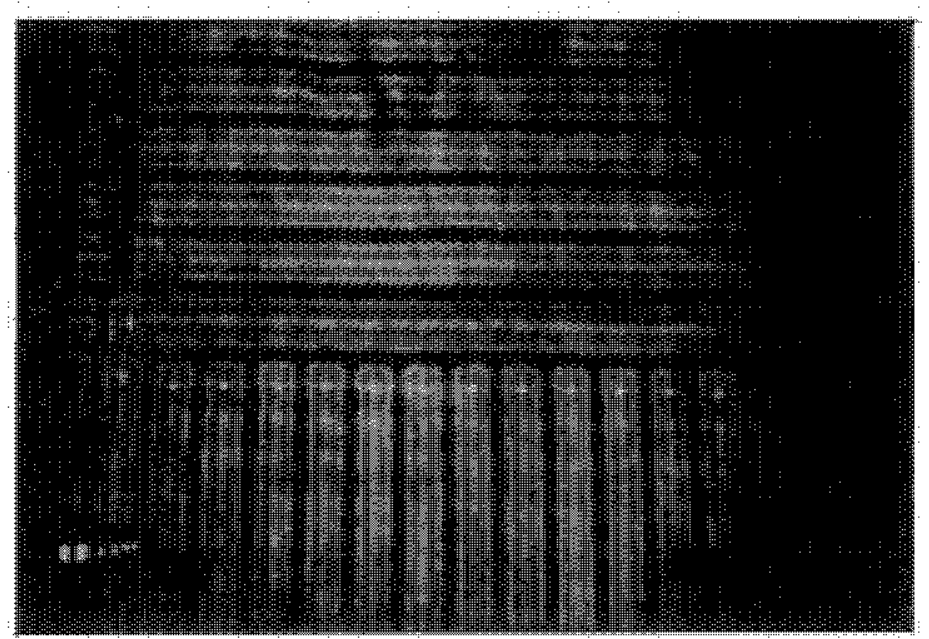

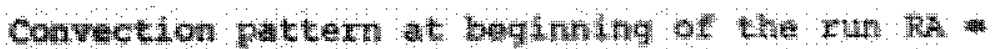
17,600 (radge 1 seflected Laht others are erandetect

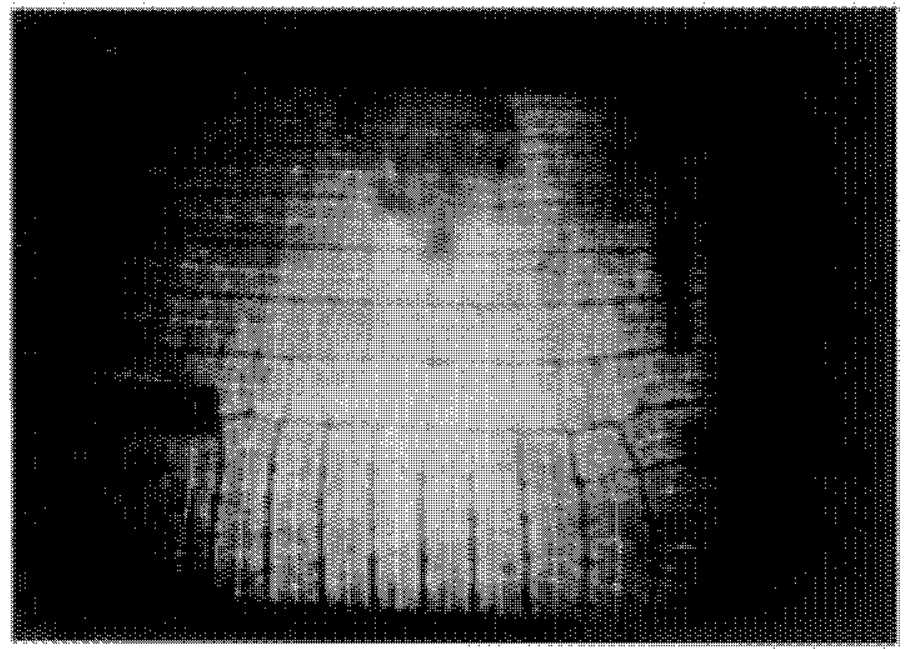

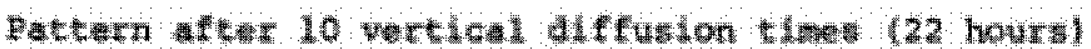

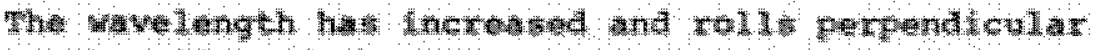

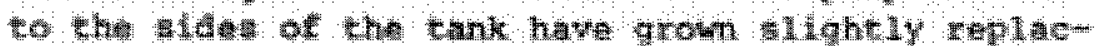
trg the prependlcular rouly

Mrise:

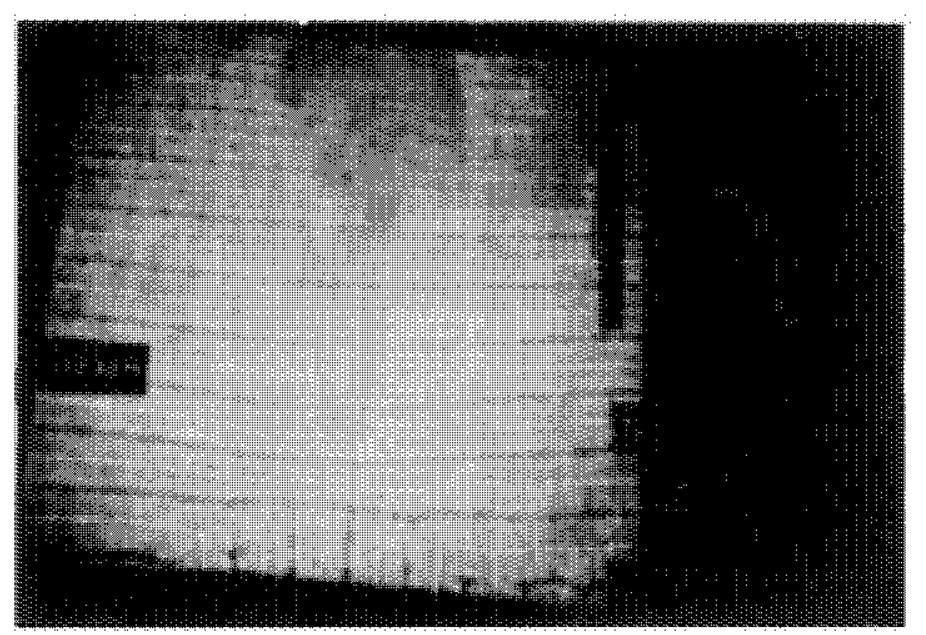

packern after 17 vertical Niffusion thes 1325

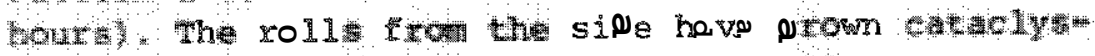
nucally replacing the perTendlcwlor ralls. 
1) The boundary was unstable to finite perturbations. These were attributed to the sidewalls of the tank and impurities in the fluid.

2) The instability led to one regime eroding the other.

3) Subcritical cross rolls of finite amplitude in the neighborhood of the boundary played an important role in the erosion process.

4) The erosion evolved in two stages. The first was a period of growth for the cross rolls (increase in the width of the boundary) with little erosion. The second was a rapid erosion of one region by the other. The transition from one stage to the other occurred cataclysmically.

$5)$ The $x$-dependence of the instability could not be represented as a sum of harmonics of the fundamental $e^{i \alpha x}$.

For two of the runs, one at $R=17,600$ and the other at $R=9,000$, the parallel rolls dominated. As has been observed by Gollub and Steinman (1981), rolls which intersect a wall and whose axes are parallel to the wall are partially replaced by "wall rolls". These have axes perpendicular to the wall and typically extend only a short distance into the tank, depending on the tank dimensions. These can 'be seen in Figure 4c; because of the geometry of the initial state, wall rolls developed in the perpendicular roll region near the boundary, but not on the parallel roll side of the boundary. The wall roll closest to the boundary appeared to be driven by the rolls on the other side of the boundary because this one grew faster than others further away.

Although the instability was initiated at the side walls, once established, it seemed to grow faster the longer it became. This may have been because at a later time the cross rolls were more established, or because its growth rate was related to its amplitude which was related to its length.

The effect of increased Rayleigh number is not well established. It appears that although instability was always characterized by initial adjustment followed by cataclysmic erosion at the lower Rayleigh number this was less extreme. At the higher Rayleigh number, during the period of initial adjustment, the wave number decreased from 3.1 to 2.4. Since the number of perpendicular rolls decreased from 16 to 11, it seems unlikely that this is explained i n terms of readjustment to fit within the box. If this effect took place at the lower value of Rayleigh number, it was much less pronounced.

One of the runs at $R=17,600$ ended with the perpendicular rolls dominating. Unlike the previous runs, impurities in the region of perpendicular rolls near the boundary, forced the boundary to bulge into the region of parallel rolls. How this affected the mechanics is unclear; however, the resulting time evolution closely paralleled the other two runs, in that it was a two-stage process.

$\underline{\text { Conclusions }}$

Based on the experiments, it seems likely that the analytic solution is an unstable equilibrium plan-form. A conjecture is that during the initial adjustment stage, the plan-form was evolving towards the equilibrium solution. The basis for this is that a thicker boundary is closer to the equilibrium solution in phase space, provided the time rate of change in the amplitudes are not too large. During the erosion stage, the plan-form is evolving away from the equilibrium solution very rapidly. 
Whether one region is preferred over the other cannot be concluded. Although not enough experiments were made, it seems likely, and this is conjecture, that neither region is preferred.

The width of the boundary was found analytically to be, at small Rayleigh number, $\frac{z d}{E}$, where $E$ is the amplitude of the temperature perturbation a 10 . The width could not be measured experimentally since it was impossible to measure amplitudes as a function of position. One method might have been to use the width of the cross roll region. This does not really answer the question of whether the experimental plan-form corresponds to the analytical solution.

The exact method by which one region replaces the other is unknown, except that cross rolls play an important role. This suggests that the quantity $R_{-}-R_{C},\left(R_{C}\right.$ is the critical Rayleigh number for the onset of cross rolls given by Busse, 1967 as 22,600) might be an important parameter characterizing the rate at which the boundary grows.

More experiments need to be performed for many different Rayleigh numbers. Obtaining the amplitude as a function of distance again would be helpful in answering how the plan-form evolves. A particular problem with the experiments was the asymmetry of wall roll formation across the boundary. A more symmetric experiment would have the boundary along a tank diagonal. Finally, the plan-form evolves so slowly, that a shorter vertical diffusion time is needed if many runs are to be performed.

The analytic results need to be extended. Previously mentioned, a

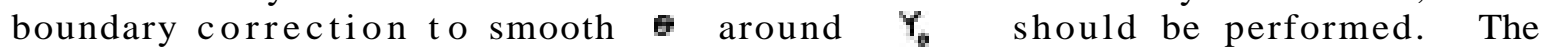
calculation should also be generalized to rolls with arbitrary wave number since the experiments indicate a smaller wave nmumber is preferred at higher Rayleigh number. A stability analysis also needs to be performed; the experiments indicate that the phase portrait around the solution is very complicated and certainly the study of imperfect plan-forms is rich in new phenomena.

\section{$\underline{\text { References }}$}

Busse, F. H., 1967. On the stability of two dimensional convection in a layer heated from below. J. Math. \& Phys., 46, 140-149.

Chen, M. M. and J. A. Whitehead, 1968. Evolution of two dimensional periodic Rayleigh convection cells of arbitrary wavenumber. J. Fluid Mechanic, 31. $1-15$.

Gollub, J. P. and J. F. Steinman, 1981. Doppler imaging of the onset of turbulent convection. Submitted to Phys. Rev. Lett.

Malkus, W.V.R. and G. Veronis, 1952. Finite amplitude convection. J. Fluid Mechanics, 4, 225-260.

Newe11, A. C. and J. A. Whitehead, 1963. Finite bandwidth, finite amplitude convection. J. Fluid Mech. 38, 279-303.

Siggia, E.D. and A. Zippelius, 1961. Dynamics of defects in Rayleigh-Benard convection. Submitted to Phys. Rev. A. 
Acknowledgements

I wish to thank Dr. J. Whitehead and R. Frazel for their assistance in the experimental portion of this work. Prof. L. Howard's guidance in the analytic portion and in particular finding a solution to the equations is thankfully acknowledged. Prof. W.V.R. Malkus's encouragement and insights are thankfully appreciated.

\section{SPIN-UP PROBLEM WITH A RADIAL CHANNEL ON THE BOTTOM}

Bruce D. Long, Jr.

\section{A Geophysical Motivation}

Why a radial channel on the bottom? This variation on the ordinary spin-up problem is motivated i $n$ part by a problem from physical oceanography -- that of coastal upwelling. In the usual two-dimensional steady-state formulation we consider a stratified ocean of uniform undisturbed depth $\mathrm{H}$ bounded laterally by a coast and driven by a uniform longshore wind stress (Fig. 1 The wind stress causes a transport of light surface water within a thin Ekman layer away from the coast, and to conserve mass an upwelling of heavier water must occur at the coast. A shorewards transport in a bottom Ekman layer and a slow pumping of fluid from the surface Ekman layer into the interior closes the circulation. This secondary circulation requires the sea surface to slope upwards in the seaward direction and thus engenders a longshore geostrophic current in the direction of the applied wind stress

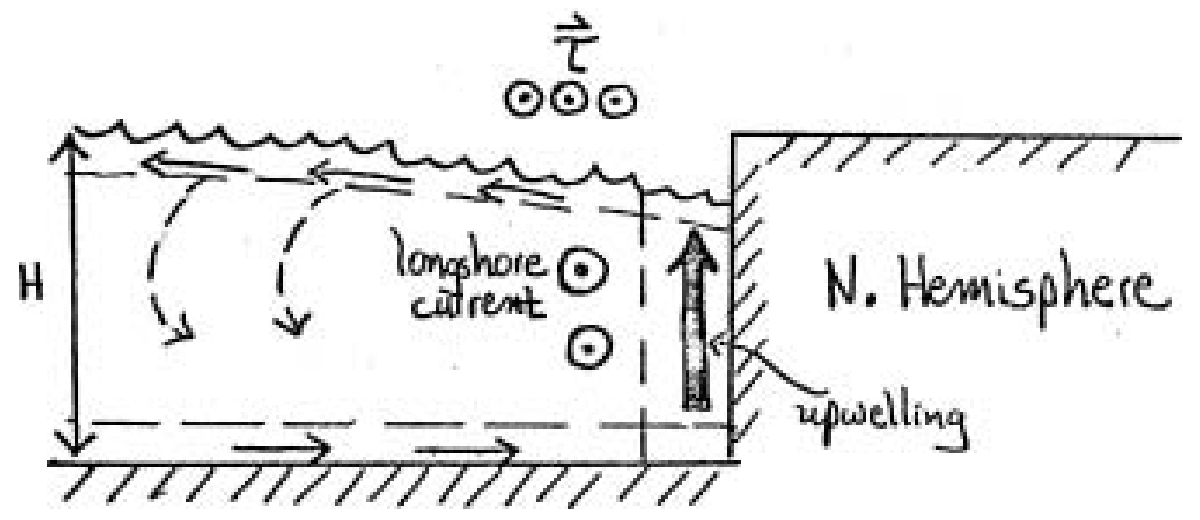

FIGURE 1 .

The flow in the bottom Ekman layer represents a balance between the horizontal pressure gradient, the Coriolis acceleration, and bottom friction. If we could somehow "turn-off" the Coriolis acceleration, the bottom flow could run down the pressure gradient and the increased shoreward transport in turn could lead to enhanced upwelling. One way to realize such a situation is to introduce some variation in the bottom topography in the third, hitherto suppressed direction. A submarine canyon trending perpendicularly to the coast whose width is smaller than the internal Rossby radius of deformation $R_{p}=\sqrt{g^{2}} / f$ where $g^{\prime}$ is the reduced gravity $\left(g^{\prime}=\frac{1}{\rho} \frac{2 \rho}{3 z} g H\right)$ and $f$ is the Coriolis parameter, removes the rotational constraint on the shoreward leg of the secondary circulation cell and allows a larger transport through the cell. This should lead to relatively more intense upwelling at the head of 
the canyon. The introduction of a localized regime where a large ageostrophic transport can occur should have a dramatic effect on fluid systems which depend critically on secondary circulations. One such system is that considered in the spin-up problem.

\section{The Spin-up Problem}

The spin-up problem for a rotating homogeneous fluid can be posed as follows: A cylinder containing a viscous fluid initially in a state of solid body rotationabouta vertical axis withangular velocity $\Omega-\Delta \Omega$ has its rotation rate suddenly increased to $\Omega$. How does the fluid come to a new state of solid body rotation and what is the characteristic time for this to occur? Greenspan and Howard (1963) show that for a fluid bounded at top and bottom by solid impervious walls the characteristic time for the new sta e to be reached -- the so-called spin-up time-- is given by the geometric mean of the diffusive time scale $\mathrm{L}^{2} / \nu$ and the inverse of the rotation rate $1 / \Omega$, where $L$ is the half-height of the container and $r$ is the kinematic viscosity of the fluid. Defining the Ekman number $E=v / L^{2} \Omega$, the spin-up time can be written as $T=\Omega^{-1} \mathbb{E}^{-1 / 2}$. The physical mechanism for spin-up involves a secondary circulation set up within the fluid, which enables the fluid to reach its new state much faster than it would if viscous diffusion of vorticity alone were acting. In a time of order $\Omega^{-1}$ Ekman layers are established on the horizontal boundaries, which suck fluid in from the essentially inviscid interior as fluid within the layers is forced outwards by the increased centrifugal force. To conserve mass these must be a slow inward flow in the interior. A parcel of fluid in this inviscid domain conserves its angular momentum and thus increases its angular velocity as it drifts inward. This leads to the eventual spin-up of the interior. It is apparent that nny mechanism allowing a faster secondary circulation of the fluid, i.e., a stronger radial flow, should lead to a faster spin-up. One possibility is to replace the impervious horizontal plates with a permeable medium (Kroll and Veronis, 1970). Here we will consider the effect of having a single narrow radial channel on the bottom. The width of the channel is of the order $\mathrm{LE}^{1 / 2}$, which insures that the horizontal flow through it will be essentially radial, and will represent a balance between the radial pressure gradient and lateral friction -- the rotational constraint is removed and the radial flow can be quite large down the channel. This increased radial flow also increases the downward suction of fluid from the interior and should lead to a faster spin-up. Because the character of the bottom is no longer axially symmetric we expect the interior flow to exhibit contributions from zonal wave numbers other than the simple wave number zero zonal flow in the "classical" spin-up problem.

A somewhat idealized model is developed which predicts that the channel does indeed decrease the spin-up time, with greater efficacy for faster rotation rates. In this elementary analysis the effect of the presence oj higher wave numbers on the spin-up time is considered negligible. A series of experiments whose parameter range rather imperfectly matches that of the theory will then be examined, with good qualitative and acceptable quantitative agreement with the model. First some theory.

\section{Theoretical Model}

Consider a configuration as shown in Figure 2. A cylinder of fluid of half-height L bounded by impervious walls rotates with constant angular velocity $\Omega-\Delta \Omega$. A "wedge" centered at $\theta=0$ is cut out of the bot $\mathrm{am}$ 
which subtends an angle (small!) of $\theta_{0}$ and has a thickness h. We require $\theta_{a} L / h \propto_{I}$. At time $t=0$ the angular velocity of the container is instantaneously increased to $\Omega$. We seek an approximate solution for the timedependent motion of the fluid. The procedure closely follows that of Kroll and Veronis (1970) but here we cannot assume axial symmetry and must keep terms in $/ / \%$.

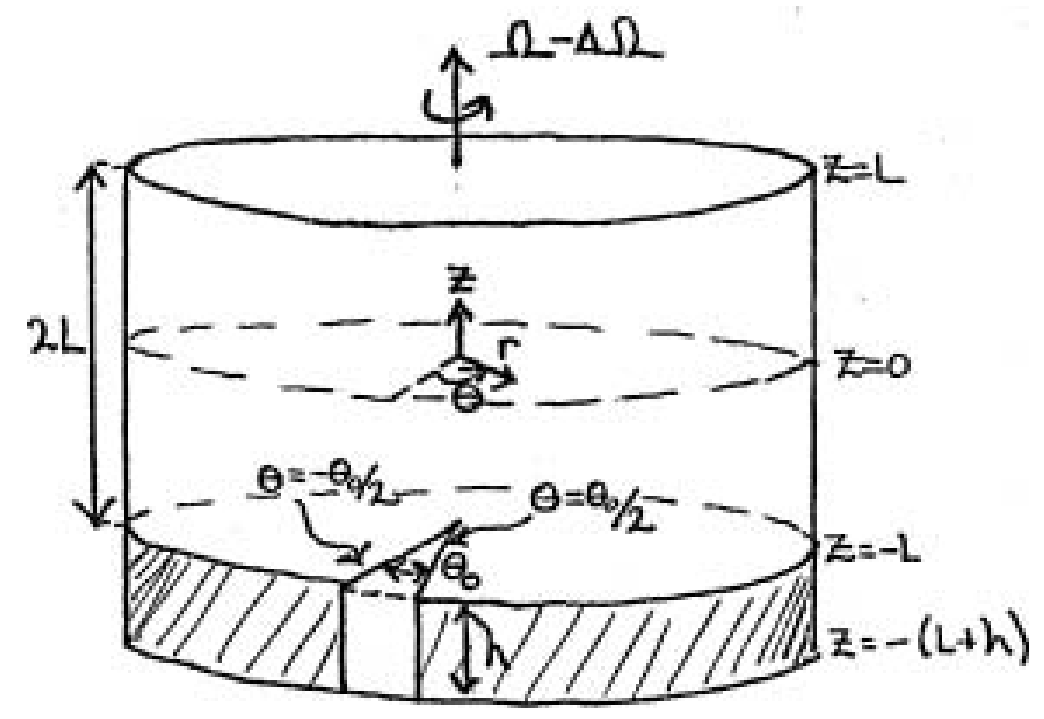

FIGURE 2.

The full equations of motion in cylindrical coordinates $r, \theta, z$ for a frame moving at the new rotation rate (see, e.g., Batchelor, 1967) are, in nondimensional form:

$$
\begin{aligned}
& \frac{\partial u}{\partial t}+\epsilon\left(u \frac{\partial u}{\partial r}+\frac{x}{r} \frac{\partial u}{\partial \theta}+w \frac{\partial u}{\partial z}-\frac{v^{2}}{r}\right)-2 v=-\frac{\partial p}{\partial r}+E\left(\nabla^{2} u-\frac{u}{r^{2}}-\frac{2}{r^{2}} \frac{\partial v}{\partial \theta}\right) \\
& \frac{\partial v}{\partial t}+6\left(u \frac{\partial v}{\partial r}+\frac{v}{r} \frac{\partial v}{\partial \theta}+w \frac{\partial v}{\partial v}+\frac{u v}{r}\right)+2 u=-\frac{1}{r} \frac{\partial p}{\partial \theta}+E\left(\nabla^{2} v-\frac{v}{r^{2}}+\frac{2}{r^{2}} \frac{\partial u}{\partial t}\right) \\
& \frac{\partial u}{\partial t}+\epsilon\left(u \frac{\partial w}{\partial r}+\frac{y}{v} \frac{\partial w}{\partial \theta}+w \frac{\partial u}{\partial z}\right)=-\frac{\partial p}{\partial z}+E \nabla^{2} w \\
& \frac{1}{r} \frac{\partial}{\partial r}(r u)+\frac{1}{r} \frac{\partial w}{\partial \theta}+0
\end{aligned}
$$

where

$$
\nabla^{2}(\cdot)=\frac{1}{r} \frac{\partial}{\partial r}\left(r \frac{\partial}{\partial r}(\cdot)\right)+\frac{1}{r^{2}} \frac{\partial^{2}}{\partial \theta^{2}}(\cdot)+\frac{\partial^{2}}{\partial t^{2}}(\cdot)
$$

Here $\mathrm{u}, \mathrm{v}$, and $\mathrm{w}$ are the radial, azimuthal, and vertical components of velocity respectively, and the centrifugal and gravitational forces (potential forces) are absorbed in the pressure term p. Velocity has been scaled as $\Delta \Omega L$, time by $\Omega^{-1}$, length by $L$, and pressure by $P L \Omega(\Delta \Omega L)$. The nondimensional 
parameters $\epsilon$ and $E$ are the Rossby number $(\Delta \Omega / \Omega$ ) and the Ekman number $\nu \mathcal{S}^{-1} L^{-2} \quad$ respectively.

In order to proceed any further we must make a number of assumptions. We will first of a 11 assume that the channel is so narrow that flow in the radial direction behaves essentially like that in a permeable medium or Hele-Shaw cell, i.e., it obeys Darcy's law.

Using the same nondimensionalization we have for the channel

$$
\begin{array}{ll}
u_{c}=-N \frac{\partial p_{c}}{\partial r} & v_{c} \sim \frac{\partial p_{c}}{\partial \theta} \sim \frac{\partial p_{c}}{\partial z} \sim 0 \\
\frac{1}{r} \frac{\partial}{\partial r}\left(r u_{c}\right)+\frac{\partial w_{c}}{\partial z}=0 &
\end{array}
$$

where $N=k \Omega / \nu=k L^{-2} E^{-1}$ and $k$ is the permeability. Just exactly what the permeability for our channel of non-constant width should be we leave as an open question for now. By ignoring the variation in width with $r$ we introduce a hopefully not too serious flaw in our model that greatly simplifies the further development. We naturally keep $\epsilon$ sufficiently small that the problem reduces to a linear one. We also consider the flow to have a boundarylayer character, $\mathbf{1}$.e., that the dependent variables outside the channel can be split into an interior and boundary layer part (e.g., $u=u_{\mathbf{v}}+\tilde{u}$

where $\tilde{R}$ vanishes as we leave the boundary layer). This requires that withi $n$ the boundary layers at $z= \pm 1$ we have $3 / \partial z=\mp E^{-1 / 2} \partial / \partial \xi$, where $\partial / \partial \zeta$ is of order one. The sidewall boundary layer is ignored since it only has an effect within the viscous time scale $L^{2} / \nu \gg T$. Since we expect the change i n spin-up time not to be excessively dramatic, we assume that $\mathrm{T}$ is of order $\Omega^{-1} E^{-1 / 2}$ as in the ordinary problem and write

$$
\frac{\partial}{\partial t}=E^{1 / 2} \frac{\partial}{\partial \tau}
$$

where again $\partial / \diamond \tau$ is of order one.

We progress by solving for the interior and boundary layer flows and matching them to the boundary conditions and to the bottom channel flow. For the interior we now have:

$$
\begin{aligned}
& E^{1 / 2} \frac{\partial u_{z}}{\partial r}-2 v_{1}=-\frac{\partial p_{r}}{\partial r}+E\left[\frac{\partial}{\partial r}\left(\frac{1}{r} \frac{\partial}{\partial r}\left[r u_{1}\right]\right)+\frac{1}{r^{2}} \frac{\partial^{2} u_{I}}{\partial \theta^{2}}+\frac{\partial^{2} u_{x}}{\partial r^{2}}-\frac{2}{r^{2}} \frac{\partial v_{z}}{\partial \theta}\right] \\
& {\left[y_{2} \frac{\partial u_{2}}{\partial \tau}+2 u_{r}=-\frac{1}{r} \frac{\partial P_{x}}{\partial \theta}+E\left[\frac{\partial}{\partial r}\left(\frac{1}{r} \frac{\partial}{\partial r}\left[r v_{I}\right]\right)+\frac{1}{r^{2}} \frac{\partial^{2} v_{2}}{\partial \theta^{2}}+\frac{\partial v_{2}}{\partial \partial^{2}}+\frac{2}{r^{2}} \frac{\partial u_{2}}{\partial \theta}\right]\right.} \\
& E^{1 / 2} \frac{\partial \omega_{2}}{\partial E}=-\frac{\partial p_{x}}{\partial \theta}+E\left[\frac{1}{r} \frac{\partial}{\partial r}\left(r \frac{\partial w_{x}}{\partial r}\right)+\frac{1}{r^{2}} \frac{\partial^{2} w_{r}}{\partial \theta^{2}}+\frac{\partial w_{1}}{\partial r^{2}}\right] \\
& \frac{1}{r} \frac{\partial}{\partial r}\left(r u_{I}\right)+\frac{1}{r} \frac{\partial v_{2}}{\partial \theta}+\frac{\partial w_{2}}{\partial z}=0
\end{aligned}
$$


$-263-$

For the boundary layer at $z= \pm 1$ we have

$$
\begin{aligned}
& E^{1 / 2} \frac{\partial \tilde{u}}{\partial \tau}-2 \tilde{v}=-\frac{\partial \tilde{p}}{\partial r}+E\left[\frac{\partial}{r r}\left(\frac{1}{r} \frac{\partial}{\partial r}[r \tilde{\sigma}]\right)+\frac{1}{r^{2}} \frac{\partial^{2} \tilde{u}}{\partial \theta^{2}}-\frac{2}{r^{2}} \frac{\partial \tilde{v}}{\partial \theta}\right]+\frac{\partial^{2} \tilde{u}}{\partial \xi^{2}}
\end{aligned}
$$

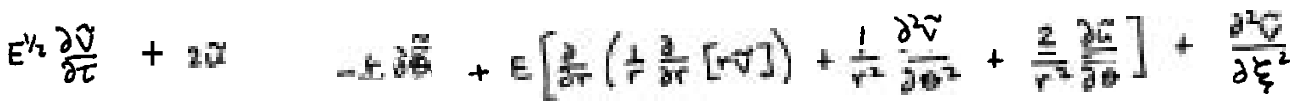

$$
\begin{aligned}
& \frac{\partial \tilde{\sigma}^{2}}{\partial \tau}= \pm E^{-1 / 2} \partial \xi+E\left[\frac{1}{r} \frac{\partial}{\partial r}\left(r \frac{\partial \tilde{\omega}}{\partial r}\right)+\frac{1}{r^{2}} \frac{\partial^{2} \tilde{\omega}}{\partial \theta^{2}}\right]+\frac{\partial^{2} \tilde{\omega}}{\partial \xi_{\xi}^{2}} \\
& \frac{1}{r} \frac{\partial}{\partial r}(r \sigma)+\frac{1}{r} \frac{\partial \sigma}{\partial \theta} \mp E^{-1 / 2} \frac{\partial \tilde{\sigma}}{\partial \zeta}=0
\end{aligned}
$$

( increases positive upwards at $\mathrm{z}=\mathbf{1}$ and positive downwards at $\mathrm{z}=+\mathbf{1}$.)

To solve this set of equations we will employ a series expansion in powers of the small quantity $\mathrm{E}^{1 / 2}$ and equate $1 \mathrm{ike}$ powers of $\mathrm{E}^{1 / 2}$, For example

$$
u=\sum_{n=0}^{\infty} E^{n / 2}\left(u_{I_{n}}+\tilde{u}_{n}\right)
$$

Plugging the expansions into (6) and (7) we obtain Interior

$\mathrm{B}^{\circ}$ :

$$
\begin{array}{ll}
-2 v_{I_{0}}=-\frac{\partial P_{I_{0}}}{\partial r} & \frac{\partial \rho_{I_{0}}}{a t}=0 \\
2 u_{I_{0}}=-\frac{\partial}{\partial P_{I_{0}}} & \frac{1}{r} \frac{\partial}{r}\left(r u_{I_{0}}\right)+\frac{1}{r} \frac{\partial v_{I_{0}}}{\partial \theta}+\frac{\partial W_{I_{0}}}{\partial a}=0
\end{array}
$$

$\mathrm{E}^{1 / 2 \text {; }}$

$$
\begin{array}{ll}
\frac{\partial u_{\tau_{0}}}{\partial \tau}-2 v_{x_{1}}=-\frac{\partial p_{z_{1}}}{\partial t} & \frac{\partial w_{2}}{\partial \tau}=-\frac{\partial p_{z_{1}}}{\partial \tau} \\
\frac{\partial v_{z_{2}}}{\partial \tau}+2 u_{z_{1}}=-\frac{1}{r} \frac{\partial p_{x_{1}}}{\partial \theta} & \frac{1}{r} \frac{\partial}{\partial r}\left(r u_{z_{1}}\right)+\frac{1}{r} \frac{\partial v_{z_{1}}}{\partial \theta}+\frac{\partial w_{z_{1}}}{\partial z}=0
\end{array}
$$

Boundary layer at $Z= \pm 1$ :

$\mathrm{E}^{-1 / 2:} \quad \frac{\partial \tilde{p}_{0}}{\partial \xi}=0 \quad \frac{\partial \tilde{\omega}_{0}}{\partial \xi}=0$ 
Eo:

$$
\begin{aligned}
& -2 \tilde{v}_{0}=-\frac{\partial \tilde{p}_{0}}{\partial r}+\frac{\partial^{2} \tilde{u}_{0}}{\partial \xi^{2}} \\
& 2 \tilde{r}_{0}=-\frac{1}{r} \frac{\partial \tilde{p}_{0}}{\partial \theta}+\frac{\partial^{2} \tilde{v}_{0}}{\partial \xi_{q}^{2}} \\
& \pm \frac{\partial \tilde{p}_{0}}{\partial \xi}+\frac{\partial^{2} \tilde{w}_{0}}{\partial \xi^{2}}=0 \\
& \frac{1}{r} \frac{\partial}{\partial r}\left(r \tilde{w}_{0}\right)+\frac{1}{r} \frac{\partial \tilde{v}_{0}}{\partial \theta}=\frac{\partial \tilde{w}_{0}}{\partial \xi}=0
\end{aligned}
$$

The set (9) implies that there is no vertical shear on the order zero velocity comvonents

$$
\frac{\partial \vec{v}_{w_{0}}}{\partial z}=0
$$

In particular we note that $\left.w_{x_{0}}\right|_{z=-1}=\left.w_{x_{0}}\right|_{z=+1}$

Cross-differentiating the horizontal momentum equations in (9) and using the continuity equation we obtain the vorticity equation:

$$
\frac{\partial}{\partial \tau}\left[\frac{1}{r} \frac{\partial}{\partial r}\left(r v_{z_{0}}\right)-\frac{1}{r} \frac{\partial u_{z_{0}}}{\partial \theta}\right]=\left.w_{x_{1}}\right|_{z=+1}-\left.w_{z_{1}}\right|_{z=-1}
$$

Substituting in the geostrophic relations from (8) we get an equation relating the pressure to the interior vertical velocity at the boundaries.

$$
\frac{\partial}{\partial \tau}\left[\frac{1}{r} \frac{\partial}{\partial r}\left(r \frac{\partial p_{0}}{\partial r}\right)+\frac{1}{r^{2}} \frac{\partial^{2} p_{20}}{\partial \theta^{2}}\right]=2\left(\left.w_{2}\right|_{2 z+1}-\left.w_{z 1}\right|_{z=-1}\right)
$$

The boundary layer equations (10) imply that $P_{0}$ and $\tilde{w}_{0}$ equal zero, since a 11 boundary layer quantitatives must vanishas $\xi$. The Ekman layer equations (11) have the solution

$$
\begin{aligned}
& \tilde{u}_{0}^{2}=e^{-\xi}\left[A^{ \pm}(r, \theta, \tau) \cos \xi+B^{ \pm}(r, \theta, \tau) \sin \xi\right] \\
& \tilde{v}_{0}^{2}=e^{-\xi}\left[\theta^{2}(r, \theta, \tau) \cos \xi-A^{ \pm}(r, \theta, \tau) \sin \xi\right]
\end{aligned}
$$

where + or - indicate top or bottom boundary layer respectively, and integrating the continuity equation from $z=0$ to $\infty$ we have

$$
\left.\tilde{\psi}_{1}^{2}\right|_{\xi \rightarrow 0}=\mp \frac{1}{2 r}\left\{\frac{\partial}{\partial r}\left[r\left(A^{t}+B^{t}\right)\right]+\frac{\partial}{\partial \theta}\left(B^{t}-A^{2}\right)\right\}
$$


On the bottom away from the channel we require that $u$ and $v$ vanish:

$$
u=u_{I_{0}}+\left.\tilde{u}^{-}\right|_{z_{0}=0}=0
$$

etc.

$$
\text { Setting } \zeta=0 \text { in (14) we get }
$$

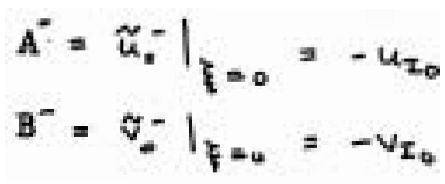

bottom, away from channel

but since u, and $v_{\text {Io }}$ do not vary with height

$$
\mathrm{A}^{-}=\mathrm{A}^{+}, \mathrm{B}^{-}=\mathrm{B}^{+} \quad \text { away from channel }
$$

Over the channel the top boundary condition remains unchanged

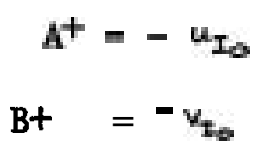

top, over channel

but on the bottom we match $\mathbf{u}$ i $n$ the channel and in the free fluid

$$
\left.u_{c}\right|_{z=-1}=u_{z_{0}}+\left.\tilde{u}_{0}^{-}\right|_{\xi=0}
$$

which yields

$$
\begin{aligned}
& A^{-}=u_{1} l_{z=-1}-u_{\Sigma_{0}} \\
& B^{-}=-v_{I_{\infty}}
\end{aligned}
$$

bottom, over channel

Substituting these values of $A$ and $B$ into (15) and rewriting in terms of the pressure we get, away from channel:

$$
\left.\tilde{\omega}_{1}^{ \pm}\right|_{p=0}= \pm \frac{1}{4}\left[\frac{1}{r} \frac{\partial}{\partial r}\left(r^{2} \frac{p_{r 0}}{\partial r}\right)+\frac{1}{r^{4}} \frac{\partial^{2} p_{x_{0}}}{\partial \theta^{2}}\right]
$$

Over the channel $\left.\mathbb{4}^{*}\right|_{\xi \rightarrow \infty}$ remains unchanged, while

$$
\left.\tilde{w}_{1}\right|_{\xi=0}=-\frac{1}{4}\left[\frac{1}{r} \frac{\partial}{\partial r}\left(r \frac{\partial p_{0}}{\partial r}\right)+\frac{1}{r^{2}} \frac{\partial^{2} r_{0}}{\partial \theta^{2}}\right]+\frac{1}{2 r} \frac{\partial}{\partial r}\left(\left.r u_{0}\right|_{t=-1}\right)
$$


It is now time to solve for $\mathbf{u}_{\mathbf{c}}$ and $\mathbf{w}_{\mathbf{c}}$. From (5) $\mathbf{u}_{\mathbf{c}}$ is given by

$$
u_{e}=-N \frac{\partial p_{c}}{\partial r}
$$

Integrating the last of (5) from $z=-\left(\mathbf{l}+\frac{h}{b}\right)$ to $z=-1$, using $\frac{\partial p_{c}}{\partial z}=0$, and requiring $\mathbf{w}_{\mathbf{c}}=0$ at $z=-\left(1+\frac{h}{L}\right)$ yields

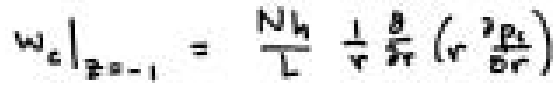

We also match the radial pressure gradient at $z=-1$

$$
\frac{\partial \rho_{c}}{\partial r}=\frac{\partial p_{2}}{\partial r}
$$

An equation in terms of PIo alone is now obtained by matching the vertical velocities at the boundaries. Away from the channel:

$$
\begin{aligned}
& \left.w\right|_{z=-1}=0=\left.w_{\tau_{0}}\right|_{z=-1}+E^{1 / L}\left(\left.w_{\tau_{1}}\right|_{z=-1}+\left.\tilde{w}_{1}\right|_{z=-\infty}\right) \\
& \left.w\right|_{z=+1}=0=\left.w_{z_{0}}\right|_{z=+1}+E^{K}\left(\left.w_{z_{1}}\right|_{z=1}+\left.\tilde{w}_{1}^{+}\right|_{z=0}\right)
\end{aligned}
$$

Subtracting and using $\left.w_{p_{2}}\right|_{r=-1}=\left.w_{2}\right|_{2=+1}$ gives

$$
\begin{aligned}
& \left.w_{\Sigma_{1}}\right|_{2=+1}-\left.w_{z_{1}}\right|_{2+-1}=-\left(w_{1}+\left.\right|_{\xi=0}-w_{1}||_{\xi=0}\right) \\
& =-\frac{1}{2}\left[\frac{1}{r} \frac{\partial}{\partial r}\left(r^{2} \frac{P_{z}}{\partial r}\right)+\frac{1}{r^{2}} \frac{\partial^{2} P_{2}}{\partial \theta^{2}}\right]
\end{aligned}
$$

Thus using this result in (13)

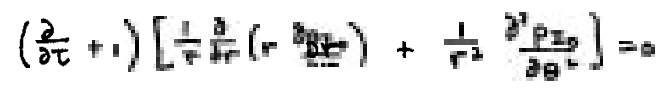

away from channel

Over the channel:

$$
\begin{aligned}
& \left.w\right|_{z=-1}=\left.w_{0}\right|_{z=-1}=\left.w_{z}\right|_{z=-1}+E^{x / 2}\left(\left.u_{x_{1}}\right|_{z=-1}+\left.\tilde{w}_{1}\right|_{\xi=0}\right) \\
& \left.w\right|_{z=+1}=0=\left.w_{2}\right|_{2=+1}+E^{1 / 2}\left(\left.w_{2,1}\right|_{2 n+1}+\left.w_{1}^{*}\right|_{\xi=0}\right)
\end{aligned}
$$


$-267-$

Subtracting

$$
\begin{aligned}
& \left.w_{21}\right|_{z+1}-\left.w_{21}\right|_{z=-1}=-\left.E^{-1 / 2} w_{c}\right|_{z=-1}-\left(\left.\tilde{w}^{+}\right|_{\xi=0}-\tilde{w}-\left.\right|_{z-\infty}\right) \\
& =-\frac{1}{2}\left[\frac{1}{r} \frac{\partial}{\partial r}\left(r^{\partial} \frac{\partial \tau_{r}}{\partial r}\right)+\frac{1}{r^{2}} \frac{\partial^{2} p \tau_{p}}{\partial \theta^{2}}\right]-N\left(\frac{h}{L E^{1 / 2}}+\frac{1}{2}\right) \frac{1}{r} \frac{\partial}{\partial r}\left(r \frac{\partial p \tau_{0}}{\partial r}\right)
\end{aligned}
$$

Again substituting into (13)

$$
\begin{array}{r}
\left(\frac{\partial}{\pi}+1\right)\left[\frac{1}{r} \frac{\partial}{\partial r}\left(r \frac{\partial P g}{\partial r}\right)+\frac{1}{r^{2}} \frac{\partial^{2} P_{n g}}{\partial \theta^{2}}\right]=-2 N\left(\frac{h}{L E^{1 / h}}+\frac{1}{2}\right) \frac{1}{r} \frac{\partial}{\partial r}\left(r^{\partial} \frac{P_{x_{g}}}{\partial r}\right) \\
\text { for }-\frac{\theta_{g}}{2}<\theta<\frac{\theta_{0}}{2}
\end{array}
$$

To combine (21) and (22) into one equation we employ the Heaviside step fundton $\mathrm{H}(\theta)$ and write

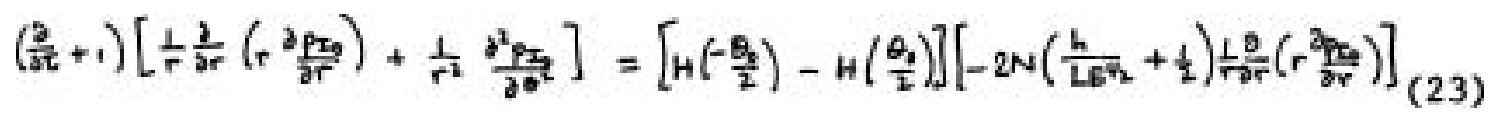

In an attempt to solve (23) we expand P Io in a Fourier series (and drop the irksome subscript for now) as

$$
P=\sum_{k=-\infty}^{\infty} P_{k}(r, \tau) e^{i k \theta}
$$

and get

$$
\begin{aligned}
\left(\frac{\partial}{\tau}+1\right) \sum_{k=0}^{\infty} e^{i k \theta} & {\left[\frac{1}{r} \frac{\partial}{\partial r}\left(r \frac{\partial p_{k}}{\partial r}\right)-\frac{k^{2}}{r} p_{k}\right]=} \\
& \sum_{k=-\infty}^{\infty} e^{i k \theta}\left[H\left(-\frac{\theta_{k}}{2}\right)-H\left(\frac{\theta_{q}}{2}\right)\right]\left[-2 N\left(\frac{h}{L E^{2} h}+t\right)+\frac{\partial}{r r}\left(r \frac{\partial p_{k}}{\partial r}\right)\right]
\end{aligned}
$$

Now mulitply both sides by $\mathbf{e}^{-110}$, integrate from 0 to $2 \pi$, and use the orthogonality of the expansion functions to find

$$
\begin{aligned}
\left(\frac{\partial}{r}+1\right)\left[\frac{1}{r} \frac{\partial}{\partial r}\left(r \frac{\partial p l}{\partial r}\right)-\frac{l^{2}}{r^{2}} p_{l}\right]= \\
\qquad \sum_{k=-\infty}^{\infty} \frac{\sin \left[(k-l) \frac{\theta^{2}}{2}\right]}{\pi(k-l)}\left[-2 N\left(\frac{h}{L \pi^{4}}+\frac{1}{2}\right) \frac{1}{r} \frac{\partial}{\partial r}\left(r \frac{\partial p_{b}}{\partial r}\right)\right]
\end{aligned}
$$


To simplify things a bit we take the limit of small $\theta_{p}$, where $\sin \left[(k-l) \frac{\theta_{\Omega}}{2}\right] \approx$ $(\kappa-e) \boldsymbol{\theta}_{0}$ (we reach the same juncture by replacing the combination of step function $\stackrel{2}{s}$ with a delta function of amplitude $\theta_{0}$ at the onset). Then

$$
\left(\frac{\partial}{\partial t}+1\right)\left[\frac{1}{r} \frac{\partial}{8 r}\left(r^{2} \frac{p_{l}}{\partial r}\right)-\frac{l^{2}}{r^{2}} p_{l}\right]=\frac{-N \theta_{0}}{\pi}\left(\frac{h}{L L^{1 / 2}}+\frac{1}{2}\right) \sum_{k=-\infty}^{\infty} \frac{1}{r} \frac{\partial}{\partial r}\left(r \frac{\partial p_{k}}{\partial r}\right)
$$

The term on the right-hand side involves a coupling of all wave numbers -- it appears we have a rather hopeless equation. But since at $\tau=0$ only wave number zero is excited, it seems reasonable to ignore the other wave numbers in the sum, solve the resulting (easy) equation for po, and check the adequacy of our bold maneuver later. Sweeping it under the rug for now we have

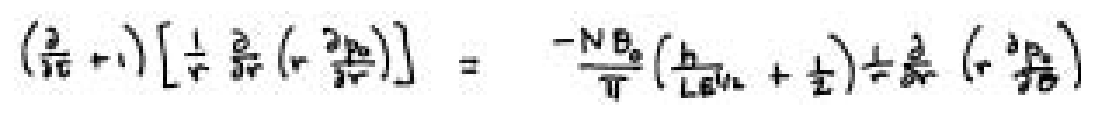

which has the solution

$$
P_{0}=q_{0}(r) \exp \left[-\left(1+\frac{N Q}{\pi}\left[\frac{h}{L^{4} H_{2}}+\frac{1}{2}\right]\right) \tau\right]
$$

where $q_{o}$ is an arbitrary function of $r$. The solution which fits the initial condition that $v_{z_{0}}=-r$ at $=0$ is

$$
P_{0}=-\left(r^{2}+\alpha_{0}\right) \exp [-(1+\gamma) \tau]
$$

where $\alpha_{0}$ is a constant and

$$
\gamma=\frac{N \theta_{0}}{\pi}\left[\frac{b}{L G^{1 / 2}}+\frac{1}{2}\right]
$$

Since in taking a zonal average only the $p_{0}$ term remains in $p=\sum_{p_{k}} e^{i k \theta}$ the zonally averaged azimuthal velocity (denoted by an overbar) is given by

$$
\bar{V}_{x_{0}}=-r e^{-(1+r) \tau}
$$

In dimensional terms

$$
\bar{V}_{I_{b}}=-\Delta \Omega r e^{-t^{1 / 2} \Omega(1+r) \tau}=-\Delta \Omega r_{e}-t / \tau
$$


$-269-$

and we see that the spin-up time for the zonally averaged flow is

$$
T=\frac{1}{\Omega E^{1 / 2}\left[1+\frac{N \theta_{0}}{T}\left(\frac{h}{L E / 2}+\frac{1}{2}\right)\right]}
$$

It is heartening to see that this result could have been obtained from the permeable medium result of Knoll and Veronis by multiplying their $\mathrm{N}$ by the ratio of the area of the channel to that of the bottom of the cylinder $\boldsymbol{a}_{\mathbf{n}} / \boldsymbol{\pi}$. The channel decreases the spin-up time as compared to the ordinary Ekman spinup case. But was it legitimate to ignore all higher wave numbers in the righthand side of (27)?

Using our result for po (and still ignoring higher wave numbers) we have from (27):

$$
\left(\frac{\partial}{\partial t}+1\right)\left[\frac{1}{r} \frac{\partial}{\partial r}\left(r \frac{\partial r}{\partial r}\right)-\frac{\ell^{2}}{r^{2}} P_{l}\right]=4 \gamma e^{-(1+\gamma) \tau}
$$

Letting $p_{\ell}=q_{\ell}(\mathbf{r}) s_{L}(\tau)$ and using the usual tricks of separation of variables

$$
\begin{aligned}
& \frac{d S_{l}}{d \tau}+S_{l}=\frac{4 \gamma}{m} e^{-(1+r) \tau} \\
& \frac{1}{r} \frac{d}{d r}\left(r \frac{d q_{l}}{d r}\right)-\frac{l^{2}}{r^{2}} q_{l}=m
\end{aligned}
$$

where $\mathrm{m}$ is the separation constant. The solution to (35) satisfying $\mathrm{p}_{2}=0$
at $\boldsymbol{\tau}=0$ for $\ell$ is

$$
S_{l}=\frac{4}{m} e^{-\tau}\left(1-e^{-\gamma \tau}\right)
$$

Equation (36) has the general solution

$$
\begin{aligned}
& q_{e}=\alpha_{l}^{\prime} r^{l}+\beta_{l}^{\prime} r^{-l}+\frac{m r^{2}}{4-l^{2}} \quad l \neq \pm 2 \\
& q_{ \pm 2}=\alpha_{ \pm 2}^{\prime} r^{ \pm 2}+\beta_{ \pm 2}^{\prime} r^{-2}+\frac{m}{4} r^{2} \ln r
\end{aligned}
$$

where $\alpha_{L}^{\prime}$ and $\beta_{L}^{\prime}$ are constants. To avoid a blow-up a the origin we require $\ell_{l}^{\prime}=0$ for $\ell>0$ and $\alpha_{l}^{\prime}=0$ for $L<0$. Thus we have $(\ell \neq 0)$ (making $\ell$ positive)

$$
\begin{aligned}
& P_{ \pm l}\left(\alpha_{ \pm 2} r^{l}+\frac{r^{2}}{4-l^{2}}\right) 4 e^{-\tau}\left(1-e^{-\gamma \tau}\right) \quad l \neq 2 \\
& P_{ \pm L}=\left(\alpha_{12} r^{2}+\frac{r^{2}}{4} \ln r\right) 4 e^{-\tau}\left(1-e^{-r \tau}\right)
\end{aligned}
$$


The constants $\alpha$ must be determined from consideration of the sidewall boundary layer - a tâsk we leave for a later date. What we are interested in is whether remains small compared to $\rho_{0}$ for a time $\tau \sim(1+\boldsymbol{\gamma})^{-1}$, which would validate our method of obtaining $T$. The ratio $P_{e}$ to $P_{*}$ can be written

$$
\frac{P_{l}}{P_{0}}=f(r)\left(e^{\gamma \tau}-1\right)
$$

The unknown function $f(r)$ is hopefully no larger than order one. Thus a most at $\tau=(1+8)^{-1}$

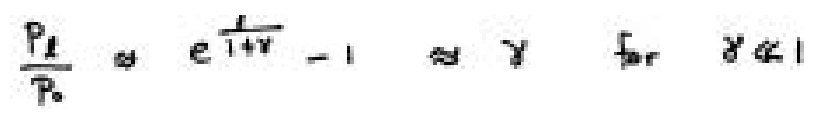

In the experiments $\gamma$ was on the order of .25 , so our estimate of $\mathrm{T}$ should be reasonably good, if we can trust this rather shaky argument, although the interesting development of the higher wave numbers in the flow remains as of now unexplored.

Experimental Method

A series of experiments was conducted in a cylindrical plexiglass tank with a rigid top of inside diameter $29.3 \mathrm{~cm}$. A disk of thickness 1.23 was placed at the bottom. The disk had a thin wedge cut out of it. Ideally the resulting channel would have radial walls, but due to the finite width of a bandsaw blade this state is only approximatmely reached. The nominal value of

$\theta$ for the wedge is 0.034 radians. With the disk in place the half-height of the tank was $4.50 \mathrm{~cm}$. The apparatus was placed on a rotating table spun counterclockwise with a movie camera mounted above in the table frame. The angular velocity of the table could be determined to an accuracy of $0.01 \mathrm{~s}^{-1}$.

A thymol blue indicator solution was used for the working fluid, with a kinematic viscosity at $23^{\circ} \mathrm{C}$ of about $.96 \times 10^{-2} \mathrm{~cm}^{2} \mathrm{~S}^{-1}$. The indicator solution is used i $n$ the method of flow visualization (Baker, 1966). It is yellowish when acidic and deep blue when basic. The fluid is titrated until it is slightly acidic. Conducting wires connected to the positive terminal of a DC power supply are stretched across the cylinder in a pattern shown in Figure 3. A negative lead enters the fluid at the top center of the tank. When the power supply is turned on the fluid around the positive wires turns blue, forming a neutrally buoyant tracer. One wire crosses just above the channel in order to see what sort of radial flow the channel induces. The criss-crossed wires allow some measure of the zonally averaged azimuthal flow. By measuring the angle $\phi$ between the tracer lines and the wire lines as a function of time after the angular velocity is increased by $\Delta \Omega$ we can obtain a measure of the spin-up time of the zonally averaged azimuthal flow. For we can rewrite (31) a s

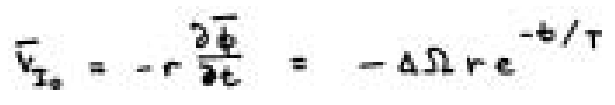

Integrating this from $\mathbf{t}=\mathbf{0}$ to $\mathbf{t}=\mathbf{t}$ we get

$$
\bar{\phi}(t)=T \Delta \Omega\left(1-e^{-t / T}\right)
$$


As $t \rightarrow \infty$ we get the final angle subtended by the tracer lines

$$
\bar{\phi}_{\text {fin a l }}=T \Delta \Omega
$$
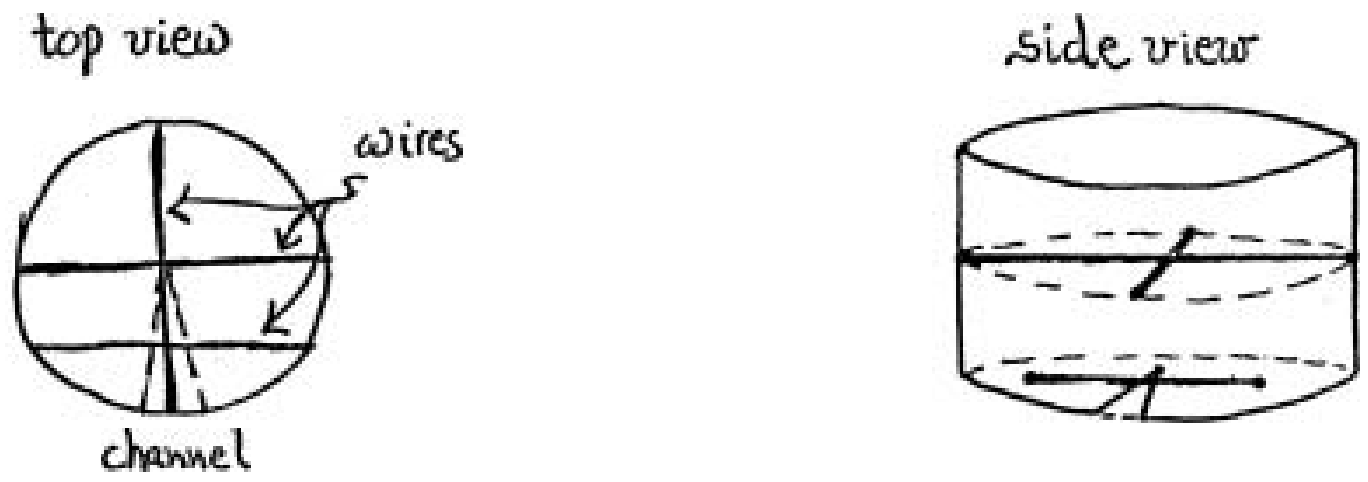

FIGURE 3.

Thus by measuring the average value of the four central angles as a function of time we can compare experiment with theory. Several runs were made with different values of $\Omega$ and $\Delta \Omega$. As a check of the apparatus some control runs were done with the channel either filled with putty or covered by cellophane tape of a thickness less than that of an Ekman layer.

\section{Experimental Results}

Figure 4 shows a typical sequence of photos taken when the channel is uncovered. The channel runs from the center to the bottom of the photos. Note the distortion of the cross-channel line marker in a pattern suggesting enhanced radial flow over the channel. Several potential difficulties are also evident -- the distortion of the marker lines used in measuring from straight lines and a slow drift of the center of the pattern from the center of the tank. The slow drift may be due to imperfect leveling of the bottom disk, as it is also apparent when the channel is not present. The distortion of the lines indicated that different parts of the fluid are spinning-up at different rates. Both imperfections are hopefully minimized by taking the average of four values of $\phi$ to obtain $\bar{\phi}$.

Although numerous runs were made with a range in $\Omega$ of about 1 to $4 \mathrm{~s}^{-1}$, only two runs with $\Omega$ relatively large were subjected to careful quantitative scrutiny. The rudimentary theoretical model predicts that the channel-induced reduction in spin-up time should, other parameters being fixed, be more significant (and thus measureable) at large $\Omega$. Runs both with and without a channel were made at $\Omega=4.46 \mathrm{~s}^{-1}, \Delta \Omega=0.12 \mathrm{~s}^{-1}$ and $\Omega 3.16 \mathrm{~s}^{-1}, \Delta \Omega=0.09 \mathrm{~s}^{-1}$ and $\bar{\xi}$ determined as a function of time. In both cases $\boldsymbol{\xi}$ was about 0.03 , at the limits of applicability of the linear theory. Equation (39) shows that a plot of $\bar{P}$ vs. $e^{-t / T}$ should be linear, with y-intercept $T \Delta \Omega$. In order to determine $\mathrm{T}$ it is thus imperative to know $\Delta \Omega$ with some degree of accuracy. Unfortunately, the available equipment allowed a relative error in the measurement of $\Delta \Omega$ on the order of $10 \%$. A way was found to bypass this 

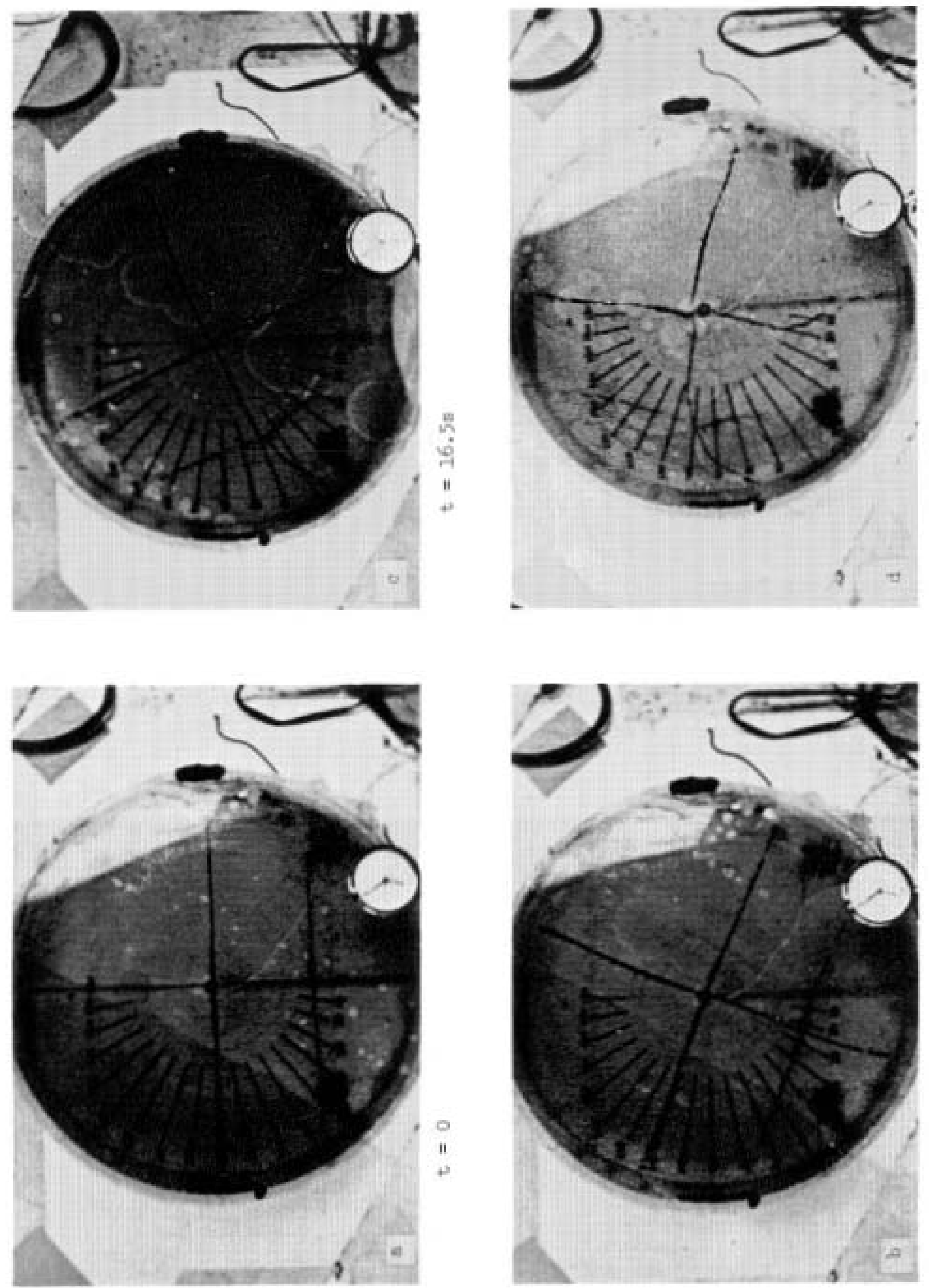

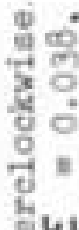

จ

ले

II

혁뭉

द्रुष :-

- 8

சأ

m

고ำ

夏

से

불붕

ए人

녕. ․․ㅁำ

$\geq \frac{9}{4}$

b.

空击的

if 
difficulty, however. A plot was made of $\bar{\phi}$ vs. $e^{-t / T_{\mathbf{k}}}$, where $T_{\hat{B} k}$ is the theoretical spin-up time with no channel on the bottom. As $e^{-t / 2}$ approaches 1 the slope of the curve should aproach $-T_{5 k} \Delta \Omega$. Since $T_{\xi k}$ is known, measuring the slope near the right-hand side of the plot gives an improved value of A $\Omega$.

In Figure 5 the filled circles are the data points in the case with the channel, the open circles are the data points without the channel (scaled to match the slight difference in $4 \Omega$ between the two cases) and the straight line is the theoretical curve for ordinary Ekman spin-up without a channel for the two runs. We see a significant drop-off of $\bar{\phi}$ from that of the ordinary spin-up problem for large $\mathbf{t}$, indicating a faster spin-up time. Using

$$
\begin{aligned}
& \begin{array}{ccll}
\phi_{\text {fimal }}=\Delta \Omega T & \text { we find: } & T=15.8 \mathrm{~s} & \mp=0.72 \\
\operatorname{Run} 1 & Q=4.46 \mathrm{~s}^{-1} & T_{E_{k}}=21.8 \mathrm{~S} & T_{\text {ak }}
\end{array}
\end{aligned}
$$

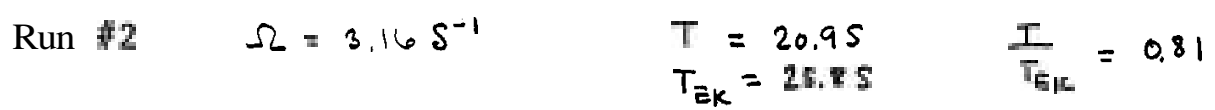

In Figure $6 \bar{\phi}$ is plotted against $e^{-t / T}$ for the two runs yielding a reasonably good straight line. As predicted the relative effect of the channel is more pronounced at higher $\Omega$ Since $\frac{h}{L E K} \gg \frac{1}{2} \quad$ (33) predicts that $\frac{T \in}{T}=1$ should be proportional to $\Omega^{3 / 2}$ :

$$
\frac{\left(\frac{T E_{K}}{T}-1\right)_{2}}{\left(\frac{T_{E_{K}}}{T}-1\right)_{1}} \approx\left(\frac{\Omega_{2}}{\Omega_{1}}\right)^{3 / 2}
$$

The ratio on the left has a value of 0.62 , while that on the right is 0.60 -which is an acceptable agreement.

In order to compare the quantitative agreement between the theoretical value of $\mathrm{T}$ from (33) and the experimentally measured one we need a value for the permeability $k$ of the channel. For a Hele-Shaw cell the permeability is given by

$$
k=\frac{S^{2}}{12}
$$

where $S$ is the width of the cell. If we use this for the channel we are still faced with a choice for the value of s since it varies ideally as a linear function of $r$ and i n practice as a function of the machinist's capability on the bandsaw. If for $s$ we use the width of the channel at the sidewall we find $\mathrm{k}=0.021 \mathrm{~cm}^{2}$ while, if we use the mean width of the channel (measured), $\mathrm{k}=1.6 \times 10^{-3} \mathrm{~cm}^{2}$. Alternately, we can use the values of $\mathrm{T}$ found experimentally to deduce the implied value of $\mathrm{k}$, using $\mathrm{N}=\mathrm{kh} / \nu$ (and 33 ). If this is done Run indicates a value of $\mathrm{k}=2.8 \times 10^{-3} \mathrm{~cm}^{2}$, while Run 2 yields $\mathrm{k}=2.9 \times 10^{-3} \mathrm{~cm}^{2}$. This is in fair agreement with the value of $\mathrm{k}$ obtained by using the mean width of the channel.

For Further I gati

Two runs obviously do not make a very convincing case. More runs are needed over a wider range of $\Omega$, with $\theta_{\text {, }}$ and $h$ possibly also being varied. The interesting developnent of the higher wave numbers in the flow field, which was ignored in obtaining an estimate of $T$, merits further theoretical 

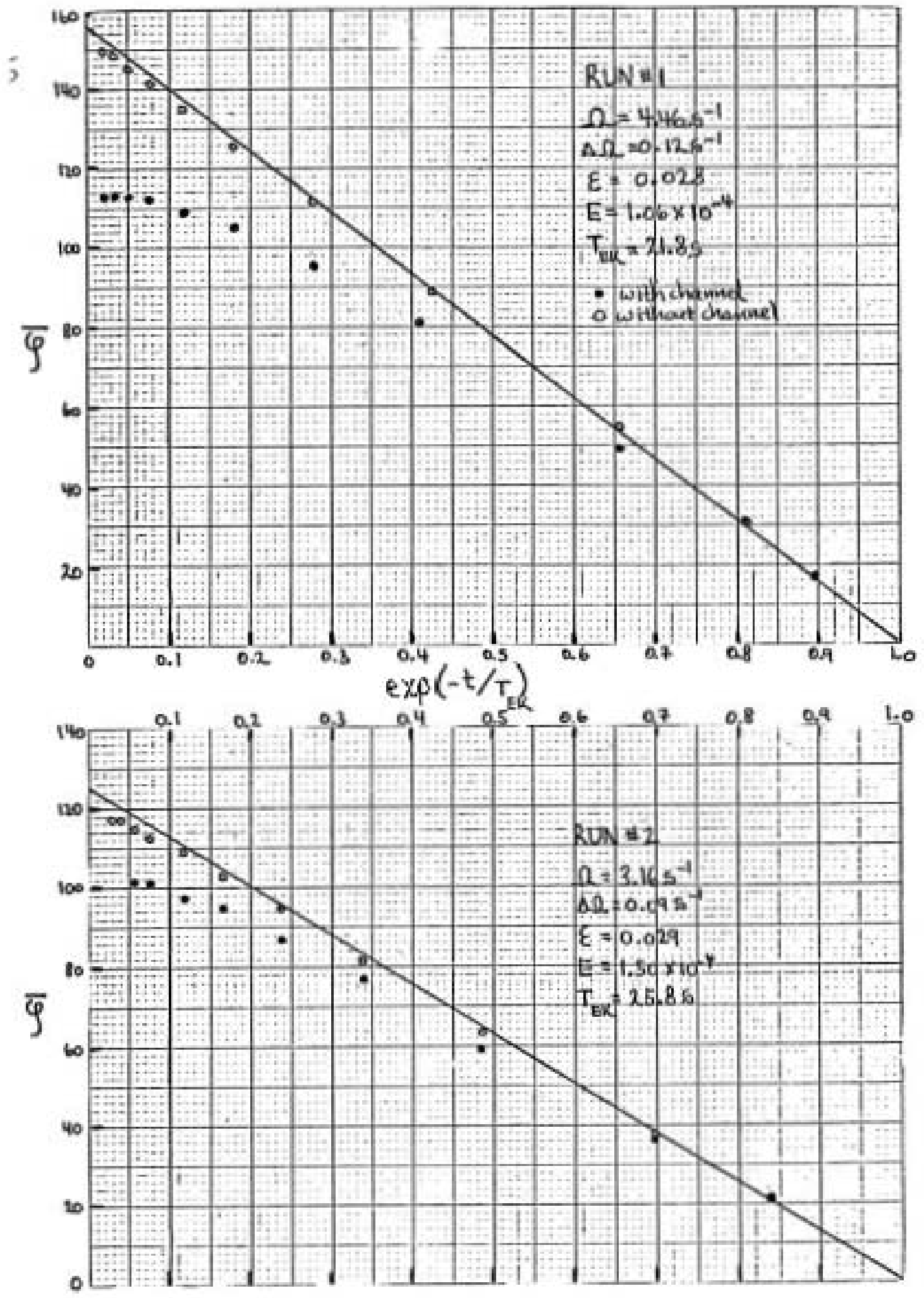

Figure 5 

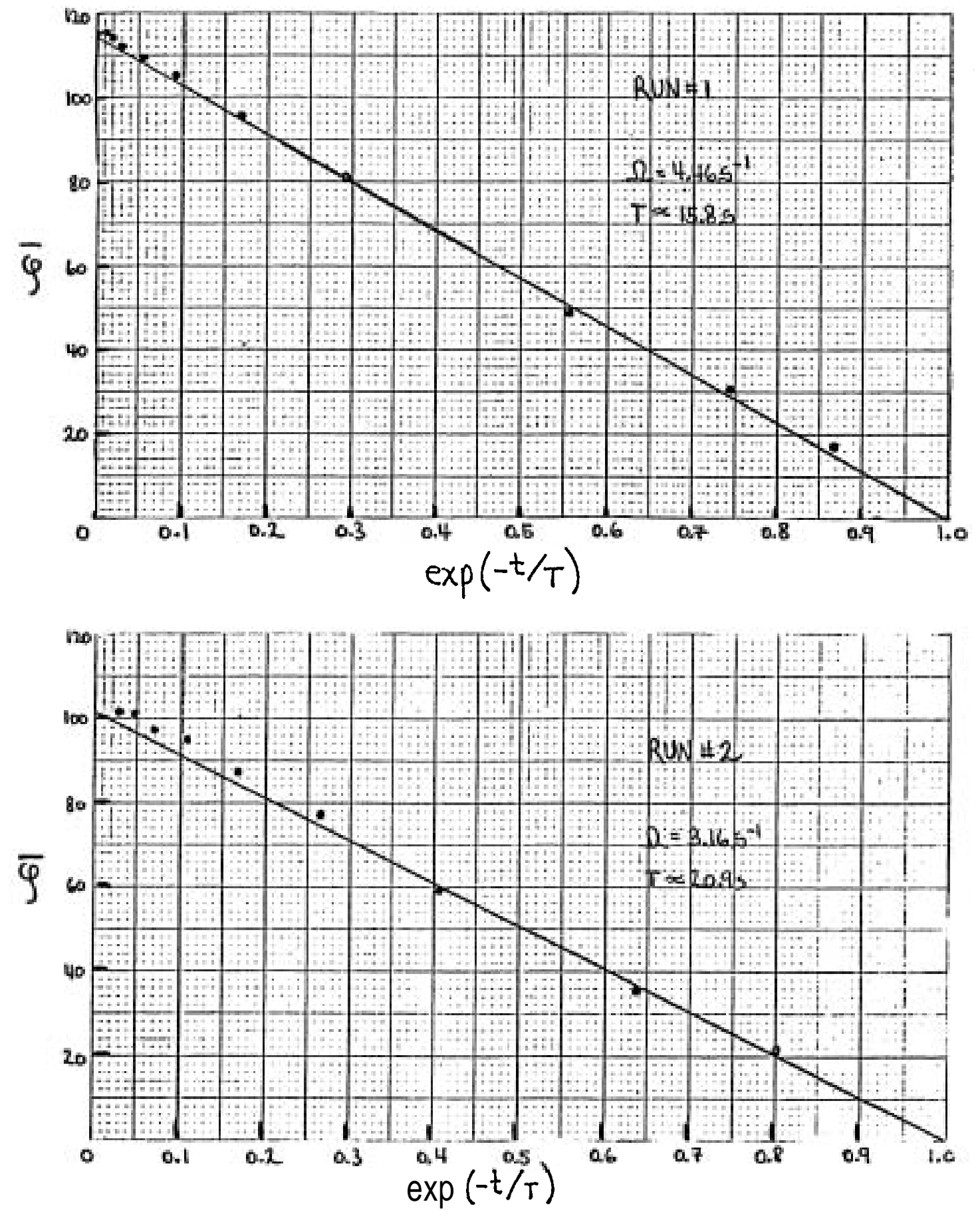

Figure 6 
and experimental consideration, and perhaps the assistance of a computer. For an experiment with a truly oceanographic application density stratification must be introduced, with the internal radius of deformation larger than the width of the channel (canyon?). We can go over to a steady state problem by keeping constant but rotating the top plate relative to the bottom one, mimicking the wind stress in the upwelling problem.

\section{ACKNOWHDGEMENIS}

I would like to thank Dr. George Veronis for suggesting this problem and providing guidance and inspiration along the way, Dr. Rory Thompson for his friendly advice and assistance, and Mr. Robert Frazel for help with the experiments.

\section{REFERENCES}

Baker, D. J., 1966. A technique for the precise measurement of small fluid velocities. J. Fluid Mech., 26, 573-575.

Batchelor, G. K., 1967. An Introduction to Fluid Dynamics. Cambridge University Press.

Greenspan, H. P. and L. N. Howard, 1963. On a time dependent motion of a rotating fluid. J. Fluid Mech., 17, 385-404.

Krol1, J. and G. Veronis, 1970. The spin-up of a homogeneous fluid bounded below by a permeable medium. J. Fluid Mech., 40, 225-239.

Pedlosky, J., 1979. Geophysical Fluid Dynamics, Springer Verlag.

\section{A TALE OF TWO MEIHODS}

P. H. Coullet and E. A. Spiegel

\section{INTRODUCTION}

This summer we have heard a lot about bifurcations in convection, be they stationary, Hopf or multiple. There have been two kinds of method used for these problems by various lecturers, namely, any of several asymptotic procedures and the direct method of modal expansion and reduction to normal form. To deal with multiple bifurcations in the asymptotic method based on multitiming and amplitude expansion a mixing of orders was needed, as we saw. That is, if you want a nondegenerate amplitude equation, you can expect to have an $\varepsilon$ in it, whether you get this by reconstitution, as in the original course, or insinuate it in by some iteration scheme as Howard and Knobloch did i n their lectures. You can also be conservative, accept the degenerate amplitude equation, and use higher order information to select a solution as Reiss e t al. do at Northwestern. These asymptotic methods work alright, but they do not seem to have the power of what we call the direct method to reveal clearly what is really going on.

In the direct method, one reduces the problem to one of ordinary differential equations at the outset by an expansion in normal modes- As Guckenheimer described in his lectures, we can reduce the order by going onto a center manifold. Then we can use coordinate transformations to simplify the nonlinear terms and get the normal form. However, this direct route is, to 
our taste, too wedded to the modal expansion. If you are doing a convection problem which is nonBoussinesq one has nonstandard boundary conditions, the modes are not so simple and the expansions are unwieldy, as one of us has had the misfortune to learn. Of course, when such messy things occur every scheme is in some trouble, but the direct method encounters it at once and in a way that is hard to deal with.

In short, there are things we like and things we do not like in both approaches and that is the tale we want to tell of these two methods. We have looked for a happy ending and want to try this one out on you. We have decided to take what we like and discard what we dislike from each approach and make a hearty hybrid. Or if you prefer, the ending is that two methods marry and produce a new scheme. We shallexplain the new scheme by working out the Hopf bifurcation for thermohaline convection.

\section{FORMULATION OF A TRACTABLE RROBLEM}

The rmohaline convection in Boussinesq approximation in two dimensions is governed by these equations:

$$
\begin{aligned}
& \partial_{t} \nabla^{2} \Psi=\sigma \nabla^{4} \Psi-\sigma R \partial_{\times} \Theta+\sigma \zeta S \Phi_{x}+J\left(\Psi, \nabla^{x} \Psi\right) \\
& \partial_{t} \Theta=-\partial_{x} \Psi+\nabla^{2} \Theta+J(\Psi, \Theta) \\
& \partial_{t} \Phi=\partial_{x} \Psi+\sigma \nabla^{2} \Phi+J(\Psi, \Phi)
\end{aligned}
$$

Here(1) and $\Phi$ are the deviations of the temperature and salinity from their static values, $x$ is the horizontal coordinate, $z$ is the vertical coordinate, $\nabla^{2}$ is the Laplacian and

$$
J(\Psi, x)=\partial_{x} \Psi \partial_{z} \chi-\partial_{z} \Psi \partial_{k} \chi
$$

The four parameters, R, S, $\sigma$ and $\tau$ that appear in the equations are respectively the Rayleigh number, the salt Rayleigh number, the Prandt 1 number and the Lewis number; $R$ is the usual $R_{T}$ and $S$ is $R_{s} / \tau$.

le t

$$
M=\left(\begin{array}{ccc}
\sigma \nabla^{4} & -\sigma R \partial x & \sigma \tau S \partial_{x} \\
-\partial_{x} & \nabla^{2} & 0 \\
\partial_{x} & 0 & \sigma \nabla^{2}
\end{array}\right)
$$

and

$$
\mathscr{L}=\left(\begin{array}{ccc}
\nabla^{2} & 0 & 0 \\
0 & 1 & 0 \\
0 & 0 & 1
\end{array}\right)
$$

The governing equations may be written as

$$
\partial_{t} \mathscr{L} u=\mathcal{L} u+\mathcal{V}(u, u)
$$


where

$$
u=\left(\begin{array}{c}
\Psi \\
\Theta \\
\Phi
\end{array}\right)
$$

and

$$
\mathcal{N}(F, G)=\left(\mathscr{L} \partial_{z} F\right)\left(E^{+} \partial_{x} G\right)-\left(\mathscr{L} \partial_{x} F\right)\left(E^{+} \partial_{z} G\right)
$$

with

$$
E=\left(\begin{array}{l}
1 \\
0 \\
0
\end{array}\right) \quad, E^{+}=(1,0,0)
$$

To these equations we associate some simple boundary conditions:

$$
\begin{aligned}
& \Psi=0, \nabla^{2} \Psi=0 \text { on } z=0,1 \text { and } x=0,2 \pi / a \\
& (A)=0, \Phi=0 \quad z=0,1 \\
& \partial x \Theta=0, \quad \partial_{x} \Phi=0 \text { on } x=0,2 \pi / a
\end{aligned}
$$

\section{STABILITY THEORY}

The linear equation is

$$
\partial_{t} \not u=\mathcal{M} u
$$

and it has solutions

$$
u=\left(\begin{array}{cc}
u_{\text {mn }} \operatorname{sinmax} & \sin n \pi z \\
v_{\text {mn }} \operatorname{cosmax} & \sin a \pi z \\
v_{\text {mn }} \operatorname{cosmax} & \sin a \pi z
\end{array}\right) e^{\eta^{t}}
$$

This kind of formula recurs frequently and we introduce a shorthand notation to deal with it.

Let

and

$$
\underline{I}_{m n}=\left(\begin{array}{ll}
\operatorname{sinmax} & \text { sin } n \pi z \\
\operatorname{cosmax} & \text { sin } n \pi z \\
\operatorname{cosmax} & \text { sin } n \pi z
\end{array}\right)
$$

$$
U_{m n}=\left(\begin{array}{c}
u_{m n} \\
v_{m n} \\
w_{m n}
\end{array}\right)
$$

Then we rewrite $\mathbf{( 3 . 2 )}$ as

$$
u=\tau_{m n} \star \Xi_{m n} e^{\eta t}
$$


Note that the operation signified by * is commutative, associated and distributive.

The linear problem for various modes may then be written as

$$
M_{m n} U_{m n}=\eta L_{m n} U_{m n}
$$

where no summation convection is used herein, and

$$
\begin{aligned}
& M_{m n}=\left(\begin{array}{ccc}
\sigma q_{m m}^{4} & \sigma R_{m a} & -\sigma S_{m a} \\
-m a & -q_{m a}^{2} & 0 \\
m a & 0 & -6 q_{m n}^{2}
\end{array}\right) \\
& L_{m n}=\left(\begin{array}{ccc}
-q_{m n}^{2} & 0 & 0 \\
0 & 1 & 0 \\
0 & 0 & 1
\end{array}\right)
\end{aligned}
$$

with

$$
q_{m n}^{2}=m^{2} a^{2}+n^{2} \pi^{2}
$$

In other words, we have the block diagonal representation

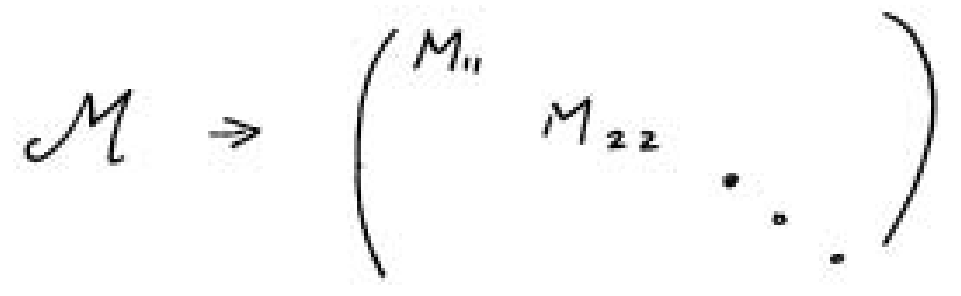

The condition that (3.1) have a solution is $\prod_{m n} \operatorname{det}\left(M-\eta L_{m \rightarrow}\right)=0$. and we are led to

$$
\begin{gathered}
\Pi_{m n}\left\{\eta^{3}+q_{m n}^{2}(\sigma+\sigma+1) \eta^{2}+\left[q_{m n}^{4}(\sigma+\sigma+\sigma \tau)-\sigma m^{2} a^{2} Q_{-1}\right] \eta\right. \\
\left.+m^{2} \sigma \tau a^{2}\left[\frac{8_{m a}^{6}}{m^{2} a^{2}}-Q_{0}\right]\right\}=0
\end{gathered}
$$

where

$$
Q_{m}=R+S / \sigma^{m}
$$


$-280-$

If (3.11) is satisfied, it must be true that

$$
\begin{gathered}
\eta^{3}+z^{2}(\bar{v}+\sigma+1) y^{2}+\left[q^{4}(\tau+\sigma+\sigma \tau)-\frac{\sigma a^{2}}{\bar{\sigma}^{2}} Q .\right] \eta y \\
+\sigma \sigma \alpha^{2}\left[\frac{g^{*}}{a^{2}}-Q_{0}\right]=0
\end{gathered}
$$

where

$$
\mathrm{q}^{2}=\mathrm{q}_{11}^{2}
$$

Of course, other roots of (3.11) may exist besides those in (3.13), but we now restrict our attention to values of $R$ and $S$ and a such that (3.11) is fulfilled only for $m=1, n=1$, and for one value of a.

When, for real w, we have

$$
\eta=i \omega
$$

we have marginal stability. Then (3.13) gives the well known results of Ven ranis:

$$
\begin{gathered}
\sigma(\sigma+1) R+\sigma(\sigma+\sigma) \tau S=(\sigma+\tau)(\sigma+1)(\sigma+1) R_{0} \\
\omega^{2}=\sigma \tau a^{2}\left(R_{0}-R_{0}\right) /(\sigma+\tau+1) q^{2} \\
R_{0}=\sigma^{6} / a^{2}
\end{gathered}
$$

Condition (3.16) tells us when overstability starts, provided that $\omega^{2}>0$. We want to study the amplitude equation for the time periodic solution that bifurcates at that point, with $\omega^{2}>0$. When (3.16) is satisfied (3.13) becomes

$$
\left(y^{2}+\omega^{2}\right)\left(\eta+\sigma^{2}(\sigma+\sigma+1)\right)=0
$$

If we move slightly of condition (3.1) and divide (3.13) by $\left[y+\boldsymbol{g}^{2}(\sigma+2+1)\right]$ we get, on assuming that $|y| \ll \xi^{2}(\tau+\sigma+1)$, the quadratic

$$
\eta^{2}-\lambda \eta+\omega^{2}=0
$$

where

$$
\lambda=\frac{a^{2}}{(\sigma+\varepsilon+1) q^{4}}\left[\sigma(\sigma+1) R+\sigma(\sigma+\sigma) \sigma S-(\sigma+\sigma)(\sigma+1)(\tau+1) R_{0}\right]
$$


We see that $\boldsymbol{\lambda}$ is very nearly the growth rate of the near-marginal modes. The definitions ( 3.17 ) and (3.18) continue to hold, hence $\eta \equiv \boldsymbol{i} \pm \mathbf{i}$ approximates the solutions of (3.20).

So much for eigenvalues. The eigenvectors of the marginal modes satisfy

$$
M \varphi=i \omega<\varphi
$$

where

$$
M=M_{u} \quad, \quad L=L_{1}
$$

We find

$$
\phi=\left(\begin{array}{cc}
1 \\
-a \frac{\left(q^{2}-i \omega\right)}{q^{4}+\omega^{2}} \\
a \frac{\left(\tau q^{2}-i \omega\right)}{\tau^{2} q^{4}+\omega^{2}}
\end{array}\right)
$$

where the normalization constant is taken so as to make the first component unity.

It is also useful in getting amplitude equations to find the solution of the adjoint equation

$$
M^{+} \phi^{+}=i \omega L^{+} \phi^{+}
$$

where the adjoints of the matrix representations of the operators are simply the transposed matrices. We find readily that

$$
\phi^{+}=\left(\begin{array}{cc}
1 \\
\sigma \operatorname{Ra}\left(\frac{q^{2}-i \omega}{q^{4}+\omega^{2}}\right) \\
-\sigma \sigma S a\left(\frac{\sigma q^{2}-i \omega}{\sigma^{2} q^{4}+\omega^{2}}\right)
\end{array}\right)
$$

Thus

$$
\phi^{t}=\left(\begin{array}{c}
\sigma^{\prime} z \\
-\sigma \lambda s
\end{array}\right) \otimes \phi
$$




\section{THE AMPLITUDE EQUATION}

We come now to the moral of this tale - a method for determining the amplitude equation. We have seen that the eigenvector of linear theory is complex, so we anticipate that the amplitude, call it $\mathbf{Z}(\mathbf{t})$, will also be complex. We also expect that 2 will satisfy an ordinary differential equation. We assume that this equation will, for small amplitude, give us back the original linear theory. Therefore we suppose that

$$
\dot{z}=(i \omega+\lambda) z+g(z, \bar{z})
$$

where we have to determine the nonlinear function $g . \quad(z$ is the complex conjugate of $Z$ and it satisfies (4.1).)

The basic idea is that a 11 the time dependence of $u$ is given by $z$ and $\bar{z}$, at least near the Hopf bifurcation. Therefore, we make the Ansatz

$$
u(t, x)=V(z, \bar{z}, x)
$$

Our task is to determine $\checkmark$ and $g$ jointly. As we shall see, since $\boldsymbol{Z}$ has replaced the time, we shall get derivatives with respect to $\boldsymbol{Z}$ that are the analogs of slow-time derivatives in asymptotic theory. This comes about because of (4.2) which is reminiscent of the Sogoliuhov and Mitropoloky approach to nonlinear oscillations. Howard, in his lecture, suggested how asymptotics might go if we used similar kinds of gambits to derive p.d.e.'s.

Here we do no asymptotics and immediately start acting like normal formalists. We introduce Taylor series. We let

$$
\begin{aligned}
V & =\sum_{k=1}^{\infty} V_{k} \\
V_{k} & =\sum_{i+j=k} V_{k}^{i j} z^{\prime} \bar{z}^{J}
\end{aligned}
$$

And we proceed similarly for $\boldsymbol{g}$. However, for purposes of presentation we shall simplify matters in two ways. First we shall compute $\mathbf{3}$ at marginal stability and ignore the small correction to it that arises when we move slightly off the marginal conditions. Second we shall admit that we know the normal form for a Hopf bifurcation and do not need to recompute it here. Then at marginal stability (4.1) is rewritten as

$$
\dot{z}=i \omega z+\alpha /\left.z\right|^{2} z
$$

and the calculation of $\alpha$ is going to provide an illustration of our method. 
We put (4.2) and (4.4) into (2.7):

$$
\begin{aligned}
& {\left[i \omega\left(z \frac{\partial}{\partial z}-\bar{z} \frac{\partial}{\partial z}\right) L-M\right] V } \\
&= \mathcal{N}(V, V) \\
& \quad-\mid z^{2} /\left(\alpha z \frac{\partial}{\partial z}+\bar{\alpha} \bar{z} \frac{\partial}{\partial z}\right) b V
\end{aligned}
$$

Now we use (4.3a) and we obtain at once

$$
\left[i \omega\left(Z \frac{\partial}{\partial z}-\bar{z} \frac{\partial}{\partial \bar{z}}\right) \not L-M\right] V_{1}=0
$$

But from $(4.3 b)$ we see that

$$
V_{1}=V_{1}^{\prime 0} Z+V^{\circ} \bar{z}
$$

and we get

$$
\begin{aligned}
& M V_{1}^{10}=i \omega L V_{1}^{\prime 0} \\
& M V_{1}^{\circ}=-i \omega V^{\circ} V_{1}^{\circ}
\end{aligned}
$$

Then

and

$$
V_{1}^{10}=\varphi \otimes=
$$

where

$$
V_{1}^{01}=\bar{\varphi} \times \overline{=}
$$

$$
\overline{-} \equiv \overline{-}_{\| !}
$$

If we want to change the normalization of $\varphi$, that factor will show up in There are several such "gauge" choices in this kind of calculation, but we shall not discuss them; it seems preferable to keep things simple in describing the essential parts of the method. This being said, we are done with the first degree.

Next, we look a t

$\left[i \omega\left(z \frac{\partial}{\partial z}-\bar{z} \frac{\partial}{\partial z}\right) L-M\right] V_{2}=M\left(V_{1}, V_{1}\right)$ 
We find

$$
\begin{gathered}
N\left(V_{1}, V_{1}\right)=z^{2} \mathcal{N}\left(V_{1}^{10}, V_{1}^{10}\right) \\
+z \bar{z}\left[N\left(V_{1}^{10}, V_{1}^{01}\right)+N\left(V_{1}^{01}, V_{1}^{10}\right)\right. \\
+\bar{z}^{2} \mathcal{N}\left(V_{1}^{01}, V_{1}^{01}\right)
\end{gathered}
$$

With the substitution

$$
V_{2}=V_{2}^{20} z^{2}+V_{2}^{\prime \prime} z \bar{z}+V_{2}^{02} \bar{z}^{2}
$$

we get

$$
\begin{aligned}
(2 i \omega L-M) V_{2}^{20} & =N\left(V_{i}^{\prime 0}, V_{i}^{\prime 0}\right) \\
-M V_{2}^{\prime \prime} & =N\left(V_{1}^{01}, V_{1}^{\prime 0}\right)+\mathcal{N}\left(V_{1}^{00}, V_{1}^{0 \prime}\right) \\
(-2 i \omega t-M) V_{2}^{02} & =N\left(V_{1}^{0,}, V_{i}^{0 \prime}\right)
\end{aligned}
$$

There are no resonances in (4.14) and they can be solved. We defer such details to the next section and turn to the third degree.

For $\sqrt{3}$ we get

$$
\begin{aligned}
& {\left[i \omega\left(z \partial_{z}-\bar{z} \partial_{\bar{z}}\right) L_{-}-\mu\right] V_{3}=\tilde{\mathcal{N}}\left(V_{1}, V_{2}\right)} \\
& -\alpha /\left.z\right|^{2} z \partial_{z} \not V_{1}-\bar{\alpha} /\left.z\right|^{2} \bar{z} \partial \bar{z} \mathscr{L} V_{1}
\end{aligned}
$$

where

$$
\tilde{N}(A, B)=\mathcal{N}(A, B)+\mathcal{N}(B, A)
$$

Now when we expand this out we observe that it can be written in the form

$$
\tilde{N}\left(\sqrt{1}, v_{2}\right)=\sum_{i+j=3} \mathcal{W}_{3}^{i j} Z^{i} \bar{Z}^{j}
$$

Moreover, we have

$$
\begin{aligned}
V_{3}= & V_{3}^{10} z^{3}+ \\
& V_{3}^{21} / \bar{z}^{2} / z+V_{3}^{\prime 2} /\left.z\right|^{2} \bar{z} \\
& +V_{3}^{03} \bar{z}^{3}
\end{aligned}
$$

Equation (4.15) gives us equation for the $\sqrt{3}^{i j}$;

$$
(3 i \omega L-M) \sqrt{3}^{10}=N_{3}^{30}
$$




$$
\begin{aligned}
& (i \omega L-M) V_{3}^{21}=\mathcal{V}_{3}^{21}-\alpha \mathcal{L} V_{1}^{\prime 0} \\
& (-i \omega L-M) V_{3}^{\prime 2}=N_{3}^{12}-\bar{\alpha} V_{1}^{0} \\
& (-3 i \omega-M) V_{3}^{03}=N_{3}^{03}
\end{aligned}
$$

Now it happens that there are no resonances in (4.19a) and (4.191) and they may be solved. Of course, we knew this when we introduced such a simple forrn of $g$ into (4.1). Had we not allowed this knowledge to be sportsmanlike, we would have introduced instead a full Taylor series like (4.3) for $g$ as well. Then (4.19a) and (4.19d) would have contained terms from 9 . We would have determined them as we now shall calculate $\boldsymbol{\alpha}$. But those particular terms in 3 do vanish and we have decided not to include them in this presentation. It is a feature of this approach that if you know the normal form, you save some effort, but if you do not happen to know it, you will find it out, along with the center manifold, automatically.

The point now is that we need to determine $\alpha$ so that (4.19b) and (4.19c) are soluble. They are just inhomogeneous versions of (4.8a) and (4.8b). The adjoint to (4.8a) is

$$
M^{+} w^{t}=i \omega \mathcal{L}^{t} W^{t}
$$

But $\boldsymbol{W}^{\top}$ is orthogonal to the first member of $(4.19 \mathrm{~b})$, with respect to the usual scalar product in Hilbert space. Our problem of solvability is resolved by the simple condition

$$
\alpha=\frac{\left\langle\mathcal{N}^{21}, W^{+}\right\rangle}{\left\langle\mathcal{L}^{10}, W^{+}\right\rangle}
$$

Then $(4.19 \mathrm{c})$ is also dealt with; one need only observe that

$$
\bar{N}_{3}^{21}=N_{3}^{12}
$$

and we find that if $\sqrt{3}^{2 \prime}$ solves $(4 \cdot 19 b)$, so, too, does

s a tisfy $(4 \cdot 19 \mathrm{c})$.

$$
\sqrt{2}_{2}^{12}=\bar{\nabla}_{3}^{21}
$$

This determines the solution in principle up to second degree. The practice remains to be discussed and we kept it somewhat separate since there are various ways to deal with it. Let us perform one now. 


\section{DETAILS OF THE CALCULATION}

The evaluation of the nonlinear terms that arose in IV was bypassed there. In fact, those evaluations arise one way or another in any method we have used to get amplitude equations. We begin with a useful formula for all those evaluations.

Let

$$
\begin{aligned}
& \Gamma_{m n}=\left(\begin{array}{l}
F_{\text {man }} \\
C_{i \operatorname{man}} \\
H_{\text {man }}
\end{array}\right) \otimes-m n \\
& \gamma_{m n}=\left(\begin{array}{l}
f_{m+1} \\
g_{m+1} \\
h_{m+1}
\end{array}\right) \&=-
\end{aligned}
$$

Then

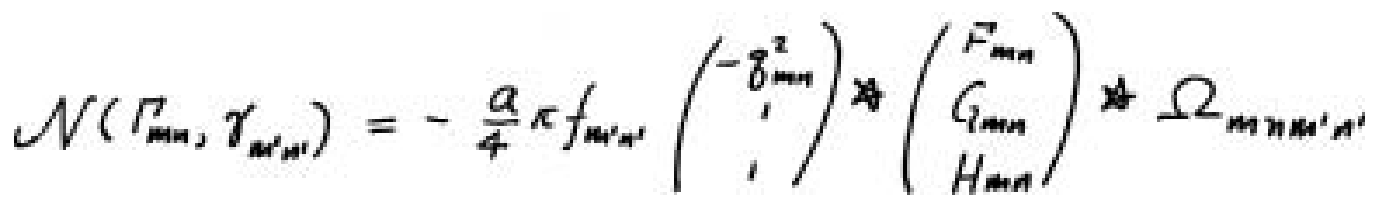

whe re

$$
\begin{aligned}
\Omega_{m n m^{\prime} n^{\prime}}= & \left(m n^{\prime}-m^{\prime} n\right)\left(\bar{\Xi}_{m+m ; n+n^{\prime}}-\bar{\Xi}_{m-n^{\prime}, n-n^{\prime}}\right) \\
& +\left(m n^{\prime}+m^{\prime} x^{\prime}\right)\left(\bar{\Xi}_{m+m^{\prime}, n-n^{\prime}}-\overline{ }_{m-m^{\prime}, n+n^{\prime}}\right)
\end{aligned}
$$

Now we return to $\boldsymbol{\xi}$ IV and we see that up to (4.12) everything is known explicitly. He begin there with our renewed effort and we use (5.2). After observing that $\Xi_{n o}=0$, we get

$$
\begin{aligned}
& \mathcal{N}\left(V_{1}^{10}, V_{1}^{10}\right)=\frac{1}{2} \pi a\left(\begin{array}{c}
-\xi^{2} \\
1 \\
1
\end{array}\right) * \phi * \equiv_{02} \\
& W\left(V_{1,}^{01}, V_{1}^{01}\right)=\frac{1}{2} \pi a\left(\begin{array}{c}
-8^{2} \\
1 \\
1
\end{array}\right) * \bar{\varnothing} * \bar{\Xi}_{02}
\end{aligned}
$$


$-287-$

Then we seek solutions of (4.1a) like

$$
V_{2}^{i}=\hat{V}_{2}^{i j} \star \bar{z}_{02}
$$

and, on doing this, we replace (4.14) by

$$
\begin{aligned}
\left(2 i \omega L_{02}-M_{02}\right) \hat{V}_{2}^{20} & =\frac{1}{2} \pi a L_{\| \varphi} \\
-M_{02} \hat{V}_{2}^{\prime \prime} & =\pi a L_{\|}\left(\mathcal{R}_{e} \varphi\right) \\
\left(-2 i \omega-M_{02}\right) \hat{V}_{2}^{2} & =\frac{1}{2} \pi a L_{11} \bar{\varphi}
\end{aligned}
$$

$M_{02}$ is defined in (3.7) and it is diagonal. We have

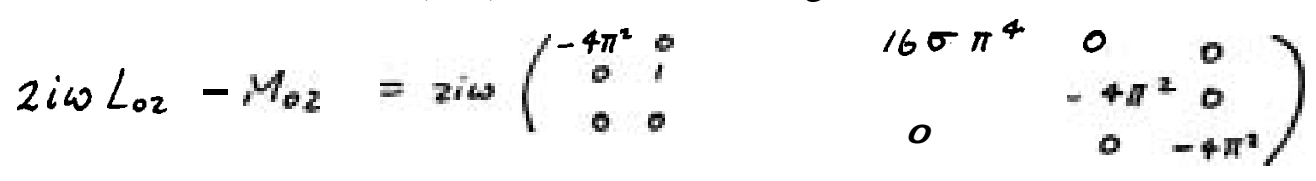

$$
=\left(\begin{array}{ccc}
-\left(8 \pi^{2} \omega i+16 \sigma \pi^{4}\right) & 0 & 0 \\
0 & 2 i \omega+4 \pi^{2} & 0 \\
0 & 0 & 2 i \omega+4 \pi^{2} \tau
\end{array}\right)
$$

and

$$
\frac{1}{2} \pi a L_{n} \varphi=\frac{1}{2} \pi a\left(\begin{array}{c}
-q^{2}\left(8^{2}-i \omega\right) /\left(q^{4}+\omega^{2}\right) \\
-a \\
a\left(\sigma q^{2}-i \omega\right) /\left(\sigma^{2} q^{4}+\omega^{2}\right)
\end{array}\right)
$$

Te find

$$
\hat{V}_{2}^{20}=\frac{\pi a}{4}\left(\begin{array}{c}
\frac{g^{2}}{\pi^{2}}\left(\frac{2 \pi^{2} \sigma-i \omega}{4 \pi^{4} \sigma^{2}+\omega^{2}}\right) \\
-\frac{a\left(8^{2}-i \omega\right)\left(2 \pi^{2}-i \omega\right)}{\left(8^{4}+\omega^{2}\right)\left(4 \pi^{4}+\omega^{2}\right)} \\
\frac{a\left(\tau \xi^{2}-i \omega\right)\left(2 \pi^{2} \tau-i \omega\right)}{\left(\tau^{2} \xi^{4}+\omega^{2}\right)\left(4 \pi^{4} \sigma^{2}+\omega^{2}\right)}
\end{array}\right)
$$


- 288 -

$$
\begin{aligned}
& \hat{V}_{2}^{20}=\frac{\pi a}{4}\left(\begin{array}{c}
\frac{g^{2}}{4 \pi^{2}}\left(\frac{2 \pi^{2} \sigma-i \omega}{4 \pi^{4} \sigma^{2}+\omega^{2}}\right) \\
-\frac{a\left(g^{2}-i \omega\right)\left(2 \pi^{2}-i \omega\right)}{\left(8^{4}+\omega^{2}\right)\left(4 \pi^{4}+\omega^{2}\right)} \\
\frac{a\left(\tau q^{2}-i \omega\right)\left(2 \pi^{2} \tau-i \omega\right)}{\left(\tau^{2} q^{4}+\omega^{2}\right)\left(4 \pi^{4} \tau^{2}+\omega^{2}\right)}
\end{array}\right) \\
& \hat{V}_{2}^{02}=\hat{V}_{2}^{20}
\end{aligned}
$$

Then

$$
V_{2}=\left(z^{2} \hat{V}_{2}^{20}+z \bar{z} \hat{V}_{2}^{\prime \prime}+\bar{z}^{2} V_{2}^{02}\right) * \overline{=}_{02}
$$

This implicitly is the center manifold to second degree, but it is in transformed coordintes compared to the standard expression.

Now we are poised to evaluate $\alpha$. Equation (4.21) indicates that we need to know that

$$
N_{3}^{21}=\tilde{N}\left(V_{1}^{\prime 0}, V_{2}^{\prime \prime}\right)+\tilde{N}\left(V_{1}^{01}, V_{2}^{20}\right)
$$

Since the first component of $\equiv_{02}=0$

$$
\begin{aligned}
& \tilde{\mathcal{V}}\left(V_{1}^{\prime 0}, V_{2}^{\prime \prime}\right)=\mathcal{N}\left(V_{2}^{\prime \prime}, V_{1}^{\prime 0}\right) \\
& =\pi a L_{u} \hat{V}_{2}^{\prime \prime} *\left(\equiv_{13}-\Xi_{1 "}\right) *\left(\begin{array}{l}
0 \\
i \\
1
\end{array}\right) \\
& =\left(\frac{\delta^{2} a^{3}}{4}\right)\left(\frac{0}{-\frac{1}{\delta^{2}+\omega^{2}}} \frac{1}{\delta \delta^{2}+\omega^{2}}\right) *\left(\equiv_{\prime \prime}-\equiv_{s}\right)
\end{aligned}
$$

$$
\tilde{W}\left(V_{1}^{0}, V_{2}^{20}\right)=\frac{\pi^{2} a^{3}}{4}\left(\begin{array}{c}
0 \\
-\frac{\left(8^{2}-i \omega\right)\left(2 \pi^{2}-i \omega\right)}{\left(8^{4}+\omega^{2}\right)\left(4 \pi^{4}+\omega^{2}\right)} \\
\frac{\left(\tau \delta^{2}-i \omega\right)\left(2 \pi^{2} z-i \omega\right)}{\left(\tau^{2} \delta^{4}+\omega^{2}\right)\left(4 \pi^{4} \tau^{2}+\omega^{2}\right)}
\end{array}\right) \times\left(\equiv_{\omega^{2}}-\equiv_{13}\right)
$$


Then

$$
N_{3}^{21}=\frac{a^{3}}{4}\left(\begin{array}{c}
0 \\
-\frac{8^{2}\left(4 \pi^{4}+\omega^{2}\right)+r^{2}\left(\delta^{2}-i \omega\right)\left(2 \pi^{2}-i \omega\right)}{\left(q^{4}+\omega^{2}\right)\left(4 \pi^{4}+\omega^{2}\right)} \\
\frac{q^{2}\left(4 \pi^{4} \tau^{2}+\omega^{2}\right)+\pi^{2}\left(\tau \delta^{2}-i \omega\right)\left(2 \pi^{2} \tau-i \omega\right)}{\left(\tau^{2} q^{4}+\omega^{2}\right)\left(4 \pi^{4} \tau^{2}+\omega^{2}\right)}
\end{array}\right)
$$

Now to apply (4.21) we need $\mathcal{W}^{+}$. We see from (4.20) that $\boldsymbol{W}^{+}$can only have modal components that are marginally stable, hence it must have the rodal

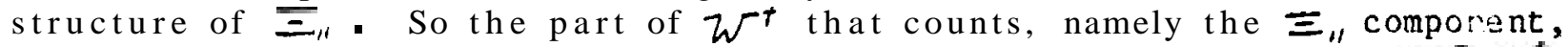
is proportional to $\phi^{+}$. Since the constant does not matter, we take $\boldsymbol{W}^{r}=\boldsymbol{\phi}^{\boldsymbol{t}}$.

$$
\begin{aligned}
\left\langle N_{3}^{21}, \phi^{+}\right\rangle= & =\frac{\sigma \sigma^{4}}{16}\left\{\frac{\left[\sigma \pi^{4} q^{2}+a^{2} \omega^{2}-\left(2 \pi^{2}+g^{2}\right) \omega^{2}\right] R\left(q^{2}-i \omega\right)}{\left(q^{4}+\omega^{2}\right)^{2}\left(4 \pi^{4}+\omega^{2}\right)}\right. \\
& \left.+\frac{\left[G \pi^{4} \tau^{2} q^{2}+a^{2} \omega^{2}-\left(2 \pi^{2}+q^{2}\right) \omega^{2}\right] \sigma s\left(q^{2}+i \omega\right)}{\left(\tau^{2} q^{4}+\omega^{2}\right)^{2}\left(4 \pi^{4} \sigma^{2}+\omega^{2}\right)}\right\}
\end{aligned}
$$

and

$$
\left\langle\mathcal{L} V_{1}^{10}, \phi^{t}\right\rangle=-\frac{1}{4}\left[q^{2}+\frac{\sigma R a^{2}(8-i \omega)}{\left(g^{4}+\omega^{2}\right)^{2}}+\frac{\sigma \tau \operatorname{Sa}^{2}\left(\tau_{8}-i \omega\right)^{2}}{\tau^{2} \varepsilon^{4}+\omega^{2}}\right]
$$

The ratio of the integrals given in (5.12) and (5.13) gives $\alpha$. That ratio, as far as can tell, has no socially redeeming features and so we feel it proper not to show it explicitly in public.

\section{CONCLUSION}

Is this a far, far better thing that we have done or will one or another asymptotic method do as well? The asymptotic methods give the same answers superficially, but the answers have different meanings. If you take the reconstituted thermohaline results in the original course and restore the original variables you perceive, with a bit of calculating, that the neglec:ed terms are of order unity i n asymptotic terms. If you do not restore the original variables you have mixed orders in the same equation. None of th: need trouble one, but we do not need to face those questions either. Here, the convergence (or lack of it) is not asymptotic but in the sense of powe 5 series. That is why the neglected terms look of order one in the asymptoti: version: They are of order one asymptotically, but they are higher order terms i n the present method nontheless. Just how negligible they really ar: needs to be discussed. But discusion is for sissies; the hero wants to compute. 
A significant question is this: Which is the best way to compute amplitude equations? The answer depends on the taste and experience' of the computer, and the fact is that our experience is limited. It appears that the differences among methods in the practical sense depend on the co-dimension. Our impression is that as the co-dimension goes up, the computational difficulty in the present scheme goes up less quickly than for, say, the method of reconstitution. They cross perhaps at co-dimension $21 / 2$ or so. But either way you compute, it is going to be necessary to automate these procedures.

Perhaps the most telling question is which scheme should you choose if your problem has a quadrivial linear problem? In that case, we think the way just outlined has some advantages. As we have seen, much of the formal manipulation can be done independently of the details and formulas for the explicit results could be given for a general form of the problem. Thus, even if the linear problem has to be solved numerically, we believe that the present scheme should be efficacious. An example that we find interesting is stellar pulsation theory.

The final question that we may raise is that of extensions. The immediate problem that comes to mind is the case where the amplitude equation is an evalution equation as i $n$ wave theory, that is, a p.d.e. Here the asymptotic method works, but we have not yet agreed on the generalization of the present method. This is not surprising. The use of normal formalism gives normal forms; if we could do that for p.d.e.'s we would be found to have something rather complex on our hands. Even the degenerate cases, integrable systems, are not yet classifiable.

A more manageable extension is the replacement of Taylor series by rational approximations. This has served well i n other contexts, but it is not an easy extension and we shall not be through with it soon.

In conclusion, the junior author thanks the senior author for allowing his name to appear on this "fellow's report"; both fellows thank their fellow participants for interest and encouragement; and the participants thank the N.S.F. for support of some individual research (in this case through Columbia University) as well as of the collective GFD program. 